\title{
Bodenschutz in der deutschen Landwirtschaft - Stand und Verbesserungsmöglichkeiten
}

\author{
Dissertation \\ zur Erlangung des Doktorgrades \\ der Fakultät für Agrarwissenschaften \\ der Georg-August-Universität Göttingen
}

\author{
vorgelegt von \\ Henning Brand-Sassen \\ geboren in Barßel
}

Göttingen, im Juli 2004 
D 7

1. Referentin/Referent: Prof. Dr. M. Köhne

2. Korreferentin/Korreferent: Prof. Dr. J. Isselstein

Tag der mündlichen Prüfung: 15. Juli 2004 


\section{Vorwort}

Die vorliegende Arbeit wurde am Institut für Agrarökonomie der Georg-AugustUniversität Göttingen angefertigt. Sie entstand in Zusammenarbeit mit dem Zentrum für Landwirtschaft und Umwelt der Universität Göttingen. Die Anfertigung der Arbeit und ihre Veröffentlichung wurde durch eine Vielzahl von Personen und Institutionen gefördert, denen ich an dieser Stelle herzlich danken möchte.

Für die Überlassung des Themas und die wissenschaftliche Betreuung bedanke ich mich bei Herrn Prof. Dr. M. Köhne. Seine konstruktiven Anregungen und Vorschläge waren für die Durchführung der Arbeit äußerst hilfreich und für die zügige Bearbeitung dieses Themas essentiell. Für die Übernahme des Korreferats danke ich Herrn Prof. Dr. J. Isselstein. Ein ganz besonderer Dank gilt den Kolleginnen und Kollegen des Instituts für Agrarökonomie. Ihre ständige Hilfsbereitschaft, der fachliche und private Gedankenaustausch sowie das äußerst angenehme Arbeitsklima haben einen großen Beitrag zum Gelingen dieser Arbeit geleistet. Herrn Dr. H.-H. Steinmann sowie den weiteren Mitarbeiterinnen und Mitarbeitern des Zentrums für Landwirtschaft und Umwelt danke ich für die konstruktive Unterstützung und Diskussionsbereitschaft in den jeweiligen Gesprächsrunden. Während der Erstellung der Arbeit war eine intensive Zusammenarbeit mit verschiedenen Personen und Institutionen unerlässlich, um der großen Vielfalt des Themenbereichs hinreichend gerecht zu werden. Bei diesen möchte ich mich an dieser Stelle, ohne die einzelnen Namen zu nennen, recht herzlich bedanken.

Abschließend gilt mein größter Dank meinen Eltern sowie der gesamten Familie und v. a. meiner Freundin Katja Steinebach für die unaufhörliche Unterstützung und den Rückhalt in jeder Phase dieser Arbeit. 



\section{Inhaltsverzeichnis}

$1 \quad$ Einleitung............................................................................... 1

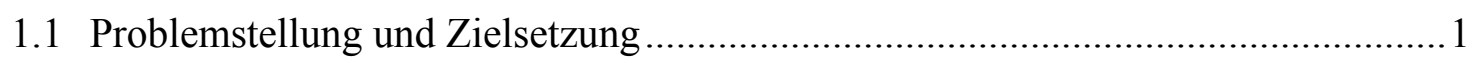

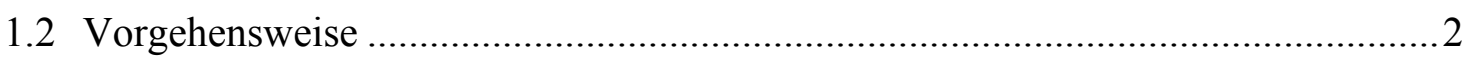

\section{Informationsbeschaffung und Datengrundlagen ................ 5}

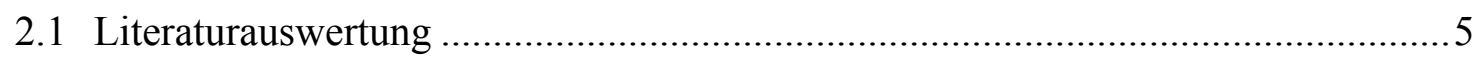

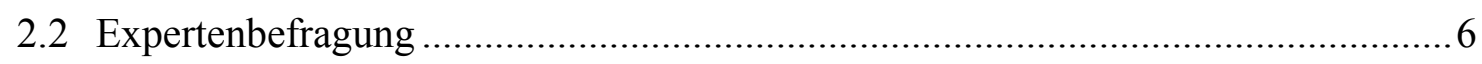

2.2.1 Wesen und Zielsetzung der Befragung ....................................................... 6

2.2.2 Ablauf und Durchführung der Befragung .................................................. 6

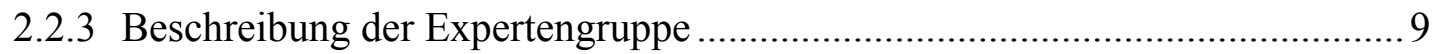

\section{Umweltwirkungen der Landwirtschaft auf den Boden .... 11}

3.1 Stand der Umweltwirkungen

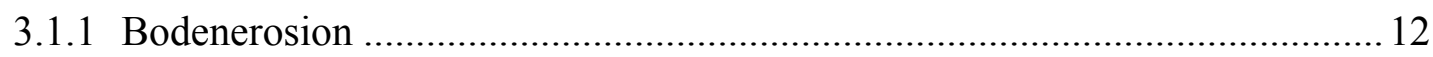

3.1.1.1 Wirkungen der Landwirtschaft auf die Bodenerosion ............................ 13

3.1.1.2 Die Abschätzung des Bodenabtrags mittels Bodenerosionsmodellen ...... 16

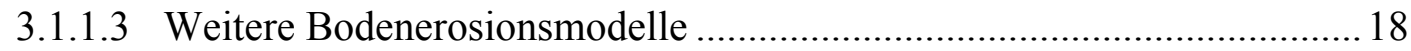

3.1.1.4 Umfang und Bedeutung der Bodenerosion durch Wasser in Deutsch-

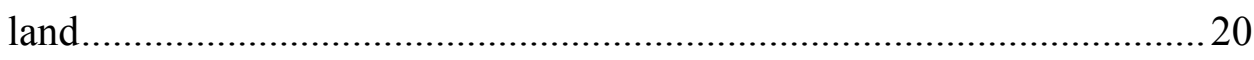

3.1.1.5 Einflussmöglichkeiten der Landwirtschaft auf die Bodenerosion ............22

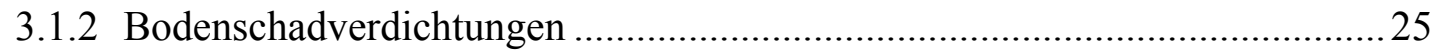

3.1.2.1 Wirkungen der Landwirtschaft auf die Bodenschadverdichtung..............27

3.1.2.2 Umfang und Bedeutung von Bodenschadverdichtungen in Deutschland. 32

3.1.2.3 Einflussmöglichkeiten der Landwirtschaft auf die Bodenschadver-

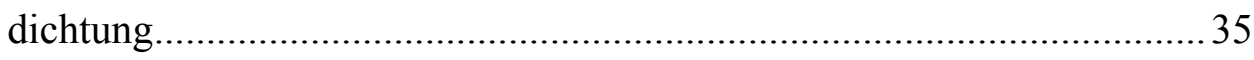

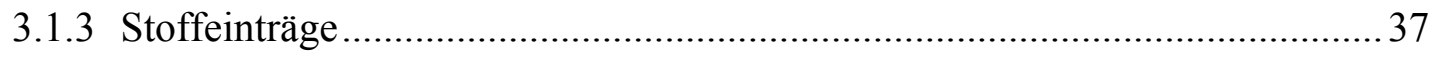

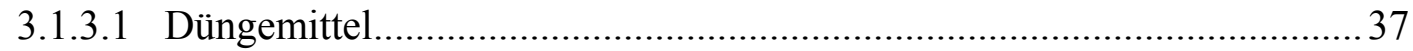

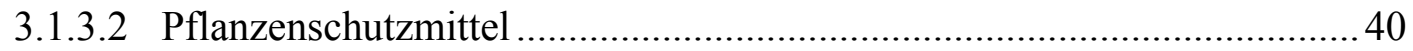

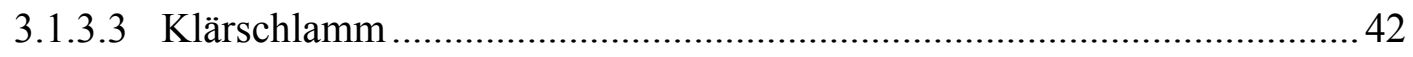

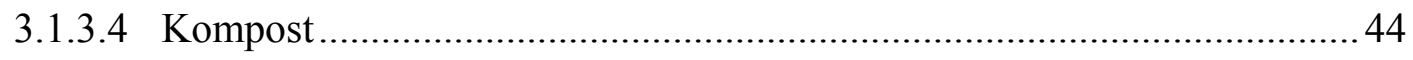

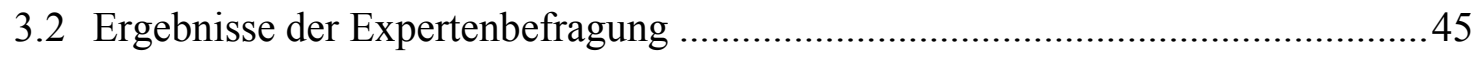




\section{Das Bodenschutzrecht unter dem Gesichtspunkt der landwirtschaftlichen Bodennutzung................................. 48}

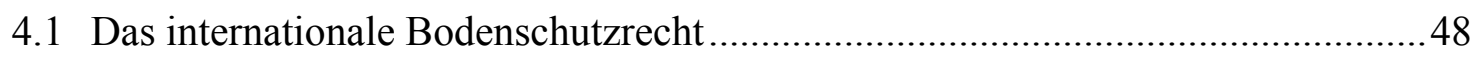

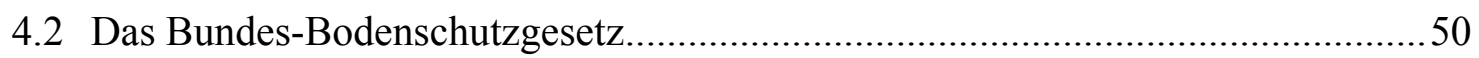

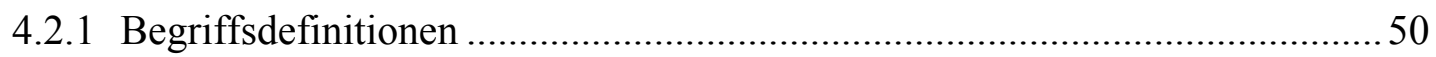

4.2.2 Entstehung des Bodenschutzrechts in Deutschland und Europa.....................52

4.2.3 Zweck und Grundsätze des Gesetzes .......................................................54

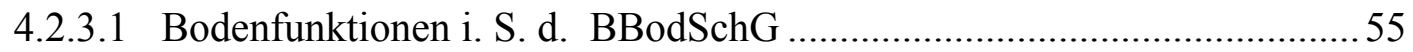

4.2.3.2 Schutz vor schädlichen Bodenveränderungen ........................................56

4.2.4 Anwendungsbereiche und Abgrenzung zu den Fachgesetzen in $\S 3$ Abs. 1

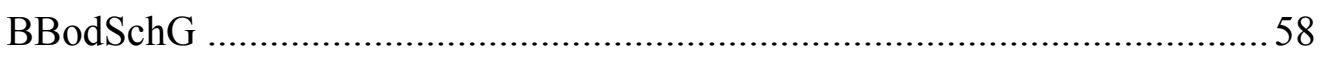

4.2.5 Landwirtschaftliche Bodennutzung im BBodSchG ...................................... 64

4.2.5.1 Die gute fachliche Praxis der landwirtschaftlichen Bodennutzung nach $\S 17$ BBodSchG .64

4.2.5.2 Die Grundsätze der guten fachlichen Praxis ......................................... 64

4.2.5.3 Die Grundsätze der guten fachlichen Praxis im Einzelnen....................... 66

4.2.5.4 Die Beratung nach $\S 17$ Abs. 1 Satz 2 BBodSchG ................................ 73

4.2.5.5 Vergleich des $\S 17$ BBodSchG mit entsprechenden Regelungen anderer

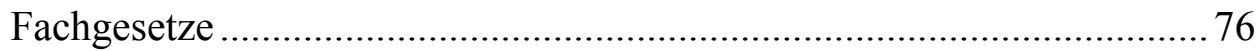

4.2.5.5.1 Vergleich mit dem Düngemittelrecht ............................................ 76

4.2.5.5.2 Vergleich mit dem Pflanzenschutzmittelrecht ................................ 78

4.2.5.5.3 Vergleich mit dem Naturschutzrecht.............................................. 79

4.2.6 Die Ausgleichsregelung nach $\S 10$ Abs. 2 BBodSchG ................................. 80

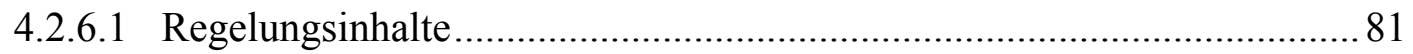

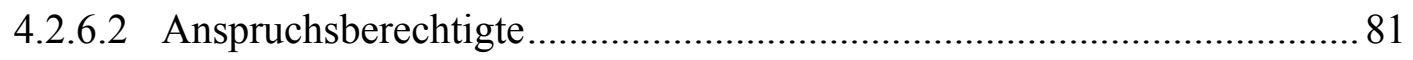

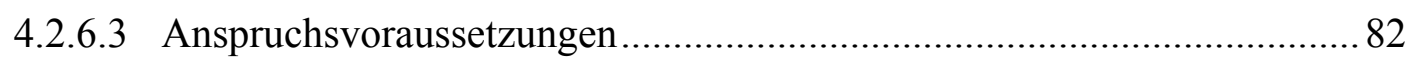

4.2.6.4 Zumutbare innerbetriebliche Anpassungsmaßnahmen ........................... 82

4.2.6.5 Der Begriff des angemessenen Ausgleichs ........................................... 83

4.2.6.6 Bedeutung des $\S 10$ Abs. 2 BBodSchG in der Praxis ............................... 83

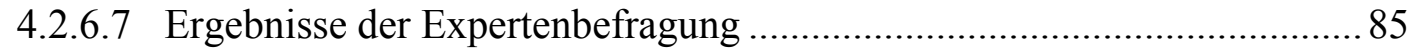

4.2.7 Die Ausweisung von Bodenschutzgebieten nach $\S 21$ Abs. 3 BBodSchG..... 85

4.3 Die Bundes-Bodenschutz- und Altlastenverordnung ....................................... 87 
4.3.1 Allgemeine Vorschriften und Anwendungsbereiche 87

4.3.2 Ergänzende Vorschriften für die Gefahrenabwehr von Bodenerosionen durch Wasser ( $\S 8,5$ BBodSchV). 88

4.4 Das Bodenschutzrecht in der rechtspolitischen Diskussion. .89

4.4.1 Stand der Diskussion um das deutsche Bodenschutzrecht. 89

4.4.2 Diskussion über fehlende Anordnungen und Grenzwerte im Bereich des physikalischen Bodenschutzes 92

4.4.3 Die Unterstützung des Bodenschutzrechts durch Cross Compliance 94

4.4.3.1 Grundsätzliche Vorüberlegungen .94

4.4.3.2 Umsetzung in der EU und in Deutschland. 95

4.4.3.3 Diskussion der Cross-Compliance und Konsequenzen für den Bodenschutz 96

4.4.4 Ergebnisse der Expertenbefragung 97

4.4.4.1 Zufriedenheit mit dem aktuellen Bodenschutzrecht und Umsetzung der Rechtsvorschriften in die landwirtschaftliche Praxis.

4.4.4.2 Bedeutung verschiedener gesetzlicher Regelwerke für den ldw.

Bodenschutz und Verbesserungsmöglichkeiten 99

4.4.4.3 Die Wirksamkeit der guten fachliche Praxis als Vorsorgeelement des Bodenschutzes

\section{Verbindungen zwischen Wasser- und Bodenschutz ....... 103}

5.1 Abgrenzung von Wasser- und Bodenschutz 103

5.2 Die EU-Wasserrahmenrichtlinie und der Gesetzentwurf zu einem Hochwasserschutzgesetz. 104

5.3 Landwirtschaft und Hochwasserschutz. 109

5.3.1 Zusammenhang zwischen Infiltration und Hochwasser ... 109

5.3.2 Einflüsse landwirtschaftlicher Maßnahmen auf den Hochwasserabfluss

5.3.3 Diskussion der Bedeutung der Landwirtschaft für einen vorbeugenden Hochwasserschutz

5.3.4 Umwandlung von Ackerland in Grünland 113

5.3.5 Ergebnisse der Expertenbefragung 116

5.4 Fazit und Folgerungen für diese Arbeit 


\section{Maßnahmen des Bodenschutzes in der Landwirtschaft und deren ökonomische Bewertung ...................................... 118}

6.1 Ergebnisse der Literaturauswertung über die ökonomischen Aspekte des

Bodenschutzes in der Landwirtschaft 118

6.1.1 Volkswirtschaftliche Aspekte des Bodenschutzes ....................................... 118

6.1.2 Betriebswirtschaftliche Aspekte des Bodenschutzes ................................. 122

6.2 Theoretische Grundlagen zur ökonomischen Bewertung und Vorgehensweise.. 126

6.2.1 Die Methodik der Kostenvergleichsrechnung........................................... 127

6.2.2 Die Methodik der Kosten-Wirksamkeits-Analyse ..................................... 128

6.2.3 Die Methodik der Kosten-Nutzen-Analyse................................................ 128

6.2.3.1 Kosten von Bodenschutzmaßnahmen ................................................... 130

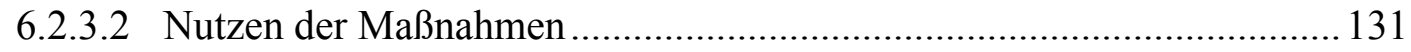

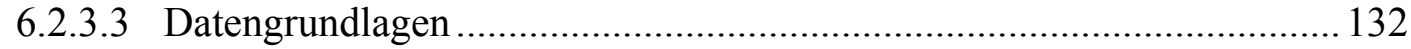

6.2.3.4 Weitere Annahmen .......................................................................... 132

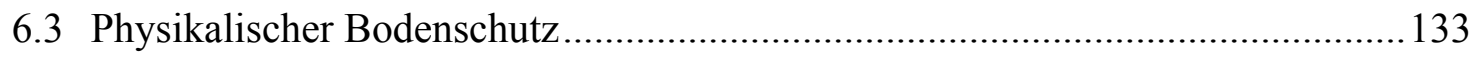

6.3.1 Maßnahmen zur Verhinderung von Bodenerosion .................................... 133

6.3.1.1 Bodenschutz durch konservierende Bodenbearbeitung ......................... 134

6.3.1.1.1 Verbreitung der konservierenden Bodenbearbeitung..................... 135

6.3.1.1.2 Umstellung auf konservierende Bodenbearbeitung ...................... 138

6.3.1.1.3 Betriebswirtschaftliche Bewertung der konservierenden

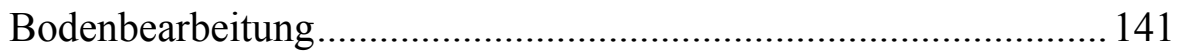

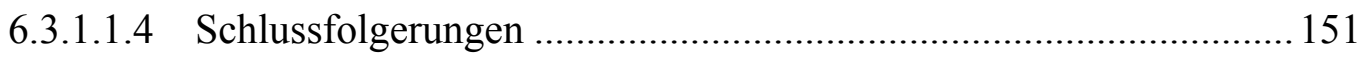

6.3.1.1.5 Vertiefung der Betrachtungsweise anhand des Göttinger INTEX-

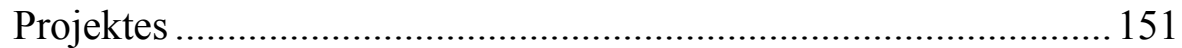

6.3.1.1.5.1 Darstellung des INTEX-Projektes ...................................... 152

6.3.1.1.5.2 Datengrundlage und Vorgehensweise zur betriebswirtschaftlichen Analyse des INTEX-Projektes ......... 153

6.3.1.1.5.3 Betriebswirtschaftliche Ergebnisse des INTEX-Projektes ...... 154

6.3.1.1.5.4 Diskussion der Ergebnisse .................................................... 161

6.3.1.2 Tiefenlockerung von Fahrspuren ...................................................... 161

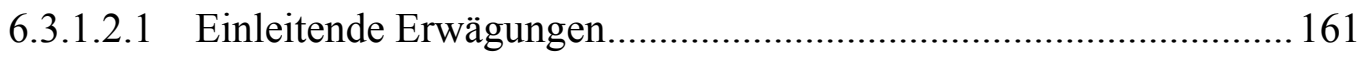

6.3.1.2.2 Betriebswirtschaftliche Analyse der Maßnahme............................ 163 


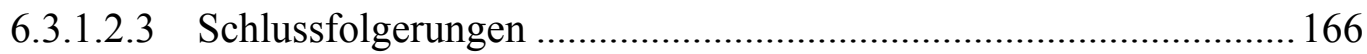

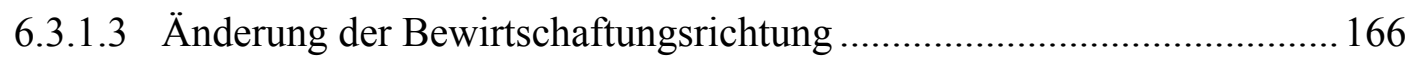

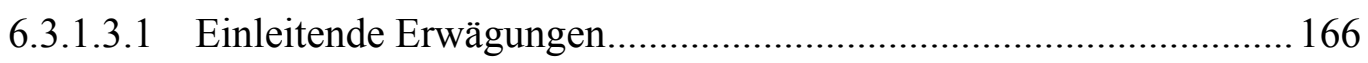

6.3.1.3.2 Betriebswirtschaftliche Analyse der Maßnahme............................ 169

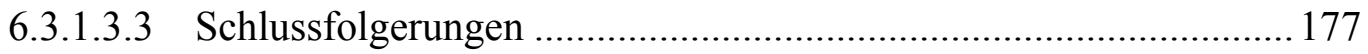

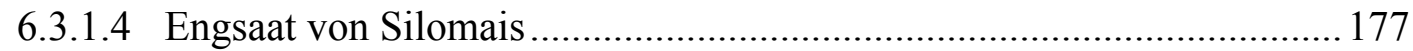

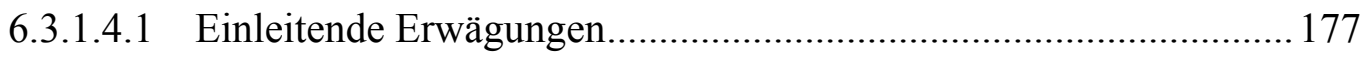

6.3.1.4.2 Betriebswirtschaftliche Analyse der Maßnahme............................ 178

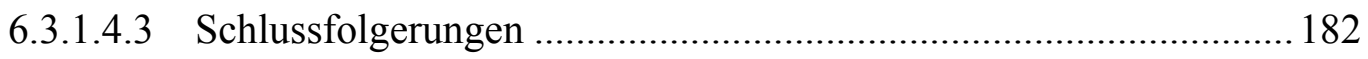

6.3.1.5 Anbau von Zwischenfrüchten und Untersaaten .................................. 183

6.3.1.5.1 Einleitende Erwägungen.......................................................... 183

6.3.1.5.2 Betriebswirtschaftliche Analyse der Maßnahme............................ 184

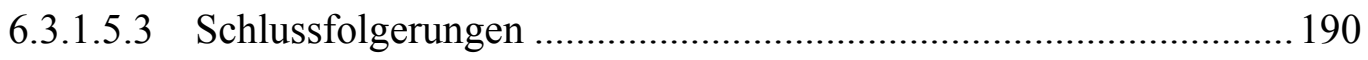

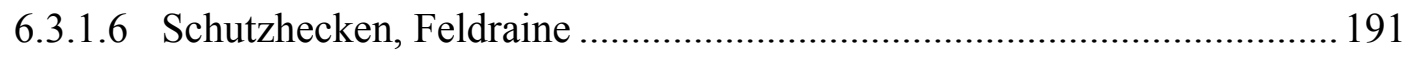

6.3.1.6.1 Einleitende Erwägungen.............................................................. 191

6.3.1.6.2 Betriebswirtschaftliche Analyse der Maßnahme........................... 192

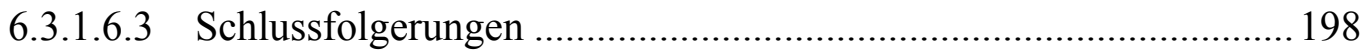

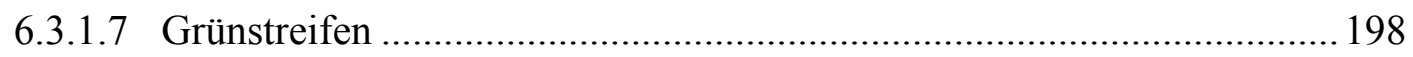

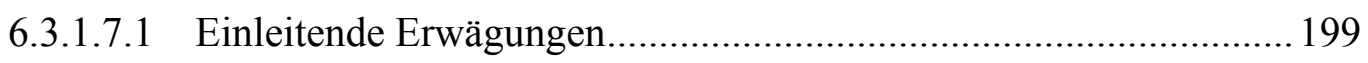

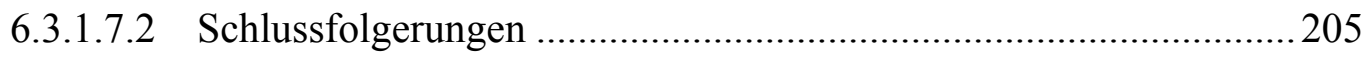

6.3.1.8 Verzicht auf den Anbau an Hanglagen .................................................205

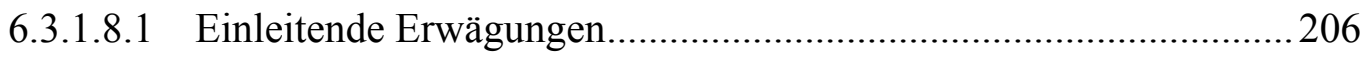

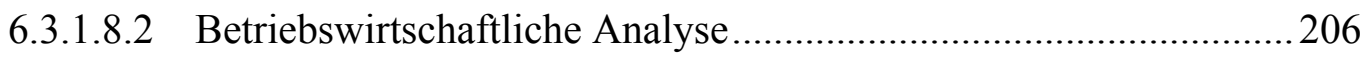

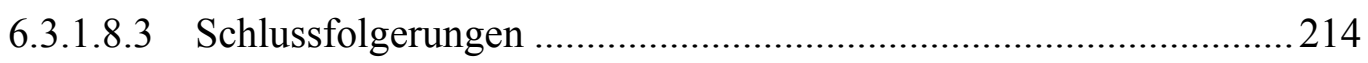

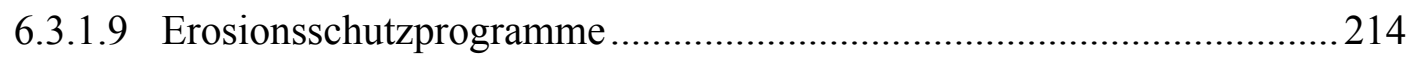

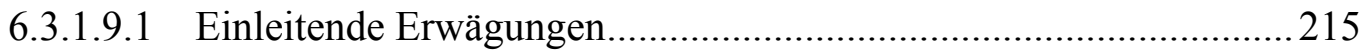

6.3.1.9.2 Betriebswirtschaftliche Analyse der Maßnahme...........................2 218

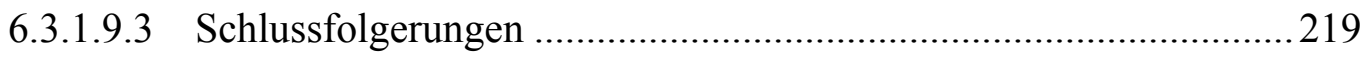

6.3.2 Maßnahmen zur Verhinderung von Bodenverdichtungen .......................... 220

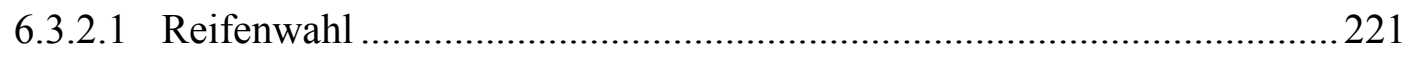

6.3.2.1.1 Einleitende Erwägungen...........................................................22

6.3.2.1.2 Betriebswirtschaftliche Analyse der Maßnahme............................ 222

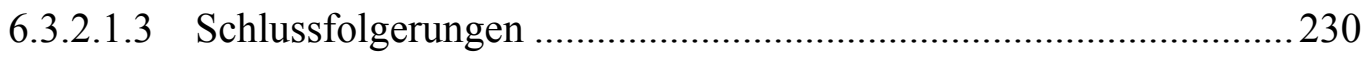

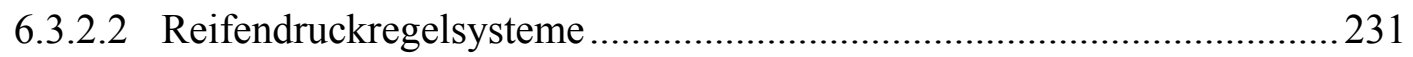




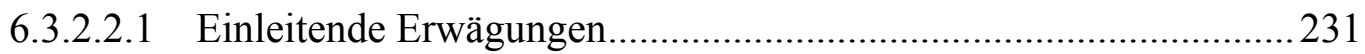

6.3.2.2.2 Betriebswirtschaftliche Analyse der Maßnahme........................... 232

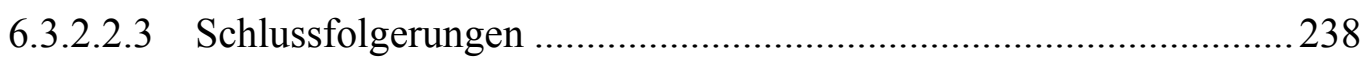

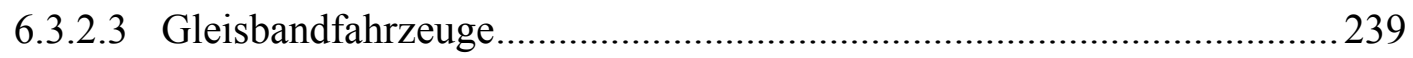

6.3.2.3.1 Einleitende Erwägungen...........................................................2239

6.3.2.3.2 Betriebswirtschaftliche Analyse der Maßnahme............................ 241

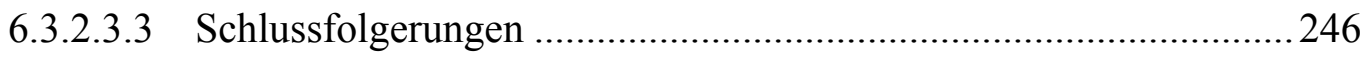

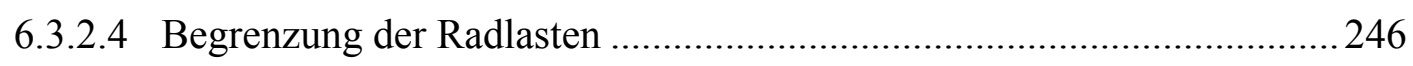

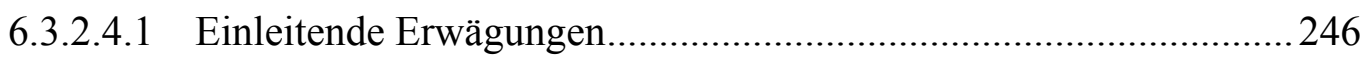

6.3.2.4.2 Betriebswirtschaftliche Analyse der Maßnahmen.........................247

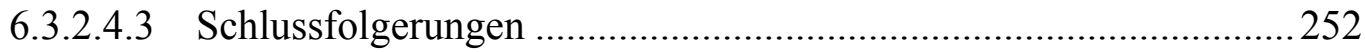

6.3.3 Weitere Maßnahmen zum physikalischen Schutz des Bodens ....................253

6.3.3.1 Stilllegung von schlaginternen Minderertragsteilflächen ...................... 253

6.3.3.1.1 Einleitende Erwägungen...........................................................253

6.3.3.1.2 Betriebswirtschaftliche Analyse der Maßnahme........................... 254

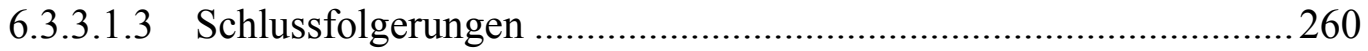

6.3.3.2 Maßnahmen zur Verminderung des Flächenverbrauchs und deren ökonomische Bewertung............................................................... 260

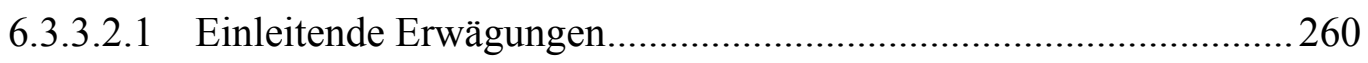

6.3.3.2.2 Betriebswirtschaftliche Aspekte der Maßnahme............................ 260

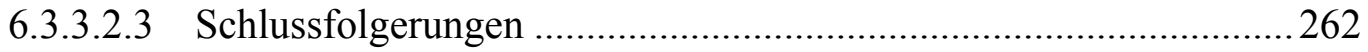

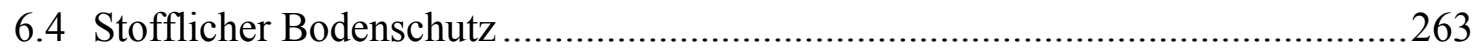

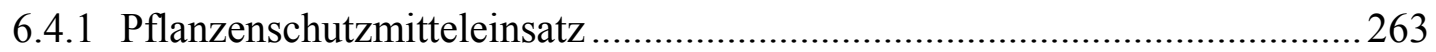

6.4.1.1 Reduzierung des Einsatzes von Pflanzenschutzmitteln ......................... 264

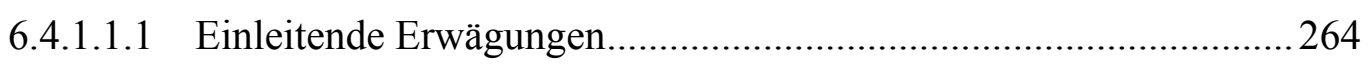

6.4.1.1.2 Betriebswirtschaftliche Analyse der Maßnahme............................266

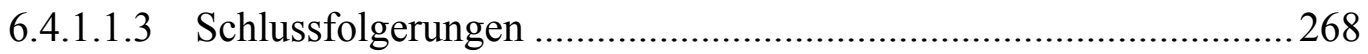

6.4.1.2 Mechanische Unkrautbekämpfung …..................................................2. 268

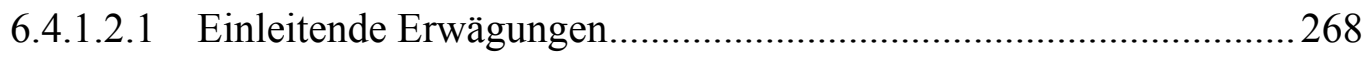

6.4.1.2.2 Betriebswirtschaftliche Analyse der Maßnahme............................269

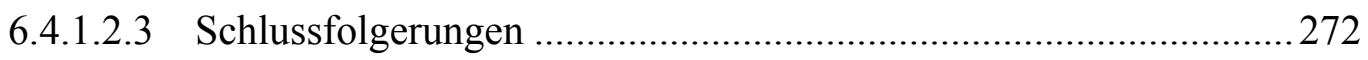

6.4.1.3 Verzicht auf Pflanzenschutzmitteleinsatz .......................................... 272

6.4.1.3.1 Einleitende Erwägungen.........................................................2273 
6.4.1.3.2 Betriebswirtschaftliche Analyse der Maßnahme...........................2 274

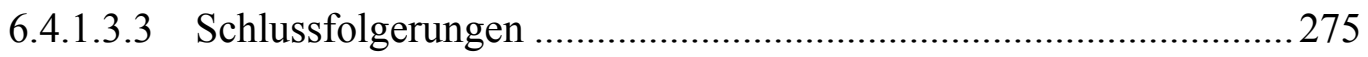

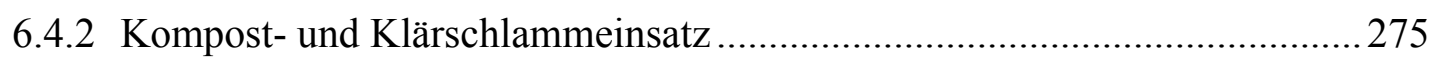

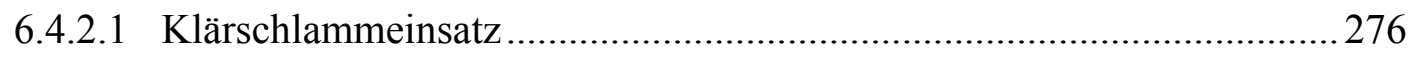

6.4.2.1.1 Einleitende Erwägungen...........................................................2 276

6.4.2.1.2 Betriebswirtschaftliche Analyse der Maßnahme...........................2276

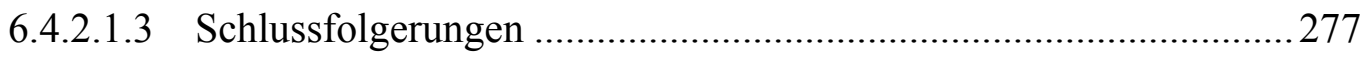

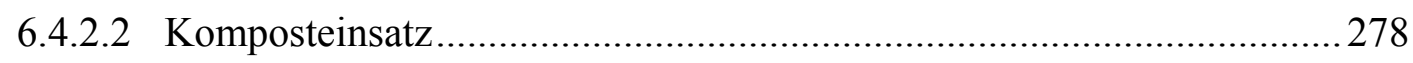

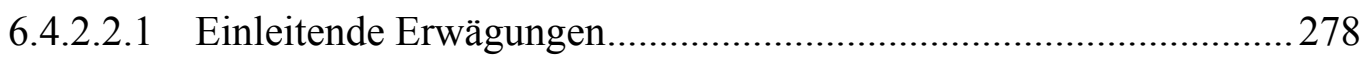

6.4.2.2.2 Betriebswirtschaftliche Analyse der Maßnahme...........................2278

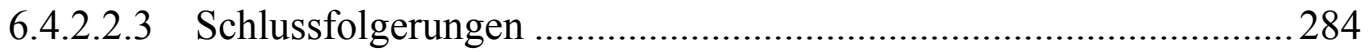

6.4.3 Zusammenfassung und Diskussion der Ergebnisse ................................... 284

6.4.3.1 Zusammenfassende Darstellung der Ergebnisse der Maßnahmen des physikalischen Bodenschutzes gegen Erosion...................................... 285

6.4.3.2 Zusammenfassende Darstellung der Ergebnisse der Maßnahmen des physikalischen Bodenschutzes zum Schutz gegen Bodenschadverdichtung.

6.4.3.3 Zusammenfassende Darstellung der Ergebnisse der Maßnahmen des stofflichen Bodenschutzes ............................................................. 290

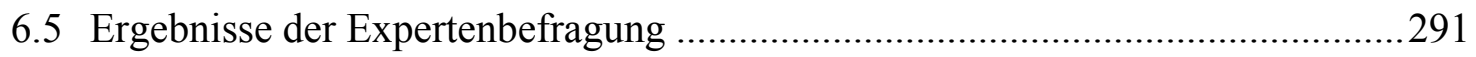

7 Schlussfolgerungen und Empfehlungen für Bodenschutzkonzepte landwirtschaftlicher Betriebe...... 294

7.1 Erweiterung von Bodenschutzkonzepte zum Schutz vor Bodenerosion 294

7.2 Erweiterung von Bodenschutzkonzepte zum Schutz vor Bodenschadverdichtungen

7.3 Weitere Empfehlungen für landwirtschaftliche Betriebe, Berater sowie Politik und Verwaltung .... 300

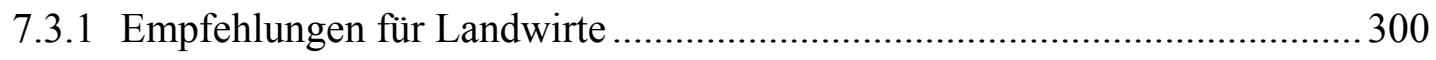

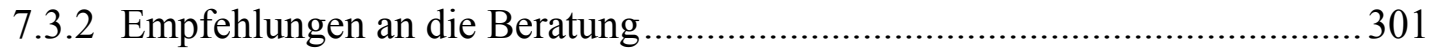

7.3.3 Empfehlungen an die Verwaltung und die Politik ...................................... 302

8 Zusammenfassung ........................................................ 304 
9 Literaturverzeichnis .......................................................... 308

10 Anhang..........................................................................I 


\section{Tabellenverzeichnis}

Tabelle 1: Einfluss der Bodenbedeckung auf den Abfluss und Bodenabtrag

(Relativwerte auf der Basis zehnjähriger Messungen)

Tabelle 2: Vergleich verschiedener Parameter nach konventioneller und konservierender

Bodenbearbeitung

Tabelle 3: Erosionsmindernde Maßnahmen und ihr Einfluss auf Wasser- und

Winderosion

Tabelle 4: Entwicklung der Flächenleistung und Radlasten von Maschinen und Geräten in der deutschen Landwirtschaft

Tabelle 5: Zu erwartende Ertragsausfälle durch Überschreiten der substratspezifischen Grenzwerte für die Lagerungsdichte

Tabelle 6: Gemessener Bodendruck unter drei verschiedenen Reifen bei einer Radlast

von $6,8 \mathrm{t}$ und verschiedenen Reifeninnendrücken

Tabelle 7: Durchschnittliche Schwermetallgehalte und prozentuale

Grenzwertausschöpfung der AbfKlärV von bundesdeutschem Klärschlamm (in $\mathrm{mg} / \mathrm{kg})$

Tabelle 8: Stärke der Auswirkungen von Landnutzungsmaßnahmen auf die

Abflussbildung und -konzentration bei konvektiven Starkniederschlägen (ohne

Forstwirtschaft)

Tabelle 9: Jährliche Kosten zur Behebung und Kompensation der

Bodenerosionsschäden (Wind- und Wassererosion) bei Getreide und Mais und einer durchschnittlichen Bodenabtragsrate von $17 \mathrm{t} / \mathrm{ha} / \mathrm{a}$ in den USA

Tabelle 10: Einfluss der Direktsaat (DS) sowie Mulchsaat (MS) auf den Pflanzenertrag im Vergleich zur Pflugbearbeitung $(\mathrm{P}=100 \%)$.

Tabelle 11: Prozentuale Veränderung des Deckungsbeitrages verschiedener

Fruchtartenfolgen bei der Umstellung auf Verfahren der Mulchsaat im

Pilotbetrieb

Tabelle 12: Kosten der Bodenbearbeitung im Falle der Pflugbearbeitung sowie Mulch-

u. Direktsaat auf Ebene der Verfahrenskosten.

Tabelle 13: Einfluss der Bodenbedeckung auf den Oberflächenabfluss sowie der

Bodenerosion durch Wasser und Wind (Relativwerte auf Basis 10-jähriger

Messungen) 
Tabelle 14: Berechnung des Substitutionswertes der Hauptnährstoffe in einer Tonne erodiertem Oberboden

Tabelle 15: Einzelbetriebliches KNV der Mulch- und Direktsaat im Verhältnis zur Pflugbearbeitung bei einem Zinssatz von $4 \%$ und mittleren Bodenabtragsraten ohne Förderung sowie Berücksichtigung externer Effekte

Tabelle 16: Spurflächensumme innerhalb einer Vegetationsperiode für verschiedene Früchte in $\%$

Tabelle 17: Verfahrenskosten der Tiefenlockerung von Fahrspuren

Tabelle 18: KW (€/t verhinderter Bodenabtrag) bei Tiefenlockerungsmaßnahmen von

Fahrspuren bei unterschiedlichen Bodenabträgen und Erfolgspotenzialen der Maßnahme.....

Tabelle 19: KW (€/t verhinderter Bodenabtrag) bei Tiefenlockerungsmaßnahmen von Fahrspuren bei Bodenabträgen von 4,6 t/ha und 8,7 t/ha und verschiedenen Erfolgspotenzialen der Maßnahme

Tabelle 20: Hangneigungsgrenzen für die Bodenbearbeitung quer zum Hang sowie in Falllinie 167

Tabelle 21: Literaturauswertung zu Ertragsminderungen auf Vorgewendeflächen 170

Tabelle 22: Gesamtauswirkungen der Bearbeitung quer zum Hang auf den Bodenabtrag bei verschiedenen Fruchtfolgen und Bodenverhältnissen sowie einer Hangneigung von $10 \%$

Tabelle 23: KNV und jährlicher monetärer Vorteil ( $€ /$ ha/a) der Bewirtschaftung quer zum Hang bei konventionellem Verfahren

Tabelle 24: KNV und jährlicher Vorteil der Bewirtschaftung quer zum Hang in Kombination mit der konservierenden Bodenbearbeitung auf einem Standort mit einem durchschnittlichen Bodenabtrag von 3,1 t/ha/a

Tabelle 25: Vergleich der Aussaatkosten bei Silomais

Tabelle 26: KNV und jährlicher Vorteil der Engsaat von Silomais bei verschiedenen möglichen Ertragssteigerungen

Tabelle 27: Kosten und N-Bindung verschiedener Zwischenfrüchte u. Untersaaten am

Beispiel von Sommergerste.

Tabelle 28: Monetärer Nutzen sowie KW durch die Verminderung des Bodenabtrages durch Zwischenfruchtanbau 
Tabelle 29: Relatives Reduktionspotenzial des Nitratgehalts durch ausgewählte Zwischenfrüchte im Boden (0-90 cm Tiefe) bei Vegetationsruhe im Herbst (Mittel von vier Erntejahren)

Tabelle 30: KNV und jährlicher Vorteil verschiedener Varianten des

Zwischenfruchtanbaus

Tabelle 31: Kalkulation des Erwerbsverlustes durch die Heckenpflanzung bei der Fruchtfolge WR-WW-WG.

Tabelle 32: KNV der Anlage von Windschutzhecken bei unterschiedlichen

Ertragssteigerungen und Förderhöhen

Tabelle 33: Maximale Hanglänge als begrenzender Faktor der Konturnutzung....

Tabelle 34: Kosten der Anlage von Grünstreifen mit unterschiedlichen Streifenbreiten

und Reihenabständen für einen Schlag mit der Größe 50*100 m.....................201

Tabelle 35: Erwerbsverluste bei der Anlage von Grünstreifen

Tabelle 36: Bisherige Untersuchungsergebnisse zur Wirksamkeit von Grünstreifen auf

die Minderung des Bodenabtrags.

Tabelle 37: Mögliche KNV für die Grünstreifennutzung auf verschiedenen Standorten

bei einem Zinssatz von $4 \%$

Tabelle 38: Einfluss der alternativen Verwendung von Hanglagen durch Grünland und deren Einfluss auf den Bodenabtrag und monetären Nutzen .....

Tabelle 39: KW (€/t verhinderter Bodenabtrag) bei der Umwandlung der Hangfläche in

Dauergrünland bei verschiedenen Fruchtfolgen und Hangneigungen .....

Tabelle 40: KNV und jährlicher Vorteil bei verschiedenen Alternativen zur

Ackernutzung am Hang.

Tabelle 41: Fördermaßnahmen im Rahmen des Erosionsschutzes in den einzelnen

Bundesländern für das Jahr 2003. Stand: Dezember 2003.

Tabelle 42: KW (in €/t verhinderter Bodenabtrag) bei der konservierenden

Bodenbearbeitung und unterschiedlichen Förderhöhen .....

Tabelle 43: Einzelbetriebliches diskontiertes KNV der Mulch- und Direktsaat bei einem

Zinssatz von $4 \%$ und mittleren Bodenabtragsraten, ink1. Förderung

(60€/ha)

Tabelle 44: Anschaffungskosten und Bodendruck unter drei verschiedenen Reifen bei einer Radlast von 6,8 t und verschiedenen Reifeninnendrücken

Tabelle 45: KW (€/bar Bodendruckabsenkung) beim Einsatz von Breit- und Terrareifen

bezogen auf den Ober- und Unterboden im Vergleich zum Standardreifen 
Tabelle 46: KNV beim Einsatz von Breit- und Terrareifen, optimistische Annahmen ..229

Tabelle 47: Jährliche Gesamtkosten von Reifendruckregelanlagen für Schlepper und Anhänger

Tabelle 48: KW beim Einsatz von Reifendruckregelsystemen bei Schleppern,

Anhängern sowie deren Kombination bezogen auf den Ober- und Unterboden .235

Tabelle 49: Veränderung der Kosten durch eine Reifendruckregelanlage am Schlepper und Güllefass im landwirtschaftlichen Betrieb

Tabelle 50: KNV beim Einsatz von Reifenreglern und verschiedenen Varianten 238

Tabelle 51: Potenzielle Einsatzzeiten für Gleisbandfahrzeuge oder Großschlepper der 200 kW-Klasse bei klassischen Arbeitsgängen.

Tabelle 52: Vergleich von Leistung und Kosten verschiedener Maschinen beim Pflügen

bei einer durchschnittlichen Schlaggröße von 40 ha.

Tabelle 53: Bodendrücke (in bar) in Tiefen von 10-40 $\mathrm{cm}$ bei verschiedenen

Bereifungsvarianten im Vergleich zum Bandlaufwerk.

Tabelle 54: KW des Raupeneinsatzes mit Pflug und Tiefgrubber im Vergleich zum

Großschlepper

Tabelle 55: Erlösvorteil bei der Minderung der Radlasten von $10 \mathrm{t}$ auf $5 \mathrm{t}$ in

Zuckerrübenfruchtfolgen bei der Verhinderung einer Bodenschadverdichtung (BSV)

Tabelle 56: KNV und durchschnittlicher jährlicher Vorteil bei der Reduzierung der

Radlasten durch einen GKRB 2

Tabelle 57: KNV und durchschnittlicher jährlicher Vorteil der Stilllegung einer

Minderertragsfläche auf verschiedenen Standorten

Tabelle 58: Ausgebrachte Wirkstoffmengen und Weizenerträge im langjährigen

Anbauvergleich bei integrierter und konventioneller Bewirtschaftung .

Tabelle 59: KNV bei Weizen im Integrierten Anbau auf verschiedenen Standorten mit

Annahmen von EL TITI.

Tabelle 60: Verfahrenskosten für unterschiedliche Unkrautbekämpfungsmaßnahmen..269

Tabelle 61: Prozentualer Bekämpfungserfolg und KW bei Klettenlabkraut bei chemischer und mechanischer Unkrautbekämpfung (Striegel) auf INTEX-

Flächen 270

Tabelle 62: KNV (\%) und durchschnittlicher jährlicher Vorteil beim Striegeleinsatz in

Winterweizen und-gerste. 
Tabelle 63: Kurz- und mittelfristige Ertragseffekte und -verluste bei verschiedenen Früchten bei einem Verbot von PSM....

Tabelle 64: Einsparbare PSM-Kosten sowie das KNV bei einem Verbot von PSM für

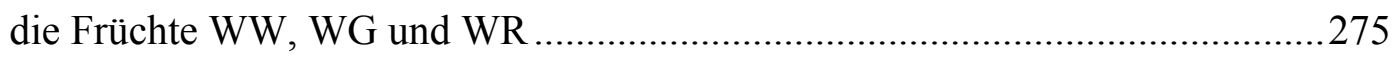

Tabelle 65: Nährstoffwert einer Klärschlammgabe (5 t TM/ha) ...................................27

Tabelle 66: Veränderung der Bodenerosion durch Wasser durch Kompostausbringung bei einer Ausbringungsmenge von $10 \mathrm{t} / \mathrm{ha} / \mathrm{a}$ auf verschiedenen Standorten........ 281

Tabelle 67: Nährstoffwert einer Kompostgabe (10 t TM/ha/a) ohne Berücksichtigung des Stickstoffs 282

Tabelle 68: KNV der Ausbringung von Kompost bei unterschiedlichen Ausbringungskosten 


\section{Abbildungsverzeichnis}

Abbildung 1: Ablauf einer typischen Expertenbefragung ............................................. 7

Abbildung 2: Arbeitsbereiche der befragten Experten ................................................ 9

Abbildung 3: Themen des Bodenschutzes in der Landwirtschaft ................................... 12

Abbildung 4: Schäden und Schadensmerkmale durch Bodenerosion .............................. 14

Abbildung 5: Mittleres jährliches Bodenabtragsrisiko pro Landkreis (in $\mathrm{t} / \mathrm{ha}$ ) .................20

Abbildung 6: Ökonomische Schäden durch Bodenverdichtungen auf landwirtschaftlich

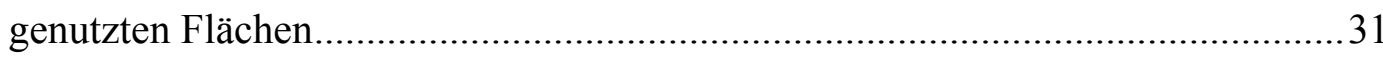

Abbildung 7: Verdichtungsgefährdung der Ackerflächen pro Landkreis, abgeschätzt aus der Summe von Vorbelastungsstufe und Spurflächensumme der jeweiligen

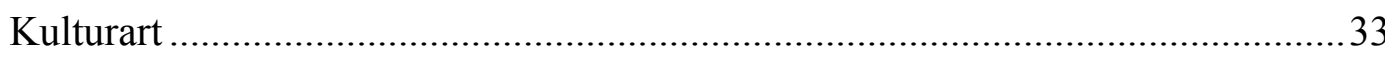

Abbildung 8: Inlandsabsatz von anorganischen Düngemitteln in Deutschland .................38

Abbildung 9: PSM-Absatz in Deutschland (in Tausend t) ................................................ 40

Abbildung 10: Beurteilung des derzeitigen Zustands des landwirtschaftlich genutzten Bodens in Bezug auf verschiedene Bodenschädigungen im Rahmen der Expertenbefragung $(\mathrm{n}=22)$

Abbildung 11: Belastungen und Einträge mit den nach Meinung der Experten höchsten

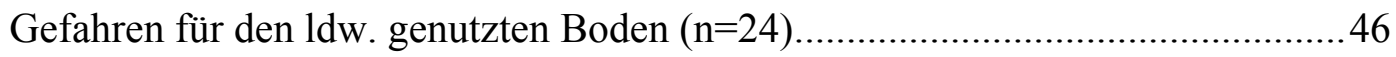

Abbildung 12: Möglichkeiten der Behebung und Kompensation von Bodenschädigungen nach Meinung der Experten $(\mathrm{n}=19)$

Abbildung 13: Subsidiäre Anwendung des BBodSchG nach $§ 17$ Abs. 3 am Beispiel der Landwirtschaft, bei schädlichen Bodenveränderungen.

Abbildung 14: Auswirkungen des Bodenschutzrechts auf die landwirtschaftliche Praxis $(\mathrm{n}=22)$ 98

Abbildung 15: Bedeutung verschiedener gesetzlicher Regelwerke für den landwirtschaftlichen Bodenschutz $(\mathrm{n}=24)$.

Abbildung 16: Mögliche Szenarien bei der Umwandlung von Ackerland in Grünland in Überschwemmungsgebieten

Abbildung 17: Beurteilung der Wirksamkeit verschiedener Maßnahmen auf den Oberflächenabfluss $(\mathrm{n}=22)$.

Abbildung 18: Markterlöse der Feldfrüchte in den INTEX-Anbausystemen (1995-2002). 
Abbildung 19: Variable Kosten des Winterweizen- und Winterrapsanbaus verschiedener Anbauverfahren an den Standorten Reinshof und Marienstein (1995-2002) ...... 156

Abbildung 20: Arbeitszeitbedarf der einzelnen Anbauverfahren auf den Standorten Reinshof und Marienstein (1995-2002; Gerste/Roggen der integrierten Systeme von 1999-2002)

Abbildung 21: Fruchtfolge-Deckungsbeiträge der Anbausysteme auf den Standorten Reinshof und Marienstein (1999-2002)

Abbildung 22: Unterschiede des pfluglosen integrierten Anbausystems gegenüber dem Pflugverfahren bei Winterweizen und Winterraps auf dem Standort Reinshof (1995-2002)

Abbildung 23: Unterschiede des pfluglosen integrierten Anbausystems gegenüber dem Pflugverfahren bei Winterweizen und Winterraps auf dem Standort Marienstein (1995-2002)

Abbildung 24: Entwicklung der durch das Erosionsschutzprogramm NRW geförderten pfluglos bewirtschafteten Fläche für beide Kammergebiete

Abbildung 25: Förderumfang der Maßnahme Konservierende Bodenbearbeitung in Sachsen seit 1993 .

Abbildung 26: Schematische Darstellung der Fahrwerke 220

Abbildung 27: Die Beziehung zwischen Luftdruck des Reifens und Bodendruck im Ober- und Unterboden

Abbildung 28: Kapitalbedarf eines Standardschleppers (157 kW), eines Großschleppers $(220 \mathrm{~kW})$ und einer Raupe $(225 \mathrm{~kW})$ bezogen auf die jährliche Einsatzfläche...243 Abbildung 29: Verfahrenskosten von Zuckerrüben bei einem SKRB-6 und GKRB-2 in Abhängigkeit von der Auslastung.

Abbildung 30: Auswirkungen von verschiedenen Ertragsminderungen auf den

Deckungsbeitrag auf einer Minderertragsfläche 258

Abbildung 31: Maschinen- und Arbeitskosten bei der Ausbringung von Kompost (Hof-Feld) 280

Abbildung 32: Zusammenfassung der Ergebnisse der KWA von Maßnahmen zum Schutz vor Bodenerosion 286

Abbildung 33: Zusammenfassung der Ergebnisse der KNA von Maßnahmen zum Schutz vor Bodenerosion

Abbildung 34: KW bei verschiedenen Maßnahmen zur Reduzierung des Bodendrucks 288 
Abbildung 35: Zusammenfassung der Ergebnisse der KNA von Maßnahmen zum Schutz vor Bodenschadverdichtungen

Abbildung 36: KNV der Maßnahmen zur Reduzierung von PSM-Austrägen in der Landwirtschaft.

Abbildung 37: Beurteilung der Bedeutung der Maßnahmen für die Schadensreduzierung mittels Expertenbefragung anhand des gewichteten Mittelwertes $(\mathrm{n}=22)$

Abbildung 38: Beurteilung des einzelbetrieblichen KNV mittels Expertenbefragung anhand des gewichteten Mittelwertes ( $\mathrm{n}=19)$

Abbildung 39: Erweitertes Bodenschutzkonzept zur Ermittlung geeigneter einzelbetrieblicher Maßnahmen in Gebieten mit hoher Bodenerosionsgefährdung.

Abbildung 40: Erweiterung des Konzeptes „Bodenschonendens Befahren“ von SOMMER um ökonomische Aspekte 298

Abbildung 41: Konzept zum Schutz vor Bodenschadverdichtungen auf landwirtschaftlichen Flächen 


\section{Übersichtenverzeichnis}

Übersicht 1: Sachgerechte Differenzierung zwischen Vorsorge und Gefahrenabwehr in der landwirtschaftlichen Bodennutzung.

Übersicht 2: Einteilung der Maßnahmen der guten fachlichen Praxis nach verschiedenen

Risikostufen. 58

Übersicht 3: Vorsorgemaßnahmen gegen Bodenschadverdichtungen nach den

Konkretisierungen der guten fachlichen Praxis ( 17 Abs. 2 BBodSchG) 70

Übersicht 4: Vorsorgemaßnahmen gegen Bodenerosion nach den Konkretisierungen der guten fachlichen Praxis der Bodennutzung (§ 17 Abs. 2 BBodSchG)

Übersicht 5: Vorgehensweise bei der Ermittlung der Wirtschaftlichkeit verschiedener

Bodenschutzmaßnahmen mittels Kostenvergleich, KWA und KNA

Übersicht 6: Bedingungen für die konservierende Bodenbearbeitung:.

Übersicht 7: Vorgehensweise bei der KNA für konservierende

Bodenbearbeitungsverfahren

Übersicht 8: Die Anbausysteme des Untersuchungszeitraums 1995-2002 im Überblick

am Beispiel der Standorte Reinshof und Marienstein.....

Übersicht 9: Schwierigkeiten bei der Bearbeitung quer zum Hang 168

Übersicht 10: Vorgehensweise bei der KNA für die Bewirtschaftung quer zum Hang.. 169

Übersicht 11: Vorgehensweise bei der KNA für die Engsaat von Silomais .................... 178

Übersicht 12: Vorgehensweise bei der KNA für den ZUA............................................ 185

Übersicht 13: Vorgehensweise bei der KNA zur Pflanzung von Hecken......................... 192

Übersicht 14: Vorgehensweise bei der KNA für die Nutzung von Grünstreifen............200

Übersicht 15: Vorgehensweise bei der KNA beim Verzicht des Anbaus an Hanglagen 207

Übersicht 16: Vorgehensweise bei der KNA für verschiedene bodenschonende Reifen 223

Übersicht 17: Vorgehensweise bei der KNA für Reifendruckregelanlagen.

Übersicht 18: Vorgehensweise bei der Analyse für den Einsatz von Raupenschleppern

Übersicht 19: Vorgehensweise bei der KNA für die Maßnahme der Reduzierung der Radlasten

Übersicht 20: Vorgehensweise bei der Analyse für die Stilllegung von

Minderertragsteilflächen

Übersicht 21:Vorgehensweise bei der KNA für die Reduzierung des PSM-Einsatzes durch das Schadschwellenprinzip 
Übersicht 22: Vorgehensweise bei der KNA für die Maßnahme der mechanischen

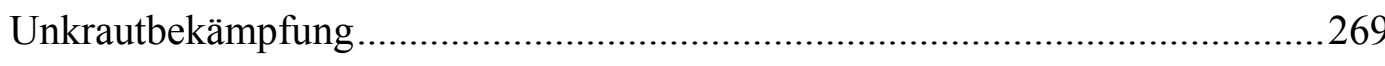

Übersicht 23: Vorgehensweise bei der KNA für die Maßnahme des Verzichts auf PSM-

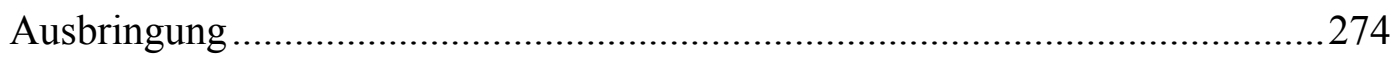

Übersicht 24: Vorgehensweise bei der KNA für die Kompostausbringung ....................279 


\section{Abkürzungsverzeichnis}

ABAG

AbfKlärV

Abs.

$\mathrm{AF}$

AFP

Art.

AUP

BA

BauGB

$\mathrm{BB}$

BBodSchG

BBodSchV

Beg.

bez.

BG

BGB

BGB1.

BImSchG

BioAbfV

$\mathrm{BM}$

BMVEL

BNatSchG

Bsp.

bspw.

BÜK

BVB

B-W

BWaldG

ca.

$\mathrm{CaO}$
Allgemeine Bodenabtragsgleichung

Klärschlammverordnung

Absatz

Ackerfläche

Agrarinvestitionsförderprogramm

Artikel

Agrarumweltprogramm

Bodenabtrag

Baugesetzbuch

Bodenbearbeitung

Bundesbodenschutzgesetz

Bundesbodenschutzverordnung

Beginn

bezüglich

Berufsgenossenschaft

Bürgerliches Gesetzbuch

Bundesgesetzblatt

Bundesimmissionsschutzgesetz

Bioabfallverordnung

Bullenmast

Bundesministerium für Verbraucherschutz, Ernäh-

rung und Landwirtschaft

Bundesnaturschutzgesetz

Beispiel

beispielsweise

Bodenübersichtskarte

Bundesverband Boden

Baden-Württemberg

Bundeswaldgesetz

circa

Kalk 
CREAMS

DDR

DMG

dt

DüngeVO

DVWK

ebd.

et al.

evtl.

f.

ff.

FA

FlurbG

FM

FUL

GAK

GBO

GenTG

ggf.

GIS

GNG

ha

HEKUL

i. d. R.

i. S. d.

inkl.

INT

INTEX

k. A.

$\mathrm{K}_{2} \mathrm{O}$
Chemicals, Runoff and Erosion from Agricultural

Management Systems

Deutsche Demokratische Republik

Düngemittelgesetz

Dezitonne

Düngeverordnung

Deutsche Vereinigung für Wasserwirtschaft, $A b-$ wasser und Abfall

ebenda

et alii

eventuell

folgende (Seite)

fortfolgende Seiten

Färsenaufzucht

Flurbereinigungsgesetz

Festmasse

Förderprogramm Umweltschonende Landbewirt-

schaftung

Gemeinschaftsaufgabe Küstenschutz

Grundbuchordnung

Gentechnikgesetz

gegebenenfalls

Geografisches Informationssystem

Gesamtnährstoffgehalt

Hektar

Hessisches Kulturlandschaftsprogramm

in der Regel

im Sinne des

inklusive

integriert

Integriert-Extensiv

Keine Angabe

Kali 


\begin{tabular}{|c|c|}
\hline Kart. & Kartoffeln \\
\hline KNA & Kosten-Nutzen-Analyse \\
\hline KNV & Kosten-Nutzen-Verhältnis \\
\hline $\mathrm{kPa}$ & Kilo Pascal \\
\hline $\mathrm{KrW}-/ \mathrm{AbfG}$ & Kreislaufwirtschafts- und Abfallgesetz \\
\hline KTBL & $\begin{array}{l}\text { Kuratorium für Technik und Bauwesen in der } \\
\text { Landwirtschaft }\end{array}$ \\
\hline KW & Kostenwirkung \\
\hline KWA & Kosten-Wirkungs-Analyse \\
\hline KWV & Kosten-Wirkungs-Verhältnis \\
\hline LABO & Länderarbeitsgemeinschaft Boden \\
\hline LAWA & Länderarbeitsgemeinschaft Wasser \\
\hline ldw. & landwirtschaftlich \\
\hline $1 \mathrm{fdm}$. & laufender Meter \\
\hline 1S & lehmiger Sand \\
\hline LWK & Landwirtschaftskammer \\
\hline $\mathrm{m}^{2}$ & Quadratmeter \\
\hline mech. & mechanisch \\
\hline MEKA & Marktentlastungs- und Kulturlandschaftsausgleich \\
\hline MS & Mulchsaat \\
\hline $\mathrm{MSmSb}$ & Mulchsaat mit Saatbettbereitung \\
\hline $\mathrm{MSoSb}$ & Mulchsaat ohne Saatbettbereitung \\
\hline MUSLE & Modified Universal Soil Loss Equation \\
\hline $\mathrm{N}$ & Stickstoff \\
\hline NAU & Niedersächsisches Agrar- und Umweltprogramm \\
\hline Nds. & Niedersachsen \\
\hline $\mathrm{NH}_{4}$ & Ammoniak \\
\hline NK & Nutzen-Kosten \\
\hline NK-Diff. & Nutzen-Kosten-Differenz \\
\hline $\mathrm{NO}_{3}$ & Nitrat \\
\hline $\mathrm{Nr}$. & Nummer \\
\hline NRW & Nordrhein-Westfalen \\
\hline $\mathrm{P}_{2} \mathrm{O}_{5}$ & Phosphat \\
\hline
\end{tabular}




\begin{tabular}{|c|c|}
\hline pers. & persönlich(e) \\
\hline $\mathrm{pF}$ & Wasserspannung \\
\hline PflSchG & Pflanzenschutzgesetz \\
\hline PLANAK & $\begin{array}{l}\text { Planungsausschuss für die Gemeinschaftsaufgabe } \\
\text { „Verbesserung der Agrarstruktur und des Küsten- } \\
\text { schutzes“ }\end{array}$ \\
\hline PSM & Pflanzenschutzmittel \\
\hline RBA & Relativer Bodenabtrag \\
\hline Red. & Reduzierung \\
\hline RGB1. & Reichsgesetzblatt \\
\hline RL & Richtlinie \\
\hline Rnd. Nr. & Randnummer \\
\hline ROG & Raumordnungsgesetz \\
\hline R-P & Rheinland-Pfalz \\
\hline $\mathrm{RR}$ & Reifendruckregelanlage \\
\hline RS & Radschlepper \\
\hline RUSLE & Revised Universal Soil Loss Equation \\
\hline $\mathrm{S}$ & Sand \\
\hline S-A & Sachsen-Anhalt \\
\hline SA & Streifenabstand \\
\hline SB & Saatbettbearbeitung \\
\hline SG & Sommergerste \\
\hline SKRB-6 & Sechsreihiger selbstfahrender Köpfrodebunker \\
\hline $\mathrm{sL}$ & sandiger Lehm \\
\hline SRU & Sachverständigenrat für Umweltfragen \\
\hline $\mathrm{t}$ & Tonne \\
\hline TM & Trockenmasse \\
\hline TS & Trockensubstanz \\
\hline u. a. & und andere \\
\hline UFD & Unterfußdüngung \\
\hline USA & Vereinigte Staaten von Amerika \\
\hline USLE & Universal Soil Loss Equation \\
\hline USt & Umsatzsteuer \\
\hline
\end{tabular}


u. U. unter Umständen

UVP Umweltverträglichkeitsprüfung

v. a. vor allem

Verm. Verminderung

VO Verordnung

WEPP Water Erosion Prediction Project

WG Wintergerste

WHG Wasserhaushaltsgesetz

WR Winterraps

WRRL EU-Wasserrahmenrichtlinie

WW Winterweizen

z. B. zum Beispiel

ZF Zwischenfrucht

ZR Zuckerrüben

ZUA Zwischenfrucht- und Untersaatenanbau 


\section{Einleitung}

\subsection{Problemstellung und Zielsetzung}

Der Boden erfüllt für die Gesellschaft lebenswichtige ökologische und ökonomische Funktionen. Er dient als Grundlage der Nahrungsmittelproduktion, als Standort für Siedlungs- und Industrieflächen sowie als Abbaumedium und Archiv der Kulturgeschichte. Für die Landwirtschaft stellt er aufgrund seiner Produktionsfunktion nicht nur die Bewirtschaftungsgrundlage, sondern auch die generationenübergreifende Werterhaltung der landwirtschaftlichen Betriebe dar.

Trotz der hohen Bedeutung des Bodens für die Landwirtschaft und die gesamte Gesellschaft ist er verschiedenen Gefahren ausgesetzt. Die Gefahren lassen sich in stoffliche Schädigungen $^{1}$ sowie physikalische Schädigungen ${ }^{2}$ einteilen. Bezüglich der physikalischen Schädigungen bestehen in Deutschland bisher erhebliche Wissensdefizite, was v. a. mit den komplexen und z. T. kaum wahrnehmbaren Prozessen der Bodenerosion und Bodenschadverdichtung einhergeht. Deshalb und aufgrund der Tatsache, dass von physikalischen Beeinträchtigungen des landwirtschaftlich genutzten Bodens nachweislich hohe ökologische und ökonomische Schäden ausgehen können, konzentriert sich die Untersuchung in dieser Arbeit vorrangig auf die Bodenerosion und Bodenschadverdichtung.

Mit dem Bundes-Bodenschutzgesetz (BBodSchG) vom 17.3.1999 und der BundesBodenschutzverordnung (BBodSchV) ist der Boden v. a. aufgrund der geringen Transparenz seiner Schädigungen sehr spät als drittes Umweltmedium neben Luft und Wasser in einem bundeseinheitlichen Rahmen rechtlich geregelt worden. Die Landwirtschaft trägt die Verantwortung für den Boden als größter Flächennutzer im Wesentlichen durch die Einhaltung der guten fachlichen Praxis nach $\S 17$ BBodSchG. Einige gesellschaftliche Gruppen kritisieren das derzeitige Instrument der guten fachlichen Praxis und fordern strengere Grenzwerte und Auflagen. Diese Diskussion wird derzeit ebenfalls bei der Festsetzung der Regelungen der Cross-Compliance geführt.

\footnotetext{
${ }^{1}$ Stoffeinträge durch organische und anorganische Düngemittel sowie Pflanzenschutzmittel.

${ }^{2}$ Bodenerosion durch Wasser und Wind, Bodenschadverdichtung oder Bodenversiegelung.
} 
Eine sozioökonomische Begleitforschung zum Problembereich Landwirtschaft und Bodenschutz fehlt zum größten Teil. ${ }^{3}$ Außerdem ist bei den z. Zt. herrschenden schwierigen Rahmenbedingungen und dem zunehmenden Wettbewerbsdruck auf einzelbetrieblicher Ebene davon auszugehen, dass kostenverursachende Maßnahmen ohne betriebswirtschaftlich errechenbaren Nutzen immer weniger Akzeptanz finden. Maßnahmen des Bodenschutzes sind hiervon besonders betroffen, weil einem mittlerweile großen Angebot von Schutzmaßnahmen mit nur ungenügend wissenschaftlich bewiesenen Nutzeneffekten eine Gegenüberstellung von Kosten und Nutzen weitestgehend fehlt. Trotzdem werden von Seiten der Politik in einigen Bereichen sehr weitreichende Forderungen an die Landwirtschaft gestellt, wie es z. B. derzeit bei dem geplanten und bereits durch das Bundeskabinett verabschiedete Ackerbauverbot in bestimmten Überschwemmungsgebieten durch ein Artikelgesetz zum Hochwasserschutz der Fall ist.

Hieraus leitet sich das Ziel dieser Arbeit ab. Neben einer Darstellung des aktuellen Bodenschutzrechts in Deutschland und der Diskussion der Defizite und Verbesserungsmöglichkeiten werden in dieser Arbeit verschiedene Bodenschutzmaßnahmen erläutert und betriebswirtschaftlich bewertet. Diese Bewertung geschieht anhand von Kostenvergleichen, Kosten-Wirkungs-Analysen (KWA) sowie Kosten-Nutzen-Analysen (KNA). Abschließend werden auf dieser Basis Empfehlungen und Vorschläge für die praktische Landwirtschaft, die landwirtschaftliche Beratung sowie für Verwaltung und Politik gegeben.

\subsection{Vorgehensweise}

Im Kapitel 2 werden zunächst die Datengrundlagen dieser Arbeit dargestellt und erläutert. Neben der Vorgehensweise bei der Literaturrecherche wird die Durchführung der Expertenbefragung dieser Arbeit dargestellt und erläutert. Die Expertenbefragung wurde mit Experten aus der Wissenschaft, der Beratung und der Verwaltung durchgeführt und dient zur Gewinnung von zusätzlichen Kenntnissen der Themen dieser Arbeit sowie der Validierung von Ergebnissen des Kapitels 6.

\footnotetext{
${ }^{3}$ Vgl. Frielinghaus (2003), S. 65, Hollmann (2003), S. 97 sowie Stahl, Schmidt U. Gierke (2001), S. 110
} 
Das Kapitel 3 beschreibt die Situation des Bodens in Hinblick auf die landwirtschaftliche Nutzung. Dabei werden die Wirkungen der Landwirtschaft auf die einzelnen Bodengefährdungen, in diesem Fall die Bodenerosion, Bodenschadverdichtungen und verschiedene Stoffeinträge sowie deren Umfang und Einflussmöglichkeiten, dargestellt. Ergänzend werden die Erkenntnisse aus der Expertenbefragung zu diesen Themen herangezogen.

Kapitel 4 beschäftigt sich mit dem für die Landwirtschaft relevanten Bodenschutzrecht in Deutschland. Nach einem kurzen Einblick in das Bodenschutzrecht verschiedener europäischer Länder wird das Bundes-Bodenschutzgesetz (BBodSchG) erörtert. Der Schwerpunkt dieser Betrachtung liegt auf der guten fachlichen Praxis in der landwirtschaftlichen Bodennutzung nach $\S 17$ BodSchG, der Ausgleichsregelung nach $\S 10$ Abs. 2 BBodSchG und der Ausweisung von Bodenschutzgebieten nach $\S 21$ Abs. 3 BBodSchG. Weiterhin erfolgt ein Vergleich des Bodenschutzrechts mit den Regelungen des Düngemittel- Pflanzenschutzmittel- und Naturschutzrechts. Daraufhin wird die BBodSchV, das untergesetzliche Regelwerk zum BBodSchG, und deren Auswirkungen auf die Landwirtschaft, dargestellt. Bevor die Ergebnisse der Expertenbefragung thematisiert werden, findet eine ausführliche Diskussion zum Bodenschutzrecht statt.

Die rechtlichen, naturwissenschaftlichen und ökonomischen Verbindungen zwischen dem Wasser- und Bodenschutz sind Gegenstand des Kapitels 5. Hierbei spielen v. a. die möglichen Auswirkungen der EU-Wasserrahmenrichtlinie und das geplante Hochwasserschutzgesetz mit dem darin vorgesehenen Ackerbauverbot eine zentrale Rolle. Dieses Kapitel wird am Ende mit den Ergebnissen der Expertenbefragung abgerundet.

Das Kapitel 6 stellt den Schwerpunkt dieser Arbeit dar. Nach einer Beschreibung und Erläuterung der einzelnen Bewertungsmethoden, dem Kostenvergleich, der KWA und der KNA werden verschiedene landwirtschaftliche Maßnahmen des physikalischen und stofflichen Bodenschutzes auf einzelbetrieblicher Ebene analysiert und somit eine Basis für eine vergleichende Bewertung geschaffen. Auch in diesem Kapitel dienen die Ergebnisse der Expertenbefragung als Ergänzung und Diskussionsgrundlage für die betriebswirtschaftliche Einordnung der Maßnahmen.

Im Kapitel 7 werden aufgrund der vorangegangenen betriebswirtschaftlichen Bewertung einzelner Maßnahmen Zukunftsperspektiven und Entwicklungsmöglichkeiten von Bodenschutz- und Hochwasserschutzkonzepten diskutiert. Die bisherigen Ergebnisse der 
Arbeit fließen in Kapitel $8 \mathrm{zu}$ allgemeinen Empfehlungen für die landwirtschaftliche Praxis, die Beratung, Verwaltung und Politik zusammen, woraus allgemeine Schlussfolgerungen für den landwirtschaftlichen Bodenschutz gezogen werden. Das Kapitel 9 fasst abschließend die gesamte Arbeit zusammen. 


\section{Informationsbeschaffung und Datengrundlagen}

Im Folgenden werden die verwendeten Datengrundlagen in systematisierter Art wiedergegeben und die Vorgehensweise bei der Informationsbeschaffung dargestellt. In dieser Arbeit stützt sich die Informationsbeschaffung in erster Linie auf eine umfangreiche Literaturauswertung sowie eine Expertenbefragung von Vertretern aus Wissenschaft, Verwaltung und Beratung.

\subsection{Literaturauswertung}

Die Literaturauswertung spielt in dieser Arbeit eine wichtige Rolle. Die verwendete Literatur im weiteren Sinne kann in diesem Zusammenhang in folgende Bereiche eingeteilt werden: Monografien, Fachzeitschriftenartikel, Dissertationen und Habilitationen, Diplomarbeiten, Tagungsbände, Versuchszwischen- oder -abschlussberichte, mehrbändige ergänzbare Handbücher, Internet, Datensammlungen und Gesetzestexte. Eine wichtige Rolle spielen jedoch auch nicht veröffentlichte Informationen wie mündliche oder schriftliche Auskünfte aus Hoch- oder Fachhochschulen, Verwaltungen, aus der gewerblichen Wirtschaft sowie von Beratern und Landwirten.

In Bereichen des allgemeinen sowie speziellen physikalischen Bodenschutzes bestehen z. T. erhebliche Wissenslücken. Dies liegt vor allem an der mangelnden Vielfalt von boden- und pflanzenbaulichen Versuchen sowie an der geringeren Veröffentlichungsfrequenz.

Ansonsten kann festgestellt werden, dass im Bereich des Bodenschutzrechts eine intensive Veröffentlichungstätigkeit von rechtswissenschaftlicher Seite festzustellen ist, die z. T. sehr spezielle Sachverhalte aufgreift. Eine abschließende Wertung des neuen Bodenschutzrechts fehlt bisher jedoch.

Aus agrarökonomischer Sicht ist die geringe bisherige Untersuchungsvielfalt zu bemängeln. Wie in Kapitel 6.1.2 deutlich wird, gibt es nur sehr wenige Studien, die sich mit dem landwirtschaftlichen Bodenschutz aus betriebswirtschaftlicher Sicht beschäftigen. Außerdem beschäftigen sich die Studien oftmals nur mit speziellen Fragestellungen. 


\subsection{Expertenbefragung}

Im Folgenden wird das Wesen, die Zielsetzung sowie die Vorgehensweise der in dieser Arbeit durchgeführten Expertenbefragung beschrieben. Die Expertenbefragung gehört zu den qualitativen Forschungsmethoden und beschränkt sich auf eine relativ kleine Anzahl von Befragten. Dabei stehen oft Fragen über zukünftige Entwicklungen im Vordergrund der Forschung.

\subsubsection{Wesen und Zielsetzung der Befragung}

In der sozialwissenschaftlichen Literatur wird die Expertenbefragung häufig gar nicht oder nur am Rande behandelt. Eine Expertenbefragung ist nach ATTESLANDER eine „mündliche oder schriftliche teilstandardisierte Methode mit deren Hilfe sowohl qualitative als auch quantitative Aspekte bearbeitet werden können“ “ ${ }^{4}$. In dieser Arbeit dient die Expertenbefragung der Gewinnung von Erkenntnissen über Sachverhalte und zukünftige Entwicklungen im landwirtschaftlichen Bodenschutz sowie der Validierung mit den unterschiedlichen Bewertungsmethoden ermittelten Ergebnissen zur Ermittlung der Vorzüglichkeit von Bodenschutzmaßnahmen. Die Befragung soll ferner die aus der Literaturrecherche gewonnenen Daten zur ökonomischen Bewertung von Bodenschutzmaßnahmen erweitern und ggf. Datenlücken schließen. Aufgrund der Vielzahl der Themenbereichen ist dies allerdings nur begrenzt möglich, da nur wenige der Befragten alle Bereiche mit ihrem Fachwissen abdecken können.

\subsubsection{Ablauf und Durchführung der Befragung}

Der Ablauf einer typischen Expertenbefragung ist in der folgenden Abbildung dargestellt, dem auch diese Befragung Folge leistet.

\footnotetext{
${ }^{4}$ ATteSLANDER (2000), S. 105 ff.
} 


\section{Abbildung 1: Ablauf einer typischen Expertenbefragung}

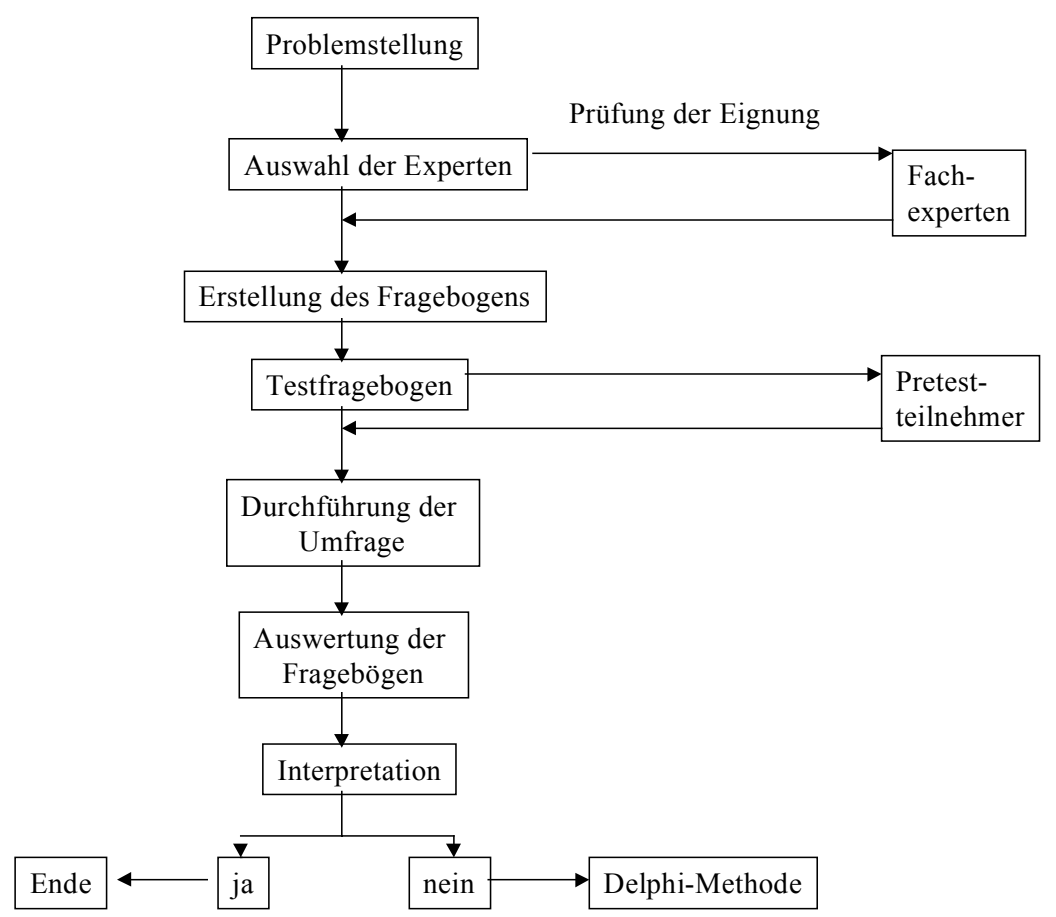

Quelle: Eigene Darstellung

Die Expertenauswahl ist einer der wichtigsten Faktoren einer Expertenbefragung. Die Zusammensetzung der Expertengruppe kann der Abbildung 2 in Kapitel 2.2.3 entnommen werden. Die Auswahl erfolgte hauptsächlich im Zusammenhang mit der Literaturrecherche. Demzufolge wurde von den Publikationen auf die Eignung des Experten geschlossen. Außerdem wurden durch persönliche Gespräche Kontakte mit den Beratern und der Verwaltung geknüpft und hierdurch die Eignung für die Befragung überprüft. Auf das Problem der regionalen Tätigkeitsfelder der Experten konnte aufgrund der kleinen zur Verfügung stehenden Auswahl nicht eingegangen werden. Anders als bei der quantitativen Befragung dürfte dieser Faktor keine große Rolle spielen. Nachdem eine für Expertenbefragungen typische Anzahl von 26 Teilnehmern ${ }^{5}$ ausgewählt wurde, begann die Erstellung des Fragebogens. Dabei wurde ein teilstandardisierter Fragebogen ${ }^{6}$ mit folgenden Bereichen entwickelt:

\footnotetext{
${ }^{5}$ Vgl. BEREKOVEN (1999), S. 269 f.

${ }^{6}$ siehe Anhang
} 
- Zustand des landwirtschaftlich genutzten Bodens,

- Maßnahmen zum Schutz des Bodens und deren ökonomische Bewertung,

- Bodenschutzrecht in Deutschland,

- Boden- und Hochwasserschutz und

- Zukünftige Ausrichtung des Bodenschutzes.

$\mathrm{Zu}$ Beginn eines Kapitels konnten die Experten angeben, ob sie ihre fachliche Kompetenz für geeignet hielten. Dies war deshalb notwendig, weil der Fragebogen analog zu dieser Arbeit sehr unterschiedliche Fachgebiete bearbeitet. Aufgrund der verschiedenen Themengebiete war nahezu auszuschließen, dass die Experten den ganzen Fragebogen bearbeiteten konnten, was auch nicht geschah. Der Fragebogen umfasste 19 Seiten, was bei einer Expertenbefragung die Obergrenze darstellen sollte, um die Bereitschaft der Teilnehmer nicht zu beeinträchtigen. Nach der Erstellung des Fragebogens wurde ein Pretest bei $10 \%$ der Stichprobe durchgeführt. Da dieser Pretest ohne weitere Schwierigkeiten beendet wurde, konnte mit der Befragung begonnen werden.

Die Befragung erfolgte schriftlich auf dem postalischem Wege. Das Anschreiben ist im Anhang dargestellt. Die eingeräumte Bearbeitungsfrist betrug drei Wochen. Danach wurde noch eine E-Mail mit einem Erinnerungsschreiben mitsamt des Fragebogens versendet, um den Rücklauf zu erhöhen. Letztlich wurden 26 Fragebögen zurückgesendet. Die Möglichkeit, die Befragung nach der Delphi-Methode ${ }^{7}$ durchzuführen, wurde nach der Auswertung der zum größten Teil eindeutigen Ergebnisse ausgeschlossen, weil der zusätzliche Aufwand bei den Befragten unverhältnismäßig gewesen wäre.

Die Auswertung des Fragebogens wurde mit dem Statistikprogramm SPSS Version 11.5 durchgeführt. Aufgrund der geringen Stichprobe ist die Tiefe der methodischen Vorgehensweise eingeschränkt, was jedoch dem Wesen der qualitativen Befragung entspricht. ${ }^{8}$ Daher beschränkt sich die Auswertung im weitesten Sinne auf die deskriptive Statistik. Die Ergebnisse der Expertenbefragung sind den entsprechenden Themengebieten dieser Arbeit zugeteilt. Sie finden sich in den Kapiteln 3.2, 4.2.6.7, 4.4.4, 5.3.5. sowie 6.5.

\footnotetext{
${ }^{7}$ Bei der Delphi-Methode werden den Experten i. d. R. die Ergebnisse der ersten Befragungsrunde vorgelegt. Diese sollen dazu nochmals Stellung nehmen. Dieses Verfahren dient der Konsensfindung bei stark streuenden Ergebnissen in der ersten Befragungsrunde.

${ }^{8}$ Vgl. BEREKOVEN (1999), S. $269 \mathrm{f}$.
} 


\subsubsection{Beschreibung der Expertengruppe}

Wie Abbildung 2 zeigt, setzte sich die Expertengruppe aus Personen verschiedener Arbeitsbereiche wie Wissenschaft, landwirtschaftlicher Fachberatung, landwirtschaftlichen Fachbehörden sowie Verbänden zusammen. Die entsprechende Zugehörigkeit wurde im letzten Teil des Fragebogens erfragt. Da hier Mehrfachnennungen möglich waren, kann nicht von der Anzahl der Nennungen auf die Gruppengröße geschlossen werden. Der Schwerpunkt der Expertengruppe liegt bei der Beratung in den verschiedenen Arbeitsbereichen mit insgesamt 22 Nennungen, gefolgt von den Fachbehörden bzw. der Administration (20 Nennungen) und der Verbandsarbeit (3 Nennungen).

\section{Abbildung 2: Arbeitsbereiche der befragten Experten}

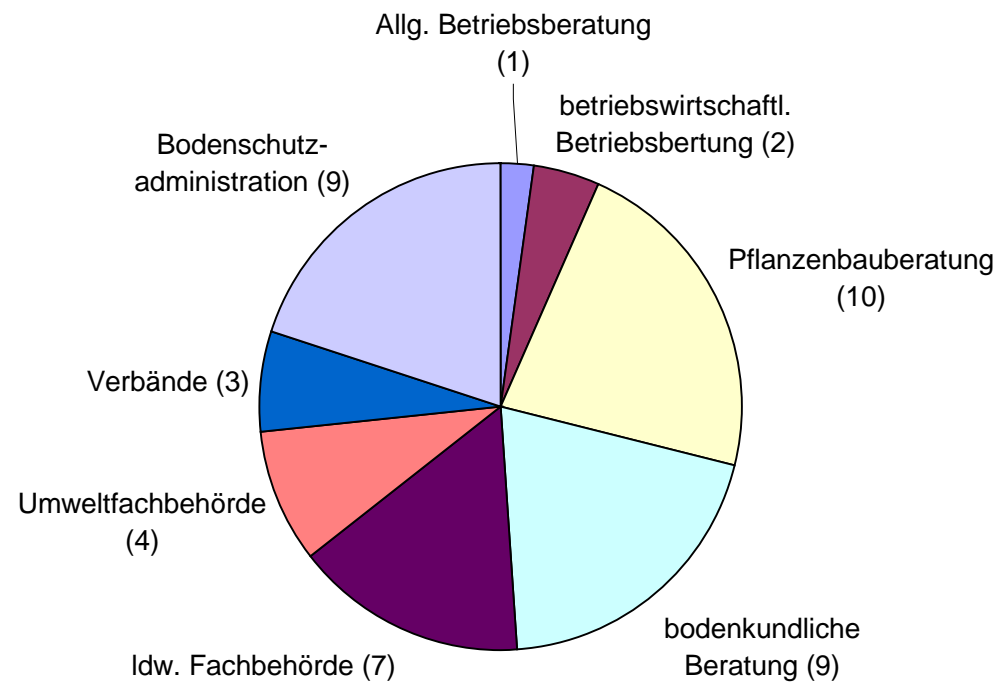

Quelle: Eigene Darstellung

Die Qualifikation der Experten zur Teilnahme an dieser Befragung kann den Nennungen zu den von ihnen bearbeiteten Bodenschutzthemen entnommen werden. ${ }^{9}$ Der größte Bereich ist dabei der physikalische Bodenschutz. Dazu gehört der Bereich der Bodenerosion, womit sich 16 Experten zum Zeitpunkt der Befragung beschäftigten, gefolgt von der Bodenverdichtung mit 13 Nennungen und der Bodenversiegelung mit drei Nennungen.

\footnotetext{
${ }^{9}$ Bei dieser Frage waren ebenfalls Mehrfachnennungen möglich.
} 
Im Bereich des stofflichen Bodenschutzes beschäftigen sich 11 Experten mit der Düngemittelproblematik, zehn mit der Klärschlamm- und Kompostproblematik sowie fünf mit der Schwermetall- und vier mit der Pflanzenschutzmittelproblematik.

Im folgenden Kapitel wird die Beziehung zwischen der Landbewirtschaftung und der daraus resultierenden Gefahr für den Boden dargestellt. Hierzu gehört eine Erläuterung des aktuellen Zustands des ldw. genutzten Bodens sowie der Einflussmöglichkeiten der Landwirtschaft, diesen Zustand zu verbessern. 


\section{Umweltwirkungen der Landwirtschaft auf den Boden}

Gegenstand dieses Kapitels sind die einzelnen Gefährdungen des Bodens. Neben den naturwissenschaftlichen Grundlagen stehen auch die Verbreitung sowie die Bedeutung der Bodenschädigungen im Mittelpunkt. Der Inhalt dieses Kapitels bildet somit eine wichtige Informations- und Argumentationsgrundlage für die Schaffung von Bodenschutzmaßnahmen in der Landwirtschaft.

\subsection{Stand der Umweltwirkungen}

Im Folgenden wird die aktuelle Situation der landwirtschaftlichen Nutzflächen in Deutschland bezüglich unterschiedlicher Bodenschädigungen dargestellt. Diese lassen sich allgemein in physikalische und stoffliche Bodenschädigungen einteilen. Hierauf basierend sind in der unteren Abbildung 3 die verschiedenen Themengebiete des landwirtschaftlichen Bodenschutzes dargestellt. Auf die schädlichen Bodenveränderungen wird in den nächsten Kapiteln 3.1.1.1 und 3.1.2.1 ausführlich eingegangen, da sie für diese Arbeit von zentraler Bedeutung sind. Die Landwirtschaft ist jedoch auch vom Flächenverbrauch in Deutschland betroffen. Momentan werden in Deutschland 105 ha/Tag zumeist landwirtschaftliche Nutzfläche verbraucht. ${ }^{10}$ Hinzu kommen noch die Flächen für Ausgleichs- und Ersatzmaßnahmen. Der Flächenverbrauch kann sowohl durch Überbauung als auch durch Abgrabung stattfinden, wie in Abbildung 3 dargestellt ist.

\footnotetext{
${ }^{10}$ Vgl. StATistisches BUNDESAMT (2003)
} 


\section{Abbildung 3: Themen des Bodenschutzes in der Landwirtschaft}

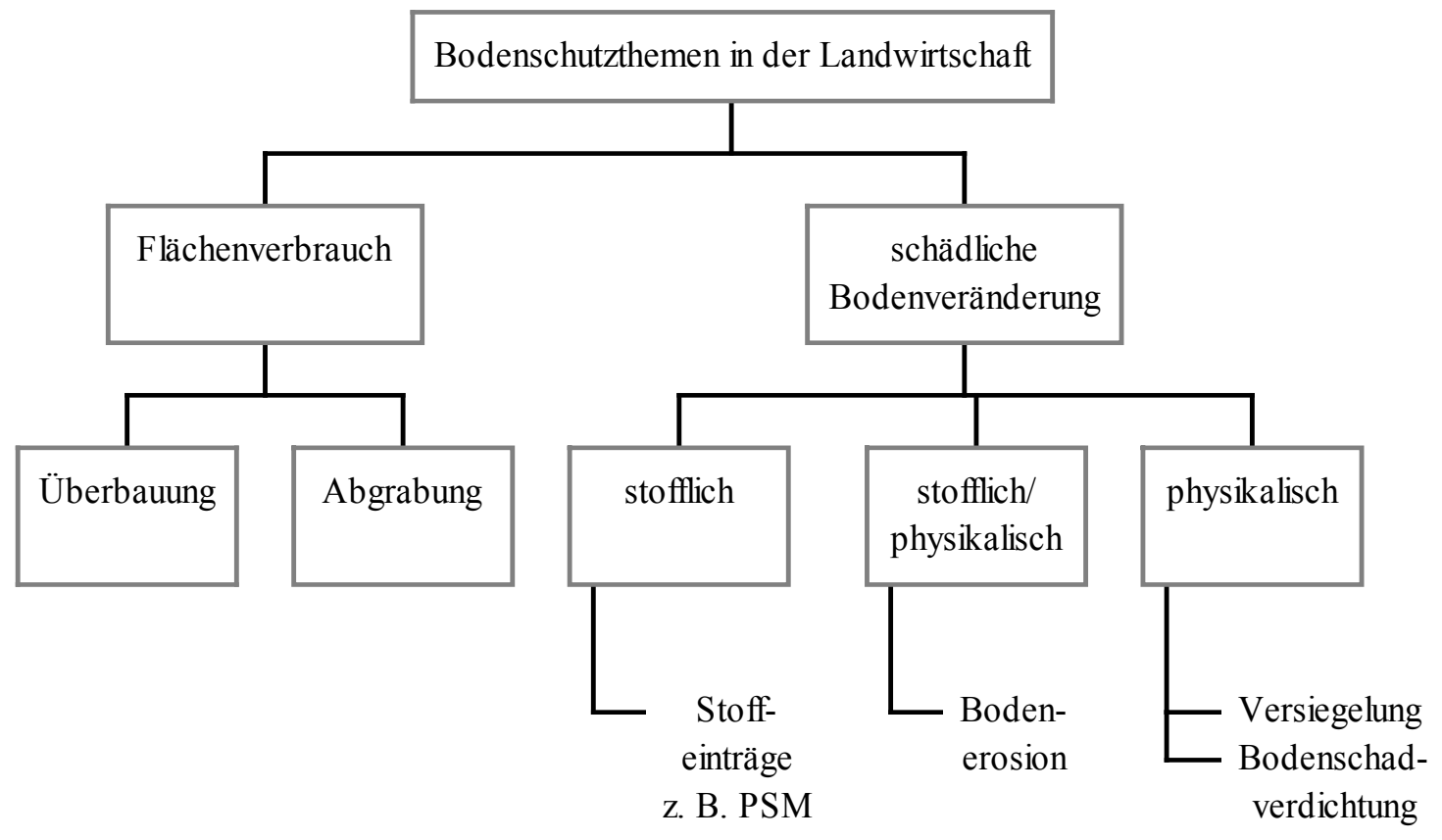

Quelle: Eigene Darstellung

\subsubsection{Bodenerosion}

Die Bodenerosion ist einerseits ein natürlicher und andererseits ein durch landwirtschaftliche Tätigkeit anthropogen beeinflusster Prozess. Die Bodenerosion kann in Wind- und Wassererosion unterteilt werden. Bei der Winderosion werden die Bodenteilchen durch die Geschwindigkeit des Windes und dadurch entstehenden Druck- und Hubkräften in Bewegung gesetzt. Durch diese Bewegung werden wiederum andere Bodenteilchen angestoßen und abgetragen.

Für die Winderosion hat das BMVEL ${ }^{11}$ im entsprechenden Bund-Länderpapier Orientierungswerte entwickeln lassen, die Hinweise darüber geben, ab wann hierdurch Schädigungen auftreten können:

- Windgeschwindigkeit

$>8 \mathrm{~m} /$ sec. $^{12}$

- Bodenanfälligkeit

v. a. Feinstsand und Anmoor, trocken

- Windoffenheit in der Landschaft

$<5 \mathrm{~km}$ Flurelemente je $\mathrm{km}^{2}$ in waldarmen Regionen

\footnotetext{
${ }^{11}$ Vgl. BMVEL (2001), S. 49

12 gemessen in $10 \mathrm{~m}$ Höhe, dies entspricht $5-6 \mathrm{~m} / \mathrm{sec}$. an der Bodenoberfläche.
} 
- Bodenoberfläche

$<25 \%$ Bodenbedeckung

Die Wassererosion entsteht durch das Aufschlagen von Regentropfen auf unbedeckte Bodenpartikel. Diese werden durch das abfließende Oberflächenwasser samt der darin gelösten Stoffe abgeschwemmt. Das Ausmaß des Bodenabtrags hängt von vielen Faktoren ab. Die Oberflächenbedeckung, die Hangneigung, die Bodenart und der Strukturzustand des Bodens spielen dabei sehr wichtige Rollen. Diese Faktoren und die Schädigungen des landwirtschaftlich genutzten Bodens sind Gegenstand der Kapitel 3.1.1.2 und 3.1.1.4. Für den Bereich der Wassererosion gelten nach dem Bund-Länder-Papier des BMVEL $^{13}$ folgende Orientierungswerte für den Eintritt von Schädigungen ${ }^{14}$ :

- Niederschlag $>7,5 \mathrm{~mm} / \mathrm{m}^{2}$ oder $>5 \mathrm{~mm} / \mathrm{h}$

- Bodenanfälligkeit sL, $1 \mathrm{~S}$ oder $\mathrm{S}$

- Hanglängen

$>50 \mathrm{~m}$

- Hangneigung

$>4 \%$

- Bodenbedeckung

$<30 \%$

\subsubsection{Wirkungen der Landwirtschaft auf die Bodenerosion}

Die Schädigungen von Bodenerosionen können entweder auf der Fläche des Abtrags (Onsite-Schaden) oder auf benachbarten Flächen außerhalb des Schlages durch Bodenabtrag (Offsite-Schaden) ${ }^{15}$ entstehen. Die Systematik der Bodenschädigungen ist in Abbildung 4 dargestellt. Beide Schadenskategorien können unter kurzfristiger und langfristiger Sichtweise gesehen werden.

\footnotetext{
${ }^{13}$ Vgl. BMVEL (2001), S. 47

${ }^{14}$ Vgl. FrielinghaUs ET AL. (2002), S. 16

15 Bspw. zeigen Untersuchungen von FriELINGHAUS zu Bodenabträgen infolge von Wassererosion auf Testflächen in Brandenburg und Mecklenburg-Vorpommern, dass im Durchschnitt mit einer Tonne Boden etwa 0,3 kg Stickstoff und 0,3 kg Phosphor abgetragen wurden (Vgl. FriELINGHAUs (1998), S. 210 f.)
} 


\section{Abbildung 4: Schäden und Schadensmerkmale durch Bodenerosion}

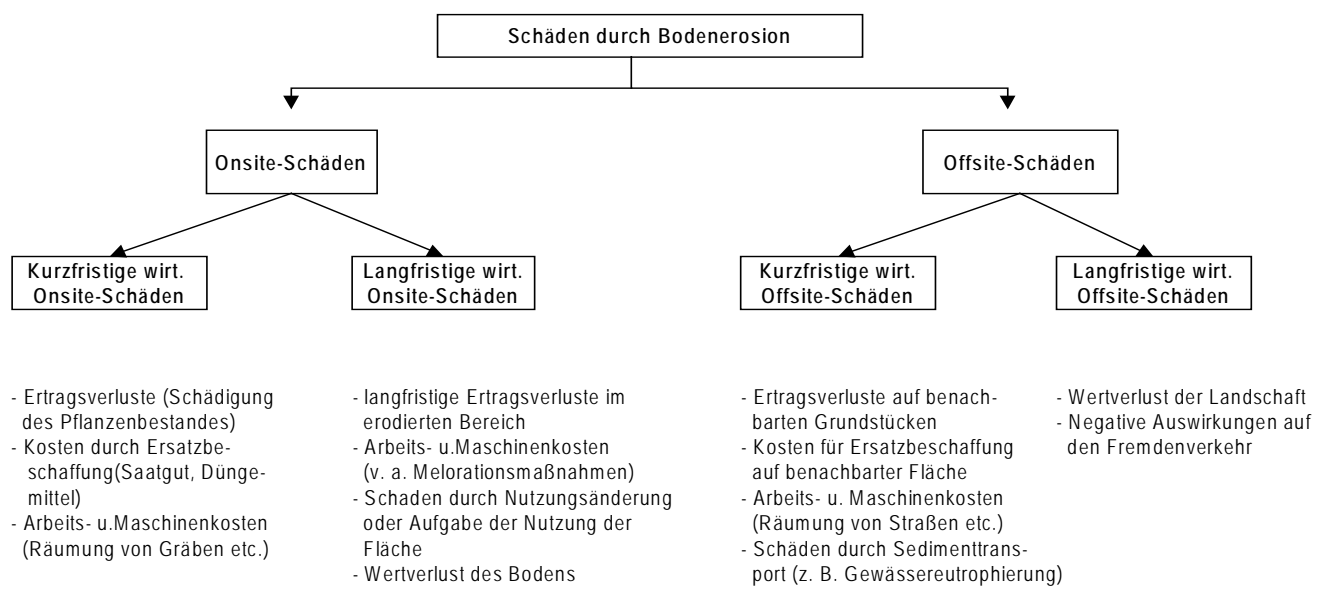

Quelle: Eigene Darstellung

$\mathrm{Zu}$ den kurzfristigen Onsite-Schäden gehören z. B. die auf dem erodierten Schlag entstandenen Ertragsrückgänge, die erheblich sein können. Dies liegt vor allem daran, dass die eventuell entstehenden Mehrerträge auf den Akkumulationsflächen die Mindererträge auf den Reduktionsflächen nicht ausgleichen können. Dadurch kommt es auf der Gesamtfläche auch dann zu Ertragsrückgängen, wenn der Boden nur innerhalb des einzelnen Schlages bewegt wird. Die Ausprägung der Ertragsrückgänge hängt wiederum von der Mächtigkeit des Oberbodens auf der Reduktionsfläche ab. Außerdem zählen zu den Onsite-Schäden noch die Arbeits- und Maschinenkosten zur Umlagerung des Bodens, Kosten für Neuansaat etc. Die langfristigen Schäden bestehen in erster Linie aus den langfristigen Ertragsverlusten, Arbeits- und Maschinenkosten für langfristige Meliorationsmaßnahmen, Schäden durch Nutzungsänderung oder -aufgabe sowie aus dem Wertverlust durch den Rückgang der natürlichen Ertragsfähigkeit.

Die Offsite-Schäden sind schwerer zu quantifizieren, da oft die direkte UrsacheWirkungsbeziehung fehlt. Bereits 1991 weisen JAROSCH u. ZEDDIES ${ }^{16}$ auf das Problem

\footnotetext{
${ }^{16}$ Vgl. JAROSCH u. ZEDDIES (1991), S. 103
} 
der Zurechnung der Kosten hin. Doleschel u. HeIßENHUBER ${ }^{17}$ bearbeiten in ihrem Forschungsprojekt jenes Problem der externen Kosten und schätzen in einer Modellrechnung für Bayern die externen Kosten der Beseitigung von Sedimentablagerungen in Gewässern etc. auf rund 15,3 Mio. € pro Jahr. Derartige Ansätze wurden allerdings nicht weiter verfolgt. Auch FLEIGE ${ }^{18}$ weist in diesem Zusammenhang v. a. auf das Problem hin, die ökologischen Schäden der Bodenerosion vollständig und genau zu monetarisieren.

Typische kurzfristige Offsite-Schäden sind Ertragsverluste auf benachbarten Grundstücken, Kosten für Ersatzbeschaffung auf der benachbarten Fläche (z. B. Saatgut- oder Düngerkosten), Arbeits- und Maschinenkosten, sowie die Schäden durch den Sedimentund Stofftransport und die daraus resultierende Eutrophierung der Gewässer. Aus langfristiger Sicht spielen bei den Offsite-Schäden v. a. der Wertverlust der Landschaft und die negativen Auswirkungen auf Gesellschaft sowie Fremdenverkehr eine Rolle. Diese Werte lassen sich jedoch nur sehr schwer quantifizieren und sind nur durch Befragungen im Rahmen von direkten Bewertungsmethoden, wie z. B. der Kontingenten Bewertungsmethode, abschätzbar.

Um die Wirkungen der Landwirtschaft auf die Bodenerosion darstellen zu können, muss das Gefährdungspotenzial zu errechnen oder abschätzbar sein. Bodenerosion ist allerdings nur mit einem sehr hohen Aufwand messbar. Die Probleme liegen v. a. in folgenden Tatsachen:

- Die Gesamterosion wird sehr stark von Einzelereignissen determiniert.

- Es gibt eine Vielzahl von Einflussfaktoren auf die Bodenerosion.

- Es existieren verschiedene Betrachtungsebenen (z. B. Teilschlag, Einzelschlag, Flur, Gemarkung, ganze Regionen).

Die durch die Landbewirtschaftung verursachte Bodenerosion hat in den letzten fünf Jahrzehnten zugenommen. Dabei sind Bodenerosionsereignisse häufig durch folgende Faktoren verstärkt worden:

\footnotetext{
${ }^{17}$ Vgl. Doleschel und HeißENHUber (1991), S. 207

${ }^{18}$ Vgl. Fleige (1999), S. 122
} 
- Vergrößerung der bewirtschafteten Flächen bei gleichzeitiger Reduktion von Landschaftsstrukturelementen,

- Zunahme des Mais- und Weizenanbaus, Abnahme des Ackerfutterbaus,

- Zunahme der Bewirtschaftungs- und Befahrintensität,

- Zunahme des Umbruchs von natürlichem Grünland sowie der Tiefe und der Intensität der wendenden Bodenbearbeitung,

- Zunahme des Einsatzes großer Maschinen aufgrund des technischen Fortschrittes sowie der Entwässerung von Ackerflächen. ${ }^{19}$

Das folgende Kapitel 3.1.1.2 beschäftigt sich daher mit der Abschätzung der potenziellen Bodenerosionsgefährdung durch Wassererosion, insbesondere anhand der Allgemeinen Bodenabtragsgleichung (ABAG).

\subsubsection{Die Abschätzung des Bodenabtrags mittels Bodenerosionsmodellen}

Für die betriebswirtschaftliche Betrachtung des Problems der Bodenerosion in der Landwirtschaft ist die Frage der Bewertung des Bodenabtrags, bzw. die Kenntnis von Toleranzgrenzen, grundlegend. In diesem Zusammenhang ist auf SCHWERTMANN et al. ${ }^{20} \mathrm{zu}$ verweisen, die den tolerierbaren Bodenabtrag (in t/ha und Jahr) als den Bodenverlust sehen, bei dem der natürliche Ertrag des Bodens in einem Zeitraum von 300 bis 500 Jahren keine wesentliche Minderung erfährt. Hierfür wird folgende Faustformel genannt, die auf der in Untersuchungen festgestellten Korrelation zwischen der Gründigkeit des Bodens und dessen Ertragsmesszahl basiert:

\section{Tolerierbarer Bodenabtrag: $\quad$ Acker- oder Grünlandzahl $\quad$ [t/ha und Jahr]}

Das Konzept des tolerierenden Bodenabtrags stand in der Vergangenheit sehr häufig aufgrund seiner Pauschalierung in der Kritik, kann jedoch als grober Richtwert sehr schnell und einfach vom Landwirt ermittelt werden.

\footnotetext{
${ }^{19}$ Vgl. Frielinghaus, BeESE u. Ellerbrock (1999)

${ }^{20}$ Vgl. SCHWERTMANN, VOGL u. KAINZ (1987)
} 
In die Betrachtung muss die Tatsache mit einbezogen werden, dass die Bodenneubildung unter mitteleuropäischen Bedingungen (v. a. Klima und Bodennutzung) nur etwa $<1 \mathrm{t} /$ ha/a beträgt. Hieraus ist zu schließen, dass Bodenerosion an sich nicht zu tolerieren wäre. ${ }^{21}$

Neben dem Toleranzgrenzenkonzept wurden bereits zahlreiche Bodenerosionsmodelle entwickelt. Die ABAG ist eine der ersten Modelle zur Abschätzung des Bodenabtrags. Sie ist den empirischen Bodenerosionsmodellen zuzuordnen und geht auf die Universal Soil Loss Equation (USLE) von WiSCHMEIER u. SMITH aus dem Jahr 1965 zurück. Sie wurde anschließend von SCHWERTMANN et al. zunächst auf die bayerischen, dann auf die deutschen Verhältnisse übertragen.

Die ABAG ermöglicht es mit folgender Gleichung, den langjährigen mittleren Bodenabtrag (A) zu ermitteln:

$$
\mathbf{A}=\mathbf{R} \times \mathbf{K} \times \mathbf{L} \times \mathbf{S} \times \mathbf{C} \times \mathbf{P} \quad \text { (in } \mathrm{t} / \mathrm{ha} \text { und Jahr) }
$$

Nach der Gleichung bestimmen demnach sechs Faktoren den Bodenabtrag und können wie folgt beschrieben werden:
R: Regen- und Oberflächenabflussfaktor
K: Bodenerodierbarkeitsfaktor
L: Hanglängenfaktor
S: Hangneigungsfaktor
C: Bedeckungs- und Bearbeitungsfaktor
P: Erosionsschutzfaktor

Wie aus obiger Beschreibung ersichtlich wird, können einige der Faktoren nicht bzw. nur mit großem Aufwand verändert werden. Hierzu zählen z. B. die Niederschlagsmenge oder der Hangneigungsfaktor. Von den dargestellten Faktoren ist der C-Faktor am stärksten durch den Landwirt und seiner Fruchtfolgeplanung zu beeinflussen. Durch die Entwicklung eines PC-Programms ${ }^{22}$ mit den wesentlichen Elementen der ABAG ist die Verbreitung in Landwirtschaft und Beratung noch stärker vereinfacht worden.

\footnotetext{
${ }^{21}$ Vgl. Frielinghaus (1998), S. 38

${ }^{22} \mathrm{Bei}{ }^{\mathrm{PC}} \mathrm{ABAG}$ handelt es sich um ein excelbasiertes Programm.
} 
Die der ABAG zugrundeliegenden Algorithmen sind in verschiedenen anderen weiterentwickelten Erosionsmodellen (RUSLE, MUSLE, dABAG) zu finden. Nach MosimanN ist die ABAG ,,das einzige wissenschaftlich gut abgesicherte, praxistaugliche Modell zur

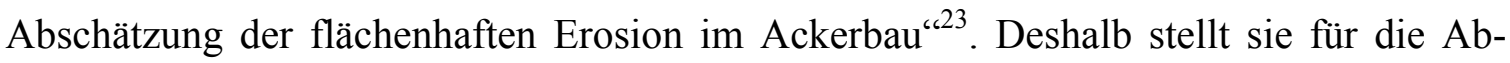
schätzung der Erosion in der Praxis ,eine Grundlage ohne Alternative dar“24. Gerade aus diesen Gründen ist die ABAG und deren Anwenderprogramme zum Einsatz in der landwirtschaftlichen Fachberatung sowie auf landwirtschaftlichen Betrieben prädestiniert.

Trotz allem sind bei der ABAG einige Schwachstellen zu finden, die vor allem darin begründet sind, dass sie den linearen Bodenabtrag in Erosionsrinnen und -gräben nicht berücksichtigt, wie es bei den physikalisch basierten Modellen ${ }^{25}$ der Fall ist. Außerdem berücksichtigt sie die durch Fremdwasser (z. B. Abfluss von Straßen oder benachbarten Schlägen), Hangwasseraustritte oder die durch schmelzenden Schnee entstehende Erosion nicht. Diese Kritikpunkte werden durch die Verwendung von neueren physikalischen Bodenerosionsmodellen aufgegriffen und verbessert, die, wie das nächste Kapitel zeigt, sehr aufwendig sind.

\subsubsection{Weitere Bodenerosionsmodelle}

Aufgrund der Kritik an der ABAG wurden weitere, stärker prozessorientierte physikalische Modelle entwickelt. Sie konzentrieren sich auf die Simulation von Einzelereignissen, wobei eine räumliche Übertragbarkeit gewährleistet ist. Der Vorteil dieser Modelle besteht darin, dass durch Ermittlung der Deposition des abgetragenen Bodenmaterials Onsite- und Offsiteschäden errechnet werden können. Außerdem können Risiken exakter abgeschätzt und Gegenmaßnahmen definiert werden. Beispiele für physikalisch begründete Modelle sind CREAMS (Chemicals, Runoff and Erosion from Agricultural Management Systems), WEPP (Water Erosion Prediction Project) oder EROSION 3-D. ${ }^{26}$

Physikalische Bodenerosionsmodelle sind bisher in der Vergangenheit selten angewendet worden. Bekannte Beispiele finden sich dafür in der Gemeinde Rheurdt und Schwalmtal, die beide am Niederrhein liegen. Beide Gemeinden hatten auf einigen Flächen in der

\footnotetext{
${ }^{23}$ Mosimann (1995), S. 4

${ }^{24}$ ebd.

${ }^{25}$ Vgl. Kapitel 3.1.1.3

${ }^{26}$ Vgl. LANDESANSTALT FÜR UMWELTSCHUTZ (2003)
} 
Vergangenheit hohe Bodenverluste durch Wassererosion infolge einzelner Niederschlagsereignisse zu verzeichnen. Die Kommunen wurden durch die hohen OffsiteKosten belastet. Die Kosten durch den Sedimenteintrag eines einzelnen Bodenerosionsereignisses 1998 betrugen für die Gemeinde Rheurdt $40.000 €$. Des weiteren kommt noch die Unterhaltung von 48 Sediment- und Sandfängen für das $250 \mathrm{~km}^{2}$ große Gebiet hinzu. ${ }^{27}$ Daraufhin wurde ein Arbeitskreis ins Leben gerufen, der mit Hilfe der Bodenerosionsmodellierung (Erosion-3D und d-ABAG) Schutzmaßnahmen für die Gemeindegebiete entworfen hat. Zusammenfassend ist jedoch für die physikalischen Modelle festzustellen, dass die Nachteile dieser Modelle beim hohen Aufwand und den nötigen spezifischen Kenntnissen des Anwenders liegen.

Für die Bodenerosion durch Wind existieren keine analogen Modelle. Die Hauptforschungsstandorte für Winderosion sind die USA und Kanada, wo seit 50 Jahren intensive Forschungsarbeit auf den Gebieten der Prozessaufklärung und Entwicklung von Schutzkonzepte betrieben wird. Die Winderosion ist als folgende Funktion darzustellen: ${ }^{28}$

\section{$\mathbf{W E}=\mathbf{f}(\mathbf{K}, \mathbf{V}, \mathbf{Z}, \mathbf{L}, \mathbf{U})$}

$\mathrm{K}=$ Bodenerodierbarkeit (Körnung ${ }^{29}$, Bodenfeuchte, Humusgehalt, Krusten)

$\mathrm{V}=$ Vegetationsbedeckung

$\mathrm{Z}=$ Rauhigkeit der Oberfläche

$\mathrm{L}=$ Feldlänge

$\mathrm{U}=$ Klima (Windgeschwindigkeit, Niederschlag, Verdunstung)

Die Übertragbarkeit dieser Gleichung auf mitteleuropäische oder deutsche Verhältnisse ist nicht ohne weiteres gegeben, da die Untersuchungsintensität zur Übertragung sehr gering ist.

\footnotetext{
${ }^{27} \mathrm{Vgl}$. SCHMidT (2002)

28 Vgl. WoodrufF et al. (1965), S. 12

${ }^{29}$ Der erosionsresistente Durchmesser der Körner oder Aggregate des Bodens liegt allgemein zwischen 0,63 bis etwa $1 \mathrm{~mm}$.
} 


\subsubsection{Umfang und Bedeutung der Bodenerosion durch Wasser in Deutschland}

Um Anforderungen an Schutzmaßnahmen von landwirtschaftlicher Seite stellen zu können, ist es zunächst notwendig, die Gefährdungsgebiete der Schädigung zu kennen.

Hierbei muss berücksichtigt werden, dass es z. Zt. nur flächendeckende Daten für die Verbreitung der potenziellen Abtrags- bzw. Verdichtungsgefährdung gibt. Zudem ist es schwierig und v. a. aufwendig, Aussagen über die tatsächliche Bodenerosion auf den landwirtschaftlichen Nutzflächen zu machen. Hierzu wären eine jährliche Anpassung der Datengrundlage sowie direkte Messungen des Bodenabtrags notwendig. In der folgenden Abbildung ist das mittlere Bodenabtragsrisiko durch Wassererosion erstmals für das gesamte Bundesgebiet dargestellt.

\section{Abbildung 5: Mittleres jährliches Bodenabtragsrisiko pro Landkreis (in t/ha)}
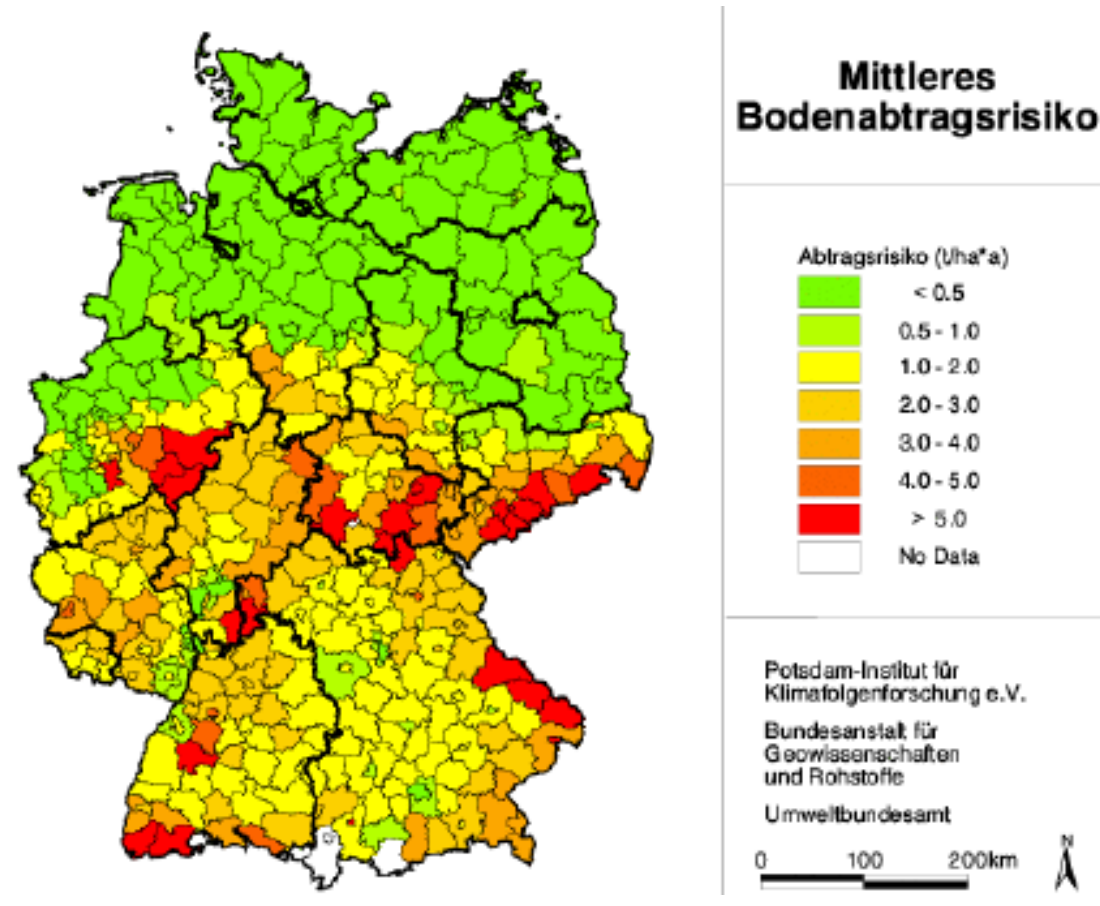

Quelle: ERHARD et al. (2002), S. 70

Das mittlere Bodenabtragsrisiko auf Basis der Landkreise wurde mit Hilfe der ABAG erstellt. In der Abbildung 5 ist zu erkennen, dass das Abtragsrisiko von Norden nach Süden zunimmt. Die höchsten Werte ( $>5 \mathrm{t} / \mathrm{ha} / \mathrm{a})$ sind vor allem in den Mittelgebirgsregionen Mittel- und Südwestdeutschlands, im Erzgebirge sowie im Bayerischen Wald festzustellen. Dies liegt zum einen an den dort vorherrschenden Löss- und lösshaltigen Böden und zum anderen an der Kombination von hohen Niederschlägen und Hang- 
neigungen. Bei der Abbildung 5 ist jedoch zu berücksichtigen, dass der Anteil der Ackerfläche in diesen Landkreisen sehr gering ist. Deshalb werden in der Veröffentlichung von ERHARD et al. auch zusätzlich noch die Werte des Bodenabtragsrisikos auf Ebene der Landkreise aufsummiert.

Da die Veröffentlichung des potenziellen Bodenabtrags für das gesamte Bundesgebiet von ERHARD ET AL. z. Zt. die neueste und umfangreichste Datengrundlage ist, werden ältere Schätzungen und Berechnungen für zum größten Teil nur einzelne Bundesländer nicht rezitiert. Überdies wurde die Reduzierung der Bodenfruchtbarkeit durch einen ständigen Bodenabtrag schon sehr früh untersucht. ${ }^{30}$

Wie eine Expertenbefragung zum Thema Umweltindikatoren in der Landwirtschaft zeigt, sollte die Bodenerosion grundsätzlich nicht $10 \mathrm{t} / \mathrm{ha} / \mathrm{a}$ überschreiten. ${ }^{31}$ Dabei ist zu berücksichtigen, dass die Auswirkungen von Erosionsereignissen eine hohe Variabilität aufweisen. Die Spannweite der punktuell tatsächlich ermittelten Bodenabträge beträgt z. B. bei der Wassererosion 0,5-54 t/ha/Ereignis und bei der Winderosion 0,8-172 t/ha/Ereignis. ${ }^{32}$ Diese Werte lassen sich jedoch nicht auf großflächige Gebiete übertragen, weshalb es letztlich keine Kartierungen von tatsächlichen Bodenabträgen in Deutschland gibt.

Für das Bundesland Bayern liegt die erste ausführliche und mehrjährige Studie über eine flächendeckende Abschätzung der Bodenabträge vor. ${ }^{33}$ Mit Hilfe der ABAG wurde ein Mittel des Bodenabtrages auf der Basis von 13125 Rasterflächen ermittelt. 1986 betrug demnach der mittlere Bodenabtrag auf Ackerflächen (incl. Dauerkulturen) 11 t/ha/a. Auf die gesamte landwirtschaftlich genutzte Fläche bezogen betrug dies 6,0 t/ha. In 2001 erfolgte eine neue Berechnung innerhalb dieses Modells, wobei der veränderten Nutzungsstruktur der landwirtschaftlich genutzten Flächen Rechnung getragen wurde. ${ }^{34}$ Nach dieser Untersuchung werden in Bayern auf den Ackerflächen (incl. Dauerkulturen) etwa 7,1 t/ha (- $35 \%$ ) abgetragen, bezogen auf die gesamte landwirtschaftlich genutzte Fläche bedeutet dies ein Abtrag von 3,8 t/ha (-36,7\%). Der Rückgang ist nach den Autoren v. a. durch die Nutzungsänderung der Ackerflächen (Mulchsaat bei Hopfen, Zwischenfrucht-

\footnotetext{
${ }^{30}$ Vgl. hierzu Richter (1965), S. XVI u. 592, SCHMIDT (1991), S. 283

${ }^{31}$ Vgl. TREMEL (2000), S. 146

${ }^{32}$ Vgl. RATHE (1998), zitiert in GUNREBEN et al. (2003), S. $13 \mathrm{f}$.

${ }^{33}$ Vgl. AuERSWALD U. SCHMIDT (1986)

${ }^{34}$ Vgl. KÖNIGER U. SCHWAB (2001)
} 
anbau etc.) zu erklären. Allerdings könnten Veränderungen der Erfassungsgenauigkeit durch die 2001 angewendete GIS-Technologie dieses Ergebnis etwas verzerren.

WERNER et al. $^{35}$ gehen vergleichend hierzu mittels einer Abschätzung durch die ABAG von einem mittleren Bodenabtrag von $8,7 \mathrm{t} / \mathrm{ha} / \mathrm{a}$ für die alten Bundesländer und 4,6 t/ha/a für die neuen Bundesländer aus.

BRUNOTTE $^{36}$ geht für das Bundesland Niedersachsen von einem durchschnittlichen jährlichen Bodenabtrag durch Wasser von 5 t/ha/Jahr aus. Dieser Wert deckt sich also mit den Schätzungen von WERNER et al.

\subsubsection{Einflussmöglichkeiten der Landwirtschaft auf die Bodenerosion}

Bei den einzelnen Einflussgrößen der ABAG wird bei ERHARD et al. deutlich, dass sowohl der R-Faktor als auch der LS-Faktor den größten Einfluss auf die Bodenerosionsrate haben. ${ }^{37}$ Hierbei ist zu berücksichtigen, dass es sich bei beiden Faktoren um natürliche Faktoren handelt, die durch den einzelnen Landwirt nicht zu beeinflussen sind. Der aus landwirtschaftlicher Sicht wichtigster Faktor ist der C-Faktor. Er ist v. a. in Nordostdeutschland bzw. den Bundesländern Bayern und Baden-Württemberg am höchsten. In diesen Gebieten ist der hohe Anteil an Brache ${ }^{38}$ sowie ein hoher Anteil an Sommerblattsowie Reihenfrüchten signifikant. Wie hoch der Einfluss der Bodenbedeckung auf den Bodenabtrag in der ldw. Praxis einzuschätzen ist, zeigen FRIELINGHAUs et al. anhand von zehnjährigen Abtragsmessungen.

\footnotetext{
${ }^{35} \mathrm{Vgl}$. WERNER et al. (1994), S.81

${ }^{36}$ Vgl. BRUNOTTE (1990), zitiert in GUNREBEN et al. (2003), S.13

${ }^{37}$ Vgl. ERHARD et al. (2002), S. 71

${ }^{38}$ Die Schwarzbrache hat den höchsten C-Faktor, der eins beträgt.
} 
Tabelle 1: Einfluss der Bodenbedeckung auf den Abfluss und Bodenabtrag (Relativwerte auf der Basis zehnjähriger Messungen)

\begin{tabular}{ccccc}
\hline $\begin{array}{c}\text { Boden- } \\
\text { bedeckung } \\
\%\end{array}$ & $\begin{array}{c}\text { Pfanzen- } \\
\text { rückstände i. d. } \\
\text { TM (t/ha) }\end{array}$ & $\begin{array}{c}\text { Oberflächenab- } \\
\text { fluss } \\
\%\end{array}$ & $\begin{array}{c}\text { Bodenabtrag } \\
\text { Wassererosion } \\
\%\end{array}$ & $\begin{array}{c}\text { Bodenabtrag } \\
\text { Winderosion } \\
\%\end{array}$ \\
\hline 0 & 0 & 45 & 100 & 100 \\
$>25-30$ & 0,5 & 40 & 25 & 15 \\
$>30-50$ & 2 & $<30$ & 8 & 3 \\
$>50-70$ & 4 & $<30$ & 3 & $<1$ \\
$>70$ & 6 & $<30$ & $<1$ & $<1$ \\
\hline
\end{tabular}

Quelle: FrielinghaUS ET AL. (2002), S. 33

Durch eine Bodenbedeckung von 70 \%, was durch den Anbau von Winterzwischenfrüchten realisiert werden kann, ist eine fast vollständige Reduzierung des Bodenabtrages möglich. Außerdem wird der Oberflächenabfluss bereits bei einer Bodenbedeckung von $30-50 \%$ auf höchstem Niveau reduziert.

Eine weitere Einflussmöglichkeit der Landwirtschaft auf die Bodenerosion besteht durch die Anwendung der konservierenden Bodenbearbeitung. Die Bodenbearbeitung hat einen sehr hohen Einfluss auf die Bodenerosion, da man mit ihr verschiedene erosionssteuernde Parameter (z. B. Bodenbedeckung, Oberflächenstruktur) beeinflussen kann. Dieser Einfluss konnte in einem Praxisversuch wie folgt dokumentiert werden:

Tabelle 2: Vergleich verschiedener Parameter nach konventioneller und konservierender Bodenbearbeitung

\begin{tabular}{lccc}
\hline & konventionell & $\begin{array}{c}\text { konservierend } \mathrm{A}^{39} \text { mit } \\
\text { Mulchsat }\end{array}$ & $\begin{array}{c}\text { konservierend } \mathrm{B}^{40} \\
\text { mit Mulchsaat }\end{array}$ \\
\hline Bodenabtrag (g) & 318 & 138 & 26 \\
Abfluss (1) & 21 & 12 & 3 \\
Infiltrationsrate (\%) & 49 & 71 & 92 \\
Aggregatstabilität (\%) & 30 & 43 & 49 \\
Humusgehalt (\%) & 2,0 & 2,6 & 2,5 \\
Bedeckungsgrad (\%) & 1 & 30 & 70 \\
\hline
\end{tabular}

Quelle: NiTSCHE ET AL. (2000)

Die Allgemeingültigkeit dieser Messungen kann dadurch dokumentiert werden, dass sie Eingang in das Bund-Länderpapier zur guten fachlichen Praxis (gfP) zur Vorsorge gegen

\footnotetext{
39 einmalige Stoppelbearbeitung, Grubbern zur Senfaussaat zum Herbst und Saatbettbereitung (Flachgrubber, $3 \mathrm{~cm}$ ).

${ }^{40}$ nur Senfaussat mit Schleuderstreuer und einmaliger Saatbettbereitung.
} 
Bodenschadverdichtungen und Bodenerosion gefunden haben. ${ }^{41}$ Sie zeigen, dass die konservierende Bodenbearbeitung durch die Erhöhung des Bodenbedeckungsgrades den Bodenabtrag fast gänzlich beseitigen kann. Dazu ist allerdings ein begleitender Zwischenfruchtanbau notwendig.

In der folgenden Tabelle sind zusammenfassend verschiedene erosionsmindernde Maßnahmen zusammengetragen.

Tabelle 3: Erosionsmindernde Maßnahmen und ihr Einfluss auf Wasser- und Winderosion

\begin{tabular}{lcc}
\hline \multicolumn{1}{c}{ Maßnahme } & Wassererosion & Winderosion \\
\hline Landschaftsgestaltung: & & \\
Bearbeitung quer zum Hang & ++ & ++ \\
Verkürzung der Schlaglänge & ++ & ++ \\
Windschutzhecken & 0 & ++ \\
\hline Bodennutzung: & & \\
Fruchtfolgegestaltung: & +++ & +++ \\
Umwandlung von Acker in Grünland & ++ & ++ \\
Ausweitung des mehrjährigen Ackerfutterbaus & + & + \\
Verminderung des Hackfruchtanteils & & \\
Anderung des Anbauverfahrens: & ++ & ++ \\
Mulchsaat & + & + \\
Untersaaten & + & - \\
Spurlockerung & + & 0 \\
Niederdruckreifen & + & - \\
Dränung & & \\
\hline
\end{tabular}

Quelle: Verändert nach AuERSwALD (2002), S. 423

Es wird deutlich, dass die Umwandlung von Acker in Grünland den höchsten positiven Einfluss auf die Erosionsminderung hat. Die Maßnahmen der Landschaftsgestaltung weisen prinzipiell einen hohen positiven Einfluss auf die Erosionsminderung auf. Maßnahmen der Änderung von Anbauverfahren werden unterschiedlich beurteilt. Die Mulchsaat hat einen hohen positiven Einfluss, Untersaaten, Spurlockerung, Niederdruckreifen und Dränung haben in Bezug auf die Wassererosion nur einen geringen positiven Einfluss und auf die Winderosion bezogen keinen oder einen negativen Einfluss. Analog zu diesem geht das nächste Kapitel auf das Problem der Bodenschadverdichtungen auf landwirtschaftlich genutzten Böden ein.

\footnotetext{
${ }^{41}$ Vgl. BMVEL (2001)
} 


\subsubsection{Bodenschadverdichtungen ${ }^{42}$}

Die Bodenschadverdichtung ist nicht nur ein Zustand, sondern auch ein Prozess. ${ }^{43}$ Der Zustand beschreibt den Grad der Dichtlagerung, d. h. den Wert des Porenvolumens, der Porenziffer bzw. der Lagerungsdichte. Der Prozess beschreibt die Änderung der gerade genannten Kennzahlen. Demzufolge kann der Prozess der Bodenschadverdichtung nur mit der Kenntnis der Vorbelastung des Bodens korrekt angegeben werden. Von dem Ergebnis der Messung des Verdichtungsprozesses kann allerdings nicht ohne weiteres auf die Beeinträchtigung der Bodenfunktionen geschlossen werden. Dies erschwert die Analyse von Bodenschadverdichtungen dahingehend, dass keine allgemeingültigen Aussagen getroffen werden können, da Böden unterschiedliche Empfindlichkeiten gegenüber Verdichtungsimpulsen aufweisen. Bodenschadverdichtungen können in der Ackerkrume, in der Krumenbasis und im Unterboden entstehen. Dabei ist zu beachten, dass die Reversibilität der Verdichtungen in dieser Reihenfolge auch stark abnehmend ist. Innerhalb der Ackerkrume sind durch tiefere Bodenbearbeitungsmaßnahmen gewisse Auflockerungen des Bodens zu erreichen. Die Struktur der Krumenbasisverdichtung mit Tiefenlockerungsmaßnahmen kann zwar zerstört werden, der Ausgangszustand des Bodens lässt sich jedoch nicht herstellen. Unterbodenverdichtungen sind gänzlich irreversibel. ${ }^{44}$ Hierfür spricht ebenfalls, dass nahezu ausgeschlossen werden kann, dass unter unseren Klimabedingungen die Unterbodenverdichtungen durch starke Frosteinwirkung zurückgehen. ${ }^{45}$

In der Vergangenheit war bisher ungeklärt, welche Bedingungen erfüllt sein müssen, damit eine schädliche Bodenveränderung nach $\S 2$ Abs. 3 BBodSchG vorliegt. Nach neuesten Ergebnissen ist davon auszugehen, dass eine schädliche Bodenveränderung durch Bodenschadverdichtung im Sinne des BBodSchG vorliegt, wenn:

- die Luftkapazität im Unterboden $5 \%$ unterschreitet,

- die gesättigte Wasserleitfähigkeit weniger als $10 \mathrm{~cm}$ am Tag beträgt und

- das Ergebnis einer Feldgefügeansprache vor Ort in den Bewertungsstufen 4 bis 5 liegt. $^{46}$

\footnotetext{
${ }^{42}$ Im Gegensatz zur Bodenverdichtung ist die Bodenschadverdichtung ein Prozess, der negative Auswirkungen auf die Produktionsfunktion sowie auf die Regelungs- und Lebensraumfunktion hat.

${ }^{43}$ Vgl. EHLERS, SCHMidTKE u. RAUBER (2003), S. 10

${ }^{44}$ Vgl. hierzu HAKANSSON u. REEDER (1994), SOMMER (1974) u. HORN et al. (2000)

${ }^{45}$ Vgl. HARTGE u. HORN (2002), S. 37

${ }^{46}$ Vgl. Agra-Europe (2003), S. L 25
} 
Bereits 1971 hat in diesem Zusammenhang CzERATZKI ${ }^{47}$ optimale Werte des Porenvolumens für die Hauptbodenarten zusammengefasst. Demnach beträgt das optimale Porenvolumen für Tonböden 47 \%, für Schluffböden 45 \% und für Sandböden $40 \%$.

Die mechanische Belastung des Bodens wird mit den Kennziffern der Radlast (t), dem mittleren Kontaktflächendruck (kPa, bar) und der Überrollhäufigkeit dargestellt. Die durch die Radlast der Maschine eingebrachten Drücke verbreiten sich im Boden dreidimensional als sog. Druckzwiebeln. Dies führt bei Überlappung der Druckzwiebeln zu weiteren Schub- und Schwerkräften und einer weiteren Druckerhöhung. Es ist durch die Erhöhung der Reifenaufstandsfläche zwar möglich, den Kontaktflächendruck zu vermindern. Die Druckfortpflanzung in die Tiefe bleibt aber dennoch bestehen, da die entscheidende Größe hierfür die absolut aufgebrachte Last ist. ${ }^{48}$ Außerdem war in der Vergangenheit zu beobachten, dass die Aufstandsfläche der Maschinen in gleichem Maße wie die Lasten erhöht wurden, was nicht zu einer Reduzierung der in den Boden eingebrachten Drücke geführt hat. ${ }^{49}$

Die Verdichtungsempfindlichkeit von Ackerböden hängt im wesentlichen von den folgenden drei Faktoren ab. Die Verdichtungsempfindlichkeit steigt z. B. mit zunehmendem Tongehalt und zunehmender Bodenfeuchte. Doch auch die Porenvolumina der Böden spielen in dieser Betrachtung eine Rolle. Je lockerer der Boden gelagert ist oder je intensiver er bearbeitet wurde, desto empfindlicher reagiert er auf den auftreffenden Bodendruck und somit auf Schadverdichtungen. ${ }^{50}$

Eine Bodenschadverdichtung wirkt sich negativ auf die Produktionsfunktion des Bodens aus und kann zu Ertragsminderungen von bis zu $35 \%^{51}$ und höheren Produktionskosten führen. Der Grund hierfür liegt v. a. in folgenden Auswirkungen:

- Rückgang der Wasserleitfähigkeit im gesättigten und ungesättigten Boden. Dies führt zum einen zu Wasserstau und zum anderen zu Wassermangel im Sommer.

- Eingeschränktes Infiltrationsvermögen. Dies führt zu einer erhöhten Erosionsgefahr, Lachgasemissionen und anderen Störungen des Gasaustausches.

\footnotetext{
${ }^{47}$ Vgl. CZERATZKi (1972), S. 30

${ }^{48}$ Vgl. HORN (2003)

${ }^{49} \mathrm{Vgl}$. EHLERS, SCHMidTKE u. RAUBER (2003)

${ }^{50} \mathrm{Vgl}$. SOMMER (2000), S. $378 \mathrm{ff}$.

${ }^{51}$ Vgl. HORN (2001), S. 81
} 
- Zu geringe Luftkapazität und Luftdurchlässigkeit. Dies führt zu einer gestörten Boden- und Wurzelatmung. Außerdem wird die Wurzelausbreitung gehemmt und somit das Pflanzenwachstum behindert.

- Unzureichender Gaswechsel und Diffusion. Dies führt zu Sauerstoffmangel, Fehlen von aeroben Bodenlebewesen und Denitrifikation. ${ }^{52}$

Für die Messung von Bodenschadverdichtungen stehen verschiedene Geräte zur Verfügung. Die weiteste Verbreitung hat die Penetrometersonde erfahren, die bei der Anwendung, d. h. dem Einführen in den zu untersuchenden Boden, den Eindring- bzw. Durchdringungswiderstand misst. Außerdem werden z. Zt. verschiedene Penetrometersonden entwickelt, die an landwirtschaftlichen Geräten, wie z. B. Grubbern, angebracht werden können und die Widerstände im Boden des gesamten Schlages messen können.

\subsubsection{Wirkungen der Landwirtschaft auf die Bodenschadverdichtung}

Bei der Landbewirtschaftung werden durch das Befahren der Flächen mit Maschinen und Geräten verschiedene physikalische Kräfte wie Druck- und Schubspannungen in den Boden eingeleitet. Übertreffen diese Kräfte die Eigenstabilität des Bodens, kommt es zur Bodenschadverdichtung. Bodenverdichtungen haben neben zahlreichen im folgenden beschriebenen Nachteilen auch gewünschte Effekte, wie z. B. die Rückverfestigung eines zu lockeren Saatbettes. Führt man sich die Entwicklung der modernen Landtechnik vor Augen, ist zu erkennen, dass in den letzten Jahren allein aufgrund der steigenden Radlasten die Bodenschadverdichtungen auf landwirtschaftlich genutzten Flächen zugenommen haben müssen.

\footnotetext{
${ }^{52}$ Vgl. SOMMER et al. (2001), S. 16
} 
Tabelle 4: Entwicklung der Flächenleistung und Radlasten von Maschinen und Geräten in der deutschen Landwirtschaft

\begin{tabular}{lcc}
\hline & $\mathbf{1 9 7 0}$ & $\mathbf{2 0 0 0}$ \\
\hline Flächenleistung (ha/h): & & \\
\hline Pflügen & $0,8-1$ & $2-3$ \\
& $(50 \mathrm{~kW})$ & $(120 \mathrm{~kW})$ \\
\hline Mähdrusch & $1-2$ & $2-5$ \\
& $(3 \mathrm{~m})$ & $(6 \mathrm{~m})$ \\
\hline Rübenernte & 0,2 & 1 \\
& $(1-$ reihig) & $(6-$ reihig) \\
\hline Radlast (t): & & \\
Schlepper & $1-2$ & $2-3$ \\
Mähdrescher (beladen) & $2-3$ & $4-8$ \\
Rübenroder (beladen) & $1-3$ & $6-10$ \\
\hline
\end{tabular}

Quelle: Sommer, Lebert u. Brunotte (2003), S. 178

Die größte potenzielle Gefahr für den Boden geht demnach von den heutigen Rübenrodern mit Radlasten von bis zu 10 t, und von Mähdreschern aus. Zu beachten sind allerdings auch die Rahmenbedingungen des Einsatzes. So nimmt z. B. die Gefährdung einer Bodenschadverdichtung mit zunehmenden Feuchtegehalt und Schluffanteil im Boden stark zu. Dies führt deshalb v. a. auch bei der im Herbst stattfindenden Zuckerrübenernte zu Problemen. Diese werden weiterhin durch die Tatsache verschärft, dass die hohen Anschaffungskosten von Rübenrodern durch eine hohe Auslastung in den Maschinenringen oder Lohnunternehmen von über 500 ha/a ausgeglichen werden, obwohl die Abschreibungsschwelle bei den KTBL-Datensammlungen nur bei etwa 250 ha/a liegen.

Neben den Radlasten und Kontaktflächendrücken führt v. a. die Überrollhäufigkeit dazu, dass der Boden immer stärker verdichtet wird. Zapf ${ }^{53}$ hat in diesem Zusammenhang ermittelt, dass beim ordnungsgemäßen Winterweizenanbau nur $27 \%$ der Fläche unbefahren bleiben und $58 \%$ drei- bis sechsmal überfahren werden. Die höchste Überrollhäufigkeit betrug 23. Beim Silomaisanbau werden $43 \%$ bis zu neun Mal und $29 \%$ mehr als neun Mal überfahren, bei Maximalwerten von 24 Überrollungen.

In der Rechtsprechung wird das Problem der Begrenzung der Verdichtung besonders deutlich. In einem Urteil des OLG NAUMBURG ${ }^{54}$ ging es um die Pflicht zur ordnungsgemäßen Bewirtschaftung von Pachtflächen. Ein Landwirt (A) hatte mit einem anderen (B)

\footnotetext{
${ }^{53} \mathrm{Vgl.} \mathrm{ZAPF} \mathrm{(1997)}$

${ }^{54}$ Vgl. OLG NAUMBURG (2002)
} 
einen Pflugtausch vereinbart, bekam den Schlag jedoch mit sichtbaren Bodenverdichtungen und Fahrspuren, die durch eine nasse Silomaisernte entstanden waren, zurück. Daraufhin verklagte er den B auf Schadenersatz. Dieser sollte u. a. die Kosten für die Beseitigung der Bodenverdichtungen enthalten. Das Gericht entschied, dass die Durchführung der Ernte nicht gegen die gfP verstoßen hat, zumal der Landwirt (B) schon mit der Ernte begonnen hatte, als der Regen einsetzte. Daraufhin unterbrach er die Ernte für 4 Tage, bevor er sie fortsetzte. Bei der Bewirtschaftung von Ackerflächen sind Bodenverdichtungen, nach Meinung des Gerichts nicht grundsätzlich zu vermeiden. Der Landwirt (B) hätte jedoch die Fahrspuren wieder durch Tiefenlockerung beseitigen müssen. Deshalb wurde er u. a. zu Schadensersatz in Höhe der Kosten der Tiefenlockerungsmaßnahme verurteilt.

Bodenschadverdichtungen führen durch den Eingriff in den Bodenhaushalt insofern zu ökonomischen Beeinträchtigungen, als dass v. a. die Erträge nachhaltig negativ beeinflusst werden. Diese Auswirkungen sind innerhalb einer Literaturrecherche in der sich im Anhang befindlichen Übersicht A-1 zu ersehen. ${ }^{55}$

Die betriebswirtschaftlichen Wirkungen von Bodenschadverdichtungen sind nicht exakt zu erfassen. Beispiele hierfür sind die Verzögerung der Abreife der Pflanzen wie auch die daraus resultierenden steigenden Trocknungskosten. Außerdem werden die Ertragsreduzierungen durch den technischen Fortschritt überlagert und hängen sehr stark von den Rahmenbedingungen wie Witterung, Produktionstechnik sowie Pflanzenarten ab. ${ }^{56}$ SoMMER $^{57}$ weist 1985 darauf hin, dass zur Erarbeitung der kausalen Zusammenhänge zwischen der Belastung des Bodens und dessen Auswirkungen auf das Pflanzenwachstum noch erhebliche Forschungsanstrengungen nötig sind. Nach Sichtung der Literatur in diesem Bereich ist diese These auch in der Gegenwart noch gültig, wenngleich er später konstatiert, dass die Funktion des Ertrages in Abhängigkeit vom PV eine Parabel darstellt, deren Scheitelpunkt von der Bodenfeuchte abhängt. ${ }^{58}$ Es fehlen v. a. wissenschaftlich fundierte Kenntnisse, um die Ursache-Wirkungsbeziehung befriedigend darzustellen. Außerdem gibt es keinen allgemeingültigen Verdichtungszustand, von dem ab mit Er-

\footnotetext{
${ }^{55} \mathrm{Vgl}$. Hierzu auch Kapitel 4.

${ }^{56}$ Vgl. LindSTROM u. VOORHEES (1994), S. 276 u. HANUS (1990), S.149

${ }^{57}$ Vgl. SOMMER (1985), S. 381

${ }^{58}$ Vgl. SOMMER et al. (1994), S. 191
} 
tragseinbußen $\mathrm{zu}$ rechnen ist. ${ }^{59}$ Eine diesbezügliche Zusammenfassung aber auch der Hinweis auf die Schwierigkeiten bei dem Nachweis der Kausalkette Bodenschadverdichtung und ökonomisch verwertbarem Ertrag ist in DÜRR et al. ${ }^{60} \mathrm{zu}$ finden. PETELKAU hat in diesem Zusammenhang Richtwerte entwickelt ${ }^{61}$, die allerdings nur als grober Anhaltspunkt für Ertragseinbußen verwendet werden können und einer erneuten Überprüfung bedürfen.

Tabelle 5: Zu erwartende Ertragsausfälle durch Überschreiten der substratspezifischen Grenzwerte für die Lagerungsdichte

\begin{tabular}{cc}
\hline $\begin{array}{c}\text { Zunahme der Lagerungsdichte } \\
\left(\mathbf{g} / \mathbf{c m}^{\mathbf{3}}\right)\end{array}$ & $\begin{array}{c}\text { Minderung des Ertrages } \\
(\mathbf{\%})\end{array}$ \\
\hline 0,10 & $5-7$ \\
0,15 & $10-12$ \\
0,20 & $15-20$ \\
0,25 & $25-30$ \\
0,30 & $>40$ \\
\hline
\end{tabular}

Quelle: Petelkau (1987), zit. in Hanus (1990), S. 150

SOMMER $^{62}$ gibt bei einmaliger starker Verdichtung Mindererträge bei Zuckerrüben von $11 \%$, bei Winterweizen von $12 \%$ und bei Wintergerste von $26 \%$ an. Zudem sind Nachwirkungen in den folgenden zwei Jahren gemessen worden.

Bei den Auswirkungen von Bodenschadverdichtungen auf den Ertrag müssen ferner Direkt- und Nachwirkungen unterschieden werden. Tabelle 5 zeigt bspw. die Direktwirkungen von Bodenschadverdichtungen. Es muss allerdings davon ausgegangen werden, dass auch in den Folgejahren der Erstverdichtung Ertragseinbußen auftreten. In Feldversuchen weist HORN ${ }^{63}$ bspw. im Jahr der Verdichtung des Bodens Ertragsminderungen von bis zu $50 \%$ nach, die in den Folgejahren immer noch $15 \%$ betragen. $\mathrm{Zu}$ den Effekten der Nachwirkung von Bodenschadverdichtungen sind zudem noch erhöhte Maschinenkosten wegen der höheren Widerstände zu berücksichtigen. ${ }^{64}$ Hierzu gibt es allerdings keine weiteren Hinweise in der Literatur.

\footnotetext{
${ }^{59}$ Vgl. HANUS (1990), S. 150

${ }^{60}$ Vgl. DÜRR et al. (1995), S. 56

${ }^{61} \mathrm{Vgl}$. Tabelle 5

${ }^{62}$ Vgl. SOMMER (1985), S. 381

${ }^{63}$ Vgl. HORN (1999)

${ }^{64}$ Vgl. SOMMER et al. (1994a)
} 
Ein weiterer Nachteil der Bodenschadverdichtung ist die geringere Nährstoffeffizienz der Pflanzen. Helal et al. ${ }^{65}$ weisen in Gefäßversuchen nach, dass Sommerweizen (Sommergerste) aus verdichtetem Boden $21 \%(31 \%)$ weniger N, $18 \%$ (26\%) weniger P und $17 \%(24 \%)$ weniger $\mathrm{K}$ aus dem verdichteten Boden entzieht und die Kornerträge demzufolge $15 \%$ (26\%) geringer sind.

In der folgenden Abbildung 6 ist der durch den anthropogenen Einfluss entstehende ökonomische Schaden auf landwirtschaftlichen Nutzflächen qualitativ zusammenfassend dargestellt.

\section{Abbildung 6: Ökonomische Schäden durch Bodenverdichtungen auf landwirtschaft-} lich genutzten Flächen

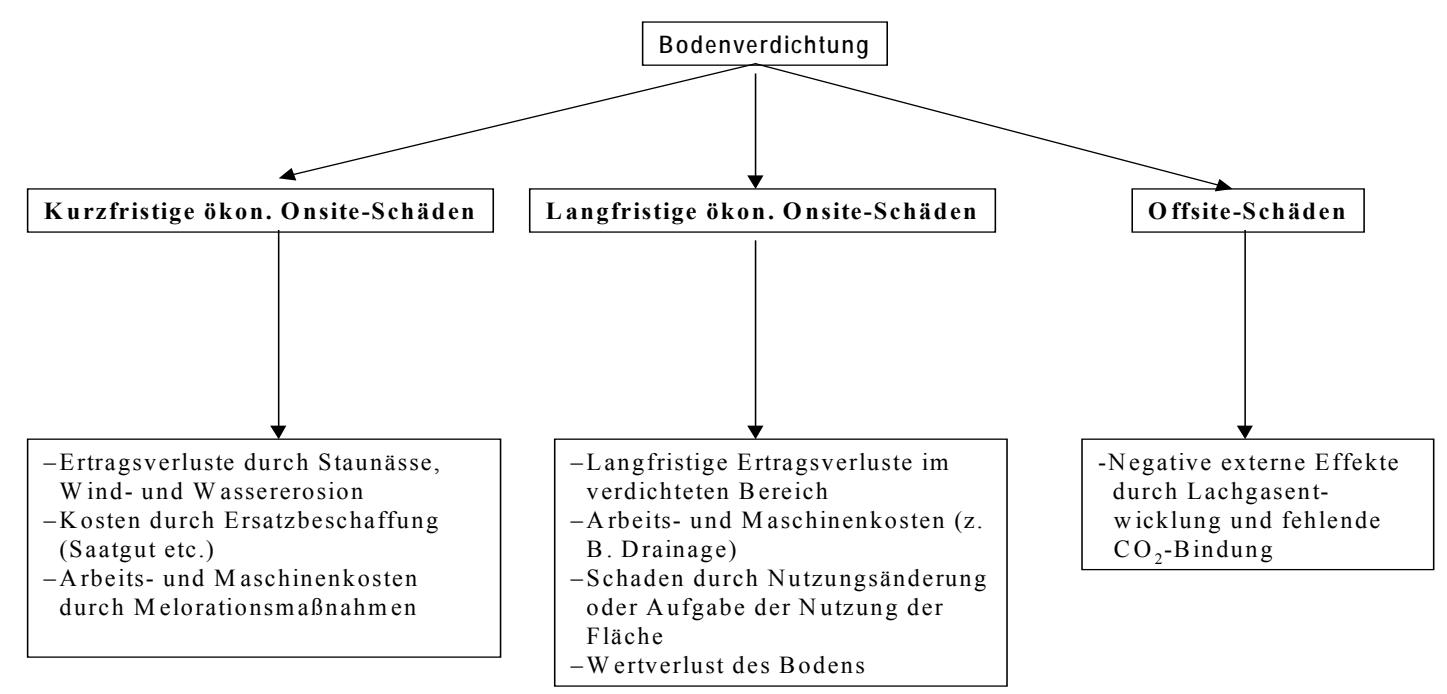

Quelle: Eigene Darstellung

Analog zu den ökonomischen Schäden der Bodenerosion lassen sich die Schäden der Bodenschadverdichtung ebenfalls in Onsite- und Offsite-Schäden sowie in zwei zeitlichen Betrachtungsebenen einteilen.

Kurzfristige ökonomische Onsite-Schäden ergeben sich durch Ertragsverluste, Kosten für Ersatzbeschaffungen, zusätzliche Arbeits- und Maschinenkosten sowie eine geringere 
Nährstoffeffizienz der Pflanzen. Die langfristigen ökonomischen Onsite-Schäden bestehen aus den eben genannten Schäden wie die langfristigen Ertragsverluste, Arbeits- u. Maschinenkosten, Kosten durch Nutzungsänderung oder Aufgabe der Nutzung sowie dem allerdings kaum zu quantifizierenden Wertverlust des Bodens ${ }^{66}$. Die Offsite-Schäden bestehen aus den negativen externen Effekten, z. B. durch Lachgasbildung, fehlende $\mathrm{CO}_{2}$-Bindung und indirekt auch durch einen erhöhten Oberflächenabfluss. Dies ist darin begründet, dass die Bodenschadverdichtung ein Parameter für die Entstehung von Bodenerosion ist. Durch die Verdichtung des Bodens wird die Wasseraufnahmefähigkeit gesenkt, dadurch fließt mehr Oberflächenwasser ab, was Bodenerosion induziert.

\subsubsection{Umfang und Bedeutung von Bodenschadverdichtungen in Deutschland}

Innerhalb der letzten 40 Jahre erhöhte sich der Mechanisierungsgrad in der Landwirtschaft stetig. Der Einsatz von Ackerschleppern mit den entsprechenden zugehörigen Maschinen zeichnet sich durch eine fortlaufende Gewichtszunahme aus, welche gerade im Bereich der Radlasten von Erntemaschinen signifikant ist. Analog zum Kapitel 3.1.1.4 fehlen auch bei der tatsächlichen Verbreitung der Bodenschadverdichtung in Deutschland flächendeckende Daten. Anders als bei der Bodenerosion kann bei der Bodenschadverdichtung die Gefährdung nicht mit einer ähnlichen empirischen Gleichung wie der ABAG ermittelt werden. Zur Erstellung der folgenden Abbildung haben ERHARD et al. ${ }^{67}$ daher empirische, bodenartabhängige Regressionsgleichungen entwickelt und auf die Bodenübersichtskarten (BÜK 1000) angewandt. In die Regressionsgleichungen fließen Parameter wie Trockenrohdichte, Luftkapazität, nutzbare Feldkapazität, Totwasser- und Humusgehalt sowie die Vorbelastung bei pF 1,8 sowie die jeweiligen Spurflächensummen der Kulturarten ein.

\footnotetext{
${ }^{66}$ Vgl. JAROSCH U. ZEDDIES (1994)

${ }^{67}$ Vgl. ERHARD et al. (2002), S. 42 f.
} 


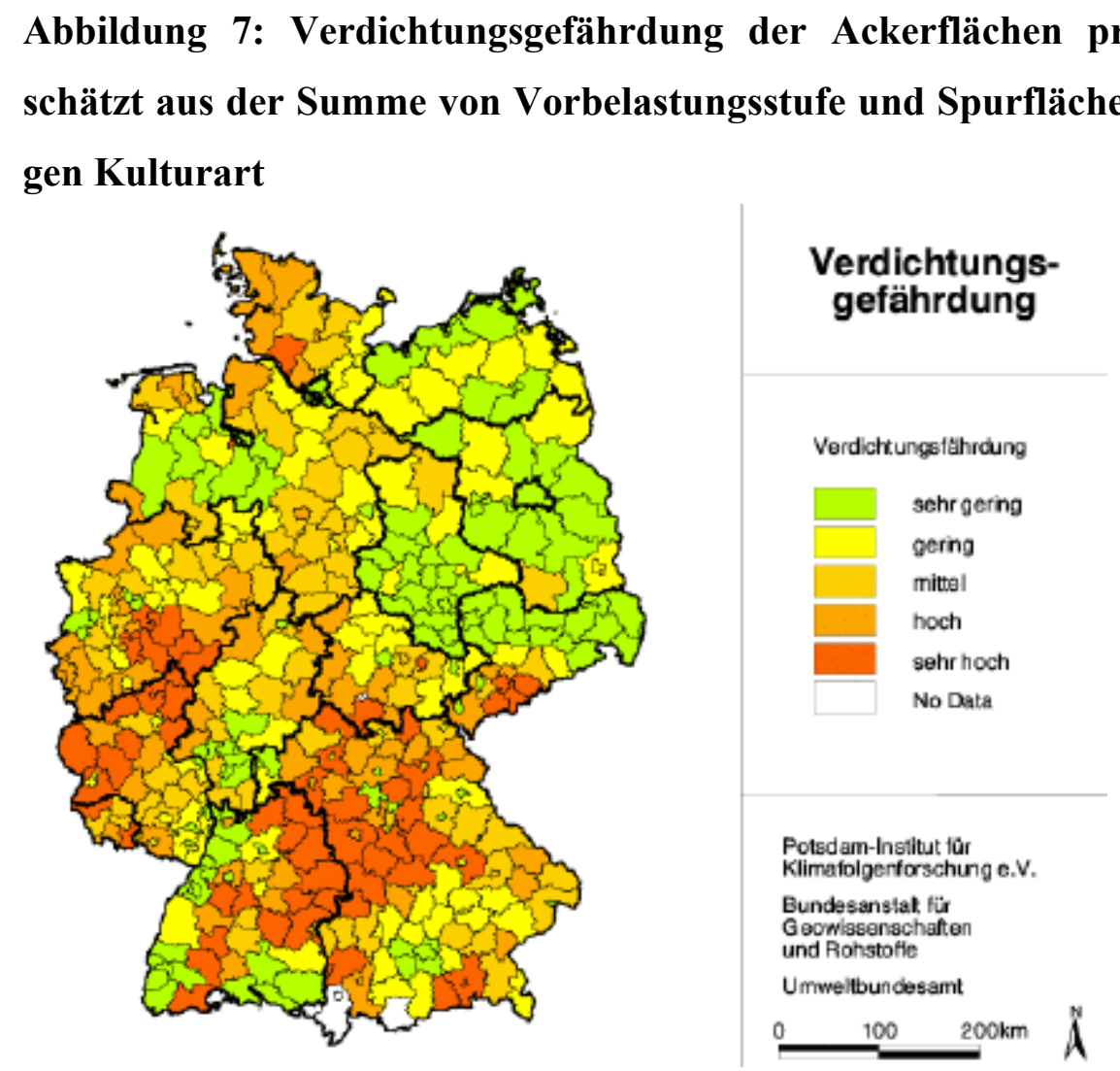

Quelle: ERHARD et al. (2002), S. 80

In der Abbildung 7 wird deutlich, dass die Verdichtungsgefährdung in Deutschland wesentlich stärker zu differenzieren ist als die Erosionsgefährdung. Die Verdichtungsgefährdung wird bspw. neben den Bodenarten Lehm, Löss und Lösslehm sehr stark von den angebauten Kulturarten bestimmt. Die Spurflächensummen sind bei Zuckerrüben, Silomais und Kartoffeln um ein Drittel höher als bei Getreide. ${ }^{68}$ Eine wichtige Rolle hierbei spielt wiederum die Art der Bewirtschaftung sowie der Anteil des organischen Düngers an der Gesamtdüngermenge.

Es wird deutlich, dass die höchste Verdichtungsgefährdung in Mittelgebirgsregionen im Sauerland sowie in der bayrisch-schwäbische Alp erreicht werden, wo hohe Vorbelastungsstufen und regional hohe Anteile an Hackfrüchten zusammentreffen. In SchleswigHolstein und Südniedersachsen sind ebenfalls hohe Verdichtungsgefährdungen zu erkennen, was mit den angebauten Kulturen mit hohen Spurflächensummen zusammenhängt. ${ }^{69}$ Die Autoren machen allerdings generelle Einschränkungen bei der Aussagekraft dieser

\footnotetext{
${ }^{68} \mathrm{Vgl}$. hierzu ZAPF (1998)

${ }^{69}$ Vgl. ERHARD et al. (2002), S. 80 f.
} 
Abbildung. Bspw. ist nicht abschließend zu erklären, warum die Verdichtungsgefährdung in den intensiven Zuckerrübenanbaugebieten der Leipziger und Magdeburger Börde gering ist. Die Autoren führen dies auf Unvollständigkeiten der Daten- und Bewertungsgrundlagen zurück. Ein Erklärungsansatz wären die geringen Niederschläge und somit die geringen Bodenfeuchten in diesen Gebieten.

Neben dem aktuellen Forschungsvorhaben von ERHARD et al. gibt es weitere ältere und speziellere Studien zum Bereich der Bodenschadverdichtungen auf landwirtschaftlichen Nutzflächen. Bspw. zeigen Studien von 144 niedersächsischen Ackerbaustandorten, dass durch den Einsatz moderner und schwerer Landtechnik die Schlepperradsohle im Zeitraum von 1952 bis 1982 von $25 \mathrm{~cm}$ Tiefe auf die Tiefe von $35 \mathrm{~cm}$ vorangetrieben wurde. Die Lagerungsdichte stieg dabei von 1,58 auf $1,63 \mathrm{~g} \mathrm{~cm}^{-3} \cdot{ }^{70}$ EHLERS, SCHMIDTKE UND RAUBER $^{71}$ untersuchten ebenfalls die Situation der südniedersächsischen Lössböden unter Ackernutzung und stellen hohe bis sehr hohe Verdichtungen in der Krumenbasis fest und weisen nach, dass Verdichtungen in eine Tiefe von 40 bis $45 \mathrm{~cm}$, an einigen Standorten in Tiefen bis $80 \mathrm{~cm}$ vorangetrieben wurden. Außerdem messen sie auf einem Standort Schadverdichtungen, deren Ausmaße über die Proctordichte ${ }^{72}$ hinausgehen. Als Hauptgrund sehen die Autoren den Einsatz von sechsreihigen Köpf-Rode-Bunkern zur Zuckerrübenernte unter zu feuchten Bedingungen. Als Konsequenz aus den Ergebnissen fordern sie eine Begrenzung der Radlasten für landwirtschaftliche Fahrzeuge und Geräte. Die kritische Betrachtung der Ergebnisse wird von ISENSEE $^{73}$ geführt. Analog zu EHLERS, SCHMIDTKE UND RAUBER vergleichen GIESKA et al. ${ }^{74}$ die aktuelle physikalische Degradierung des Ober- und Unterbodens in der Hildesheimer Börde anhand der Aggregatstabilität und der Messung der Dichte mit Messungen aus den sechziger Jahren. Zwar gab es nach ihren Ergebnissen vor einigen Jahrzehnten bereits Degradationserscheinungen, jedoch haben sich diese in den letzten Jahren verstärkt. Bspw. weist der Unterboden der untersuchten Standorte eine 95 \%ige Proctordichte auf. Diese Ergebnisse scheinen demnach diejenigen von EHLERS, SCHMIDTKE und RAUBER, zumindest für intensiv genutzte Ackerbaustandorte mit Zuckerrübenanbau, zu bestätigen.

\footnotetext{
${ }^{70}$ Vgl. BMVEL (2001), S.22

${ }^{71}$ Vgl. EhLERS, SCHMidTKe U. RAUber (2003), S. $11 \mathrm{ff}$.

72 Die Proctordichte ist eine definierte Dichte des Bodens, die unter bestimmten Laborbedingungen maximal erreicht werden kann.

${ }^{73} \mathrm{Vgl}$. ISENSEE (2003), S. 64

${ }^{74}$ Vgl. GIESKA et al. (2003)
} 
ZAPF $(1997)^{75}$ stellt für Bayern fest, dass bei hohen Bodenfeuchten und praxisüblicher Erntetechnik bei der Fruchtfolge WR-WW-WG auf ca. 30 \% der ldw. genutzten Fläche Schädigungen des Unterbodens gemessen wurden. Bei Zuckerrüben wurden bis zu einer Tiefe von $50 \mathrm{~cm}$ auf $70 \%$ der Anbaufläche Schadverdichtungen festgestellt.

Da jedoch momentan keine Vorsorge-, Prüf- oder Maßnahmenwerte für Bodenschadverdichtungen vorliegen, ist es nicht möglich, eine Systematik für schädliche Bodenveränderungen in Hinblick auf die Bodenschadverdichtung zu erstellen.

\subsubsection{Einflussmöglichkeiten der Landwirtschaft auf die Bodenschadverdichtung}

Der Anspruch an die Arbeitsproduktivität landwirtschaftlicher Produktionsverfahren führt zu größeren Fahrzeugen mit höheren Radlasten. Der Landwirt hat durch verschiedene Maßnahmen die Möglichkeit, folgende Einflussfaktoren auf die Bodenschadverdichtung positiv zu beeinflussen, z. B.

- Radlasten,

- Bewirtschaftungsform und -zeitpunkt,

- Reifeninnendruck,

- Kontaktflächendruck,

- Walkbarkeit,

- Radschlupf und

- Zugkraftbedarf. ${ }^{76}$

Wie bereits in Kapitel 1.1.2 dargestellt, spielen die eingesetzten Radlasten eine zentrale Rolle. Auch wenn die Wirkungen der Radlasten durch die Verbreiterung der Aufstandsfläche vermindert werden kann, werden durch steigende Radlasten die Schadverdichtungsprozesse stärker im Boden vorangetrieben.

Die Bewirtschaftungsform ist ein weiterer entscheidender Einflussfaktor. Es haben einige Feldversuche gezeigt, dass konservierend bestellte Flächen durch eine bessere Bodenstruktur tragfähiger sind. ${ }^{77}$ Der Bewirtschaftungszeitpunkt spielt insofern eine Rolle, als

\footnotetext{
${ }^{75}$ Vgl. ZAPF, R. (1997), S. 28

${ }^{76}$ Vgl. TIJINK (2003), S. 40

${ }^{77}$ Vgl. hierzu EHLERS (2003), S. 15 und BMVEL (2001), S. 31
} 
dass die Tragfähigkeit des Bodens von der Bodenfeuchte determiniert wird. Feuchte Böden sind verdichtungsanfälliger als trockene Böden.

Der Reifeninnendruck beeinflusst die Aufstandsfläche des Reifens und den Kontaktflächendruck, der wiederum die Unterbodenschadverdichtung beeinflusst. Der Radschlupf und die damit zusammenhängenden Größen Walkbarkeit und Zugkraftbedarf spielen für die Verdichtung des Oberbodens bzw. der Krumenbasisverdichtung eine weitere entscheidende Rolle. Je höher der Radschlupf ist, desto stärker wird der Oberboden verdichtet.

Neben diesen grundlegenden Einflussfaktoren bestehen mittlerweile technische Möglichkeiten, Bodenverdichtungen zu reduzieren. Diese sind im Einzelnen:

- Reifendruckregelanlagen,

- Breit- bzw. Terrareifen,

- Verminderung der Fahrspuren und

- Tiefenspuranzeiger.

Diese Maßnahmen werden allerdings bzgl. ihrer Wirksamkeit und ihres Nutzens in den entsprechenden wissenschaftlichen Kreisen kontrovers diskutiert. ${ }^{78}$ Dies gilt vor allem für die technische Möglichkeit, die Spurtiefe mittels Sensoren zu messen und mit deren Hilfe auf die Schadverdichtungsprozesse zu schließen. ${ }^{79} \mathrm{Zu}$ einigen der obigen Maßnahmen bestehen bereits wissenschaftlich abgesicherte Ergebnisse. Dies zeigt die folgende Tabelle 6 am Beispiel der Verteilung des Bodendrucks gleicher Radlasten durch unterschiedliche Bereifung und Reifeninnendruck in den Ober- sowie Unterböden.

Tabelle 6: Gemessener Bodendruck unter drei verschiedenen Reifen bei einer Radlast von 6,8 t und verschiedenen Reifeninnendrücken

\begin{tabular}{lccc}
\hline \multicolumn{1}{c}{ Radlast } & $\mathbf{6 , 8}$ t & $\mathbf{6 , 8 ~ t}$ & $\mathbf{6 , 8 ~ t}$ \\
\hline Reifenart & $23,1 \mathrm{R} 26 \mathrm{BIB}^{\prime} \mathrm{X}$ & $30,5 \mathrm{LR} 32 \mathrm{BIB}^{\prime} \mathrm{X}$ & $73 \times 44,0-32 \mathrm{NHS}$ Terra \\
Reifeninnendruck & $2,4 \mathrm{bar}$ & $1,3 \mathrm{bar}$ & $1,2 \mathrm{bar}$ \\
\hline Bodendruck $(\mathbf{2 0} \mathrm{cm})$ & $>2 \mathrm{bar}$ & $1,5 \mathrm{bar}$ & $1 \mathrm{bar}$ \\
Bodendruck $(\mathbf{4 0} \mathrm{cm})$ & $1 \mathrm{bar}$ & $0,4 \mathrm{bar}$ & $0,3 \mathrm{bar}$ \\
\hline
\end{tabular}

Quelle: BMVEL (2001), S. 28

\footnotetext{
${ }^{78}$ Vgl. hierzu HORN (2003) und EHLERS, SCHMIDTKE U. RAUBER (2003)

${ }^{79}$ Vgl. HoRN (2001), S. 75
} 
Es ist zu erkennen, dass die Bodendrücke mit zunehmender Reifenbreite und abnehmendem Reifeninnendruck sich spürbar verringern. Bei dem eingesetzten Terrareifen sind die Bodendrücke am geringsten. Ein Vorteil dieser Bereifung ist neben der höheren Breite auch die Möglichkeit, mit geringeren Reifeninnendrücken zu fahren und somit den Kontaktflächendruck überproportional zu mindern.

Die Begrenzung der Radlasten landwirtschaftlicher Fahrzeuge ist ein Ansatz, der die Hauptursache von Bodenschadverdichtungen in modernen Ackerbausystemen sehr stark reduzieren würde. Diese Diskussion wird später im Kapitel 4.4.2 aufgegriffen.

\subsubsection{Stoffeinträge}

Im diesem Kapitel wird die Wirkung der Stoffeinträge auf den ldw. genutzten Boden dargestellt. Stoffeinträge gelangen hauptsächlich durch organische und anorganische Düngemittel sowie PSM in den Boden. Daher werden diese drei Kategorien im Folgenden betrachtet.

\subsubsection{Düngemittel}

Die Verwendung von anorganischen Düngemitteln in der deutschen Landwirtschaft seit 1990 wird in der folgenden Abbildung 8 dargestellt. Für Stickstoffdüngemittel ist ein weitestgehend konstanter Verbrauch festzustellen, die Verwendung von Phosphat- und Kalidüngemitteln ist jedoch rückläufig. Bei der Phosphatdüngung ist im Zeitraum von 1980 bis heute ein Rückgang um fast 75 \% für das gesamte Bundesgebiet festzustellen. Im Bundesdurchschnitt werden z. Zt. 19 kg $\mathrm{P}_{2} \mathrm{O}_{5} /$ ha über Mineraldünger ausgebracht. ${ }^{80}$

$\mathrm{Zu}$ bedenken ist in diesem Zusammenhang, dass die Weltvorräte an Rohphosphaten endlich sind. Die Schätzungen, wie hoch die derzeitigen Vorräte an verwendbaren Rohphosphaten zu bilanzieren sind, gehen sehr weit auseinander. Hinzu kommt, dass die Frage der Endlichkeit von qualitativ hochwertigen Rohphosphaten abhängt.

\footnotetext{
${ }^{80}$ Vgl. BuHSE (2003), S. 22
} 
Abbildung 8: Inlandsabsatz von anorganischen Düngemitteln in Deutschland

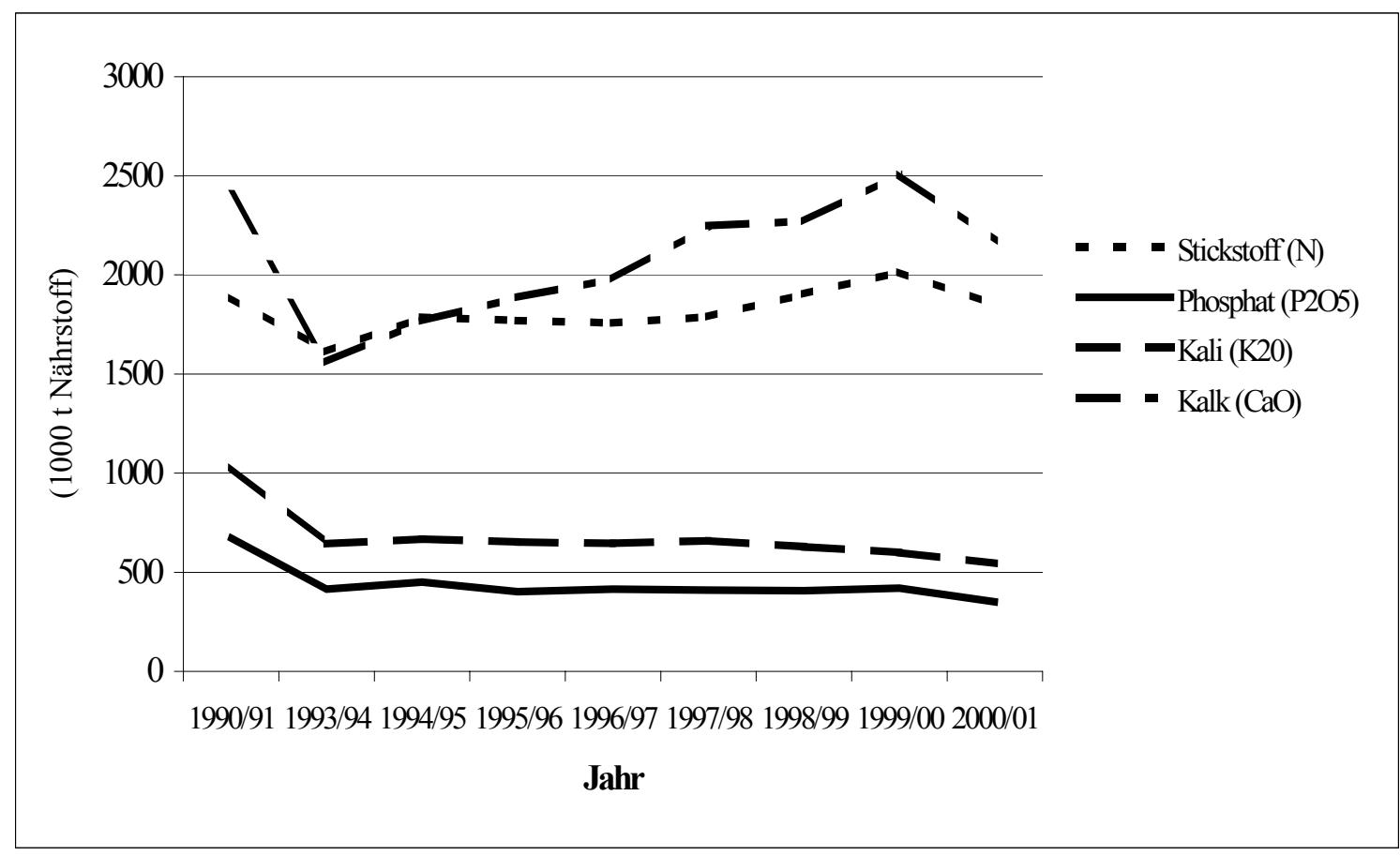

Quelle: BMVEL (2003)

Der Kalkeinsatz ist Mitte des letzten Jahrzehnts zunächst angestiegen, für das Wirtschaftsjahr 2000/01 besteht allerdings ein Rückgang.

In Deutschland werden jährlich etwa 29 Mio. t TS Wirtschaftsdünger mit verschiedenen Ausbringungstechniken, die Gegenstand des Kaptitels 3.1.3.1 sind, ausgebracht. ${ }^{81}$ Diese Ausbringungstechniken weisen unterschiedliche Verlustraten auf. Diese Verluste in Form von $\mathrm{NH}_{4}-\mathrm{N}$ belasten v. a. die Atmosphäre und führen zur Geruchsbelästigung der Bevölkerung. Aber auch hinsichtlich der Versorgung mit $\mathrm{P}_{2} \mathrm{O}_{5}$ weisen einige Böden sehr hohe Werte auf, die zu Umweltbelastungen bspw. durch Auswaschung führen können.

Das Hauptproblem, das von Düngemitteln ausgeht ist jedoch die N-Belastung. In Deutschland besteht aktuell ein Gesamtbilanzüberschuss von Stickstoff (anorganischer und organischer $\mathrm{N}$ ) in Höhe von $118 \mathrm{~kg} \mathrm{~N} / \mathrm{ha} / \mathrm{a}^{82}$, vor zwei Jahrzehnten betrug dieser Überschuss noch $160 \mathrm{~kg} \mathrm{~N} / \mathrm{ha}^{83}$. Die Gründe für die immer noch recht hohen Salden in Deutschland liegen in der geringen Ausnutzungseffizienz und dem schlechten Verhältnis zwischen Output und Input von $33 \%$ bei den organischen Düngemitteln. Allerdings ist

\footnotetext{
${ }^{81}$ Vgl. BANNIK (2002), S.17

${ }^{82} \mathrm{Vgl}$. AgRA-EUROPE (2003a), L 7

${ }^{83}$ Vgl. Gutser u. EBertseder (2002), S. 4 f.
} 
zu berücksichtigen, dass Deutschland im EU-Vergleich mit anderen Staaten im Mittelfeld liegt, bspw. weisen die Benelux-Staaten sowie Dänemark deutlich höhere N-Salden auf. Hohe Einsparpotenziale sind insbesondere bei intensiv viehhaltenden Betrieben durch den verstärkten Einsatz von verlustmindernder Ausbringungstechnik zu erwarten. Langfristig wird ein durchschnittlicher Bilanzüberschuss von 50 bzw. $80 \mathrm{~kg} \mathrm{~N} /$ ha angestrebt. $^{84}$ Im Grunde können nur Marktfruchtbaubetriebe und Futterbaubetriebe unter 0,5 GV/ha diesen Wert erreichen. ${ }^{85}$ Der SACHVERSTÄNDIGENRAT FÜR UMWELTFRAGEN (SRU) fordert in diesem Zusammenhang v. a. eine dringend notwendige Harmonisierung der Bilanzierungsmodelle auf der Ebene der europäischen Gemeinschaft. ${ }^{86}$

Neben den Bodenverlusten bei der Bodenerosion gehen v. a. auch $\mathrm{N}$ und P verloren. Mit einer Tonne Boden geht ein Verlust von 0,2 $\mathrm{kg} \mathrm{N}, 0,3-1,8 \mathrm{~kg}_{2} \mathrm{O}_{5}$ sowie $6,7 \mathrm{~kg} \mathrm{~K}_{2} \mathrm{O}$ einher. ${ }^{87}$ Dieser Abtrag führt wiederum zu externen Kosten durch die Belastung der Oberflächengewässer.

Die weitere Erforschung der Offsite-Schäden durch erodierte Flächen der Landwirtschaft hat sich sehr stark auf die hierdurch entstehende Gewässereutrophierung konzentriert. So zeigen Schätzungen von WERNER et al., dass 38 \% (1991: 58 \%) der Phosphoreinträge in die Nord- u. Ostsee auf Bodenerosion und 12,7 \% (1991: $12 \%$ ) auf den allgemeinen Oberflächenabfluss zurückzuführen sind. Bei den diffusen Stickstoffeinträgen resultieren 5,3 \% (1991: $11 \%$ ) aus der Bodenerosion und 3,7 \% (1991: $6 \%$ ) aus dem Oberflächenabfluss. Die Schwankungen zwischen beiden Schätzungen zeigen eine Ungenauigkeit, die jedoch die Aussagekraft nur gering herabsetzt. Es muss ebenfalls berücksichtigt werden, dass Binnengewässer noch direkter und somit stärker von den intervallartigen Schadstoffströmen betroffen sind. Einträge oder Atmosphärenbelastungen bei der Winderosion können nicht hinreichend abgeschätzt werden.

\footnotetext{
${ }^{84} \mathrm{Vgl}$. AGRA-EurOPE (2003a), L 7

${ }^{85}$ Vgl. GutSER u. EBERTSEDER (2002), S. $4 \mathrm{f}$.

${ }^{86}$ Vgl. SRU (2004), S. 14

${ }^{87}$ Vgl. Kapitel 6.3.1.1.3
} 


\subsubsection{Pflanzenschutzmittel}

In der folgenden Abbildung 9 ist der PSM-Absatz von 1990 bis 2001 dargestellt. Die PSM werden dabei in die einzelnen Untergruppen Herbizide, Fungizide, Insektizide sowie Wachstumsregler eingeteilt.

\section{Abbildung 9: PSM-Absatz in Deutschland (in Tausend t)}

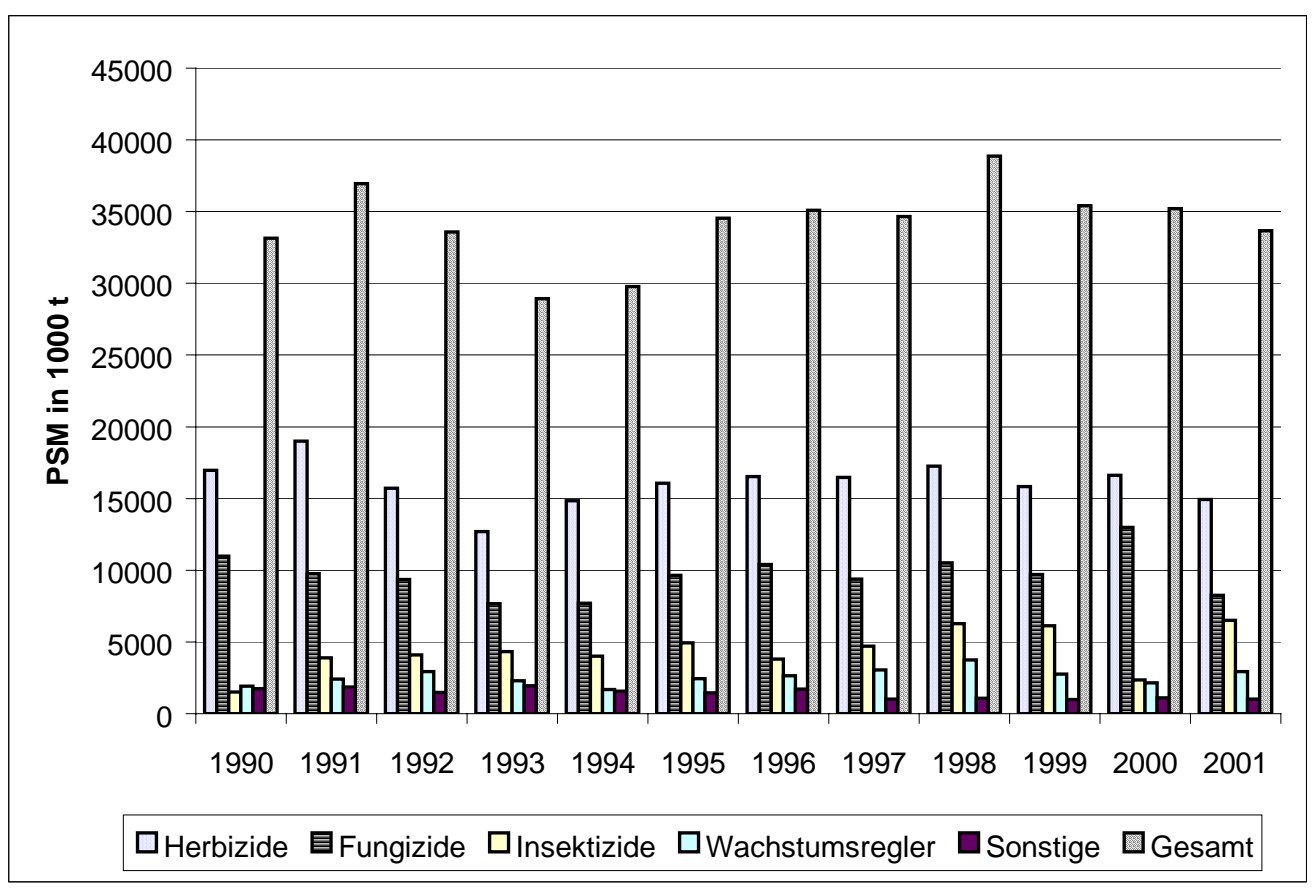

Quelle: UMwELtBUnDESAMT (2002)

Auffallend ist der Rückgang der abgesetzten PSM bis 1993 auf etwa 29.000 t. Seit 1994 ist bei den Herbiziden ein Anstieg zu erkennen. Laut UMwELtBundeSAmT ${ }^{88}$ sind die Gründe hierfür v. a. die Wiederinkulturnahme von stillgelegten Flächen, Umstellungen in den neuen Bundesländern sowie witterungsbedingte Schwankungen. Seit 1998 ist ein Rückgang in der abgesetzten Gesamtmenge zu erkennen. Ca. $50 \%$ der eingesetzten PSM bestehen aus Herbiziden, $26 \%$ aus Fungiziden und $3 \%$ aus Insektiziden. Die sonstigen Pflanzenschutzmittel setzen sich v. a. aus den inerten Gasen zur Konservierung von pflanzlichen Produkten zusammen.

In der öffentlichen Diskussion geht es i. d. R. um die Risiken, die vom Einsatz der PSM ausgehen. Diese Risiken werden gerade auch in der Bevölkerung als sehr hoch angese-

\footnotetext{
${ }^{88}$ Vgl. UMWELTBUNDESAMT (2002)
} 
hen, so dass der Einsatz von Pflanzenschutzmitteln negativ besetzt ist. Anhand des in der Biologischen Bundesanstalt entwickelten Modells SYNOPS kann jedoch gezeigt werden, dass das Umweltrisikopotenzial von Pflanzenschutzmitteln in den letzten Jahren sehr stark abgenommen hat und eine Differenzierung zwischen einzelnen Pflanzenschutzmitteln unumgänglich ist. Die Risiken durch die Lang- und Kurzzeitkonzentrationen von Herbiziden im Boden werden nach diesem Modell, hier der Vergleich des Jahres 2001 mit dem Referenzjahr 1987, auf 37 \% bzw. 15 \% reduziert. Eine ähnliche Risikominderung ergibt sich bei den Insektiziden ( $25 \%$ und $14 \%$ ). Lediglich bei den Fungiziden steigt das Risiko sehr stark an (47\% und $211 \%)$. Die Substitution von Triazinen durch Sulfonylharnstoffe hat eine sehr deutliche Reduktion des Risikos für den Boden (2,9\% und 0,4\% gegenüber 1987) zur Folge. ${ }^{89}$ Aufgrund dieser Ergebnisse ist es erstaunlich, dass es innerhalb der Europäischen Union kein einheitliches Ausbringungsverbot für Triazine wie z. B. Atrazin, ein Herbizid für den Maisanbau, gibt.

Die Wirkung von PSM im Boden ist äußerst unterschiedlich und hängt von vielen Faktoren, wie z. B. der Löslichkeit des Wirkstoffs ab. ${ }^{90}$ Insgesamt kommen etwa $20 \%$ der applizierten Menge direkt mit dem Boden in Kontakt und sind somit potenziell abtragsgefährdet. Die Bodenerosion spielt bei dem Transport von PSM-Verbindungen in Oberflächengewässer eine bedeutende Rolle. ${ }^{91}$ Für das mittlerweile in Deutschland verbotene Atrazin wurden auf Flächen bis $3 \%$ Hangneigung Austräge bis zu $2 \%$ der Aufwandmenge gemessen. Bei Hangneigungen von 10-15\% betrugen die Austräge $5 \%$. Nach Meinung von HoPPE sind diese Messungen für fast alle Herbizide repräsentativ. Bei einer mittleren Ausbringungsmenge von 2,5 kg/ha sind somit $0,5 \mathrm{~kg} / \mathrm{ha}$ potenziell abtragsgefährdet. Je nach vorherrschender Hangneigung können nach den Berechnungen von HALL 0,025-0,01 kg/ha ausgetragen werden.

Andere Schätzungen wie hier von FISCHER gehen davon aus, dass etwa 0,1-0,2 \% der Aufwandmengen durch Erosion und Oberflächenabfluss in Oberflächengewässer gelangen können. Insgesamt kann daher mit einem Abtrag von 40 t/a in Deutschland gerechnet

\footnotetext{
${ }^{89}$ Vgl. GuTSCHE (2002), S. 3

${ }^{90}$ Zur detaillierten Beschreibung der Wirkung von PSM im Boden siehe HopPE (1996), S. 27 ff.

${ }^{91} \mathrm{Vgl}$. HALL et al. (1983), S. 336 f. zit. in HOPPE (1996)
} 
werden, wovon 2-4 t/a in die oberirdischen Gewässer gelangen. ${ }^{92}$ Eine sehr detaillierte Übersicht über gemessene PSM im Grundwasser geben auch DOMROESE et al. ${ }^{93}$

Der Eintrag von PSM in den Boden geschieht nicht nur durch die Anwendung auf dem Feld, sondern ebenso durch die Reinigung der Pflanzenschutzmittelgeräte oder Unfälle. Diese Eintragspfade sind jedoch nicht Gegenstand der folgenden Betrachtungen.

Aus landwirtschaftlicher Sicht kann bei bestimmten Produktionsverfahren gänzlich auf PSM verzichtet werden. Der komplette Verzicht, der Einsatz von mechanischen Unkrautbekämpfungsverfahren und die konsequente Verfolgung der Ziele des Integrierten Anbaus werden im Kapitel 6.4.1 näher untersucht.

\subsubsection{Klärschlamm}

In Deutschland werden jährlich 4,1 Mio. t Klärschlamm produziert, etwa $44 \%$ des anfallenden Klärschlamms werden landwirtschaftlich verwertet. Dabei variiert dieser Anteil regional sehr stark, z. B. werden in Niedersachsen etwa $75 \%$ des anfallenden Klärschlamms landwirtschaftlich verwertet. An die Landwirte fließen dadurch ca. 50-75 Mio. $€ / \mathrm{a}$ an Zusatzeinkommen. ${ }^{94}$ Es werden jährlich zwischen 0,3 und $3 \%$ der Ackerfläche benötigt, um den Klärschlammanfall zu verwerten. Weitere Möglichkeiten der Verwertung des anfallenden Klärschlamms sind die thermische Verwertung sowie die Deponierung.

Die Wirkung des Klärschlamms im Falle der landwirtschaftlichen Verwertung ist umstritten und wird daher immer wieder in der politisch geprägten Öffentlichkeit diskutiert. In diesem Zusammenhang muss beachtet werden, dass Klärschlämme unterschiedliche Qualitäten, v. a. in Hinsicht auf die Schwermetallkonzentrationen, aufweisen. In der Tabelle 7 sind daher die durchschnittlichen Schwermetallgehalte sowie die jeweilige Ausnutzung der Grenzwerte der AbfKlärV dargestellt.

\footnotetext{
${ }^{92}$ Vgl. Fischer (1996), zit. in FredE U. DABBERT (1998), S. 14

${ }^{93}$ Vgl. hierzu DOMROESE et al. (2001)

${ }^{94}$ Vgl. MeLSA (2002), S. 4
} 
Tabelle 7: Durchschnittliche Schwermetallgehalte und prozentuale Grenzwertausschöpfung der AbfKlärV von bundesdeutschem Klärschlamm (in mg/kg)

\begin{tabular}{lccccccc}
\hline \multicolumn{1}{c}{ Schwermetalle } & Blei & $\begin{array}{c}\text { Cadmi- } \\
\text { um }\end{array}$ & Chrom & Kupfer & $\begin{array}{c}\text { Nickel } \\
\text { Quecksil- } \\
\text { ber }\end{array}$ & Zink \\
\hline$\varnothing$ Schwermetallgehalte & 63 & 1,4 & 46 & 274 & 23 & 1 & 809 \\
$(\mathrm{mg} / \mathrm{kg})$ & $7 \%$ & $14 \%$ & $5 \%$ & $34 \%$ & $11 \%$ & $12 \%$ & $32 \%$ \\
$\begin{array}{l}\text { Prozentuale Grenzwertaus- } \\
\text { schöpfung nach AbfKlärV }\end{array}$ & $7 \%$ & & & & & & \\
\hline
\end{tabular}

Quelle: GunREBEN (2002), S. 2

An der Tabelle 7 ist ersichtlich, dass die Grenzwerte der AbfKlärV durch die Ausbringung von durchschnittlich belastetem Klärschlamm noch weit unterschritten sind. Problematisch sind daher nur die hochkonzentrierten Klärschlämme. Ein weiteres Problem bei der Klärschlammentsorgung stellen spezielle Stoffe wie z. B. persistente Kohlenwasserstoffe dar, deren Wirkung im Boden noch nicht hinreichend untersucht worden ist.

Um die Risiken für die Landwirte abzusichern, wurde ein sog. gesetzlicher Klärschlammfonds nach § 9 DMG eingerichtet, in denen die Klärschlammproduzenten regelmäßig einzahlen müssen und dessen Volumen etwa 128 Mio. $€$ betragen muss. Innerhalb des freiwilligen Klärschlammfonds der Klärschlammproduzenten sind seit 1990 in insgesamt 14 Fällen Entschädigungsleistungen in Höhe von insgesamt $19.429 €$ gezahlt worden.

Aus Sicht der Düngung ist v. a. der hohe Anteil an Phosphor ein wichtiges Argument für die Ausbringung von Klärschlämmen. Wenn die gesamte Menge des in kommunalen Anlagen produzierten Klärschlamms landbaulich verwertet würde, könnte diese Menge den Bedarf an Phosphat in Deutschland zu einem Drittel decken. ${ }^{95}$ Zudem weist dieses Phosphat eine hohe Pflanzenverfügbarkeit auf. Bei einer durchschnittlichen Aufbringungsmenge von $5 \mathrm{t} \mathrm{TM} / \mathrm{ha}$ ergibt sich eine Nährstoffzufuhr von $75 \mathrm{~kg} \mathrm{P} / \mathrm{ha}, 41 \mathrm{~kg} \mathrm{~N} / \mathrm{ha}$, $35 \mathrm{~kg} \mathrm{Mg} / \mathrm{ha}$ sowie $460 \mathrm{~kg} \mathrm{Ca} / \mathrm{ha}$.

Z. Zt. wird auf verschiedenen Ebenen eine Diskussion über die Zukunft der landwirtschaftlichen Klärschlammverwertung z. T. sehr emotional geführt. An dieser Stelle wird diese komplexe Diskussion nicht im Ganzen aufgegriffen, da das Problem der Klärschlammausbringung in Bezug auf den Bodenschutz nur aufgrund der Auswirkungen auf

\footnotetext{
${ }^{95}$ Vgl. MÖNICKE U. KÜHN (2003), S.33
} 
den Einzelbetrieb und nicht aus volkswirtschaftlicher Sicht untersucht wird. ${ }^{96}$ Es ist allerdings nach einem Konzept der Bundesregierung vorgesehen, die Grenzwerte für Klärschlämme zu senken und auch Wirtschaftsdünger in dieses Konzept mit einzubeziehen. Dies hätte zur Folge, dass derzeit v. a. Schweinegülle aufgrund der hohen Zink- und Kupfergehalte nicht mehr vollständig ausgebracht werden dürfte. Die Bundesregierung ist sich allerdings sicher, dass durch Kupfer- und Zinkminderungstechniken bei der Herstellung von Futtermitteln und -zusatzstoffen die Gehalte drastisch reduziert werden können. Ein Verbot der ldw. Klärschlammausbringung hätte nach Schätzungen für das Bundesland Bayern zur Folge, dass die Abwasserkosten für die Bevölkerung um $0,07 € / \mathrm{m}^{3}$ für Anlagen für mehr als 20.000 Einwohner und um $0,18 € / \mathrm{m}^{3}$ für Anlagen für weniger als 20.000 Einwohner steigen würden. Dies liegt an den höheren Kosten der thermischen Beseitigung von Klärschlamm. ${ }^{97}$

\subsubsection{Kompost}

In Deutschland werden jährlich etwa 3,5-4 Mio. t Biokompost hergestellt. ${ }^{98}$ Etwa $40 \%$ des anfallenden Biokomposts wird landwirtschaftlich verwertet. Biokompost zeichnet sich v. a. durch seine hohen Anteile an organischer Substanz (24-51\% TM) aus. Dem Boden werden durch Kompostaufbringung jedoch auch alle anderen Hauptnährstoffe zugeführt. Innerhalb von drei Jahren können nach $\S 6$ Abs. 1 BioAbfV maximal 30 Tonnen Biokompost TM/ha ausgebracht werden, wenn bestimmte Grenzwerte für Schwermetalle nicht überschritten werden. Durch diese Menge werden im Durchschnitt $390 \mathrm{~kg} \mathrm{~N}$, $210 \mathrm{~kg} \mathrm{P}_{2} \mathrm{O}_{5}, 330 \mathrm{~kg} \mathrm{~K} 20,1.200 \mathrm{~kg} \mathrm{CaO}$ sowie $210 \mathrm{~kg} \mathrm{MgO}$ ausgebracht. Dabei ist zu beachten, dass der Stickstoff im Biokompost zum großen Teil an die organische Substanz gebunden und somit nicht als unmittelbar pflanzenverfügbar anzusehen ist. Deshalb sind im Anwendungsjahr lediglich fünf Prozent des Gesamtstickstoffs in der Düngebedarfsermittlung anrechenbar. In den Folgejahren beträgt die durch Mineralisierung stattfindende Freisetzung des Stickstoffs jeweils etwa drei Prozent. ${ }^{99}$

Biokompost hat im Bezug auf den Bodenschutz zahlreiche positive Effekte auf den Boden:

\footnotetext{
${ }^{96}$ An dieser Stelle sei auf das Programm „Gute Qualität und sichere Erträge“ der Bundesregierung hingewiesen.

${ }^{97}$ Vgl. AGRA-Europe (2004b), S. L 15
} 
- Erhöhung des Humusgehalts,

- Aktivierung des Bodenlebens,

- Verbesserung der Bodenstruktur und des Wasserhaushalts,

- Aggregatstabilisierung des Bodens durch Kalkzufuhr und

- direkte Verringerung der Erosion.

Diese Vorteile werden im Kapitel 6.4.2.2.2 näher untersucht.

\subsection{Ergebnisse der Expertenbefragung}

Im Folgenden werden die Ergebnisse der Expertenbefragung zu den Gefährdungen des Bodens durch landwirtschaftliche Nutzung dargestellt. Im ersten Teil des Fragebogens wurden Fragen zum gegenwärtigen Zustand des landwirtschaftlich genutzten Bodens gestellt. Die Experten sollten diesen sowohl für physikalische als auch für stoffliche Bodenbelastungen einschätzen. Die Ergebnisse sind in der Abbildung 10 dargestellt.

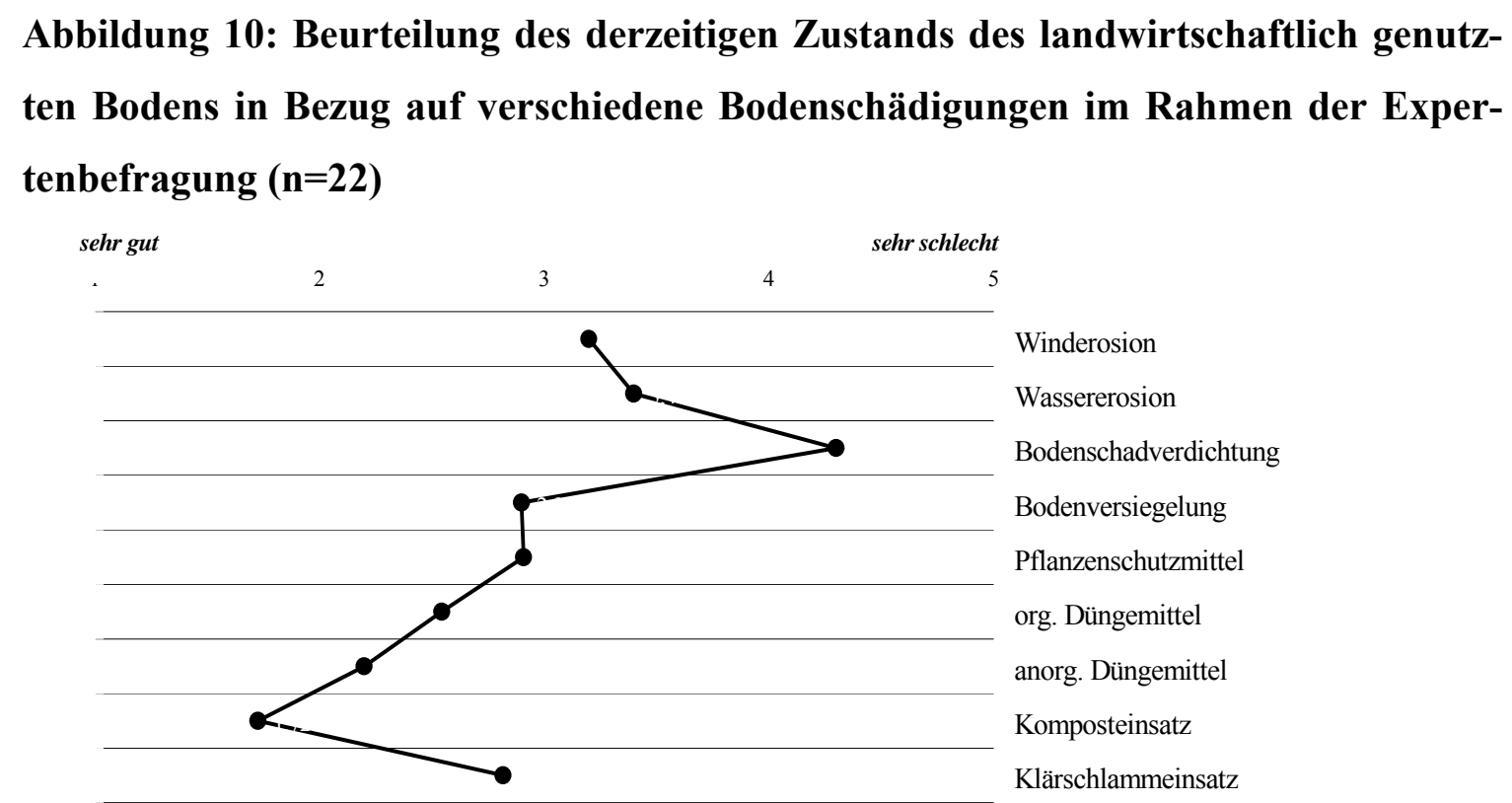

Quelle: Eigene Darstellung

\footnotetext{
${ }^{98}$ Vgl. AID (2003), S. 5

${ }^{99}$ Vgl. ebd. S. 7 und KuHLMAnN et al. (1995), S. 40
} 
Es wird deutlich, dass die Experten den Zustand des Bodens in Bezug auf die stofflichen Schädigungen als eher gut einschätzen. Probleme sehen sie demnach eher bei den physikalischen Schädigungen, v. a. bei der Bodenschadverdichtung.

Weiterhin sollten die Experten einschätzen, welche Einträge bzw. Bodenbelastungen ihrer Meinung nach die größten Gefahrenpotenziale für den ldw. genutzten Boden bilden. Dabei waren drei Kategorien vorgegeben, so dass eine Rangfolge erzeugt wurde. Die Rangfolge wurde dann mit den Werten drei, für das höchste Gefährdungspotenzial, sowie zwei und eins für die folgenden Kategorien bewertet und den entsprechenden Häufigkeiten zugeordnet. Das Ergebnis ist in der Abbildung $11 \mathrm{zu}$ sehen.

\section{Abbildung 11: Belastungen und Einträge mit den nach Meinung der Experten höch- sten Gefahren für den ldw. genutzten Boden $(n=24)$}

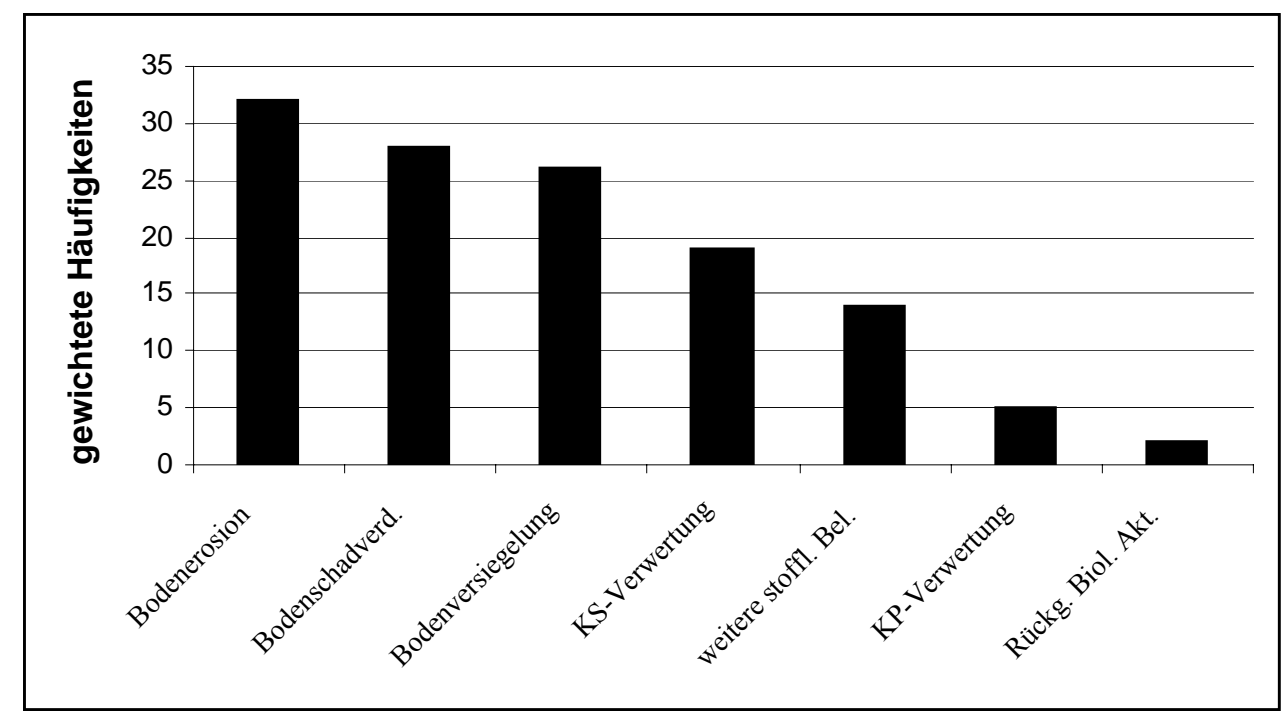

Quelle: Eigene Darstellung

Es ist zu erkennen, dass nach Meinung der Experten v. a. die physikalischen Bodenschädigungen wie Bodenerosion sowie -schadverdichtung und -versiegelung die höchsten Gefahrenpotenziale aufweisen. Bei den stofflichen Bodenschädigungen sind sie der Meinung, dass das höchste Gefahrenpotenzial bei der Klärschlammverwertung besteht.

Die letzte Frage zu dem Thema des Zustandes des ldw. genutzten Bodens enthielt die Bitte, wiederum eine Rangfolge aufzustellen. In diesem Fall sollte sie bzgl. der Behebungsund Kompensationsmöglichkeiten der Bodenschädigungen von landwirtschaftlicher Seite 
aufgestellt werden. Die Bewertung wurde analog der zuletzt beschriebenen vorgenommen. In der Abbildung 12 wird das Ergebnis dargestellt.

\section{Abbildung 12: Möglichkeiten der Behebung und Kompensation von Bodenschädi- gungen nach Meinung der Experten (n=19)}

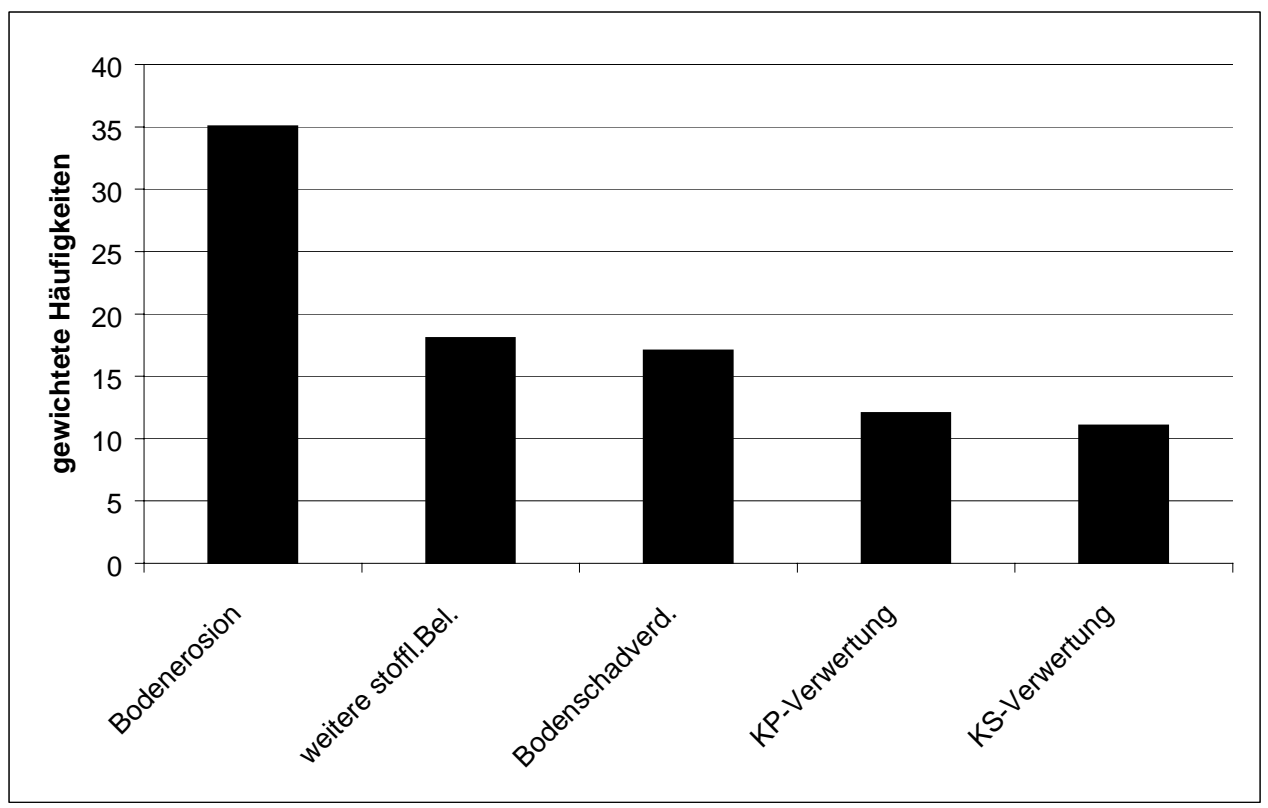

Quelle: Eigene Darstellung

Es wird deutlich, dass die Experten der Auffassung sind, dass Bodenerosion auf den ldw. genutzten Flächen am ehesten zu beheben ist. An zweiter Stelle nennen sie die weiteren stofflichen Belastungen, worunter in erster Linie die Belastungen durch organische und anorganische Düngemittel zu subsumieren sind. Eine ähnliche Behebungs- und Kompensationsmöglichkeit werden den Schädigungen durch Bodenschadverdichtungen zugemessen, gefolgt von den Schädigungen der ldw. Kompost- und Klärschlammverwertung. Diese Gewichtung schlägt sich auf den weiteren Verlauf dieser Arbeit nieder. 


\section{Das Bodenschutzrecht unter dem Gesichtspunkt der land- wirtschaftlichen Bodennutzung}

Das im Jahr 1999 in Kraft getretene Bodenschutzrecht mit dem BBodSchG und der BBodSchV ist zwar in erster Linie ein Gesetz zur Sanierung von Altlasten, beinhaltet jedoch auch Regelungen, welche die Landwirtschaft betreffen. Nach einem allgemeinen Überblick über das internationale, das europäische und das deutsche Bodenschutzrecht geht dieses Kapitel auf die speziellen Regelungen für die Landwirtschaft ein. Außerdem wird die Bedeutung der BBodSchV für die Landwirtschaft erläutert. Am Schluss werden die Regelungen für die Landwirtschaft kritisch diskutiert und mit den Ergebnissen der Expertenbefragung abgerundet.

\subsection{Das internationale Bodenschutzrecht}

Auf der internationalen Ebene bestehen lediglich Absichtserklärungen hinsichtlich eines effektiveren weltweiten Bodenschutzes. Deshalb versuchen diese Erklärungen v. a. auf globale Bodenprobleme, wie etwa die zunehmende Wüstenbildung, einzugehen. Zu nennen ist hierbei die Bodencharta des Europarates von 1972, die Weltbodencharta der FAO von 1981 und die Konferenz von Rio im Jahr 1992 mit ihrem Aktionsprogramm „Agenda 21“. Dieses Aktionsprogramm sieht Handlungsanweisungen für einen integrierten Ansatz der Planung und Bewirtschaftung von Landressourcen sowie für die Bekämpfung der o. g. Wüstenbildung vor. ${ }^{100}$ Mit diesen internationalen Absichtserklärungen und Handlungsanweisungen konnten weder nationale noch internationale Verbindlichkeiten erzeugt werden. Das große Problem bleibt neben der Konsensfindung auf globaler Ebene die Vollzugsbereitschaft der einzelnen Unterzeichnerländer und somit ein eklatantes Vollzugsdefizit. Deshalb sind auch keine Auswirkungen auf das deutsche Bodenschutzrecht erkennbar.

Auf der EU-Ebene gibt es nur wenige spezifische Bodenschutzregelungen. Die meisten europarechtlichen Regelungen haben nur einen mittelbaren Bezug zum Bodenschutz. Ausnahmen sind hingegen die beiden folgenden Vorschriften:

${ }^{100}$ Vgl. FrenZ (2000), S. 37 


\section{1) Die Klärschlamm-Richtlinie}

Sie dient zur Regelung der Verwendung von Klärschlamm in der Landwirtschaft, ohne dass Böden, Vegetation, Tier und Mensch gefährdet werden.

\section{2) Die Richtlinie über Umweltverträglichkeitsprüfungen (UVP-Richtlinie)}

Diese Richtlinie schreibt v. a. eine Bewertung der unmittelbaren sowie mittelbaren Auswirkungen einer Anlage oder eines Eingriffs in Natur und Landschaft auf den Umweltfaktor Boden vor.

Seit April 2002 verfügt auch die EU über eine Bodenschutzstrategie mit dem Titel „Hin zu einer spezifischen Bodenschutzstrategie“،101. Hierbei handelt es sich um eine Mitteilung der Kommission an den Rat, das Europäische Parlament, den Wirtschafts- und Sozialausschuss sowie den Ausschuss der Regionen. Ziel des Papiers ist es, einen Plan zur Entwicklung einer gemeinschaftlichen Bodenschutzstrategie zu entwickeln. In der Mitteilung wird neben einer Beschreibung der Hauptgefahren des Bodens in der EU auch auf die Probleme in den Beitrittsländern eingegangen. Die Komponenten der zukünftigen Bodenschutzstrategie sollen aus der Vorsorge, dem vorausschauenden Handeln sowie einer Umwelthaftung bestehen. Für die landwirtschaftliche Bodennutzung enthält die Mitteilung v. a. Maßnahmen, die im Rahmen der Gemeinsamen Agrarpolitik gefördert werden sollen:

- Ökologische Landwirtschaft,

- Schutz der Terassenkultur,

- Sichere Verwendung von Pestiziden,

- Verwendung zertifizierter Komposte und

- Aufforstung.

Für die deutsche Landwirtschaft sind daher zunächst alle Förderungen, bis auf den Schutz der Terassenkultur, maßgebend. Bis Juni 2004 will die Kommission weiterhin Rechtsvorschriften für die Entwicklung eines gemeinsamen Bodenüberwachungssystem erlassen. ${ }^{102}$

\footnotetext{
101 Vgl. EUROPÄISCHE KOMMISSION (2002) S.179

${ }^{102}$ Vgl. AgRA-Europe (2004a), E 10
} 
Die Strategie der Kommission ist zunächst zu begrüßen. In der EU ist eine Harmonisierung des Rechts auf vielen Ebenen nötig. Neben dem Wasserschutzrecht sollte auch das Bodenschutzrecht in Europa weiter vereinheitlicht werden, um Unstimmigkeiten und Wettbewerbsnachteile in den einzelnen Mitgliedsstaaten zu vermeiden. Am Ende bleibt allerdings die Frage nach dem Vollzug der Bodenschutzstrategie. Erstens haben noch nicht alle bisherigen Mitgliedstaaten ein Bodenschutzgesetz und entsprechende Regelungen wie Österreich oder Deutschland, zweitens sind einige neue Mitgliedsstaaten mit z. T. sehr hohen Bodenschutzdefiziten in die EU eingetreten. Daher wird es noch ein langer Weg sein, bis die EU einheitliche Bodenschutzregelungen besitzt und diese in den Mitgliedsstaaten auch mitgetragen werden.

\subsection{Das Bundes-Bodenschutzgesetz}

Das am 1.3.1999 in Kraft getretene Bundes-Bodenschutzgesetz (BBodSchG) regelt die Vorsorge, die Gefahrenabwehr und die Sanierung schädlicher Bodenveränderungen. Hiermit stellt der Gesetzgeber den Boden als drittes und letztes Umweltmedium unter den Schutz eines bundeseinheitlichen Gesetzes. In diesem Kapitel wird auf diese Regelungen eingegangen. Dabei steht die landwirtschaftliche Bodennutzung im Zentrum der Überlegungen, v. a. die $\S \S 17,10$ Abs. 2 und $\S 21$ Abs. 3 BBodSchG werden näher betrachtet. Außerdem wird die gfP nach $\S 17$ BBodSchG mit denen des Dünge- und Pflanzenschutzmittelrechts sowie des novellierten Naturschutzrechts verglichen.

\subsubsection{Begriffsdefinitionen}

Der Gesetzgeber sieht nach $\S 2$ Abs. 1 BBodSchG den Boden als Symbiose zwischen der oberen Schicht der Erdkruste und den in ihr enthaltenen flüssigen und gasförmigen Bestandteilen an. Die Meinungen darüber, ob es sich in dem $\S 2$ Abs. 1 BBodSchG um eine Definition oder eine Beschreibung des Bodenbegriffs handelt, gehen allerdings auseinander. Die hieraus resultierende Frage, ob es sich im Falle einer Definition nicht um eine einseitige Betrachtung handelt, da Böden eine große Heterogenität aufweisen, muss aus Sicht des Bodenschutzes bejaht werden. Da es keinen allgemeinen Aufbau von Böden gibt, müssen die jeweiligen relevanten Bodenschichten geschützt werden. ${ }^{103}$ Des Weite-

\footnotetext{
${ }^{103}$ Vgl. hierzu Versteyl U. SONDERMANN (2002), S. 68
} 
ren ist es v. a. aus verfassungsrechtlichen Gesichtspunkten ${ }^{104}$ nötig, den Boden i. S. d. G. hinreichend vom Grundwasser zu trennen, welches dem Anwendungsbereich des Wasserrechts, also dem Wasserhaushaltsgesetz (WHG), zuzuordnen ist. Dies geschieht im BBodSchG durch die in der Naturwissenschaft verbreitete Unterscheidung zwischen gesättigter und ungesättigter Zone des Bodens. Demnach ist das Grundwasser als das unterirdische Wasser anzusehen, welches in der gesättigten Zone verbleibt. ${ }^{105}$ Das im Boden gelöste Wasser, die sog. Bodenlösung, gehört zum Regelungsbereich des Bodenschutzrechts. Es kann aus Haftwasser (Wasser, welches den Kapillarkräften der Bodenteilchen unterliegt) oder Sickerwasser (Wasser, welches nur der Schwerkraft unterliegt) bestehen. Haftwasser gehört zur Bodenlösung, Sickerwasser nur dann, wenn es noch nicht in die gesättigten tieferen Bodenschichten abgeflossen ist. Nach $\S 2$ Abs. 2 BBodSchG erfüllt der Boden ferner folgende Funktionen:

1. natürliche Funktionen

2. Archiv der Natur- und Kulturgeschichte

3. Nutzungsfunktionen.

Unter der natürlichen Funktion versteht der Gesetzgeber, dass der Boden als Lebensraum für verschiedenste Arten dient (Lebensraumfunktion), und er einen elementaren Bestandteil des Naturhaushalts darstellt sowie als Abbau-, Ausgleichs- oder Aufbaumedium zu sehen ist (Regelungsfunktion). Die Nutzungsfunktion des Bodens besteht hauptsächlich in der Produktion von Biomasse und Rohstoffen, Bereitstellung von Siedlungs- und Erholungsflächen sowie als Standort für die landwirtschaftliche Produktion (Produktionsfunktion).

Die landwirtschaftliche Produktionsfunktion ist aus quantitativen Gesichtspunkten besonders hervorzuheben, da in Deutschland etwa 53,5 \% der Gesamtfläche landwirtschaftlich genutzt wird. Hintergrund der Einbeziehung der land- und forstwirtschaftlichen Produktionsfunktion in $\S 2$ Abs. 2 Nr. 3c BBodSchG ist auch die aus der Schädigung von Böden resultierende drohende Unterversorgung mit gesunden Nahrungsmitteln und die Verhinderung einer Anreicherung von Schadstoffen in Mensch und Tier. ${ }^{106}$ Allein

\footnotetext{
${ }^{104}$ Der Bund verfügt hinsichtlich des Wasserrechtes nur über eine Rahmengesetzgebungskompetenz gemäß Art. 75 Abs. 1 Nr. 4 GG.

${ }^{105}$ Vgl. hierzu BT-DrUCKS. 13/6701, S. 28

${ }^{106}$ Vgl. BT- DRUCKS. 13/6701, S. 29
} 
hieraus, aber auch aus der Tatsache, dass die land- und forstwirtschaftliche Nutzung nur ohne Verlust der Ertragsfähigkeit und somit der Bodenfruchtbarkeit nachhaltig erhalten werden kann, erwächst die Notwendigkeit eines bodenschonenden Produktionsprozesses, wie z. B. der konservierenden Bodenbearbeitung.

Der Begriff der schädlichen Bodenveränderungen ist ein zentraler Begriff des BBodSchG. Bei der Prüfung, ob eine schädliche Bodenveränderung vorliegt, müssen zwei Aspekte berücksichtigt werden.

- $\quad$ Beeinträchtigung der Bodenfunktionen

- $\quad$ Erhebliche Schäden des einzelnen bzw. der Allgemeinheit.

Die negative Einwirkung auf den Boden kann somit stofflicher als auch nicht-stofflicher Art sein. Für den Bereich der Bodenschadverdichtung wurden bereits Ansätze zur konkreten Definition einer schädlichen Bodenveränderung erstellt. ${ }^{107}$

Verdachtsflächen sind nach $\S 2$ Abs. 4 BBodSchG Grundstücke, die von schädlichen Bodenveränderungen betroffen sind. Hierbei sind allerdings nicht Grundstücke im Sinne des Bürgerlichen Rechts gemeint ${ }^{108}$, sondern die tatsächlich belasteten Flächen. Dies trägt dazu bei, dass die Akzeptanz von Grundstückseigentümern oder -nutzern an Bodenschutzmaßnahmen steigt, da die jeweiligen Nutzungsinteressen nicht in dem Maße tangiert werden. Ein Verdacht besteht nur dann, wenn auch konkrete Anhaltspunkte vorliegen. Reine Behauptungen oder Vermutungen von Personen reichen noch nicht aus. ${ }^{109}$

\subsubsection{Entstehung des Bodenschutzrechts in Deutschland und Europa}

Bis Mitte der 80er Jahre stand der Bodenschutz in Deutschland noch am Rande der umweltpolitischen und -rechtlichen Aktivitäten. Er gewann durch die aufkommende Altlastenproblematik der Wiedervereinigung Deutschlands und die Ausmaße des Waldsterbens zunehmend an Bedeutung. Ein zentraler Grund für die Vernachlässigung des Bodenschutzes war der Eigentumscharakter der Böden und die daraus resultierende Nachhaltig-

\footnotetext{
${ }^{107}$ Vgl. Kapitel 6.3.2

${ }^{108}$ Grundstücke sind im Zivilrecht räumlich abgegrenzte Teile der Erdoberfläche, die in einem Grundbuchblatt eingetragen sind oder nach $\S 3$ Abs. 3 GBO gebucht sind.

${ }^{109}$ Vgl. BR-DRUCKS. 702/96, S. 87
} 
keitsannahme, der Interessenidentität zwischen Bodennutzung und Bodenschutz, in Bezug auf die Grundeigentümer im Gegensatz zu den Medien Luft und Wasser. Der Boden wurde frühzeitig partiell von anderen Rechtsvorschriften unter Schutz genommen, so z. B. durch das WHG von 1960, das Abfallgesetz von 1972 und schließlich durch das BNatSchG.

In Deutschland ist als konkreter Beginn der Entwicklung eines einheitlichen Bodenschutzrechts der 28.02.1983 mit einer gemeinsamen Erklärung der zuständigen Bundesminister, das Bodenschutzrecht z. B. dem Naturschutzrecht gleichzustellen, zu nennen. ${ }^{110}$ Daraufhin folgte die vom Bundeskabinett am 6. 02. 1985 verabschiedete Bodenschutzkonzeption der Bundesregierung. Sie enthielt im Wesentlichen zwei Ansätze:

1. Stoffeinträge in den Boden zu minimieren. Dies sollte z. B. durch eine Schaffung von Kreislauf- und Reststoffmanagementsystemen geschehen.

2. Den Bodenverbrauch zu verringern. Dies sollte v. a. durch flächensparendes Bauen und sparsamere Nutzung von Bodenschätzen geschehen.

Bis in die 90er Jahre hinein wurden nach Vorlage der Bodenschutzkonzeption der Bundesregierung über 50 Vorschriften über den Schutz des Bodens geschaffen, die über einzelne Rechtsgebiete verteilt waren. ${ }^{111}$

Im Folgenden bildete sich die „Bund/Länder-Arbeitsgruppe Bodenschutz“, welche dann in einem Maßnahmen- und Leitlinienkatalog einzelne Schutzmaßnahmen konkretisierte, den Zeit- und Kostenrahmen festlegte und Leitlinien in den Bereichen Rechtsetzung, Verwaltungsvollzug, Information und Forschung erarbeitete. Dieser Maßnahmenkatalog diente im Folgenden als Grundlage der am 8.12.1987 vom Bundeskabinett beschlossenen „Maßnahmen zum Bodenschutz“.

Auf Ebene der Bundesländer waren kurze Zeit später ebenfalls dementsprechende Bemühungen festzustellen. So verabschiedete Baden-Württemberg am 24.07.1991, Sachsen am 12.08.1991 und Berlin am 10.10.1995 jeweils ein Landesbodenschutzgesetz, welche durch das BBodSchG vom 17.03.1998 jedoch überarbeitungsbedürftig wurden.

\footnotetext{
${ }^{110}$ Vgl. KNOPP u. LÖHR (2000), Rnd. Nr. 9

${ }^{111}$ Vgl. HOLZWARTH, RADTKE u. HiLgER (2000)
} 
Das BBodSchG wurde auf Initiative des Bundesumweltministeriums ins Parlament eingebracht. Hierzu legte das Ministerium am 22.03.1986 den ersten Referentenentwurf vor. Im darauffolgenden Gesetzgebungsverfahren gab es zunächst zahlreiche Diskussionen auf Bundes- und Länderebene, die dazu führten, dass sich der Vermittlungsausschuss über ein halbes Jahr mit den Entwürfen beschäftigen musste, bevor es zu einer Einigung im Bundestag und -rat kam. Am 1.03.1999 trat das BBodSchG in Kraft. Seitdem wurden die zu diesem Zeitpunkt bestehenden Landesbodenschutzgesetze entsprechend angepasst, und viele Bundesländer erließen nach und nach eigene Landesbodenschutzgesetze.

Auf europäischer und internationaler Ebene gab es in der Vergangenheit zwar zahlreiche Anstrengungen bezüglich des Bodenschutzes, ${ }^{112}$ jedoch fehlt es bisher auf beiden Ebenen an verbindlichen Richtlinien zum Schutz des Bodens, die über reine Absichtserklärungen hinaus gehen. Zudem liegen in den wenigsten europäischen Ländern Erfahrungen mit rechtlichen Bodenschutzregelungen vor. Die Ausgestaltung der Bodenschutzpolitik in der EU ist vollkommen unterschiedlich. Während in den derzeitigen Beitrittsländern an Aktionsprogrammen gearbeitet wird, sieht man bspw. in Dänemark und Schweden den Bodenschutz als integralen Bestandteil des allgemeinen Umweltschutzes an. In Großbritannien liegt ein Konsultationspapier vor, welches eine Gesamtstrategie für den nationalen Bodenschutz enthält. Frankreich hat einen nationalen Aktionsplan für Bodenbearbeitung und Bodenschutz entwickelt, welcher auf der Grundlage eines Bodenüberwachungsnetzes entsprechende Maßnahmen vorsieht. In Deutschland liegt demnach mit dem BBodSchG das umfassenste Konzept zum Schutz des Bodens vor. ${ }^{113}$ Gerade aus Sicht der EU wäre eine einheitliche Bodenschutzgesetzgebung wünschenswert, dennoch liegt eine derartig gemeinsam verfolgte Bodenschutzpolitik in weiter Zukunft.

\subsubsection{Zweck und Grundsätze des Gesetzes}

Im Folgenden werden zunächst der Zweck und verschiedene Grundsätze des BBodSchG erläutert. Hierzu erfolgt eine Beschreibung der Bodenfunktionen, bevor dann ausführlicher auf den zentralen Begriff der schädlichen Bodenveränderungen eingegangen. In die-

\footnotetext{
112 Vgl. hierzu z. B. „Europäische Bodencharta“ der EG, „World Soil Charta“ der FAO und der „World Soils Policy“ der UN jeweils von 1982. Siehe außerdem: „Towards a thematic strategy for soil protection“, Mitteilung der Kommission an den Rat, das Europäische Parlament, den Wirtschafts- und Sozialausschuss sowie an den Ausschuss der Regionen von 2002.

${ }^{113}$ Vgl. EUROPÄISCHE KOMMISSION (2002), S. 23 f.
} 
sem Zusammenhang spielt im Bodenschutzrecht die Unterscheidung zwischen Vorsorge und Gefahrenabwehr zum Schutz vor schädlichen Bodenveränderungen eine wichtige Rolle.

\subsubsection{Bodenfunktionen i. S. d. BBodSchG}

Die Schutzwirkung des BBodSchG bezieht sich nach $\S 1$ Abs. 1 BBodSchG nicht auf den Boden an sich, sondern auf die in $\S 2$ Abs. 2 festgelegten Bodenfunktionen. Der Boden soll folglich, anders als in den Bodenschutzgesetzen der Bundesländer BadenWürttemberg, Berlin und Sachsen, ${ }^{114}$ durch einen funktionalen Ansatz geschützt werden. Bezüglich der daraus folgenden Schutzstellung der ökonomischen Nutzungsfunktionen wurde dem Gesetzgeber des Öfteren vorgeworfen, hiermit das Ziel des Bodenschutzgesetzes zu verfehlen und eine zu starke anthropozentrische Sichtweise zu besitzen. Hierzu ist jedoch anzumerken, dass als erste zu schützende Funktion die natürliche Funktion des Bodens genannt wird, jedoch eine Rangfolge bezüglich der Priorität der Schutzwürdigkeiten von Bodenfunktionen nicht hinreichend gegeben ist. ${ }^{115}$ Bei der Einteilung der Bodenfunktionen geht der Gesetzgeber allein von dem heutigen und somit sehr spezifischen Stand der Bodenforschung aus, trifft aber keine exakte wissenschaftliche Abgrenzung der einzelnen Funktionen, die in einem sehr engen Wirkungszusammenhang stehen. ${ }^{116}$ Dieser ist gerade bei den Zusammenhängen zwischen der landwirtschaftlichen Nutzungsfunktion und den natürlichen Funktionen gegeben. Als „Kernfrage des Bodenschutzrechts“ leitet sich die Notwendigkeit ab, aus dem Spannungsverhältnis zwischen den ökologischen und ökonomischen Bodenfunktionen, eine Balance zwischen den Bodenfunktionen herzustellen.

Aus den im BBodSchG aufgeführten Bodenfunktionen ergibt sich zwangsläufig ein Spannungsfeld zwischen einer nach dem BBodSchG nicht bedingt vorrangig ${ }^{117} \mathrm{zu}$ behandelnden ökologischen und einer ökonomischen Sichtweise. Dementsprechend kann

\footnotetext{
${ }^{114}$ Vgl. hierzu Kap. 4.2.2

${ }^{115}$ Vgl. FRENZ (2000), S. 44

${ }^{116}$ Vgl. BT-DRUCKS. 13/6701, S. 32

${ }^{117}$ In $\S 1$ S. 3 BBodSchG wird zwar der Schutz der natürlichen Funktionen betont, wobei die Nutzungsfunktionen nicht erwähnt werden. Dies ist aber noch kein hinreichender Beweis für eine Rangfolge.

Vgl. hierzu VERSTEYL U. SONDERMANN (2002), S. 76
} 
eine landwirtschaftliche Nutzung nur als nachhaltig ${ }^{118}$ bezeichnet werden, wenn die natürlichen Bodenfunktionen geschützt werden.

\subsubsection{Schutz vor schädlichen Bodenveränderungen}

Der Schutz vor schädlichen Bodenveränderungen kann zum einen durch Vorsorgemaßnahmen, zum anderen durch Gefahrenabwehrmaßnahmen gewährleistet werden. Die Beziehungen zwischen Vorsorge und Gefahrenabwehr werden in Übersicht 1 verdeutlicht.

\footnotetext{
${ }^{118}$ Unter Nachhaltigkeit ist in diesem Zusammenhang die Vereinbarkeit der ökologischen, sozialen und ökonomischen Ziele wirtschaftlichen Handels zu verstehen. Das BBodSchG trifft keine abschlie- Bende Erläuterung des Begriffs „Nachhaltigkeit“. Frenz interpretiert Nachhaltigkeit i. S. d. G. als „dauerhafte und umfassende Gewährleistung der Funktionen des Bodens“. Vgl. FrENZ (2000), S. 23
} 


\section{Übersicht 1: Sachgerechte Differenzierung zwischen Vorsorge und Gefahrenabwehr in der landwirtschaftlichen Bodennutzung}

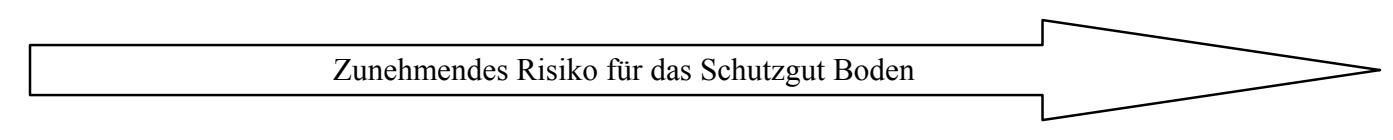

\begin{tabular}{|c|c|c|}
\hline \multicolumn{2}{|c|}{ Vorsorge } & \multirow{2}{*}{\begin{tabular}{l}
\multicolumn{1}{|c}{ Gefahrenabwehr } \\
Schadenseintritt ist hinreichend \\
wahrscheinlich.
\end{tabular}} \\
\hline $\begin{array}{l}\text { Schadenseintritt ist aufgrund prak- } \\
\text { tischer Vernunft ausgeschlossen, } \\
\text { wohl aber möglich. }\end{array}$ & $\begin{array}{l}\text { Schadenseintritt ist bei Anhalten } \\
\text { weiterer Wirkungen (z. B. Boden- } \\
\text { erosion) zu befürchten. }\end{array}$ & \\
\hline $\begin{array}{l}\text { Bereich des Restrisikos } \\
=>\text { Vorsorge }\end{array}$ & $\begin{array}{l}\text { Beginn des unerwünschten Risikos } \\
=>\text { Vorsorge, in Einzelfällen } \\
\text { Gefahrenabwehr }\end{array}$ & $\begin{array}{l}\text { Beginn des nicht hinnehmbaren } \\
\text { Risikos } \\
=>\text { Gefahrenabwehr }\end{array}$ \\
\hline $\begin{array}{l}\S 17 \text { BBodSchG } \\
=>\text { Beratung }\end{array}$ & $\begin{array}{l}\S 8 \text { BBodSchV }^{119} \\
=>\text { Beratung u. Anordnung }\end{array}$ & $\begin{array}{l}\S 5 \text { und } \S 8 \mathrm{BBodSchV}^{120} \\
=>\text { Anordnung }\end{array}$ \\
\hline \multicolumn{3}{|c|}{ Maßnahmen der guten fachlichen Praxis } \\
\hline
\end{tabular}

Quelle: In Anlehnung an FRIELINGHAUs (2002b), S. 15

Es wird in Übersicht 1 deutlich, dass aus Sicht des deutschen Bodenschutzrechts zwischen der Vorsorge vor schädlichen Bodenveränderungen und der Gefahrenabwehr von schädlichen Bodenveränderungen unterschieden werden muss. Bzgl. der Vorsorge vor schädlichen Bodenveränderungen ist die gfP nach $§ 17$ BBodSchG und deren Konkretisierungen alleiniger Maßstab. Erst wenn eine schädliche Bodenveränderung eingetreten ist, besteht die Möglichkeit, behördliche Anordnungen zu treffen. Die Maßnahmen des landwirtschaftlichen Bodenschutzes können ebenfalls in die beiden Kategorien Vorsorge und Gefahrenabwehr eingeteilt werden.

\footnotetext{
${ }^{119}$ Vgl. Kapitel 4.3.2

${ }^{120}$ Vgl. ebd.
} 


\section{Übersicht 2: Einteilung der Maßnahmen der guten fachlichen Praxis nach verschie-} denen Risikostufen

\begin{tabular}{l|l|l}
\hline \multicolumn{1}{c|}{ niedrig } & \multicolumn{1}{c}{ mittel } & \multicolumn{1}{c}{ hoch } \\
\hline - FF-Gestaltung & - Mulchsaat (MSoSb) & - Windschutzstreifen \\
- Zwischenfrüchte & $\begin{array}{l}\text { - Dauerhafte kons. Boden- } \\
\text { bearbeitung }\end{array}$ & - Reduzierung der Überfahrten \\
- Untersaaten & - Reduzierung von Achslasten & - Schlagunterteilung \\
- Mulchsaat (MSmSb) & & \\
- Kalkung & & \\
\hline
\end{tabular}

Quelle: In Anlehnung an Frielinghaus (2002b), S. 19

Die Übersicht 2 zeigt eine Aufteilung einiger Maßnahmen der guten fachlichen Praxis nach Risikoparametern schädlicher Bodenveränderungen. Bei den Risikostufen „niedrig“ und „, mittel“ reichen vorsorgende Maßnahmen aus. Lediglich bei der Risikostufe „hoch“ müssen umfangreichere Gefahrenabwehrmaßnahmen, wie z. B. die Anlage von Windschutzstreifen, ergriffen werden. Der Fachausschuss des Bundesverbandes Boden (BVB) „Gefahrenabwehr bei Bodenerosion“ hat im Sinne eines Maßnahmenwerts nach BBodSchV die Abgrenzung zwischen Vorsorge und Gefahrenabwehr bei der Bodenerosion wie folgt festgesetzt: Schädliche Bodenveränderungen durch Bodenerosion und somit die Notwendigkeit von Gefahrenabwehrmaßnahmen liegen dann vor, wenn der flächenbezogene jährliche Bodenabtrag größer ist als der Quotient aus Bodenzahl geteilt durch zwei. Bei einer Ackerfläche mit einer Ackerzahl von 60 müssen demnach dann Gefahrenabwehrmaßnahmen eingeleitet werden, wenn der jährliche Bodenabtrag 30 t/ha beträgt.

\subsubsection{Anwendungsbereiche und Abgrenzung zu den Fachgesetzen in $\S 3$ Abs. 1 BBodSchG}

In $\S 3$ Abs. 1 BBodSchG ist der Anwendungsbereich des Gesetzes geregelt. Hiernach ist das BBodSchG bei einer schädlichen Bodenveränderung nur dann anzuwenden, wenn die in $\S 3$ Abs. 1 genannten Rechtsbereiche keine entsprechenden bodenbezogenen Regelungen enthalten. Aus dieser Subsidiaritätsklausel ergibt sich eine Prüfungshierarchie, die für die in der Landwirtschaft relevanten Rechtsbereiche ${ }^{121}$ dargestellt wird.

\footnotetext{
${ }^{121}$ Aus dem $\S 3$ Abs. 1 Nummern 2, 3, 8 und 10 BBodSchG werden die Vorschriften über den Betrieb von Abfallbeseitigungsanlagen, die Vorschriften über die Beförderung gefährlicher Güter, den Verkehrswegebau sowie das Bergrecht, ausgeklammert.
} 


\section{Abbildung 13: Subsidiäre Anwendung des BBodSchG nach § 17 Abs. 3 am Beispiel der Landwirtschaft, bei schädlichen Bodenveränderungen}

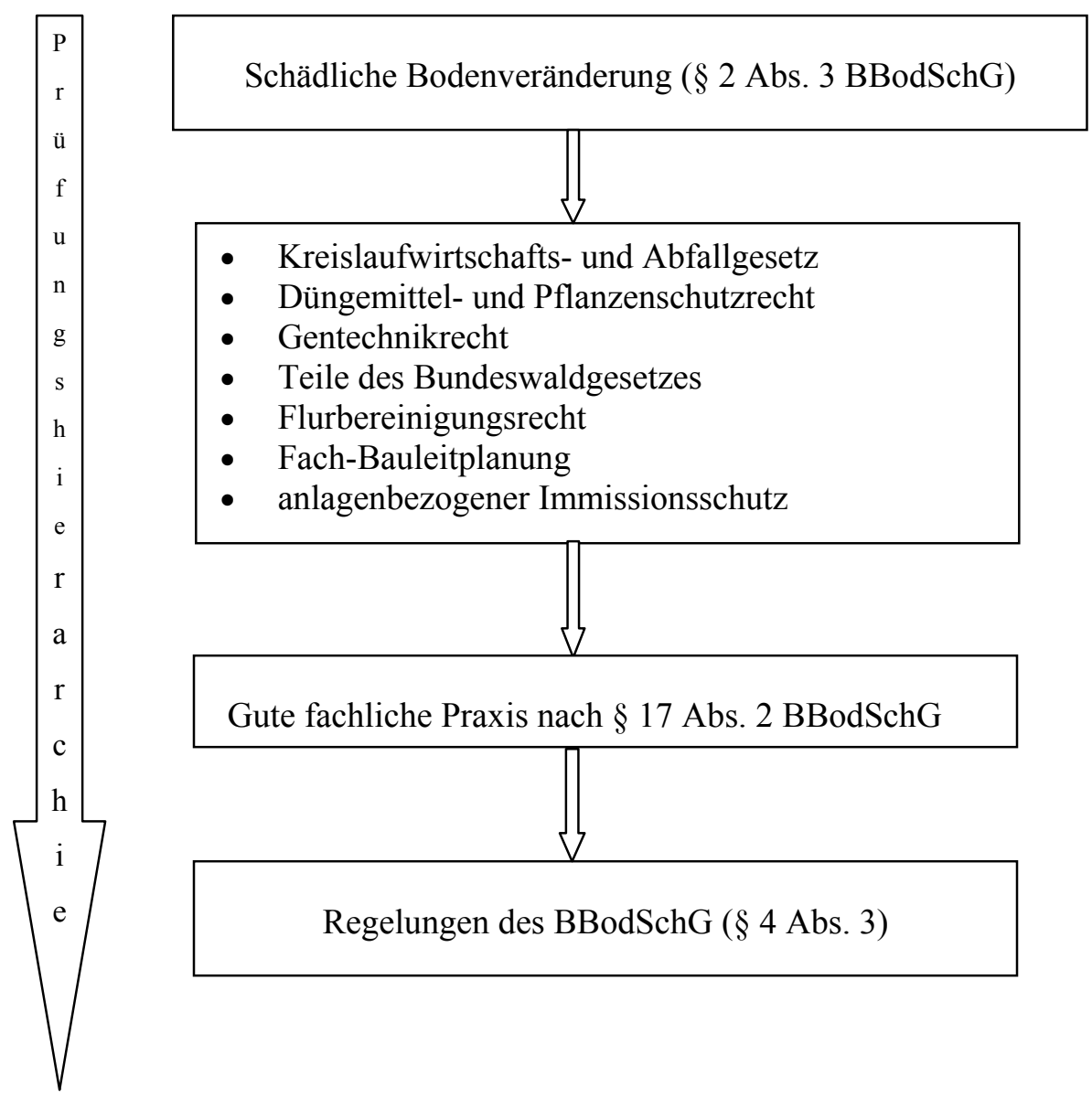

Quelle: Eigene Darstellung

Bei Vorliegen einer schädlichen Bodenveränderung auf einer landwirtschaftlich genutzten Fläche ist zunächst zu prüfen, ob die unten näher erläuterten bodenschutzrelevanten Regelungen der Fachgesetze tangiert werden. Ist dies nicht der Fall, ist zu prüfen, ob gegen die Grundsätze der guten fachlichen Praxis verstoßen wurde. Ein Verstoß gegen die Grundsätze der guten fachlichen Praxis ist nach dem Ermessen der zuständigen Bodenschutzbehörde festzustellen. Liegt auch hier kein Verstoß vor, werden die allgemeinen Vorschriften zur Gefahrenabwehr des Gesetzes verwendet.

Im Folgenden werden die einzelnen dem BBodSchG vorrangigen Rechtsbereiche näher erläutert und deren Auswirkung auf die landwirtschaftliche Praxis dargestellt.

\section{Kreislaufwirtschafts- und Abfallgesetz und Klärschlammverordnung}

Hierunter sind die Vorschriften des Kreislaufwirtschafts- und Abfallgesetzes (KrW(AbfG), die das Aufbringen von Abfällen zur Verwertung als Sekundärrohstoff- oder 
Wirtschaftsdünger im Sinne $\S 1$ Düngemittelgesetz (DMG) regeln, deren Rechtsverordnungen sowie der Klärschlammverordnung zu subsumieren. ${ }^{122}$

Im KrW-/AbfG ist v. a. $\S 8 \mathrm{KrW}-/$ AbfG betroffen, der den Bereich der landwirtschaftlichen Düngung regelt. Hierdurch können u. a. Verbote oder Beschränkungen bei der Ausbringung, Untersuchungen des Wirtschaftsdüngers oder Bodens sowie Maßnahmen zur Vorbehandlung des Düngers angeordnet werden. Voraussetzung hierfür ist jedoch nach $\S 8$ Abs. 2 Nr. 2 S. 2 KrW-/AbfG die Überschreitung der guten fachlichen Praxis nach $\S 1$ a DMG. Außerdem ist der $\S 5$ Abs. $3 \mathrm{KrW}-/$ AbfG zu beachten, der vorschreibt, dass die Verwertung des Düngers ordnungsgemäß und schadlos zu erfolgen hat. Hierunter ist auch zu verstehen, dass das BBodSchG beachtet werden muss, was jedoch nichts am Vorrang des KrW-/AbfG ändert. ${ }^{123}$

Die Klärschlammverordnung (AbfKlärV) geht nach $\S 3$ Abs. 1 BBodSchG dem BBodSchG ebenfalls vor. Die AbfKlärV regelt das Aufbringen von Klärschlamm auf landwirtschaftlich genutzte Böden. Diese Verordnung enthält jedoch in $§ 9$ keine Rechtsfolgenregelungen bei Überschreitungen. Da es sich in solch einem Fall um eine schädliche Bodenveränderung nach $\S 2$ Abs. 3 handelt, würde hier das BBodSchG greifen. ${ }^{124}$

\section{Düngemittel- und Pflanzenschutzrecht}

Wie schon oben erwähnt, haben nicht nur die Vorschriften des DMG sondern auch die des Pflanzenschutzgesetzes (PflSchG) Vorrang vor dem BBodSchG. Im Düngemittelrecht ist das DMG und dessen Rechtsverordnungen, die Düngeverordnung (DüngeVO) zu beachten. Ein Vorrang des Düngemittelrechts ist nur dann gegeben, wenn es sich bei den entsprechenden Stoffen um Düngemittel i. S. d. DMG handelt. Eine weitere Voraussetzung ist die sog. zulässige Zuführung von Stoffen in oder auf den Boden, die nach $\S 1 \mathrm{a}$ Abs. 1 S. 1 DMG nach guter fachlicher Praxis, welche in der DüngeVO näher bestimmt wird, ausgeführt werden muss. Ist dies nicht der Fall, besitzt das DMG lediglich in $\S 10$ Ordnungswidrigkeitsvorschriften jedoch existieren keine Vorschriften zum weiteren Umgang mit dem belasteten Boden. Da es sich in diesen Fällen aller Wahrscheinlichkeit nach

\footnotetext{
${ }^{122}$ Vgl. FrenZ (2000), S. 100

${ }^{123}$ Vgl. ebd., S. 101

124 Vgl. ebd., S. 103
} 
um eine schädliche Bodenveränderung i. S. d. BBodSchG handelt, gelten dann die Vorschriften des BBodSchG.

Im Bereich des Pflanzenschutzmittelrechts ist das PflSchG dem BBodSchG im Bezug auf schädliche Bodenveränderungen vorrangig. Der Boden ist nach $§ 2$ Nr. 6 PflSchG direkt in den Schutzbereich des Gesetzes einbezogen. Entsprechend der Abgrenzung im Fall des Düngemittelrechts ist die Vorrangigkeit des PflSchG vor dem BBodSchG auf die Pflanzenschutzmittel nach $\S 2$ Nr. 9 PflSchG beschränkt. Wenn die Anforderungen des PflSchG an die gfP nicht erfüllt werden und es zu einer schädlichen Bodenveränderung kommt, ist das BBodSchG zuständig. ${ }^{125}$

\section{Gentechnikrecht}

Das Gentechnikrecht wird in Zukunft sicherlich allein im Hinblick auf den Anbau gentechnisch veränderter Pflanzen eine steigende Bedeutung für die Landwirtschaft haben und ist daher in die ausführlichere Beschreibung der einzelnen vorrangigen Rechtsbereiche des $\S 3$ Abs. 1 BBodSchG aufgenommen worden. Hier ist das Gentechnikgesetz (GenTG) gegenüber dem BBodSchG vorrangig, zumal es im Gegensatz zum Pflanzenschutz- oder Düngemittelrecht in vielen Bereichen, wie etwa der Gefahrenabwehr, abschließende Regelungen enthält. Der Boden wird in diesem Gesetz zwar nicht explizit genannt, aber unter dem Begriff ,,sonstige Umwelt“ $§ 1$ Abs. 1 GenTG subsummiert. ${ }^{126}$ Des Weiteren regelt das Gesetz das Inverkehrbringen und die Freisetzung von gentechnisch veränderten Organismen. Eine Genehmigung wird nicht erteilt, wenn es unvertretbare schädliche Einwirkungen bei der Freisetzung geben könnte. Da das Gesetz jedoch nichts darüber enthält, wann solche Einwirkungen für den Boden schädlich sind, muss auf die schädlichen Bodenveränderungen nach $\S 2$ Abs. 3 BBodSchG verwiesen werden. ${ }^{127}$ An diesem Punkt ist abschließend auf die Novellierung des GenTG zu verweisen, welches am 11.02.2004 vom Bundeskabinett beschlossen wurde und aufgrund der restriktiven Vorgaben zur Anwendung der „Grünen Gentechnik“ in der öffentlichen Diskussion steht.

\footnotetext{
${ }^{125}$ Vgl. Frenz (2000), S. 109

${ }^{126}$ Vgl. HOLZWARTH ET AL. (2000), § 3 Rnd. Nr. 12

${ }^{127}$ Vgl. FrenZ (2000), S. 110
} 


\section{Wald- und Forstrecht}

Da das Wald- und Forstrecht nicht unmittelbar für die Landwirtschaft relevant ist, wird dieser Rechtsbereich nur kurz angesprochen. Die wesentliche Rechtsgrundlage bilden hier das Bundeswaldgesetz (BWaldG), bzw. die Forstgesetze der Bundesländer. So wird z. B. der Waldboden vom BWaldG geschützt. Außerdem enthält es als Rahmengesetz des Bundes das Instrument des „Schutzwaldes“ nach $\S 12$ Abs. 1, in dem Maßnahmen zu ergreifen sind, die z. B. Bodenerosion verhindern oder eindämmen sollen.

\section{Flurbereinigungsrecht}

Nach $\S 3$ Abs. 1 Nr. 7 BBodSchG gehen die Vorschriften des Flurbereinigungsgesetzes (FlurbG) auch in Verbindung mit dem Landwirtschaftsanpassungsgesetz (LandwAnpG) denen des BBodSchG vor. Die Vorrangigkeit betrifft vor allem die $\S \S 37,38$ FlurbG, in denen die Aufgaben der Flurbereinigung sowie die allgemeinen Grundsätze erläutert werden. So sollen z. B. nach $\S 37$ Abs. 1 FlurbG im Rahmen der Flurbereinigung neben der Herbeiführung von besseren wirtschaftlichen Bedingungen für die teilnehmenden Betriebe auch „bodenschützende sowie -verbessernde“ Maßnahmen ergriffen werden. Damit wird deutlich, dass der Gesetzgeber auch im FlurbG den Boden vor Windund Wassererosion schützen will, hierfür aber keine konkreten Maßnahmen nennt. ${ }^{128}$ Diese sind aus dem BBodSchG abzuleiten. Diese Vorgehensweise ist auf das LandwAnpG übertragbar. Die speziellen Verfahren und Pläne sind, wie auch beim FlurbG, vom Regelungsbereich des BBodSchG ausgenommen. ${ }^{129}$

\section{Vorschriften des Bauplanungs- und Bauordnungsrechts}

Die Landwirtschaft ist vom Bauplanungs- und Bauordnungsrecht auf zwei Arten betroffen. Auf der einen Seite nimmt sie selbst Flächen für die Bebauung in Anspruch. Auf der anderen Seite wird sie durch die hohe zusätzliche Flächeninanspruchnahme durch die allgemeine Bebauung und Ausweisung von Baugebieten zurückgedrängt. Hierbei kommt die Problematik der Ausgleichsflächen für Bebauungsmaßnahmen noch erschwerend hinzu.

\footnotetext{
${ }^{128}$ Vgl. BT-DrUCKS. 13/6701, S. 32

${ }^{129}$ Vgl. FrenZ (2000), S. 112 f.
} 
Nach $\S 3$ Abs. 1 Nr. 9 BBodSchG gehen die Regelungen des Bauplanungs- und Bauordnungsrechts, wenn sie schädliche Einwirkungen auf den Boden regeln, denen des BBodSchG vor. Im Mittelpunkt steht hierbei das Baugesetzbuch (BauGB). Nach $\S 1 \mathrm{a}$ Abs. 1 BauGB sollen z. B. Bodenversiegelungen auf ein nötiges Maß begrenzt werden und soll mit Grund und Boden sparsam umgegangen werden. Weiterhin schreibt die Umweltverträglichkeitsprüfung nach $\S 1 \mathrm{a}$ Abs. 2 Nr. 3 BauGB die Einbeziehung von Bodenschutzbelangen in die Planungsphase vor. Außerdem werden diese im Bauordnungsrecht der Länder einbezogen. Auf eine nähere Darstellung der Zusammenhänge wird an dieser Stelle verzichtet.

\section{Bundes-Immissionsschutzgesetz}

Das Verhältnis von Bundes-Immissionsschutzgesetz (BImSchG) und BBodSchG wird sowohl in $\S 3$ Abs. 2 Nr. 11 als auch in $\S 3$ Abs. 3 BBodSchG und $\S 1$ BImSchG geregelt. In $\S 1$ BImSchG zielt der Gesetzgeber ebenfalls auf den Schutz des Bodens ab. Deshalb kommt es zu einer Verzahnung zwischen beiden Rechtsbereichen. Generell sagt $§ 3$ Abs. 2 Nr. 11 aus, dass das BImSchG dann Vorrang vor dem BBodSchG hat, wenn es Einwirkungen auf den Boden regelt. Hierbei ist $\S 3$ Abs. 3 BBodSchG zu berücksichtigen, der eine Brücke zum Immissionsschutzrecht schlägt. Demnach gelten durch Immissionen verursachte schädliche Bodenveränderungen gleichzeitig als schädliche Umwelteinwirkungen i. S. d. $\S 3$ Abs. 1 BImSchG. Die Beziehungen zwischen dem BBodSchG und dem BImSchG werden an dieser Stelle aufgrund der geringen Bedeutung für das weitere Vorgehen nicht weiter ausgeführt.

\section{Naturschutz- und Wasserrecht}

Zwei für die Landwirtschaft sehr wichtige Rechtsbereiche, das Naturschutz- und Wasserrecht, sind in $\S 3$ Abs. 1 nicht aufgeführt. Zunächst gilt daher, dass die drei Rechtsbereiche nebeneinander anzuwenden sind. Im Falle einer Kollision zwischen den Rechtsbereichen gilt ein allgemeiner Rechtsgrundsatz: Die speziellere Rechtsnorm ist der allgemeineren vorzuziehen. Hinweise hierzu finden sich im BBodSchG in $\S 4$ Abs. 4 S. 3 und $\S 7$ Abs. 6. Diese Hinweise beziehen sich ausschließlich auf die Schnittstellen zwischen 
Bodenschutz- und Wasserrecht. ${ }^{130}$ Bezüglich der Schnittstelle Bodenschutz- und Naturschutzrecht finden sich keine Hinweise.

\subsubsection{Landwirtschaftliche Bodennutzung im BBodSchG}

Wie bereits in Kapitel 4.2.1 angeführt wurde, beträgt der Anteil der landwirtschaftlich bewirtschafteten Fläche etwa 53,5 \% der Gesamtfläche Deutschlands. Daher kommt der Landwirtschaft eine besondere Bedeutung bzgl. des Bodenschutzes zu. Dieser Verantwortung kommt der Landwirt nach, indem er seine Bodennutzung nach den Grundsätzen der gfP gestaltet. Im Folgenden werden deswegen zunächst die Grundsätze der gfP dargestellt und deren Inhalte erläutert. Außerdem wird auf die Beratung nach $\S 17$ Abs. 1 BBodSchG eingegangen. Im Anschluss daran findet ein Vergleich der gfP der Bodennutzung mit denen anderer Rechtsbereiche statt.

\subsubsection{Die gute fachliche Praxis der landwirtschaftlichen ${ }^{131}$ Bodennutzung nach $\S 17$ BBodSchG}

Die gfP des $\S 17$ BBodSchG wird im Umweltrecht häufig als ,,auffällige Sonderregelung ${ }^{\text {“132 }}$ bezeichnet und steht sowohl in der rechtswissenschaftlichen als auch in der umweltwissenschaftlichen Diskussion in der Kritik. Das zentrale Element des $§ 17$ stellen die Grundsätze der guten fachlichen Praxis dar, welche im Folgenden näher erläutert werden. Diese Grundsätze erfuhren zwei Jahre nach dem Erlass des BBodSchG für die Problembereiche Bodenerosion und Bodenschadverdichtung eine Konkretisierung in einem Bund-Länder-Papier, welches v. a. als Beratungsgrundlage dienen soll. ${ }^{133}$

\subsubsection{Die Grundsätze der guten fachlichen Praxis}

Im BBodSchG wird in $\S 7$ die Vorsorge gegen schädliche Bodenveränderungen behandelt. Der landwirtschaftliche Betrieb erfüllt diese Vorsorgepflichten, wenn er die gfP in der ldw. Bodennutzung nach $\S 17$ Abs. 2 BBodSchG einhält.

\footnotetext{
${ }^{130}$ Vgl. hierzu Kapitel 5.1

${ }^{131}$ Hierbei ist zu berücksichtigen, dass der $\S 17$ BBodSchG nur für die Landwirtschaft, nicht jedoch für die Forstwirtschaft Anwendung findet.

${ }^{132}$ SMEDDinCK U. HoGENMÜLLER (2000), S. 298

${ }^{133}$ Vgl. BMVEL (2001)
} 
Die gfP ist wie die „ordnungsgemäße Landwirtschaft“ oder der „Stand der Technik“ ein unbestimmter Rechtsbegriff. ${ }^{134}$ Unbestimmte Rechtsbegriffe werden nicht vom Bundesgesetzgeber abschließend und allgemeinverbindlich definiert. Eine behördliche Anordnung zur Durchsetzung der guten fachlichen Praxis zur Vorsorge gegen schädliche Bodenveränderungen war zwar im Gesetzgebungsverfahren angedacht, hat aber am Schluss keine Zustimmung gefunden. ${ }^{135}$ Die fehlende Konkretisierung führt auch dazu, dass strafrechtliche Sanktionierungen gegen Verstöße der gfP nicht geahndet werden können, da eine klare Bestimmung der Rechtspflichten fehlt. ${ }^{136}$

Bei der Ausgestaltung der gfP muss berücksichtigt werden, dass eine vollständige und gleichzeitige Erfüllung der Grundsätze der gfP nicht immer möglich ist. Es gibt zahlreiche Zielkonflikte, wie z. B. den Zielkonflikt zwischen Betriebswirtschaft und Ökologie bei der Zuckerrübenernte. Außerdem sind schädigende Wetterereignisse, wie z. B. Starkregen oder Stürme, zwar statistisch aber nicht temporär vorhersehbar. Weiterhin ist die Produktion von Nahrungsmitteln, verglichen mit einer industriellen Herstellung von Gütern, ein sehr komplexes und von vielen Einflussfaktoren beherrschtes Verfahren. Daher wird das Instrument der gfP der landwirtschaftlichen Bodennutzung gerecht. Die ausführliche Diskussion um die gfP wird in Kapitel 4.4 geführt.

In Bezug auf die Gefahrenabwehr gemäß $§ 4$ Abs. 3 BBodSchG ist bei der Ermittlung der Zuständigkeit des jeweiligen Fachrechts die Rangfolge in Abbildung 13 im Kapitel 4.2.4 zu berücksichtigen. Für den Landwirt bedeutet dies in der Praxis eine hohe Rechtssicherheit. Denn durch die Erfüllung der Grundsätze der guten fachlichen Praxis wird der Vorsorge- und Gefahrenabwehrpflicht stets genüge getan. Dies gilt vor allem im Zusammenhang mit den Konkretisierungen der Grundsätze. Kommt der Landwirt also den Grundsätzen der guten fachlichen Praxis in der Bodennutzung nach, hat er, auch bei Eintreten einer landwirtschaftstypischen Bodengefahr wie z. B. einer Bodenverdichtung sowohl die Vorsorge- als auch die Gefahrenabwehrpflicht erfüllt. Bei Gefahren, die keinen direkten Bezug zur landwirtschaftlichen Bodennutzung aufweisen, wie z. B. einem Alt-

\footnotetext{
${ }^{134}$ Vgl. Agra-Europe (1998), S. L18, andere Meinung

${ }^{135}$ Vgl. VersteyL (2002), S. 362

${ }^{136}$ BT-DRUCKS. 14/9852, S. 141
} 
lastenfund auf einer landwirtschaftlich genutzten Fläche, gilt dieser Zusammenhang wiederum nicht. ${ }^{137}$

Die sieben Grundsätze der gfP sind in $\S 17$ Abs. 2 nicht erschöpfend ${ }^{138}$ dargestellt und dienen als Handlungsanweisungen ${ }^{139}$. Ursprünglich sollte die gfP durch Ziele gekennzeichnet werden. Der Begriff Ziel wurde jedoch im Laufe des Gesetzgebungsverfahrens in Grundsatz umformuliert. ${ }^{140}$ Dies zeigt eine gewisse Verstärkung von einer reinen Absichtserklärung und Zielsetzung zu einer Durchsetzungsbereitschaft des Gesetzgebers. Die Grundsätze werden im Folgenden dargestellt. Sie enthalten im wesentlichen die Elemente des physikalischen Bodenschutzes. Der stoffliche Bodenschutz wird somit weiterhin durch die entsprechenden Fachgesetze gewährleistet. Grundlage der Zusammenstellung ist der Beschluss der Agrarminister des Bundes und der Länder vom 23.09.1997 sowie ein Beschluss der Agrarminister der Länder vom 1.10.1993. ${ }^{142}$

\subsubsection{Die Grundsätze der guten fachlichen Praxis im Einzelnen}

Im Folgenden werden die Grundsätze der gfP in der ldw. Bodennutzung im Einzelnen erläutert:

\section{Bodenbearbeitung}

Nach $\S 17$ Abs. 2 S. 2 Nr. 1 hat die Bodenbearbeitung „,.. unter Berücksichtigung der Witterung grundsätzlich standortangepasst zu erfolgen... “. Die Bodenbearbeitung stellt i. S. d. G. diejenigen Maßnahmen dar, die zu einer Veränderung der Bodenstruktur führen. Hierzu zählt das Pflügen, Eggen, Walzen, Fräsen oder Grubbern. Der Begriff Bodenbearbeitung ist nach FRENZ ${ }^{143}$ jedoch so weit zu fassen, dass auch die Fruchtarten-, Sorten- und Fruchtfolgenwahl sowie die Entscheidung des Landwirts, einen Schlag als Grünland oder Acker zu nutzen, hierunter zu fassen ist. Die Begründung für den letzten Punkt

\footnotetext{
${ }^{137}$ Vgl. KoBes (1998), S. 786

${ }^{138}$ Dies wird in diesem Satz durch den Zusatz „insbesondere“ gekennzeichnet. Dieser Zusatz stellt einen Kompromiss dar zwischen der Bundesregierung, die eine abschließende Aufzählung gefordert hatte und der Opposition, die den Bundesländern die Möglichkeit eröffnen wollte, die Grundsätze weiter durch Verwaltungsvorschriften zu konkretisieren, vergleiche hierzu VERSTEYL (2002), S. 365.

${ }^{139}$ Vgl. PEINE (2002), S. 525

${ }^{140}$ Vgl. VERSTEYL (2002), S. 364

${ }^{141}$ Vgl. hierzu BT-DRUCKS. 13/7778, S. 85

${ }^{142}$ Vgl. VERSTEYL (2002), S. 365

${ }^{143}$ Vgl. FrenZ (2000), S. 591
} 
entnimmt der Verfasser aus dem Zusammenhang zwischen standortangepaßter Bodenbearbeitung und dem Klima. Für eine standortangepasste Bodenbearbeitung ist demnach das langfristige Klima entscheidend. Dieses wiederum wirkt sich auf die Anbauentscheidung des einzelnen Landwirts aus. Die Entscheidung für eine bestimmte Fruchtfolge bzw. für oder gegen Grünland bzw. Acker und somit einer standortangepassten Bodenbearbeitung hängt demzufolge auch vom Klima ab. In der landwirtschaftlichen Praxis sind jedoch noch weitere Einflussfaktoren zu nennen. So spielt bspw. die Bodenart als Einflussgröße der Staunässebildung eine entscheidende Rolle dafür, ob ein Schlag als Wiese genutzt oder Ackerfrüchte angebaut werden können. Die allgemeinen Ziele der Bodenbearbeitung sind nach FRENZ ${ }^{144}$ :

- Schaffung eines guten Wachstums für die Pflanzen,

- Schaffung eines physikalisch günstigen Bodengefüges im Saatbett, in der Ackerkrume sowie im Unterboden und

- Mechanische Bekämpfung von Unkraut und Ausfallgetreide.

Generell muss bei der Bodenbearbeitung zwischen der konventionellen (Einsatz des Pfluges) und verschiedenen konservierenden Bodenbearbeitungsverfahren unterschieden werden. Welche dieser Verfahren als standortangepasst anzusehen ist, hängt von vielen standortbedingten Faktoren ab.

Der Einschub des Wortes „grundsätzlich“ in $§ 17$ Abs. 2 Satz 2 Nr. 1 BBodSchG zeigt, dass der Begriff der standortangepassten Bodenbearbeitung interpretiert werden muss und dass auch Ausnahmen akzeptiert werden können. So kann ein Bearbeitungsverfahren auch dann zur guten fachlichen Praxis zählen, wenn es aus ökologischen und ökonomischen Gründen innerhalb bestimmter Grenzen nicht standortangepasst ist. ${ }^{145}$

\section{Erhalt und Verbesserung der Bodenstruktur}

Nach $\S 17$ Abs. 2 Satz 2 Nr. 2 BBodSchG soll ,die Bodenstruktur erhalten oder verbessert“ werden. Unter Bodenstruktur ist nach Meinung von FRENZ ${ }^{146}$ die ,räumliche Anordnung der festen Bodenbestandteile und deren Zusammenhalt" gemeint. Die Boden-

\footnotetext{
${ }^{144}$ Vgl. ebd., S. 591 f.

145 Vgl. Frenz (2000), S. 593

${ }^{146}$ Vgl. ebd., S. 592
} 
struktur wird hauptsächlich durch zu schwere Maschinen geschädigt, die bei zu hohen Bodenfeuchten zum Einsatz kommen, ${ }^{147}$ wie z. B. bei der Zuckerrübenernte im Herbst. Hinzu kommen negative Auswirkungen durch eine falsche Reifenwahl oder einen zu hohen Reifeninnendruck. Eine der wichtigsten Auswirkungen aus der Schädigung der Bodenstruktur ist die Entstehung von Bodenschadverdichtungen mit den daraus resultierenden Konsequenzen für den Bodenhaushalt ${ }^{148}$.

Der Gesetzgeber fordert in diesem zweiten Punkt der Grundsätze der gfP einen Erhalt oder eine Verbesserung der Bodenstruktur. Durch den Begriff „Verbesserung“ setzt er indirekt voraus, dass die Situation der landwirtschaftlich genutzten Böden in Bezug auf die Bodenstruktur vielfach nicht befriedigend ist und verbessert werden muss. Dies kann durch z. B. tiefwurzelnde Pflanzen oder konservierende Bodenbearbeitung geschehen.

\section{Verhinderungen von Bodenverdichtungen ${ }^{149}$}

Gemäß $§ 17$ Abs. 2 Satz 2 Nr. 3 BBodSchG sollen „Bodenverdichtungen, insbesondere durch Berücksichtigung der Bodenart, Bodenfeuchtigkeit und des von den zur landwirtschaftlichen Bodennutzung eingesetzten Geräten verursachten Bodendrucks, so weit wie möglich vermieden werden“. Diese Forderung weist, wie oben angesprochen, starke Zusammenhänge mit den Punkten 1 und 2 des $\S 17$ Abs. 2 BBodSchG auf. Die Konsequenzen für den Bodenhaushalt und somit für den Ertrag der betroffenen Flächen sind nahezu identisch. Auch die Strategien zur Vermeidung von Bodenschadverdichtungen sind identisch mit denen in Punkt 2 vorgestellten Maßnahmen.

In diesem Zusammenhang ist jedoch auf einen für die landwirtschaftlichen Produktionsabläufe sehr wichtigen Sachverhalt hinzuweisen: Es widerspricht nicht den Grundsätzen der guten fachlichen Praxis, wenn ein Landwirt seinen Boden in einem extrem niederschlagsreichen Jahr z. B. zur Ernte befährt. Dazu ist in $\S 17$ Abs. 2 Satz 2 Nr. 3 BBodSchG die Einschränkung „so weit wie möglich“ angegeben, welche die Abwägung von ökonomischen Interessen und technischen Möglichkeiten sowie die Einbeziehung der

\footnotetext{
${ }^{147}$ Vgl. hierzu Kapitel 3.1.2.1

${ }^{148}$ Vgl. hierzu Kap. 3.1.2.1 und Kapitel 3.1.2.3

149 Der Gesetzgeber verwendet im BBodSchG den Begriff Bodenverdichtung. In der naturwissenschaftlichen Literatur wird hingegen der Begriff Bodenschadverdichtung verwendet. Nicht jede Bodenverdichtung ist anthropogen verursacht und hat negative ökologische und ökonomische Beeinträchtigungen zur Folge. Auch wenn das BBodSchG von „Bodenverdichtungen“ spricht, wird im Folgenden vom Verfasser synonym der Begriff „Bodenschadverdichtung“" verwendet.
} 
ökologischen Auswirkungen erfordert. Außerdem geht dieser Absatz des BBodSchG von einer landwirtschaftlichen Bodennutzung aus, welche eine Fortsetzung der Nutzung voraussetzt. Auch die Tatsache, dass es sich um Grundsätze der guten fachlichen Praxis handelt, schließt ein Befahrungsverbot aus, da ein Grundsatz nur einen Handlungsrahmen vorgeben kann. ${ }^{150}$

Das Bund-Länder-Papier zur Konkretisierung der Grundsätze der gfP nennt zur Vorsorge gegen Bodenschadverdichtung Maßnahmen, die wie in Übersicht 3 gegliedert werden können:

${ }^{150}$ Vgl. Frenz (2000), S, 593 f. 


\section{Übersicht 3: Vorsorgemaßnahmen gegen Bodenschadverdichtungen nach den Kon-} kretisierungen der guten fachlichen Praxis (§ 17 Abs. 2 BBodSchG)

\begin{tabular}{|c|c|c|c|}
\hline $\begin{array}{c}\text { Anwendung und } \\
\text { Weiterentwicklung } \\
\text { technischer Maß- } \\
\text { nahmen } \\
\end{array}$ & $\begin{array}{l}\text { Anpassung von Ar- } \\
\text { beitsverfahren }\end{array}$ & $\begin{array}{c}\text { Verbesserung der } \\
\text { Tragfähigkeit des } \\
\text { Bodens }\end{array}$ & $\begin{array}{l}\text { Begrenzung der } \\
\text { mechanischen Be- } \\
\text { lastung }\end{array}$ \\
\hline $\begin{array}{l}\text { Vergrößerung } \\
\text { der Radauf- } \\
\text { standsfläche }\end{array}$ & $\begin{array}{l}\text { Fahren außer- } \\
\text { halb der Furche } \\
\text { beim Pflügen } \\
\text { (Onland- } \\
\text { Pflügen) }\end{array}$ & $\begin{array}{l}\text { Nichtwendende } \\
\text { und schonende } \\
\text { Bodenlockerung }\end{array}$ & $\begin{array}{l}\text { - } \quad \text { Technische Aus- } \\
\text { stattung }\end{array}$ \\
\hline \multirow{4}{*}{$\begin{array}{l}\text { Anpassung des } \\
\text { Reifeninnen- } \\
\text { drucks durch } \\
\text { Reifendruck- } \\
\text { regelanlagen } \\
\end{array}$} & $\begin{array}{l}\text { Zusammenlegen } \\
\text { von Arbeitsgän- } \\
\text { gen }\end{array}$ & $\begin{array}{l}\text { Anbau von Zwi- } \\
\text { schenfrüchten }\end{array}$ & $\begin{array}{l}\text { - Überbetriebli- } \\
\text { cher Maschinen- } \\
\text { einsatz }\end{array}$ \\
\hline & $\begin{array}{l}\text { Einsatz zapfwel- } \\
\text { lenangetriebener } \\
\text { Geräte } \\
\end{array}$ & $\begin{array}{l}\text { - Organische } \\
\text { Düngung }\end{array}$ & $\begin{array}{l}\text { - Spurtiefenanzei- } \\
\text { ge }\end{array}$ \\
\hline & $\begin{array}{l}\text { Substitution von } \\
\text { Herbst- oder } \\
\text { Winterfurche } \\
\text { durch eine } \\
\text { Sommerfurche } \\
\end{array}$ & & $\begin{array}{l}\text { - Überrollhäufig- } \\
\text { keit }\end{array}$ \\
\hline & $\begin{array}{l}\text { Trennung von } \\
\text { Spur- und An- } \\
\text { baufläche (con- } \\
\text { trolled-traffic) }\end{array}$ & & \\
\hline
\end{tabular}

Quelle: BMVEL (2001), S. 27 ff.

Einige der in der Übersicht 3 dargestellten Maßnahmen zur Vorsorge gegen Bodenschadverdichtungen werden im Kapitel 6 ausführlich hinsichtlich der ökologischen und betriebswirtschaftlichen Parameter untersucht.

\section{Vermeidung von Bodenabträgen}

In $\S 17$ Abs. 2 Satz 2 Nr. 4 BBodSchG fordert der Gesetzgeber, dass „Bodenabträge durch eine standortangepasste Nutzung, insbesondere durch Berücksichtigung der Hangneigung, der Wasser- und Windverhältnisse sowie der Bodenbedeckung, möglichst vermieden werden“ sollen. Unter Bodenabtrag versteht der Gesetzgeber in diesem Fall die Bodenerosion durch Wasser und Wind. FrENZ ${ }^{151}$ schließt aus dem Begründungsauf- 
wand $^{152}$ des Gesetzgebers, dass er hier den Schwerpunkt bei den Grundsätzen der guten fachlichen Praxis gesetzt hat. So gibt er, um die Dringlichkeit des Problems zu erläutern, an, dass in den nord- und ostdeutschen Tiefebenen bis zu $30 \%$ der AF besonders anfällig für Winderosion sind und auf $15 \%$ der AF bedenkliche Winderosionswerte erreicht wurden. Die Problematik der lößreichen hügeligen Gebiete mit ihren hohen Abträgen wird ebenfalls angesprochen.

In der Übersicht 4 werden Vorsorgemaßnahmen aus dem Bund-Länder-Papier zur Konkretisierung der Grundsätze der gfP zu dem Problembereich Bodenerosion dargestellt.

${ }^{152}$ Vgl. hierzu BT-Drucks. 13/6701, S. 43 


\section{Übersicht 4: Vorsorgemaßnahmen gegen Bodenerosion nach den Konkretisierungen der guten fachlichen Praxis der Bodennutzung (§ 17 Abs. 2 BBodSchG)}

\begin{tabular}{llll}
\hline $\begin{array}{c}\text { Allgemeine acker- und } \\
\text { pflanzenbauliche Maß- } \\
\text { nahmen }\end{array}$ & $\begin{array}{c}\text { Erosionsmindernde Bo- } \\
\text { denbearbeitungs- und Be- } \\
\text { stellverfahren }\end{array}$ & $\begin{array}{c}\text { Erosionsmindernde Flur- } \\
\text { gestaltung }\end{array}$ \\
\hline - Zwischenfruchtanbau & - $\begin{array}{l}\text { Strohverteilung und } \\
\text { Stoppelbearbeitung }\end{array}$ & $\begin{array}{l}\text { Erosionsschutzstreifen } \\
\text { quer zum Gefälle oder } \\
\text { der Hauptwindrichtung }\end{array}$ \\
\hline - Untersaaten in Reihen- \\
früchten, z. B. Mais
\end{tabular}

Quelle: BMVEL (2001), S. 57 ff.

Analog zur Vorgehensweise der Übersicht 3 werden die Vorsorgemaßnahmen zur Verminderung der Bodenerosion im Kapitel 6 aus naturwissenschaftlicher und betriebswirtschaftlicher Sicht analysiert.

\section{Erhaltung der naturbetonten bodenschützenden Strukturelemente}

In $\S 17$ Abs. 2 Satz 2 Nr. 5 BBodSchG fordert der Gesetzgeber, dass „die naturbetonten Strukturelemente der Feldflur, insbesondere Hecken, Feldgehölze, Feldraine, Ackerterrassen, die zum Schutz des Bodens notwendig sind“, erhalten werden müssen. Im Zuge einer Flurbereinigung lassen sich Strukturelemente in der Feldflur neu anlegen. Flurgehölze erfüllen neben dem Schutz vor Erosion noch weitere Funktionen, wie z. B. die Schaffung von Lebensräumen von Nützlingen und die Verbesserung des Kleinklimas des Bodens (abiotische Wirkung). Bei diesem Grundsatz stellt sich aber auch die Frage, ob hiermit in das Naturschutzrecht eingegriffen wird. Nach Ansicht von FrENZ ist die Kom- 
petenz der Grundsätze der guten fachlichen Praxis jedoch zu schwach, um einen Konflikt zwischen Bodenschutz- und Naturschutzrecht zu schaffen. ${ }^{153} 154$

\section{Erhalt und Förderung der biologischen Aktivitäten des Bodens}

In $\S 17$ Abs. 2 Satz 2 Nr. 6 BBodSchG geht es darum, dass ,die biologische Aktivität des Bodens durch entsprechende Fruchtfolgegestaltung erhalten oder gefördert wird“. Der Gesetzgeber sieht hier v. a. den Anbau von Zwischenfrüchten, die Erweiterung der Fruchtfolgen und die Schaffung eines ausgeglichenen Nährstoffkreislaufs als Maßnahmen zur Steigerung der biologischen Aktivität und somit auch der Bodenfruchtbarkeit.

\section{Erhaltung des standorttypischen Humusgehalts}

Nach $\S 17$ Abs. 2 Satz 2 Nr. 7 BBodSchG soll ,der standorttypische Humusgehalt des Bodens, insbesondere durch eine ausreichende Zufuhr an organischer Substanz oder durch Reduzierung der Bearbeitungsintensität erhalten“ werden. Dies kann durch Stallmist, Stroh, pflanzliches Material oder Gülle erfolgen. Die Kompost- und Klärschlammaufbringung führen in einem gewissen Maße auch zu einer Erhöhung des Humusgehaltes. Sie stehen jedoch aufgrund verschiedener Belastungspotenziale z. Zt. sehr in der öffentlichen Diskussion. ${ }^{155}$

\subsubsection{Die Beratung nach $\S 17$ Abs. 1 Satz 2 BBodSchG}

Die Grundsätze der guten fachlichen Praxis sollen durch die nach Landesrecht zuständigen landwirtschaftlichen Beratungsstellen (z. B. Landwirtschaftskammern, -ämter) vermittelt werden. Hierin sehen Peine, Versteyl und Frenz, dass keine Pflicht zur Vermittlung und somit zur Durchsetzung der Vorsorgeanforderungen besteht. ${ }^{156}$ Dies ist gerade im Gesetzgebungsverfahren durch den Bundesrat kritisiert worden. ${ }^{157}$ Die Gesetzesentwürfe der damaligen Oppositionsparteien SPD und Bündnis 90/DIE GRÜNEN hatten in

\footnotetext{
${ }^{153}$ Vgl. FRENZ (2000), S. 597

${ }^{154}$ Zum Verhältnis zwischen dem BBodSchG und dem novellierten BNatSchG siehe Kapitel 4.2.5.5.3.

${ }^{155}$ Zur Diskussion der Klärschlamm- und Kompostaufbringung in der Landwirtschaft siehe Kapitel 3.1.3.3.

${ }^{156}$ Vgl. Peine (2002), S. 526, Versteyl (2002), S. 362 und FrenZ (2000), S. 587

${ }^{157}$ Vgl. BT-DRUCKS. 13/6701 S. 55
} 
ihren Vorschlägen eine Grundlage für behördliche Anordnungen zur Durchsetzung der guten fachlichen Praxis enthalten. ${ }^{158}$

Bei $\S 17$ Abs. 1 Satz 2 BBodSchG handelt sich um eine Soll-Vorschrift. ${ }^{159}$ Der Gesetzgeber setzt somit auf das Prinzip der Kooperation. Dies bedeutet jedoch auch, dass die zuständigen Behörden keine Maßnahmen anordnen oder Bußgelder bei Nichtbeachtung verhängen können. ${ }^{160}$ Das Kooperationsprinzip setzt folglich ein Interesse und eine Mitwirkungsabsicht des Landwirts voraus, welches nur dadurch gewährleistet wird, dass er das Informations- und Beratungsangebot erhält. Nach Meinung der damaligen Bundesregierung ist das Kooperationsprinzip geeigneter, um auf die verschiedenen komplexen und sich ändernden Umweltbedingungen bezüglich des landwirtschaftlich genutzten Bodens zu reagieren. ${ }^{161}$ PEINE und VERSTEYL ziehen den Schluss, dass die Beratung nach $\S 17$ Abs. 1 Satz 2 das „einzige vorsorgebezogene Handlungsinstrument“ des Gesetzes darstellt. ${ }^{162}$ Hieraus ist folglich die hohe Bedeutung dieses Instrumentes für den vorsorgenden Bodenschutz abzuleiten.

Da das Bundesrecht einen Vorrang vor den landesrechtlichen Bodenschutzgesetzen hat, ist es den Bundesländern nicht möglich, eigene Rechtsvorschriften wohl aber Verwaltungsvorschriften zur Konkretisierung der gfP zu erlassen. ${ }^{163}$ Diese Vorrangigkeit kann am Beispiel des $\S 11$ Abs. 2 BodSchG BW verdeutlicht werden. Das Bodenschutzgesetz Baden-Württemberg wird momentan neu überarbeitet. Die alte Fassung sieht für die Bodenschutzbehörde die Möglichkeit vor, Maßnahmen zur Durchsetzung der in $\S 11$ Abs. 1 BodSchG BW gestellten Anforderungen an die Landwirte anzuordnen. Dies wurde durch das Inkrafttreten des BBodSchG ungültig. ${ }^{164}$

In den einzelnen Bodenschutzgesetzen der Länder sind mittlerweile die Zuständigkeiten für die Vermittlung der Grundsätze der guten fachlichen Praxis in der landwirtschaftlichen Bodennutzung geregelt worden. Sie liegen bei den Landwirtschaftskammern, -ämtern bzw. bei den Landesanstalten für Landwirtschaft.

\footnotetext{
${ }^{158}$ Vgl. BT-DruCKS. 13/7891, S. 49

${ }^{159}$ Vgl. VersteyL (2002), S. 362

${ }^{160}$ Zur Diskussion des Kooperationsprinzips siehe auch Kap. 4.4.2.

${ }^{161}$ Vgl. BT-DRUCKS. 13/6701, S. 65

162 VERSTEYL (2002), S. 363

${ }^{163}$ Vgl. FrENZ (2000), S. 588

${ }^{164}$ Schriftliche Auskunft HeRR NOTTER am 31.1.2003
} 
Als Grundlagen der Beratung können u. a. folgende Veröffentlichungen dienen:

- Bund-Länder-Papier: die Grundsätze und Handlungsempfehlungen zur guten fachlichen Praxis in der ldw. Bodennutzung ${ }^{165}$,

- KTBL-Arbeitsgruppe „Bodenbearbeitung und Bodenschutz“: Schlussfolgerungen für die gfP $^{166}$,

- Auswertungskarten zur Erosions- und Verdichtungsgefährdung: Bodenkarten im Maßstab von 1:50.000 ${ }^{167}$ und

- Verschiedene in den Ländern erarbeitete Handlungskonzepte: z. B. Informationshefte zum ldw. Bodenschutz im Land Brandenburg (ZALF) ${ }^{168}$.

Die Vermittlung der Grundsätze der gfP durch die ldw. Fachbehörden beschränkt sich mittlerweile nicht mehr nur auf Artikel in ldw. Fachzeitschriften, Feldtage und allgemeine Informationsveranstaltung für Landwirte und deren Berater. Es werden in einigen Bundesländern wie z. B. Nordrhein-Westfalen von der Landwirtschaftskammer Großparzellenversuche zur konservierenden Bodenbearbeitung durchgeführt und deren Ergebnisse auf verschiedene Weise mit den Beratern und Landwirten diskutiert. ${ }^{169}$

Eine flächendeckende Bodenschutzberatung gibt es z. Zt. nicht, obwohl es in der Expertenbefragung eine Mehrheit von etwa $73 \%$ der Befragten ${ }^{170}$ gab, die der Aussage „Es fehlt in Deutschland eine flächendeckende Bodenschutzberatung zur Durchsetzung vorsorgender Bodenschutzkonzepte für die Landwirtschaft" entweder teilweise zustimmten oder völlig zustimmten. Hierzu ist zu sagen, dass eine differenziertere Frage, z. B. nach der Notwendigkeit einer Bodenschutzberatung in speziell geschädigten oder gefährdeten Gebieten, sicherlich noch mehr Zustimmung erbracht hätte. Dies wäre zugleich ein realistischerer Ansatz, der die Effizienz von Bodenschutzberatung unter dem Aspekt der finanziellen Schwierigkeiten der Länderhaushalte noch steigern würde.

\footnotetext{
${ }^{165}$ Vgl. BMVEL (2001)

${ }^{166}$ Vgl. KTBL (1998)

${ }^{167}$ Die Auswertungskarten liegen mittlerweile für fast alle Bundesländer vor und können i. d. R. über die entsprechenden geologischen Landesämter bezogen werden.

${ }^{168}$ Vgl. FRIELINGHAUS et al. (2002a)

${ }^{169}$ Vgl. NigGeSCHULZE (2003)

${ }^{170} \mathrm{n}=26$
} 


\subsubsection{Vergleich des $\S 17$ BBodSchG mit entsprechenden Regelungen anderer Fachgesetze}

Die Verflechtungen zwischen dem Düngemittel- und Bodenschutzrecht waren bereits Gegenstand des Kapitels 4.2.4. An dieser Stelle wird ein Vergleich der gfP nach $\S 17$ BBodSchG und den wichtigsten Regelungen zur gfP im landwirtschaftlichen Fachrecht vorgenommen.

\subsection{Vergleich mit dem Düngemittelrecht}

Die gfP bei der Düngung von landwirtschaftlichen Flächen wird in der DüngeVO vom 26.01.1996 umfassend geregelt. Ein Indiz dafür, dass die gfP in der Anwendung innerhalb der Landwirtschaft ein flexibles Instrument darstellt, zeigen die Änderungen und Anpassungen der DüngeVO in der Vergangenheit. Außerdem steht eine Novellierung an, die am Ende dieses Abschnittes erläutert wird. Die letzte Änderung der DüngeVO trat am 20.03.2003 in Kraft. Sie war auf eine Verletzung der EU-Nitratrichtlinie zurückzuführen. Demnach dürfen im Betriebsdurchschnitt auf Ackerland maximal $170 \mathrm{~kg} \mathrm{~N} / \mathrm{ha}$ und auf Grünland maximal $210 \mathrm{~kg} \mathrm{~N} / \mathrm{ha}$ jährlich ausgebracht werden. Bei der Berechnung des Betriebsdurchschnitts müssen allerdings nicht bewirtschaftete Flächen sowie Bracheflächen abgezogen werden. Außerdem dürfen Ausbringungsverluste bis zu $20 \%$ des Gesamtgehaltes nicht mehr bei der Ermittlung der Obergrenzen angerechnet werden.

Neben diesen Neuerungen stellt die DüngeVO folgende Anforderungen. Düngemittel sind so auszubringen, dass

- die Nährstoffe von den Pflanzen weitestgehend ausgenutzt werden können,

- Nährstoffverluste weitestgehend vermieden werden und

- Stickstoffdüngemittel bedarfsgerecht ausgebracht werden.

Die Verordnung räumt bei der Ausbringung von Wirtschaftsdüngern tierischer Herkunft Ausbringungsverluste von $20 \%$ des Gesamtstickstoffgehalts ein. Die Maschinen und Geräte zur Ausbringung der Düngemittel müssen nach $§ 2$ DüngeVO geeignet sein und den anerkannten Regeln der Technik entsprechen. Die Maschinen und Geräte müssen eine gleichmäßige Verteilung, verlustarme Ausbringung voraussetzen und über eine sachgerechte Mengenbemessung verfügen. Außerdem darf bei der Ausbringung kein direkter 
Eintrag in Oberflächengewässer erfolgen. Es dürfen nur dann stickstoffhaltige Düngemittel ausgebracht werden, wenn der Boden dafür aufnahmefähig ist.

Nach $\S 3$ DüngeVO dürfen Wirtschaftsdünger tierischer Herkunft, Sekundärrohstoffdünger sowie vergleichbare Mehrnährstoffdünger in der Zeit vom 15. November bis zum 15. Januar grundsätzlich nicht ausgebracht werden. Die zuständige Behörde kann hierzu jedoch Ausnahmen, z. B. im Einzelfall, erlassen. Ausbringungsverluste sind bei der Ausbringung dieser Düngemittel generell so weit wie möglich zu vermeiden und auf unbestelltem Ackerland sofort einzuarbeiten. Außerdem dürfen sie nach der Hauptfruchternte nur

1. zu Feldgras, Grassamen, Untersaaten, Herbstaussaaten einschl. Zwischenfrüchten oder

2. bei Strohdüngung in gewissen Mengen

ausgebracht werden.

Nach § 4 DüngeVO sind die Grundsätze der Düngebedarfsermittlung zu berücksichtigen. Diese wird i. d. R. schlagbezogen durchgeführt. Folgende Einflussfaktoren sind dabei zu berücksichtigen:

- Standorteigenschaften,

- Bodenfruchtbarkeit: die im Boden verfügbaren Nährstoffmengen und -gehalte,

- durch Bewirtschaftung zugeführte und pflanzenverfügbare Nährstoffmengen,

- Anbaubedingungen, wie z. B. Kulturart, Vorfrucht, Bodenbearbeitung und Beregnung und

- Ergebnisse regionaler Feldversuche.

Nach $\S 5$ DüngeVO müssen Betriebe, die mehr als 10 ha LF bewirtschaften, Nährstoffvergleiche erstellen. Diese können nach zwei verschiedenen Verfahren, der Feld-StallBilanz oder der Hoftor-Bilanz, erstellt werden.

Der derzeit diskutierte Entwurf für eine Novellierung der DüngeVO sieht zahlreiche Punkte vor, die von den beteiligten sehr unterschiedlich bewertet werden. Hierzu zählt vor allem:

- ein geplanter Abstand von 2 m beim Düngen zu Gewässern und Nachbarflächen, 
- Ausbringungsverbot organischer Düngemittel ab einer Außentemperatur von $30^{\circ} \mathrm{C}$,

- Erweiterung der Sperrzeiten für Ackerflächen auf den Zeitraum 15. Oktober bis zum 15. Januar,

- Verpflichtung zur Erstellung der Hoftorbilanz bei Grünlandbetrieben, die FeldStallbilanz entfällt und

- Verbot der Gülleausbringung mit Pralltellern.

In den Ausführungen zur DüngeVO wird deutlich, dass diese Regelungen wesentlich konkreter gefasst sind und mit z. T. strengen Grenzwerten und Anforderungen die gfP beim Düngen regeln. Außerdem finden regelmäßige stichpunktartige Kontrollen zur Einhaltung der DüngeVO statt. Hierbei wird v. a. das Vorliegen der Nährstoffvergleiche überprüft. Dabei ist jedoch anzumerken, dass der Landwirt nur bestraft werden kann, wenn er keine oder nur unzureichende Nährstoffvergleiche erstellt hat. Ein Überschreiten der Düngerhöchstmengen hat für ihn keine rechtliche Konsequenzen. Die geplante Neuregelung der DüngeVO bleibt abzuwarten, allerdings ist festzustellen, dass es zu erheblichen zusätzlichen Regelungen und Auflagen für landwirtschaftliche Betriebe kommen wird.

\subsection{Vergleich mit dem Pflanzenschutzmittelrecht}

Die Grundsätze für die Durchführung der guten fachlichen Praxis im Pflanzenschutz wurden wenige Monate nach dem Erlass des ersten Gesetz zur Änderung des Pflanzenschutzgesetzes vom 14.05.1998 als Ergänzung vom BMVEL veröffentlicht. ${ }^{171}$ Die Veröffentlichung enthält acht Grundsätze, von denen die Kernpunkte im Folgenden erläutert werden sollen. Sie enthalten Regelungen zur

1. Zulassung von Pflanzenschutzmitteln,

2. Prüfung von Pflanzenschutzgeräten und

3. Sachkunde des Anwenders.

Die Grundsätze enthalten indirekt Anforderungen, die mit dem Bodenschutz verknüpft sind. Bspw. sollen die Bodenbearbeitung, das Anbausystem und die Fruchtfolgen stand-

${ }^{171}$ Vgl. hierzu BMVEL (1998) 
ortgerecht und situationsbezogen gestaltet werden, damit der Befall durch Schadorganismen nicht gefördert wird.

Bezüglich der Verwendung von Pflanzenschutzgeräten ist eine Prüfung in amtlich anerkannten Kontrollstellen vorzunehmen. Beim Einsatz sind zahlreiche Hinweise zu beachten. Bspw. sollen Spritzeinsätze bei Windgeschwindigkeiten über $5 \mathrm{~m} / \mathrm{s}$, Temperaturen über $25{ }^{\circ} \mathrm{C}$ oder relativen Luftfeuchten unter $30 \%$ unterbleiben. Die Grundsätze der guten fachlichen Praxis fordern darüber hinaus die Aufzeichnung der getätigten Arbeitsgänge mittels Schlagkarteien. Außerdem beinhaltet die gfP beim Pflanzenschutz die Einhaltung der jeweiligen Abstandsauflagen von Oberflächengewässern und Saumstrukturen. Diese müssen in einem aufwendigen Verfahren für jeden Schlag und dem verwendeten Pflanzenschutzmittel berechnet werden. Die Abstände können durch den Einsatz von abdriftarmen Düsen durch den Landwirt beeinflusst werden.

\subsection{Vergleich mit dem Naturschutzrecht}

Durch das novellierte Bundesnaturschutzgesetz vom 25.03.2002 geht der Gesetzgeber in $\S 5$ BNatSchG auf die landwirtschaftliche Bodennutzung ein und definiert anhand der gfP das Verhältnis zwischen Landwirtschaft und Naturschutz. Dabei ist dieses Verhältnis durch die Definition von Mindeststandards im Gegensatz zum vorherigen Naturschutzgesetz wesentlich stringenter. Hinzu kommt, dass Maßnahmen, die über die gfP hinaus gehen, nicht mehr per se ausgleichspflichtig sind. ${ }^{172}$

In $\S 5$ Abs. 4 BNatSchG listet der Gesetzgeber sieben Grundsätze auf, die u. a. auch die landwirtschaftliche Bodennutzung tangieren. Hierzu gehören:

Grundsatz Nr. 1: „Bei der landwirtschaftlichen Nutzung muss die Bewirtschaftung standortangepasst erfolgen und die nachhaltige Bodenfruchtbarkeit und langfristige Nutzbarkeit der Flächen gewährleistet werden.“

Grundsatz Nr. 5: „Auf erosionsgefährdeten Hängen, in Überschwemmungsgebieten, auf Standorten mit hohem Grundwasserstand sowie auf Moorstandorten ist ein Grünlandumbruch zu unterlassen.“

\footnotetext{
${ }^{172}$ Vgl. Fischer-HÜFTLE et al. (2003), § 5 Rnd. Nr. 7
} 
Grundsatz Nr. 6: „Die natürliche Ausstattung der Nutzfläche (Boden, Wasser, Flora, Fauna) darf nicht über das zur Erzielung eines nachhaltigen Ertrages erforderliche Maß hinaus beeinträchtigt werden.“

Grundsatz Nr. 7: „Eine schlagspezifische Dokumentation über den Einsatz von Dünge- und Pflanzenschutzmitteln ist nach Maßgabe des landwirtschaftlichen Fachrechts durchzuführen.“

Bei den o. g. Grundsätzen sind sowohl Übereinstimmungen (Nr. 1) als auch Ergänzung (Nr. 5, 6 und 7) zu den Grundsätzen der guten fachlichen Praxis im BBodSchG festzustellen. Die Grundsätze des BNatSchG müssen innerhalb der Ländergesetzgebung noch konkretisiert und an regionale Gegebenheiten angepasst werden.

Bei der Implementierung in die Ländergesetze ist anders als bei der gfP im Bodenschutzrecht die Durchsetzbarkeit der Grundsätze der gfP zu berücksichtigen. ${ }^{173}$ Die Länder müssen bspw. zunächst die in Grundsatz Nr. 5 genannten Begriffe „erosionsgefährdete Hänge“ oder „Überschwemmungsgebiete etc. definieren, bevor sie ein Umbruchverbot für Grünland in diesen Gebieten einführen. Diese Aufgabe wird vom Bundesgesetzgeber, der beim Naturschutzrecht die Rahmengesetzgebungskompetenz innehat, nicht wahrgenommen.

Bei Grundsatz Nr. 7 ist zu berücksichtigen, dass die Länder in diesem Punkt keinen Regelungsspielraum besitzen. Dies liegt an dem Begriff der „Maßgabe des landwirtschaftlichen Fachrechts“. Das landwirtschaftliche Fachrecht in diesem Bereich ist das Pflanzenschutz- und Düngemittelrecht, was aufgrund der konkurrierenden Gesetzgebung vom Bundesgesetzgeber als dessen Aufgabe wahrgenommen wird. Deshalb werden die Maßgaben allein vom Bundesgesetzgeber bestimmt.

\subsubsection{Die Ausgleichsregelung nach $\S 10$ Abs. 2 BBodSchG}

Die Ausgleichsregelung nach $\S 10$ Abs. 2 BBodSchG wurde im Gesetzgebungsverfahren, u. a. nach den Forderungen der landwirtschaftlichen Berufsstandsvertreter, in das Gesetz als Mindestabsicherung vor nicht selbst verschuldeten schädlichen Bodenveränderungen

${ }^{173}$ Vgl. ebd., § 5 Rnd. Nr. 28 
in das BBodSchG eingebracht. Im Folgenden wird neben der Erläuterung dieses Paragrafen eine Abschätzung der Bedeutung für die Landwirtschaft vorgenommen.

\subsubsection{Regelungsinhalte}

Die Ausgleichsregelung nach $\S 10$ Abs. 2 BBodSchG ist neben dem $\S 17$ BBodSchG die zweite Sonderregelung für die Land- und Forstwirtschaft in diesem Gesetz. Sie tritt in Kraft, wenn die zuständige Behörde Anordnungen zur Beschränkung der land- und forstwirtschaftlichen Bodennutzung zur Erfüllung der Gefahrenabwehrpflichten nach $\S 4$ BBodSchG getroffen hat. $\mathrm{Ob}$ und in welchem Umfang ein hieraus resultierender Härteausgleich gewährt wird, liegt in der Kompetenz der Bundesländer. ${ }^{174}$ Die Vorlage für diesen Paragrafen stellt $§ 19$ Abs. 4 Wasserhaushaltsgesetz (WHG) dar, in dem Ausgleichsleistungen für Nutzungsbeschränkungen in Wasserschutzgebieten geregelt werden. Die Ausgleichsleistungen werden hier schon gewährleistet, wenn noch keine entschädigungspflichtige Enteignung vorliegt. ${ }^{175}$

$\S 10$ Abs. 2 BBodSchG geht davon aus, dass der Boden für den landwirtschaftlichen Betrieb die Existenzgrundlage darstellt und dass eine Einschränkung der Nutzung eine Existenzgefährdung für diesen zur Folge haben kann. Deshalb können diese Betriebe besonders hart von Nutzungsbeschränkungen getroffen werden, welches die Sonderregelung rechtfertigen soll. Als Schäden der Nutzungsbeschränkung dürfen nur die direkt durch den Eingriff der Behörde entstandenen Nachteile gesehen werden, Summationsoder Distanzschäden werden durch $\S 10$ Abs. 2 nicht entschädigt. ${ }^{176}$

\subsubsection{Anspruchsberechtigte}

Anspruchsberechtigte sind ausschließlich Land- und Forstwirte, die Zustandsstörer ${ }^{177}$ sind. Da $\S 10$ Abs. 2 BBodSchG nicht die Frage klärt, wann ein Landwirt Verursacher ist, also ob die Bedingung der Einhaltung der guten fachlichen Praxis ausreicht, muss diese

\footnotetext{
${ }^{174}$ Hierzu dient z. B. in Niedersachsen $\S 12$ NBodSchG, der in einer Verordnung die Höhe des Ausgleichs bestimmt. Diese ist z. Zt. noch in Bearbeitung.

${ }^{175}$ Vgl. hierzu KÖHN (2002)

${ }^{176}$ Vgl. FrenZ (2000), S. 453 f.

${ }^{177}$ Der Zustandsstörer ist der Eigentümer oder Besitzer eines Grundstücks, welches die Gefahr einer schädlichen Bodenveränderung aufweist. Im Gegensatz dazu ist derjenige, der eine Altlast oder schädliche Bodenveränderung verursacht hat der Verhaltensstörer.
} 
mit den allgemeinen Anforderungen des Gesetzes beantwortet werden. ${ }^{178}$ Dies geschieht in $\S 4$ Abs. 3 BBodSchG.

\subsubsection{Anspruchsvoraussetzungen}

Eine Grundvoraussetzung für die Zahlung eines Ausgleichs an einen Landwirt ist eine behördliche Anordnung zur Beschränkung der land- und forstwirtschaftlichen Bodennutzung. Hiermit sind sowohl Anbau- als auch Nutzungsverbote gemeint. Die Maßnahme muss außerdem eine besondere Härte, ein sog. Sonderopfer, darstellen, die über die allgemeine Belastung erheblich hinausgeht. Hierzu müssen die allgemeinen Belastungen anderer Grundstückseigentümer oder - besitzer, denen Nutzungsbeschränkungen auferlegt wurden, ermittelt und in Relation mit den Belastungen des einzelnen Landwirts gesetzt werden. Die Einschränkungen müssen ferner mit einer drohenden Existenzgefährdung des landwirtschaftlichen Betriebs einhergehen.

\subsubsection{Zumutbare innerbetriebliche Anpassungsmaßnahmen}

Der Landwirt hat innerhalb seines wirtschaftlichen Handelns die Möglichkeit und die Pflicht nach $\S 254$ BGB, Anpassungsmaßnahmen zu ergreifen, um die Schwere der behördlichen Anordnungen abzumildern. Deshalb ist nach $\S 10$ Abs. 2 Satz 1 BBodSchG nur „für die nach zumutbaren innerbetrieblichen Anpassungsmaßnahmen verbliebenen“ wirtschaftlichen Schäden (z. B. Ertragsminderungen oder -ausfälle) ein Ausgleich zu zahlen. Die Zumutbarkeit der Anpassungsmaßnahmen soll sich vor allem nach betriebswirtschaftlichen Gesichtspunkten richten. Zumutbare Anpassungsmaßnahmen sind im Einzelnen: ${ }^{179}$

- zumutbare Reduzierung der Erzeugung,

- Umstellung der Produktion auf ökologischen Landbau und

- Substitution von Nutzpflanzen durch Zierpflanzen.

In einem solchen Fall müssen deshalb die Kosten für die Umstellung der Betriebsabläufe erfasst werden. Die Zumutbarkeit von Maßnahmen richtet sich nach folgenden Kennzeichen des Betriebes:

\footnotetext{
${ }^{178}$ Vgl. Frenz (2000), S. 454

${ }^{179}$ Vgl. BT-Drucks. 13/6701, S. 41
} 
- Produktionsprogramm des Gesamtbetriebes und

- Möglichkeit des Flächentausches.

\subsubsection{Der Begriff des angemessenen Ausgleichs}

Nach $\S 10$ Abs. 2 BBodSchG ist ein angemessener Ausgleich zu zahlen. Durch den angemessenen Ausgleich dürfen nur wirtschaftliche Beeinträchtigungen beglichen werden, die eine direkte Verbindung zur behördlichen Anordnung besitzen. Außerdem muss der Ausgleich nicht den vollständigen Schaden abdecken und auch nicht zwangsläufig finanzieller Natur sein. Er kann auch durch die Zuweisung eines neuen Grundstücks erfolgen. ${ }^{180}$ Ein erster Regierungsentwurf sah z. B. folgende Berechnung des angemessenen Ausgleichs vor, die zur Anwendung kommen könnte: ${ }^{181}$

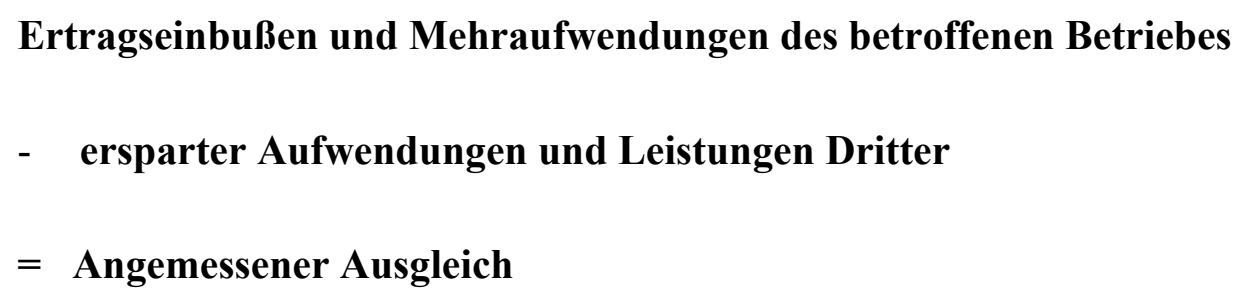

Da der Ausgleich jedoch von den Ländern zu zahlen ist, wurde diese Regelung nicht übernommen, um ihnen einen gewissen Spielraum zu lassen. Der ordentliche Rechtsweg steht bei Streitigkeiten generell nicht offen.

\subsubsection{Bedeutung des $\S 10$ Abs. 2 BBodSchG in der Praxis}

Der Gesetzgeber hat im Laufe des Gesetzgebungsverfahrens die Kosten für den Vollzug des Bundes-Bodenschutzgesetzes abgeschätzt und kommt im Fall der Ausgleichsregelung des $\S 10$ Abs. 2 BBodSchG nach den Schätzungen des Umweltbundesamtes zu dem Schluss, dass die Ausgleichsregelung in der worst-case-Situation Mehrkosten in Höhe von etwa 24 Mio. $€$ pro Jahr für die Länderhaushalte bedeuten würde. Es wurde jedoch davon ausgegangen, dass die Kosten deutlich darunter liegen, was dem Wesen einer

\footnotetext{
${ }^{180}$ Vgl. Frenz (2000), S. 458

${ }^{181}$ Vgl. Frenz (2000), S. 457
} 
worst-case-Abschätzung entspricht. ${ }^{182}$ Die einzelnen möglichen ausgleichsfähigen Flächen und Kostenpositionen sind folgende ${ }^{183}$ :

- $\quad 15.000$ ha Straßenbegleitböden

- $\quad 50.000$ ha Ackerflächen

- $\quad 5.000$ ha Erwerbsgartenbau

- $\quad 130.000$ ha Grünland
1,5 Mio. €/a

7,5 Mio. €/a

2,5 Mio. $€ / a$

13 Mio. $€ /$ a.

Die Studie schließt lediglich die durch Immissionen entstandenen Kosten ein. Die Ermittlung der Kosten wurde in Anlehnung an LATACZ-LOHMANN ${ }^{184}$ durchgeführt. Bei der Kostenermittlung des Ausgleichs bei Grünland wurden immissionsbedingte Mehrkosten von 0,19 €/Kuh und Tag angenommen. Bei der Berechnung des Erwerbsverlustes auf Ackerflächen wurde eine Senkung des Standarddeckungsbeitrages von $145 €$ /ha/a angenommen.

Die Studie ist als grobe Abschätzung der möglichen ausgleichsfähigen Schäden zu sehen und gibt höchstens diesbezügliche Anhaltspunkte. Sie sollte daher nicht als Hauptargument gegen die Existenz des $\S 10$ Abs. 2 BBodSchG herangezogen werden, zumal die Erkenntnis der Expertenbefragung in der Vollzugspraxis dafür spricht, dass diese Regelung in der Vollzugspraxis nahezu bedeutungslos ist. ${ }^{185}$ Dies wird sowohl durch den Aufbau des $\S 10$ Abs. 2 BBodSchG und seine vielen Einschränkungen, sowie der äußerst angespannten Haushaltslage aller öffentlicher Kassen deutlich. Daher ist davon auszugehen, dass auf der Ebene der unteren Bodenschutzbehörden zuerst alle Möglichkeiten der Verwehrung von Ausgleichszahlungen an landwirtschaftliche Betriebe ausgeschöpft werden. Außerdem fehlen in einigen Bundesländern noch Verordnungen, die $\S 10$ Abs. 2 BBodSchG in Länderrecht umsetzen. ${ }^{186}$

Abzuwarten bleibt, inwieweit in Zukunft eine erhöhte Dringlichkeit besteht, der Ausgleichspflicht nach $\S 10$ Abs. 2 BBodSchG nachzukommen. Vor dem Hintergrund der Verschärfung des EU-Rechts, bspw. im Bereich der Futtermittelrichtlinie und Kontami-

\footnotetext{
${ }^{182}$ Vgl. BT-DRUCKS. 13/6701, S. 4, 5

${ }^{183}$ Vgl. BACHMANN, V. BORRIES U. DinKELBERG (1995), S. 4

${ }^{184}$ Vgl. LATACZ-LOHMANN (1993)

${ }^{185}$ Vgl. Kapitel 4.2.6.7

${ }^{186}$ Vgl. NEIDHART (2003)
} 
nantenVO, sowie der gewachsenen Anforderungen an den Verbraucherschutz, ist zu erwarten, dass derartige Konfliktfälle zunehmen werden.

\subsubsection{Ergebnisse der Expertenbefragung}

In der Expertenbefragung wurde die Problematik der Ausgleichsregelung des $\S 10$ Abs. 2 BBodSchG in Form von zwei Thesen zu denen die Experten Stellung nehmen sollten und einer Frage behandelt. Zunächst wurde folgende These aufgestellt: „Die Ausgleichsregelung nach \& 10 Abs. 2 BBodSchG ist eine ungerechtfertigte Sonderregelung für die Landwirtschaft". Bei dieser Frage, die von 18 Experten beantwortet wurde, lehnten 11,5 \% der Befragten diese These völlig ab und 42,3 \% stimmten ihr nicht zu. Eine Zustimmung ergab sich lediglich bei 15,4\% der Befragten.

Die zweite These bezog sich auf die bisherige Bedeutung der Ausgleichsregelung des $\S$ 10 Abs. 2 BBodSchG in der Vollzugspraxis. Sie lautete: „Die Ausgleichsregelung nach $\S 10$ Abs. 2 BBodSchG hat in der Vollzugspraxis keine Bedeutung, da es kaum Fälle gibt, in denen ein Härteausgleich für Landwirte gezahlt wird“. Dieser These stimmten 19,2 \% der 16 Befragten völlig zu und 30,8 \% stimmten ihr zu. 7,7 \% der Befragten stimmten nicht zu bzw. 3,8 \% lehnten sie völlig ab.

Abschließend wurde $\mathrm{zu}$ diesem Themengebiet die Frage nach den zukünftigen Ausgleichszahlungen durch $\S 10$ Abs. 2 BBodSchG gestellt. Bei dieser Frage war das Ergebnis allerdings nicht eindeutig. Es antworteten insgesamt 10 Experten auf diese Frage. 19,2 \% waren der Meinung, dass die Zahlungen in Zukunft steigen werden. 15,4 \% waren der Meinung, dass die Zahlungen unverändert bleiben werden und 3,8 \% gaben an, die Zahlungen werden sinken.

\subsubsection{Die Ausweisung von Bodenschutzgebieten nach $\S 21$ Abs. 3 BBodSchG}

$\S 21$ Abs. 3 BBodSchG erlaubt es den Ländern, u. a. Gebiete auszuweisen, in denen „flächenhaft schädliche Bodenveränderungen auftreten oder zu erwarten sind“. Sie können in diesen Fällen gefahrenabwehrende Maßnahmen einleiten, die auf das gesamte Gebiet ausgedehnt werden können. Eine derartige Ausweisung kann auch Auswirkungen auf landwirtschaftliche Betriebe haben, wie das unten angeführte Beispiel des Landkreises Goslar in Niedersachsen zeigt. 
In den bisher ausgewiesenen Landesbodenschutzgebieten wird die Festlegung der Bodenplanungsgebiete durch jeweilige Rechtsverordnungen vorgesehen. Hierbei gibt es jedoch Kompetenzunterschiede in den Ländern: z. B. können in Niedersachsen die unteren Bodenschutzbehörden ${ }^{187}$ die Bodenplanungsgebiete ausweisen. Der Erlass dieser Verordnungen ist an die Praxis der Ausweisung von Landschaftsschutz- und Naturschutzgebieten angelehnt. So ist die Verordnung öffentlich bekannt zu geben und es sind die Träger öffentlicher Belange, an dem Verfahren zu beteiligen. Je nach Art und Maß der schädlichen Bodenveränderung können in einem Bodenplanungsgebiet folgende Maßnahmen festgelegt werden:

- Dauerhafte oder zeitlich befristete Nutzungsverbote oder -beschränkungen,

- Verbote des Einsatzes bestimmter Stoffe,

- Abdeckungs- oder Bepflanzungsgebote für den Boden,

- Regelungen zur Verwendung oder Ablagerung von ausgehobenem oder abgeschobenem Bodenmaterial und

- Handlungs- und Duldungspflichten der Grundstückseigentümer bzw. der Nutzer bezüglich Maßnahmen zur Vermeidung oder Verhinderung von schädlichen Bodenveränderungen.

Im Folgenden wird als Exkurs das Beispiel des Bodenplanungsgebiets Goslar dargestellt, welches das erste seiner Art in Deutschland ist.

\section{Beispiel: Bodenplanungsgebiet ${ }^{188}$ Landkreis Goslar}

Ein Beispiel für die Anwendung des $§ 21$ Abs. 3 BBodSchG stellt der Landkreis Goslar dar, in dem aufgrund von sowohl geogenen als auch anthropogenen Einflüssen, v. a. durch die lange Bergbaugeschichte, Abbau von Blei-, Zink- und Silbererzen, die Vorsorgewerte der BBodSchV bezüglich der Schwermetalle Blei, Zink, Kupfer und Nickel um mehr als das tausendfache überschritten wurden. Auch die sich hierin befindlichen landwirtschaftlichen Nutzflächen wiesen zu hohe Prüfwerte auf.

\footnotetext{
${ }^{187}$ nach $\S 9$ Abs. 3 NBodSchG

188 Im niedersächsischen Bodenschutzgesetz wird der Begriff Bodenplanungsgebiet und nicht Bodenschutzgebiet verwendet.
} 
Dieses Gebiet wurde vor diesem Hintergrund am 1.10.2001 nach § 4 NBodSchG durch eine Verordnung ${ }^{189}$ der unteren Bodenschutzbehörde, in diesem Fall der Landkreis Goslar, zu einem Bodenplanungsgebiet erklärt, welches eine Größe von $50 \mathrm{~km}^{2}$ umfasst.

Nach $\S 11$ Abs. 4 dieser Verordnung, die abweichend erst vollständig am 1.10.2005 in Kraft treten wird, müssen die Landwirte dafür Sorge tragen, dass die Schadstoffe nicht vom Boden in die Nutzpflanzen gelangen. Die untere Bodenschutzbehörde kann in Einzelfällen den Anbau von Nutzpflanzen untersagen. Weizen darf nur angebaut werden, wenn die untere Bodenschutzbehörde die Eignung der Weizensorte geprüft hat.

Die Anlage 1 der Verordnung enthält einige Maßnahmen zur Umsetzung der obigen Forderungen. Z. B. soll die Erosion und der damit verbundene unerwünschte Schadstofftransport durch Einbringung von Mulch oder Kompost sowie durch das Bearbeiten quer zum Hang vermieden werden. Um eine Vermischung der belasteten Schichten des Bodens zu erreichen, vorausgesetzt die Schadstoffgehalte liegen nicht über den Vorsorgegrenzwerten, sollen die Landwirte einen Tiefenumbruch durchführen. Außerdem sollte der pH-Wert bei Ackerland durch Kalkung auf mindestens 7,2 und bei Grünlandflächen auf mindestens 6,7 angehoben werden, um die Mobilität der Schwermetalle im Boden zu senken. Der Einsatz schadstoffarmer Düngemittel sollte selbstverständlich sein. Um einen Export von belasteten Boden zu vermeiden, sollte das Erntegut möglichst schmutzfrei eingeholt werden.

\subsection{Die Bundes-Bodenschutz- und Altlastenverordnung}

Die Bundes-Bodenschutz- und Altlastenverordnung (BBodSchV) vom 12.07.1999 ist das untergesetzliche Regelwerk des BBodSchG. Im Folgenden werden die wesentlichen Inhalte der BBodSchV dargestellt und deren Auswirkungen auf die Landwirtschaft beleuchtet.

\subsubsection{Allgemeine Vorschriften und Anwendungsbereiche}

Die BBodSchV besteht aus acht Teilen, die in erster Linie der Untersuchung und Bewertung von altlastverdächtigen Flächen, der Sanierung von schädlichen Bodenveränderun-

${ }^{189}$ AMTSBLATT FÜR DEN LANDKREIS GOSLAR (2001) 
gen und Altlasten sowie der Vorsorge gegen das Entstehen schädlicher Bodenveränderungen enthalten. Die Bedeutung dieser Teile für die Landwirtschaft ist aufgrund der Fokussierung auf Altlastenfälle eher begrenzt. Der sechste Teil der BBodSchV ist jedoch für die Landwirtschaft sehr bedeutend und geht explizit auf die Gefahrenabwehr von schädlichen Bodenveränderungen durch Bodenerosion ein und wird deshalb nachfolgend näher behandelt.

\subsubsection{Ergänzende Vorschriften für die Gefahrenabwehr von Bodenerosio- nen durch Wasser ( $\S$ 8, 5 BBodSchV)}

$\S 8$ BBodSchV dient zunächst der allgemeinen Identifikation einer schädlichen Bodenveränderung durch Wassererosion. Hierzu wird in diesem Paragrafen festgelegt, dass erhebliche Mengen an Bodenmaterial abgeschwemmt worden sind und dies auch zukünftig zu erwarten ist. Dabei geht $\S 8$ Abs. 4 Satz 2 BBodSchV davon aus, dass Bodenabträge zu erwarten sind, wenn aus den Standort- und Niederschlagsdaten des Gebietes ersichtlich wird, dass in einem Zeitraum von zehn Jahren erneut mit Bodenabträgen zu rechnen ist. Außerdem muss der Ursprungsort des Abtrages eindeutig bestimmt werden können. Diese Bewertung ist jeweils einzelfallbezogen und unter Einbeziehung jeweiliger Standortbedingungen durchzuführen.

Die Anforderungen an die Untersuchung und Bewertung von verdächtigen Flächen werden im Anhang 4 der BBodSchV bestimmt. Die Anforderungen und Empfehlungen im Anhang sehen bspw. vor, dass zur Erfassung der Bodenerosion die „Kartieranleitung zur Erfassung aktueller Erosionsformen“ des DVWK ${ }^{190}$ oder weitere Erosionsprognosemodelle zum Einsatz kommen können. Für die Ermittlung der Wiedereintrittswahrscheinlichkeit von Bodenabträgen sind v. a. langjährige Aufzeichnungen von Niederschlagsverteilungen des Deutschen Wetterdienstes heranzuziehen.

Wenn die Analyse abgeschlossen ist und eine schädliche Bodenveränderung durch Wassererosion vorliegt, wird der zuständigen Beratungsstelle (i. d. R. Landwirtschaftskammer bzw. -amt) die Gelegenheit gegeben, Maßnahmen zur Gefahrenabwehr zu empfehlen oder anzuordnen. Bei Anordnungen muss die entsprechende landwirtschaftliche Fachbehörde allerdings ihre Zustimmung geben.

${ }^{190}$ Vgl. DVWK (1996) 
Dies wird im dritten Teil der BBodSchV, d. h. von $\S 5$ BBodSchV, geregelt. Dabei wird in Absatz 5 auf land- und forstwirtschaftliche Flächen eingegangen. Treten bspw. auf diesen Flächen schädliche Bodenveränderungen auf, kommen nach $§ 5$ BBodSchV vor allem Schutz- und Beschränkungsmaßnahmen zur Anwendung. Diese können durch die Anpassung der Nutzung oder Bewirtschaftung oder die Veränderung der Bodenbeschaffenheit vollzogen werden. Hierfür müssen allerdings zwei Voraussetzungen erfüllt werden. Die getroffenen Maßnahmen müssen dokumentiert werden und es muss, wie bereits oben dargestellt, das Einvernehmen der zuständigen landwirtschaftlichen Fachbehörde für die Durchführung der Maßnahme eingeholt werden.

\subsection{Das Bodenschutzrecht in der rechtspolitischen Diskussion}

Nicht nur während der Gesetzgebungsphase wurde das entstehende Bodenschutzrecht von vielen Seiten stark kritisiert, sondern auch einige Jahre nach dem In-Kraft-Treten des Gesetzes gibt es noch eine Vielzahl an Fachleuten, die z. T. elementare Kritik am Bodenschutzrecht üben. In diesem Kapitel wird daher versucht, ein Zwischenfazit nach nunmehr fünf Jahren Bodenschutzgesetzgebung zu ziehen. Dabei werden die z. T. sehr konträren Auffassungen über das Bodenschutzrecht zusammengefasst und diskutiert. Die Ergebnisse der Expertenbefragung dienen hierbei zur Abrundung der Diskussion.

\subsubsection{Stand der Diskussion um das deutsche Bodenschutzrecht}

Die Kritik seitens der Wissenschaft und Verbände richtet sich in erster Linie an die Regelungen für die landwirtschaftliche Bodennutzung. Dies wird in einem Zitat des derzeitigen Direktors des Umweltbundesamtes deutlich: „Es gibt in Deutschland keine nachhaltige Bodennutzung. Das BBodSchG reguliert die Gefahrenabwehr und versucht gröbste Schäden abzuwenden. “191 Die Meinung von PEINE, einem der schärfsten Kritiker des aktuellen Bodenschutzrechts, zur angeblich fehlenden Vorsorge gestaltet sich ähnlich. ${ }^{192}$ Seiner Meinung nach sieht der Gesetzgeber zwar die Probleme, die durch landwirtschaftliche Bodennutzung verursacht würden, er war jedoch nicht in der Lage, die notwendigen Instrumente zur Durchsetzung der Vorsorgeanforderungen bereit zu stellen. PEINE wirft dem Gesetzgeber vor, dass das Gesetz ein reines Altlastensanierungsgesetz ist und nicht

\footnotetext{
${ }^{191}$ SCHENKEL (2000), S. 1

192 PEINE (2002), S. 526
} 
ansatzweise Versiegelungsvorschriften beinhaltet, um somit den fortschreitenden Bodenverbrauch zu begrenzen. Dies ist besonders vor dem Hintergrund der zukünftigen Entwicklung zu sehen. Es kann z. B. davon ausgegangen werden, dass die Altlastenproblematik an Bedeutung verlieren wird. Der zweite große Regelungsbereich, der Stoffeintrag durch Landwirtschaft und Industrie, wird ebenfalls an Bedeutung verlieren, weil die Zahl der Emittenten und die emittierten Schadstoffe abnehmen. Umso wichtiger ist es, dass das BBodSchG sich dem Problembereich der Flächenversiegelung widmet. Dem ist jedoch auch entgegenzuhalten, dass derartige Regelungen durch das BauGB getroffen werden. Hierzu kann bereits jetzt auf $\S 1$ a Abs. 1 BauGB, der sogenannten Bodenschutzklausel des BauGB, verwiesen werden. Dieser Artikel stellt jedoch lediglich ein Gebot dar, dass mit dem Boden sparsam und schonend umgegangen werden soll.

PEINE bemängelt ebenfalls, dass $§ 17$ BBodSchG nicht sachgerecht normiert ist. ${ }^{193}$ Dies liegt vor allem daran, dass die Handlungsempfehlungen auf sieben Grundsätze beschränkt sind und nicht vollständig ausgeführt werden. Abschließend sieht er das BBodSchG als „Lückenfüller“, nicht jedoch als komplettes eigenständiges Bundesgesetz.

MÜLLER ${ }^{194}$ kritisiert v. a. die fehlenden Instrumente der Vorsorge und Gefahrenabwehr im Bund-Länder-Papier, welches die Konkretisierungen der Grundsätze der guten fachlichen Praxis für die Problembereiche Bodenerosion und Bodenverdichtung enthält. Als Konsequenz fordert MÜLLER sowohl eine unterstützende Beratung der Landwirte als auch entsprechende Anordnungskompetenzen der Bodenschutzbehörden.

Die Entstehung und der Erlass des Bodenschutzgesetzes im Jahr 1999 hat auf verschiedenen Ebenen und bei unterschiedlichen Berufsgruppen zahlreiche Erwartungen geweckt, welche durch die individuellen Ziele entstanden sind. Bspw. war aus Sicht des Deutschen Bauernverbandes die Notwendigkeit eines eigenständigen Bodenschutzgesetzes v. a. aus den Gründen der Altlastenproblematik, der Verringerung des Bodenverbrauchs, der Zersplitterung des Bodenschutzrechts und einer fehlenden Ausgleichsregelung gegeben. ${ }^{195}$ Auf der Basis dieser Erwartungshaltung konnte jedoch nur eine Ernüchterung folgen. Das Gesetz regelt zwar die Vorsorge und Sanierung von Altlasten ausreichend, den Problembereich Bodenversiegelung jedoch nur unzureichend, außerdem gibt es differierende

\footnotetext{
${ }^{193}$ Vgl. PeIne (2003), S. 409

${ }^{194}$ Vgl. MÜLLER (2002), S. 241

${ }^{195}$ Vgl. LATTEN (1998), S. 43 f.
} 
Landesbodenschutzgesetze, die zu einem uneinheitlichen Vollzug führen. Die Ausgleichsregelung ist zwar im Gesetz verankert, in der Vollzugspraxis jedoch bedeutungslos. ${ }^{196}$

Mit der Diskussion um die Vorsorgeaspekte des Bodenschutzrechts geht auch die Diskussion um die gfP der Bodennutzung nach $\S 17$ BBodSchG einher. Der SACHVERSTÄNDIGENRAT FÜR UMWELTFRAGEN (SRU) spricht sich in einem Sondergutachten ${ }^{197}$ von 1996, also während der Gesetzgebungsphase des heutigen Bodenschutzgesetzes, gegen ordnungsrechtliche Vorschriften im Bodenschutzrecht und für die landwirtschaftliche Beratung aus. In einem neuen Sondergutachten fordert der SRU jedoch allgemeine gesetzliche Schutzvorgaben mit Standortbezug gegenüber Bodenerosion und -schadverdichtung. Für andere Umwelteinwirkungen sieht er nicht diese Notwendigkeit. ${ }^{198}$ Nach dem In-KraftTreten des BBodSchG wurde die Kritik gegen das Instrumentarium der gfP jedoch weiter vorangetrieben.

Der Wissenschaftliche BeiRAt Bodenschutz plädierte bereits mehrmals für eine Konkretisierung der gfP. ${ }^{199}$ Auch der NABU fordert in bestimmten Bereichen der landwirtschaftlichen Bodennutzung eine Konkretisierung der gfP. Diese könnte u. a. durch eine Verpflichtung der ganzjährigen Bodenbedeckung und die Pflicht zu hangparalleler Bewirtschaftung in erosionsgefährdeten Gebieten gewährleistet werden. ${ }^{200}$

MÜLLER $^{201}$ ist der Meinung, dass die fehlende Möglichkeit der Konkretisierung und Durchsetzung der gfP die entscheidende Schwachstelle des Gesetzes ist. Er führt in diesem Zusammenhang das Düngemittelrecht an. Das DMG habe bspw. nicht zu einer Reduzierung der verwendeten Düngermenge in der ldw. Praxis geführt, deshalb sei unmittelbar die DüngeVO als Konkretisierung herangezogen worden. Bei dieser Argumentation muss allerdings angeführt werden, dass beide Bereiche zwar auf rechtlicher Ebene miteinander verglichen werden können, einem Vergleich auf naturwissenschaftlichtechnischer Basis halten sie jedoch nicht Stand. Es muss berücksichtigt werden, dass die Umsetzung der bestehenden DüngeVO nicht an naturwissenschaftlich-technische, son-

\footnotetext{
${ }^{196}$ Vgl. Kapitel 4.2.6.7

${ }^{197}$ Vgl. SRU (1996), S. 82

${ }^{198}$ Vgl. BT-Drucks. 14/9852, S. 142

${ }^{199}$ Vgl. Agra-Europe (2000), S. L 11

${ }^{200}$ Vgl. WEINS (2001), S. 248

${ }^{201}$ Vgl. MÜLLER (2002), S. 105
} 
dern an administrative Grenzen stößt. Bspw. sind hinreichende Analysemethoden zur Ermittlung von Nitratgehalten etc. schon seit längerem bekannt und können flächendeckend eingesetzt werden. Im Bereich des physikalischen Bodenschutzes werden derartige Messmethoden derzeit entwickelt. Der flächendeckende Einsatz ist jedoch noch nicht möglich. An dieser Stelle ist auch der Vorteil der nicht abgeschlossenen Definition der gfP sehr offensichtlich. Der Gesetzgeber kann bei einem sich neu darstellenden Stand der Forschung reagieren und derartige Anforderungen an die gfP knüpfen. Als Nachteil dieser Vorgehensweise ist zu nennen, dass innerhalb der Landwirtschaft dadurch eine z. T. erhebliche Rechtsunsicherheit über lange Zeiträume entstehen kann. Außerdem kann es zu erhöhten Verwaltungskosten kommen, da im Falle von Rechtsstreitigkeiten die Interpretation der gfP durch die Gerichte vorgenommen werden muss.

Ein weiterer Kritikbereich, der in Kapitel 4.4.2 näher diskutiert wird, ist die Frage, ob es neben den Vorsorge- und Maßnahmewerten im stofflichen Bodenschutz auch im Bereich des physikalischen Bodenschutzes Grenzwerte zur Vorsorge gegen schädliche Bodenveränderungen geben soll. Derartige Diskussionen über Vorsorgemaßnahmen werden u. a. seitens einiger Fachexperten aus dem Bereich Bodenwissenschaft, aber auch vom Deutschen Institut für Normung (DIN) geführt.

\subsubsection{Diskussion über fehlende Anordnungen und Grenzwerte im Bereich des physikalischen Bodenschutzes}

Wie im vorigen Kapitel bereits kurz beschrieben, steht die Diskussion über fehlende Bestimmungen und Grenzwerte als Ergänzung der gfP schon seit längerem im Mittelpunkt der öffentlichen Auseinandersetzung mit dem Bodenschutzrecht in Deutschland.

Generell müssen Grenzwerte für den physikalischen Bodenschutz folgende Bedingungen erfüllen,

- Anwendbarkeit aus naturwissenschaftlicher und landtechnischer Sicht,

- Kontrollier- und Durchsetzbarkeit aus organisatorisch-legislativer Sicht,

- Vereinbarkeit mit dem europäischen Recht,

- Förderungsmöglichkeiten aus agrarpolitischer Sicht und

- Verursachung möglichst geringer Transaktionskosten. 
Im Kapitel 4.4.1 wurde bereits deutlich, dass die Festlegung von Grenzwerten voraussetzt, dass diese durch entsprechende Messungen auch überprüft werden können. Gerade bei dem Problem der Bodenschadverdichtung sind derartige Messtechniken, die flächendeckend einsetzbar sind, derzeit noch in der Entwicklung. Bspw. wurde in Großbritannien vor kurzem ein Penetrometer entwickelt, das an der Spitze der Grubberschare angebracht werden kann und dort den Widerstand und die Feuchtigkeitsstufe während des Fahrens misst. Eine weitere Entwicklung ist z. B. der Spurtiefenmesser, der die Eindringtiefe des Reifens in den Boden misst. Hiergegen gibt es allerdings bodenwissenschaftliche Vorbehalte, die an dieser Stelle nicht näher ausgeführt werden. Die zukünftige Entwicklung bleibt abzuwarten, denn geeignete Messmethoden sind die Basis für die verlässliche Durchführung und Kontrolle. Auch wenn die EU bisher nur Absichtserklärungen und Strategiepapiere bzgl. des Bodenschutzes vorgelegt hat, muss davon ausgegangen werden, dass auch momentan diskutierte Gesetzes- oder Verordnungsänderungen im deutschen Bodenschutzrecht eine EU-Konformität aufweisen müssen. Ein generelles Problem der Festsetzung von Grenzwerten sind die Kosten des Verwaltungs- und Kontrollaufwandes. Bei dem Problembereich Bodenschadverdichtung ist eine Festsetzung von Achslastbegrenzungen zu bestimmten Feuchtigkeitszuständen des Bodens mit vertretbaren Kontrollaufwendungen nicht vereinbar. Auf der anderen Seite wäre eine generelle Achslastbegrenzung zwar etwas besser kontrollierbar, dafür hätte solch eine Regelung eine unverhältnismäßige Erhöhung der Produktionskosten zur Folge, da sie die standortspezifischen Gegebenheiten der zu bearbeitenden Schläge in keiner Weise berücksichtigen würde und daher als suboptimal zu bezeichnen ist.

Weiterhin ist bei der komplexen und der Standortvariabilität unterworfenen landwirtschaftlichen Bodennutzung festzustellen, dass eine Normung der Produktionsverfahren per se nicht sachgerecht ist. Anders als industrielle Herstellungsprozesse ist die bodenbezogene Produktion von Nahrungsmitteln vielfach nicht das Ergebnis der Anwendung von Patentrezepten und normbaren Einflussgrößen, sondern basiert auf der langjährigen Erfahrung und auf dem fachlichen Wissen des Betriebsleiters, welches Bodenbeeinträchtigungen nicht gänzlich verhindern kann. Deshalb sind Grenzwerte und zusätzliche Anordnungen, wenn deren Implementierung unumgänglich sind, so weit wie möglich an die regionalen Gegebenheiten anzupassen und unter der Prämisse möglichst geringer Transaktionskosten zu sehen. 


\subsubsection{Die Unterstützung des Bodenschutzrechts durch Cross Compliance}

Abschließend wird in diesem Kapitel auf das Instrument der „Cross Compliance“ (CC) eingegangen, das in Hinblick auf die deutsche wie auch auf die europäische Bodenschutzpolitik zukünftig eine große Rolle spielen wird.

\subsubsection{Grundsätzliche Vorüberlegungen}

Für den Begriff der CC gibt es keine entsprechende deutsche Übersetzung. Das Instrument der CC stammt aus den USA und kann als eine Kopplung verschiedener umweltrelevanter Vorgaben an den Erhalt direkter Einkommensübertragungen gesehen werden. ${ }^{202}$ Das Ziel des CC in der europäischen Agrarpolitik ist es, einen Mindeststandard an Umweltleistungen flächendeckend zu erreichen. Im Gegensatz zur gfP wendet man bei der $\mathrm{CC}$ die oben angesprochene Kopplung der Einkommenstransfers an die Einhaltung von Umweltstandards als finanzielles Druckmittel an. Die nötige räumliche Differenzierung wird dadurch geschaffen, dass die Mitgliedstaaten über die Ausgestaltung geeigneter CCMaßnahmen entscheiden. Diese Vorgehensweise birgt allerdings die Gefahr, dass innerhalb der EU unterschiedlich strenge Umweltstandards implementiert werden und für die Mitgliedsstaaten Wettbewerbsvor- oder -nachteile entstehen können. Ein weiterer bisher noch ungeklärter Streitpunkt bei der Umsetzung der CC ist die Entwertung von Agrarumweltprogrammen. Je nach der Höhe der Anforderungen im Rahmen der CC kann sie dazu führen, dass diese über die Anforderungen der AUP hinaus gehen und diese somit keine Berechtigung mehr hätten. Die Folge wäre ein Einkommensverlust innerhalb der Landwirtschaft in Höhe der ansonsten gezahlten Prämien in den überflüssig gewordenen Agrarumweltprogrammen. LATACZ-LOHMANN u. BUCKWELL sehen bei der Maßnahme der CC außerdem die Gefahr von Ineffizienzen in Bezug auf mögliche Wohlfahrtseffekte. $^{203}$

Ähnlich wie bei der gfP können bei den CC-Regelungen Kontrolldefizite entstehen. Außerdem führt eine hinreichende Kontrolle der Einhaltung einer Bandbreite von Umweltstandards zu hohen Kontrollkosten.

\footnotetext{
${ }^{202}$ Vgl. LATACZ-LOHMANN u. BUCKWELl (1998), S. 429

${ }^{203}$ Vgl. LATACZ-LOHMANN u. BUCKWELL (1998), S. 429
} 
In den USA war und ist das Konzept der CC sehr erfolgreich. Für den Bereich des Bodenschutzes gibt es bspw. weniger abstrakte staatliche Anordnungen und dafür Bodenschutzberater, die einzelbetriebliche Lösungen erarbeiten. Das Conservation Reserve Program (CRP), welches in Kapitel 6.3.1.9 näher erläutert wird, leistet dabei eine finanzielle Förderung. Dies führt neben einer hohen Akzeptanz der Landwirte zu einer möglichst geringen Kontrollnotwendigkeit.

\subsubsection{Umsetzung in der EU und in Deutschland}

Die Option für die Mitgliedsstaaten, durch CC Direktzahlungen an Umweltauflagen zu koppeln, wurde mit den Beschlüssen der Agenda 2000 eingeführt. Zum Stand der Umsetzung der CC in den einzelnen Mitgliedsstaaten wird auf das INSTITUTE FOR EUROPEAN ENVIRONMENTAL POLICY (IEEP) verwiesen. ${ }^{204}$

Im Rahmen der CC wird in Deutschland zukünftig die Einhaltung von 19 bereits geltenden europäischen Rechtsvorschriften, die z. T. seit langem in nationales Recht umgesetzt worden sind, überwacht. Die Rechtsvorschriften kommen aus den Bereichen:

- Gesundheit von Mensch, Tier und Pflanzen (inkl. Pflanzenschutz, Tiergesundheit und Tierschutz),

\section{- Lebensmittelsicherheit und}

- Umweltschutz (inkl. Boden- Wasser- und Naturschutz).

Die einzelnen Kriterien zur Einhaltung der Rechtsvorschriften, d. h. die Frage, ob diese Kriterien im einzelnen über die gfP hinaus gehen sollen, wird derzeit in Bund-LänderArbeitsgruppen diskutiert. Neben der direkten Bindung der Direktzahlungen an CC sind diese zugleich davon abhängig, ob der Landwirt seine Flächen in einem ,guten landwirtschaftlichen und ökologischen Zustand“ hält. Derzeit ist in diesem Zusammenhang v. a. die Bodenbedeckung als Indikator in der Diskussion. Zum einen soll hierzu eine jährliche Bodenbedeckungsrate von 50-75 \% der AF eines Betriebes, eine ausgeglichene Humusbilanz und die jährliche einmalige Zerkleinerung des Aufwuchses bei Stilllegungsflächen gehören. ${ }^{205}$

\footnotetext{
${ }^{204}$ IEEP (2003)

205 Vgl. SCHÄFER (2004)
} 


\subsubsection{Diskussion der Cross-Compliance und Konsequenzen für den Boden- schutz}

Aus Sicht des Bodenschutzes ist die Maßnahme der CC zunächst zu begrüßen, da sie zu einer Werterhöhung des Standards der guten fachlichen Praxis führt. Die Landwirte werden generell die Gefahr des Verlustes oder der Kürzung ihrer Direktzahlungen sowie eine wahrscheinlich höhere Kontrollintensität in ihre Planungen mit einbeziehen müssen. Dadurch sinkt zunächst der Anreiz, die Einhaltung der verschiedenen Grundsätze der gfP zu umgehen. Für die Politik besteht z. Zt. die Möglichkeit, höhere bzw. konkretere Standards an Umweltauflagen durchzusetzen. Derzeit entwickelt das Bundeslandwirtschaftsministerium eine Verpflichtungsverordnung, die eine Voraussetzung zum Erhalt der Direktzahlungen darstellt. Demnach sind aus Sicht des Bodenschutzes v. a. folgende Maßnahmen vorgesehen:

- Flächen mit einer Hangneigung von $2^{\circ}$ bis $10^{\circ}$ Hangneigung sollen mindestens zur Hälfte erosionsvermindernd bewirtschaftet werden.

- Flächen mit einer Hangneigung von über $10^{\circ}$ müssen nach dem Verordnungsentwurf vom 1. November bis zum 15. Februar mit Pflanzen bewachsen sein und Pflanzenreste dürfen in dieser Zeit nicht untergepflügt werden.

- Die Anbauverhältnisse müssen mindestens drei Fruchtfolgeglieder aufweisen, die einen Anteil von mindestens $20 \%$ haben. $^{206}$

Vorausgesetzt, diese Standards werden mit einer geeigneten Kontrolle gekoppelt, ergibt sich hieraus ein höherer Nutzen für den Bodenschutz. Die Auswirkungen der Standards auf den einzelnen Betrieb hängen von den KNV der Umsetzung dieser Maßnahmen ab. Sollte es weiterhin zu einer Pflicht des Zwischenfruchtanbaus oder der Auflage der konservierenden Bodenbearbeitung kommen, können diese in einem Teil der Gebiete recht kostengünstig in den Betrieben implementiert werden. Voraussetzung hierfür sind bspw. Standorte mit ausreichenden Niederschlägen und geeigneten Böden. ${ }^{207}$

Auf einzelbetrieblicher Ebene ist generell zu berücksichtigen, dass bei der Erhöhung oder beim Erlass neuer Bodenschutzauflagen die Wettbewerbsfähigkeit der Betriebe berück-

\footnotetext{
${ }^{206}$ Vgl. AGRA-Europe (2004c), S. L 1 f.

${ }^{207}$ Vgl. Kapitel 6.3.1.5 und Kapitel 6.3.1.1
} 
sichtigt werden muss. Dies bedeutet, dass eine Auseinandersetzung mit den KNV der geforderten Maßnahmen, wie es in dieser Arbeit geschieht, stattfinden muss. Hierzu gehört ferner der Vergleich mit anderen EU-Staaten. Es darf aus Sicht der Wettbewerbsfähigkeit der landwirtschaftlichen Betriebe in Deutschland nicht zu einer Verzerrung durch zu hohe Umweltauflagen kommen. Dies sollte nur dann geschehen, wenn zu hohe und nicht tolerierbare Belastungen vorliegen. Generell ist der EU-einheitlichen Verabschiedung von Umweltstandards Vorrang zu geben.

\subsubsection{Ergebnisse der Expertenbefragung}

Im Folgenden werden die Ergebnisse der Expertenbefragung zum Themenbereich des Bodenschutzrechts in Deutschland dargestellt.

\subsubsection{Zufriedenheit mit dem aktuellen Bodenschutzrecht und Umsetzung der Rechtsvorschriften in die landwirtschaftliche Praxis}

Die erste Frage beschäftigte sich damit, wie hoch die Zufriedenheit der Experten mit dem aktuellen Bodenschutzrecht ist. Insgesamt war ein Experte $(3,8 \%)$ sehr zufrieden, 11 $(42,3 \%)$ zufrieden, 10 (38,5 \%) waren unzufrieden und ein Experte $(3,8 \%)$ sehr unzufrieden. Drei Experten (11,5 \%) äußerten sich nicht zu dieser Frage.

Da die Frage nach dem bisherigen Erfolg zum Zeitpunkt der Befragung sehr wichtig erschien, wurde diese Frage im Verlauf des Fragebogens noch einmal mit einer geringen Abweichung gestellt. Diesmal sollten die Experten ihre Meinung zum Erfolg des Bodenschutzrechts im Speziellen äußern. Hier wird deutlich, dass die Ergebnisse der ersten Frage zu relativieren sind. Nur vier Experten $(15,4 \%)$ zeigten, dass sie den bisherigen Erfolg als hoch ansehen. 15 Befragte (57,7 \%) hingegen fanden den bisherigen Erfolg niedrig und zwei $(7,7 \%)$ stuften ihn sehr niedrig ein. Fünf Experten (19,2 \%) antworteten nicht auf diese Frage.

Auf die Frage, ob bisher schon Auswirkungen des Bodenschutzrechts erkennbar seien, gestaltet sich die Situation wie in der Abbildung 14 ersichtlich ist: 


\section{Abbildung 14: Auswirkungen des Bodenschutzrechts auf die landwirtschaftliche Praxis $(\mathbf{n}=\mathbf{2 2})$}

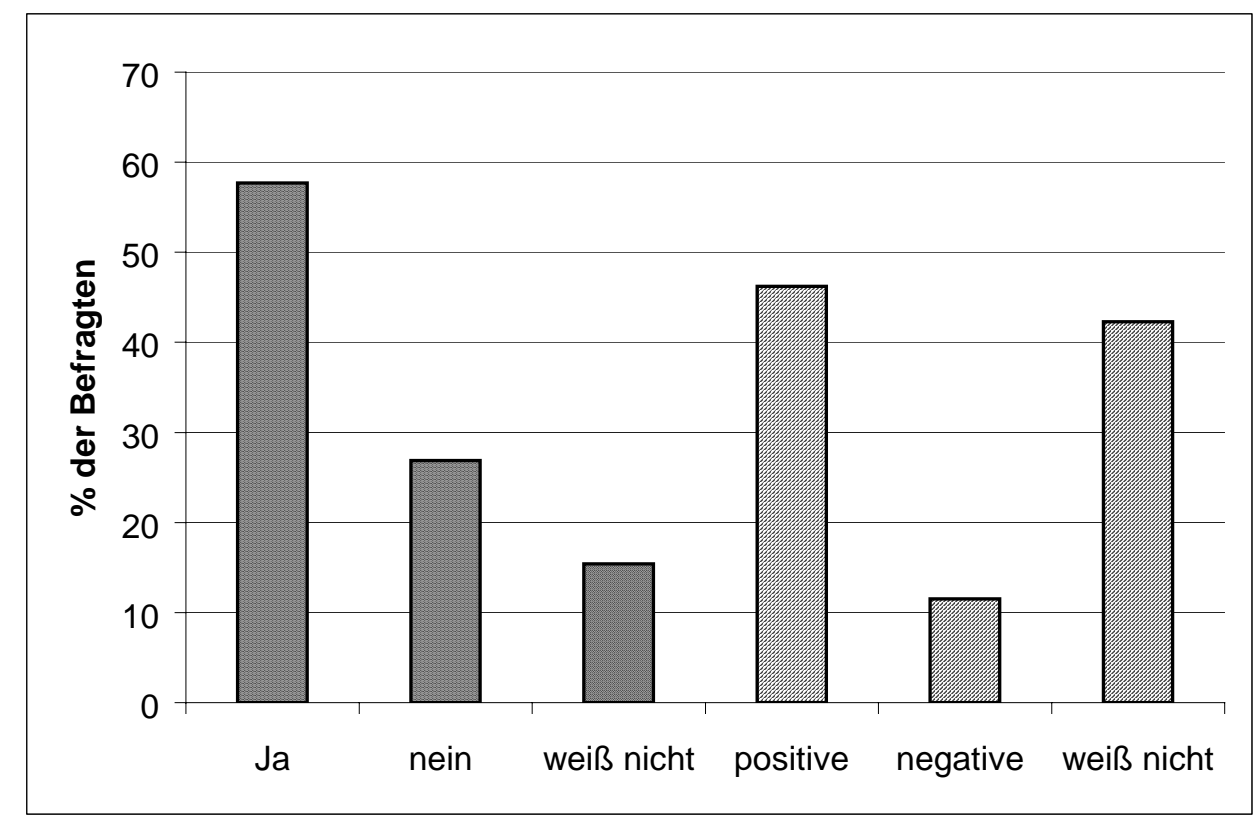

Quelle: Eigene Darstellung

Die Abbildung 14 zeigt, dass die Mehrheit der Experten (57,7\%) Auswirkungen des Bodenschutzrechts auf die landwirtschaftliche Praxis erkennen. Davon finden 46,2 \%, dass diese Auswirkungen positiv sind, und nur 11,5 \%, können negative Auswirkungen erkennen. Die Experten konnten ferner von der Verbalisierung der Auswirkungen Gebrauch machen. Als negative Auswirkung nannte bspw. ein Experte die Unsicherheit in der Landtechnikindustrie und die Erhöhung des technischen Aufwandes für Bodenschutzmaßnahmen. Als positive Auswirkungen wurden v. a. die stärkere Sensibilisierung der Landwirte für den Bodenschutz (4 Nennungen) und die stärkere Umsetzung von Erosionsschutz in den Betrieben (4 Nennungen) genannt.

$\mathrm{Zu}$ diesem Themenbereich können Ergebnisse aus einer Landwirtebefragung von FRIEDRICH et al. ${ }^{208}$ angefügt werden. Sie wurde auf 85 Betrieben in NRW in 2000 und 2001 durchgeführt und beschäftigte sich u. a. mit den Auswirkungen der Bodenschutzgesetzgebung. In der Befragung wird deutlich, dass $11 \%$ aller Befragten noch gar nichts über das BBodSchG gehört hatten. Der wichtigste Informationspfad ist die Beratung und Bera- 
tungsveranstaltungen. $31 \%$ der Befragten gaben an, dass sie sich ,unzureichend“ bzw. „nicht informiert“ fühlten. Außerdem ergab die Befragung, dass 33 \% der Befragten Konfliktpotenziale bei Erosionsproblemen zwischen der Gesetzgebung und der Bewirtschaftung sahen, und $8 \%$ erwarteten Einschränkungen der Bewirtschaftung.

Die Ergebnisse zeigen, dass die Beratung den wichtigsten Informationspfad zwischen der Gesetzgebung und Landwirtschaft darstellt. Es bleibt festzuhalten, dass zum Zeitpunkt der Befragung ein recht hohes Informationsdefizit bei den Landwirten vorherrschte und Beratungsbedarf bestand.

4.4.4.2 Bedeutung verschiedener gesetzlicher Regelwerke für den Idw. Bodenschutz und Verbesserungsmöglichkeiten

Die Experten sollten ferner Stellung zu der Bedeutung verschiedener gesetzlicher Regelwerke für den ldw. Bodenschutz nehmen. Die Ergebnisse sind in der Abbildung 15 zusammengefasst. 


\section{Abbildung 15: Bedeutung verschiedener gesetzlicher Regelwerke für den landwirt- schaftlichen Bodenschutz $(n=24)$}

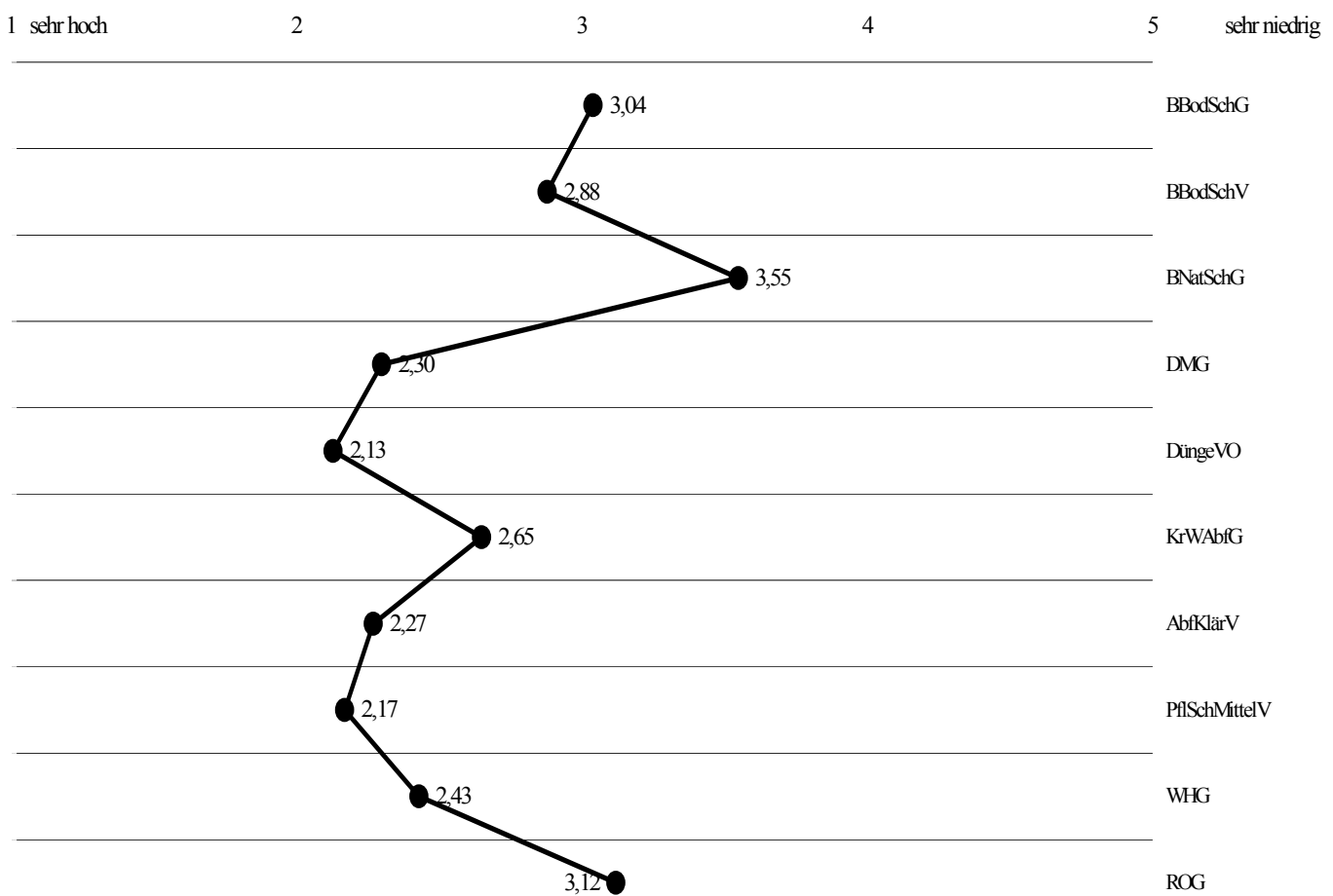

Quelle: Eigene Darstellung

Es wird deutlich, dass v. a. die Regelwerke für bedeutsam erachtet werden, welche den stofflichen Bodenschutz regeln. Hierzu zählen die DüngeVO, das DMG, die AbfKlärV sowie die PflSchMittelV. Das Bodenschutzrecht wird hingegen als mittelmäßig bedeutsam und das BNatSchG als weniger bedeutsam angesehen. Beim letzten Punkt ist die Frage zu stellen, ob die Experten schon das novellierte Bundesnaturschutzgesetz vor Augen hatten.

Die nächste Frage beschäftigte sich mit dem Reformbedarf der gesetzlichen Regelwerke in der obigen Abbildung. Dazu sollten die Experten die einzelnen Regelwerke in eine Reihenfolge mit sinkendem Reformbedarf bringen. Das Ergebnis überrascht aufgrund der Tatsache, dass die Klärschlammverordnung den höchsten Reformbedarf hat. Dann folgt bereits das BBodSchG und die DüngeVO. An vierter Stelle liegen sowohl das Raumordnungsgesetz als auch die BBodSchV.

Lediglich zwei Experten haben eine Stellungnahme zu dieser Frage abgegeben und bemängelt, dass es gerade in der BBodSchV keine verbindlichen Anordnungen zur Boden- 
erosion und -schadverdichtung gibt und eine höhere Harmonisierung der einzelnen Regelwerke in Bezug auf den stofflichen Bodenschutz gefordert.

Eine weitere Frage beschäftigte sich mit den generellen Mängeln des Bodenschutzrechts, die als offene Frage formuliert war. Nach einer Zusammenfassung der Antworten ergibt sich folgende, nach Häufigkeiten geordnete Reihenfolge:

1. Umsetzung des Bodenschutzrechts in die Praxis,

2. Keine Mängel,

3. Fehlender physikalischer Bodenschutz,

4. Instrument der guten fachlichen Praxis,

5. Zu viele Ungenauigkeiten und

6. Fehlende Anordnungen.

Es ist zu erkennen, dass die Experten hauptsächlich die Umsetzung des Bodenschutzrechts in die Praxis monieren. Allerdings sind nur unwesentlich weniger Experten der Meinung, dass das Bodenschutzrecht keine Mängel hat.

\subsubsection{Die Wirksamkeit der guten fachliche Praxis als Vorsorgeelement des Bo- denschutzes}

Im Folgenden wurden die Experten nach ihrer Meinung zur guten fachlichen Praxis nach $\S 17$ BBodSchG als einziges Vorsorgeelement im Bodenschutzrecht gefragt. Insgesamt wurden hierzu drei direkte Fragen gestellt. Zusätzliche Informationen zu der Expertenmeinung über die gfP sind den obigen Kapiteln zu entnehmen. Die erste Frage bezog sich auf die Wirksamkeit der gfP als Vorsorgeinstrument. Dabei ist festzustellen, dass die Experten die gfP als ein in der Praxis wirksames Vorsorgeinstrument sehen. Drei Experten $(11,5 \%)$ sahen die gfP als sehr wirksam an und über die Hälfte, nämlich 15 Experten (57,7 \%), als wirksam. Fünf Experten (19,2 \%) befanden die gfP in Bezug auf die Wirksamkeit als Vorsorgeinstrument nicht wirksam und zwei $(7,7 \%)$ sehr unwirksam. Ein Experte (3, $8 \%)$ nahm zu dieser Frage keine Stellung.

Die nächste Frage beschäftigte sich mit den Konkretisierungen der gfP im Bund-LänderPapier. Die Experten sollten zum Ausdruck bringen, ob sie diese Konkretisierungen für ausreichend halten. Es ergab sich ein uneinheitliches Bild. 12 Experten (46,2 \%) fanden, 
dass die Konkretisierungen ausreichen, 10 Experten (38,5\%) hingegen waren der Meinung, dass sie nicht ausreichen und zwei (7,7 \%) äußerten, dass sie völlig unzureichend seien. Wiederum zwei (7,7 \%) äußerten sich zu dieser Frage nicht.

Die letzte Frage aus diesem Themengebiet befasste sich mit der Wirksamkeit des Kooperationsprinzips nach $\S 17$ BBodSchG, um die Grundsätze der gfP an die Landwirte heranzutragen. Das Ergebnis gestaltet sich wie folgt: Die Mehrheit mit 14 Experten $(53,8 \%)$ fand, dass dieses Instrument hinreichend wirksam ist und 9 Experten sahen dieses Instrument als nicht hinreichend wirksam an. Drei Experten $(11,5 \%)$ äußerten sich nicht zu dieser Frage.

Es bleibt also festzuhalten, dass die Wirksamkeit der gfP in der landwirtschaftlichen Praxis tendenziell ein wirksames Vorsorgeinstrument darstellt. Die darauf aufbauenden Konkretisierungen des Bund-Länder-Papiers werden deutlich kritischer in Bezug auf ihre Vollständigkeit gesehen. In der Befragung besteht die Tendenz, dass die Wirksamkeit des Kooperationsprinzips mehrheitlich positiv gesehen wird. 


\section{Verbindungen zwischen Wasser- und Bodenschutz}

Zwischen den Umweltmedien Wasser und Boden bestehen sowohl hohe naturwissenschaftliche als auch rechtliche Zusammenhänge. Daher werden im folgenden Kapitel die Verbindungen zwischen Wasser- und Bodenschutz erläutert. Hierzu wird v. a. auf die EU-Wasserrahmenrichtlinie und auf den Bereich Landwirtschaft und Hochwasserschutz eingegangen. Als Beispiel wird eine KWA und KNA für die Maßnahme der Umwandlung von Ackerland in Grünland dargestellt, die in dem Gesetzentwurf zum Hochwasserschutzgesetz verankert werden soll.

\subsection{Abgrenzung von Wasser- und Bodenschutz}

Die Schädigungen von Oberflächengewässern und von Grundwasser und Boden weisen aufgrund ihrer natürlichen Komplexität innerhalb des Naturhaushalts einen sehr starken Zusammenhang auf. So können z. B. durch Bodenerosion verursachte stoffliche Belastungen in Oberflächengewässer transportiert werden und diese schädigen. Eine Schädigung des Grundwassers geht wiederum unweigerlich mit einer Schädigung des Bodens einher. Daher kommen Bodenschutzmaßnahmen vielfach dem Wasserschutz zugute und umgekehrt.

In Kapitel 4.2.1 wurde bereits die bodenschutzrechtliche Unterscheidung zwischen dem Grundwasser und dem Boden dargestellt. Laut Art. 75 Abs. 1 Nr. 4 GG hat der Bundesgesetzgeber bezüglich des Wasserhaushalts im Gegensatz zum Bodenrecht nur eine Rahmenkompetenz. Der Gesetzgeber darf somit bestimmte Regelungen wasserrechtlicher Sachverhalte nicht in das BBodSchG einbringen. Das BBodSchG nimmt aber an zahlreichen Stellen Bezug auf das Wasserrecht. ${ }^{209}$ Bspw. enthält $§ 1$ Satz 2 BBodSchG die Forderung, dass die durch schädliche Bodenveränderungen verursachten Gewässerverunreinigungen saniert werden müssen. Dies ist zwar eine direkte Vorschrift für die Sanierung von Gewässern, allerdings liegt hier das Augenmerk auf der indirekten Gewässerverunreinigung, die durch eine schädliche Bodenveränderung hervorgerufen wurde. Auch die Gefahrenabwehr für durch schädliche Bodenveränderungen hervorgerufenen Gewässer-

\footnotetext{
${ }^{209}$ Vgl. LABO u. LAWA (2000), S. 2
} 
beeinträchtigungen geschieht nach Maßgabe des Bodenschutzrechts. ${ }^{210}$ Das Sickerwasser ist aus dem WHG ausgeklammert und unterliegt somit dem Rechtsbereich des Bodens. ${ }^{211}$ Ausdrücklich nicht dem BBodSchG unterliegende Gewässerverunreinigungen sind v. a. diejenigen, die nicht durch schädliche Bodenverunreinigungen hervorgegangen sind oder die keinem verursachenden Grundstück zugeordnet werden können. ${ }^{212}$

Für die Landwirtschaft ist v. a. für den Gefahrenbereich der Bodenerosion eine ordnungsgemäße Trennung des Wasserrechts vom Bodenschutzrecht wichtig. Das BBodSchG regelt grundsätzlich die Gefahrenabwehr und Sanierung bei Bodenerosionen, da es sich hierbei um eine Schädigung der Bodenfunktionen handelt. Dies geschieht durch $\S 17$ Abs. 2 Nr. 4 BBodSchG sowie durch $\S 2$ Nr. 10 und $\S 8$ sowie Anhang 4 BBodSchVO. Das Wasserrecht enthält in § 19 WHG jedoch Vorschriften für das Problem der Bodenerosion im Speziellen: Zum einen für die Bodenerosion in Wasserschutz- und Überschwemmungsgebieten, zum anderen für die Bodenerosion im Bereich der Bewirtschaftung von Gewässern. Für diese Bereiche sind beide Rechtsvorschriften nebeneinander anwendbar.

\subsection{Die EU-Wasserrahmenrichtlinie und der Gesetzentwurf zu einem Hochwasserschutzgesetz}

Mit der am 22.12.2000 in Kraft getretenen Richtlinie 2000/60 EG des Europäischen Parlaments und Rates zur Schaffung eines Ordnungsrahmens für Maßnahmen der Gemeinschaft im Bereich der Wasserpolitik, der EU-Wasserrahmenrichtlinie (WRRL), kommen verschiedene Veränderungen auf die deutsche Wasser- und Landwirtschaft zu, die heute noch nicht hinreichend abzuschätzen sind. Die WRRL beschränkt sich nicht nur, wie es das bisherige deutsche Wasserrecht, auf Wasserschutzgebiete und den Trinkwasserschutz, sondern hat einen inhaltlich umfassenderen und flächendeckenderen Ansatz. ${ }^{213}$ Die WRRL weist im Verhältnis zu anderen EU-Richtlinien wie der FFH- oder Vogelschutzrichtlinie auch einen höheren Flächenbezug auf. Bspw. beträgt der in NRW betroffene Flächenanteil $8 \%$ der Landesfläche. ${ }^{214}$

\footnotetext{
${ }^{210}$ Vgl. LABO u. LAWA (2000), S. 3

${ }^{211}$ Vgl. hierzu Kapitel 4.2.1

${ }^{212}$ Vgl. LABO u. LAWA (2000), S. 5

${ }^{213}$ Vgl. KÖHN (2002), S. 111

${ }^{214}$ Vgl. Helmer (2004), S. 18
} 
Die WRRL ist an die Mitgliedstaaten gerichtet und legt einen strikten Umsetzungsrahmen fest. Vier Jahre nach Inkrafttreten ist die Bestandsaufnahme abzuschließen. Zwei Jahre später muss ein Messnetz und eine Überwachung für die betroffenen Flächen installiert werden. Die Maßnahmenprogramme müssen neun Jahre nach dem Inkrafttreten in allen Bundesländern aufgestellt, zwischen den Flussgebieten aufeinander abgestimmt werden und nach weiteren drei Jahren der Vollzug gewährleistet sein. ${ }^{215}$

Die Auswirkungen der WRRL auf die landwirtschaftlichen Betriebe abzuschätzen ist schwierig, da die WRRL an einigen Stellen zu ungenau und an anderen wiederum zu restriktiv ist. Dies führt dazu, dass es abzuwarten bleibt, wie die Richtlinie in Deutschland umgesetzt wird, was derzeit noch nicht vollständig geschehen ist.

Die Landwirtschaft ist vor allem durch diffuse ${ }^{216}$ oder punktförmige Einträge von Düngeund Pflanzenschutzmitteln, der Wasserentnahme für Bewässerungs- und Beregnungszwecke sowie den Einfluss der Landbewirtschaftung auf die Grundwasserneubildung von der EG-Wasserrahmenrichtlinie betroffen. Dies kann an einem Beispiel verdeutlicht werden: Momentan erreichen lediglich 14 \% der Gewässer bezüglich der Nitratbelastung und 19 \% der Gewässer bezüglich der Phosphatbelastung in Deutschland das Qualitätsziel der WRRL ,gut". 217

Durch die WRRL soll in allen sog. aquatischen Ökosystemen eine gute Qualität erreicht werden $^{218}$. Für das Oberflächenwasser bedeutet dies einen guten ökologischen und chemischen Zustand und für das Grundwasser einen guten chemischen sowie mengenmäßigen Zustand. Nach Meinung der LÄNDERARBEITSGEMEINSCHAFT WASSER (LAWA) ${ }^{219}$ ist dieses Ziel nur dann zu erreichen, wenn die gegenwärtige landwirtschaftliche Praxis geändert wird. Weitere Ziele sind wie folgt zu kennzeichnen:

- Durchsetzung des Verschlechterungsverbots und die Verbesserung aller aquatischen Ökosysteme, der damit zusammenhängenden Landökosysteme und Feuchtgebiete,

\footnotetext{
${ }^{215}$ Vgl. BoseniUs (2001), S. 27

${ }^{216}$ Nach Auskunft der LAWA erfolgen $72 \%$ der Stickstoff- und $66 \%$ der Phosphateinträge Deutschlands in Oberflächengewässer diffus, wovon $80 \%$ der N- und 70 \% der P-Einträge aus der Landwirtschaft stammen. Vgl. LAWA 2002, S. 4

${ }^{217}$ Vgl. HeLMER (2004), S. 18

${ }^{218}$ Vgl. hierzu Anhang V der WRRL, wo die Qualitätskriterien für die Oberflächengewässer definiert werden.

${ }^{219}$ Vgl. LAWA (2002), S. 4
} 
- Förderung einer nachhaltigen Wassernutzung,

- Maßnahmen zur schrittweisen Reduzierung bzw. Einstellung von Emissionen,

- Schutz des Grundwassers vor Verschmutzungen und die schrittweise Reduzierung der vorhandenen Verschmutzungen und

- Maßnahmen gegen Überschwemmungen und Dürren.

Die 2009 aufzustellenden Maßnahmenprogramme müssen grundlegende und ergänzende Maßnahmen enthalten. Die grundlegenden Maßnahmen ${ }^{220}$ sind Mindestanforderungen, die eingehalten werden müssen. Sie ergeben sich aus bestehenden Rechtsverordnungen wie z. B. der Klärschlammrichtlinie, der Nitratrichtlinie oder der Richtlinie über Pflanzenschutzmittel. Die ergänzenden Maßnahmen ${ }^{221}$ bestehen aus verschiedenen rechtlichen, administrativen, wirtschaftlichen und steuerlichen Instrumenten, die bisher nur wenig konkretisiert wurden und noch erweiterbar sind.

Die Bewirtschaftungspläne sollen Angaben zum Ist-Zustand der Flussgebiete in Form von Kartierungen oder Analysenergebnissen enthalten. Des Weiteren sollen die ergriffenen Maßnahmen zur Verbesserung bzw. zum Erhalt der Wasserqualität dokumentiert werden. Nach Art. 14 Abs. 1 sind im Rahmen der Aufstellung von Bewirtschaftungsplänen sowohl die Öffentlichkeit als auch die betroffenen Landwirte zu informieren und anzuhören. $^{222}$

Die LAWA ${ }^{223}$ macht in einem Arbeitspapier gemeinsam mit der LABO und dem BMVEL in diesem Zusammenhang deutlich, dass in vielen Gebieten Deutschlands eine Umsetzung der WRRL nur mit einer Änderung der derzeitigen landwirtschaftlichen Bodennutzung einhergehen kann. Hierzu werden in diesem Papier zahlreiche Vorschläge zur Umsetzung einer grundwasserschonenden und standorttypischen Bodennutzung gemacht, auf die allerdings hier nicht näher eingegangen wird, da sie zum größten Teil Gegenstand dieser Arbeit sind. ${ }^{224}$ Die konkreten Umsetzungsvorschläge der LAWA sind folgende: ${ }^{225}$

\footnotetext{
${ }^{220}$ Vgl. hierzu Anhang VI, Teil A der WRRL

${ }^{221}$ Vgl. ebd. Teil B

${ }^{222}$ Vgl. KÖHN (2002), S. 111

${ }^{223}$ Vgl. LAWA (2002), S. 4 ff.

${ }^{224}$ Vgl. Kapitel 6.3.1.1
} 


\section{Landwirtschaftliches Ordnungsrecht}

- Änderung der Düngeverordnung

Hierdurch sollen Stickstoffbilanzüberschüsse reduziert werden. Z. B. soll der Grenzwert von $50 \mathrm{mg} / \mathrm{l}$ Nitrat auch im Oberflächenwasser erreicht werden. Dies kann durch verschiedene Änderungen der DüngeVO erreicht werden, z. B. durch eine Verlängerung des Ausbringungsverbots (z. B. 1. Oktober bis 31. Januar) oder eine Erhöhung der Güllelagerkapazitäten von mind. 6 Monaten, ganzjährige Bodenbedeckung etc.

- Pflanzenschutzrecht

Pflicht zur Nachrüstung von Pflanzenschutzspritzgeräten zur Ermöglichung einer Reinigung auf dem Feld oder eine Pflicht zur schlagspezifischen Aufzeichnung des Pflanzenschutzmitteleinsatzes

\section{Sonstige Maßnahmen mit Synergieeffekten}

Diese Maßnahmen können sein: Umwandlung von leicht erodierbaren Flächen in Grünland, Gewässerrandstreifen von mind. 10 m, Rückbau von Drainageleitungen, Einbindung des Gewässerschutzes in die Berufsausbildung

\section{Neuordnung der nationalen Agrarförderpolitik}

Vorschläge im Rahmen der GAK im Rahmen des Modulationsgesetzes ${ }^{226}$.

Die LAWA spricht sich im Rahmen der Neuordnung der nationalen Agrarförderpolitik v. a. für die Umwandlung der an die Produktion gekoppelten Prämien in Grünland- oder Futterflächenprämien aus. Dies führe zu einer Anpassung der Tierhaltung an die Futterfläche und somit zu einer gewässerverträglichen Grünlandbewirtschaftung. ${ }^{227}$

\footnotetext{
${ }^{225}$ Auf der Grundlage eines Berichts der Bund-Länder-Arbeitsgruppe „Eckpunkte für eine zukunftsfähige Agrar- und Verbraucherpolitik“ im Rahmen der gemeinsamen Agrarminister und Umweltministerkonferenz am 13. Juni 2001 in Potsdam.

${ }^{226}$ Vgl. LAWA (2002), S. 4 ff.

${ }^{227}$ Vgl. LAWA (2002), S. 8
} 
Aus diesem Abschnitt ist das Fazit zu ziehen, dass Boden- und Wasserschutzmaßnahmen eng miteinander verbunden sind. Werden neue Anforderungen an die Landwirtschaft im Boden- und Wasserschutzrecht gestellt, ist daher zu prüfen, ob es Synergieeffekte gibt. Außerdem wird speziell bei der WRRL deutlich, dass sich die Landwirtschaft und ihre Berufsstandsvertreter bei der Festsetzung der Maßnahmenprogramme aktiv beteiligen müssen, um überzogene und unverhältnismäßige Umsetzungen zu vermeiden

\section{Gesetzentwurf zum Hochwasserschutzgesetz}

Am 8.8.2003 wurde vom Bundesumweltminister ein „Entwurf eines Gesetzes zur Verbesserung des vorbeugenden Hochwasserschutzes“'228 vorgelegt. Dieses wurde am 11.02.2004 vom Bundeskabinett beschlossen. Bei diesem Gesetzentwurf handelt es sich um den Entwurf eines Artikelgesetzes. Dieses Artikelgesetz sieht Änderungen des WHG, BauGB, ROG, Bundeswasserstraßengesetzes, Gesetzes über den Deutschen Wetterdienst und des Gesetzes über die Umweltverträglichkeitsprüfung vor.

Für die Landwirtschaft ist v. a. der Artikel 1, die Änderung des WHG und hier insbesondere der $\S 31 \mathrm{~b}$ wichtig, der die Ausweisung von Überschwemmungsgebieten bestimmt und deren Nutzung vorschreibt. In Absatz 3 ist ein Ackerbauverbot in Überschwemmungsgebieten, die mindestens einmal in 100 Jahren überschwemmt werden, bis zum 31.12.2012 vorgesehen. Außerhalb von Abflussbereichen können die Bundesländer allerdings Ausnahmen für Flächen vorsehen, bei denen keine Bodenerosion und keine Schadstoffeinträge in Gewässer erwartet werden können. Diese Flächen müssen so bewirtschaftet werden, dass

1. eine ganzjährige „Gründecke“ sichergestellt ist,

2. eine konservierende Bodenbearbeitung erfolgt und

3. die Ausbringung von Dünge- und Pflanzenschutzmitteln im Hinblick auf mögliche Überflutungen eingeschränkt wird.

Der Entwurf des Artikelgesetzes, der u. a. auch Bebauungsverbote in Überschwemmungsgebieten vorsieht, ist prinzipiell auf eine hohe Zustimmung gestoßen. Allerdings gibt es zahlreiche Diskussionen über die Forderung des BMU, den Ackerbau auf erosi- 
onsgefährdeten Ackerflächen bis 2012 zu verbieten. Nach Schätzungen des DBV wären in Deutschland etwa 90.000 ha und somit 7,5 \% der AF von dem Verbot betroffen. Ein Ackerbauverbot würde nach den Berechnungen zu Ertrags- und Vermögensverlusten von 3,6 Mrd. $€$ führen. Der DBV geht somit von Ertrags- und Vermögensverlusten von $40.000 € /$ ha AF aus. Im Folgenden wird speziell auf den Zusammenhang zwischen Landwirtschaft und Hochwasserschutz aus naturwissenschaftlicher und ökonomischer Sicht eingegangen.

\subsection{Landwirtschaft und Hochwasserschutz}

Überschwemmungen von Flüssen zählen zu den immer wiederkehrenden Katastrophen, welche neben hohen ökonomischen Verlusten auch das Leben vieler Menschen bedrohen. Die Zusammenhänge, die diese Katastrophen verursachen, sind sehr komplex und bis heute nicht gänzlich durch die Wissenschaft zu erklären. Ein wesentlicher Faktor ist dabei das abfließende Wasser durch starke Regenfälle sowie durch die Schneeschmelze. Die Landwirtschaft stellt in diesem Fall als größter Flächennutzer eine sehr wichtige Einflussgröße dar. Die Größe, die sie am stärksten beeinflusst ist die Infiltrationsrate der landwirtschaftlich genutzten Flächen.

\subsubsection{Zusammenhang zwischen Infiltration und Hochwasser}

Die Prozesse der Bodenschadverdichtung, Verschlämmung sowie der Verlust der biologischen Aktivität führen zu einem Verlust an der Infiltrationskapazität landwirtschaftlich genutzter Böden. ${ }^{229}$ Die Entstehung dieser Prozesse ist auf viele Gründe zurückzuführen, die in dem Kapitel 3.1.1 behandelt werden. Die Infiltrationskapazität ist bei der Frage, ob die Landwirtschaft durch die Gestaltung ihrer Produktionssysteme einen aktiven Beitrag zum vorbeugenden Hochwasserschutz leisten kann, der zentrale und wichtigste Begriff. Die Infiltration hängt v. a. vom spezifischen Bodengewicht, Lebendverbau, Porenvolumen, sowie der -verteilung, von der Aggregatstabilität, der Basensättigung und der Wasserhaltekapazität $a b .{ }^{230}$ Flächendeckende Daten über die Infiltration auf Schlägen sind kaum vorhanden, da es sich bei der Messung der Infiltration um ein sehr zeit- und arbeitsintensives Verfahren handelt. Außerdem schwanken die Infiltrationsraten aufgrund ihrer

\footnotetext{
${ }^{229}$ Vgl. SchefFer, Schachtschabel U. AuERSWALd (1998)

${ }^{230}$ Vgl. SCHNUG U. HANEKLAUS (2002), S. 198
} 
bestimmenden Parameter sehr stark. So können sich innerhalb eines Schlages zahlreiche unterschiedliche Infiltrationsraten bestimmen lassen. Typische Werte für die Infiltration von Wasser sind bei Sandböden 20-30 mm/h, Lehmböden 10-20 mm/h und bei Tonböden 1-5 mm/h. ${ }^{231}$ Eine verringerte Infiltration wirkt sich, wie SPAROVEK et al. für das Einzugsgebiet der Schunter in Niedersachsen zeigen, auf die Intensität von Hochwasserereignissen aus. Es stellte sich heraus, dass unter Infiltrationsraten von rund $15 \mathrm{~mm} / \mathrm{h}$ die Intensität der Überflutungen stark zunahm. ${ }^{232}$

Den hohen Einfluss der Mulch- und Direktsaat auf die Infiltrationsrate des Bodens zeigen SCHMIDT et al. ${ }^{233}$. In einem Beregnungsversuch bei Zuckerrüben auf einer 19-\% Hangfläche zeigen sie, dass die Endinfiltrationsrate bei der Pflugvariante bei 31,5\% und bei der Mulchsaatvariante bei $82 \%$ liegt.

\subsubsection{Einflüsse landwirtschaftlicher Maßnahmen auf den Hochwasserab- fluss}

Im Folgenden werden die Einflüsse der landwirtschaftlichen Maßnahmen zur Reduzierung des Wasserabflusses ausführlich beschrieben. Die Maßnahmen und deren grundlegende Bewertung sind in der nächsten Tabelle 8 dargestellt.

\footnotetext{
${ }^{231}$ Vgl. ebd.

${ }^{232}$ Vgl. SpAROVEK et al. (2002)

${ }^{233}$ Vgl. SCHMIDT et al. (1999), S.20
} 
Tabelle 8: Stärke der Auswirkungen von Landnutzungsmaßnahmen auf die Abflussbildung und -konzentration bei konvektiven Starkniederschlägen (ohne Forstwirtschaft)

Auswirkungen von Änderungen auf

Landnutzung

Abflussbildung Abflusskonzentration

\begin{tabular}{lcc}
\hline Ackernutzung & & \\
Fruchtfolge & ++ & $+/++$ \\
Bodenbearbeitung & ++ & $+/++$ \\
Düngung & $0 /+$ & $0 /+$ \\
Pflanzenschutz & $0 /+$ & 0 \\
Grünland & & $0 /+$ \\
Narbendichte & $+/+$ & \\
\hline Landeskulturelle Maß- & & ++ \\
nahmen & & $+/++$ \\
Dränung & ++ & $+/++$ \\
Wegebau & $0 /+$ & $0 /+$ \\
Strukturelemente & $0 /+$ & \\
Uferrandstreifen & 0 & $+/++$ \\
\hline Flurbereinigung & & $+/++$ \\
Flächengröße & $0 /+$ & + \\
Bewirtschaftungsrichtung & $0 /+$ & ++ \\
Melioration* & $+/++$ & \\
\hline
\end{tabular}

* Tieflockerung, -umbruch, -düngung

Stärke der Auswirkungen sind immer relativ innerhalb einer Landnutzungs- oder Maßnahmengruppe zu verstehen; Richtung der Auswirkung ist nicht berücksichtigt.

$0 \quad$ keine bzw. vernachlässigbare Auswirkungen

$+\quad$ mittlere Auswirkungen

$+\quad$ starke Auswirkungen

Quelle: DVWK (1999), zit. in FELDWISCH (2002), S. 357

In der Tabelle 8 wird deutlich, dass es innerhalb der landwirtschaftlichen Ackernutzung sehr effiziente Maßnahmen gibt, die Abflussbildung und -konzentration von Oberflächenwasser positiv im Sinne des Hochwasserschutzes zu beeinflussen. Im Bereich der Ackernutzung sind dabei v. a. die Fruchtfolgegestaltung und Bodenbearbeitung zu nennen. Bei der Fruchtfolgegestaltung können z. B. über die Anbaufruchtauswahl sowie den Zwischenfruchtanbau höhere Transpirationsleistungen von 100 bis zu $300 \mathrm{~mm}$ pro Jahr erreicht werden, über den Bodenbedeckungsfaktor kann die Infiltrationsrate um mehrere Zehnerpotenzen erhöht werden. Die hohe Bedeutung der Bodenbearbeitung resultiert v. a. durch die Erhöhung der Infiltration durch konservierende Bodenbearbeitung 
und die Möglichkeit über die Verhinderung von Bodenschadverdichtungen den Oberflächenabfluss zu minimieren. Die Düngung kann in bestimmten Fällen eine positive Wirkung auf den Oberflächenabfluss und die Abflusskonzentration ausüben. V. a. wenn organische Dünger oder Sekundärrohstoffdünger wie Kompost oder Häckselgut ausgebracht werden, wird die Oberflächenverschlämmung vermindert, das Bodenleben und somit die Infiltration gefördert. Pflanzenschutzmaßnahmen wirken sich nur positiv auf den Oberflächenabfluss aus, wenn sie mechanisch durchgeführt werden. Ansonsten bestehen keine Auswirkungen.

Die Grünlandnutzung reduziert im Verhältnis zur Ackernutzung allein durch die höhere Porenkontinuität den Oberflächenabfluss, trotzdem sind gerade bei hohen Viehbesatzdichten auch auf Grünlandflächen Verdichtungen und Narbenschädigungen festzustellen, die den positiven Effekt z. T. erheblich relativieren. Bspw. können auf hängigem Grünland mit intensiver Beweidung und daraus resultierenden Narbenschäden ähnlich hohe Abflusswerte wie beim Ackerland erzielt werden. ${ }^{234}$

Von den landeskulturellen Maßnahmen beeinflussen v. a. Drainagen den Oberflächenabfluss und dessen Konzentration. Bei den Wegen und Strukturelementen kommt es stark auf deren Anteil an. Da sie i. d. R. weniger als $10 \%$ des Gesamtanteils ausmachen, ist ein Einfluss nur marginal feststellbar. Strukturelemente fördern jedoch die Abflusskonzentration, da sie als lineare Abflussbahnen dienen. Die Elemente der Flurbereinigung wirken sich stärker auf die Abflusskonzentration aus. Dies ist z. B. auf großen Schlägen ohne Strukturelemente oder bei der Bewirtschaftung quer zum Hang (Verminderung der Fahrspurerosion) der Fall. Meliorationsmaßnahmen in Form von Lockerungsmaßnahmen haben aus Sicht des Hochwasserschutzes einen positiven Effekt auf das Abflussgeschehen.

Da die direkte Messung von Infiltrationsraten sehr aufwendig ist, werden i. d. R. Computermodelle verwendet. Hierzu gibt es bereits einige gut untersuchte Modelle, die in der Praxis angewandt werden. Ein Beispiel hierfür ist die Modellierung des Oberflächenabflusses $^{235}$ auf Einzugsgebietsebene (Lumdaeinzugsgebiet, Größe $129 \mathrm{~km}^{2}$ ) ${ }^{236}$. Hier wurde mit Hilfe des Abfluss-Simulationsprogramms AGNPS z. B. der direkte Oberflächenab-

\footnotetext{
${ }^{234}$ Vgl. FELDWISCH (1999), S. 44

${ }^{235}$ Eine Zusammenstellung verschiedener Niederschlags-Abfluss-Modelle sowie deren jeweilige Einsatzgebiete ist bei FELDWISCH (1999), S. 79 dargestellt.

${ }^{236}$ Vgl. RODE (1995)
} 
fluss bei Substitution der gesamten Hackfrüchte (2,5\% der Gesamtfläche) durch Sommergerste simuliert. Durch eine solche Maßnahme kann nach dem Modell der Oberflächenabfluss um $2 \%$ gemindert werden. Diese Wirkung stellt sich jedoch ebenfalls ein, wenn alle Hackfrüchte in einem Mulchsaatverfahren angebaut werden würden. Eine Minderung um $8 \%$ ergibt sich rechnerisch, wenn in diesem Gebiet die gesamte landwirtschaftliche Nutzfläche (Ackerflächen 29,5 \%, Grünlandflächen 27 \%) theoretisch gesehen in eine Grasbrache umgewandelt würde. Wenn auf der gesamten Ackerfläche statt einer wendenden eine konservierende Bodenbearbeitung durchgeführt wird, senkt dies den Direktabfluss um $4 \%$.

\subsubsection{Diskussion der Bedeutung der Landwirtschaft für einen vorbeugen- den Hochwasserschutz}

Die Einflussmöglichkeiten der Landnutzung bei der Verminderung von Hochwasserhäufigkeiten müssen, um eine sachgerechte Beurteilung durchführen zu können, differenziert werden. Bei hohen Wiederkehrwahrscheinlichkeiten ( z. B. alle fünf Jahre), hat die Landnutzung eine hohe Einflussmöglichkeit. Geringere Wiederkehrwahrscheinlichkeiten können durch höhere Niederschlagsmengen bedingt sein. Mit höheren Niederschlagsmengen in kurzer Zeit schwinden die Einflussmöglichkeiten der Landnutzung. Allerdings sollten die Maßnahmen der Landnutzung nicht unabhängig voneinander gesehen werden, da sich innerhalb dieser zahlreiche Multiplikatoreffekte ergeben könnten. Den derzeit in der politischen Diskussion stehenden extremen Lösungen, wie z. B. das Ackerbauverbot in Überschwemmungsgebieten, stehen zunächst zahlreiche zu differenzierende Maßnahmen zur Verfügung, die einen effektiven und kosteneffizienteren Hochwasserschutz ermöglichen. ${ }^{237}$ Daher ist an die Politik zu appellieren, dann von den Maximalforderungen Abstand zu nehmen, wenn andere geeignete Maßnahmen zur Verfügung stehen.

\subsubsection{Umwandlung von Ackerland in Grünland}

Im Folgenden wird eine KNA für die Maßnahme der Umwandlung von Ackerland in Grünland durchgeführt. Die Vorgehensweise wird in Kapitel 6.2 dargestellt. Sie orientiert sich an den in Kapitel 6 berechneten Maßnahmen zur Reduzierung der Bodenerosion und -schadverdichtung. 
Die Umwandlung von Ackerland in Grünland wird v. a. als Maßnahme in Überschwemmungsgebieten von der Politik gefordert, da es sich hierbei um eine effiziente Maßnahme handelt, um den Oberflächenabfluss zu reduzieren. Dies liegt zum einen an der ganzjährigen Bodenbedeckung und zum anderen an der Vielzahl an Bodenlebewesen, die unter Grünland zu einer wesentlich höheren Infiltration des Niederschlags führen. Aus diesem Grund kann ab 2004 im Rahmen der GAK diese Maßnahme in Überschwemmungsgebieten mit einem Höchstbetrag von bis zu $574 €$ /ha gefördert werden. Die einzelbetrieblichen Auswirkungen einer Umwandlung des Ackerlands in Grünland werden in diesem Abschnitt in einer KNA dargestellt.

Die Kosten bei der Umwandlung von Ackerland in Grünland bestehen zunächst in dem Erwerbsverlust durch den Verlust der Ackerfläche in Höhe des FruchtfolgeDeckungsbeitrags. Unter der Voraussetzung der Standard-Fruchtfolge WR-WW-WG ergibt sich ein Fruchtfolgedeckungsbeitrag in Höhe von $564 € /$ ha. ${ }^{238}$ Dieser Deckungsbeitrag wird im Folgenden mit denen verschiedener Grünlandnutzungsformen verglichen. Hierbei muss im Einzelfall allerdings geprüft werden, ob für den Betrieb die jeweilige Grünlandnutzungsform grundsätzlich durchführbar ist. Entscheidungskriterien können dabei das Vorhandensein von Wirtschaftsgebäuden, Möglichkeit des Bezugs zusätzlicher Milchlieferrechte und Mutterkuhprämienrechte oder Verpachtungsmöglichkeiten sein. Ist dies nicht der Fall, besteht die Möglichkeit der Stilllegung der Fläche.

In der folgenden Abbildung 16 sind verschiedene Szenarien für die Folgenutzung einer in Grünland umgewandelte Ackerfläche dargestellt. Dabei sind die zu erzielenden Deckungsbeiträge und die diskutierten Ausgleichszahlungen in Höhe von $574 € /$ ha dargestellt. Ausgehend von der Standardfruchtfolge mit einem Deckungsbeitrag von $564 € /$ ha liefert die Färsenaufzucht (FA) einen negativen Deckungsbeitrag von etwa $-750 € /$ ha, die Bullenmast (BM) etwa $-860 € /$ ha und die Mutterkuhhaltung $50 € /$ ha.

Bei der Verpachtung wurde angenommen, dass die Ausgleichszahlungen zunächst nicht an den Eigentümer in voller Höhe zurückfließen. In der Praxis wird sich jedoch ein Wert zwischen $100 € /$ ha und $674 € /$ ha ergeben. Zu berücksichtigen ist hierbei, dass in dem

\footnotetext{
${ }^{238}$ Vgl. LWK HANNOVER (2002)
} 
Überschwemmungsgebiet viel Grünland freiwerden kann, was sich wiederum senkend auf den Pachtpreis auswirkt. ${ }^{239}$

\section{Abbildung 16: Deckungsbeiträge von Produktionsverfahren bei der Umwandlung von Ackerland in Grünland in Überschwemmungsgebieten}

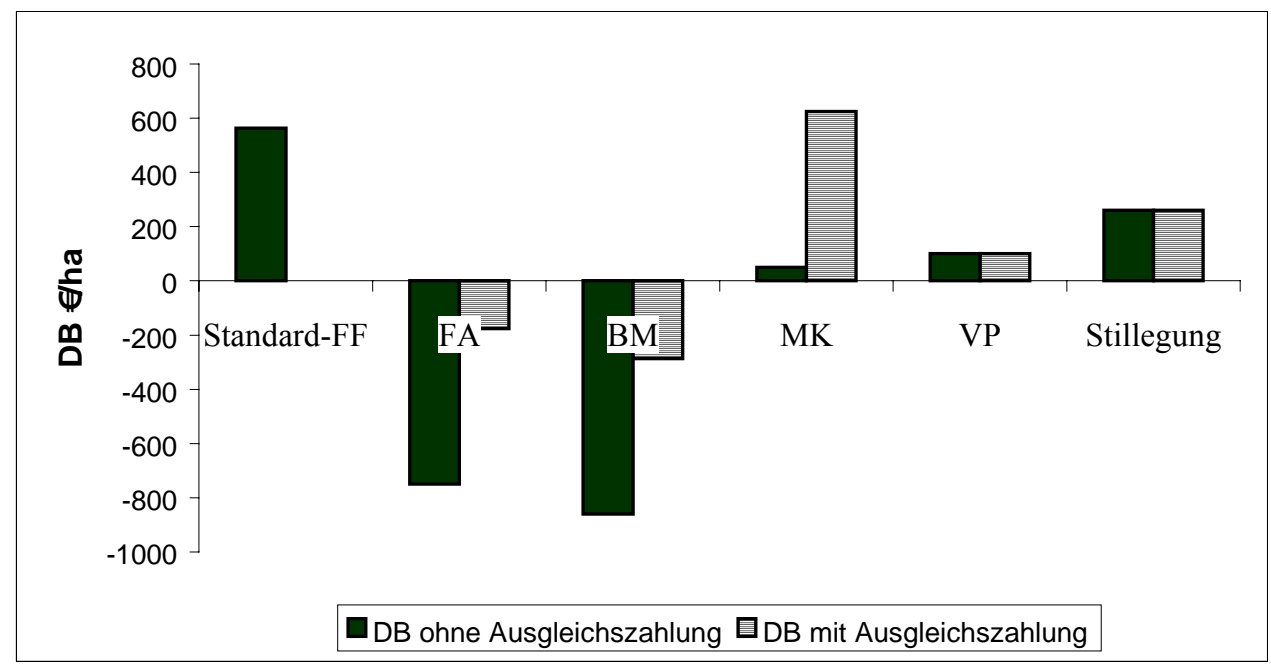

Quelle: FID (2002), STROTDREES (2003), sowie eigene Berechnungen

An dieser Stelle ist ersichtlich, dass keine wirtschaftlich gleichwertige Alternative zur ackerbaulichen Nutzung besteht. Hieran ändern auch Direktzahlungen nichts. Hierdurch kommt zwar der DB der Mutterkuhhaltung in die Nähe des DB der Standardfruchtfolge, jedoch müssen an dieser Stelle die obigen Einschränkungen zur Mutterkuhhaltung gemacht werden. Weiterhin ist zu berücksichtigen, dass mit der Standardfruchtfolge nicht die deckungsbeitragsstärkste Fruchtfolge wie z. B. ZR-WW-WW angenommen wurde. In vielen fruchtbaren Überschwemmungsgebieten wird diese oder ähnliche Fruchtfolgen angebaut, was dazu führt, dass die Verluste auf der Ebene der landwirtschaftlichen Betriebe noch höher ausfallen. Aufgrund der Eindeutigkeit der Ergebnisse und der Tatsache, dass die Nutzeneffekte zumeist aus externen Effekten bestehen und daher nicht direkt monetarisiert werden können, wird im Folgenden auf eine Berechnung der KNV verzichtet.

Die Ergebnisse dieser Betrachtung haben gezeigt, dass den Landwirten andere Alternativen angeboten werden müssen, damit sie unter dem bestehenden Wettbewerbsdruck einen nachhaltigen Hochwasserschutz betreiben können. Diese Alternativen werden im 
Kapitel 6 berechnet und bestehen z. B. aus der konservierenden Bodenbearbeitung, dem Zwischenfruchtanbau oder dem Anbau von Strukturelementen. Diese Maßnahmen wurden bereits in Tabelle 8 in Kapitel 5.3.2 als sehr wirkungsvoll erachtet.

\subsubsection{Ergebnisse der Expertenbefragung}

Die Expertenbefragung beschäftigte sich in einem Abschnitt mit den Zusammenhängen von Landwirtschaft und Hochwasserschutz, sowie den Möglichkeiten der Landwirtschaft, den Hochwasserschutz zu unterstützen. Die erste Frage bezog sich auf die Effektivität verschiedener vorgegebener Maßnahmen auf den Oberflächenabfluss. Die Ergebnisse können der Abbildung 17 entnommen werden.

\section{Abbildung 17: Beurteilung der Wirksamkeit verschiedener Maßnahmen auf den Oberflächenabfluss $(\mathrm{n}=\mathbf{2 2})$}

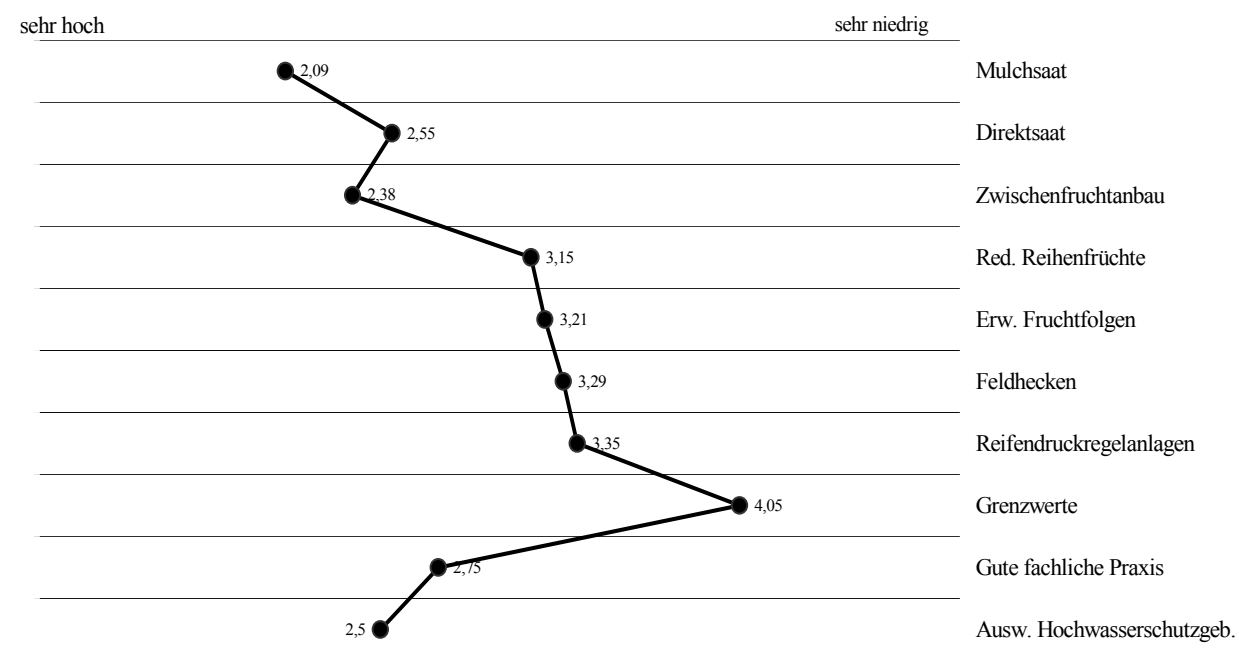

Quelle: Eigene Darstellung

In der Abbildung ist zu erkennen, dass die Experten v. a. der konservierenden Bodenbearbeitung und dem Zwischenfruchtanbau eine gute Wirksamkeit der Reduzierung des Oberflächenabflusses bescheinigen. Auch die Ausweisung von Hochwasserschutzgebieten wird positiv bewertet. Skeptisch sind die Experten bei der allgemeinen Verwendung von Grenzwerten. An dieser Stelle ist jedoch erkennbar, dass sehr hohe Ressentiments gegenüber Grenzwerten vorliegen. Es kommt v. a. darauf an, welche Grenzwerte gemeint sind. Bspw. können Grenzwerte für die Mindestbodenbedeckung sehr sinnvoll sein. 


\subsection{Fazit und Folgerungen für diese Arbeit}

Die Umweltmedien und Rechtsbereiche Wasser und Boden weisen hohe Zusammenhänge auf. Eine exakte Trennung beider Bereiche ist daher unumgänglich. Außerdem sind aus Sicht des Bodenschutzes wichtige Rechtsvorschriften im Bereich des Wasserschutzes zu beachten. Die WRRL wird definitiv durch zusätzliche Maßnahmen in den Wassereinzugsgebieten einen Einfluss auf den vorsorgenden Bodenschutz ausüben, der sich allerdings noch nicht abschließend feststellen lässt. Eine weitere enge Verknüpfung des Wasser- und Bodenschutzes wird sich ergeben, wenn der Entwurf zum vorsorgenden Hochwasserschutz in dieser Form in Kraft tritt. Aus dem oben genannten und aus der Tatsache, dass es zahlreiche Maßnahmen gibt, die sowohl dem Wasser- als auch dem Bodenschutz dienen, entsteht die Notwendigkeit der betriebswirtschaftlichen Analyse dieser Maßnahmen, die im Kapitel 6 durchgeführt wird. 


\section{Maßnahmen des Bodenschutzes in der Landwirtschaft und deren ökonomische Bewertung}

Der Hauptschwerpunkt dieser Arbeit ist die ökonomische Bewertung von Bodenschutzmaßnahmen. Die Landwirtschaft verursacht auf der einen Seite unvermeidliche Bodenschädigungen, auf der anderen Seite bestehen diesbezügliche Reduktionsmöglichkeiten. Dieses Kapitel beschäftigt sich nach einer umfassenden Literaturrecherche mit verschiedenen Bodenschutzmaßnahmen. Dabei werden die Maßnahmen, soweit möglich, auf ihre Kosten-Nutzenstruktur untersucht.

\subsection{Ergebnisse der Literaturauswertung über die ökonomischen Aspekte des Bodenschutzes in der Landwirtschaft}

Die Bedeutung des Bodenschutzes in der Landwirtschaft und der daraus resultierende Handlungsbedarf für Wissenschaft und Praxis wurde analog zur Entwicklung der Bodenschutzgesetzgebung in der landwirtschaftlichen Betriebswirtschaftslehre sehr spät erkannt. Diese späte Entwicklung spiegelt sich in der folgenden Literaturauswertung wieder. Sie stellt die wichtigsten Forschungsergebnisse der letzten zwei Jahrzehnte dar. Zunächst steht die volkswirtschaftliche Literatur im Vordergrund. Die Ausführungen beschränken sich im wesentlichen auf das Problem der externen Effekte. Danach folgt eine ausführliche Literaturstudie über die betriebswirtschaftlichen Untersuchungen des landwirtschaftlichen Bodenschutzes. Es erfolgt eine Aufteilung der Inhalte dieser Studie auf das Kapitel 6.1.2 sowie auf die einzelnen Kapitel, welche die jeweiligen Bodenschutzmaßnahmen behandeln.

\subsubsection{Volkswirtschaftliche Aspekte des Bodenschutzes}

Schädliche Bodenveränderungen weisen nicht nur monetäre Auswirkungen auf den eigentlichen Belastungsort, sondern auch auf die Umgebung und das Umfeld, auf. Sie verursachen somit negative externe Effekte.

Sowohl von stofflichen als auch von physikalischen Bodenbelastungen können negative externe Effekte ausgehen. Aufgrund der Komplexität des Naturhaushalts und der oft nicht sichtbaren und schleichenden externen Einträge und Belastungen ist es aus naturwissenschaftlicher Sicht schwierig, diese hinreichend zu analysieren und zu quantifizieren. Er- 
schwerend kommt aus ökonomischer Sicht hinzu, dass eine Monetarisierung der quantifizierten Schäden aufgrund obiger Probleme mit hohen Unsicherheiten und einer zu hohen Zahl von Unbekannten verbunden ist.

Um diesem Problem im Bereich Bodenerosion zu begegnen, schlug CLARK ${ }^{240}$ vor, zunächst alle externen Effekte zu addieren und zu bewerten und dann pauschal ein Drittel der Kosten der erodierten Fläche zuzuordnen. Dies ist eine sehr vereinfachte Methode, welche die quantitative Verteilung der einzelnen Effekte allerdings überhaupt nicht berücksichtigt. Deshalb fand sie bisher auch nur wenig Anwendung.

In Deutschland gibt es nur wenige Studien, die sich mit der Problematik der negativen externen Effekte von Bodenbelastungen beschäftigen. In einer internationalen Expertengruppe $^{241}$ wurde für verschiedene Länder versucht, die externen Kosten für die Gesellschaft durch die Landwirtschaft abzuschätzen. Die Gruppe kommt zu dem Ergebnis, dass in Deutschland von der Landwirtschaft insgesamt externe Kosten von $115 €$ /ha ausgehen. Es liegen im Gegensatz zu Großbritannien und den USA jedoch keine Kostenschätzungen speziell über den Bereich der Bodenbelastungen vor.

Für die alten Bundesländer existiert eine ausführliche Studie über externe Kosten von stoffl. Bodenbelastungen von HÜBLER UND SCHABLITZKI ${ }^{242}$. In dieser auf die gesamte Wirtschaft bezogene Studie spielt die Landwirtschaft in Bezug auf die Kostenverursachung eine bedeutende Rolle. Die externen Kosten werden von den Autoren in Schadenskosten, Ausweichkosten, Vermeidungskosten sowie Planungs- und Überwachungskosten aufgeteilt. Die Autoren berechnen für 1991 bspw. Ausweichkosten für die gesamte landwirtschaftliche Klärschlammverwertung hin zur Verbrennung von 90,5-137,5 Mio. €. Auf der Grundlage verschiedener Messungen im alten Bundesgebiet bezüglich der Überschreitung von Schadstoffen nach der Klärschlammverordnung kommen die Autoren zu dem Ergebnis, dass etwa 0,8 \% bis maximal 1,1\% der LF in der BRD die Anforderungen der Klärschlammverordnung nicht erfüllen. Die aus diesem Zusammenhang zu treffenden Nutzungsbeschränkungen auf den landwirtschaftlichen Flächen bewerten sie bei einem Belastungsgrad von 0,8 \% der LF mit 19,3 Mio. $€$ und bei 1,1 \% der LF mit 26,6 Mio. $€$. Dem Modell liegt hier zugrunde, dass die Nutzungsbeschränkungen zu Kosten von

\footnotetext{
${ }^{240}$ Vgl. hierzu CLARK et al. (1985)

${ }^{241}$ Vgl. PRETTY ET AL. (2002)

${ }^{242}$ Vgl. HÜBLER U. SCHABLITZKI (1991)
} 
$200 € /$ ha führen. Die jährlichen Schäden der Volkswirtschaft durch sog. bodenbedingte Krankheiten ${ }^{243}$ wie z. B. Nierenerkrankungen und deren Folgen (Krankenhausaufenthalte, Arbeitsunfähigkeitstage, Sterbefälle oder Frührente) schätzen die Autoren auf ca. 32,9 Mio. $€^{244}$

Eine weitere Studie zum Bereich der externen Kosten in der landwirtschaftlichen Bodennutzung stammt von WAIBEL U. FLEISCHER ${ }^{245}$. In dieser werden die gesamtwirtschaftlichen Kosten des Einsatzes von Pflanzenschutzmitteln (PSM) in der Landwirtschaft im Rahmen einer ausführlichen KNA bestimmt. Für die alten Bundesländer werden für die menschliche Gesundheit, das Trinkwasser und für bestimmte Tier- und Pflanzenarten jährliche Folgekosten in Höhe von 129 Mio. € errechnet. Die höchsten Kosten verursacht die Instandhaltung und Gewährleistung der Trinkwasserversorgung mit 65,4-95,1 Mio. $€ / a$, gefolgt von den Kosten der staatlichen Kontrolle mit 33,7 Mio. €/a, den Kosten durch Gesundheitsbelastungen sowie der Lebensmittelüberwachung mit jeweils 11,8 Mio. €/a. Außerdem ermitteln sie für den Rückgang der Biodiversität durch den Herbizideinsatz einen Betrag von 5,11 Mio. €/a und für die Giftschäden an Honigbienen 1-2 Mio. €/a. Einige negative externe Effekte, wie z. B. die Schädigung der Bodenfruchtbarkeit und Langzeitwirkungen auf die menschliche Gesundheit werden aufgrund der schwierigen Bewertung und des fehlenden Datenmaterials nicht monetarisiert. Die Studie kommt abschließend zu dem Ergebnis, dass die gesamtgesellschaftlichen Kosten um etwa $23 \%$ höher sind als die einzelbetrieblichen Kosten des Pflanzenschutzmitteleinsatzes. Nach dem Erscheinen der Studie wurden zahlreiche Kritikpunkte, wie z. B. die Vorgehensweise bei den Modellrechnungen, diskutiert. ${ }^{246}$

DOLESCHEL $u$. HEIßENHUBER ${ }^{247}$ beschäftigen sich explizit mit den externen Kosten der Bodenerosion und stützen sich in vielen Teilen ihrer Arbeit auf die Bodenerosionsforschung in den USA. Neben der Aufzählung und Analyse der durch Bodenerosion ausgelösten negativen externen Effekten (z. B. im Bereich der Vorfluter, Oberflächengewässer, Grundwasserqualität) wird auch das Problem deutlich, einzelne Symptome quantitativ auf die primäre Ursache zurückzuführen. Die Autoren beschränken sich in ihrer Arbeit letzt-

\footnotetext{
${ }^{243}$ Erkrankungen aus der Aufnahme von bodenbürtig belasteten Nahrungsmitteln.

${ }^{244}$ Vgl. Zur kritischen Betrachtung der Vorgehensweise siehe FrOMM (1997), S. 203 f.

${ }^{245}$ Vgl. WAIBEL U. FLEISCHER (1998)

${ }^{246}$ Zur ausführlichen Kritik und Diskussion siehe SCHMITZ U. KISSLING (1999).

${ }^{247}$ Vgl. DOLESCHEL U. HeißENHUBer (1991), S. 190 ff.
} 
lich auf die Kosten der technischen Sedimentbeseitigung. Als Datengrundlage dienen die durchschnittlichen Bodenabträge auf Ackerland in Bayern, $8 \mathrm{t} / \mathrm{ha} / \mathrm{a}$, wodurch sich ein jährlicher Gesamtabtrag von 17 Mio. t. für Bayern ergibt. Die durch Bodenerosion entstehenden Sedimentbereinigungskosten werden der Kostendatei für Maßnahmen des Naturschutzes und der Landschaftspflege ${ }^{248}$ entnommen. Die Autoren kommen zu dem Ergebnis, dass sich die jährlichen Kosten der Sedimentbereinigung in Bayern auf rund 15 Mio. $€$ belaufen. Dies entspricht in Bayern einer Kostenbelastung des Ackerlandes von ca. $7 € /$ ha.

Eine detaillierte Kostenschätzung für den Bereich der Bodenerosion legen PIMENTEL et al. $^{249}$ in einer Studie für die USA vor (vgl. Tabelle 9). Da eine solche Schätzung für Deutschland nicht vorliegt, kann sie nur bedingt Anhaltspunkte für die Kostenbewertung der Bodenerosion für die landwirtschaftlich genutzte Fläche in Deutschland liefern.

Tabelle 9: Jährliche Kosten zur Behebung und Kompensation der Bodenerosionsschäden (Wind- und Wassererosion) bei Getreide und Mais und einer durchschnittlichen Bodenabtragsrate von $17 \mathrm{t} / \mathrm{ha} / \mathrm{a}$ in den $\mathrm{USA}^{250}$

\begin{tabular}{ccc}
\hline & Berücksichtigte Kostenpositionen & $\begin{array}{c}\text { Kosten } \\
\text { (US-\$/ha/a) }\end{array}$ \\
\hline Onsite- & Bewässerung & 30 \\
Schäden & Düngung & 100 \\
& Zufuhr von Boden & 16 \\
\hline Offsite- & Verschiedene Bereiche & 50 \\
Schäden & & \\
\hline Gesamt & & $\mathbf{1 9 6}$ \\
\hline
\end{tabular}

Quelle: PIMENTEL et al. (1995), S. 1119

Dabei nehmen PIMENTEL et al. in der Modellrechnung an, dass die Ertragsminderungen bspw. bei Mais in 20 Jahren $20 \%$ betragen. Sie versuchen in einer Hochrechnung ferner, ihre Ergebnisse auf die gesamte Volkswirtschaft der USA zu übertragen. Demnach entstehen jährliche Gesamtkosten von 43,9 Mrd. \$ durch Bodenerosion. Die Onsite-Schäden betragen $62 \%$ (27 Mrd. \$) und die Offsite-Schäden betragen $38 \%$ (16,9 Mrd. \$). Die

\footnotetext{
${ }^{248}$ Vgl. hierzu HuNDSDORFER (1988), S. 93

${ }^{249}$ Vgl. PiMENTEL et al. (2000), S. $1117 \mathrm{ff}$.

${ }^{250}$ PIMENTEL et al. nehmen einen jährlichen Bodenabtrag von $17 \mathrm{t} / \mathrm{ha}$ *a sowie einen Betrachtungszeitraum von 20 Jahren an.
} 
Höhe der Offsite-Schäden sieht RICHTER ${ }^{251}$ typisch für Industrieländer an, deren enge Infrastruktur sehr anfällig für Erosionsschäden sei.

Ferner schätzen die Autoren, dass es Kosten von ca. 40 \$/ha/a verursachen wird, den Bodenabtrag durch Wasser und Wind von 17 t/ha/a auf 1 t/ha/a zu reduzieren. Die Autoren betonen das besonders gute KNV von 0,19, wobei die Annahme getroffen wird, dass eine Tonne vor dem Abtrag geschützter Boden einen Nutzen von 2,1 US-\$ darstellt. Aufgrund dieser Ergebnisse fordern die Autoren eine Ausdehnung des in den USA verbreiteten „Conservation Reserve Programs، ${ }^{\text {252. }}$.

Eine ähnliche, allerdings auf eine Region in Kanada beschränkte Modellstudie liegt von STONEHOUSE ${ }^{253}$ vor, die an dieser Stelle allerdings nicht weiter ausgeführt wird, da derartige Modellberechnungen nicht Gegenstand dieser Arbeit sind und deren Ergebnisse lediglich eingeschränkt nutzbar sind.

In einer Studie von WERNER UND WODSAK ${ }^{254}$ werden Schätzungen zu den Stickstoff- und Phosphoreinträgen in die Nord- und Ostsee durchgeführt. Danach gehen 38 \% der Phosphoreinträge auf den erosionsbedingten Bodenabtrag und $12 \%$ auf den Oberflächenabfluss zurück. Bei den diffusen Stickstoffeinträgen wurden $5 \%$ mit dem Bodenabtrag durch Wassererosion und $3 \%$ mit dem Oberflächenabfluss erklärt. Für den Bereich der Winderosion liegen derartige Schätzungen nicht vor.

\subsubsection{Betriebswirtschaftliche Aspekte des Bodenschutzes}

Wie bereits in der Einleitung dargestellt wurde, fehlt größtenteils eine sozioökonomische Begleitforschung zum Bereich des landwirtschaftlichen Bodenschutzes. Dies gilt auch für die landwirtschaftliche und umweltorientierte Betriebslehre. Im Folgenden wird der bisherige Stand der Forschung wiedergegeben. Dabei liegt der Fokus zunächst auf verschiedenen grundlegenden und umfangreichen Untersuchungen. Einige speziell für die Bodenschutzmaßnahmen relevanten Publikationen werden allerdings nicht an dieser Stelle, sondern in den entsprechenden Unterkapiteln des Kapitels 3 aufgeführt.

\footnotetext{
${ }^{251}$ Vgl. RiCHTER, G. (1998), S. 112

${ }^{252}$ Vgl. hierzu auch Kapitel 6.3.1.9

${ }^{253}$ Vgl. STONEHOUSE (1997)

${ }^{254}$ Vgl. WERNER U. WODSAK (1994)
} 
Die Schwerpunkte der betriebswirtschaftlich orientierten Forschung waren in erster Linie auf den Teilbereich der Maßnahmen zur Verhinderung der Bodenerosion beschränkt. Bereits in den 40er und 50er Jahren, in denen die Ertragssicherung und -steigerung von landwirtschaftlichen Nutzflächen im Vordergrund standen, wurden zahlreiche Untersuchungen, z. T. Feldversuche mit betriebswirtschaftlichem Hintergrund, angelegt, um die Beziehung zwischen dem Ertrag und der Anlage von Windschutzstreifen zu erforschen. ${ }^{255}$ Die Ergebnisse sind i. d. R. standortbezogen und uneinheitlich, so dass sie hier nicht im Einzelnen genannt werden. Die aktuellen Forschungsergebnisse auf diesem Gebiet werden in Kap. 6.3.1.6 verwendet.

In den frühen 80er Jahren wurde auf dem Gebiet der landwirtschaftlichen Betriebswirtschaftslehre damit begonnen, die erosionsbedingten Bodenschädigungen zu untersuchen. Bspw. weisen HeißENHUBER und SCHMIDTLEIN ${ }^{256}$ auf die steigende Anbaufläche des Silomaises in den 70er und 80er Jahren und die damit verbundenen Bodenschädigungen durch Bodenerosion hin. Hierzu quantifizieren sie mit Hilfe der ABAG eine Steigerung des durchschnittlichen Bodenabtrags durch Erhöhung des Silomaisanteils von unter 10 t/ha/a auf über 40 t/ha/a. Auf dieser Grundlage bewerten Sie anhand einer Näherung einen Ertrags- oder Nährstoffverlust von 3,5 bis $6 € / t$ Bodenabtrag und ermitteln die aus theoretischer Sicht ökonomisch optimale Fruchtfolge. Ein Problem sehen die Autoren jedoch bei einer derartigen Herangehensweise, die zwar die Bodenbelastung ökonomisch bewertet, sie jedoch auch in einem erheblichen Maße zulässt. Deshalb muss nach Ansicht der Autoren vielmehr ein Toleranzgrenzenkonzept angewendet werden, in dem das Ziel nicht die Maximierung des Gesamtdeckungsbeitrages, sondern die Erreichung eines tolerierbaren Bodenabtrags durch Erosionsschutzmaßnahmen ist.

Um die betriebswirtschaftliche Bewertung von Erosionsschutzmaßnahmen geht es auch in einer Studie von JAROSCH und ZEDDIES ${ }^{257}$. Sie vergleichen bezogen auf den Problembereich der Wassererosion verschiedene Auswirkungen von erosionsmindernden Maßnahmen auf die Fruchtfolge ZR-WW-KM-WW. Die Mulchsaat von Zuckerrüben und Mais erbringt demzufolge die höchste Reduktion des Bodenabtrages von bis zu $80 \%$.

\footnotetext{
${ }^{255} \mathrm{Vgl}$. BORCHERT (1987)

${ }^{256}$ Vgl. HEIßENHUBER U. SCHMIDTLEIN (1988)

${ }^{257}$ Vgl. JAROSCH U. ZEDDIES (1994)
} 
Außerdem ermitteln die Autoren Kosten für verschiedene Erosionsschutzmaßnahmen, die im Kapitel 6 als Vergleichsbasis verwendet werden.

$\mathrm{SCHACH}^{258}$ beschäftigt sich ebenfalls mit der Bewertung von Erosionsschutzmaßnahmen. Anhand von einzelbetrieblichen linearen Programmierungsmodellen errechnet er den optimalen Einsatz von Erosionsschutzmaßnahmen. Er ermittelt für eine Tonne nicht abgetragenen Boden einen Wert von etwa $7 € /$ t. Trotz der vergleichbaren Vorgehensweise liegt dieser Wert über dem von HEIßENHUBER und SCHMIDTLEIN sowie über dem in dieser Arbeit verwendeten Wert ${ }^{259}$. Der Unterschied liegt darin, dass Schach die Kosten für Saatgut berücksichtigt hat. Die Frage, ob und inwieweit das Saatgut von der Fläche abgeschwemmt wurde, ist allerdings nur durch Betrachtung des betreffenden Schlages zu klären, und Wahrscheinlichkeiten für derartige Abschwemmungen sind nur sehr schwer abschätzbar.

FLEIGE $^{260}$ beschäftigt sich in seiner Arbeit neben der ökologischen auch mit der ökonomischen Bewertung von Erosionsschäden auf einem Standort in Ostholstein, der in der Jungmoränenlandschaft südlich von Kiel liegt. Zunächst werden auf einem Gutsbetrieb Daten zum langjährigen Bodenabtrag erhoben und mit den allgemeinen Toleranzgrenzen verglichen. FLEIGE schätzt für dort typische landwirtschaftliche Betriebe mit der Fruchtfolge WW-WG-WR mit vorwiegend Parabraunerden mittleren Erosionsgrades den Schaden auf 0,97 Mio. $€(2309,5 € /$ ha) innerhalb von 30 Jahren, was einer wirtschaftenden Generation entspricht. Dieser Schaden steigt bei starker Erosionsgefährdung des Bodens auf etwa 1,64 Mio. $€(3904,8 € /$ ha) an. Hierbei ist zu beachten, dass es sich bei den Kosten nur um langfristige einzelbetriebliche Onsite-Kosten, z. B. langfristige Ertragseinbußen, Wertverlust des Bodens und zusätzliche Arbeits- und Maschinenkosten, handelt.

Für die langfristige Bewertung des Bodens infolge von Erosionsereignissen wendet BRUNOTTE $^{261}$ zwei verschiedene Ansätze an. Der erste Ansatz geht davon aus, dass zwischen der Mächtigkeit des Oberbodens und der Ertragsfähigkeit ein direkter Zusammenhang besteht. Die Beeinträchtigung des Wasserhaushalts spielt in diesem Zusammenhang eine entscheidende Rolle, denn die Beeinträchtigungen der Nährstoffversorgung können nicht

\footnotetext{
${ }^{258}$ Vgl. SCHACH (1987)

${ }^{259}$ Vgl. hierzu Kapitel 6.3.1.1.3

${ }^{260}$ Vgl. Fleige (1999)

${ }^{261}$ Vgl. BrunOtTE in SOMMER et al. (1994), S. 319
} 
durch zusätzliche Düngung ausgeglichen werden. BRUNOTTE kommt aufgrund verschiedener Versuchsergebnisse bei Mais und Zuckerrüben zu dem Schluss, dass der Nutzen der konservierenden Bodenbearbeitung in Bezug auf die Erhaltung des Oberbodens auf 0,05-1 $€ /$ ha beziffert wird, und somit eher als marginal anzusehen ist.

Der zweite Ansatz von BRUNOTTE, ein Verkehrswertansatz, richtet sich nach den Marktpreisen für den Boden, der seiner Ansicht nach vor allem von den Bodenzahlen abhängt. Ausgehend von einer Löß-Parabraunerde mit einem Marktpreis von $20.000 € /$ ha würde der Marktpreis sich bei einer durch Bodenerosion (60 cm Bodenabtrag) ausgelösten Veränderung hin zur Löß-Pararendzina auf $12.500 €$ /ha verringern, da die Wertzahl des Bodens von 75 auf 55 BP gesunken ist. Es ist allerdings davon auszugehen, dass dieser Prozess etwa 600 Jahre dauert, so dass sich ein jährlicher Nutzen aus der Bodenwerterhaltung von $12,5 € /$ ha ergäbe.

BRUNOTTE führt jedoch auch Kritik dieser Vorgehensweise an. Bspw. können die Bodenabtrags-Ertrags-Beziehungen nicht für tiefgründige Böden aufrecht erhalten werden. Dort kann es durch die Bodenentwicklungen (Humusanreicherung durch Wurzeln) im Unterboden zu einer gegenläufigen Tendenz kommen. An der Vorgehensweise von BRUNOTTE ist außerdem zu kritisieren, dass die wirtschaftliche Ertragsfähigkeit und somit die Bodenpreise aufgrund unsicherer Märkte und der Agrarpolitik nicht exakt vorausgesehen werden können. Der Verkehrswert des Bodens ist gegenwärtig auch zahlreichen Verzerrungen unterworfen, so dass diesem Preis nicht die tatsächliche landwirtschaftliche Ertragsfähigkeit zugrunde liegt, sondern andere Faktoren den Preis bestimmen. Bspw. verzerren die Ausweisung von Bau- oder Bauplanungsgebieten den Preis nachhaltig. In viehstarken Regionen ergeben sich höhere Bodenpreise durch die Notwendigkeit der Betriebe, Güllenachweisflächen vorzuhalten. Dementsprechend liegen die Ertragswerte landwirtschaftlicher Böden wesentlich niedriger als deren Verkehrswerte. ${ }^{262}$

HEIßENHUBER und SCHMIDTLEIN ${ }^{263}$ behandeln in ihrer Studie ebenfalls den Wertverlust des Bodens durch Erosion und dessen Berechnungsmöglichkeiten anhand einer Ertragswertermittlung. Die Autoren gehen wie folgt vor. Sie ermitteln den Grenzkaufpreis eines Hektars und treffen die Annahme, dass sich dieser Wert parallel zur Ertragsfähigkeit ent-

\footnotetext{
262 Vgl. hierzu BAHRS (1999) S. 155 f.

${ }^{263}$ Vgl. HeißENHUBER u. SCHMidTLEIn (1988), S. $112 \mathrm{f}$.
} 
wickelt. Dies setzt ein entsprechendes Verhältnis zwischen Oberbodendicke und Ertrag bzw. Bodenwert voraus. Der Grenzkaufpreis wird wie folgt errechnet: Von dem Fruchtfolgedeckungsbeitrag werden der entsprechende Lohnansatz, ein Gewinn- und Risikozuschlag sowie die sonstigen Kosten, wie z. B. Grundsteuer, Berufsgenossenschaftsbeitrag abgezogen. Durch Kapitalisierung erhalten die Autoren den oberen Kaufpreis bzw. Grenzkaufpreis. Dieser Ansatz geht wie der von BRUNOTTE vereinfachend von einer linearen Beziehung zwischen der Ertragsfähigkeit und der Mächtigkeit des Oberbodens aus, was in Wirklichkeit nicht der Fall ist. Bspw. ist beim Verlust der oberen Schichten des Bodens eine geringere bzw. keine Ertragswirkung festzustellen als bei dem Verlust der unteren Oberbodenschichten. In Ermangelung weiterer Studien und aus Gründen der Vereinfachung sowie um der Kritik an dem Konzept von BRUNOTTE in dieser Arbeit zu entgehen, wird die Vorgehensweise von HeIßENHUBER und SCHMIDTLEIN in Kapitel 6.3.1.1.3 verwendet, um den Wertverlust des Bodens durch Erosion zu ermitteln.

\subsection{Theoretische Grundlagen zur ökonomischen Bewertung und Vorge- hensweise}

Im folgenden Kapitel werden die einzelnen methodischen Bewertungsansätze der bodenschützenden Maßnahmen dieser Arbeit dargestellt. Es handelt sich dabei um drei Bewertungsansätze: die Kostenvergleichsrechnung (KVR), die Kosten-Wirksamkeits-Analyse (KWA) und die Kosten-Nutzenanalyse (KNA). Da die Berechnungen für einzelne landwirtschaftliche Betriebe durchgeführt werden, ist dabei zu berücksichtigen, dass sich die folgende Darstellung der Bewertungsmethoden auf die einzelwirtschaftliche Ebene beschränkt. Auch wenn z. B. die KNA in erster Linie ein volkswirtschaftliches Instrumentarium ist, sind zwischen einzel- und volkswirtschaftlichen Kosten-Nutzenüberlegungen wichtige Gemeinsamkeiten zu erkennen:

1. die Suche nach alternativen Handlungsmöglichkeiten,

2. der Vergleich von Handlungsalternativen,

3. die Wahl der effizientesten Handlungsmöglichkeit und

4. die in die Zukunft gerichtete Betrachtungsweise.

Die Verknüpfung der oben vorgestellten Methoden in dieser Arbeit ist in der Übersicht 5 dargestellt. 


\section{Übersicht 5: Vorgehensweise bei der Ermittlung der Wirtschaftlichkeit verschiede- ner Bodenschutzmaßnahmen mittels Kostenvergleich, KWA und KNA}

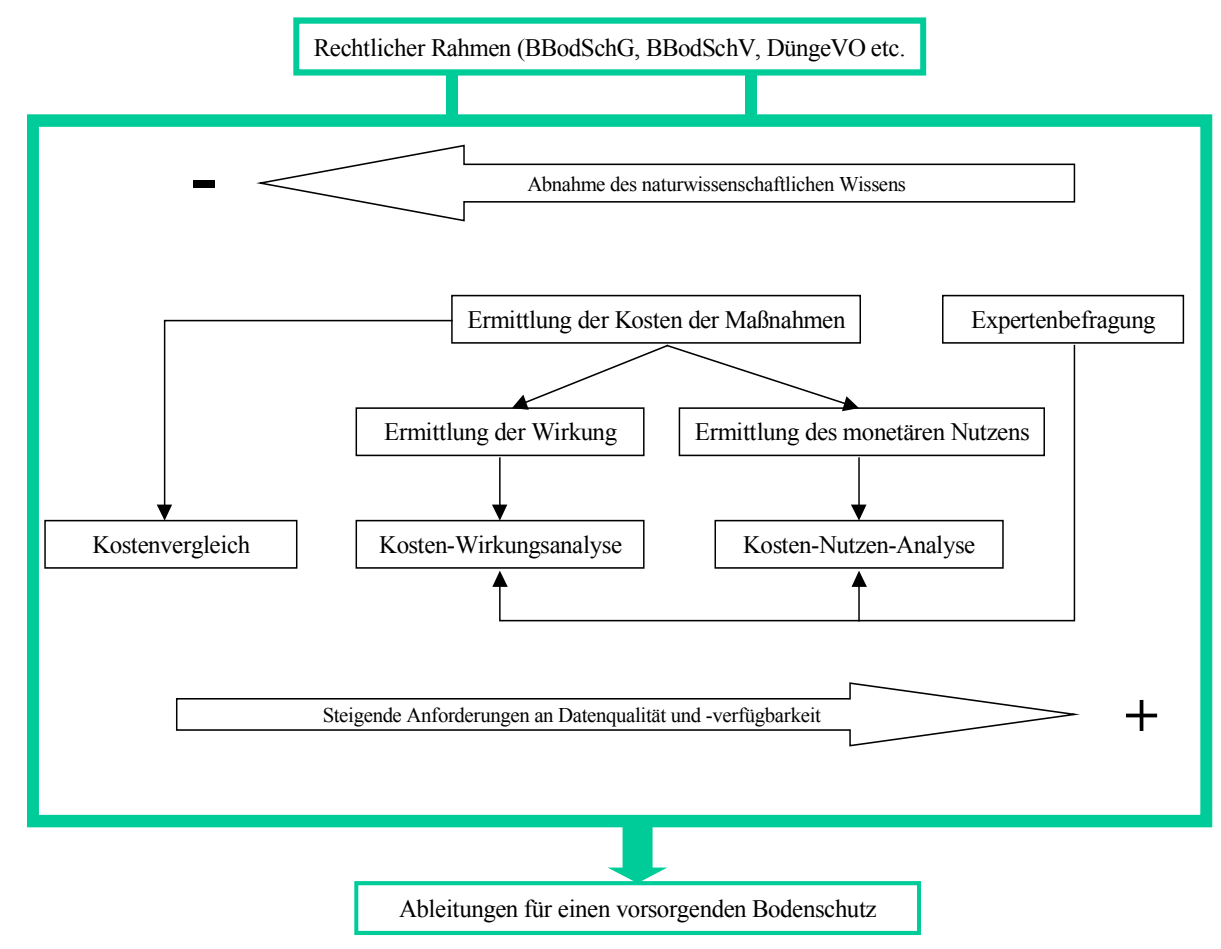

Quelle: Eigene Darstellung

Die in der Übersicht 5 angesprochenen Methoden werden im folgenden Kapitel erläutert.

\subsubsection{Die Methodik der Kostenvergleichsrechnung}

Die KVR ist die einfachste Bewertungsmethode. Sie dient zur Ermittlung der relativen Vorzüglichkeit einer Maßnahme auf Ebene der Gesamtkosten. Die KVR wird in dieser Arbeit angewendet, wenn der Nutzen weder anhand der Wirksamkeit noch monetär bestimmt werden kann. ${ }^{264}$

Bei der KVR ist diejenige Maßnahme zu empfehlen, welche die geringsten Kosten aufweist. Die Grundvoraussetzung für eine KVR ist demzufolge die Tatsache, dass der jeweilige Nutzen bei den verschiedenen Maßnahmen gleich hoch sein muss. 
Da durch die KVR lediglich die relative Vorzüglichkeit ermittelt werden kann, bedarf es bei den in dieser Arbeit durchgeführten KVR bei einigen untersuchten Maßnahmen ergänzender Erläuterungen.

\subsubsection{Die Methodik der Kosten-Wirksamkeits-Analyse}

Die KWA dient in ihrer ursprünglichen Form der Ermittlung der relativen Effizienz von Maßnahmen oder Maßnahmenbündeln. Sie findet in dieser Arbeit lediglich auf einzelbetrieblicher Ebene Anwendung, und zwar dann, wenn der Nutzen der betrachteten Maßnahmen quantifiziert, jedoch nicht monetarisiert werden kann. ${ }^{265}$ Bei der KWA werden wie bei der KVR zunächst alle Kosten der einzelnen Maßnahme erfasst. Der Nutzen kann in unterschiedlich skalierten, meist jedoch physikalischen Größen angegeben werden. ${ }^{266}$ Dadurch wird die Grundlage für eine Rangfolge einzelner Maßnahmen geschaffen. ${ }^{267}$

Zunächst muss das Ziel der KWA definiert werden. Im Fall dieser Arbeit ist es das Ziel, eine höchstmögliche Reduktion der entsprechenden Bodenbelastung mit kostenminimalen Maßnahmen zu erreichen. Die Bestimmung der verschiedenen alternativen Maßnahmen hat sich an diesem Ziel zu orientieren.

Die nötigen Daten für die Wirksamkeitsanalyse werden zum größten Teil aus Literaturauswertungen entnommen, die Kostenanalyse basiert auf den Werten des KTBL oder anderen Kostendateien ${ }^{268}$. Die zeitliche Angleichung geschieht durch die Diskontierung der Kostenströme, damit die zu unterschiedlichen Zeitpunkten anfallenden Kostengrößen vergleichbar sind. Es wird dabei ein marktüblicher Zinssatz von $4 \%$ unterstellt. Die hieraus entstehende Kennzahl (z. B. Kosten in $€ /$ t verhinderter Bodenabtrag oder Kosten in $€ /$ bar Druckabsenkung) ist folglich ein Vergleichswert der Kostenwirkung der jeweiligen Bodenschutzmaßnahmen.

\subsubsection{Die Methodik der Kosten-Nutzen-Analyse}

Aus betriebswirtschaftlicher Sicht sind Maßnahmen des Bodenschutzes als Investitionen eines landwirtschaftlichen Betriebes zu sehen, deren Umfänge von den damit verbunde-

\footnotetext{
265 Vgl. Übersicht 5

${ }^{266}$ Vgl. MÜHLENKAMP (1994), S. 7

${ }^{267}$ Zur Durchführung einer KWA im Allgemeinen siehe HANUSCH (1994) und WILHELM (1999), S. 37

${ }^{268}$ Vgl. ROTH u. BERGER (1999)
} 
nen Nutzen und Kosten innerhalb eines bestimmten Zeitraumes abhängen. Gemäß dem Grenzproduktivitätsprinzip ist es das Ziel des Unternehmers, innerhalb dieses Zeitraumes den Überschuss des Nutzens über die dafür aufgewendeten Kosten zu maximieren, wobei i. d. R. externe Effekte keine Berücksichtigung finden. ${ }^{269}$ Deshalb bedient das Grenzproduktprinzip lediglich eine kurzfristige Sichtweise. Es ist folglich davon auszugehen, dass ein Landwirt, vorausgesetzt er hat vollkommene Information über die Nutzenbeträge, eine bodenschützende Maßnahme dann unterlässt, wenn das Kosten-Nutzen-Verhältnis $(\mathrm{KNV})$ sich in einem Bereich größer eins bewegt. Da die vollkommene Information über den Nutzenbeitrag bei vielen Bodenschutzmaßnahmen allerdings fehlt, kommt es zu einer Verzerrung des KNV. Das KNV wird sich in diesem Fall tendenziell schlechter darstellen, als es in der Realität ist. Dies muss bei der Interpretation der Ergebnisse berücksichtigt werden.

Die KNA dient nicht nur der Ermittlung der relativen, sondern auch der absoluten Vorzüglichkeit einer Maßnahme oder eines Maßnahmenbündels. Außerdem wird die KNA dabei eingesetzt, das Produktionsprogramm oder - niveau zu ermitteln. ${ }^{270}$

Bei der KNA erfolgt eine Gegenüberstellung sämtlicher monetär bewertbarer Kosten und Nutzen. Der Nutzen wird in dieser Arbeit mit dem indirekten Verfahren ermittelt, d. h. es werden tatsächliche, berichtigte oder unterstellte Preise herangezogen. Die Kosten werden analog zur KWA den Daten des KTBL oder anderen Kostendateien entnommen. Liegen keine derartigen Daten vor, werden Preisempfehlungen der Hersteller herangezogen, wie es bspw. im Kapitel 6.3.2.2 der Fall ist. Bei der KNA werden beide betrachteten Größen diskontiert. Der hierfür verwendete Zinssatz beträgt 4 \%. Es wird neben dem KNV auch der durchschnittliche jährliche monetäre Vorteil errechnet. Dieser Wert gibt Auskunft über die Höhe des Nutzen- bzw. Kostenüberschusses im Mittel der Jahre und dient daher als weiterer Vergleichswert zur wirtschaftlichen Beurteilung der Bodenschutzmaßnahme.

Die Anwendbarkeit der KNA ist in dieser Arbeit jedoch nicht für jede Bodenschutzmaßnahme gegeben. Dies liegt hauptsächlich daran, dass die KNA die höchsten Anforderun-

\footnotetext{
${ }^{269}$ Vgl. WerbeCK U. WinK (1994), S. 403

${ }^{270}$ Vgl. Mishan (1975), S. 9
} 
gen an das naturwissenschaftliche Wissen und an die Qualität der verwendeten Daten stellt. $^{271}$

In dieser Arbeit werden ausschließlich die einzelbetrieblichen Kosten- und Nutzenpositionen betrachtet. Wenn möglich, werden in ergänzenden Erwägungen die externen Effekte, die sich bspw. in einem gesamtgesellschaftlichen Nutzen äußern, mit berücksichtigt. ${ }^{272}$

\subsubsection{Kosten von Bodenschutzmaßnahmen}

Bei der Implementierung von Bodenschutzmaßnahmen ist bspw. zu berücksichtigen, dass die Landwirte die tatsächlichen Schäden nicht oder nur ungenau mikroökonomisch schätzen können. Die Kosten der einzelnen Bodenschutzmaßnahmen bestehen zunächst aus den zusätzlichen direkten Kosten für deren Durchführung. Diese Kosten können z. B. beim Anlegen von Hecken, durch Anschaffung von Reifendruckregelanlagen, TerraBereifung oder Mulchsaattechnik entstehen. Einzelne Maßnahmen können indirekte Kosten auslösen, so z. B. bei Ertragsrückgängen durch Schattenwirkungen von Hecken. Die indirekten Kosten haben innerhalb der Berechnungen dieser Arbeit den Charakter, dass sie i. d. R. in der Zukunft anfallen und deren Höhe nicht genau bestimmt werden kann. Deshalb werden in diesem Fall Daten aus Forschungsstudien herangezogen, aus denen sich die indirekten Kosten ableiten lassen. Wenn die Forschungsstudien starke Schwankungen aufweisen, wird diesem Umstand in entsprechenden variierenden Annahmen Rechnung getragen.

Die Kosten der Maßnahmen können auf zwei Wegen ermittelt werden. Zum einen könnten sie durch Messungen der Arbeitszeit und Ermittlung der Maschinenkosten, oder zum anderen durch normierte Daten des KTBL bzw. Verrechnungssätze entsprechend ansässiger Maschinenringe ermittelt werden. In dieser Arbeit wird letzterer Weg eingeschlagen, da sich die Berechnungen nicht an real existierenden Betrieben orientieren, sondern eine allgemeine Gültigkeit besitzen sollen. Außerdem ist z. B. bei Kostenvergleichen nicht die absolute Höhe der Kosten, sondern deren Relation entscheidend. Zu beachten ist hierbei, dass diese Kosten den gegenwärtigen Stand der Technik symbolisieren. Durch weiteren technischen Fortschritt, die zukünftige Agrarpolitik und sich eventuell ergebene

\footnotetext{
${ }^{271}$ Vgl. Übersicht 5

${ }^{272}$ Eine weitere Betrachtung der Problematik der externen Effekte findet sich in Kapitel 6.1.1.
} 
Kostendegressionspotenziale, z. B. Pachtmöglichkeit von Acker- und Grünlandflächen, können sich für einige Betriebe veränderte Kostensituationen ergeben, die in dieser Arbeit jedoch nicht abgeschätzt und daher nicht berücksichtigt werden können.

Bei den ausgewiesenen oder verrechneten Preisen ist zu berücksichtigen, dass es sich um Bruttopreise handelt. Sie enthalten daher die von pauschalierenden Landwirten zu verwendenden Steuersätze von 16 \% (bei Handelsdüngern, Pflanzenschutzmitteln, Gebäuden und Maschinen), 7 \% (bei Futtermitteln, Saatgut, Tieren) oder $9 \%$ (bei pauschalierenden Landwirten). Ein Sonderfall stellt die Berücksichtigung der Umsatzsteuer beim Pflanzmaterial von Hecken dar. In diesem Fall würden, wie in Kapitel 6.3.1.6 deutlich wird, $5 \%$ Umsatzsteuer fällig werden.

Bei der Durchführung von Bodenschutzmaßnahmen ist weiterhin davon auszugehen, dass innerbetrieblich verursachte Kosten auftreten können. Sofern diese Kosten der Bodenschutzmaßnahme zugeordnet werden können, werden sie quantifiziert und finden Eingang in die entsprechende Berechnung. Ansonsten weisen ergänzende Erwägungen auf die Zuordnungsprobleme hin.

\subsubsection{Nutzen der Maßnahmen}

Analog zu den zuvor behandelten Kosten von Bodenschutzmaßnahmen ist auch beim Nutzen, die Aufteilung in direkten und indirekten Nutzen zu vollziehen. Der direkte Nutzen ist unmittelbar aus den Auswirkungen der Maßnahme abzuleiten, wie etwa ein höherer Ertrag bei der Anwendung von bodenschonenden Fahrwerken. Beim indirekten Nutzen ist diese Beziehung nicht vorhanden, wie das Beispiel der Kosteneinsparungen bei der Durchführung von konservierenden Bodenbearbeitungsverfahren zeigt. ${ }^{273}$

Ein generelles Problem ergibt sich bei der monetären Bewertung des Nutzens mittels des indirekten Verfahrens, worauf bereits im Kapitel 6.2.3 eingegangen wurde. Bspw. fallen bei einer Umstellung von konventioneller auf konservierende Bodenbearbeitung bereits im ersten Jahr Aufwendungen in Form von Verfahrenskosten an. Der monetär zu bewertende Nutzen kann jedoch u. U. erst nach einigen Jahren auftreten, wenn es z. B. zu ei-

\footnotetext{
${ }^{273}$ Vgl. hierzu Übersicht A-2.
} 
nem Starkregenereignis kommt. Deshalb ist eine Diskontierung der Kosten- und vor allem der Nutzenströme unabdingbar.

\subsubsection{Datengrundlagen}

Die Datengrundlage für die Ermittlung der Gesamtkosten von Bodenschutzmaßnahmen besteht in dieser Arbeit hauptsächlich aus den Kostendateien des KTBL für die allgemeine Betriebsplanung sowie für die Landschaftspflege ${ }^{274}$ und KTBL-Programmen $^{275}$, Daten der Ämter für Agrarstruktur, persönlichen Mitteilungen oder einzelnen speziellen Kostendateien $^{276}$. Außerdem werden die Richtwertdeckungsbeiträge der LWK Hannover verwendet. $^{277}$

Die Datengrundlage zur Quantifizierung und Monetarisierung des Nutzens sind wesentlich differenzierter und bestehen aus verschiedenen pflanzenbaulichen, bodenkundlichen und betriebswirtschaftlichen Versuchsergebnissen von Universitäten, Landwirtschaftskammern sowie Lehr- und Versuchsanstalten. Eine weitere Datengrundlage, v. a. zur Validierung und Ergänzung der Ergebnisse, beinhaltet die Expertenbefragung, die in Kapitel 2.2 erläutert ist, dar.

\subsubsection{Weitere Annahmen}

Aufgrund der hohen Vielfalt an betrachteten und untersuchten Bodenschutzmaßnahmen und des Mangels an ausreichenden Versuchsergebnissen ist eine Konzentration auf einen definierten Modellbetrieb nicht vorgesehen. Die einzelnen Maßnahmen erfordern jeweils eine spezielle Betrachtung und unterschiedliche Voraussetzungen an naturräumlichen Gegebenheiten, Standorte sowie Anbauverfahren. Allerdings werden bei den betrachteten Maßnahmen Daten aus typischen gefährdeten Regionen verwendet, wie bspw. Daten und Annahmen aus den Lößgebieten des tertiären Hügellandes bei den Maßnahmen gegen Wassererosion oder solche aus der Nordwestdeutschen Tiefebene bei den Maßnahmen gegen Winderosion.

\footnotetext{
${ }^{274}$ Vgl. hierzu KTBL (2002) u. KTBL (1998)

${ }^{275}$ Vgl. MAKOST 3.0 und KTBL (2000a)

${ }^{276}$ Vgl. ROTH u. BERGER (1999)

${ }^{277}$ Vgl. LWK HANNOVER (2002)
} 
Ferner werden verschiedene Fruchtfolgen, die für die entsprechenden Gebiete typisch sind, vorausgesetzt. Hinsichtlich der Bodenbearbeitung werden verschiedene Varianten untersucht, neben der Bearbeitung mit Pflug werden ebenso verschiedene Varianten der konservierenden Bodenbearbeitung miteinander verglichen. Betriebsgrößen werden ebenfalls maßnahmen- und regionsspezifisch vorausgesetzt. Dementsprechend wird der Maschinenbesatz angepasst. Die Leistung der Maschinen liegt generell oberhalb der Abschreibungsschwelle, so dass die Kosten hierfür den variablen Maschinenkosten zugerechnet werden können.

Die Maßnahmen des Bodenschutzes werden im Folgenden in die des physikalischen sowie in die des stofflichen Bodenschutzes eingeteilt.

\subsection{Physikalischer Bodenschutz}

Im Folgenden werden Bodenschutzmaßnahmen zur Reduzierung der physikalischen Schädigungen des Bodens detailliert beschrieben und anhand der in Kapitel 6.2 dargelegten Methoden auf deren betriebswirtschaftliche Vorzüglichkeit untersucht. Unter physikalischem Bodenschutz wird in diesem Zusammenhang der Schutz des Bodens vor Bodenerosion und -schadverdichtung verstanden. Auf die Flächenversiegelung durch Überbauung und Abgrabung wird aufgrund der fehlenden einzelbetrieblichen Einflussnahme nicht eingegangen.

\subsubsection{Maßnahmen zur Verhinderung von Bodenerosion}

Erosionsschutzmaßnahmen sind vorwiegend beim Anbau von erosionsfördernden Kulturen wie z. B. Mais oder Rüben, sowie in Hanglagen erforderlich. Die landtechnischpflanzenbauliche Forschung hat mittlerweile zahlreiche Maßnahmen für die landwirtschaftliche Praxis entwickelt und begleitet. Sie reichen von der einfachen Lockerung der Fahrspuren über die Mulch- u. Direktsaat, dem Anbau von Zwischenfrüchten bis hin zur Aufgabe der ackerbaulichen Nutzung und dem Ersatz durch Grünland. Durch die Anwendung derartiger Schutzmaßnahmen entstehen dem Betrieb i. d. R. verschiedene Kosten. Diese können z. B. durch die Anpassung und Veränderung der Produktionstechnik (Bodenbearbeitung, Bestelltechnik oder Bereifung), oder durch zusätzliche variable Arbeits- und Maschinenkosten verursacht werden. Bei einer Produktionsumstellung, z. B. von Silomais zu Grünland, sind, neben der Veränderung der Produktionskosten auch die 
Kosten der Substitution der entfallenen Futternährstoffe zu berücksichtigen. Wird die landwirtschaftliche Nutzung einer Fläche aufgegeben, sind die Deckungsbeitragsverluste der jeweils wettbewerbsschwächsten Frucht oder langfristig die Fruchtfolgedeckungsbeiträge anzusetzen. Durch eine Veränderung der Schlaggröße und -struktur müssen zusätzlich Arbeits- und Maschinenkosten berücksichtigt werden, die aus veränderten Rüst- und Wegezeiten entstehen.

Durch die Einführung von Erosionsschutzmaßnahmen lassen sich u. a. die Schäden auf den Bodenabtrags und -eintragsflächen um einen beträchtlichen Teil reduzieren. Dieser Nutzen entsteht v. a. aus der Vermeidung von Nährstoffverlusten, Ertragsminderungen und von Wertminderungen der Flächen. Bei der Kompensation der Nährstoffverluste treten v. a. Ersatzbeschaffungskosten und zusätzliche Ausbringungskosten auf. Die Erosionsschutzmaßnahmen verhindern langfristig die Abnahme der Bodenfruchtbarkeit und somit Ertragsminderungen sowie den daraus resultierenden Wertverlust des Bodens. Dieser letztgenannte Effekt darf jedoch nicht überbewertet werden, da der Kaufpreis des Bodens eine Funktion weiterer zahlreicher Parameter ist.

Weiterhin besteht der Nutzen aus der Einsparung von Sanierungsmaßnahmen und der Vermeidung von externen Effekten (Eutrophierung von Gewässern, Minderung des Wertes der Naturlandschaft). Der Nutzen durch die Einsparung von Sanierungsmaßnahmen wird vereinfacht durch die Normdaten des $\mathrm{KTBL}^{278}$ ermittelt, die positiven Nutzeneffekte durch die Verminderung von obigen externen Effekten können durch die Erhaltung verschiedener Pflanzen- und Tierarten quantifiziert, aber nur schwer monetarisiert werden.

Im Folgenden werden die einzelnen Maßnahmen zur Reduzierung der Bodenerosion auf landwirtschaftlichen Nutzflächen dargestellt und anhand der in Kapitel 6.2 erläuterten Maßnahmen aus ökonomischer und ökologischer Sicht bewertet.

\subsubsection{Bodenschutz durch konservierende Bodenbearbeitung ${ }^{279}$}

Die konservierende Bodenbearbeitung ist der Oberbegriff für Bodenbearbeitungsverfahren, die ohne eine wendende Bearbeitung des Pfluges auskommen. Hierzu zählen z. B.

\footnotetext{
${ }^{278}$ Vgl. hierzu KTBL (1998)

${ }^{279}$ In dieser Arbeit wird der Begriff „,konservierende Bodenbearbeitung“ synonym für die Begriffe ,reduzierte“, ,pfluglose“ oder ,nicht wendende“" Bodenbearbeitung verwendet.
} 
die Mulchsaatverfahren, solange sie ohne den Pflug auskommen oder Direktsaatverfahren. In der Literatur finden sich auch andere Begriffe wie z. B. die Festbodenmulchwirtschaft nach EHLERS ${ }^{280}$. Im Folgenden wird neben einer ausführlichen Darstellung der Verbreitung der konservierenden Bodenbearbeitung auch auf die Umstellungsprobleme und die betriebswirtschaftliche Analyse dieser Verfahren eingegangen.

\subsection{Verbreitung der konservierenden Bodenbearbeitung}

Die konservierende Bodenbearbeitung wird in großen Teilen Nordamerikas und Kanadas seit vielen Jahrzehnten flächendeckend betrieben. In Europa hingegen werden überwiegend Pflugsysteme angewendet. ${ }^{281}$ Die konservierende Bodenbearbeitung hat in der Vergangenheit in Deutschland an Bedeutung gewonnen. Dies liegt zum einen an der finanziellen Unterstützung in einigen Bundesländern durch Erosionsschutzprogramme im Rahmen der KULAP oder des Modulationsgesetzes, zum anderen aber auch an einzelbetrieblichen Vorteilen, wie z. B. der Verminderung von Arbeitsspitzen bei der Aussaat oder der Reduktion der Verdunstung in niederschlagsarmen Gebieten.

Der Anteil der konservierenden Bodenbearbeitung in Deutschland ist bisher nicht erfasst worden. Er beträgt nach Meinung der Befragten in der Expertenbefragung ${ }^{282}$ für das gesamte Bundesgebiet etwa $8 \%$ der AF und wird in den nächsten zehn Jahren auf $24 \%$ der AF ansteigen.

Eine Studie zur Verbreitung der konservierenden Bodenbearbeitung liegt für Thüringen vor. Der Anteil der konservierenden Bodenbearbeitung in Thüringen wird von WERNER, REICH UND DEGNER ${ }^{283}$ im Rahmen einer Umfrage ${ }^{284}$ auf insgesamt $38 \%$ der AF geschätzt. Dabei entfallen 37,1 \% auf die Mulch- und 0,9\% auf die Direktsaat. Die Anteile variieren jedoch sehr stark. Deshalb ist darauf hinzuweisen, dass im Thüringer Becken der Anteil der konservierenden Bodenbearbeitung etwa $54 \%$ beträgt. Dies deutet auf die zahlreichen Vorteile konservierender Bodenbearbeitungsverfahren in Gebieten mit geringen Jahresniederschlägen und starker Sommertrockenheit hin.

\footnotetext{
${ }^{280}$ Vgl. EHLERS (1996), S. $221 \mathrm{ff}$.

${ }^{281}$ Vgl. KÖLLER U. LINKE (2001), S. 15 ff.

${ }^{282}$ Vgl. hierzu Kapitel 2.2, n= 17

${ }^{283}$ Vgl. WERNER, REICH UND DEGNER (1999), S. 52

$284 \mathrm{n}=127.000$ ha, 95 Betriebe.
} 
Die Gründe für den Pflugeinsatz sind vor allem die vorhandene Technik $(60 \%$ der Befragten), die Vermeidung des Ertragsrisikos (> 50 \%), Unkraut- und Schädlingsbekämpfung (50\%) sowie die Beseitigung von Radspuren und die Einarbeitung von Ernterückständen (20\%). Die Gründe für die Durchführung einer pfluglosen Bearbeitung sahen $80 \%$ der Befragten in der Senkung der Bearbeitungskosten, $45 \%$ im Erosionsschutz und $25 \%$ in der besonderen Bodeneignung ihrer Schläge. ${ }^{285}$

Für das Bundesland Sachsen liegen Schätzungen vor, dass etwa 30 \% der AF konservierend bestellt werden. Laut dem Evaluierungsbericht über das Programm „Umweltgerechte Landbewirtschaftung ${ }^{6286}$ werden ca. $20 \%$ der AF innerhalb des Erosionsschutzprogramms gefördert.

Die Akzeptanz konservierender Bodenbearbeitungsverfahren ist für die alten Bundesländer eher als gering einzustufen. Anhand einer umfangreichen Umfrage aus dem Jahr 1999 im Raum Südniedersachsen wird dies deutlich. ${ }^{287}$ Etwa $80 \%$ der befragten Betriebe ( $\mathrm{n}=$ 450) setzen bei Getreide nach Getreide den Pflug ein. Nach Getreide zu Zuckerrüben setzen $50 \%$ der Betriebe den Pflug ein, und $35 \%$ organisieren die Bodenbearbeitung in einem variablen Pflug/Mulchsaat-System. Etwa $20 \%$ der landwirtschaftlichen Betriebe führen nach Getreide zu Zuckerrüben eine konsequente Mulchsaat durch. Nach Zuckerrüben zu Getreide liegt der Mulchsaatanteil noch etwas höher. Hierbei ist zu berücksichtigen, dass Verfahren der konservierenden Bodenbearbeitung zum Zeitpunkt der Umfrage nicht finanziell gefördert wurden. An den hohen Anteilen kann ein Umdenkprozess abgeleitet werden, jedoch muss auch berücksichtigt werden, dass die Mulchsaat allein aus dem Gesichtspunkt der guten fachlichen Praxis ein vielfaches betragen müsste. Die hohen Anteile der konservierenden Bodenbearbeitung in den neuen Bundesländern sind z. T. mit den Förderprogrammen (Sachsen), mit der langjährigen Anwendung bereits zu DDRZeiten und der Reduzierung von Arbeitsspitzen zu erklären.

Die Grenzen der Anwendung der konservierenden Bodenbearbeitung können der Übersicht 6 entnommen werden. Sie bestehen hauptsächlich aus den Standortbedingungen, der Bodenart, und der Fruchtfolgegestaltung. Bei EHLERS ${ }^{288}$ findet sich eine diffe-

\footnotetext{
${ }^{285}$ Vgl. WERNER, REICH UND DEGNER (1999), S.52 f.

${ }^{286} \mathrm{Vgl}$. SÄCHSISCHE LANDESANSTALT FÜR LANDWIRTSCHAFT (2001)

${ }^{287}$ Vgl. BRUNOTTE et al. (1999), S. $72 \mathrm{f}$.

${ }^{288}$ Vgl. EHLERS (1996), S. 224
} 
renziertere Einteilung der Böden für das gesamte Bundesgebiet bezüglich der Eignung für eine konsequente und langfristige konservierende Bodenbearbeitung. Grundlage ist dabei eine Ermittlung von Trockengebieten, bei denen sich die konservierende Bodenbearbeitung aufgrund der Verbesserung des Wasserhaushalts stärker etablieren könnte. Zur Abgrenzung dieser Trockengebiete geht EHLERS von einer 700 mm-JahresniederschlagIsohyete aus. Humusarme und mischkörnige Sandböden, Tonböden mit nicht aufweitbaren Tonmineralen und staunasse Böden sind seiner Meinung nach nicht für die konservierende Bodenbearbeitung geeignet. Eine spezifische Einteilung der Eignung von Böden für die konservierende Bodenbearbeitung in Thüringen findet sich bei WERNER, REICH U. DEGNER $^{289}$ in Übersicht 6.

\section{Übersicht 6: Bedingungen für die konservierende Bodenbearbeitung:}

Vorteilhafte Standorte

- Kalkreiche Tonböden

- Humose Sandböden

- Steinige Böden

- $\mathrm{Zu}$ Verschlämmung neigende Böden

- Erosionsgefährdete Standorte

- Trockene Standorte

- Leicht zu erwärmende Böden

\section{Nachteilige Standorte}

- Nicht quellfähige Tonböden

- Zu Dichtlagerung neigende Sandböden und feinsandige Schluffböden

- Staunasse Böden

- Kalte und untätige Böden

Quelle: Verändert nach FELDWISCH (1999), S. 34

TEBRÜGGE UND BÖHRNSEN ${ }^{290}$ beziffern allein die direktsaatfähige Fläche in Deutschland auf 28,5 \% der AF, da auf dieser Fläche Wintergetreide, Hülsenfrüchte, Hackfrüchte, Raps und Silomais angebaut werden und eine Umstellung problemlos erfolgen kann.

Bei der Mulchsaat werden in der landwirtschaftlichen Praxis verschiedene Varianten unterschieden. Zunächst ist eine Mulchsaat mit oder ohne Saatbettbereitung denkbar, dann muss zwischen der Saat in Strohmulch oder in die abgestorbene Zwischenfrucht unterschieden werden.

\footnotetext{
${ }^{289}$ Vgl. WERNER, REICH UND DEGNER (1999), S. 51

${ }^{290}$ Vgl. TeBrÜGGE U. BöHRNSEN (1994), S. 111
} 


\subsection{Umstellung auf konservierende Bodenbearbeitung}

Bei der Umstellung eines landwirtschaftlichen Betriebes auf konservierende Bodenbearbeitung sind verschiedene Möglichkeiten denkbar. Von einem kompletten Umstieg der gesamten Ackerfläche ist jedoch zunächst abzuraten, da die konservierende Bodenbearbeitung höhere Anforderungen an das pflanzenbau-technische Wissen des Betriebsleiters stellt und $u$. U. sehr gravierende Probleme mit sich führen kann. Die praktische Umsetzung der einzelnen konservierenden Bodenbearbeitungsverfahren ist der Abbildung A-1 im Anhang zu entnehmen. Dort sind die verschiedenen Varianten der Maschinennutzung der konservierenden Bodenbearbeitung im Vergleich zur konventionellen Bodenbearbeitung und zur Direktsaat dargestellt.

Außerdem hängt die Umstellungsvariante maßgeblich von den natürlichen Standortbedingungen, der Maschinenausstattung sowie der Risikobereitschaft des Betriebsleiters ab. Kürzlich entwickelte Punktesysteme zur Einordnung des landwirtschaftlichen Betriebes bezüglich der Umstellungsfähigkeit dienen dem Landwirt zu Orientierung und zum ersten Einstieg in die neue Technik. ${ }^{291}$ Hierbei werden vor allem oben genannte Einflussfaktoren abgefragt. Diese Methode ist zwar nicht wissenschaftlich begründet, jedoch im Hinblick auf die Akzeptanz in der landwirtschaftlichen Praxis als zielführend zu bezeichnen.

Zunächst empfiehlt sich der Umstieg auf konservierende Bodenbearbeitung bei Einzelfrüchten, wie z. B. bei Weizen nach Raps mit der betriebseigenen Technik, bevor der konservierend bestellte Anteil an der AF auf z. B. $70 \%$ ausgedehnt wird. Wie das Beispiel des Bundeslandes Sachsen zeigt, ist auf Großbetrieben derzeit schon zu beobachten, dass die Bodenbearbeitung und Aussaat komplett dauerhaft konservierend geschieht. In diesen Fällen reicht die betriebseigene Technik vieler Familienbetriebe, z. B. eine Kreiseleggen-Drillkombination, jedoch nicht mehr aus, um zufriedenstellende Ergebnisse bei den auflaufenden Früchten und entsprechende Arbeitsleistungen zu gewährleisten. Bei einem Umstieg auf konservierende Saattechnik sind weiterhin die hohen Anschaffungskosten zu berücksichtigen, die z. B. bei Universaldrillmaschinen mit 3 m Arbeitsbreite bei 20.000-28.000 $€^{292}$ in der Basisversion liegen. Die Anschaffungskosten für vergleichbare Direktsaatmaschinen liegen nach KTBL mit etwa $33.000 €$ noch höher. Kleineren

\footnotetext{
${ }^{291}$ Vgl. hierzu KiRCHNER (2003), S. 56 f.

${ }^{292}$ Auskunft des Unternehmens Horsch, Schwandorf.
} 
Betrieben ist daher zu raten, sich zu Maschinengemeinschaften zusammenzuschließen oder eventuell die Erledigung der Aussaat komplett auszulagern.

Es ist davon auszugehen, dass die konservierende Bodenbearbeitung tendenziell nicht zu Ertragsrückgängen führen muss. TEBRÜGGE UND BÖHRNSEN ${ }^{293}$ stellen in einer Literaturrecherche bezüglich der Auswirkung der Direktsaat auf den Ertrag von Winterweizen fest, dass die Direktsaat keine Ertragsrückgänge zur Folge hat. Allerdings müssen derartige Betrachtungen immer vor dem Hintergrund der Standortvariabilität gesehen werden, wie Kapitel 6.3.1.1.5.2 zeigt.

Bezüglich der Veränderungen der Ertragsstruktur durch die Umstellung zur konservierenden Bodenbearbeitung liegen langjährige Untersuchungen von TEBRÜGGE ${ }^{294}$ auf verschiedenen Standorten Hessens unter Praxisbedingungen vor. Sie werden in der Tabelle 10 dargestellt:

Tabelle 10: Einfluss der Direktsaat (DS) sowie Mulchsaat (MS) auf den Pflanzenertrag im Vergleich zur Pflugbearbeitung $(\mathrm{P}=100 \%)$

\begin{tabular}{|c|c|c|c|c|c|c|c|c|c|c|}
\hline \multicolumn{11}{|c|}{ Ertragsdifferenzen gegenüber $P=100$} \\
\hline \multirow{2}{*}{$\begin{array}{l}\text { Standort } \\
\text { Ver- } \\
\text { suchs- } \\
\text { dauer } \\
\text { System }\end{array}$} & \multicolumn{2}{|c|}{$\begin{array}{l}\text { O.heim } \\
\text { 19 Jahre }\end{array}$} & \multicolumn{2}{|c|}{$\begin{array}{l}\text { B.köbel } \\
19 \text { Jahre }\end{array}$} & \multicolumn{2}{|c|}{$\begin{array}{l}\text { Gießen } \\
10 \text { Jahre }\end{array}$} & \multicolumn{2}{|c|}{$\begin{array}{l}\text { W.born } \\
19 \text { Jahre }\end{array}$} & $8 \mathrm{~J}$. & $19 \mathrm{~J}$. \\
\hline & DS & MS & DS & MS & DS & MS & DS & MS & DS & MS \\
\hline WW & 1,6 & $-0,7$ & 0,3 & 1,9 & $-0,6$ & 0,0 & $-4,9$ & 3,5 & 3,1 & 7,0 \\
\hline WG & 1,7 & $-0,3$ & & & 14,2 & $-1,8$ & 1,1 & 9,6 & $-5,2$ & $-8,6$ \\
\hline WR & & & & & & & $-4,3$ & $-4,7$ & & \\
\hline Raps & & & & & & & 5,4 & $-3,5$ & 10,9 & 17,6 \\
\hline Hafer & & & & & -14 & $-4,1$ & 1,6 & 19,3 & & \\
\hline SG & & & & & & & $-5,6$ & 4,3 & & \\
\hline Erbse & & & & & & & -9 & 6,5 & & \\
\hline ZR & 13,8 & 3,65 & -15 & 2,9 & & & & & & \\
\hline Silomais & & & & & -12 & $-3,1$ & & & & \\
\hline Kö.mais & & & $-0,7$ & & & & & & & \\
\hline
\end{tabular}

Quelle: Verändert nach TEBRÜGGE (2003), S. 57

In Tabelle 10 wird die Heterogenität der Erträge konservierender Bodenbearbeitung und Direktsaat deutlich. Dennoch ist davon auszugehen, dass im Mittel der Standorte und

\footnotetext{
${ }^{293}$ Vgl. TEBRÜGGE U. BÖHRNSEN (1994), S. 103

${ }^{294}$ Vgl. TeBRÜGGE (2003), S. 57
} 
Feldfrüchte beide Bearbeitungsverfahren zu keinen signifikanten Ertragsrückgängen führen.

Ein generelles Problem der konservierenden Bodenbearbeitung ist der höhere Unkrautdruck. Durch die fehlende phytosanitäre Wirkung des Pfluges kommt es zu einem stärkeren Auflaufen von Unkräutern, dem mit zusätzlichen Herbizidmaßnahmen begegnet werden muss. Diese Mehraufwendungen können bis zu 30 \% betragen. ${ }^{295}$ Besonders der Befall mit der Tauben Trespe ist auf pfluglos bewirtschafteten Flächen signifikant. Hinzu kommt, dass es bisher keine wirkungsvollen selektiven Herbizide zur Bekämpfung des Problemunkrauts gibt. ${ }^{296}$ Deshalb wäre eine Verbesserung der Wirksamkeit von Herbiziden sowohl aus ökologischer als auch aus ökonomischer Sicht wünschenswert.

Abschließend sind in der Tabelle 11 die Ergebnisse des Pilotprojektes „Reduzierte Bodenbearbeitung“ des MLUR Brandenburg dargestellt. Es werden die Änderungen der DB im Zeitraum 1998-2003 nach der Umstellung auf konservierende Bodenbearbeitung, in diesem Fall Mulchsaat, dargestellt.

Tabelle 11: Prozentuale Veränderung des Deckungsbeitrages verschiedener Fruchtartenfolgen bei der Umstellung auf Verfahren der Mulchsaat im Pilotbetrieb

\begin{tabular}{c|ccccccc}
\hline \multicolumn{7}{c}{ Nachfrucht } \\
\hline $\begin{array}{l}\text { Vorfrucht } \\
\text { WW }\end{array}$ & WW & WG & Roggen & Erbsen & WR & ZR & Kart. \\
WG & & +9 & & +10 & & $+11-15$ & $+2-3$ \\
Roggen & & & $+10-11$ & +10 & $+7-10$ & $+11-15$ & $+2-3$ \\
Erbsen & & +7 & & & & \\
WR & +8 & +7 & $+8-9$ & & & \\
ZR & +8 & & & +16 & & \\
Kart. & +8 & +8 & $+6-8$ & +16 & & \\
\hline
\end{tabular}

Quelle: MLUR Brandenburg (2003), S. 51

Auffallend ist, dass alle Deckungsbeiträge sich positiv verändert haben. Lediglich der DB der Kartoffel reagiert nicht so stark auf die Umstellung. Die Gründe liegen in diesem Fall bei den hohen Gesamtkosten des Verfahrens und den geringen Kostenanteilen der Pflugarbeit.

\footnotetext{
${ }^{295}$ Vgl. Kapitel 6.3.1.1.3

${ }^{296}$ Vgl. hierzu KLINGEBIEL (2003)
} 


\subsection{Betriebswirtschaftliche Bewertung der konservierenden Bodenbearbei- tung}

Im Folgenden wird zuerst eine KWA, dann eine KNA für konservierende Bodenbearbeitungsverfahren durchgeführt. Die Übersicht 7 dient sowohl zur Darstellung der Vorgehensweise für die Durchführung der KNA als auch zur generellen Übersicht über dieses Kapitel.

\section{Übersicht 7: Vorgehensweise bei der KNA für konservierende Boden-}

\section{bearbeitungsverfahren}

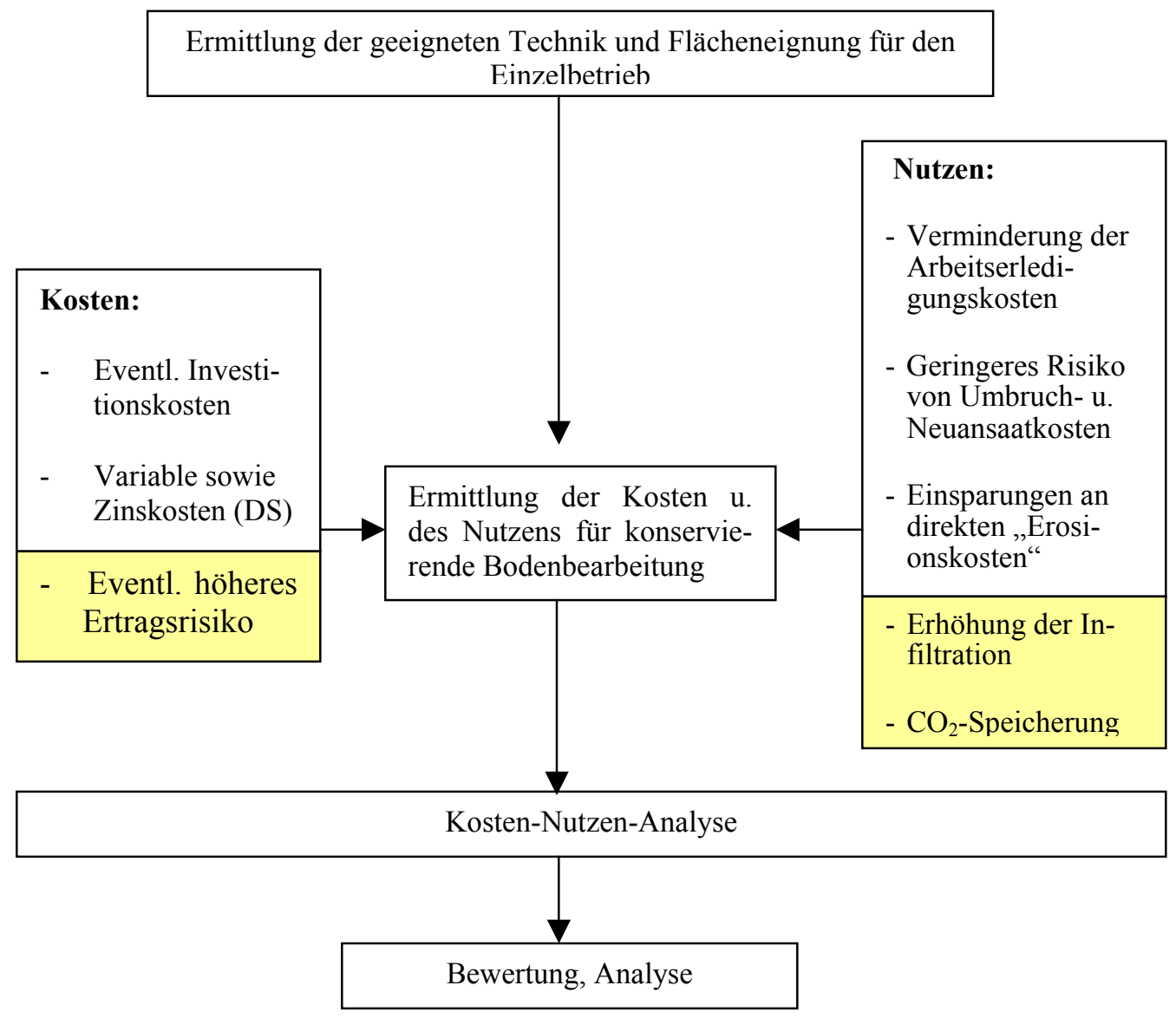

Quelle: Eigene Darstellung, Schraffierung: Positionen konnten aufgrund fehlender Daten in der Berechnung nicht berücksichtigt werden

Zunächst werden die Kosten zur Durchführung einer Mulch- und Direktsaat für den Einzelbetrieb ermittelt. Diese sind der Tabelle $12 \mathrm{zu}$ entnehmen. 
Tabelle 12: Kosten der Bodenbearbeitung im Falle der Pflugbearbeitung sowie Mulch- u. Direktsaat auf Ebene der Verfahrenskosten ${ }^{297}$

\begin{tabular}{lcccc}
\hline & $\begin{array}{c}\text { Var. Kosten } \\
(\boldsymbol{\epsilon} / \mathbf{h a})\end{array}$ & $\begin{array}{c}\text { Fixe Kosten } \\
(\boldsymbol{\epsilon} / \mathbf{h a})\end{array}$ & $\begin{array}{c}\text { Arbeitskosten } \\
(\boldsymbol{\epsilon} / \mathbf{h a})\end{array}$ & $\begin{array}{c}\text { Summe } \\
(\boldsymbol{\epsilon} / \mathbf{h a})\end{array}$ \\
\hline Pflugbearbeitung & & & & \\
Grubber (2,5 m) & 12,32 & 10,51 & 9,75 & \\
Pflug (4-Schar, 1,4 m) & 33,65 & 21,44 & 24,6 & \\
Saatbettkombination 2,5 m & 9,3 & 6,13 & 9,9 & \\
Drillmaschine (2,5 m) & 7,41 & 9,64 & 13,05 & \\
Gesamtkosten (€/ha) & $\mathbf{6 2 , 7}$ & $\mathbf{4 7 , 7}$ & $\mathbf{5 7 , 3}$ & $\mathbf{1 6 7 , 7}$ \\
\hline Mulchsaat (ohne ZF) & & & & \\
Grubber (2,5 m) & 12,32 & 10,51 & 9,75 & \\
Scheibenegge & 13,21 & 13,28 & 7,2 & \\
Anbauspritze (15 m) & 17,03 & 3,85 & 4,05 & \\
Drillmaschine (2,5 m) & 7,41 & 9,64 & 13,05 & \\
Gesamtkosten (€/ha) & $\mathbf{5 0 , 0}$ & $\mathbf{3 7 , 3}$ & $\mathbf{3 4 , 0}$ & $\mathbf{1 2 1 , 3}$ \\
\hline Direktsaat & & & & \\
Anbauspritze (15 m) & 22,7 & 3,85 & 4,05 & \\
Direktsaatmaschine (4 m) & 21,1 & 24,66 & 6,75 & \\
\hline Gesamtkosten (€/ha) & $\mathbf{4 3 , 8}$ & $\mathbf{2 8 , 5}$ & $\mathbf{1 0 , 8}$ & $\mathbf{8 3 , 1}$ \\
\hline
\end{tabular}

Quelle: Eigene Berechnungen nach KTBL (2002)

Bei den in Tabelle 12 dargestellten Kosten der konventionellen Bodenbearbeitung mit Pflug und den beiden konservierenden Verfahren der Mulch- und Direktsaat ist ein Kostenvorteil der konservierenden Bodenbearbeitungsverfahren zu erkennen. Dieser ist mit der Einsparung der Verfahrenskosten des Pflügens, die je nach Maschinenausstattung Arbeits- und Maschinenkosten in Höhe von 66-120 €/ha ausmachen können, zu erklären. Den Einsparungen von 46,4 €/ha bzw. 84,6 €/ha ${ }^{298}$ können bei der konservierenden Bodenbearbeitung Kosten für die Behandlung durch ein Totalherbizid in Höhe von ca. $31 € /$ ha gegenüberstehen. Diese Kosten fallen jedoch nicht zwingend an und können durch einen Arbeitsgang mit einem geeigneten Bodenbearbeitungsgerät ersetzt werden. Im Gegensatz zu Nachauflaufherbiziden sind Totalherbizide in der Regel schneller abbaubar und die Umweltbelastungen folglich geringer. ${ }^{299}$ In der folgenden KNA wird bei der Mulchsaat von zus. PSM-Kosten in Höhe von 24,93 €/ha und bei der Direktsaat von zusätzlichen Kosten in Höhe von 29,45 €/ha ausgegangen. Bei dem Wechsel von konventioneller zur konservierender Bodenbearbeitung ist bei einigen Betrieben von einem hö-

\footnotetext{
${ }^{297}$ Lohnansatz: $15 € / \mathrm{h}$; Schlaggröße: 5 ha

${ }^{298}$ Diese Einsparungen stellen einen Nutzeneffekt dar, der in der KNA Berücksichtigung findet.

${ }^{299}$ Vgl. TEBRÜGGE U. BÖHRNSEN (1994), S. 109
} 
heren Risiko auszugehen. Dieses wird jedoch weder bei der KWA noch bei der KNA berücksichtigt.

Zur Durchführung der konservierenden Bodenbearbeitung gibt es verschiedene Möglichkeiten. Eine Mulchsaatmaschine mit geeigneten Säscharen ist gerade in der Phase der Umstellung auf dieses Verfahren nicht zwingend notwendig, da mit der vorhandenen Maschinenausstattung gearbeitet werden kann. In diesem Fall kommen keine zusätzlichen Investitionskosten auf den landwirtschaftlichen Betrieb zu. Da es jedoch das Ziel der gfP ist, in einigen Gebieten dauerhaft bzw. regelmäßig zu bestimmten Früchten konservierende Bodenbearbeitungsverfahren zu implementieren, ist die Anschaffung entsprechender Mulch- oder Direktsaatmaschinen in das betriebswirtschaftliche Kalkül einzubeziehen. In der KNA dieses Kapitels werden die Daten des KTBL herangezogen, d. h. es ist bei der Direktsaat eine entsprechende Technik vorausgesetzt worden.

Der primäre Nutzen der konservierenden Bodenbearbeitung entsteht aufgrund der Minderung des Bodenabtrags durch Wind- und Wassererosion durch die Erhöhung des Bodenbedeckungsgrades, was in der folgenden Tabelle dargestellt ist.

Tabelle 13: Einfluss der Bodenbedeckung auf den Oberflächenabfluss sowie der Bodenerosion durch Wasser und Wind (Relativwerte auf Basis 10-jähriger Messungen)

\begin{tabular}{ccccc}
\hline $\begin{array}{c}\text { Bodenbede- } \\
\text { ckung }\end{array}$ & $\begin{array}{c}\text { Pflanzenrück- } \\
\text { stände i. d. TM }\end{array}$ & $\begin{array}{c}\text { Oberflächen- } \\
\text { abfluss }\end{array}$ & $\begin{array}{c}\text { Bodenabtrag } \\
\text { Wassererosion }\end{array}$ & $\begin{array}{c}\text { Bodenabtrag } \\
\text { Winderosion }\end{array}$ \\
$\mathbf{\%}$ & t/ha & $\mathbf{\%}$ & $\mathbf{\%}$ & $\mathbf{\%}$ \\
\hline 0 & 0 & 45 & 100 & 100 \\
$25-30$ & 0,5 & 40 & 25 & 15 \\
$30-50$ & 2 & $<30$ & 8 & 3 \\
$50-70$ & 4 & $<30$ & 3 & $<1$ \\
$>70$ & 6 & $<30$ & $<1$ & $<1$ \\
\hline
\end{tabular}

Quelle: FriELINGHAUS et al. (2002), S. 33

Dabei ist es aufgrund einer Vielzahl von bodenphysikalischen Untersuchungen und den zur Verfügung stehenden Erosionsmodellen keine Schwierigkeit, den verminderten Bodenabtrag abzuschätzen, direkte Messungen sind jedoch sehr zeitaufwendig und kostenintensiv. Die Höhe des verminderten Bodenabtrags hängt v. a. von den tatsächlichen Bode- 
nabträgen auf dem Standort, der Eintrittswahrscheinlichkeit erosiver Niederschläge sowie der ganzjährig realisierten Bodenbedeckung des Verfahrens ab. ${ }^{300}$

Wissenschaftlich abgesicherte Untersuchungen haben in der Vergangenheit gezeigt, dass der Bodenverlust durch Mulchsaatverfahren um etwa $95 \%$ gemindert und durch Direktsaatverfahren gänzlich vermieden werden kann. ${ }^{301}$ Eine andere Untersuchung aus dem Kraichgau zeigt, dass im Mulchsaatverfahren bei Mais der Bodenabtrag um $95 \%$ und bei Direktsaat um $98 \%$ gemindert werden kann. ${ }^{302}$

\section{Kosten-Wirksamkeits-Analyse}

Derartige Ergebnisse müssen jedoch vor dem Hintergrund der Standortvariabilität gesehen werden. Bei einer durchschnittlichen geschätzten Bodenabtragsrate in Deutschland von $8,7 \mathrm{t} / \mathrm{ha} / \mathrm{a}$ (West) bzw. 4,6 t/ha/a (Ost) ${ }^{303}$ durch Bodenerosion würde eine Umstellung zur konservierenden Bodenbearbeitung eine Senkung der Bodenabtragsrate in Höhe von 8,2 bzw. 4,4 t/ha/a bedeuten. Bei der Saldierung der Kosteneinsparungen und Mehrkosten (PSM) entstehen $-21,47 € /$ ha für die Mulchsaat und $-55,15 € /$ ha für die Direktsaat. Es ergeben sich folgende $\mathrm{KW}:^{304}$

- Mulchsaat: - 4,9 bzw. $-2,7 € / t$ verhinderter Bodenabtrag

- Direktsaat: - 12,5 bzw. - 6,7 €/t verhinderter Bodenabtrag

Die Ergebnisse sind so zu interpretieren, dass es im Fall der Mulchsaat je nach Standort $4,9 €$ bzw. 2,7 $€$ freiwerden, um eine Tonne Bodenabtrag zu verhindern. An der KW ist abzusehen, dass die Direktsaat eine höhere Effizienz bei der Verminderung der Bodenerosion aufweist. Die Ergebnisse müssen unter verschiedenen Einschränkungen gesehen werden, deshalb reicht eine derart isolierte Betrachtungsweise nicht aus. Eine entsprechende Einschränkung ist bspw. das unterschiedliche Erosionspotenzial verschiedener Flächen und die vereinfachte Annahme des Erosionsminderungspotenzials der Maßnahme, die nur sehr schwer auf einem Einzelschlag ermittelt werden kann. Die Ergebnisse machen jedoch deutlich, dass die Kosten der Maßnahmen bezogen auf deren Wirksam-

\footnotetext{
${ }^{300}$ Vgl. hierzu Kapitel 3.1.1

${ }^{301}$ Vgl. hierzu SCHMidT et al. (1999), S. 20, BRunOtTe, Roth U. Hollmann (1994), S. 304

${ }^{302}$ Vgl. Landesanstalt FÜr PflanZenbau (2002), S. 3

${ }^{303}$ Vgl. Kapitel 3.1.1.1 ff.

${ }^{304}$ Beispielberechnung bei der Mulchsaat: $-21,47 € / \mathrm{ha}: 4,4 \mathrm{t} / \mathrm{ha}=-4,9 € / \mathrm{t}$
} 
keit auf Standorten mit hohen Erosionsraten günstiger sind als auf Standorten mit geringen Erosionsraten und geben somit einen Anhaltspunkt der Wirksamkeit der Mulch- und Direktsaat.

\section{Kosten-Nutzen-Analyse}

Im Folgenden wird eine KNA für beide Verfahren der konservierenden Bodenbearbeitung durchgeführt, wobei die Ergebnisse der Tabelle 12 bzgl. der Kostenermittlung vorausgesetzt und an dieser Stelle um die Monetarisierung des Nutzens erweitert werden.

Untersuchungen zeigen, dass beim Einsatz von konservierenden Bodenbearbeitungsverfahren geringere Arbeitserledigungskosten ${ }^{305}$ entstehen. Weiterhin reduziert die konservierende Bodenbearbeitung das Risiko von Umbruch- und Neuansaatkosten ${ }^{306}$ bei Starkregenereignissen. Bei extremen Starkregenereignissen kann es dazu kommen, dass der Pflanzenbestand geschädigt bzw. der Feldaufgang reduziert wird. Ob ein Umbruch oder eine Neuansaat durchgeführt werden muss, kann nur vor Ort im Einzelfall entschieden werden. Dabei müssen vom Betriebsleiter die dafür notwendigen Kosten abgeschätzt und mit den zusätzlich zu erwartenden Erlösen gegenübergestellt werden. Hierbei ist jedoch zu berücksichtigen, dass durch die verspätete Aussaat Mindererträge unvermeidbar sind, die im Folgenden allerdings nicht weiter berücksichtigt werden. ${ }^{307}$ Es ist davon auszugehen, dass durch die Anwendung der konservierenden Bodenbearbeitung keine Umbruch- und Neuansaatkosten mehr anfallen. ${ }^{308}$

Diese aus der Anwendung von Mulch- und Direktsaatverfahren resultierenden Kosteneinsparungen müssen allerdings vor dem Hintergrund der Eintrittswahrscheinlichkeit von Starkregenereignissen gesehen werden. Für die folgende KNA wird angenommen, dass ohne konservierender Bodenbearbeitung eine Neuansaat, bzw. ein Umbruch alle zehn Jahre erforderlich wäre. Aufgrund des in der Tabelle A-1 ermittelten Wertes von $134,7 € /$ ha wird von einem gerundeten Wert von $135 € /$ ha ausgegangen. In den folgenden

\footnotetext{
${ }^{305}$ Vgl. hierzu Übersicht A-2 und Tabelle 12

${ }^{306}$ Die Kosten für eine Neuansaat der Fruchtfolge WR-WW-WG sind in Tabelle A-1 dargestellt.

${ }^{307}$ Auf die hohe Streuung der Ergebnisse in Bezug auf Ertragsminderungen durch eine spätere Aussaat geht BRUNS ein. Er gibt Spannen von $3 \%$ und $5 \%$ bis zu $15 \%$ und $18 \%$ an.

Vgl. BRUNS (1980), S. 20

${ }^{308}$ Vgl. BrunotTe, Roth U. Hollmann (1994), S. 315 f.
} 
Berechnungen geht dieser Nutzen als ein alle zehn Jahre eintretender Nutzen in Höhe von jeweils $135 € /$ ha ein.

Durch die Minderung der Bodenerosion wird der Verlust von Oberboden vermindert. Daher wird im Folgenden der monetäre Wert einer Tonne nicht erodierten Oberbodens ermittelt. Hierbei sind generell folgende Positionen zu beachten:

- Monetärer Wert der gelösten Hauptnährstoffe,

- Monetärer Wert der verwendeten PSM,

- Saatgutkosten und

- Variable Maschinenkosten zur PSM- und Düngerausbringung sowie Aussaat.

Im Folgenden werden die Saatgutkosten nicht weiter berücksichtigt da keine Daten über die Beziehung zwischen Starkregenereignissen und Saatgutabfluss vorliegen. Außerdem hängt der Abfluss und die dadurch entstehende Unwirksamkeit von verschiedenen Faktoren ab, die an dieser Stelle nicht berücksichtigt werden können. Eine doppelte Berücksichtigung von Nährstoff-, Pflanzenschutzmittelkosten und deren Ausbringungskosten lässt bei der Berechnung des Nutzens durch die Reduzierung des Risikos von Starkregenereignissen und bei der Ermittlung des Werts einer Tonne nicht erodierten Oberbodens aus Gründen der Vereinfachung nicht vermeiden. Diese würde bei einer Wiederkehrwahrscheinlichkeit eines Starkregenereignisses von 10 Jahren dementsprechend alle 10 Jahre auftreten. Aufgrund der Tatsache, dass die in beiden Berechnungen verwendeten naturwissenschaftlichen Daten bezüglich des Abschwemmungsgrades und der Nährstoffund PSM-Bindung im Boden nur als Richtwerte zu sehen sind, ergeben sich unweigerlich Überschneidungen und Unschärfen bei der Wirkung des Nutzens.

Zunächst wird der monetäre Wert der im Boden gelösten Düngemittel ermittelt. Zur Berechnung werden die Daten von HeißENHUBER und SCHMIDTLEIN ${ }^{309}$ sowie BeHRENDT et al. ${ }^{310}$ verwendet und mit aktuellen Preisen ergänzt. Ausgehend von der Annahme, dass sich die gelösten Düngemittel im Saatbett befinden und eine durchschnittliche Dichte von $130 \mathrm{t} / \mathrm{cm} / \mathrm{ha}$ vorherrscht, beträgt die Masse des betrachteten Oberbodens $650 \mathrm{t} / \mathrm{ha}$. Die Vorgehensweise ist der folgender Tabelle zu entnehmen.

\footnotetext{
${ }^{309}$ Vgl. HeißENhuber u. SCHMidTlein (1988), S. 113

${ }^{310}$ Vgl. BEHRENDT et al. (1999)
} 
Tabelle 14: Berechnung des Substitutionswertes der Hauptnährstoffe in einer Tonne erodiertem Oberboden

\begin{tabular}{cccccc}
\hline $\begin{array}{c}\text { Haupt- } \\
\text { nährstoffe }\end{array}$ & $\begin{array}{c}\text { GNG je t } \\
\text { Oberboden }\end{array}$ & $\begin{array}{c}\text { Anreiche- } \\
\text { rungsfak- }_{\text {tor }^{311}}\end{array}$ & $\begin{array}{c}\text { GNG im } \\
\text { erod. Boden } \\
\text { u. Abfluss- } \\
\text { wasser } \\
(\mathrm{kg})\end{array}$ & \multicolumn{2}{c}{$\begin{array}{c}\text { Nährstoffwert } \\
\text { ingesamt }\end{array}$} \\
\hline $\mathrm{N}$ & $0, \mathrm{~kg}$ Nährstoff & $€ / \mathrm{t}$ Boden \\
\hline $\mathrm{P}_{2} \mathrm{O}_{5}$ & 0,5 & 1,0 & 0,2 & 0,66 & 0,1 \\
$\mathrm{~K}_{2} \mathrm{O}$ & 2,4 & 1,5 & 1,4 & 0,52 & 0,8 \\
\hline Gesamt & 1,5 & 6,7 & 0,24 & 1,6 \\
\hline
\end{tabular}

Quelle: HeißENHUBER u. SCHMIDTLEIN (1988), S. 113, FID (2002) sowie eigene Berechnungen

Die Tabelle 14 zeigt, dass der Substitutionswert der Hauptnährstoffe $\mathrm{N}, \mathrm{P}_{2} \mathrm{O}_{5}$ und $\mathrm{K}_{2} \mathrm{O}$ einer Tonne erodiertem Oberbodens mit 2,5€ anzusetzen ist. Unter Berücksichtigung der Arbeitserledigungskosten ergibt sich ein Wert von 2,6€/t Boden.

Die Kosten für PSM und deren Ausbringungskosten werden der Tabelle A-2 entnommen, die entsprechende Werte enthält. Als Beispielfruchtfolge dient WR-WW-WG. Wie bereits in Kapitel 3.1.3.2 dargestellt wurde, sind ca. 20 \% der applizierten PSM-Menge potenziell abtragsgefährdet. Demnach können in Verbindung mit der Tabelle A-2 bei einem etwas höheren Abschwemmungsgrad von $25 \%$ maximal Kosten von 42,5€/ha entstehen. Dies entspricht einem Wert von 0,07 €/t Boden. Demzufolge weist eine Tonne Oberboden in diesem Fall einen Wert von 2,7 €/dt für Düngung und PSM auf. Hierbei ist zu berücksichtigen, dass dieser Wert nur ein Anhaltspunkt und eine standortangepasste Berechnung unerlässlich ist.

Ein weiterer Nutzenaspekt, gerade bei einem dauerhaften Einsatz von konservierenden Bodenbearbeitungsverfahren ist die langfristige Werterhaltung des Bodens für den landwirtschaftlichen Betrieb. Die bisher dazu angefertigten Studien und deren kritische Betrachtung wurden bereits in Kapitel 6.1.2 dargelegt. Der Wertverlust des Bodens durch Bodenerosion wird nach der Methode von HEIßENHUBER und SCHMIDTLEIN ${ }^{313}$ ermittelt.

\footnotetext{
${ }^{311}$ Der Anreicherungsfaktor ist der Nährstoffgehalt im Sediment/Nährstoffgehalt im Oberboden.

${ }^{312}$ Anmerkung: Es handelt sich hierbei um die Nährstoff- sowie Ausbringungskosten.

${ }^{313}$ Vgl. HEIßENHUBER u. SCHMIDTLEIN (1988)
} 
Für einen ldw. Betrieb mit der Fruchtfolge WR-WW-WG errechnet sich gegenwärtig ein Fruchtfolgedeckungsbeitrag von $564 € /$ ha. Abzüglich des Lohnansatzes (147,5€/ha), des $10 \%$ Gewinn- und Risikozuschlages (56,0 €/ha) sowie der sonstigen Kosten (25€/ha) ergibt sich eine Grundrente von 335,5 €/ha. Bei einem durchschnittlichen Zinssatz von $3 \%{ }^{314}$ führt die Kapitalisierung zu einem Grenzkaufpreis von $11.071,5 € /$ ha.

Ausgehend von einer Oberbodendicke von $40 \mathrm{~cm}$ Schluffauflage ergibt dies bei einer Dichte von 130 t/cm/ha Oberboden eine Masse von 5200 t/ha. Bei einem Abtrag einer Tonne Oberboden werden in diesem Beispiel somit Kosten durch den Wertverlust von $\mathbf{2 , 1} € / \mathbf{t}$ verursacht.

Zusammenfassend ist daher festzustellen, dass durch die Verhinderung des Verlustes einer Tonne Oberbodens ein Nutzen von rd. 4,8 €/t entstehen kann. Es ist allerdings in diesem Zusammenhang darauf hinzuweisen, dass der Nutzen unter Berücksichtigung der gesamten Offsite-Schäden durch Bodenerosion noch höher ist. Es sei allein an die in Kapitel 6.1.2 durch HEIßENHUBER ermittelten externe Kosten der Sedimentbeseitigung in Bayern von $7 € /$ ha erinnert. Diese werden im Folgenden allerdings nicht berücksichtigt, da es sich hier um eine einzelbetriebliche Betrachtungsweise handelt.

Eine weitere nicht monetarisierbare Kostenkomponente ist die Kohlenstoffanreicherung im Boden. Die konservierende Bodenbearbeitung führt zu einer positiven Beeinflussung des $\mathrm{CO}_{2}$ - und somit des Klimahaushaltes. TEBRÜGGE ${ }^{315}$ geht bspw. davon aus, dass durch konservierende Bodenbearbeitung unter mitteleuropäischen Verhältnissen etwa $1 \mathrm{t} \mathrm{Hu}$ mus pro Hektar und Jahr neu gebildet wird. Hierdurch werden 0,58 t Kohlenstoff im Boden gebunden, woraus ansonsten etwa 2,1 t $\mathrm{CO}_{2}$ gebildet würden. Bei einer flächendeckenden Anwendung der konservierenden Bodenbearbeitung, auf etwa $70 \%$ der AF Deutschlands, könnten somit jährlich der Ausstoß von 18 Mio. t $\mathrm{CO}_{2}$ verhindert werden. Dieses Einsparpotenzial könnte bei der Einführung eines Kohlenstoff-Zertifikathandels für die landwirtschaftlichen Betriebe einen erheblichen Zusatznutzen bedeuten, wie bspw.

\footnotetext{
${ }^{314}$ An dieser Stelle wird abweichend von der KNA mit einem Zinssatz von $3 \%$ gerechnet, um der Besonderheit des Bodenmarktes Rechnung zu tragen.

${ }^{315}$ Vgl. TEBRÜGGE (2003), S.54
} 
in Kanada, dort zahlen die Energieversorger den Landwirten in einigen Gebieten 20 \$, wenn diese Direktsaat betreiben. ${ }^{316}$

Des weiteren wirkt sich die konservierende Bodenbearbeitung sehr stark auf die Infiltrationsrate des Bodens aus, wie bereits in Kapitel 3.1.1.1 deutlich wurde. Ähnliche Ergebnisse liegen aus den Lößgebieten des Kraichgaus vor. Im Mittel von fünf Versuchen bei Silomais wurde der Bodenabtrag und die Infiltrationsrate gemessen. Bei der Pflugvariante betrug der mittlere Bodenabtrag 22 t/ha. Auf der Mulchsaat-Parzelle ergab sich bei ähnlicher Regenmenge ein Bodenabtrag von $1 \mathrm{t} / \mathrm{ha}(-97 \%)$ und bei der Direktsaat-Parzelle ein Bodenabtrag von 0,4 t/ha. (-99\%). ${ }^{317}$ Die konservierende Bodenbearbeitung ist somit ein aktiver Beitrag der Landwirtschaft zum vorsorgenden Hochwasserschutz ${ }^{318}$, was einen erheblichen externen Nutzeneffekt darstellt, welcher der Gesellschaft noch wesentlich deutlicher kommuniziert werden muss.

\section{Zusammenfassende Bewertung und Berechnung der KNV}

Bei der KNA werden für die Mulchsaat Kosten in Höhe von 121,3€/ha und für die Direktsaat Kosten in Höhe von 83,1 €/ha angenommen. ${ }^{319}$ Da diese Kosten niedriger als beim Pflugeinsatz liegen, entsteht ein Nutzen in Höhe der Differenz beider Kostenpositionen. Dieser liegt bei der Mulchsaat bei 46,4 €/ha und bei der Direktsaat bei 84,6 €/ha. Als Kosten verbleiben nur die eventuell auftretenden höheren PSM-Kosten, die bei der Mulchsaat 24,93 €/ha und bei der Direktsaat 29,45€/ha betragen sollen. ${ }^{320}$

Der Nutzen besteht weiterhin aus der Verhinderung der Kosten des Bodenabtrags sowie des Wertverlustes des Bodens zusammen. Die Effektivität der Mulchsaat in Bezug auf die Verminderung des Bodenabtrages betrage wie bei der KWA $95 \%$ und die der Direktsaat $98 \%$. Der Nutzen ist abhängig vom Standort, wobei in dieser Berechnung zwei Standorte mit einem durchschnittlichen Bodenabtrag von 8,7 bzw. 4,6 t/ha/ $/ \mathrm{a}^{321}$ angenommen werden. Im ersten Fall ergibt sich ein Nutzen von $67,52 € / \mathrm{ha}^{322}$ bei der Mulchsaat und $106,2 € /$ ha bei der Direktsaat. Im zweiten Fall weist der Nutzen eine Höhe von $86,07 € /$ ha

\footnotetext{
${ }^{316}$ Vgl. FARMERS WEEKLY (2001)

${ }^{317}$ Vgl. LandeSanstalt FÜr Pflanzenbau (2002), S. 5

${ }^{318}$ Vgl. hierzu Kapitel 5

${ }^{319}$ Vgl. Tabelle 12

${ }^{320}$ Annahme: Glyphosateinsatz mit Anbauspritze (15 m) und Traktor (67 kW)

${ }^{321}$ Diese Werte entsprechen den von WERNER et al. ermittelten.

${ }^{322}$ Rechenweg: 4,6 t/ha*0,95*4,8 €/h/t+46,4 €/ha $=67,52 € / \mathrm{ha}$
} 
bei der Mulchsaat sowie 125,4 €/ha bei der Direktsaat auf. Der Betrachtungszeitraum beträgt 25 Jahre. Außerdem wird angenommen, dass alle zehn Jahre ein Umbruch und die darauffolgende Neuansaat nicht stattfinden muss und dadurch ein entsprechender alle zehn Jahre eintretender Nutzen von $135 €$ /ha entsteht. Dies bedeutet, dass der Nutzen alle zehn Jahre um $135 € /$ ha höher ist. Insgesamt handelt es sich daher um eine optimistische Sichtweise, die am Schluss einige Einschränkungen erfahren muss. Die KNV beider Verfahren unter obig angenommen Verhältnissen sind in der Tabelle 15 aufgeführt. Die Berechnungen können aus dem Anhang, Tabelle A-21 bis Tabelle A-24 entnommen werden.

Tabelle 15: Einzelbetriebliches KNV der Mulch- und Direktsaat im Verhältnis zur Pflugbearbeitung bei einem Zinssatz von $4 \%$ und mittleren Bodenabtragsraten ohne Förderung sowie Berücksichtigung externer Effekte

\begin{tabular}{lcccc}
\hline & $\begin{array}{c}\text { Mulchsaat } \\
\text { Bodenabtrag (t/ha) }\end{array}$ & $\begin{array}{c}\text { Mulchsaat } \\
(4,6)\end{array}$ & $\begin{array}{c}\text { Direktsaat } \\
(8,7)\end{array}$ & $\begin{array}{c}\text { Direktsaat } \\
(4,6)\end{array}$ \\
\hline KNV in \% & $\mathbf{0 , 3 2}$ & $\mathbf{0 , 2 6}$ & $\mathbf{0 , 2 5}$ & $\mathbf{0 , 2 2}$ \\
$\varnothing$ jährl. Vorteil $(€ /$ ha/a) & 52 & 71 & 86 & 106 \\
Berücksichtigung von externen Ef- & & & & \\
fekten (7 $€$ /ha/a): & & & & \\
KNV in \% & $\mathbf{0 , 3 0}$ & $\mathbf{0 , 2 4}$ & $\mathbf{0 , 2 4}$ & $\mathbf{0 , 2 1}$ \\
$\varnothing$ jährl. Vorteil $(€ /$ ha/a) & 59 & 78 & 93 & 113 \\
\hline
\end{tabular}

Quelle: Eigene Berechnungen, Zinssatz $4 \%$

Die Tabelle 15 zeigt die KNV der Mulch- und Direktsaat auf zwei verschieden Standorten. Hierbei ist neben den guten KNV zu sehen, dass beide Verfahren, sowohl die Mulchals auch die Direktsaat auf beiden Standorten einen Überschuss des Nutzens über die Kosten aufweisen. Dabei ist die Direktsaat immer rentabler als die Mulchsaat.

Abschließend ist festzustellen, dass eine Berücksichtigung der Verminderung der externen Kosten zu einer weiteren Verbesserung der KNV der Mulch- und Direktsaat führt. Wenn bspw. der von DOLESCHEL und HeIßENHUBER ${ }^{323}$ ermittelte Nutzen der Verminde- 
rung von Sedimentbereinigungskosten in Höhe von $7 € /$ ha in die Berechnungen einbezogen wird, verbessert sich die KNV und auch die jährlichen Vorteile. ${ }^{324}$

\subsection{Schlussfolgerungen}

Es bleibt festzuhalten, dass es sich bei der Mulch- und Direktsaat um sehr gute Bodenschutzmaßnahmen handelt. Da in der Berechnung optimistische monetäre Nutzenannahmen gemacht worden sind, ist darauf zu verweisen, dass hohe jährliche monetäre Vorteile vorhanden sind. Die verwendeten Nutzeneffekte sind zwar ausreichend von wissenschaftlichen Studien bestätigt, allerdings herrschen große Spannen vor. Aus diesen Spannen müssen für die einzelbetriebliche Betrachtung die entsprechenden Werte verwendet und u. U. angepasst werden.

Wichtig sind in diesem Zusammenhang auch die nichtmonetären Betrachtungen. Bspw. erfordert die konservierende Bodenbearbeitung neben den bereits beschriebenen natürlichen Bedingungen eine höhere Betriebsleiterqualifikation und somit ein höheres Wissen bezüglich pflanzenbaulicher Zusammenhänge. Wenn dieses Wissen nicht im Einzelbetrieb vorhanden ist, sollte es durch die Beratung zugekauft werden. Ebenfalls ist davon auszugehen, dass gewisse Lerneffekte erst dazu führen werden, dass eine konservierende Bodenbearbeitung reibungslos funktioniert. Hierdurch können ebenfalls hohe Kosten entstehen, die nicht vorhersehbar sind. Sogar psychologische Effekte spielen bei der Durchführung der konservierenden Bodenbearbeitung eine Rolle. In Regionen mit geringer Verbreitung muss der als „Pionier“ zu bezeichnende Landwirt, der dieses Verfahren zuerst einführt, mit zahlreichen Vorurteilen kämpfen. Der im Gegensatz zur Pflugbearbeitung mit Pflanzenresten bedeckte Boden bspw. wird häufig als mangelnde Fachkenntnis gedeutet. Im Folgenden werden die gewonnenen Kenntnisse am Beispiel des Göttinger INTEX-Projektes weiterhin vertieft.

\subsection{Vertiefung der Betrachtungsweise anhand des Göttinger INTEX-Projektes}

Im Folgenden werden die Ergebnisse des Göttinger INTEX-Projektes als Exkurs angeführt, um die Problematik der konservierenden Bodenbearbeitung zu vertiefen und die

\footnotetext{
${ }^{324}$ Die diesbezüglichen Berechnungen werden aufgrund der geringen dafür notwendigen Transferleistung nicht im Anhang aufgeführt.
} 
betriebswirtschaftliche Bewertung im Kapitel 6.3.1.1.3 zu ergänzen. Dabei erfolgt dahingehend eine Erweiterung, dass eine Verknüpfung von konservierender Bodenbearbeitung und integrierter Wirtschaftsweise stattfindet.

\subsection{Darstellung des INTEX-Projektes}

Das Göttinger INTEX-Projekt wurde von 1990-2002 auf den beiden Versuchsbetrieben Reinshof und Marienstein, beide in der Nähe von Göttingen im südlichen Niedersachsen, durchgeführt. Zunächst werden diese beiden Standorte im Folgenden näher beschrieben.

\section{Standortbedingungen}

Die Versuchsflächen des Betriebes Marienstein (ca. 160 - 210 m ü. NN) sind durch variierende Bodenverhältnisse gekennzeichnet. Folgende Bodentypen sind dort zu finden: Ranker, Braun- Parabraunerden, Pseudogleye sowie Kolluvien bzw. PseudogleyKolluvien. Außerdem treten schwach bis stark geneigte Hänge mit einer durchschnittlichen Neigung von $10 \%$ auf. Die Ackerzahlen betragen 41-78 BP. Der Standort Marienstein hat ähnliche klimatische Bedingungen zu verzeichnen wie der Reinshof, der nachfolgend vorgestellt wird.

Die Versuchsflächen des Reinshof liegen südlich von Göttingen im Leinetal (ca. 150 m über NN). Die Versuchsflächen bestehen aus Auenböden, Gley-Auenböden, tiefe Pararendzinen, Parabraunerden und vereinzelt auch Gley-Schwarzerden. ${ }^{325}$ Die durchschnittliche Lufttemperatur beträgt $8,6{ }^{\circ} \mathrm{C}$, die jährliche Niederschlagsmenge $635 \mathrm{~mm}$. Die Ackerzahlen liegen bei 77 bis 93 BP.

\section{Anbausysteme}

Eine Charakterisierung der unterschiedlichen Anbausysteme während des Projektzeitraums liefert die Übersicht 8:

\footnotetext{
${ }^{325}$ Zur genaueren Beschreibung der Versuchsflächen und Standorte siehe TEIWES (1997).
} 
Übersicht 8: Die Anbausysteme des Untersuchungszeitraums 1995-2002 im Überblick am Beispiel der Standorte Reinshof und Marienstein

\begin{tabular}{|c|c|c|c|}
\hline & & \multicolumn{2}{|l|}{ Anbausysteme } \\
\hline & Ordnungsgemäß & Integriert-flexibel & Integriert-pfluglos \\
\hline $\begin{array}{l}\text { Bodenbearbei- } \\
\text { tung }\end{array}$ & Pflug & Pflug (flexibel) & $\begin{array}{l}\text { nicht-wendend (Grub- } \\
\text { ber) }\end{array}$ \\
\hline Fruchtfolge & Raps-Weizen-Gerste & $\begin{array}{l}\text { Raps-Zwischenfrucht* - } \\
\text { Hafer**-Weizen-Gerste }\end{array}$ & $\begin{array}{l}\text { Raps-Zwischenfrucht* - } \\
\text { Hafer**-Weizen- } \\
\text { Roggen }\end{array}$ \\
\hline N-Düngung & Gute fachliche Praxis & Bilanzausgleich & Bilanzausgleich \\
\hline Pflanzenschutz & $\begin{array}{l}\text { Gute fachliche Praxis. } \\
\text { Anwendung von Herbi- } \\
\text { ziden, Fungiziden, In- } \\
\text { sektiziden sowie } \\
\text { Wachstumsreglern }\end{array}$ & $\begin{array}{l}\text { Mech. Unkrautbekämp- } \\
\text { fung, reduzierter Pflan- } \\
\text { zenschutz nach Be- } \\
\text { kämpfungsschwellen, } \\
\text { keine Wachstumsregler }\end{array}$ & $\begin{array}{l}\text { Reduzierter Pflanzen- } \\
\text { schutz nach Bekämp- } \\
\text { fungsschwellen, keine } \\
\text { Wachstumsregler }\end{array}$ \\
\hline Sonstiges & & 3 m Randstreifen & 3 m Randstreifen \\
\hline
\end{tabular}

* : Zwischenfrucht aus Ausfallraps

** : Anstelle von Hafer wurde auf den Praxisflächen auch Mais angebaut

Quelle: StEINMANN (2002), S. 25

Im Folgenden liegt der Fokus vor allem auf dem Vergleich des pfluglosen mit den Verfahren, in denen der Pflug eingesetzt wurde.

6.3.1.1.5.2 Datengrundlage und Vorgehensweise zur betriebswirtschaftlichen Analyse des INTEX-Projektes

Zunächst werden anhand von Deckungsbeitragsrechnungen die Unterschiede der einzelnen Verfahren ORD, Int-flexibel und Int-pfluglos untersucht. Dabei setzt sich das Mengengerüst wie folgt zusammen: die Daten über die Naturalerträge, die Saatgut-, Düngeund Pflanzenschutzmittelaufwendungen wurden den jeweiligen INTEX-Schlagkarteien entnommen. Bei der Stickstoffdüngung wurden die tatsächlich verwendeten Mengen, bei der Phosphor-, Kalium- und Kalkdüngung die Entzugswerte der gesamten Fruchtfolge verwendet. Für die beiden Betriebe Marienstein und Reinshof wurde eine einheitliche Maschinenausstattung unterstellt.

Die Preise für Verkaufsprodukte, Düngemittel und Saatgut sowie die Trocknungskosten wurden den Richtwertdeckungsbeiträgen der LWK Hannover aus dem Jahr 2000 entnommen. Die Preise für Pflanzenschutzmittel stammen aus den Preislisten der RHG- 
Hannover $^{326}$. Die Daten für die variablen Maschinenkosten stammen aus dem KTBLTaschenbuch $^{327}$. Das eingesetzte Umlaufkapital wurde mit $6 \%$ verzinst, bei Saatgut beträgt der Verzinsungszeitraum 9 Monate, für N-Dünger und Pflanzenschutzmittel 6 Monate. Die Ausgleichszahlungen wurden in der Höhe des Jahres 2002 angenommen.

Die Werte für die Arbeitszeit für eine Schlaggröße von 2 ha wurden aus dem KTBLTaschenbuch entnommen. Der Lohnansatz beträgt 10,23 €/Akh.

\subsection{Betriebswirtschaftliche Ergebnisse des INTEX-Projektes}

Zunächst wird die Erlössituation der beiden Betriebe Marienstein und Reinshof für die einzelnen Verfahren für den Zeitraum 1995-2002 dargestellt, hieran kann auch die Wirkung der Naturalerträge abgelesen werden.

Abbildung 18: Markterlöse der Feldfrüchte in den INTEX-Anbausystemen (19952002)

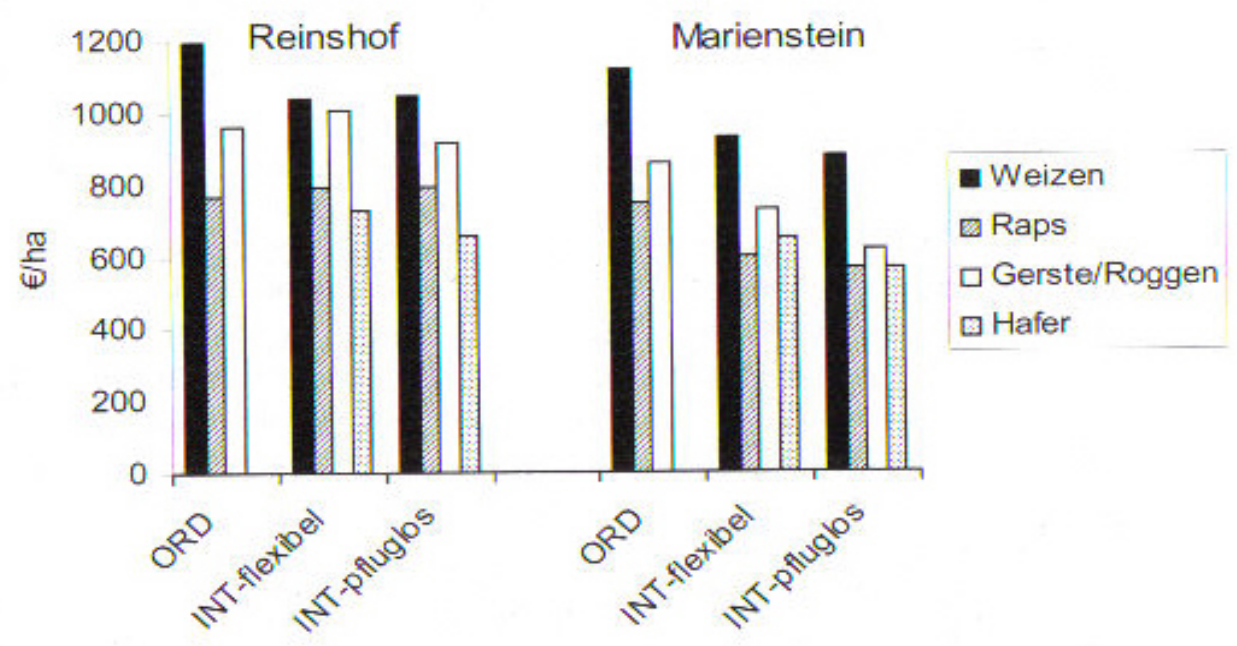

Quelle: BRAND-SASSEN u. STEINMANN (2002), S. 84

Die Ergebnisse zeigen zunächst das unterschiedliche Erlösniveau der beiden Betriebe. In Marienstein führt die integrierte Bewirtschaftungsweise zu deutlichen Erlösminderungen. Die Erlöse der integrierten Varianten liegen am Standort Reinshof um 9-12 \% und bei

\footnotetext{
${ }^{326}$ Vgl. RHG-HANNOVER (2000)
}

${ }^{327}$ Vgl. KTBL-Taschenbuch (2000) 
Marienstein um 20-28 \% niedriger. Dabei liegen die Erlöse der pfluglosen Variante in Marienstein für alle Früchte unter denen der anderen Verfahren. Beim Standort Reinshof sind die Erlöse für Weizen und Gerste geringfügig höher als in der Pflugvariante. Sie liegen trotzdem unter den Erlösen der ordnungsgemäßen Variante. Als Fazit kann festgehalten werden, dass die pfluglosen Verfahren i. d. R. zu geringeren Erlösen führen und der Erfolg dieser Verfahren maßgeblich von der Qualität des Standorts abhängt.

In der folgenden Abbildung 19 wird für die einzelnen Anbauverfahren die Struktur der variablen Kosten betrachtet und werden mögliche Einsparpotenziale für den integriertpfluglosen Anbau dargestellt. 
Abbildung 19: Variable Kosten des Winterweizen- und Winterrapsanbaus verschiedener Anbauverfahren an den Standorten Reinshof und Marienstein (1995-2002)
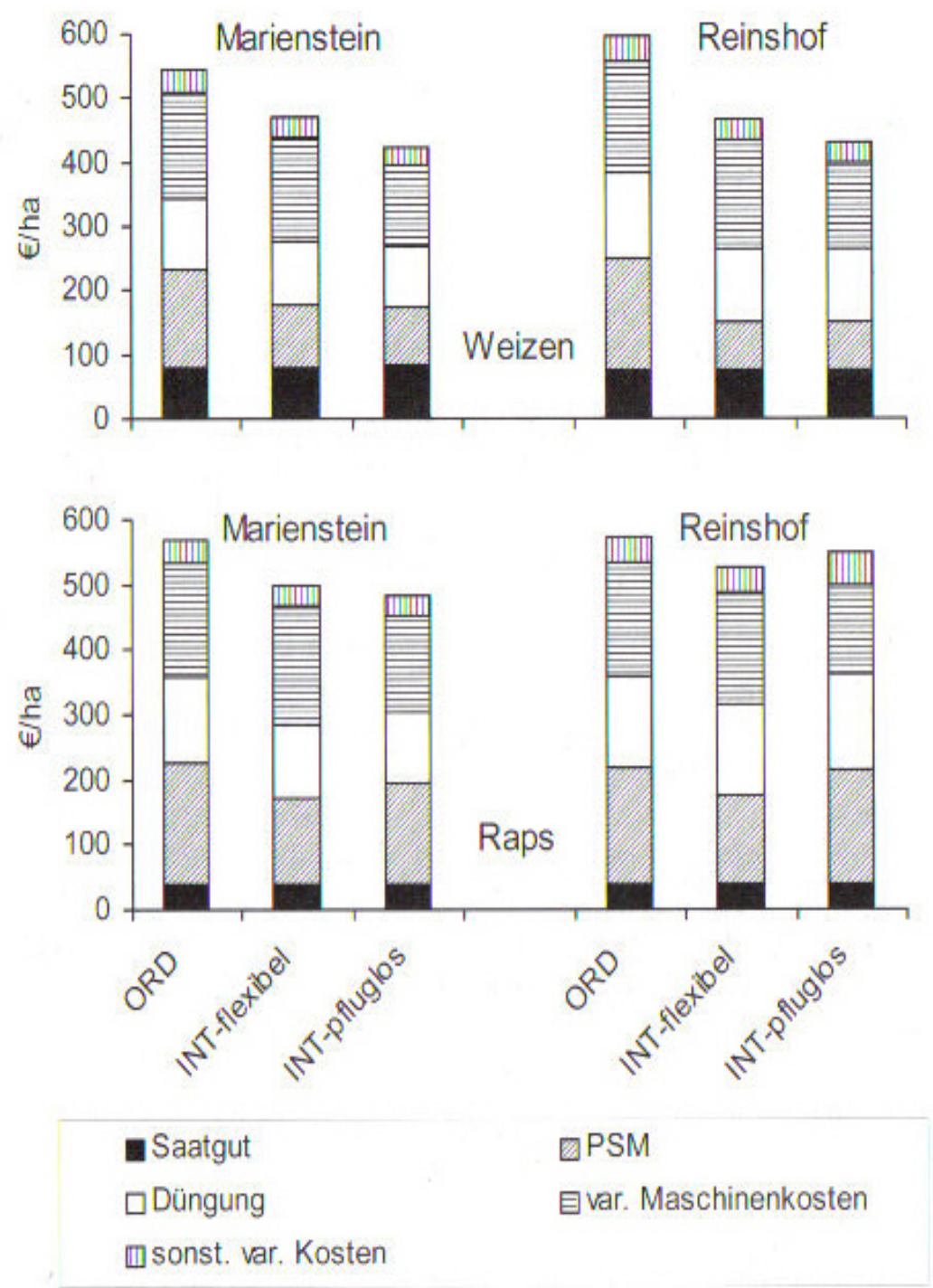

Quelle: BRAND-SASSEN u. STEINMANN (2002), S. 86

Die Einsparmöglichkeiten der integrierten Anbausysteme lagen bei Weizen bezogen auf die Düngung bei $15 \%$, Pflanzenschutzmittel bei 40-55 \% und bezogen auf die Maschinenkosten bei 0-23 \%. Beim Weizen sind die variablen Kosten bei der pfluglosen Variante am geringsten. Dies gilt ebenfalls für den Rapsanbau am Standort Marienstein.

Die notwendige Arbeitszeit korreliert sehr stark mit den Maschinenkosten. Sie wird im Folgenden dennoch für die Anbauverfahren dargestellt, da sie für viele landwirtschaftliche Betriebe einen limitierenden Faktor darstellt. 
Abbildung 20: Arbeitszeitbedarf der einzelnen Anbauverfahren auf den Standorten Reinshof und Marienstein (1995-2002; Gerste/Roggen der integrierten Systeme von 1999-2002)
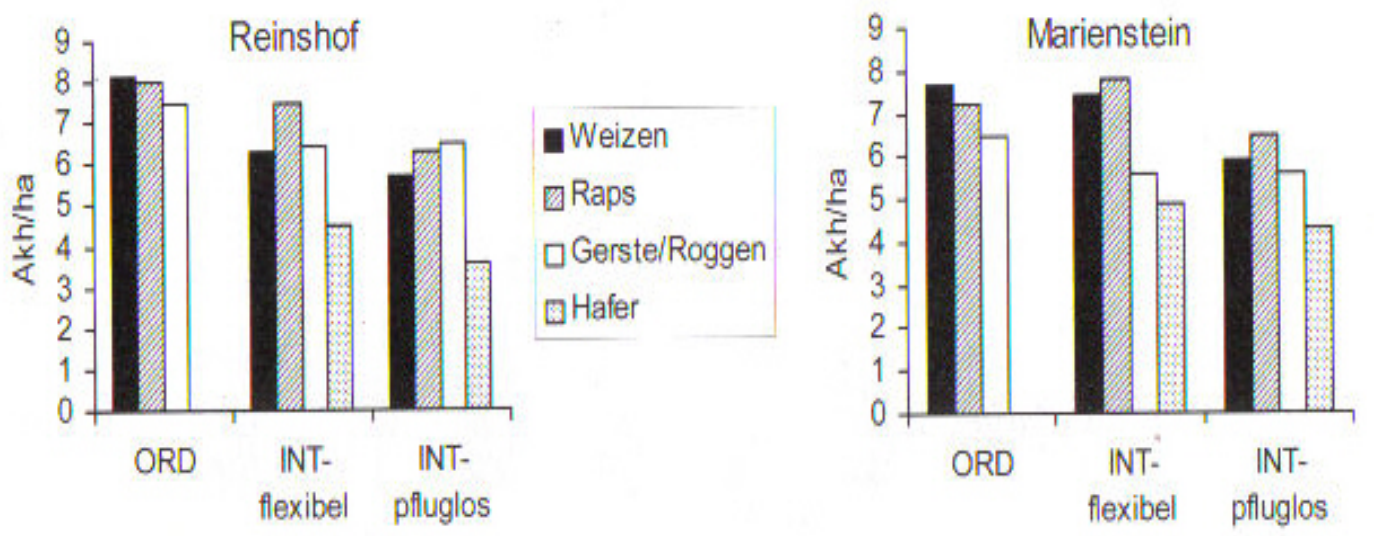

Quelle: BRAND-SASSEN u. STEINMANN (2002), S. 88

Insgesamt kann aufgrund der Abbildung 20 festgehalten werden, dass die integrierten Anbauverfahren und vor allem die pfluglosen Verfahren zu niedrigeren Arbeitszeiten führen. Die Ausnahme bildet dabei der Rapsanbau bei der Variante INT-flexibel, welche auf die langjährige mechanische Unkrautbekämpfung zurückzuführen ist. 


\section{Abbildung 21: Fruchtfolge-Deckungsbeiträge der Anbausysteme auf den Standor- ten Reinshof und Marienstein (1999-2002)}

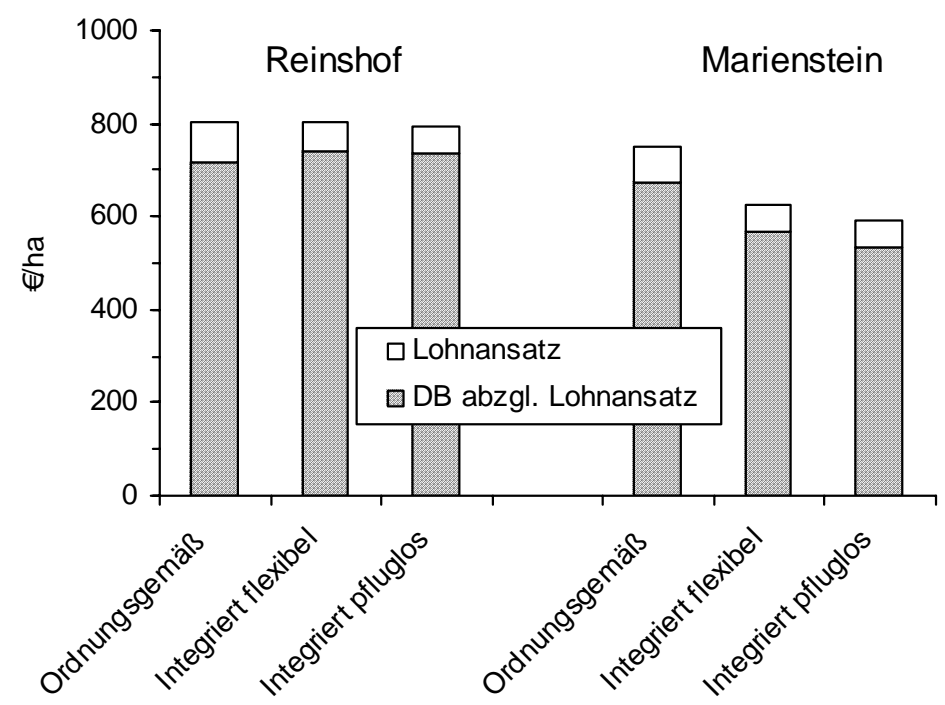

Quelle: BRAND-SASSEN u. StEINMANN (2002), S. 89

Die Fruchtfolge-Deckungsbeiträge auf dem Betrieb Reinshof liegen generell höher als auf dem Standort Marienstein. Nach Abzug des Lohnansatzes waren die Deckungsbeiträge der integrierten Systeme um $20 € /$ ha höher als die der ordnungsgemäßen Verfahren. Auf dem Standort Marienstein lagen die Fruchtfolge-Deckungsbeiträge der integrierten Verfahren um $110 € /$ ha (flexibel) und $143 €$ /ha niedriger als die der ordnungsgemäßen Verfahren.

Die betriebswirtschaftlichen Auswirkungen des völligen Pflugverzichts auf dem Standort Reinshof wird in Abbildung 22 dargestellt. Diese Abbildung stellt zusammenfassend einige ausgewählte Deckungsbeitragspositionen dar und vergleicht die Ergebnisse des Pflugverfahrens mit denen des pfluglosen Verfahrens.

Die Ergebnisse zeigen, dass bei Weizen ein geringer Vorteil des pfluglosen Verfahrens vorhanden ist, beim Winterraps ist dies nicht der Fall. Beim Pflanzenschutzmittelaufwand des Weizens konnten sogar geringfügige Einsparungen von $9 € /$ ha realisiert werden, beim Winterraps mussten Mehraufwendungen in Höhe von 30 \% getätigt werden. 


\section{Abbildung 22: Unterschiede des pfluglosen integrierten Anbausystems gegenüber dem Pflugverfahren bei Winterweizen und Winterraps auf dem Standort Reinshof (1995-2002)}
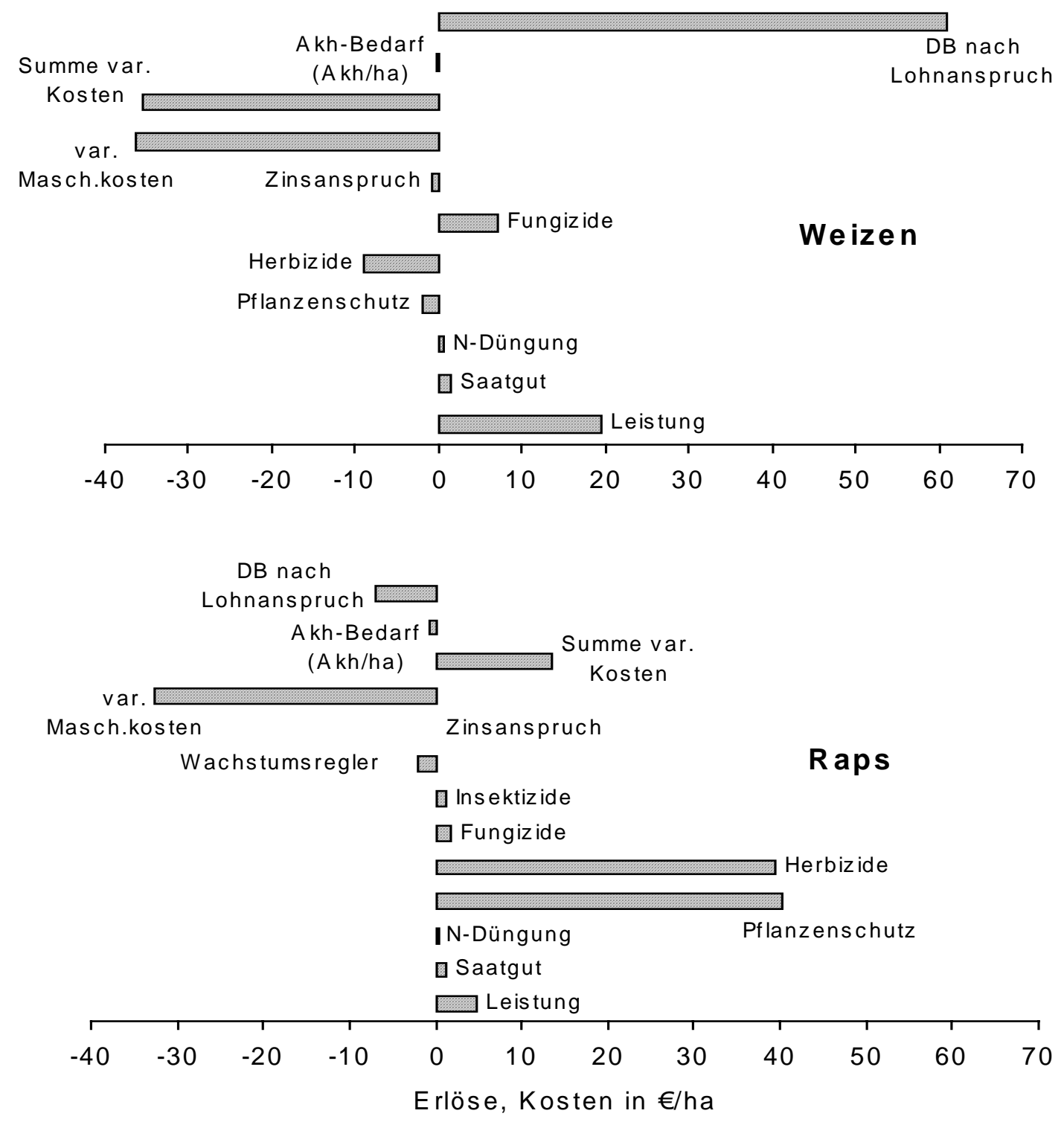

Quelle: BRAND-SASSEN u. STEINMANN (2002), S. 91

Analog zum Standort Reinshof werden die Ergebnisse für Marienstein in der Abbildung 23 dargestellt. 


\section{Abbildung 23: Unterschiede des pfluglosen integrierten Anbausystems gegenüber dem Pflugverfahren bei Winterweizen und Winterraps auf dem Standort Marien- stein (1995-2002)}

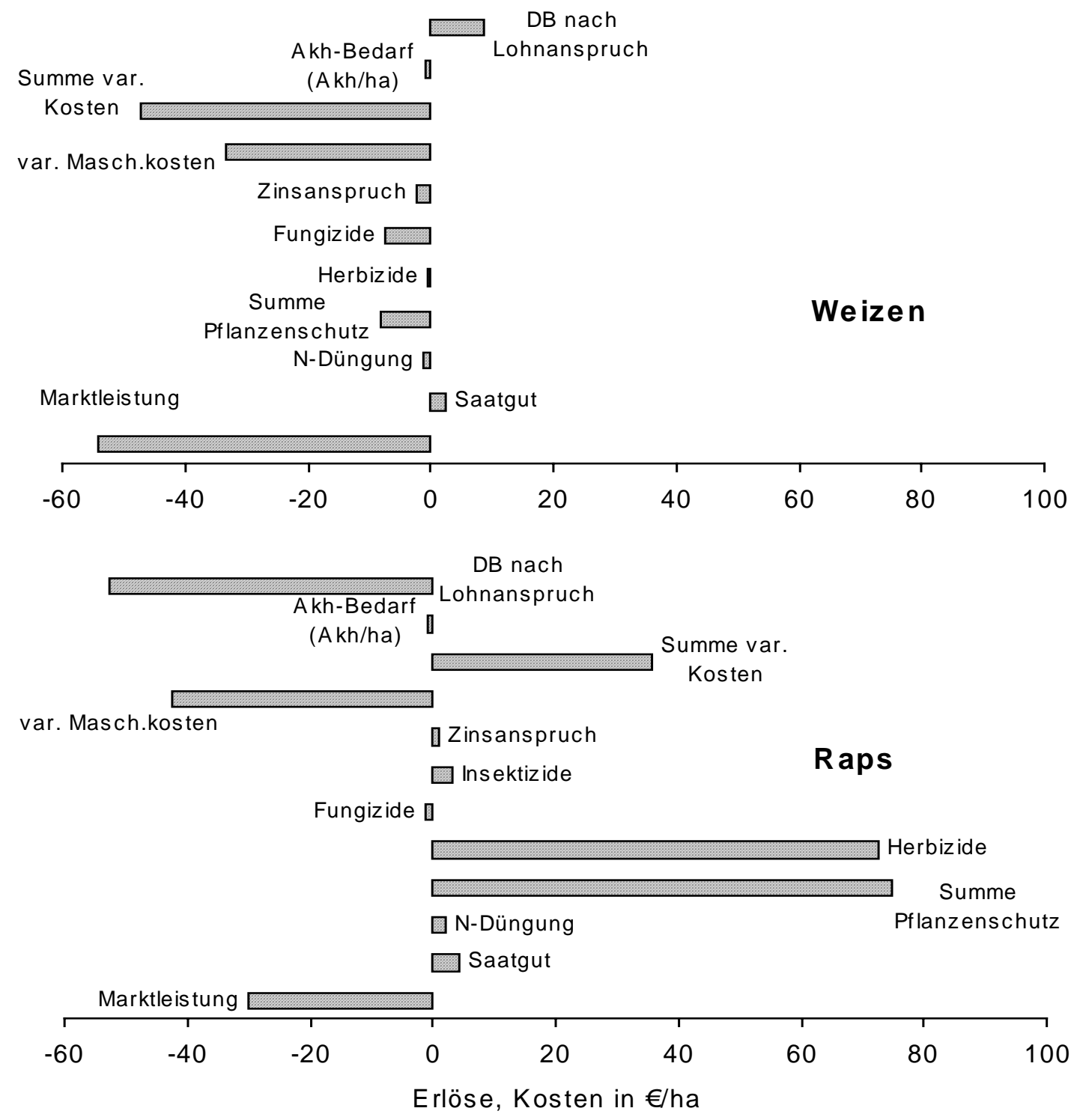

Quelle: BRAND-SASSEN u. STEINMANN (2002), S. 92

Die Ergebnisse zeigen für den Standort Marienstein lediglich beim Weizen geringe ökonomische Vorteile. Beim Raps war der PSM-Einsatz bedeutend höher und die Markleistung lag ca. $30 € /$ ha niedriger. Hieraus resultiert ein um ca. $60 € /$ ha geringerer Deckungsbeitrag. 


\subsection{Diskussion der Ergebnisse}

Die Ergebnisse der Untersuchungen zeigen, dass für die integrierten Anbauverfahren prinzipiell Einsparpotenziale, v. a. bei den Arbeits- und Maschinenkosten, bestehen. Der integrierte pfluglose Anbau führt generell zu höheren Pflanzenschutzmittelaufwendungen, welche die Vorteile kompensieren können.

Alle Ergebnisse, gerade im Vergleich der Betriebe, zeigen, dass die Standortvariabilität eine bedeutende Rolle spielt. Deshalb ist in allen Fällen eine differenzierte Betrachtung und Wertung der Ergebnisse notwendig. Dies gilt ebenfalls für die Bewertung der Ergebnisse der Literaturauswertung im Kapitel 1.1 sowie für die nachfolgenden Untersuchungen der Bodenschutzmaßnahmen. Die Ergebnisse des INTEX-Projekts finden ferner Verwendung in den Berechnungen der Maßnahmen im Bereich des stofflichen Bodenschutzes in Kapitel 6.4.1.

\subsubsection{Tiefenlockerung von Fahrspuren ${ }^{328}$}

Durch das Befahren des Ackers entstehen Fahrspuren, die durch Schadverdichtungen des Ober- bzw. Unterbodens gekennzeichnet sind. Diese Schadverdichtungen tragen erheblich zu einer erhöhten Erosionsanfälligkeit innerhalb dieser Fahrspuren bei, obwohl sie oftmals nur etwa $5 \%$ der Ackerfläche ausmachen. ${ }^{329}$ Im Folgenden wird eine KWA für die Tiefenlockerung von Fahrspuren durchgeführt.

\subsection{Einleitende Erwägungen}

Zunächst kommt es bei der Betrachtung der Auswirkungen von Fahrspuren auf Bodenschadverdichtungen darauf an, wie hoch die Spurflächensumme und die Überrollhäufigkeiten zu beziffern sind. Die Spurflächensummen ${ }^{330}$ betragen für typische Anbaufrüchte zwischen 290 bis $580 \%$.

\footnotetext{
${ }^{328}$ Unter Tiefenlockerung wird nach DIN 1185 ein „flächenhaftes Aufbrechen und Anheben eines mind. $40 \mathrm{~cm}$ tiefen Bodenbereichs" verstanden.

${ }^{329}$ Vgl. Fleige, Horn u. Weißbach (1999), S. 33 ff.

330 Anmerkung: Eine Spurflächensumme von 290 beim Winterweizen (Gülle) bedeutet, dass der Boden theoretisch im Mittel 2,9 Mal überfahren wird, bei einer Spurflächensumme von 580 \% wird der Boden 5,8 Mal überfahren.
} 
Tabelle 16: Spurflächensumme innerhalb einer Vegetationsperiode für verschiedene Früchte in \%

\begin{tabular}{lc}
\hline \multicolumn{1}{c}{ Fruchtart } & Spurflächensumme \\
\hline Winterweizen (Gülle) & 290 \\
Winterraps & 320 \\
Wintergerste & 320 \\
Winterweizen & 350 \\
Wintergerste (Gülle) & 350 \\
Zuckerrüben & 500 \\
Silomais (Gülle) & 520 \\
Kartoffeln & 580 \\
\hline
\end{tabular}

Quelle: Zusammenfassung nach ZAPF (1997)

Diese Werte geben ebenfalls Aufschluss darüber, wie stark die hierdurch induzierten Bodenverdichtungen wirken. Durch die Erhöhung der Überrollhäufigkeit steigt die Gefahr einer höheren und tieferen Bodenschadverdichtung. ${ }^{331}$

Durch Fahr- und Pflegespuren können z. T. sehr hohe Bodenabträge induziert werden. FLEIGE, HORN U. WEIßBACH ${ }^{332}$ ermitteln innerhalb einer Vegetationsperiode in der Jungmoränenlandschaft Ostholsteins jährliche Bodenabträge in den Fahrspuren von 5,4 bis 16,1 t/ha. Rотн ${ }^{333}$ gibt für Fahrspuren bei Lößböden und Zuckerrüben Bodenabträge von 1-5 t/ha/a an. Als Maßnahmen zur Verminderung des Bodenabtrags in den Fahrspuren bietet sich daher die mechanische Lockerung der unteren Bodenschichten an. Bei diesem Verfahren werden die Fahrspuren mit einem Lockerungsschar bearbeitet, um die Infiltration und somit den Bodenabtrag zu begrenzen. Der Boden muss dabei trocken sein und der Lockerungsprozess muss die gesamte Krumentiefe erfassen sowie die Fahrspur auf das Niveau des nicht bearbeiteten Ackerbodens anheben. Seitens der Landmaschinenindustrie wurden eigens hierfür Geräte entwickelt, die nach einem ähnlichen Schema arbeiten. Ein Nachteil dieses Verfahrens liegt darin, dass der gelockerte Boden sehr anfällig auf Starkregeneinflüsse reagiert und $u$. U. abgetragen werden kann. Die Kosten dieses Verfahrens sind gering, wie Tabelle 17 in 6.3.1.2.2 zeigt. Die Tiefenlockerung ist die einzige kurzfristig durchführbare Maßnahme, um Unterbodenverdichtungen zu beseitigen. Die hierdurch geschaffene Grobporenstruktur ist allerdings nicht mit der Leistungsfähigkeit des natürlichen Porensystems vergleichbar. Ein weiteres Problem ist die Rückverfes-

\footnotetext{
${ }^{331}$ Vgl. BMVEL (2001), S. 21

${ }^{332}$ Vgl. Fleige, HoRn U. WeißBACH (1999), S. 33

333 Vgl. Roth zit. in Brunotte, Roth U. Hollmann (1994), S. 309
} 
tigung des Unterbodens, wodurch dieser, durch den Einfluss hoher Radlasten, noch anfälliger bzgl. stärkerer Verdichtungen wird. ${ }^{334}$ Es dürfen deshalb in nachfolgenden Bearbeitungsgängen nur Maschinen mit möglichst geringen Radlasten zum Einsatz kommen. Ein neues und stabiles Bodengefüge bildet sich erst im Laufe der Zeit durch biologische Prozesse. Nach Meinung von HoRN bleiben trotz dieser Maßnahmen sinkende Erträge und die Ertragsunsicherheit erhalten. ${ }^{335}$ HAKANSSON und PETELKAU ${ }^{336}$ zeigen jedoch, dass durch den Effekt der Krumenbasislockerung auf Moränestandorten mittelfristige Ertragszuwächse bei Getreide, gerade bei der partiellen Tiefenlockerung, zu verzeichnen sind. Sie bestehen bis zu fünf Jahre und betragen $6 \mathrm{dt} / \mathrm{ha}$ und fallen im fünften Jahr auf $2 \mathrm{dt} / \mathrm{ha}$ ab. Eine Kompensation der zuvor durch Bodenschadverdichtungen erzeugten Mindererträge ist demnach nicht oder nur unter sehr guten Bedingungen bis zu $30 \%$ möglich. ${ }^{337}$ Aufgrund der geringen Stichprobe bezüglich der erzielbaren Mehrerträge durch Tiefenlockerungsmaßnahmen werden die obigen Ergebnisse nicht in die Beurteilung dieser Maßnahme einbezogen. Die Lockerung der Fahrspuren darf nur unter trockenen Bedingungen und möglichst nicht unterhalb die Pflugsole durchgeführt werden, um keine neuen Verdichtungen hervorzurufen.

Im Bereich des Hochwasserschutzes wird der Tiefenlockerung ein hohes Reduktionspotenzial des Oberflächenabflusses beigemessen. ${ }^{338}$ Dieser Nutzeneffekt ist jedoch nicht eindeutig quantifizierbar, da die Auswirkungen einer einzelnen Maßnahme auf das komplexe Entstehen eines Hochwasserereignisses kaum abschätzbar sind.

\subsection{Betriebswirtschaftliche Analyse der Maßnahme}

Aufgrund der fehlenden Ergebnisse bzgl. des Nutzens dieser Maßnahme wird nur eine KWA durchgeführt. Zunächst werden die Kosten der Tiefenlockerung betrachtet. Bei der Maßnahme der Tiefenlockerung wird mit einem entsprechenden Gerät (Parapflug, Wippscharlockerer, Tiefenmeißel) der Unterboden bearbeitet. Dabei ist ein hoher Zugkraftbedarf erforderlich. Durch die Maßnahme entstehen demzufolge hohe Arbeits- sowie Ma-

\footnotetext{
${ }^{334}$ Vgl. BStMLF (2001), S. 51 u. MLUR BRANDENBURG (2003), S. 35

${ }^{335}$ Vgl. HORN (2002), S. 429

${ }^{336}$ Vgl. HaKansSON u. PetelKau (1994)

${ }^{337}$ Vgl. WERNER (1989), zit. in DÜRR et al. (1995), S. 122

${ }^{338}$ Vgl. Tabelle 11 in Kapitel 5.3.2
} 
schinenkosten. Da es sich um sehr spezielle und teure ${ }^{339}$ Bearbeitungsgeräte handelt, wird von einer Fremdmechanisierung ausgegangen, so dass für das entsprechende Bearbeitungsgerät keine festen Kosten anfallen. Diese Kosten sind in der Tabelle 17 für verschiedene Geräte dargestellt. Es besteht die Annahme, dass die Fahrspurfläche $5 \%$ der gesamten AF beträgt.

Tabelle 17: Verfahrenskosten der Tiefenlockerung von Fahrspuren ${ }^{340}$

\begin{tabular}{lccc}
\hline \multicolumn{1}{c}{ Verfahren $\left(€ / 500 \mathrm{~m}^{2}\right)$} & $\begin{array}{c}\text { Parapflug } \\
(\mathbf{1 , 5} \mathbf{~ m})\end{array}$ & $\begin{array}{c}\text { Wippscharlockerer } \\
(\mathbf{1 , 7} \mathbf{~ m})\end{array}$ & $\begin{array}{c}\text { Tiefenlockerungs- } \\
\text { gerät (1,5 m) }\end{array}$ \\
\hline Var. Maschinenkosten & 0,60 & 0,35 & 0,17 \\
Var. Schlepperkosten & 0,53 & 0,53 & 0,53 \\
(83 kW) & & & \\
Arbeitskosten & 1,23 & 0,90 & 0,90 \\
Festkosten insg. & 0,31 & 0,51 & 0,16 \\
\hline Gesamt $\left(\mathbf{\epsilon} / \mathbf{5 0 0} \mathbf{~ m}^{\mathbf{2}}\right)$ & $\mathbf{2 , 6 7}$ & $\mathbf{2 , 2 9}$ & $\mathbf{1 , 7 6}$ \\
\hline
\end{tabular}

Quelle: Eigene Berechnungen nach KTBL (2002)

Der Nutzen der Tiefenlockerung von Fahrspuren ist, wie im obigen Kapitel bereits angedeutet, sehr gering. Das hauptsächliche Problem liegt dabei in den instabilen und unsicheren Ergebnissen der Maßnahme. Aufgrund der hohen Rückverdichtungsgefährdung sind auch keine nennenswerten Ertragssteigerungen bzw. - sicherungen zu erwarten. Aufgrund dieser Tatsache wird an dieser Stelle von einer KNA dieser Maßnahme abgesehen. Stattdessen werden Überlegungen bezüglich der KW der Maßnahme gemacht. Ausgehend von einem Anteil der Fahrspuren von 5 \% der Gesamtfläche werden in der folgenden Tabelle aufgrund mangelnder Daten bezüglich der Wirkung dieser Maßnahme die Reduktionspotenziale variiert. Außerdem werden analog zu den in Kapitel 6.3.1.2.1 genannten Studien von ROTH sowie FLEIGE, HORN U. WEIßBACH verschiedene Bodenabträge durch Fahrspurerosion angenommen. Die Kosten seien gemittelt und betragen 2,24€/500 $\mathrm{m}^{2}$. Der Zinssatz beträgt $4 \%$.

\footnotetext{
${ }^{339}$ Die Listenpreise von Tiefenlockerungsgeräten mit einer Arbeitsbreite von verschiedenen Herstellern liegen zwischen 7.360-25.868 € bei Arbeitsbreiten von 2,7 m bis 4,0 m. Vgl. MEYER (2003), S. 22

${ }^{340}$ Bei einem Fahrspuranteil von $5 \%$ der bewirtschafteten Ackerfläche.
} 
Tabelle 18: KW (€/t verhinderter Bodenabtrag) bei Tiefenlockerungsmaßnahmen von Fahrspuren bei unterschiedlichen Bodenabträgen und Erfolgspotenzialen der Maßnahme ${ }^{341}$

\begin{tabular}{|c|c|c|c|}
\hline $\begin{array}{l}\text { Reduktions- } \\
\text { potenzial }\end{array}$ & $\begin{array}{l}\text { niedriger Bodenab- } \\
\text { trag }\end{array}$ & $\begin{array}{l}\text { mittlerer Bodenab- } \\
\text { trag }\end{array}$ & hoher Bodenabtrag \\
\hline & $\begin{array}{c}(1 \mathrm{t} / \mathrm{ha} / \mathbf{a}) \\
0,05 \mathrm{t} / \mathbf{5 0 0} \mathrm{m}^{2}\end{array}$ & $\begin{array}{c}(5 \mathrm{t} / \mathrm{ha} / \mathbf{a}) \\
0,25 \mathrm{t} / 500 \mathrm{~m}^{2}\end{array}$ & $\begin{array}{l}(16 \text { t/ha/a) } \\
0,8 t / 500 \text { m }^{2}\end{array}$ \\
\hline $10 \%$ & 55,98 & 44,34 & 21,70 \\
\hline $50 \%$ & 44,34 & 15,08 & 5,23 \\
\hline $100 \%$ & 30,44 & 8,13 & 2,68 \\
\hline
\end{tabular}

Quelle: Eigene Berechnungen

Die Ergebnisse zeigen, wie bereits erwartet wurde, eine große Schwankungsbreite der KW. Sie sind v. a. von den Reduktionspotenzialen der Lockerungsmaßnahme abhängig. Die KW liegen im günstigsten Fall bei 2,68 €/t verhinderter Bodenabtrag und im ungünstigsten Fall bei 55,98 €. Würden die in Kapitel 6.3.1.1.3 angenommenen Bodenabtragsraten vorausgesetzt, dann lägen die $\mathrm{KW}$, wie die Tabelle 19 zeigt, bei mindestens 4,81€/t verhinderter Bodenabtrag und höchstens bei 45,82 €/t verhinderter Bodenabtrag.

Tabelle 19: KW (€/t verhinderter Bodenabtrag) bei Tiefenlockerungsmaßnahmen von Fahrspuren bei Bodenabträgen von 4,6 t/ha und 8,7 t/ha und verschiedenen Erfolgspotenzialen der Maßnahme

\begin{tabular}{ccc}
\hline $\begin{array}{c}\text { Reduktionspoten- } \\
\text { zial }\end{array}$ & $\mathbf{4 , 6}$ t/ha & $\mathbf{8 , 7}$ t/ha \\
\hline $\mathbf{1 0} \%$ & 45,82 & 33,19 \\
$\mathbf{5 0} \%$ & 16,17 & 9,23 \\
$\mathbf{1 0 0} \%$ & 8,77 & 4,81 \\
\hline
\end{tabular}

Quelle: Eigene Berechnungen

Eine direkte Vergleichbarkeit zwischen beiden Maßnahmen ist aufgrund der ungenügenden Datengrundlage nicht gegeben. Dennoch kann festgestellt werden, dass sich die KWA beider Maßnahmen aufgrund der Berechnungen ähnlich sein könnten.

\footnotetext{
${ }^{341}$ Annahmen: Anteil der Fahrspuren an der Gesamtfläche liegt bei $5 \%$ und die Kosten der Maßnahme liegen bei 2,24 €/ha. Die KW ist analog zu Kapitel 6.3.1.1.3 als Barwert der nötigen Kosten zu verstehen, um eine Tonne Bodenabtrag zu verhindern.
} 


\subsection{Schlussfolgerungen}

Die Tiefenlockerung von Fahrspuren ist zunächst eine kostengünstige Maßnahme, der jedoch ein nur bedingt zu quantifizierender Nutzen gegenübersteht. Aus bodenwissenschaftlicher Sicht ist dieses Verfahren nicht als langfristig nachhaltig zu bezeichnen. Deshalb handelt es sich um eine kurzfristig anzuwendende Maßnahme. Allgemeingültige Empfehlungen können den Landwirten aufgrund der obigen Berechnungen allerdings nicht gegeben werden, da die Höhe des Erfolges von zahlreichen standortabhängigen Variablen abhängt. Außerdem bedarf es der Erfahrung des Landwirts bei dessen Durchführung. Von Seiten der Beratung sind daher Tiefenlockerungsmaßnahmen nur in wenigen Ausnahmen zu empfehlen, jedoch nicht als regelmäßige Maßnahme und fester Bestandteil eines Produktionssystems.

\subsubsection{3 Änderung der Bewirtschaftungsrichtung}

Eine weitere Maßnahme zur Verringerung der Bodenerosion ist die Bewirtschaftung quer zum Hang. In diesem Kapitel wird die Maßnahme zunächst vorgestellt und anhand von Vor- und Nachteilen in der landwirtschaftlichen Praxis bewertet bzw. deren Verbreitung eingegrenzt. Danach wird eine KNA dieser Maßnahme unter Berechnungen des Nutzens mit der ABAG durchgeführt.

\subsection{Einleitende Erwägungen}

Bei der Bewirtschaftung quer zum Hang muss das gesamte Produktionsverfahren, zumindest aber die Aussaat und somit die Anlage der Fahrspuren in Querrichtung zum Hang erfolgen. Hierdurch wird das vom Hang abfließende Wasser abgebremst und der Bodenabtrag über diesen Pfad vermindert. Allerdings kann diese Arbeitsweise, wie Tabelle 20 zeigt, nicht für alle Hangneigungen empfohlen werden. Die Einsatzgrenze für die Bearbeitung quer zum Hang liegt demnach bei 23,5 \%. Die Bearbeitung in Fallinie kann bei einer maximalen Hangneigung von 25,8 \% eingesetzt werden und liefert bis einer Hangneigung von 21,3\% ein befriedigendes Arbeitsergebnis. Bei der Bearbeitung quer zum Hang ist dies nur bei einer Hangneigung von 19,6 \% der Fall. 
Tabelle 20: Hangneigungsgrenzen für die Bodenbearbeitung quer zum Hang sowie in Falllinie

\begin{tabular}{lcc|cc}
\hline \multicolumn{2}{c}{ quer zum Hang } & \multicolumn{2}{c}{ in Falllinie } \\
\hline & $\begin{array}{c}\text { befriedigendes } \\
\text { Arbeitsergebnis } \\
\text { bis ...\% HN }\end{array}$ & $\begin{array}{c}\text { Einsatz- } \\
\text { grenze } \\
\% \mathrm{HN}\end{array}$ & $\begin{array}{c}\text { befriedigendes } \\
\text { Arbeitsergebnis } \\
\text { bis ...\% HN }\end{array}$ & Einsatzgrenze \\
& & & & \\
& & & & \\
\hline Bodenbearbeitung & 22,5 & 28 & 24 & 29 \\
Saat & 18,7 & 21,5 & 20,7 & 23,3 \\
Pflege & 19,8 & 23,3 & 23,8 & 28,3 \\
Ernte & 17,6 & 21,3 & 16,9 & 22,5 \\
\hline$\varnothing$ aller Arbeitsgänge & $\mathbf{1 9 , 6}$ & $\mathbf{2 3 , 5}$ & $\mathbf{2 1 , 3}$ & $\mathbf{2 5 , 8}$ \\
\hline
\end{tabular}

Quelle: Verändert nach PFAHLER (1986), S.124

Außerdem muss die kritische Hanglänge berücksichtigt werden, oberhalb derer die Querbewirtschaftung wirkungslos ist. Als Lösung dieses Problems können Grünstreifen angelegt werden. ${ }^{342}$

Weitere nicht quantifizierbare und teilweise durch die moderne Landtechnik abgeminderte oder gänzlich reduzierte Nachteile der Bearbeitung quer zum Hang sind in der Übersicht 9 dargestellt. 


\section{Übersicht 9: Schwierigkeiten bei der Bearbeitung quer zum Hang}

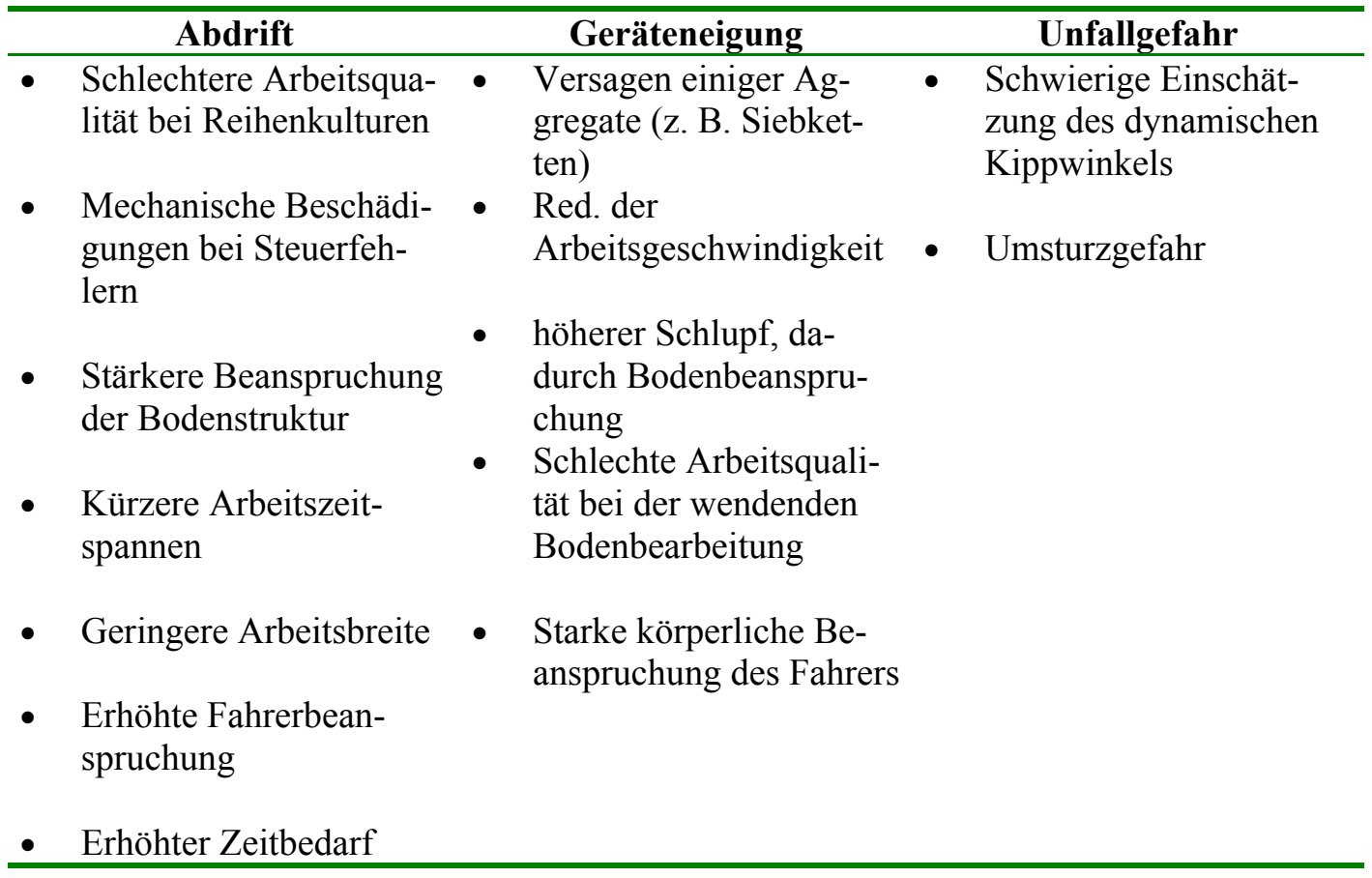

Quelle: Verändert nach PfAHLER (1986) zit. in MEYER-Roschau (1994)

Bei der Übersicht 9 ist zu beachten, dass einige Schwierigkeiten, wie die starke Beanspruchung des Fahrers oder die schlechte Arbeitsqualität, mittlerweile durch den technischen Fortschritt behoben werden konnten. Hierunter fällt z. B. die Entwicklung von Allradtraktoren, unterschiedlich einsetzbaren Lenkradübersetzungen oder automatischer Hangausgleichssysteme. Allerdings ist hierbei die Verfügbarkeit auf den landwirtschaftlichen Betrieben zu berücksichtigen, die nicht für alle o. g. Entwicklungen gegeben sein dürfte.

Bei der Bewirtschaftung quer zum Hang muss ein weiterer hemmender Faktor bedacht werden. Die Arasion führt bei konkav verlaufenen Hängen dazu, dass der gepflügte Boden durch die Schwerkraft stärker hangabwärts transportiert wird. Bei konkaven Hangformen kann die Arasion zu erheblichen Bodenabträgen führen. ${ }^{343}$ Im Folgenden wird sie aufgrund fehlender Untersuchungen und mangelnden Datenmaterials nicht weiter berücksichtigt. 


\subsection{Betriebswirtschaftliche Analyse der Maßnahme}

Die Vorgehensweise für die KNA in diesem Abschnitt wird anhand der Übersicht 10 verdeutlicht und zusammengefasst. Außerdem wird eine KWA bezüglich der Verminderung der Erosion des Bodenmaterials durchgeführt und mit den bisherigen Maßnahmen verglichen.

\section{Übersicht 10: Vorgehensweise bei der KNA für die Bewirtschaftung quer zum Hang}

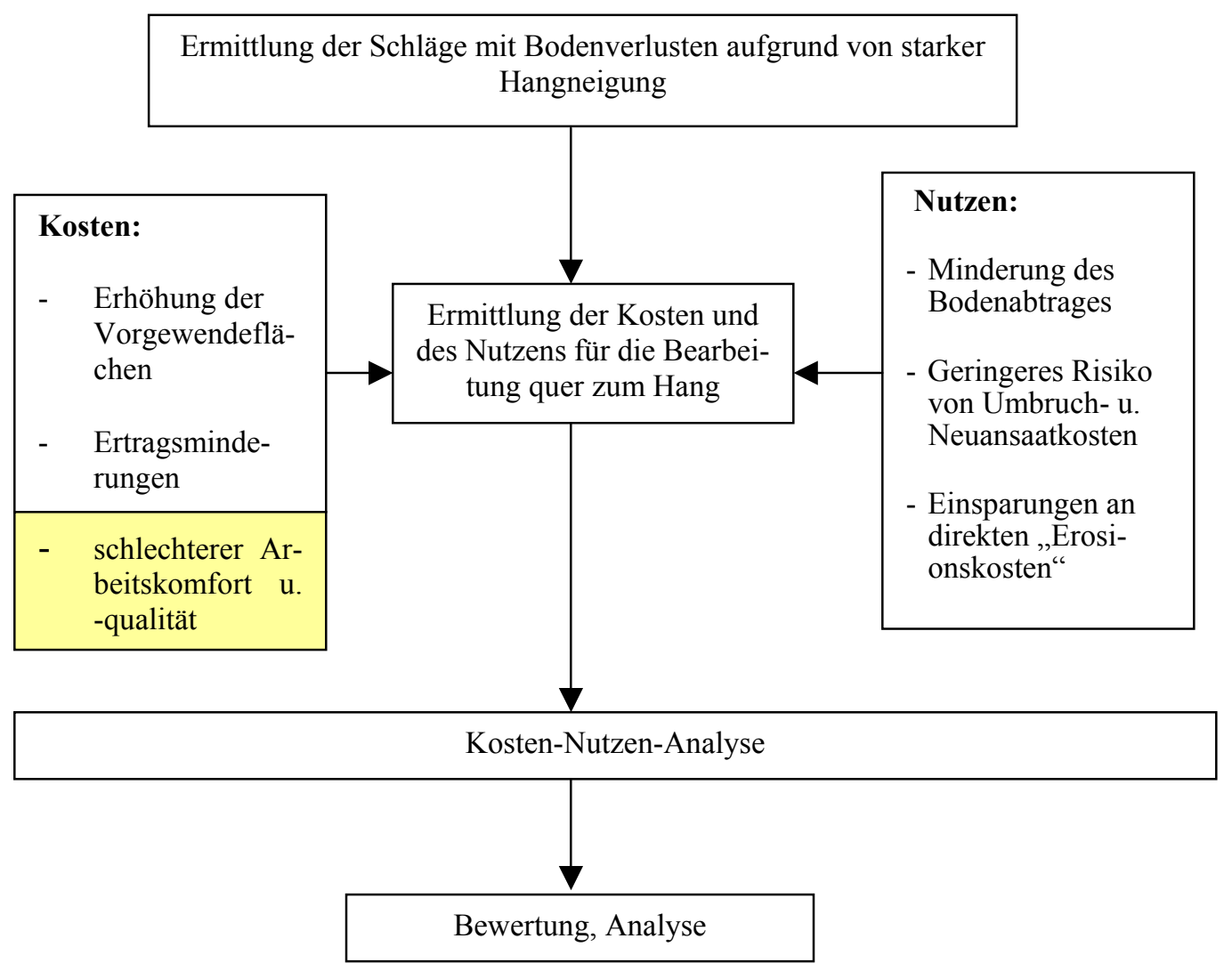

Quelle: Eigene Darstellung, Schraffierung: Positionen konnten aufgrund fehlender Daten in der Berechnung nicht berücksichtigt werden

Die Kosten der Maßnahme resultieren hauptsächlich aus der Erhöhung der Vorgewendefläche durch die Querbewirtschaftung. Diese Erhöhung hängt maßgeblich von der Schlagform ab. Im Folgenden wird davon ausgegangen, dass es sich bei den betrachteten Schlägen um längs gestreckte Rechtecke handelt, die vom Landwirt dementsprechend auch längs bewirtschaftet werden. Die Erhöhung der Vorgewendefläche kann z. B. bei 
einem Schlag mit einer Länge von 200 m und einer Breite von 100 m sowie einer Vorgewendebreite von $10 \mathrm{~m}$ durch Querbearbeitung $100 \%\left(1600 \mathrm{~m}^{2}\right)$ betragen. $^{344}$

Die durch die Erhöhung der Vorgewendefläche verursachten Schäden bestehen v. a. aus Ertragsminderungen. Die Einflussfaktoren für die Ertragsminderungen auf Vorgewendeflächen sind sehr vielfältig und reichen von den Randeinwirkungen durch Unkräuter über mechanische Beschädigungen bei Pflegearbeiten, Beschattungen von Hecken bis hin zum verzögerten Abreifen der Pflanzen. ${ }^{345}$

Ältere Schätzungen (1949-1970) von Ertragsminderungen auf Vorgewendeflächen gehen von relativen Mindererträgen über alle Fruchtarten von 13-50 \% aus. ${ }^{346}$ Neuere Untersuchungen, die Feldversuche mit einbeziehen, sind sehr selten. Sie werden in der Tabelle 21 zusammengefasst:

Tabelle 21: Literaturauswertung zu Ertragsminderungen auf Vorgewendeflächen

\begin{tabular}{|c|c|c|c|}
\hline Autor(en) & Datenquelle & Fruchtart & Ertragsminderung \\
\hline $\begin{array}{l}\text { GALL, FRIESLEBEN U. } \\
\text { PESCH (1985) }\end{array}$ & Schätzwerte & Kartoffeln & $50-80 \%$ \\
\hline KUNZE (1985) & Schätzwerte & Kartoffeln & $20-50 \%$ \\
\hline MEIWES (1984) & $\begin{array}{l}\text { Feldversuche } \\
\quad(1978-80)\end{array}$ & Winterweizen & $5,5-30 \%$ \\
\hline $\begin{array}{l}\text { JAGGARD, GUMMERSON U. } \\
\text { CLARK (1987) }\end{array}$ & $\begin{array}{c}\text { Feldversuche } \\
1982-83\end{array}$ & Zuckerrüben & $6-30 \%$ \\
\hline BRUNOTTE et al. (2000) & Feldversuche & Winterweizen & $5,6 \%$ bzw. $27,5 \%$ \\
\hline
\end{tabular}

Quelle: Eigene Zusammenstellung nach MEYER-RosCHAU (1994)

In der obigen Tabelle wird die hohe Schwankungsbreite der Ergebnisse und Annahmen deutlich. Daher ist es für die folgende Betrachtungen unerlässlich, die möglichen Ertragsminderungen zu variieren.

Bei der Bewirtschaftung quer zum Hang treten höhere Wendezeiten sowie Fahrgeschwindigkeiten auf. Die speziellen Wendezeiten und Fahrgeschwindigkeiten einiger Ackermaschinen oder Arbeitsgänge am Hang mit unterschiedlicher Hangneigung hat PFAHLER anhand von linearen Regressionsschätzungen untersucht. Allerdings erfolgte keine Unterscheidung zwischen den unterschiedlichen Bearbeitungsformen. Aufgrund

\footnotetext{
${ }^{344}$ Abzüglich der Eckflächen (400 $\left.\mathrm{m}^{2}\right)$.

${ }^{345}$ Vgl. MEYER-Roschau (1994), S. 178 ff.

${ }^{346}$ Vgl. V. SCHWARZKOPF (1979), S. 161
} 
der uneinheitlichen Ergebnisse der Literaturauswertung in Tabelle 21 werden die einzelnen durch die Ertragsminderungen beeinflussten Deckungsbeiträge ebenfalls variiert.

Bei einer Erhöhung der Vorgewendefläche ergibt sich gleichzeitig eine erhöhte Arbeitszeit für den Schlag durch die zusätzliche Wendearbeit. Die Wendezeiten für einzelne Arbeitsgänge sind durch das $\mathrm{KTBL}^{347}$ ermittelt worden. Anhand dieser Daten werden in Tabelle A-3 und Tabelle A-4 beispielhaft für typische Anbauverfahren für Getreide und Raps die zusätzlichen Arbeitskosten für die Querbewirtschaftung ermittelt. Dabei werden verschiedene Schlaggrößen, alle in Rechteckform, angenommen. Die zusätzlichen Arbeitskosten können in diesem Fall mit 9,61-57,34€/ha angegeben werden, was einer Steigerung von 14-44 \% entspricht. Hinzu kommen entsprechende variable Kosten für den Schlepper in Höhe von etwa 0,9-7,5€/ha, sodass eine zusätzliche Belastung von 10,51-64,84 €/ha für typische Getreide- oder Rapsfruchtfolgen entstehen kann.

Wird der entsprechende Schlag konservierend bewirtschaftet, kommt es darauf an, ob der Pflugverzicht durch eine Bodenbearbeitungsmaßnahme, z. B. durch den Einsatz eines Flügelschargrubbers, kompensiert werden kann oder ob zwei Arbeitsgänge notwendig sind. Im ersten und günstigsten Fall betragen die zusätzlichen Arbeits- u. Schlepperkosten 10,4-57,2 €/ha, dies entspricht einer Steigerung im Vergleich zur Falllinienbearbeitung von 21-46 \%. Dementsprechend sind bei einem Arbeitsgang durch die pfluglose Bodenbearbeitung, keine nennenswert höheren Einsparpotenziale zu erwarten. Bei mehreren zusätzlichen Arbeitsgängen müssen die zusätzlichen Kosten allerdings Eingang in die Kalkulation finden.

Abschließend ist zu erwähnen, dass in dieser Betrachtung keine Kosten für Doppelaufwendungen berücksichtigt werden. Diese Kosten entstehen bei nicht rechtwinkelig geschnittenen Schlägen. ${ }^{348}$

Der Nutzen der Bewirtschaftung quer zum Hang setzt sich wie folgt zusammen: Durch einen geminderten Zugkraftbedarf können sich geringe Einsparungen ergeben. Verlässliche Daten sind bisher nicht erhältlich, werden jedoch derzeit vom KTBL erarbeitet. ${ }^{349}$

\footnotetext{
${ }^{347}$ Vgl. KTBL (2000a)

${ }^{348}$ Vgl. MeIWES (1984), S. 127

${ }^{349}$ Vgl. Pers. Mitteilung WEIERSHÄUSER (2003)
} 
Die Auswirkungen der Bearbeitung quer zum Hang auf den Bodenabtrag werden anhand der ABAG abgeschätzt. Es werden verschiedene Bodenarten, wie sie etwa auf den Betrieben des INTEX-Projekts ${ }^{350}$ vorliegen und auch für Lößgebiete Südniedersachsens typisch sind, und verschiedene Fruchtfolgen unterschieden. Hierbei ist zu berücksichtigen, dass in die Berechnungen auch Fruchtfolgen mit einbezogen wurden, die an Standorten mit einer Hangneigung von $10 \%$ nicht der Handlungsempfehlung zur guten fachlichen Praxis entsprechen, in der Praxis jedoch immer noch zu finden sind.

${ }^{350}$ Vgl. Kapitel INTEX 
Tabelle 22: Gesamtauswirkungen der Bearbeitung quer zum Hang auf den Bodenabtrag bei verschiedenen Fruchtfolgen und Bodenverhältnissen sowie einer Hangneigung von $10 \%$

\begin{tabular}{|c|c|c|c|c|c|}
\hline $\begin{array}{l}\text { Stand- } \\
\text { ort }\end{array}$ & Fruchtfolge & $\begin{array}{c}\text { C- } \\
\text { Faktor }\end{array}$ & $\begin{array}{c}\text { Bodenabtrag o. } \\
\text { Maßnahme } \\
\text { (t/ha/a) }\end{array}$ & $\begin{array}{c}\text { Bodenabtrag m. } \\
\text { Maßnahme } \\
(\mathrm{t} / \mathrm{ha} / \mathbf{a})\end{array}$ & $\begin{array}{c}\text { Veränderung } \\
(\mathrm{t} / \mathrm{ha} / \mathbf{a}) \\
\end{array}$ \\
\hline \multirow[t]{7}{*}{${\mathrm{L} 4 \mathrm{~L} \ddot{O}^{351}}^{351}$} & Getreide & 0,07 & 3,4 & 2,8 & $-0,6$ \\
\hline & Raps-Getreide & 0,08 & 3,9 & 3,2 & $-0,7$ \\
\hline & $\begin{array}{l}\text { Kartoffel- } \\
\text { Getreide }\end{array}$ & 0,11 & 5,4 & 3,6 & $-1,8$ \\
\hline & ZR-Getreide & 0,1 & 4,9 & 4,0 & $-0,9$ \\
\hline & Mais-Getreide & 0,13 & 6,4 & 5,2 & $-1,2$ \\
\hline & $\begin{array}{l}\text { Futterbau- } \\
\text { Getreide }\end{array}$ & 0,04 & 2,0 & 1,6 & $-0,4$ \\
\hline & & & & & $\varnothing-0,93$ \\
\hline \multirow[t]{7}{*}{ SL4L"̈352 } & Getreide & 0,07 & 3,1 & 2,5 & $-0,6$ \\
\hline & Raps-Getreide & 0,08 & 3,6 & 2,9 & $-0,7$ \\
\hline & $\begin{array}{l}\text { Kartoffel- } \\
\text { Getreide }\end{array}$ & 0,11 & 4,9 & 3,3 & $-1,6$ \\
\hline & ZR-Getreide & 0,1 & 4,4 & 3,6 & $-0,8$ \\
\hline & Mais-Getreide & 0,13 & 5,8 & 4,7 & $-1,1$ \\
\hline & $\begin{array}{l}\text { Futterbau- } \\
\text { Getreide }\end{array}$ & 0,04 & 1,8 & 1,4 & $-0,4$ \\
\hline & & & & & $\varnothing-0,87$ \\
\hline \multirow[t]{7}{*}{$\mathrm{LT}^{2} \mathrm{~V}^{353}$} & Getreide & 0,07 & 1,6 & 1,3 & $-0,3$ \\
\hline & Raps-Getreide & 0,08 & 1,8 & 1,4 & $-0,4$ \\
\hline & $\begin{array}{l}\text { Kartoffel- } \\
\text { Getreide }\end{array}$ & 0,11 & 2,4 & 1,6 & $-0,8$ \\
\hline & ZR-Getreide & 0,1 & 2,2 & 1,8 & $-0,4$ \\
\hline & Mais-Getreide & 0,13 & 2,9 & 2,3 & $-0,6$ \\
\hline & $\begin{array}{l}\text { Futterbau- } \\
\text { Getreide }\end{array}$ & 0,04 & 0,9 & 0,7 & $-0,2$ \\
\hline & & & & & $\varnothing-0,45$ \\
\hline
\end{tabular}

Quelle: Eigene Berechnungen mit ${ }^{\mathrm{PC}} \mathrm{ABAG}$

Wie aus der Tabelle 22 ersichtlich ist, kann bei einer Hangneigung von $10 \%$ von einer Minderung der Bodenerosion von 0,45-0,93 t/ha/a ausgegangen werden. Für RapsGetreidefruchtfolgen liegen die Minderungen bei 0,4-0,7 t/ha/a. Hierbei ist zu berücksichtigen, dass bei einer Erhöhung der Hanglänge Zwischenelemente, wie z. B. Grünstreifen (Kapitel 6.3.1.6) angebaut werden müssten, um das Minderungspotenzial zu erhalten. Bei höheren Hangneigungen ist tendenziell zu erwarten, dass die Bodenabträge höher sind und dadurch stärker durch die Bewirtschaftungsrichtung zu beeinflussen sind.

\footnotetext{
${ }^{351}$ Annahme: $\mathrm{R}=41, \mathrm{~K}=0,55, \mathrm{LS}=2,43$, Hangneigung $10 \%$, Hanglänge $80 \mathrm{~m}$.

${ }^{352}$ Annahme: $\mathrm{R}=41, \mathrm{~K}=0,5, \mathrm{LS}=2,43$, Hangneigung $10 \%$, Hanglänge $80 \mathrm{~m}$.

${ }^{353}$ Annahme: $\mathrm{R}=41, \mathrm{~K}=0,25, \mathrm{LS}=2,43$, Hangneigung $10 \%$, Hanglänge $80 \mathrm{~m}$.
} 
Die in der obigen Tabelle dargestellte Verhinderung von Bodenabträgen stellt nicht den vollständigen Nutzen dar. Die verhinderten Bodenabträge können analog zu Kapitel 6.3.1.1.3 mit den ansonsten entgangenen Betriebsmitteln sowie den vergeblich eingesetzten Arbeits- und Maschinenkosten und dem möglichen Wertverlust, deren Nutzen sich auf 4,8 €/t beläuft, bewertet werden. Hieraus ergibt sich ein marginaler Nutzen von 2,1 $€ /$ ha/a bzw. $4,5 € /$ ha/a.

Eine weitere Nutzenkomponente der Bewirtschaftung quer zum Hang sind analog zur konservierenden Bodenbearbeitung ein geringeres Risiko für Umbruch und Neuansaat sowie die wiederholten Betriebsmitteleinsätze nach Starkregenereignissen. Diese Auswirkungen hängen sehr stark vom Standort ab. Da bei dieser Maßnahme jedoch im Gegensatz zur Vorgehensweise in 6.3.1.1.3 keine Hinweise in der Literatur bestehen, wird in diesem Fall der Nutzeneffekt nicht quantifiziert. Es ist jedoch anzunehmen, dass die Bewirtschaftung quer zum Hang die Auswirkungen von Starkregenereignissen mindert. Aus den Ergebnissen der folgenden KNA kann jedoch geschlossen werden, wie hoch dieser Nutzenbetrag sein müsste, damit ein ausgeglichenes KNV entsteht.

Zunächst werden in der KNA die Kosten in Form der Ertragsminderungen auf dem zusätzlichen Vorgewende berücksichtigt. Dabei wird von den Ergebnissen von BRUNOTTE et al. ${ }^{354}$ ausgegangen, dies bedeutet, dass die Minderungen 5 bzw. 27,5\% betragen. Bei einer zusätzlichen Vorgewendefläche von $2000 \mathrm{~m}^{2}$ ergibt sich für die Beispielfruchtfolge WR-WW-WG ein Erlösverlust ${ }^{355}$ von 6,0-37,0 €/ha.

Die KWA wird auf der Basis der Berechnungen der ABAG durchgeführt. Aufgrund der Kosten von 16,5 (Fall 1) bzw. 101,84 €/ha (Fall 2) ${ }^{356}$ und der Wirkung einer möglichen Reduktion von Bodenabträgen von $0,4 \mathrm{t} / \mathrm{ha} / \mathrm{a}$ sowie $0,7 \mathrm{t} / \mathrm{ha} / \mathrm{a}$ ergeben sich folgende $\mathrm{KW}$ :

\section{Fall 1: 23,5 - 41,2 $€ /$ t verhinderter Bodenabtrag}

\section{Fall 2: 145,5 - 254,6 $€ /$ t verhinderter Bodenabtrag}

Der Vergleich mit den KW der bisher berechneten Maßnahmen zeigt, dass die Bewirtschaftung quer zum Hang unter den angenommenen Bedingungen schlechtere KW als die

\footnotetext{
${ }^{354}$ Vgl. BRUNOTTE et al. (2000)

${ }^{355}$ Rapspreis $=25,18 € / \mathrm{dt}$; Weizenpreis $=11,3 € / \mathrm{dt}$; Gerstenpreis $=9,13 € / \mathrm{dt}$.

${ }^{356}$ Die Kosten bestehen aus dem Erlösverlust von 6,0 €/ha (Fall 1) sowie 37,0 €/ha (Fall 2) und den Kosten durch die Erhöhung der Vorgewendefläche von 10,5€/ha (Fall 1) und 64,8 €/ha (Fall 2).
} 
konservierende Bodenbearbeitung aufweist. Die KW ist im Fall 1 tendenziell mit der KW der Tiefenlockerungsmaßnahme vergleichbar.

In der folgenden KNA werden die Kosten von 16,5 €/ha (niedrig) bzw. 101,8 €/ha (hoch) bei dem konventionellen Verfahren mit Pflug mit dem oben ermittelten Nutzen von 2,1 €/ha bzw. 4,5 $€ /$ ha gegenübergestellt, woraus sich die Spanne bei den KNV und den durchschnittlichen jährlichen Vorteilen ergibt. Die KNV ergeben bei einem Zinssatz von $4 \%$ und einem Betrachtungszeitraum von 25 Jahren folgende Werte. Aufgrund der einfachen Berechnungen werden nur zwei Fälle mit jeweils einem Nutzen von 4,5 €/ha im Anhang in Tabelle A-25 und Tabelle A-26 ausführlich dargestellt.

Tabelle 23: KNV und jährlicher monetärer Vorteil (€/ha/a) der Bewirtschaftung quer zum Hang bei konventionellem Verfahren

\begin{tabular}{lcc}
\hline Kostenniveau & niedrig (16,5 €/ha) & hoch (101,8 €/ha) \\
\hline KNV in \% & 7,86 bzw. 3,67 & 48,48 bzw. 22,62 \\
$\varnothing$ jährl. Vorteil (€/ha/a) & $-14,4$ bzw. $-12,0$ & $-99,7$ bzw. $-97,3$ \\
\hline
\end{tabular}

Quelle: Eigene Berechnungen, Zinssatz 4 \%

In der Tabelle 23 wird deutlich, dass die Bewirtschaftung quer zum Hang bei konventionellen Produktionsverfahren bei weitem nicht wirtschaftlich ist. Daher ist zu prüfen, ob diese Maßnahme in Kombination mit konservierenden Bearbeitungsverfahren bessere Ergebnisse erzielen kann. Die natürlichen Bodenabträge auf den angenommenen Standorten betragen im Mittel 3,1 t/ha/a. Da die Bewirtschaftung quer zum Hang die Bodenabträge zwischen 0,4 und $0,7 \mathrm{t} / \mathrm{ha} / \mathrm{a}$ (gemittelter Nutzen 2,64 $€ / \mathrm{ha}$ ) mindern kann, verbleibt ein Bodenabtrag von 2,4-2,7 t/ha/a. Unter der Annahme, dass die Anwendung der konservierenden Bodenbearbeitung diese Bodenabträge auf ein Minimum (2-5\%) begrenzen kann, ergibt sich durch Multiplikation von 2,7 t/ha mit dem in 6.3.1.1.3 ermittelten Betrag von 4,8 $€$ /ha ein Nutzen für die Mulchsaat von 12,3€/ha und für die Direktsaat von $12,7 € /$ ha. Außerdem führt die Anwendung der konservierenden Bodenbearbeitung zur Verhinderung von Neuansaatkosten, was einen zehnjährigen Nutzen von $135 € /$ ha bedeutet.

Eine Analyse der Effektivität der beiden kombinierten Maßnahmen scheitert jedoch an der genauen Zuordnung der Nutzenpositionen. Außerdem fehlen Studien über die Beziehung beider Maßnahmen. Es kann daher nicht davon ausgegangen werden, dass eine kon- 
servierende Bodenbearbeitung am Hang ausreicht, um den Bodenabtrag ähnlich effektiv zu mindern. Daher können beide Maßnahmen nur als Maßnahmenbündel gesehen werden. Es werden die Berechnungen des Kapitels 6.3.1.1.3 verwendet und um die oben dargestellten Berechnungen ergänzt. Dies bedeutet, dass die Kosten der Querbearbeitnung in Höhe von 16,5 €/ha bzw. 101,8 €/ha und die gemittelten Kosten für eine zusätzliche Bearbeitungsmaßnahme in Höhe von 33,8 €/ha angesetzt werden. Somit ergeben sich bei der Mulchsaat Kosten in Höhe von 75,23 €/ha bzw. 160,53 €/ha und bei der Direktsaat Kosten in Höhe von 79,75 €/ha bzw.165,50 €/ha. Der Nutzen ist neben der Reduzierung der Neuansaatkosten in Höhe von $135 € /$ ha/10 Jahre auch durch die Kosteneinsparung gegenüber der Pflugbearbeitung und durch die Verminderung des Bodenabtrags in Höhe von 12,3€/ha bei der Mulchsaat und 12,7€/ha bei der Direktsaat gekennzeichnet. Außerdem kommt ein gemittelter Nutzen der Querbearbeitung in Höhe von 2,64 €/ha hinzu. Daher ergibt sich für die Mulchsaat ein Nutzen von $61,34 € /$ ha und für die Direktsaat von 99,94 €/ha. Die einzelnen Berechnungen sind der Tabelle A-27 bis Tabelle A-30 im Anhang zu entnehmen.

Tabelle 24: KNV und jährlicher Vorteil der Bewirtschaftung quer zum Hang in Kombination mit der konservierenden Bodenbearbeitung auf einem Standort mit einem durchschnittlichen Bodenabtrag von 3,1 t/ha/a

\begin{tabular}{|c|c|c|c|c|}
\hline & \multicolumn{2}{|c|}{ Mulchsaat } & \multicolumn{2}{|c|}{ Direktsaat } \\
\hline $\begin{array}{l}\text { Kostenniveau der Quer- } \\
\text { bearbeitung }\end{array}$ & $\begin{array}{c}\text { niedrig } \\
(16,5 € / \mathrm{ha})\end{array}$ & $\begin{array}{c}\text { hoch } \\
(101,8 € / \mathrm{ha})\end{array}$ & $\begin{array}{c}\text { niedrig } \\
(16,5 € / \mathrm{ha})\end{array}$ & $\begin{array}{c}\text { hoch } \\
(101,8 € / \text { ha })\end{array}$ \\
\hline $\mathrm{KNV}$ in $\%$ & 1,06 & 2,26 & 0,73 & 1,5 \\
\hline$\varnothing$ jährl. Vorteil (€/ha/a) & -4 & -89 & 30 & -55 \\
\hline
\end{tabular}

Quelle: Eigene Berechnungen, Zinssatz 4 \%

Unter der Voraussetzung, dass der Bodenabtrag fast gänzlich verhindert werden kann zeigt sich, dass die konservierenden Bodenbearbeitungsverfahren die KNV deutlich verbessern können. Einzig im Fall der Direktsaat ergibt sich bei einem niedrigen Kostenniveau ein effizientes KNV. Die Ergebnisse sind jedoch nur eingeschränkt verwendbar, da die Wechselbeziehungen beider Maßnahmen nur ungenügend abgebildet werden konnten und dienen daher nur als Beispiel. Außerdem wird sich die Vorteilhaftigkeit bei steigenden natürlichen Bodenabträgen weiter erhöhen. 


\subsection{Schlussfolgerungen}

Die Berechnungen im Kapitel 6.3.1.3.2 haben gezeigt, dass die Bewirtschaftung quer zum Hang nicht per se zu empfehlen ist. Eine Empfehlung muss zunächst die gegebenen Voraussetzungen der Schläge, v. a. Schlagform und -länge, berücksichtigen. In eine Empfehlung muss daher auch die Möglichkeit der Schlagzusammenlegung integriert werden. Sowohl die KW als auch die KNV zeigen, dass es sich zunächst um eine nicht wirtschaftliche Maßnahme handelt. Da davon auszugehen ist, dass der Nutzen der Maßnahme mit zunehmender Hangneigung und Hanglänge steigt, ist zu vermuten, dass sich diese Situation in extrem hängigen Lagen verbessern wird. Grundsätzlich ist für eine Verbesserung der Effizienz der Maßnahme zu empfehlen, diese mit der konservierenden Bodenbearbeitung zu kombinieren. Dies gilt v. a. dann, wenn die Technik und das entsprechende Wissen im Betrieb vorhanden sind.

\subsubsection{Engsaat von Silomais}

Der Silomaisanbau hat in den letzten Jahrzehnten stark zugenommen und dient in Futterbaubetrieben als eine wichtige Futterkomponente. Silomais ist eine Reihenfrucht, d. h. er weist nur eine geringe Bodenbedeckung auf, da die üblichen Reihenabstände $75 \mathrm{~cm}$ betragen. Außerdem entwickelt er sich erst spät, und der Boden ist demzufolge v. a. den erosiven Niederschlägen im Juni ungeschützt ausgeliefert.

Die Engsaat von Silomais ist eine Maßnahme, um die Bodenerosion durch die Erhöhung der Bodenbedeckung zu reduzieren und wurde vor einigen Jahren ursprünglich für Wasserschutzgebiete in Nordwestdeutschland entwickelt.

In diesem Kapitel wird die Engsaat von Silomais vorgestellt und anschließend erfolgt eine betriebswirtschaftliche Beurteilung und Analyse dieses Verfahrens. Anhand eines Kostenvergleichs, einer KWA und einer KNA wird die Wirtschaftlichkeit dieser Maßnahme untersucht.

\subsection{Einleitende Erwägungen}

Silomais wird üblicherweise mit einem Reihenabstand von $75 \mathrm{~cm}$ gelegt. Diese Pfadabhängigkeit ist durch die Lege- und Erntetechnik entstanden. Seit es durch landtechnische Weiterentwicklungen möglich ist, Mais reihenunabhängig zu ernten, entfällt die Notwen- 
digkeit der weiten Saat. Die Engsaat wird mit verschiedensten Reihenabständen angeboten. In dieser Arbeit wird von einer Weite von 37,5 cm ausgegangen, welche in der ldw. Praxis am weitesten verbreitet ist.

Die Vorteile der Engsaat liegen in einigen Gebieten Deutschlands, wie z. B. im Bundesland Thüringen in der Tatsache begründet, dass in Wasserschutzzonen II und IIIa der Maisanbau nur mit diesem Verfahren zulässig ist. Außerdem liegt ein weiterer entscheidender Vorteil in der besseren Nährstoff- und Wasserausnutzung, in einem höheren Ertrag sowie in der früheren Bodenbedeckung und somit im Erosionsschutz. Außerdem kann durch den früheren Lückenschluss im Bestand von geringeren Pflanzenschutzmittelaufwendungen ausgegangen werden.

\subsection{Betriebswirtschaftliche Analyse der Maßnahme}

Im folgenden Kapitel wird neben einer KWA auch eine KNA für die Engreihensaat von Silomais durchgeführt. Die Vorgehensweise ist der Übersicht $11 \mathrm{zu}$ entnehmen.

\section{Übersicht 11: Vorgehensweise bei der KNA für die Engsaat von Silomais}

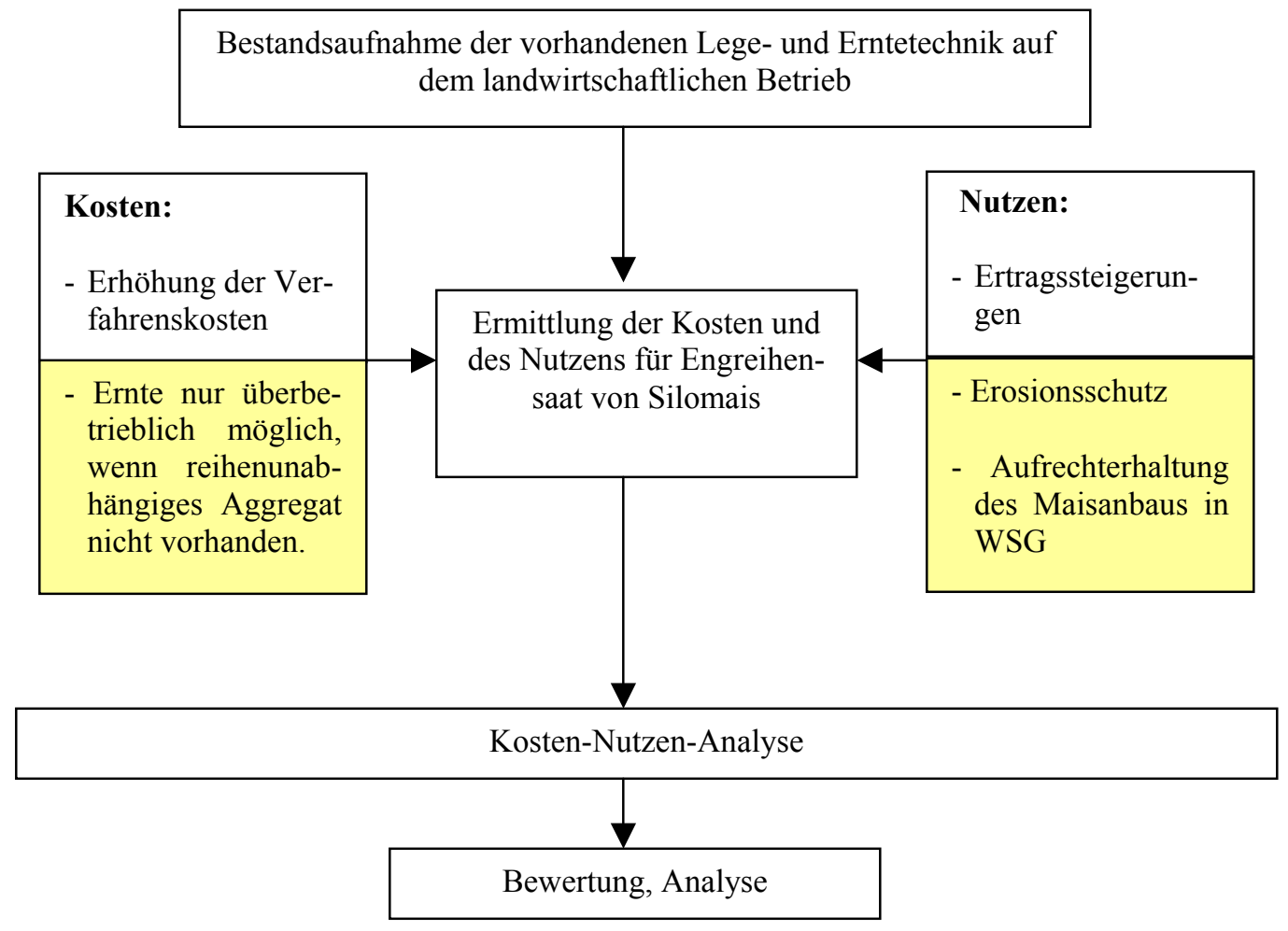

Quelle: Eigene Darstellung, Schraffierung: Positionen konnten aufgrund fehlender Daten in der Berechnung nicht berücksichtigt werden 
Im Folgenden wird für Silomais zunächst das Standardverfahren $(75 \mathrm{~cm})$ mit der Engsaat $(37,5 \mathrm{~cm})$ bezüglich der dafür aufzuwendenden Kosten verglichen. Zunächst müsste auf dem Einzelbetrieb eine Maislegemaschine angeschafft werden, welche die Voraussetzungen der Engsaat erfüllt. Um eine Vergleichbarkeit beider Maschinen zu gewährleisten, werden Maschinen mit gleicher Schlagkraft und gleichem Arbeitsergebnis (4,50 m Arbeitsbreite, 12 Legeaggregate mit Unterfußdüngung) unterstellt. Der Anschaffungspreis von $40.500 €$ liegt im Vergleich zur konventionellen Maschine um ca. $28.500 €$ höher. Dieser höhere Preis der 37,5 cm-Maschine begründet sich zum einen durch die zusätzlichen Kosten der Legeschare und zum anderen durch die aufwendigere Unterfußdüngung. Die Umrüstung einer bereits vorhandenen Maschine wird hierbei nicht berücksichtigt, da sie in einigen Fällen aufgrund technischer Probleme auch nicht erfolgen kann. Die $37,5 \mathrm{~cm}$-Legemaschine hat einen höheren Zugkraftbedarf (160 PS) als die konventionelle Legemaschine (120 PS). Im Falle der Eigenmechanisierung muss durch den geringeren Reihenabstand die Erntetechnik in der Hinsicht angepasst werden, dass ein reihenunabhängiges Schneidwerk für den Feldhäcksler angeschafft wird. Die Kosten hierfür würden sich laut KTBL-TASCHENBUCH auf etwa 22.000-30.000 € belaufen. Da die Ernte im folgenden Beispiel durch Fremdleistung erfolgen soll und in Zukunft zu erwarten ist, dass sich die Kosten für reihenabhängige und -unabhängige Schneidwerke annähern werden, wird auf einen Vergleich verzichtet.

Tabelle 25: Vergleich der Aussaatkosten bei Silomais

\begin{tabular}{l|c|c}
\hline Reihenabstände & $75 \mathrm{~cm}$ & $37,5 \mathrm{~cm}$ \\
\hline Kostenpositionen & 6-reihige Einzelkornsaat, & 12-reihige Einzelkornsaat, \\
$(€ /$ ha) & $4,5 \mathrm{~m}, \mathrm{UFD}$ & $4,5 \mathrm{~m}, \mathrm{UFD}$ \\
& $88 \mathrm{~kW}$ & $118 \mathrm{~kW}$ \\
& $0,67 \mathrm{~h} / \mathrm{ha}$ & $0,45 \mathrm{~h} / \mathrm{ha}$ \\
\hline Var. Maschinenkosten & 11,02 & 10,89 \\
& & \\
Maschinenfestkosten & & \\
$\begin{array}{l}\text { Arbeitskosten } \\
\begin{array}{l}\text { Summe Aussaatkos- } \\
\text { ten }\end{array}\end{array}$ & 21,33 & 45,02 \\
\hline
\end{tabular}

Quelle: Eigene Berechnungen nach KTBL (2002), S. 318 u. Peyker, HenKel U. HochBERG (2000)

\footnotetext{
${ }^{357}$ Bei der 6-reihigen Einzelkornsämaschine wird eine Auslastung von 333 ha/a und bei der 12-reihigen aufgrund der höheren Arbeitsgeschwindigkeit eine Auslastung von 500 ha/a angenommen.
} 
Die Berechnung zeigt, dass die Engsaat von Mais für dieses Beispiel etwa 20,62 €/ha teurer als die konventionelle Aussaat ist. Dieser Wert ist somit, als Kostenposition für die weitere Berechnung des KNV zu übernehmen. Durch die Veränderung des Pflanzschemas kommt es nicht zu einer Erhöhung des Saatguteinsatzes, der 90.000 - 100.000 Pflanzen/ha beträgt, weshalb keine zusätzlichen Saatgutkosten anzusetzen sind. Durch die Engsaat von Silomais verlieren die Pflanzen ihre Eigenschaft als Hackfrüchte. Da davon ausgegangen wird, dass in der landwirtschaftlichen Praxis kaum noch zu Silomais gehackt wird, werden eventuell anfallende höhere PSM-Kosten nicht berücksichtigt.

Der Nutzen der Engsaat des Maises liegt zunächst in dem gestiegenen Ertrag. In Feldversuchen der LWK Weser-Ems und Rheinland-Pfalz wurden Ertragssteigerungen von 7-21\% festgestellt, in einem Projekt der THÜRINGER LANDESANSTALT FÜR LANDWIRTSCHAFT $^{358}$ wurden Ertragsvorteile von $9 \%$ ermittelt. Selbst in einem extremen Trockenjahr wie 2003 waren in Hessen noch höhere Erträge von 2 \% zu realisieren, wovon in der folgenden Berechnung allerdings nicht ausgegangen wird. ${ }^{359}$

Bei einem durchschnittlichen Silomaisertrag von $450 \mathrm{dt} /$ ha bedeuten die oben dargestellten Ertragssteigerungen einen Mehrertrag in der Spanne von 31,5-94,5 dt/ha, der dem Betrieb etwa 3244,5-9761,85 MJ NEL zusätzlich an Futternährstoffen liefert. Nach dem Ersatzwertverfahren ${ }^{360}$ wird der zusätzliche Energiebetrag mit den Kosten der Energie aus Milchleistungsfuttermitteln (18\% Protein, Energiestufe III) bewertet. Bei einem durchschnittlichen Preis von $14,55 € / \mathrm{dt}^{361}$ ergeben sich Werte von $68,13-205 € /$ ha. In der KNA werden die Werte für die Ertragssteigerungen variiert. Die jeweiligen Nutzenbeiträge können der Tabelle A-5 entnommen werden.

Durch den weiteren Abstand innerhalb einer Reihe kann eine höhere Fahrgeschwindigkeit von 3-4 km/h realisiert werden, was in etwa einer Steigerung von $50 \%$ bedeutet. Diese sollte jedoch nicht völlig ausgeschöpft werden, da oberhalb von Fahrgeschwindigkeiten von 6-7 km/h eine optimale Ablage nicht mehr gewährleistet werden kann. ${ }^{362}$ Deshalb wird in den folgenden Berechnungen von einer Arbeitsersparnis von $33 \%$ ausge-

\footnotetext{
${ }^{358}$ Vgl. Peyker, HenKel U. HochberG (2000).

${ }^{359}$ Vgl. SCHMitT U. FISCH (2003), S. 10

${ }^{360}$ Vgl. KÖHNE (2000), S. 555 ff.

${ }^{361}$ Vgl. LWK-HANNOVER (2002), S. 14

${ }^{362}$ Vgl. hierzu KTBL (2000b)
} 
gangen. ${ }^{363}$ Nachteile liegen in der Praktikabilität bei der Düngung und beim Pflanzenschutz, da bei der Engsaat Fahrgassen angelegt werden müssen, deren Breite sich sowohl an der Pflanzenschutz- als auch an der Wirtschaftsdüngerausbringung orientieren muss.

Einen weiteren Nutzeneffekt, der jedoch nicht direkt zu quantifizieren ist, stellt der Erosionsschutz dar. Im Versuch der Thüringer Landesanstalt für Landwirtschaft konnte gezeigt werden, dass der Reihenschluss bei der Maisengsaat in den drei Versuchsjahren im Durchschnitt etwa 23 Tage eher erfolgte. ${ }^{364}$ Dies hat zur Folge, dass auch die vorigen Kulturperioden um 23 Tage vorverlegt werden müssten. Dies gilt allerdings nicht für die Aussaat, da die Gefahr von Spätfrösten sehr hoch sein könnte und davon auszugehen ist, dass der Landwirt bereits einen diesbezüglich optimalen Saatzeitpunkt gewählt hat. Eine exakte Berechnung des dadurch verhinderten Bodenabtrages ist aufgrund fehlender Daten nicht möglich. Allerdings lässt sich eine grobe Abschätzung des Effekts vornehmen. Ausgehend von den Daten von SCHWERTMANN et al. hat Silomais in einer Fruchtfolge mit Winterweizen und Wintergerste einen C-Faktor von $0,32642 .{ }^{365}$ Durch die Verschiebung der Entwicklung der Maispflanzen verschieben sich die Kulturperioden und dadurch der R-Faktor-Anteil. Der neue C-Faktor beträgt 0,288695. Der C-Faktor würde also noch weiter sinken, allerdings eine sehr geringe Reduzierung des Bodenabtrages bedeuten. Dieses Ergebnis ist allerdings aufgrund der sehr groben Schätzung nicht geeignet, um den Nutzen durch die Reduktion des Bodenabtrages hinreichend abzubilden und wird daher in der folgenden KNA nicht herangezogen und dient zur Darstellung der Vorgehensweise.

Zusammenfassend erfolgt eine KNA, in der die in der Tabelle 25 ermittelten Kosten von 20,62 €/ha mit verschiedenen Ertragssteigerungen als verbleibenden Nutzen gegenübergestellt werden. Dabei werden die obigen Annahmen bzgl. des Silomaisertrages von $450 \mathrm{dt} / \mathrm{ha}$ und den sich daraus ergebenden Nutzen durch die entsprechenden Ertragssteigerungen $(2 \%$-Ertragssteigerung $=19,5 € /$ ha und $22 \%$-Ertragssteigerung $=214,8 € /$ ha $)$ verwendet. Der Betrachtungszeitraum der KNA beträgt 25 Jahre und der Zinssatz 4 \%. Die KNA zeigt, dass sich gute bis sehr gute KNV ergeben. Die Berechnungen sind ausführlich, aus Platzgründen allerdings nur für die Ertragssteigerung von $2 \%$ und $22 \%$, in

\footnotetext{
${ }^{363}$ Vgl. hierzu auch PeYKer, HenKel, HochBerg (2000)

${ }^{364}$ Vgl. PeyKer, HenKel u. HochBerg (2000), S. 6

${ }^{365}$ Vgl. SCHWERTMANN et al. (1987), S. 47
} 
der Tabelle A-31 und Tabelle A-32 aufgeführt. Die einzelnen KNV sind in der Tabelle 26 zusammengefasst.

Tabelle 26: KNV und jährlicher Vorteil der Engsaat von Silomais bei verschiedenen möglichen Ertragssteigerungen

\begin{tabular}{lcccccc}
\hline Ertragssteigerung & $\mathbf{2 \%}$ & $\mathbf{6 \%}$ & $\mathbf{1 0} \%$ & $\mathbf{1 4} \%$ & $\mathbf{1 8 \%}$ & $\mathbf{2 2} \%$ \\
\hline KNV in \% & 1,06 & 0,53 & 0,21 & 0,15 & 0,12 & 0,1 \\
$\varnothing$ jährl. Vorteil ( $€ / \mathrm{ha} / \mathrm{a})$ & -1 & 18 & 77 & 117 & 155 & 194 \\
& & & & & & \\
\hline
\end{tabular}

Quelle: Eigene Berechnungen, Zinssatz $4 \%$

In der obigen Tabelle ist zu sehen, dass für die Engsaat von Mais ohne Berücksichtigung des Nutzens des Erosionsschutzes in diesem Fall Ertragssteigerungen benötigt werden, die geringfügig über $2 \%$ liegen. Es muss also für dieses Beispiel ein Nettoertrag von über $460 \mathrm{dt} / \mathrm{ha}$ erzielt werden. Da bereits fast ausschließlich KNV kleiner eins vorherrschen, ist prinzipiell kein Nutzen durch den Erosionsschutz nötig, er würde aber zu einer Verbesserung der Wirtschaftlichkeit dieser Maßnahme führen. Ein weiterer wichtiger und auf einzelbetrieblicher Ebene zu berücksichtigender Einflussfaktor auf den Erfolg dieser Maßnahme ist die Auslastung der Sämaschine im Falle der Eigenmechanisierung. Dieses Problem stellt sich jedoch auch bei der konventionellen Technik. In der landwirtschaftlichen Praxis wird in den Silomaisanbaugebieten daher die Aussaat des Maises überbetrieblich gestaltet.

\subsection{Schlussfolgerungen}

Die Engreihensaat von Silomais kann für landwirtschaftliche Betriebe empfohlen werden. Durch die Durchbrechung der Pfadabhängigkeit aufgrund der vorhandenen Erntetechnik entstehen zwar zusätzliche Kosten, diese können jedoch mit hoher Wahrscheinlichkeit durch den Nutzen der Maßnahme gedeckt werden. Je nach Betriebsgröße und Maisanbaufläche sollte jedoch über eine überbetriebliche Arbeitserledigung nachgedacht werden. Bezüglich des Erosionsschutzes dieser Maßnahme besteht noch direkter Forschungsbedarf zur Ermittlung der Reduzierung des Bodenabtrages. Hier sollten direkte Bodenabtragsmessungen durchgeführt werden, um den Erosionsschutzeffekt direkt zu messen. Diese Ergebnisse sollten auf Feldtagen oder in Rundschreiben neben den Landwirten v. a. auch den Lohnunternehmern und Maschinenringen zukommen. Gerade da es sich beim 
Silomais um eine erosionsanfällige Reihenkultur handelt und diese in vielen Betrieben unverzichtbar ist, könnte die Anwendung der Engreihensaat ein Argument bzw. ein Kompromiss für die Beibehaltung des Maisanbaus auch in kritischen Gebieten, wie z. B. leicht hängigen Lagen oder WSG, sein.

\subsubsection{Anbau von Zwischenfrüchten und Untersaaten}

In den folgenden Abschnitten werden die Maßnahmen des Zwischenfrucht- und Untersaatenanbaus dargestellt und anhand der KWA und KNA auf ihre Wirtschaftlichkeit und ökologische Effizienz hin untersucht.

\subsection{Einleitende Erwägungen}

Die Bedeutung des Zwischenfruchtanbaus in Deutschland kann nur anhand der Futterzwischenfrüchte deutlich gemacht werden. Diese wurden bis Anfang der 90er Jahre auf etwa 750.000-800.000 ha angebaut. Der Anbau von Gründüngungspflanzen wie Gelbsenf oder Ölrettich werden hingegen statistisch nicht erfasst. Nach der Einführung der EUAgrarreform 1992 sank der Anbau der Futterzwischenfrüchte auf die heutige Fläche von etwa 250.000 ha. Dies hat zum einen mit dem Instrument der Flächenstillegung, zum anderen mit der Einschränkung des Anbaus früh zu erntender Getreidevorfrüchte zu tun. ${ }^{366}$ Bei der Untersaat ist zudem die mangelnde Praxisreife von Verfahren in Getreidefruchtfolgen zu beobachten. Gute bis sehr gute Erfahrungen liegen hingegen bei Untersaaten in Mais oder Ackerbohnen vor. ${ }^{367}$

Beim Zwischenfrucht- und Untersaatenanbau (ZUA) handelt es sich um Maßnahmen, die eine höhere Bodenbedeckung gewährleisten und somit einen Erosionsschutz erzeugen. Der ZUA weist jedoch nicht nur Vorteile auf, sondern führt auch zu den folgenden Problemen, die in der landwirtschaftlichen Praxis häufig als Argument gegen den ZUA herangezogen werden:

- Zusätzlicher Arbeitsaufwand, Entstehung von Arbeitsspitzen,

- Zusätzliche Kosten,

\footnotetext{
${ }^{366}$ Vgl. LÜTKE-ENTRUP (2001), S. 8 f.

367 Vgl. BRODOWSKI U. DÖRENDAHL (2001), S. 3
} 
- Konkurrenz um Wasser bei Niederschlägen unter 500-550 mm/a bzw. Höhenlagen > $350 \mathrm{~m}$,

- Fehlende Saatmöglichkeiten bei verspäteter Hauptfruchternte und

- Durchwuchsgefahr bei lagerndem Getreide.

Durch die pflanzenbau(technische) Forschung sind allerdings in der Vergangenheit einige Lösungsmöglichkeiten entwickelt worden:

- Kombination von Stoppelbearbeitung und Zwischenfrucht- und Untersaatenaussaat um die zusätzlichen Aussaatkosten zu reduzieren,

- Aussaat kostengünstiger Zwischenfrüchte und Untersaaten (z. B. Senf, Ölrettich),

- Walzen nach der Aussaat, um bei trockenen Bedingungen die Keimung anzuregen und

- Verzicht auf wasserzehrende Bodenbearbeitungsmaßnahmen (z. B. Pflugeinsatz).

Im Folgenden werden Kosten und Nutzen der ZUA ermittelt und in einer Analyse zusammengeführt.

\subsection{Betriebswirtschaftliche Analyse der Maßnahme}

Die Vorgehensweise der KNA dieser Maßnahme ist der Übersicht 12 zu entnehmen. 


\section{Übersicht 12: Vorgehensweise bei der KNA für den ZUA}

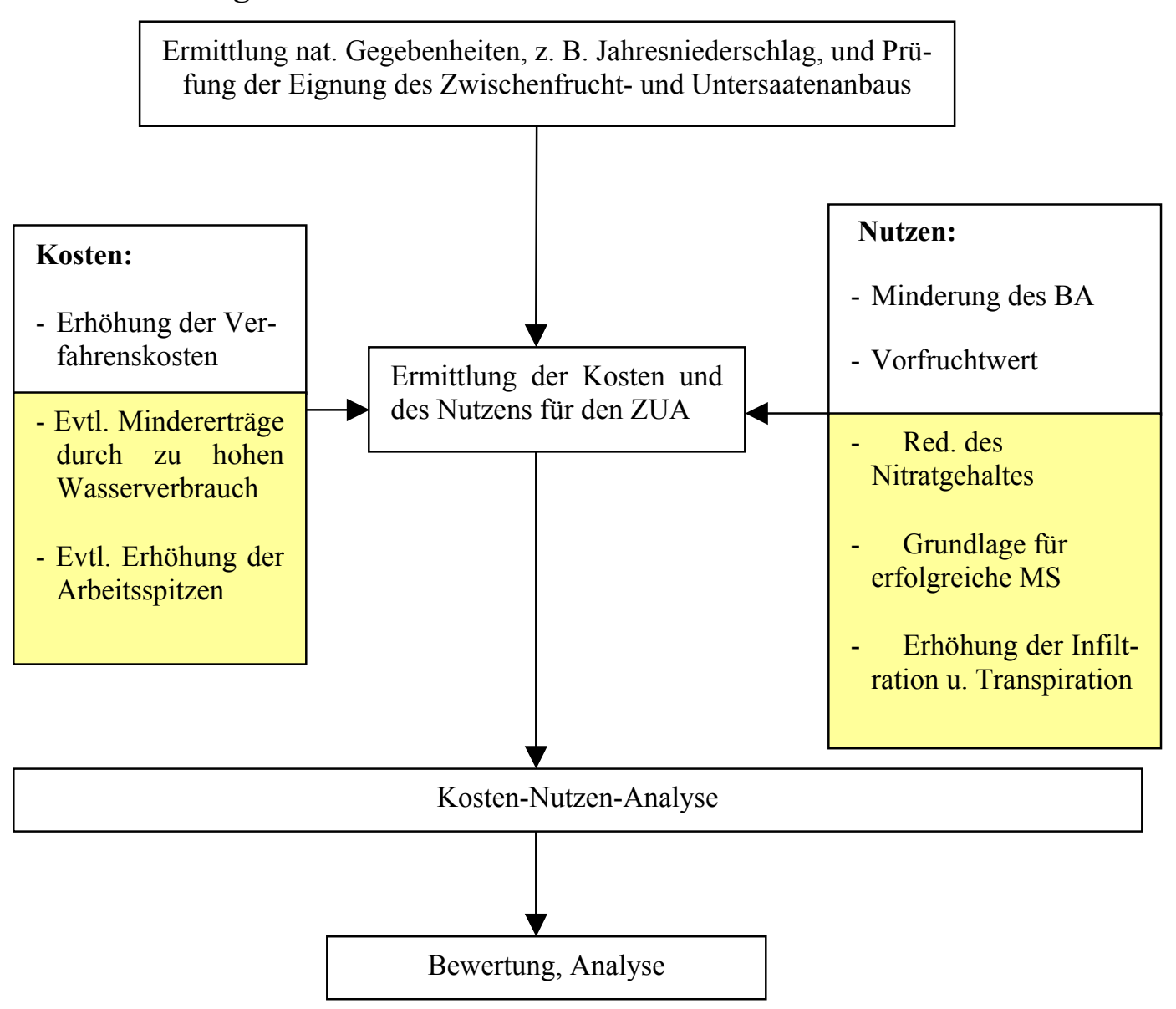

Quelle: Eigene Darstellung, Schraffierung: Positionen konnten aufgrund fehlender Daten in der Berechnung nicht berücksichtigt werden

Zunächst werden in der folgenden Tabelle v. a. die zusätzlichen Kosten und Erlöse des Zwischenfruchtanbaus dargestellt. Die Kostenspanne resultiert aus der extensiven Form des Zwischenfruchtanbaus, d. h. Blanksaat und der intensiven Form, d. h. Einsatz der Drillmaschine. Die Kosten können jedoch in Einzelfällen durch eine intensive Saatbettbereitung noch höher liegen und im Mittel $110 €$ /ha erreichen. ${ }^{368}$ 
Tabelle 27: Kosten und N-Bindung verschiedener Zwischenfrüchte u. Untersaaten am Beispiel von Sommergerste

\begin{tabular}{lccc}
\hline Zwischenfrucht & $\begin{array}{c}\text { Verfahrens- } \\
\text { kosten } \\
\text { (intensiv- ex- } \\
\text { tensiv) } \\
\text { (6/ha }\end{array}$ & $\begin{array}{c}\text { Mehrerlös } \\
\text { bei Gründer SG } \\
\text { als Vorfrucht }\end{array}$ & Mögliche N-Bindung \\
& $42-65$ & $\boldsymbol{\epsilon} / \mathbf{h a}$ & kg N/ha \\
\hline Gelbsenf & $48-71$ & 46,2 & $90-130$ \\
Phacelia & $50-73$ & 42,0 & $75-105$ \\
Ölrettich & $52-66$ & 50,0 & $135-160$ \\
Feldgras & $104-127$ & - & $90-135$ \\
Grünroggen & $0-43$ & - & $90-135$ \\
Raps (i. d. R. Auflauf- & & 44,5 & $75-135$ \\
raps & &
\end{tabular}

Quelle: KTBL (2002), S. 304, 314, Frede U. DabBert (1998), S. 63 u. BerendonK (2003), S. 3

Die Verfahrenskosten bestehen zum größten Teil aus den Saatgutkosten. Auflaufraps verursacht somit die geringsten Kosten. Die hohen Verfahrenskosten von Grünroggen resultieren aus den Saatgutkosten von rd. $100 € /$ ha. Es wird hier bereits deutlich, dass die Kosten der extensiven Verfahren zum größten Teil durch die Mehrerlöse durch den Gründüngungseffekt gedeckt werden können. Der ZUA führt allerdings zu weiteren Nutzeneffekten.

Die folgende Nutzenbetrachtung wird aufgrund fehlender Daten und Versuchsergebnisse auf den Zwischenfruchtanbau beschränkt. Zum größten Teil ist jedoch anzunehmen, dass durch den Untersaatenanbau ähnliche Nutzeneffekte zu erzielen sind. Durch den Zwischenfruchtanbau wird ein höherer Bodenbedeckungsgrad auf der genutzten Fläche erreicht. Dieser führt gerade bei Reihenfrüchten dazu, dass der Bodenabtrag vermindert wird. SCHÄFER $(1986)^{371}$ stellt z. B. in einem Versuch bei Mais auf einem hängigen Schlag fest, dass der Bodenabtrag beim Maisanbau mit den Zwischenfrüchten Senf, Klee und Gras auf 26-32 \% gegenüber der Schwarzbrache reduziert werden kann. Dies führt, wie die Tabelle 28 zeigt, bei den dort angegebenen standortabhängigen Bodenabträgen und einem Zinssatz von $4 \%$ zu einem Nutzen von 6,2 bis $32,2 € /$ ha.

\footnotetext{
${ }^{369}$ inklusive Saatgutkosten; Anbauschleuderstreuer (1,0 t) + Schlepper (54 kW), bzw. Sämaschine $(3 \mathrm{~m})+$ Schlepper (45 kW).

${ }^{370}$ Bei Sommergerste, Preis $=8,4 € / \mathrm{dt}$.

${ }^{371}$ Vgl. SCHÄFER (1986), zit. in LÜTKE-ENTRUP (2001), S. 58
} 
Tabelle 28: Monetärer Nutzen sowie KW durch die Verminderung des Bodenabtrages durch Zwischenfruchtanbau

\begin{tabular}{|c|c|c|c|}
\hline $\begin{array}{c}\text { Durchschnittlicher } \\
\text { Bodenabtrag } \\
\text { (t/ha) }\end{array}$ & $\begin{array}{c}\text { Verminderter Bo- } \\
\text { denabtrag } \\
(\mathbf{t} / \mathbf{h a} / \mathbf{a})\end{array}$ & $\begin{array}{c}\text { Monetärer Nut- } \\
\text { zen }^{372} \\
(€ / \mathbf{h a} / \mathbf{a})\end{array}$ & $\begin{array}{c}\text { KW }^{373} \\
(€ / \text { t verh. BA) }\end{array}$ \\
\hline 5 & $1,3-1,6$ & $6,2-7,7$ & $34,3^{374}-82,5$ \\
\hline 9 & $2,3-2,9$ & $11,0-14,0$ & $19,4-45,5$ \\
\hline 13 & $3,4-4,2$ & $16,3-20,2$ & $13,1-31,4$ \\
\hline 17 & $4,4-5,4$ & $21,1-26,0$ & $10,1-24,4$ \\
\hline 21 & $5,5-6,7$ & $26,4-32,2$ & $8,1-19,7$ \\
\hline
\end{tabular}

Quelle: Eigene Berechnungen

Die obige Tabelle zeigt, dass sich in diesem Beispiel die KW je nach Standort bei angenommenen Kosten von $43 € /$ ha bzw. $127 € /$ ha und der oben genannten Wirkung von 26$32 \%$ im Bereich von 8,1- 82,5€/t verhinderter Bodenabtrag bewegen. Demnach bewegen sich die KW je nach Standort in sehr guten aber auch in sehr ungünstigen Bereichen. Ein direkter Vergleich mit den bisher errechneten KW ist daher nicht aussagekräftig.

Eine weiterer Nutzeneffekt des Zwischenfruchtanbaus ist der Vorfruchtwert und somit die Verbesserung der Bodenstruktur und die Nährstofffixierung. Den Einfluss einer Zwischenfrucht auf den Ertrag hat BERENDONK ${ }^{375}$ in 5-jährigen Versuchen bei verschiedenen Zwischenfrüchten, wie bereits in Tabelle 27 gezeigt wurde, untersucht. Als Hauptfrucht diente Sommergerste, die in Monokultur angebaut wurde. LÜTKE-ENTRUP ${ }^{376}$ kann in dreijährigen Feldversuchen nachweisen, dass der Mehrertrag bei Zuckerrüben mit Zwischenfrucht Senf bzw. Phacelia 11-27 dt/ha und bei Winterweizen 0,5-4,6 dt/ha beträgt. Bei den Zuckerrüben bedeutet dies einen Mehrerlös von 52-127 €/ha und beim Winterweizen 5-50 €/ha. ${ }^{377}$ Zum Vergleich ermitteln BRUNOTTE, Roth U. HollmanN ${ }^{378}$ für Zuckerrüben einen Mehrertrag im Mittel von fünf Jahren von $30 \mathrm{dt} / \mathrm{ha}(141 € / \mathrm{ha})$ in Südniedersachsen, in der Soester Börde Mehrerträge von 11 bzw. 27 dt/ha (51,7 bzw. $€ /$ ha) und am Standort Völkenrode 60 bzw. 72 dt/ha. (282 bzw. 338,4 €/ha). Bei Winter-

\footnotetext{
${ }^{372}$ Siehe Kapitel 6.3.1.1.3, es wird der Wert 4,8 €/t verwendet.

${ }^{373}$ An dieser Stelle werden die Mittelwerte der Spalte 2 verwendet. Zur Ermittlung der KW siehe auch Kapitel 6.2.2.

${ }^{374}$ Rechenweg: $43 € / \mathrm{h}: 1,3 \mathrm{t} / \mathrm{ha} * 0,04=34,3 € / \mathrm{t}$.

375 Vgl. BERENDONK (2003), S. 3

${ }^{376}$ Vgl. LÜTKE-ENTRUP (1990), zit. in BRUNOTTE et al. (1994), S. 314

377 Angenommene Preise aus FID (2002). Weizenpreis (A-Backqualität) $=10,93 € / \mathrm{dt}$; Zuckerrübenpreis $($ A-Rüben $)=4,7 € / \mathrm{dt}$.

${ }^{378}$ Vgl. BRunOtTe, Roth u. Hollmann (1994), S. 312
} 
weizen betrugen die Mehrerträge 3,3 bzw. 3,6 dt/ha. Dies entspricht einem Mehrerlös von 36,1 bzw. $39,3 € /$ ha.

Eine andere Möglichkeit der einzelbetrieblichen Verwertung von Zwischenfrüchten besteht in der Futternutzung. Zwar wurde die Futternutzung von Zwischenfrüchten in der Vergangenheit durch den Maisanbau stark zurückgedrängt. Dennoch können einzelne Betriebe ohne ausreichendes Grünland dieses zusätzliche Grundfutter sehr gut verwerten, hinsichtlich der Proteinversorgung der Milchviehherde sind einige darauf angewiesen. Um den Nutzen der Futterlieferung der Zwischenfrüchte zu bewerten, wird der Ersatzwert $^{379}$ des zusätzlichen Futters ermittelt, da davon ausgegangen wird, dass in dem landwirtschaftlichen Betrieb lediglich ein Futterausgleich stattfindet und die Produktion deshalb nicht ausgedehnt werden kann.

Bei einem durchschnittlichen Grünertrag von 30 t/ha kann ein Bruttoenergieertrag (NEL) von etwa 31.500 MJ NEL/ha erzielt werden. Nach Abzug der Konservierungsverluste ergibt dies einen Nettoenergieertrag von 23.630 MJ NEL/ha. ${ }^{380}$ Dieser Betrag kann folglich mit den Kosten der Energie in Milchleistungsfuttermitteln (18\% Protein, Energiestufe III) bewertet werden. Bei einem mittleren Preisniveau von $14,55 € / \mathrm{dt}^{381}$ ergibt sich ein Wert von 496,23 €/ha.

Ein weiterer Nutzeneffekt des ZUA können in zusätzlichen Ausgleichszahlungen, z. B. im Rahmen der nationalen Modulation, bestehen. In Deutschland liegt der Prämiensatz für den ZUA bei $63-108 € /$ ha. $^{382}$ Da allerdings fraglich ist, ob die Prämien für den ZUA generell bestehen bleiben und Unterschiede in den einzelnen Bundesländern bestehen, wird die Berechnung der KNA ohne Prämien durchgeführt.

Ein anderer Vorteil des Zwischenfruchtanbaus ist die Reduzierung des Nitratgehalts im Boden. LÜTKE-ENTRUP ${ }^{383}$ zeigt dies in einem Versuch für einen Lösslehmstandort (Marktfruchtbaubetrieb) und einen Sandstandort (Futterbaubetrieb), in denen verschiedene Zwischenfrüchte (Phacelia, Sommerrübsen u. Welsches Weidelgras) angebaut wurden (Tabelle 29). Über einen Zeitraum von vier Jahren waren die $\mathrm{NO}_{3}$-Gehalte im Mittel aller

\footnotetext{
${ }^{379}$ Vgl. KÖHNE (2000), S. 555 ff.

${ }^{380}$ Vgl. hierzu KTBL (2002), S. 145 sowie LWK Hannover (2002), S. 54

${ }^{381}$ Vgl. LWK-HANNOVER (2002), S. 14, gemittelter Preis.

${ }^{382}$ Vgl. BMVEL (2002a), S. 3

${ }^{383}$ Vgl. LÜTKE-ENTRUP (2001), S. 58 f.
} 
drei Zwischenfrüchte um 68-85 \% geringer als bei der Schwarzbrache. Damit leistet der ZUA nicht nur einen Beitrag zum physikalischen sondern auch zum stofflichen Bodenschutz und wird daher in vielen Wasserschutzgebieten explizit gefördert.

Tabelle 29: Relatives Reduktionspotenzial des Nitratgehalts durch ausgewählte Zwischenfrüchte im Boden (0-90 cm Tiefe) bei Vegetationsruhe im Herbst (Mittel von vier Erntejahren)

\begin{tabular}{lcc}
\hline \multicolumn{1}{c}{ Zwischenfrucht } & $\begin{array}{c}\text { Lösslehm } \\
\text { (Markfruchtbau) } \\
\text { Rel. NO } \text { 3 }^{-N}-\text { Gehalte }\end{array}$ & $\begin{array}{c}\text { Sand (Futterbau) } \\
\text { Rel. NO } \text { N }_{\mathbf{3}} \text {-N- } \\
\text { Gehalte }\end{array}$ \\
\hline Schwarzbrache & $100(128 \mathrm{~kg} / \mathrm{ha})$ & $100(163 \mathrm{~kg} / \mathrm{ha})$ \\
Phacelia & 30 & 18 \\
Sommerrübsen & 15 & 20 \\
Welsches Weidelgras & 25 & 32 \\
\hline
\end{tabular}

Quelle: Verändert nach LÜTKE-ENTRUP (2001), S. 58

Dieser Nutzeneffekt wird an dieser Stelle nicht monetär bewertet, da es sich in erster Linie um einen externen Effekt handelt. Durch die verringerte Auswaschung des Nitrats kommt es zu Minderungen der Denitrifikation und stofflichen Belastungen der Oberflächengewässer und des Grundwassers. Eventuell auftretende Vorteile aus Sicht der Pflanzenernährung spiegeln sich bereits in den Mehrerträgen wieder. Außerdem ist dieser Nutzeneffekt, wenn er monetär betrachtet wird, als marginal zu bezeichnen.

Ein weiterer Nutzeneffekt des Zwischenfruchtanbaus ist die gute Kombinationsmöglichkeit mit Mulchsaatverfahren. Außerdem erhöhen Zwischenfrüchte die Transpiration um bis zu $150 \mathrm{~mm} .{ }^{384}$ Dies ist in Hinblick auf trockene Standorte ein sehr wichtiger Vorteil, um die Erträge zu steigern, bzw. Ertragsschwankungen zu reduzieren. Außerdem spielt es in einigen Gebieten für den vorsorgenden Hochwasserschutz eine wichtige Rolle, da es die Speicherkapazität des Bodens bei nachfolgenden Niederschlägen erhöhen kann. Anzunehmen ist auch, dass der Zwischenfruchtanbau zu einer Verringerung des Risikos durch Starkregeneffekte führt. Allerdings finden sich in der Literatur keine diesbezüglichen Hinweise.

\footnotetext{
${ }^{384}$ Vgl. FELDWISCH (1999), S. 33
} 


\section{Kosten-Nutzen-Analyse}

Im Folgenden wird aufbauend auf die oben getroffenen Kosten- und Nutzenannahmen eine KNA für den Zwischenfruchtanbau durchgeführt. Dabei wird von verschiedenen Varianten ausgegangen, um die Komplexität der Wirkungszusammenhänge abzubilden. Bei der Variante 1 werden die extensiven Verfahrenskosten von $43 € /$ ha und ein niedriger Nutzen von $49,3 € / \mathrm{ha}^{385}$ angenommen. In der Variante 2 wird mit den intensiven Verfahrenskosten von $127 € /$ ha und ebenfalls einem geringen Nutzen von 49,3 €/ha gerechnet. In der Variante 3 betragen die Kosten $127 € /$ ha und der Nutzen 173,2 €/ha. ${ }^{386}$ In der letzten Variante 4 betragen die Kosten $43 € /$ ha und der Nutzen 173,2 €/ha. Der Betrachtungszeitraum beträgt 25 Jahre. Bei dieser Betrachtung wurde nicht die eventuell gezahlte Förderprämie von 63-108 €/ha hinzugerechnet. Die ausführlichen Berechnungen können der Tabelle A-33 bis Tabelle A-36 entnommen werden.

\section{Tabelle 30: KNV und jährlicher Vorteil verschiedener Varianten des Zwischen-}

\section{fruchtanbaus}

\begin{tabular}{lcccc}
\hline Variante & $\mathbf{1}$ & $\mathbf{2}$ & $\mathbf{3}$ & $\mathbf{4}$ \\
\hline KNV in \% & 0,87 & 2,58 & 0,73 & 0,25 \\
$\varnothing$ jährl. Vorteil (€/ha/a) & 6,3 & -78 & 46 & 130 \\
\hline
\end{tabular}

Quelle: Eigene Berechnungen, Zinssatz $4 \%$

In der Tabelle 30 sind die Ergebnisse der KNV sowie die durchschnittlichen jährlichen Vorteile für die vier verschiedenen Varianten dargestellt. Lediglich die Variante 2 weist ein schlechtes KNV und einen jährlichen Nachteil aus. Der Grund hierfür liegt in den hohen Ansaatkosten. Außerdem ist anzumerken, dass zusätzliche Transferzahlungen die Wirtschaftlichkeit der Varianten weiter verbessern.

\subsection{Schlussfolgerungen}

Der Zwischenfruchtanbau ist aus Sicht des Bodenschutzes für landwirtschaftliche Betriebe, die eine entsprechende Wasserbilanz aufweisen, sehr zu empfehlen. Die Maßnahme

\footnotetext{
${ }^{385}$ Der Nutzen setzt sich aus 6,2 €/ha durch die Verminderung des Bodenabtrages (Standort mit einem durchschnittlichen Bodenabtrag von 5 t/ha*a) sowie 43,1 €/ha durch zusätzliche Markterlöse durch den Vorfruchtwert zusammen.

${ }^{386}$ Der Nutzen setzt sich aus 32,2 €/ha durch die Verminderung des Bodenabtrages (Standort mit einem durchschnittlichen Bodenabtrag von $21 \mathrm{t} / \mathrm{ha}^{*}$ a) sowie $141 € /$ ha durch zusätzliche Markterlöse zusammen.
} 
ist, wie am Beispiel des Zwischenfruchtanbaus anhand der KWA und KNA deutlich wird, als sehr effizient einzustufen. Dabei ist jedoch zu beachten, dass auf der Kostenseite große Unterschiede bestehen. Es sollte auf möglichst geringe Ansaatkosten, z. B. Ausbringung durch den Schleuderstreuer oder Grubber etc., geachtet werden. Für die Untersaaten gilt dasselbe. Allerdings müssen in diesem Bereich die Forschungsanstrengungen bezüglich des Nutzens dieser Maßnahme noch intensiviert werden.

\subsubsection{Schutzhecken, Feldraine}

Feldhecken dienen der Aufrechterhaltung der Leistungsfähigkeit des Naturhaushalts und zum Schutz vor Winderosion, da die Windgeschwindigkeit stark von diesen gesenkt werden kann. Durch die Zusammenlegung von Schlägen und Flurbereinigungsmaßnahmen ging die Zahl der Strukturelemente sehr stark zurück. Ehemalige Nutzfunktionen wie z. B. die Holzgewinnung oder Einzäunung und Abgrenzung von Schlägen wurden bedeutungslos. $\mathrm{Zu}$ den strukturbildenden Landschaftsbestandteilen zählen u. a. Feldgehölze, Hecken, Einzelbäume, Baumgruppen, Geländestufen, Feldraine oder Nass- und Feuchtstellen.

\subsection{Einleitende Erwägungen}

In der folgenden Analyse wird speziell für die Anpflanzung von Windschutzhecken eine KNA durchgeführt. Zur Durchsetzung von Schutzpflanzungen und der Akzeptanz von Schutzhecken in der landwirtschaftlichen Praxis ist es notwendig, den ökonomischen Nutzen der ökologischen Wirkungen dieser Elemente darzustellen. Dabei wird vom gegenwärtigen Kenntnisstand der Forschung ausgegangen. Dies bedeutet, dass z. B. für die Quantifizierung des Nutzens von Strukturelementen und Hecken nur bedingt allgemeine Aussagen gemacht werden können, da bisher nur Feldversuche auf Einzelstandorten durchgeführt worden sind. ${ }^{387}$ Die Ermittlung des KNV geschieht wie folgt:

\footnotetext{
${ }^{387}$ Vgl. hierzu GRAHLMANN (1986), S. 479
} 


\section{Übersicht 13: Vorgehensweise bei der KNA zur Pflanzung von Hecken}

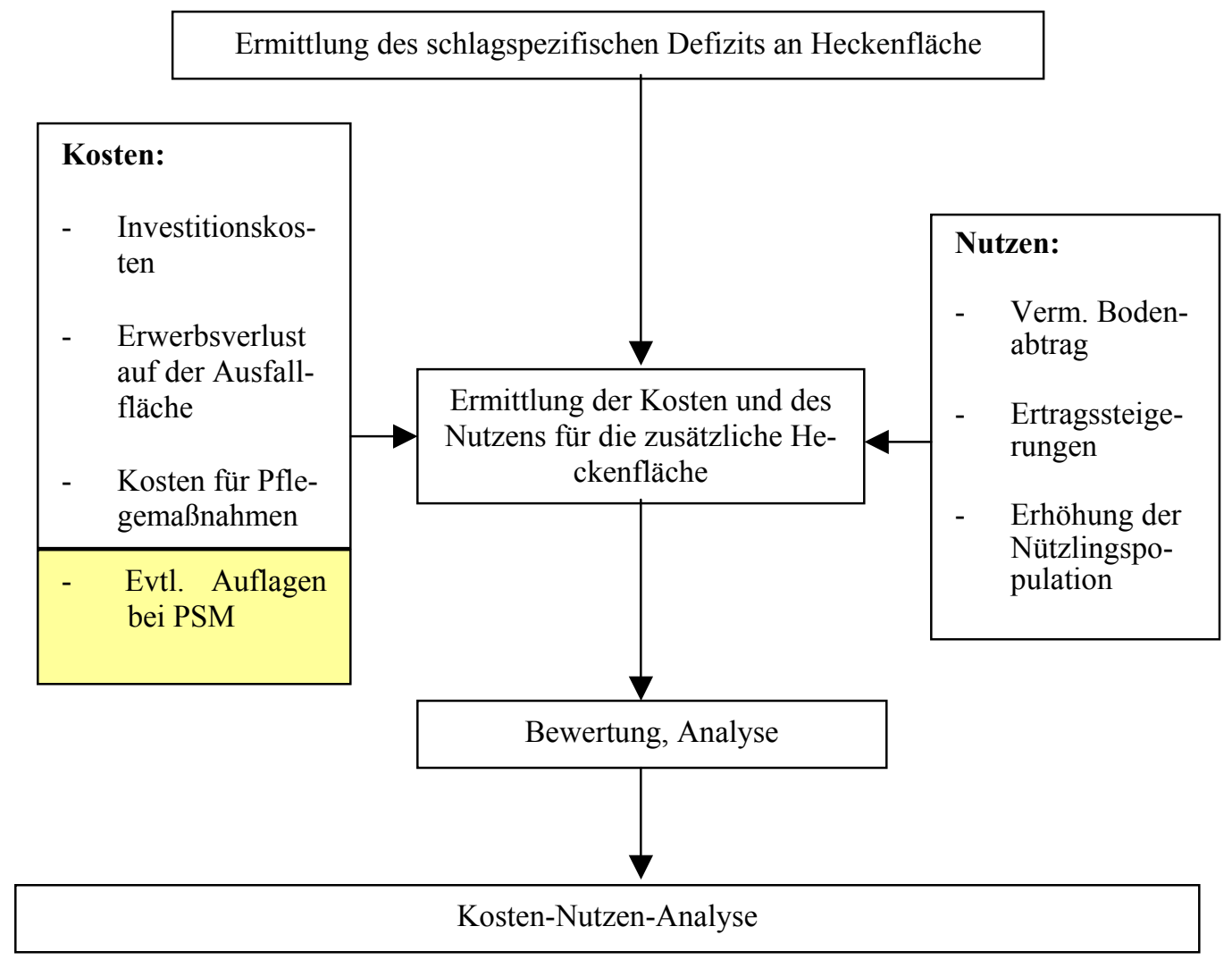

Quelle: Eigene Darstellung, Schraffierung: Positionen konnten aufgrund fehlender Daten in der Berechnung nicht berücksichtigt werden

Zunächst sollte auf der Ebene des Einzelschlages das Defizit an Strukturelementen ermittelt werden. Um einen maximalen Windschutz zu gewährleisten, sollten Hecken quer zur Hauptwindrichtung und längs zur Bearbeitungsrichtung angepflanzt werden. In dieser Betrachtung wird davon ausgegangen, dass bisher keine Hecken oder andere Strukturelemente an den Schlagrändern bestehen. Somit ist der Schlag völlig der Hauptwindrichtung ausgeliefert. Auf winderosionsanfälligen Schlägen sind, wie unten näher dargestellt wird, positive Ertragsentwicklungen durch die Minderung des Bodenabtrags und durch das verbesserte Kleinklima zu erwarten.

\subsection{Betriebswirtschaftliche Analyse der Maßnahme}

Die Investitionskosten setzen sich aus den Materialkosten (Pflanzmaterial, Baummanschetten, Draht oder Zaunpfähle), den variablen Maschinenkosten (Baum- und Strauchpflanzungen, Pflanzbewässerung und Zaunbau), den Arbeitskosten (Pflanzungen, Pflanzbewässerung sowie Leitung und Verwaltung), den Festkosten (Abschreibung für Gebäude u. Maschinen) sowie den Zinskosten zusammen. Bei der Ermittlung der Investitions- 
kosten ist letztlich noch die Umsatzsteuer zu berücksichtigen, die beim Pflanzmaterial $5 \%$ und bei den anderen Positionen $16 \%$ beträgt. In den folgenden Berechnungen ist die Umsatzsteuer jeweils enthalten.

Für die Ermittlung beispielhafter Investitionskosten für eine Windschutzhecke wird auf ROTH UND BERGER ${ }^{388}$ zurückgegriffen. Es können jedoch auch Kosten von Heckenpflanzungen im Rahmen von Flurbereinigungsmaßnahmen abgeleitet werden. Diese sind z. B. für Niedersachen in den Ämtern für Agrarstruktur nachzufragen. Das Problem ist hier jedoch, vergleichbare Durchschnittswerte zu erhalten, da Flurbereinigungsverfahren unterschiedliche Maßnahmen und Pflanzschemata erfordern. Deshalb schwanken derartige Durchschnittswerte teilweise um mehr als $100 \%{ }^{389}$

ROTH UND BERGER ermitteln für eine Neuanlage mit 14 \% Baumanteil inklusive Einzäunung Gesamtkosten von 21,95 €/lfdm. Dabei bestehen diese Gesamtkosten zu über 50 \% aus Materialkosten, wovon wiederum ein Drittel auf den Zaunbau entfallen. Diese Kosten könnten entfallen, wenn ein ungestörtes Wachstum der Hecke ohne Wildverbiss gegeben ist. Durch Erstellung der Hecke in Eigenleistung kann der Landwirt keine hohen Einsparpotenziale erwarten, da hierbei mit einem Lohnansatz von 10,74 €/Akh gerechnet wurde. In der Literatur werden diese Ergebnisse weitestgehend bestätigt. So ermittelt KNAUER ${ }^{390}$ für eingezäunte Windschutzhecken typische Gesamtkosten ${ }^{391}$ von $16,95 € / 1 \mathrm{fdm}$. Andere Quellen, wie z. B. JAROSCH U. SCHWITTEK ${ }^{392}$ geben deutlich geringere Kosten an, gehen aber von einer sehr geringen Pflanzdichte aus, die nicht die Ansprüche an eine Windschutzhecke erfüllt.

Ferner ist noch zu berücksichtigen, dass Hecken und Feldgehölze auf Pachtflächen oder auf Zukaufsflächen entstehen können. Diese Kostenpositionen müssen bei einer Gesamtkostenkalkulation ebenfalls berücksichtigt werden, werden in diese Berechnungen jedoch nicht explizit aufgenommen.

Um den Erwerbsverlust auf der Heckenfläche zu berechnen, muss zunächst festgestellt werden, wie viel Fläche langfristig der landwirtschaftlichen Nutzung entzogen wird. Es

\footnotetext{
${ }^{388}$ Vgl. Roth UND BERGER (1999), S. 5 ff.

${ }^{389}$ Vgl. Schriftliche Auskunft BIERWISCH

${ }^{390}$ Vgl. KNAUER, N. (1990), S. 54

${ }^{391}$ zweireihige Pflanzstreifen, dreijährige Pflege

392 Vgl. JAROSCH U. SCHWITTEK (1991)
} 
ist unter der derzeitigen Umweltgesetzgebung davon auszugehen, dass eine Wegnahme der Hecke nicht mehr möglich sein wird. Deshalb handelt es sich in diesem Fall um einen Dauerschaden.

Die in diesem Beispiel betrachtete hat z. B. einen Raumbedarf von $6 \mathrm{~m}^{2} / \mathrm{lfdm}$. Im nächsten Schritt wird ermittelt, welche Produktionsverfahren durch die Anpflanzung der Hecke eingeschränkt werden. Exemplarisch sei die Beispielfruchtfolge WR-WW-WG.

Tabelle 31: Kalkulation des Erwerbsverlustes durch die Heckenpflanzung bei der Fruchtfolge WR-WW-WG

\begin{tabular}{|c|c|c|c|c|}
\hline $\begin{array}{l}\text { Einzuschränkende Produktions- } \\
\text { oder Stilllegungsverfahren }\end{array}$ & $\begin{array}{c}\text { WR } \\
\text { (35 dt/ha) }\end{array}$ & $\begin{array}{c}\text { WW } \\
(80 \mathrm{dt} / \mathrm{ha})\end{array}$ & $\begin{array}{c}\text { WG } \\
(80 \mathrm{dt} / \mathrm{ha})\end{array}$ & $\begin{array}{l}\text { Rotations- } \\
\text { brache }\end{array}$ \\
\hline Anteil (\%) & 30 & 30 & 30 & 10 \\
\hline Deckungsbeitrag $(€ / \mathrm{ha})^{393}$ & 677 & 621 & 496 & 261 \\
\hline Eingesparte Arbeitszeit (Akh/ha) & 9,2 & 10 & 10,3 & 6 \\
\hline Gewogener Durchschnitt des DB (€/ha) & & & & 564 \\
\hline Einsparbare Gemeinkosten $(€ /$ ha $)$ & & & & 0 \\
\hline Vorteile aus der Arbeitsersparnis & & & & 137 \\
\hline $\begin{array}{l}(\text { Akh* } 15 € / h) \\
\text { Verbleibende Einkommensbeeinträchti- }\end{array}$ & & & & \\
\hline gung $(€ / h a)$ & & & & 427 \\
\hline
\end{tabular}

Quelle: Eigene Berechnungen nach KöHNE (2000), S. 208 f.

In Tabelle 31 wird die jährliche Einkommensbeeinträchtigung durch die Heckenpflanzung berechnet. Für einen laufenden Meter Hecke ${ }^{394}$ beträgt diese $0,25 € / \mathrm{a}$, bei entsprechender Kapitalisierung mit 25 (Dauerschaden) ergibt sich ein dauerhafter Verlust von $6,4 € / 1 \mathrm{fdm}$.

Bei der Pflege der Hecke werden zwei Maßnahmen unterschieden. Zum einen kann die Hecke manuell zurückgeschnitten ${ }^{395}$ werden, zum anderen müssen die Seiten zurückgeschnitten werden, damit ein zu starkes Hineinwachsen in den Schlag unterbleibt. Dies kann mit einem Traktor inkl. Heckenschneidwerk erfolgen. Im ersten Fall sind nach KTBL $^{396}$ bei einem Entnahmegrad von $25 \%$ des Bewuchses 6,4 Akhmin $/ \mathrm{m}^{2}$ erforderlich, die Arbeitskosten von 1,6€/m² verursachen. Dadurch entstehen Pflegekosten von 9,6 €/lfdm. Wird die Hecke mit einem Schneidwerk zurückgeschnitten, fallen minimale

\footnotetext{
393 Richtwertdeckungsbeiträge der LWK HANNOVER (2002), mittleres Ertragsniveau, ohne USt, jedoch inkl. flächenbezogener öffentlicher Zuwendungen.

${ }^{394}$ Bei einer Heckenbreite von $6 \mathrm{~m}$.

${ }^{395}$ Sog. „Auf-den-Stock-Setzen“ oder „Knicken“, um die Bewuchsdichte der Hecke zu erhalten.

${ }^{396}$ Vgl. KTBL (1998), S. 69 ff.
} 
Arbeitskosten von 0,2 Akhmin/lfdm an, was Arbeitskosten in Höhe von 0,05 €/lfdm bedeutet. Zudem entstehen bei diesem Arbeitsgang Maschinenkosten in Höhe von ebenfalls $0,05 € / \mathrm{lfdm}$, sodass sich Pflegekosten von $0,10 € / 1 \mathrm{fdm}$ ergeben. Damit die winderosionshemmende Wirkung der Hecke nicht beeinträchtigt wird, kann sie nicht wie sonst üblich alle 10 Jahre geknickt werden, sondern jedes Jahr um $25 \%$. Die Seiten werden alle fünf Jahre beschnitten.

Neben den Regelungen im Pflanzenschutzrecht existieren in den Landeswassergesetzen Abstandsauflagen zu Gewässern und terrestrischen Strukturen (Wälder, Hecken), welche Mindestabstände in Abhängigkeit von der eingesetzten Spritztechnik vorsehen. Diese Abstände differieren in den einzelnen Bundesländern zwischen 3 und $50 \mathrm{~m}$. Bei den terrestrischen Elementen gilt eine Mindestbreite von $3 \mathrm{~m}$. Hinzu kommt die Tatsache, dass die einzelnen Gemeinden Deutschlands von der BBA in ein Verzeichnis der regionalisierten Kleinstrukturanteile aufgenommen wurden. Befindet sich ein landwirtschaftlicher Betrieb in einer Gemeinde, die genügend Kleinstrukturanteile aufweist, muss er weder die technischen Auflagen noch die Abstandsauflagen einhalten. Deshalb lassen sich kaum konkrete und zu verallgemeinernde Aussagen über die Auswirkung dieser Auflagen machen.

Unter der Annahme, dass eine Abstandsauflage vorliegt, werden zwei Möglichkeiten berücksichtigt. Durch die Verbesserung der Spritztechnik entstehen dem landwirtschaftlichen Betrieb zusätzliche Kosten. Auf der anderen Seite wirkt sich das Verbot der Pflanzenschutzmittelausbringung negativ auf den Deckungsbeitrag aus, wobei es Einsparungen von Arbeits- und Maschinenkosten gibt.

Hecken können durch verschiedene Einwirkungen auf den Hauptschlag dessen Ertrag negativ beeinflussen. Negative Einflüsse bei ungünstiger Lage der Hecke sind v. a. Schattenwirkungen, Beeinträchtigungen der Manövrierfähigkeit von Schleppern und Geräten sowie schlechtes Abtrocknen und Abreifen der Früchte.

Im Folgenden wird jedoch davon ausgegangen, dass es keinen Ertragsverlust oder Mehraufwand durch den Schattenwurf der Hecke gibt, da ein solcher Beweis seitens der Naturwissenschaft auch noch nicht erbracht wurde. ${ }^{397}$ Bei der folgenden Betrachtung bleibt

\footnotetext{
${ }^{397}$ Vgl. hierzu KNAUER (1990), S. 53
} 
ebenfalls der mögliche negative Effekt der Abstandsauflagen für die Anwendung von Pflanzenschutzmitteln an Gewässern oder terrestrischen Strukturelementen unberücksichtigt.

Bei der Betrachtung des Nutzens ist zunächst die Wirkung der Hecke auf die Windgeschwindigkeit und somit auf den Bodenabtrag zu berücksichtigen. In der Literatur wird dokumentiert, dass der Schutz vor Winderosion durch Hecken bezüglich der Luvseite das drei- bis fünffache und der Leeseite das 10- bis 30fache der Heckenhöhe beträgt. ${ }^{398}$ Ein weiterer ertragsbeeinflussender Faktor ist die unproduktive Verdunstung, die durch Hecken herabgesetzt werden kann. ${ }^{399}$

Untersuchungen im norddeutschen und dänischen Küstenbereich auf Sandböden zeigen, dass auf Schlägen mit Windschutzhecke bei Getreide und Mais Mehrerträge von 5-10 \% gegenüber Schlägen ohne Windschutzhecke erzielt werden. ${ }^{400}$ JAROSCH U. SCHWITTEK gehen von einer Ertragssteigerung von $0 \%$ bzw. $6 \%$ aus. ${ }^{401}$ Untersuchungen aus den USA zeigen ebenfalls Ertragssteigerungen durch Heckenpflanzungen von 6-25 \% bei Getreide und Mais. ${ }^{402}$ Diese Ergebnisse gelten jedoch nicht für alle Heckenarten. MARXENDREWES ermittelt für Felder an Wallhecken jedoch auch Ertragsrückgänge von 2,2-7,6 \% bei Weizen. ${ }^{403}$ JAROSCH U. SCHWITTEK gehen von der Einsparung einer Insektizidspritzung pro Jahr durch die Ansiedlung von Nützlingen aus. Eine derartige Ansiedlung ist wissenschaftlich belegt, jedoch fehlen die Nachweise in Bezug auf Insektizideinsparungen.

Da in dieser Arbeit die Auswirkungen von Bodenschutzmaßnahmen untersucht werden, wird im Folgenden von einem Standort mit einer hohen Winderosionsgefährdung ${ }^{404}$ ausgegangen. Ein generelles Problem bei der Nutzenermittlung von Feldhecken stellt sich bei der zeitlichen Verzögerung der vollen Wirksamkeit dieser Maßnahme, die erst nach 10 Jahren erreicht werden kann. Deshalb wird von einem linear ansteigenden Nutzen von

\footnotetext{
${ }^{398}$ Vgl. HASSENPFLUG (1998), S. 81

${ }^{399}$ Vgl. AUERSWALD (1990), S. 539 ff.

${ }^{400}$ Vgl. KNAUER, N. (1990), S. 53

${ }^{401}$ Vgl. JaROSCH U. SCHWITTEK (1990), S. 215

${ }^{402}$ Vgl. BrandLe U. Hodges (2000), S. 3

${ }^{403}$ Vgl. MARXEN-DREWES (1987), S. $125 \mathrm{ff}$.

${ }^{404}$ Eine hohe Winderosionsgefährdung liegt bei einer Windgeschwindigkeit von $8 \mathrm{~m} / \mathrm{sec}$, gemessen in $10 \mathrm{~m}$ Höhe, bei fehlender Bodenbedeckung sowie einer windoffenen Landschaft $\left(<5 \mathrm{~km}\right.$ Flurelemente je $\left.\mathrm{km}^{2}\right)$ vor.
} 
jährlich $10 \%$ ausgegangen, da ein kontinuierliches Wachstum vorausgesetzt wird und die Wirksamkeit des Nutzens von der Heckenhöhe abhängt.

Ein weiterer sehr wichtiger Nutzeneffekt ist die öffentliche Förderung von Neuanlagen von Feldhecken in einigen Bundesländern. Die Förderung wird als Zuschuss zu den Investitionskosten gezahlt, unterscheidet sich in der Höhe jedoch erheblich. Die in einigen Bundesländern und Landkreisen angebotene Förderung von Feldhecken wird in einigen Varianten mit einer Übernahme der Investitionskosten in Höhe von $80 \%$ und $100 \%$ berücksichtigt. In der folgenden Berechnung wird von den unterschiedlichen Förderhöhen von $0 \%, 50 \%, 80 \%$ und $100 \%$ der Pflanzgutkosten sowie des Einfriedungsmaterials ausgegangen. Es ist jedoch zu berücksichtigen, dass u. U. eine Förderhöchstgrenze vorhanden ist. In der KNA wird zunächst ein Zinssatz von 4 \% zugrunde gelegt, der später variiert wird, und die Ertragssteigerungen werden auf den Fruchtfolgedeckungsbeitrag bezogen. Dabei wird eine Variation zwischen $0 \%, 5 \%$ und $10 \%$ vorgenommen. Dabei muss erwähnt werden, dass einige Nutzenpositionen, wie z. B. die Nützlingsförderung und dessen Auswirkungen auf den Insektizideinsatz, nicht mit einbezogen wurden. Unter der Berücksichtigung, dass die Einsparung einer Insektizidmaßnahme pro Jahr wie bei JAROSCH U. SCHWITTEK ${ }^{405}$ realisiert werden könnte, würde durch die Minderung der Verfahrens- und Pflanzenschutzmittelkosten ein jährlicher Nutzen über die gesamte Fruchtfolge von $15,1-24,7 € / \mathrm{ha}^{406}$ entstehen. Dieser wäre allerdings als marginal anzusehen und würde nicht zu einer Veränderung der Rangfolge der Vorzüglichkeit führen.

Wie bereits erwähnt, hängen die KNV sehr stark von den gewählten Zinssätzen ab. Deshalb werden in der Tabelle 32 die Zinssätze variiert und die entsprechenden KNV dargestellt. Die Berechnungen sind in Tabelle A-37 bis einschließlich Tabelle A-43 dargestellt.

\footnotetext{
${ }^{405}$ Vgl. JAROSCH U. SCHWITTEK (1990), S. 213

${ }^{406}$ Berechnungen nach LWK HANNOVER (2002) u. KTBL (2002) unter Berücksichtigung verschiedener Aufwandsstufen. Verwendete Maschinen: Anhängespritze, 24 m, 3000 1, Traktor: 67 kW.
} 
Tabelle 32: KNV der Anlage von Windschutzhecken bei unterschiedlichen Ertragssteigerungen und Förderhöhen

\begin{tabular}{lccccccc}
\hline & $\mathbf{1 0 / 0} \mathbf{0}^{\mathbf{4 0 7}}$ & $\mathbf{1 0 / 5 0}$ & $\mathbf{0 / 8 0}$ & $\mathbf{5 / 8 0}$ & $\mathbf{1 0 / 8 0}$ & $\mathbf{0 / 1 0 0}$ & $\mathbf{1 0 / 1 0 0}$ \\
\hline & & & & & & & \\
$\begin{array}{l}\text { KNV in \% } \\
\varnothing \text { jährl. Vorteil }\end{array}$ & 9,09 & 7,52 & $1^{408}$ & 13,16 & 6,58 & $/$ & 5,95 \\
$(€ /$ ha/a) & -326 & -292 & -295 & -250 & -138 & -267 & -222 \\
\hline
\end{tabular}

Quelle: Eigene Berechnungen, Zinssatz $4 \%$

In der obigen Tabelle wird deutlich, dass die Anlage von Windschutzhecken unter den angenommenen Bedingungen nicht wirtschaftlich ist. Es besteht in allen Fällen ein negativer jährlicher Vorteil und auch die KNV sind schlecht. Weiterhin ist die Bedeutung der Förderung, vor allem am Unterschied der Varianten 10/0 und 10/100 zu erkennen.

\subsection{Schlussfolgerungen}

Die dargestellte KNV für die Anlage von Windschutzhecken zeigt, dass sich die Vorzüglichkeit dieser Maßnahme im Wesentlichen nach der Höhe der Förderung richtet. Unter den in der Berechungen des Kapitels 6.3.1.6.2 angenommen Rahmenbedingungen ist aus einzelbetrieblicher Sicht keine der betrachteten Varianten wirtschaftlich. Aus volkswirtschaftlicher Sicht müssen jedoch die zahlreichen und nur schwer monetarisierbaren positiven Effekte berücksichtigt werden, die eine Begründung zur Bereitstellung von Fördermitteln darstellen können.

\subsubsection{Grünstreifen}

Im Folgenden wird die Wirtschaftlichkeit des Anbaus von Grünstreifen an Hanglagen anhand der KWA und KNA untersucht.

\footnotetext{
${ }^{407}$ Die erste Ziffer gibt die Ertragssteigerung und die zweite Ziffer die Förderhöhe an.

${ }^{408}$ Bei dieser Variante besteht kein Nutzen, daher kann das KNV nicht errechnet werden.
} 


\subsection{Einleitende Erwägungen}

Für Schläge mit langgestreckten Hängen besteht neben der Änderung der Bewirtschaftungsrichtung $^{409}$ die Möglichkeit, durch sog. Grünstreifen den Oberflächenabfluss und somit die Wassererosion zu begrenzen. Hierbei werden Streifen mit einer dichten Vegetation quer zur Gefällerichtung angepflanzt. Die Wirkung der Grünstreifen hängt im Wesentlichen von der Streifenbreite, dem Streifenabstand, der Bodenbedeckung, der Hangtopografie, der Abflussintensität, der Sedimentkonzentration sowie der Bewirtschaftung des Bodens ab. Außerdem wird die Wirkung von Grünstreifen durch die sog. maximale Hanglänge begrenzt. Diese gibt an, bei welcher Hangneigung noch für unterschiedliche Hanglängen ein optimaler Erosionsschutz vorliegt. Der Einsatz von Schutzstreifen wirkt demnach wie eine Hangverkürzung. ${ }^{410}$ Die maximale Hangneigung kann in folgender Tabelle ersehen werden:

\section{Tabelle 33: Maximale Hanglänge als begrenzender Faktor der Konturnutzung}

\begin{tabular}{cc}
\hline Hangneigung (\%) & Maximale Hanglänge (m) \\
\hline $1-2$ & 130 \\
$3-5$ & 100 \\
$6-8$ & 70 \\
$9-12$ & 40 \\
$13-16$ & 30 \\
$17-20$ & 20 \\
$21-25$ & 17 \\
\hline
\end{tabular}

Quelle: SCHWERTMANN (1987), S. 44

Die Wirkung von Grünstreifen auf den Bodenabtrag ist in der Tabelle 36 anhand von Forschungsergebnissen ersichtlich. Betriebswirtschaftliche Analyse der Maßnahme

In der Übersicht 14 ist die Vorgehensweise bei der KNA für die Nutzung von Grünstreifen zusammengefasst.

\footnotetext{
${ }^{409}$ Vgl. hierzu Kapitel 6.3.1.3

${ }^{410}$ Vgl. hierzu SCHWERTMANN (1987)
} 


\section{Übersicht 14: Vorgehensweise bei der KNA für die Nutzung von Grünstreifen}

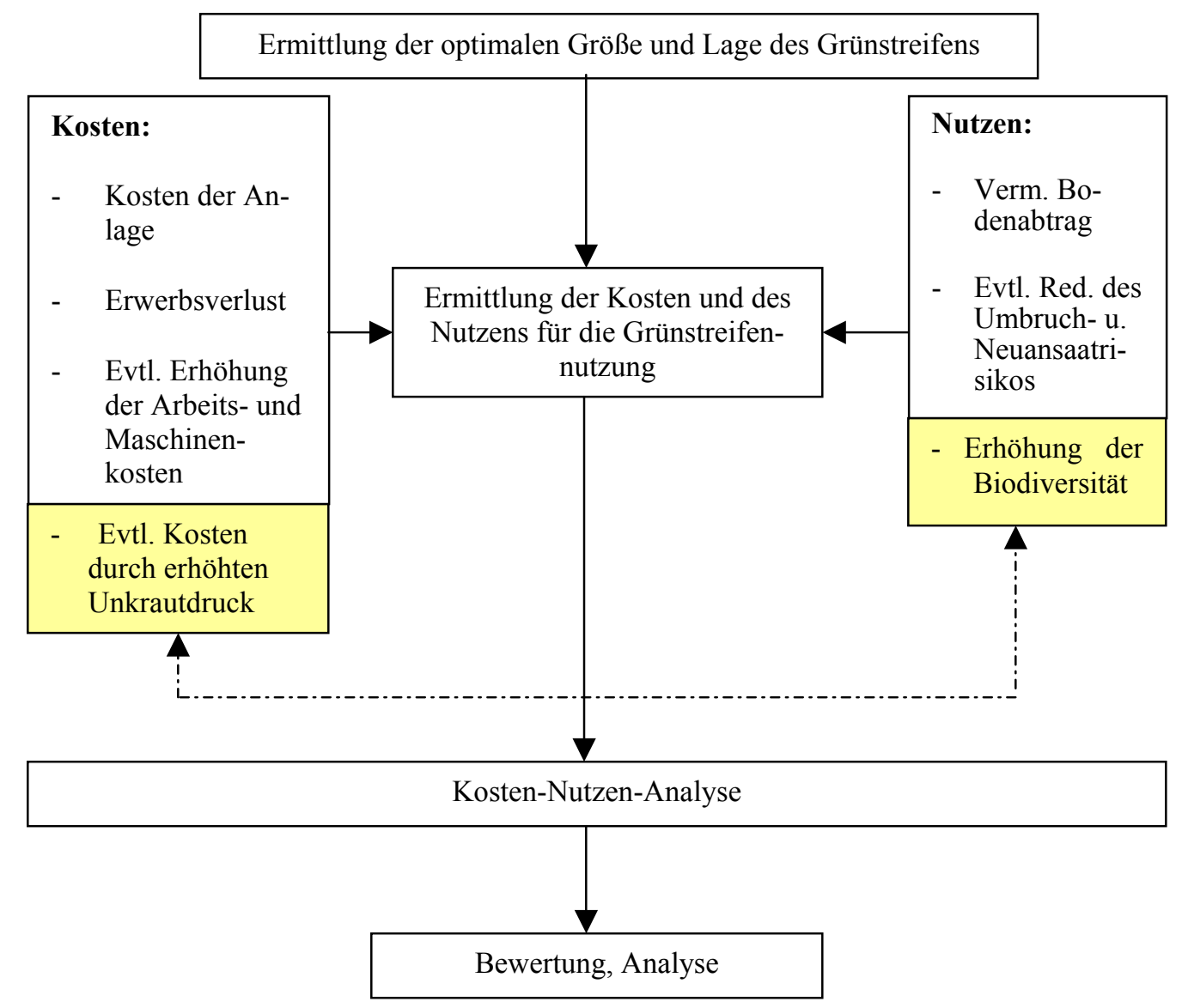

Quelle: Eigene Darstellung, Schraffierung: Positionen konnten aufgrund fehlender Daten in der Berechnung nicht berücksichtigt werden

Zunächst entstehen Kosten für die Anlage der Grünstreifen. Im einfachsten Fall erfolgt die Einsaat von dauerhaften Gräsern mit der Sämaschine, und ein Schnitt erfolgt mit dem Kreisel- oder Scheibenmähwerk. Die Verfahrenskosten hierfür liegen in diesem Fall bei etwa $0,017 € / \mathrm{m}^{2411}$. Eine Behandlung mit Herbiziden ist nicht vorgesehen, muss aber bei extremem Unkrautdruck durchgeführt werden. Außerdem wird keine Abfuhr des Mähgutes berücksichtigt, die aufgrund der geringen Menge nicht gerechtfertigt ist. Die Tabelle 34 zeigt die Anlagekosten von Grünstreifen mit unterschiedlichen Streifenbreiten und Kantenlängen.

411 Annahmen: Parzellengröße 1 ha; Kosten von $170 € /$ ha. 
Tabelle 34: Kosten der Anlage von Grünstreifen mit unterschiedlichen Streifenbreiten und Reihenabständen für einen Schlag mit der Größe 50*100 m

\begin{tabular}{cccc}
\hline $\begin{array}{c}\text { Streifenbreite } \\
(\mathbf{m})\end{array}$ & $\begin{array}{c}\text { Reihenabstand } \\
(\mathbf{m})\end{array}$ & $\begin{array}{c}\text { Fläche } \\
\left(\mathbf{m}^{\mathbf{2}}\right)\end{array}$ & $\begin{array}{c}\text { Einmalige Kosten } \\
(\mathbf{\epsilon} / \mathbf{h a})\end{array}$ \\
\hline \multirow{3}{*}{3} & 20 & 652,2 & 11,1 \\
& 40 & 348,8 & 5,9 \\
\cline { 2 - 4 } 6 & 20 & 1153,8 & 19,6 \\
& 40 & 652,2 & 11,1 \\
\hline 9 & 20 & 1551,7 & 26,4 \\
& 40 & 918,4 & 15,6 \\
10 & 20 & 1666,7 & 28,3 \\
& 40 & 1000,0 & 17,0 \\
\hline
\end{tabular}

Quelle: Eigene Berechnungen

Die Kosten liegen nach den Berechnungen im Fall der Grünstreifen mit einem Reihenabstand von $40 \mathrm{~m}$ zwischen 5,9-17 €/ha und bei einem Reihenabstand von $20 \mathrm{~m}$ bei Kosten zwischen 11,1-28,3€/ha.

Eine weitere Kostenposition ist der Erwerbsverlust auf der Streifenfläche. Die Berechnung geht von der Beispielfruchtfolge WR-WW-WG aus, wonach der Erwerbsverlust $427 € /$ ha beträgt. Weiterhin ist zu klären, wie hoch der Flächenbedarf der Grünstreifen ausfällt. Dieser ist von den Streifenbreiten und deren Reihenabständen abhängig. Die Tabelle 35 zeigt für die einzelnen Varianten die entsprechenden Erwerbsverluste auf.

Tabelle 35: Erwerbsverluste bei der Anlage von Grünstreifen

\begin{tabular}{cccc}
\hline $\begin{array}{c}\text { Streifenbreite } \\
(\mathbf{m})\end{array}$ & $\begin{array}{c}\text { Reihenabstand } \\
(\mathbf{m})\end{array}$ & $\begin{array}{c}\text { Fläche } \\
\left.\mathbf{( m}^{\mathbf{2}}\right)\end{array}$ & $\begin{array}{c}\text { Erwerbsverlust } \\
(\boldsymbol{(} / \mathbf{h a})\end{array}$ \\
\hline 3 & 20 & 652,2 & 27,9 \\
& 40 & 348,8 & 14,9 \\
\hline 6 & 20 & 1153,8 & 49,3 \\
& 40 & 652,2 & 27,9 \\
\cline { 2 - 4 } 9 & 20 & 1551,7 & 66,2 \\
& 40 & 918,4 & 39,2 \\
\hline 10 & 20 & 1666,7 & 71,2 \\
& 40 & 1000,0 & 42,7 \\
\hline
\end{tabular}

Quelle: Eigene Berechnungen

Die Erwerbsverluste können demnach von 14,9 €/ha bis 71,2 €/ha liegen und finden Eingang in die folgenden Berechnungen.

\footnotetext{
${ }^{412}$ In dieser Spalte werden die Anlagekosten von $0,017 € / \mathrm{m}^{2}$ mit der notwendigen Fläche multipliziert.
} 
Weiterhin kann die Anlage von Grünstreifen durch eine Verkleinerung des Schlages zu einer Erhöhung der Arbeits- und Maschinenkosten führen. Diese Auswirkungen können jedoch durch die Anpassung der Konturgrasanlage an die vorhandene Arbeitsbreite der Maschinen und Geräte gemindert werden. Sie hängen sehr stark von der Schlagform und -länge ab. Dabei sind zwei Fälle zu unterscheiden:

\section{Bewirtschaftung quer zum Hang}

Den geringsten Einfluss auf Beeinträchtigungen einer schlagkräftigen Bewirtschaftung ergeben sich, wenn der Schlag bereits quer zum Hang bearbeitet wird. Dennoch ergeben sich höhere Arbeits- und Maschinenkosten. Diese Unterschiede werden in den KTBLDatensammlungen offensichtlich. Bspw. unterscheiden sich die Verfahrenskosten des Pflügens (Drillens) zwischen einem 2-ha- und einem 5 ha-Schlag um etwa $8 \%$ (6\%). Da in diesem Fall jedoch unterstellt wird, dass die Grasstreifen den entsprechenden Arbeitsbreiten angepasst werden können, wird auf eine Berücksichtigung dieser Kostenpositionen verzichtet.

\section{Bewirtschaftung längs zum Hang}

Wird der Schlag längs zum Hang bearbeitet, ergeben sich durch die Anlage von Grünstreifen erhebliche Veränderungen in der Schlagstruktur. Diese äußern sich in diesem Fall nicht nur durch die Verkleinerung des Schlages sondern auch durch eine erhebliche Erhöhung der Vorgewendefläche. Dieses Problem wurde bereits in Kapitel 6.3.1.3 erläutert. Da jedoch davon auszugehen ist, dass Grünstreifen auf Schlägen mit hoher Hangneigung und entsprechenden Bodenabtragsraten angelegt werden, kann allein aus dem Aspekt der standortangepassten Bewirtschaftung im Sinne des $\S 17$ BBodSchG davon ausgegangen werden, dass auf diesen Schlägen die Bewirtschaftung quer zum Hang durchgeführt werden sollte. Deshalb wird dieser Problembereich im Folgenden nicht weiter betrachtet.

Der Nutzen der Anlage von Grünstreifen ist durch eine Literaturauswertung nicht eindeutig quantifizierbar. Bisherige Untersuchungen zeigen Minderungspotenziale des Bodenabtrags von $20-95 \%$. Die einzige Untersuchung unter Praxisbedingungen von SAUPE ${ }^{413}$ kommt zu dem Ergebnis, das nur im Zusammenhang mit der Querbearbeitung zufriedenstellende Effekte erzielt werden können. 
Tabelle 36: Bisherige Untersuchungsergebnisse zur Wirksamkeit von Grünstreifen auf die Minderung des Bodenabtrags

\begin{tabular}{lccc}
\hline \multicolumn{1}{c}{ Autor } & $\begin{array}{c}\text { Untersuchungs- } \\
\text { methode }\end{array}$ & Bedingungen & $\begin{array}{c}\text { Minderungen des Boden- } \\
\text { abtrags (\%) }\end{array}$ \\
\hline $\begin{array}{l}\text { DIEZ U. HEGE } \\
\text { (1980) }\end{array}$ & Regensimulation & $\begin{array}{l}40 \text { m Reihenabstand } \\
20 \text { m Reihenabstand }\end{array}$ & 87 \\
WILLIAMS U. & Creams-Modell & $\begin{array}{c}\text { Versch. Streifenbrei- } \\
\text { ten u. Hangneigungen } \\
\text { NICKS (1988) }\end{array}$ & $20-30$ \\
$\begin{array}{l}\text { DILLAHA ET AL. } \\
\text { (1989) }\end{array}$ & Regensimulation Brache- & 70 \\
SAUPE (1992) & $\begin{array}{c}\text { parzellen } \\
\text { Praxisbedingun- } \\
\end{array}$ & $\begin{array}{c}6 \text { m Grasstreifen } \\
3 \text { m Grasstreifen }\end{array}$ & $\begin{array}{c}\text { k. A. } \\
\text { k. A. }\end{array}$ \\
\hline
\end{tabular}

Quelle: Zusammenstellung nach SAUPE (1992)

Eine Quantifizierung des Minderungspotenzials konnte in einer Versuchsanordnung aufgrund fehlender erosiver Niederschlagsmengen nicht durchgeführt werden. Bei einer zweiten Untersuchung waren während starker Niederschläge die angelegten Streifen nur ungenügend bedeckt, so dass eine verlässliche Aussage über deren Wirkung nicht gemacht werden konnte. Hierdurch wird die Schwäche dieser Maßnahme deutlich. Durch die Tatsache, dass die stark erosiven Niederschläge bereits im Mai und Juni fallen, ist davon auszugehen, dass der Pflanzenbestand noch keine ausreichende Schutzbedeckung erreicht hat. Eigene Berechnungen mit der ABAG auf verschiedenen Standorten zeigen bei Getreide- und Getreiderapsfruchtfolgen geringere Wirksamkeiten dieser Maßnahme. ${ }^{414}$ Im Folgenden werden jedoch die Ergebnisse der obigen Praxisversuche herangezogen.

Es ist außerdem davon auszugehen, dass die Anlage von Grünstreifen auch das Risiko der Umbruch- und Neuansaatkosten senken wird. In der Literatur finden sich dahingehend jedoch keine Hinweise. Diese Vermutung muss erst durch entsprechende Feldversuche oder Modellberechnungen bestätigt werden.

Im Folgenden wird eine KWA durchgeführt. Es werden die ermittelten Kosten der Tabelle 34 und Tabelle 35 herangezogen. Zunächst wird bezüglich des Bodenabtrags mit den Ergebnissen von DIEZ und HEGE gerechnet. Dabei werden zwei verschiedene Standorte mit durchschnittlichen Bodenabträgen von 6 und 10 t/ha/a angenommen, weil am Hang tendenziell höhere Bodenabträge zu erwarten sind als die bisher durchschnittlich 
angenommenen. Die Wirksamkeit der Maßnahme ist durch die Reduktion des Bodenabtrags gekennzeichnet. Sie fließt gemäß den Ergebnissen von DIEZ u. HEGE mit 87 bzw. $95 \%$ in die Berechnungen ein. Außerdem wird eine Wirksamkeit von $20 \%$ abgebildet, welche die anderen Ergebnisse repräsentieren soll. Die Ergebnisse zeigen, dass die KW auf dem erosionsanfälligerem Standort günstiger ist. Die KW liegen dort zwischen 2,3 und 51,7 €/t verhinderter Bodenabtrag. Auf dem weniger erosionsanfälligen Standort liegt die KW zwischen 3,8 und 86,2 €/t verhinderter Bodenabtrag. Die Grünstreifen mit einem Reihenabstand von 40 m weisen nach DIEZ und HEGE eine Wirksamkeit von 87 \% auf. Für die beiden Standorte ergeben sich für diese Grünstreifen KW zwischen 2,5 und $11,9 € / \mathrm{t}$ verhinderter Bodenabtrag. Die Grünstreifen mit einem Reihenabstand von $20 \mathrm{~m}$ haben eine Wirksamkeit von 95 \%. Die KW liegen zwischen 4,3 und 18,1€/t verhinderter Bodenabtrag. Aus Platzgründen ist die Tabelle in den Anhang versetzt worden und ist dort als Tabelle A-7 zu finden.

\section{Kosten-Nutzen-Analyse}

Im Folgenden wird ausgehend von den bisherigen Ergebnissen eine KNA für die Anlage von Grünstreifen durchgeführt. Dabei werden die in Tabelle 34 und Tabelle 35 ermittelten Kosten der Anlage und des Erwerbsverlustes verwendet. Für Grünstreifen mit dem Abstand von 20 m werden Kosten von 39,0 €/ha und für Grünstreifen mit einem Abstand von 40 m werden Kosten von 20,8 €/ha veranschlagt. Der Nutzen setzt sich aus den monetären Vorteilen der Verhinderung des Bodenabtrags auf den beiden Standorten mit einem durchschnittlichen Bodenabtrag von 6 bzw. 10 t/ha zusammen. Demnach wird eine Tonne nicht abgetragenes Bodenmaterial mit 4,8€/t bewertet. Die Wirksamkeit wird mit $95 \%$ veranschlagt. Bspw. beträgt dieser Nutzen dann bei einem Bodenabtrag von $6 \mathrm{t} / \mathrm{ha} / \mathrm{a}$ und einer Streifenbreite von $3 \mathrm{~m}$ und einem Reihenabstand von $20 \mathrm{~m}$ 27,4€/ha. In diesem Zusammenhang wird die Streifenbreite ebenfalls analog zu Tabelle A-7 zwischen $3 \mathrm{~m}$, $6 \mathrm{~m}, 9 \mathrm{~m}$ und $10 \mathrm{~m}$ variiert. Die Ergebnisse sind in der Tabelle 37 zusammengetragen. Die Berechnungen können exemplarisch für beide Standorte (Streifenbreite $3 \mathrm{~m}$ und Reihenabstand $20 \mathrm{~m}$ ) in der Tabelle A-44 bis Tabelle A-47 eingesehen werden. 
Tabelle 37: Mögliche KNV für die Grünstreifennutzung auf verschiedenen Standorten bei einem Zinssatz von $4 \%$

\begin{tabular}{|c|c|c|c|c|c|c|c|c|}
\hline \multicolumn{9}{|c|}{ Standort: $6 \mathrm{t} / \mathrm{ha} / \mathrm{a}$} \\
\hline Streifenbreite & \multicolumn{2}{|c|}{$3 \mathrm{~m}$} & \multicolumn{2}{|c|}{$6 \mathrm{~m}$} & \multicolumn{2}{|r|}{$9 \mathrm{~m}$} & \multicolumn{2}{|c|}{$10 \mathrm{~m}$} \\
\hline Wirksamkeit (\%) & 8 & 95 & 87 & 95 & 87 & 95 & 87 & 95 \\
\hline KNV \% (SA 20 m) & \multicolumn{2}{|r|}{1,42} & \multicolumn{2}{|r|}{2,51} & \multicolumn{2}{|r|}{3,38} & \multicolumn{2}{|r|}{3,63} \\
\hline$\varnothing$ jährl. Vorteil (€/ha/a) & \multicolumn{2}{|r|}{-12} & & -41 & \multicolumn{2}{|r|}{-65} & \multicolumn{2}{|r|}{-72} \\
\hline KNV \% (SA 40 m) & \multicolumn{2}{|c|}{0,83} & 1,5 & & \multicolumn{2}{|c|}{2,16} & \multicolumn{2}{|l|}{2,39} \\
\hline$\varnothing$ jährl. Vorteil (€/ha/a) & 4 & & -1 & & -29 & & -35 & \\
\hline \multicolumn{9}{|c|}{ Standort: 10 t/ha/a } \\
\hline Streifenbreite & \multicolumn{2}{|c|}{$3 \mathrm{~m}$} & \multicolumn{2}{|c|}{$6 \mathrm{~m}$} & \multicolumn{2}{|r|}{$9 \mathrm{~m}$} & \multicolumn{2}{|c|}{$10 \mathrm{~m}$} \\
\hline Wirksamkeit (\%) & 87 & 95 & 87 & 95 & 87 & 95 & 87 & 95 \\
\hline KNV \% (SA 20 m) & & 0,86 & & 1,51 & & 2,03 & & 2,18 \\
\hline$\varnothing$ jährl. Vorteil (€/ha/a) & & 7 & & -23 & & -47 & & -54 \\
\hline KNV \% (SA $40 \mathrm{~m})$ & 0 , & & 0,9 & & 1,2 & & 1,43 & \\
\hline$\varnothing$ jährl. Vorteil (€/ha/a) & 21 & & 3 & & -12 & & -18 & \\
\hline
\end{tabular}

Quelle: Eigene Berechnungen, Zinssatz 4 \%

Die Maßnahme weist unter den angenommenen Bedingungen gute bis sehr schlechte KNV aus. Bei Streifenabständen von $40 \mathrm{~m}$ ergeben sich bis max. $6 \mathrm{~m}$ Streifenbreite günstige KNV. Bei Reihenabständen von $20 \mathrm{~m}$ ist dies nur auf dem Standort mit einem Bodenabtrag von 10 t/ha/a bis zu einer Streifenbreite von 3 m der Fall.

\subsection{Schlussfolgerungen}

Die Berechnungen des KNV zeigen, dass die Maßnahme der Anlage von Grünstreifen eine geeignete und effiziente Maßnahme sein kann. Hierzu müssen allerdings einige Voraussetzung erfüllt werden, wie z. B. eine möglichst optimale Anlage der Streifen, um die Arbeitsgänge nicht zu beeinflussen. Dieses Verfahren weist zudem eine gute Kombinationsmöglichkeit zur Bewirtschaftung quer zum Hang auf. In diesem Zusammenhang ergibt sich eine effiziente Möglichkeit, Landbewirtschaftung und Bodenschutz an hängigen Standorten zu vereinen. Bei dieser Maßnahme ist wiederum zu konstatieren, dass zusätzliche Forschungsanstrengungen zur konkreten Erfassung des Nutzens, z. B. in Bezug auf die Verminderung von Umbruch- und Neuansaatkosten wünschenswert wären.

\subsubsection{Verzicht auf den Anbau an Hanglagen}

Der durchschnittliche Bodenabtrag wird maßgeblich von der Hangneigung und Hanglänge bestimmt. An hängigen Flächen sind vielfach aufgrund fehlender Bodenbedeckung, Erosionsprozesse zu erkennen. Ein weiteres Problem dieser Flächen ist die geringe Ober- 
bodenmächtigkeit. Trotzdem werden Hangflächen weiterhin ackerbaulich genutzt, was zunächst einmal nicht als nachhaltig bezeichnet werden kann. Neben dem verstärkten Anbau von Zwischenfrüchten oder dem Verzicht des Anbaus von Reihenfrüchten besteht die Möglichkeit, Hangflächen zu Grünland umzuwandeln. Diese Maßnahme wird im Folgenden anhand der KWA und der KNA betrachtet.

\subsection{Einleitende Erwägungen}

Im Folgenden wird untersucht, wie sich ein Ackerbauverbot an hängigen Schlägen auf einzelbetrieblicher Ebene auswirkt und welche Nutzeneffekte entstehen können. Dazu sind zunächst die Erwerbsverluste der Fläche zu errechnen. Es sollen vier Alternativen zur Bewirtschaftung der Ausfallfläche betrachtet werden:

- die extensive Mutterkuhhaltung,

- die Bullenmast mit einer Mastdauer von 21 Monaten,

- die Stilllegung der Fläche und

- die Verpachtung.

Da davon auszugehen ist, dass durch Mutterkuhhaltung oder Stilllegung die Bodenbedeckung nahezu $100 \%$ beträgt, sind keine hohen Bodenabträge mehr zu erwarten. Bei der Mutterkuhhaltung kann es jedoch an Tränke- oder Ruhestellen zu punktuellen Abträgen kommen, die allerdings durch regelmäßiges Umtreiben und Nachsäen minimiert werden können. Bei der Nutzenbetrachtung wird daher von einer Reduktion des Bodenabtrages um 80-100\% ausgegangen.

\subsection{Betriebswirtschaftliche Analyse}

In der Übersicht 15 ist die Vorgehensweise dieses Kapitels zusammengefasst. 


\section{Übersicht 15: Vorgehensweise bei der KNA beim Verzicht des Anbaus an Hangla- gen}

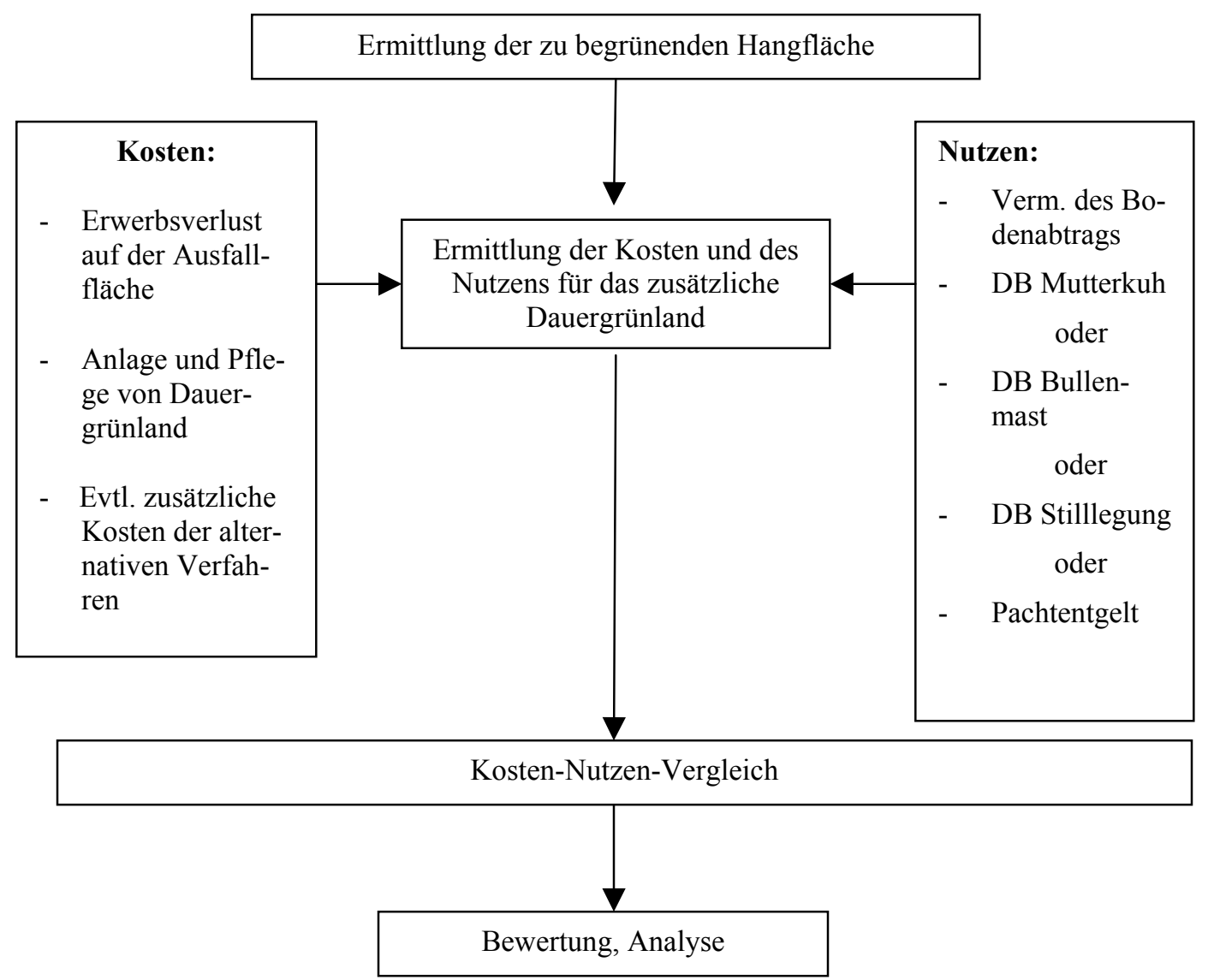

Quelle: Eigene Darstellung

Zunächst werden die Kosten dieser Maßnahme ermittelt. Da es sich um flachgründige und z. T. bereits durch Bodenerosion geschädigte Böden handelt, wird in der folgenden Betrachtung von einem geringen Ertragsniveau ausgegangen. Die DB werden der Publikation des FID der LWK Hannover entnommen. Es werden für verschiedene Fruchtfolgen die Erwerbsverluste analog zu Tabelle 31 berechnet. Die Berechnungen sind im Anhang in der Tabelle A-8 einzusehen. Nach diesen Berechnungen beträgt der jährliche Erwerbsverlust bei der Fruchtfolge 1 (WR-WW-WG) $82 € /$ ha, bei der Fruchtfolge 2 (WWWW-WW) $71 € /$ ha und bei der Fruchtfolge 3 (WR-WW-WW) $86 € /$ ha. Davon ausgehend, dass ein dauerhafter Schutz des Bodens an Hanglagen gewährt werden soll, können die Erwerbsverluste als Dauerschaden angesehen werden. Daher ergeben sich Dauerschäden von $2.050 € /$ ha im Falle der Fruchtfolge 1, $1.775 € /$ ha im Falle der Selbstfolge von Weizen und $2.150 € /$ ha bei der Fruchtfolge 3 .

Des Weiteren müssen die Kosten zur Anlage von Dauergrünland berücksichtigt werden. Nach Berechnungen mit KTBL-Daten betragen die Kosten der Anlage hierfür 
$296,2 € /$ ha. ${ }^{415}$ In den folgenden Jahren entstehen alle fünf Jahre $26 € /$ ha an Pflegekosten für die Nachsaat des Grünlandes.

Der Nutzen des Verzichts des Anbaus an Hängen liegt in erster Linie in der Reduzierung der Bodenerosion. Dazu ist jedoch eine möglichst ganzjährige Bodenbedeckung nötig. Daher werden die im Kapitel 6.3.1.8.1 genannten Alternativen nun aufgrund ihres Nutzens für landwirtschaftliche Betriebe untersucht. Außerdem erfolgt eine Berechnung zur Reduzierung des Bodenabtrages anhand der ABAG. Der hierzu gewählte Standort verfüge über eine Bodenabtragstoleranz von 3 t/ha. Es wird eine konvexe Hangform vorausgesetzt, die einen hohen LS-Faktor und somit einen entsprechenden Bodenabtrag verursacht. Es werden weiterhin unterschiedliche Hangneigungen und zwei verschiedene Wirtschaftsweisen, die Bearbeitung in Gefällerichtung und die Bearbeitung quer zum Hang, unterstellt. Die Ergebnisse der Berechnungen sind in der Tabelle 38 zusammengefasst. Es ist zu erkennen, dass die alternative Nutzung von Hanglagen den Bodenabtrag deutlich einschränkt. Je nach Wirtschaftsweise und Hangneigung werden die Reduktionspotenziale für den Bodenabtrag zwischen 90 und $95 \%$ variiert.

415 Annahmen: Blanksaat, Pflug und Saatbettkombination sowie $200 \mathrm{~kg} / \mathrm{ha}$ (Vgl. KTBL (2002), S. 150). Die Annahmen führen zu hohen Verfahrenskosten, die einzelbetrieblich sehr unterschiedlich ausfallen können. 
Tabelle 38: Einfluss der alternativen Verwendung von Hanglagen durch Grünland und deren Einfluss auf den Bodenabtrag und monetären Nutzen

\begin{tabular}{cccc}
\hline \multicolumn{4}{c}{ Langfristiger Bodenabtrag in t/ha } \\
\hline Fruchtfolge & $8 \%{ }^{1} 200 \mathrm{~m}^{2}$ & $10 \% 200 \mathrm{~m}$ & $12 \% 200 \mathrm{~m}$ \\
\hline $\begin{array}{c}\text { Bewirtschaftung quer zum Hang } \\
\text { WR-WW-WG }\end{array}$ & 4,3 & 5,8 & 7,5 \\
WW-WW-WW & 3,8 & 5,1 & 6,6 \\
WR-WW-WW & 4,3 & 5,8 & 7,5 \\
Grünland/Wald & 0,2 & 0,3 & 0,4 \\
\hline Monetärer Vorteil (€/ha) & $\mathbf{1 7 , 3 - 1 9 , 7}$ & $\mathbf{2 3 , 0 - 2 6 , 4}$ & $\mathbf{3 0 , 0}-\mathbf{3 4 , 1}$ \\
& & & \\
Bewirtschaftung in Gefällerich- & & & \\
tung & 4,9 & 6,3 & 7,9 \\
WR-WW-WG & 4,3 & 5,6 & 6,9 \\
WW-WW-WW & 4,9 & 6,3 & 7,9 \\
WR-WW-WW & 0,2 & 0,3 & 0,4 \\
Grünland/Wald & $\mathbf{1 9 , 7 - 2 2 , 6}$ & $\mathbf{2 5 , 4 - 2 8 , 8}$ & $\mathbf{3 1 , 2 - 3 6 , 0}$ \\
\hline Monetärer Vorteil (€/ha)
\end{tabular}

1 Hangneigung

2 Hanglänge

Quelle: Eigene Berechnungen mit ${ }^{\mathrm{PC}} \mathrm{ABAG}$

Der Nutzen wird durch Multiplikation des reduzierten Bodenabtrags mit dem in Kapitel 6.3.1.1.3 ermittelten Wert von $4,8 € / t$ Oberboden ermittelt.

Die obige Tabelle zeigt, dass ein Nutzen von 19,7-36,0 €/ha ${ }^{416}$ entsteht, wenn zuvor in Gefällerichtung bewirtschaftete Flächen in Grünland oder Wald umgewandelt werden. Wird eine Fläche begrünt oder bewaldet, die vorher quer zum Hang bewirtschaftet wurde, ergeben sich monetäre Vorteile von 17,3-34,1 €/ha.

Neben der Reduktion des Bodenabtrages sind die Deckungsbeiträge der Alternativen zu berücksichtigen. Diese liegen analog zu Kapitel 5.3.4 bei der Mutterkuhhaltung bei ca. $50 € /$ ha und bei der Stilllegung bei $260,5 € / \mathrm{ha}^{417}$ Außerdem wird der alternative Pachtpreis auf $50 € /$ ha festgelegt. Eine Alternative zur Flächenstillegung wäre in diesem Fall mit einer Aufforstung der Hangfläche gegeben. Bei der Beantragung einer Aufforstungs-

\footnotetext{
416 Diese Beträge entstehen durch Multiplikation der verhinderten Bodenabträge mit dem in Kapitel 6.3.1.1.3 ermittelten Wert von $4,8 € / \mathrm{t}$ nicht erodierten Boden.

${ }^{417}$ An dieser Stelle erfolgt bei der Stilllegung eine vereinfachte Betrachtung des Nutzens. Bzgl. einer weitergehenden Vorgehenswiese siehe Kapitel 6.3.3.1.
} 
prämie ist in diesem Fall zu beachten, dass die Mindestflächengröße 1 ha betragen muss $^{418}$. Außerdem dürfen keine Sonderkulturen wie z. B. Weihnachtsbäume angelegt werden. Die Prämie wird als jährlicher Zuschuss für die Dauer von bis zu 20 Jahren gezahlt. Aufgrund der oben geschilderten Sachlage könnte die „große“ Aufforstungsprämie gezahlt werden. Dies bedeutet, dass eine Förderung von bis zu $300 € /$ ha für 35 Bodenpunkte und 7,5€ für jeden zusätzlichen Bodenpunkt gezahlt würde. Die Obergrenze läge dann bei $700 € /$ ha, was 88 Bodenpunkten entspricht. Aufgrund der Tatsache, dass diese Maßnahme sehr stark in den Bereich der Forstwissenschaften hineinreicht, wird sie im Folgenden nicht weiter betrachtet.

Der weitere Nutzen der Umwandlung von Acker- in Grünland hängt v. a. davon ab, welches Produktionsverfahren auf der Grünlandfläche etabliert wird. In diesem Fall wird die Systematik des Kapitels 5.3.4 übernommen, bei dem es ebenfalls um Alternativen auf Grünland zum Ackerbau geht.

Im Folgenden wird die KWA der Umwandlung von ackerbaulich genutzten Hangflächen in Bezug auf die Erosionsminderung durchgeführt. Ausgehend von den oben dargestellten Kosten des Erwerbsverlustes sowie der Kosten der Anlage des Dauergrünlandes $\left(296,2 € /\right.$ ha) sowie den alle fünf Jahre anfallenden Pflegekosten von $130 € / \mathrm{ha}^{419}$ und den in Tabelle 38 dargestellten Erosionsminderungen ergeben sich folgende KW.

\footnotetext{
${ }^{418}$ Diese Bedingung kann gelockert werden, wenn der Anschluss an andere Waldflächen vorliegt.

${ }^{419}$ Vgl. KTBL (2002), S. 316; Spezialgrasnachsaatmaschine, $3 \mathrm{~m}, 67 \mathrm{~kW}$, Grassamen $12 \mathrm{~kg} / \mathrm{ha}$, alle fünf Jahre.
} 
Tabelle 39: $\mathrm{KW}^{420}$ (€/t verhinderter Bodenabtrag) bei der Umwandlung der Hangfläche in Dauergrünland bei verschiedenen Fruchtfolgen und Hangneigungen

\begin{tabular}{cccc}
\hline & \multicolumn{3}{c}{$\boldsymbol{€} /$ t verhinderter Bodenabtrag } \\
\hline Fruchtfolge & $8 \%{ }^{1} 200 \mathrm{~m}^{2}$ & $10 \% 200 \mathrm{~m}$ & $12 \% 200 \mathrm{~m}$ \\
\hline Bewirtschaftung quer zum Hang & & & \\
WR-WW-WG & $28,8^{421}$ & 21,4 & 16,6 \\
WW-WW-WW & 29,7 & 22,3 & 17,2 \\
WR-WW-WW & 29,7 & 22,2 & 17,2 \\
tung & & & \\
Bewirtschaftung in Gefällerich- & & & \\
WR-WW-WG & 25,1 & 19,6 & 15,7 \\
WW-WW-WW & 26,1 & 20,2 & 16,5 \\
WR-WW-WW & 25,6 & 20,3 & 16,3 \\
\hline
\end{tabular}

${ }^{1}$ Hangneigung

${ }^{2}$ Hanglänge

Quelle: Eigene Berechnungen

Die KW dieser Maßnahme sind für die gewählten Beispielstandorte als gut zu bezeichnen. Die KW liegen zwischen 16,3-29,7 €/t verhinderter Bodenabtrag und liegen im Verhältnis mit den bisher ermittelten KW im unteren Bereich.

\section{Kosten-Nutzen-Analyse}

Ausgehend von den Kosten durch den jährlichen Erwerbsverlust der Ausfallfläche in Höhe von $82 € /$ ha bei der Fruchtfolge 1, $71 € /$ ha bei der Fruchtfolge 2 bzw. $86 € /$ ha bei der Fruchtfolge 3 sowie der einmaligen Ansaatkosten von 296,2 €/ha und Nachsaatkosten in Höhe von $130 € / \mathrm{ha}^{422}$, die alle fünf Jahre anfallen, wird eine KNA durchgeführt. Hierbei muss allerdings berücksichtigt werden, dass bei der Nachsaat z. T. hohe Einsparungen z. B. durch Zusammenlegung verschiedener Arbeitsgänge erzielbar sind. Bei der Variante der Verpachtung werden die einmaligen Neuansaatkosten und der jährliche Erwerbsverlust durch den Wegfall der Ackernutzung und bei der Stilllegung nur der Erwerbsverlust berücksichtigt.

Der Nutzen setzt sich für die einzelnen alternativen Handlungsmöglichkeiten wie folgt zusammen. Zunächst ist bei allen Handlungsalternativen die Reduzierung des Bodenabtrags, dargestellt in Tabelle 38, zu berücksichtigen. Hierbei wird von der mittleren Opti-

\footnotetext{
${ }^{420}$ Kosten: WR-WW-WG: 141,2 €/ha; WW-WW-WW: 130,2 €/ha; WR-WW-WW: 145,2 €/ha.

${ }^{421}$ Rechenweg: $(296,2 € /$ ha $: 25+82 € /$ ha $+26 € /$ ha $: 4,3) * 0,04=28,8 € /$ t.

${ }^{422}$ Vgl. KTBL (2002), S. 316; Spezialgrasnachsaatmaschine, $3 \mathrm{~m}, 67 \mathrm{~kW}$, Grassamen $12 \mathrm{~kg} / \mathrm{ha}$, alle fünf Jahre.
} 
on, einer Hangneigung von $10 \%$ und einer Hanglänge von 200 m, ausgegangen. Demnach ist im Falle der Bewirtschaftung quer zum Hang und in Gefällerichtung, mit den Werten $28,9 € / \mathrm{ha}^{423}$ und $31,8 € / \mathrm{ha}^{424}$ zu rechnen. Des Weiteren werden die bereits oben dargestellten Nutzeneffekte (DB) aus der alternativen Nutzung des Ackerlandes berücksichtigt. Hieraus ergibt sich bspw. für die Mutterkuhhaltung und der Verpachtung durch Addition ein Nutzen von 81,8 €/ha und für die Stilllegung 289,4€/ha. Die Ergebnisse der Berechnungen sind in der Tabelle $40 \mathrm{zu}$ ersehen. Der Zinssatz beträgt $4 \%$ und der Betrachtungszeitraum 25 Jahre. Die ausführlichen Berechnungen sind exemplarisch für alle Varianten je einmal in der Tabelle A-48 bis Tabelle A-50 zu ersehen.

\footnotetext{
${ }^{423}$ Mittelwert; Bewirtschaftung quer zum Hang; $10 \%, 200 \mathrm{~m}$.

${ }^{424}$ Mittelwert; Bewirtschaftung in Gefällerichtung $10 \%, 200 \mathrm{~m}$.
} 
Tabelle 40: KNV und jährlicher Vorteil bei verschiedenen Alternativen zur Ackernutzung am Hang ${ }^{425}$

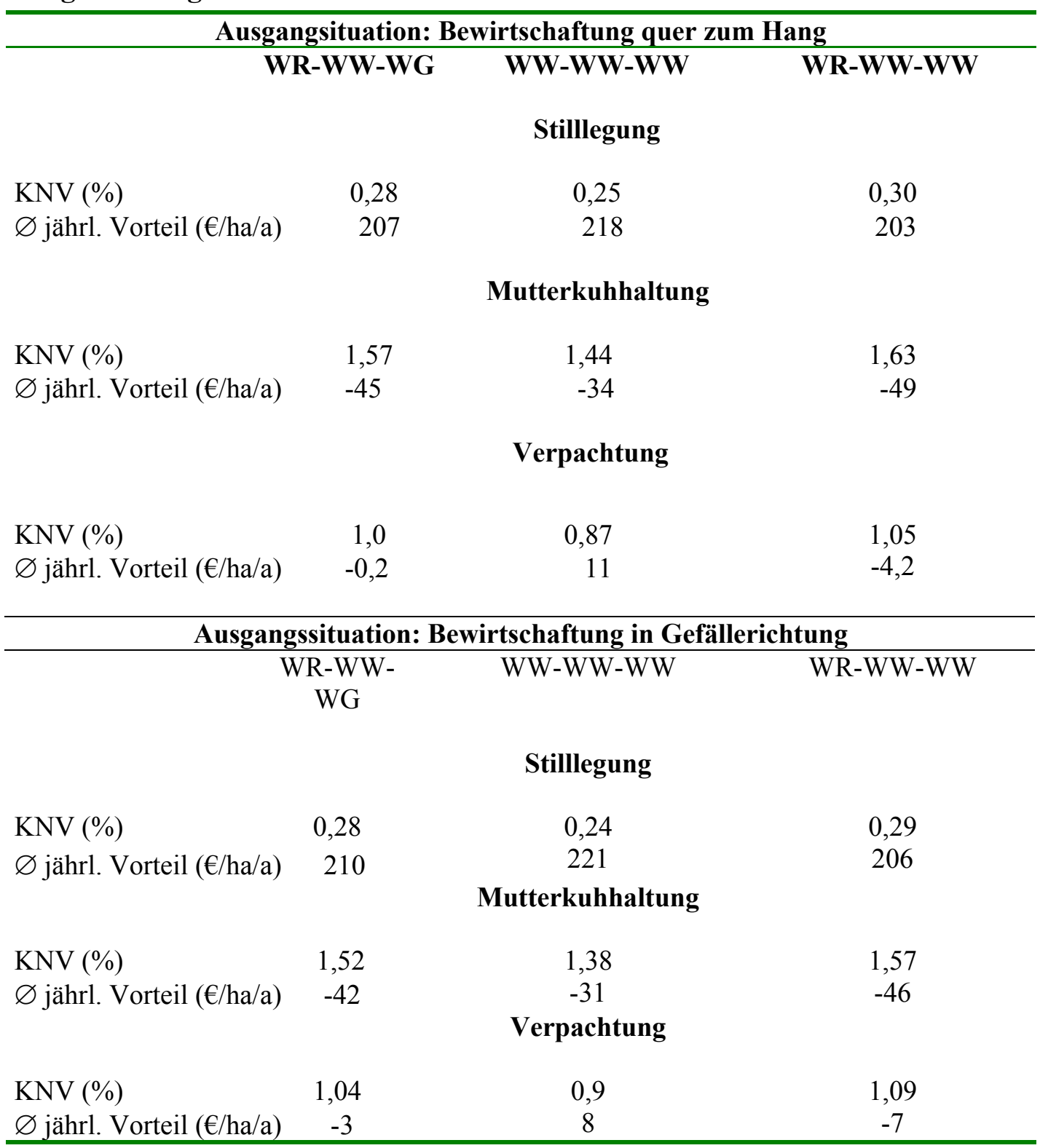

Quelle: Eigene Berechnungen

Die KNV liegen für die Stilllegung zwischen 0,24 \% und 0,30\%, für die Mutterkuhhaltung zwischen 1,38\% und 1,63\% sowie für die Verpachtung zwischen 0,8\% und $1,09 \%$. Die Interpretation und Einschränkung der Ergebnisse erfolgt im nächsten Abschnitt.

${ }^{425}$ Mit einer Hangneigung von $10 \%$ und einer Hanglänge von 200 m. 


\subsection{Schlussfolgerungen}

Die Ergebnisse zeigen, dass unter den angenommen Voraussetzungen nur die Stilllegung und die Verpachtung wirtschaftliche Alternativen sein können. An dieser Stelle müssen allerdings gerade zur Mutterkuhhaltung einige wichtige Einschränkungen gemacht werden. Da nur der DB angesetzt wurde, sind noch einige Festkosten, wie z. B. eventuell anfallende Gebäudekosten zu berücksichtigen. Ein weiterer wichtiger Punkt ist die u. U. mangelnde Verfügbarkeit von Mutterkuhprämienrechten. Im Rahmen der Entkopplung der Luxemburger Beschlüsse kann diese Erwägung allerdings eine immer geringere Rolle spielen. Die Diskussion um die Reform der Agrarpolitik ist daher weiterhin zu verfolgen und abzuwarten, inwieweit die Mutterkuhprämie entkoppelt wird. Aufgrund dieser Bewertung der Mutterkuhhaltung ist davon auszugehen, dass auch die Färsenaufzucht und die Bullenmast unter den in Kaptitel 5.3.4 verwendeten DB nicht wirtschaftlich sein können.

Außerdem wurde in der Berechnung ein Grenzstandort vorausgesetzt. Somit ist die Problematik der Stilllegung von Hangflächen auch nicht uneingeschränkt mit der von Hochwasserschutzgebieten zu vergleichen. Bei Hochwasserschutzgebieten handelt es sich i. d. R. um sehr fruchtbare Auenböden o. ä., die für die landwirtschaftlichen Betriebe die wertvollsten Böden darstellen.

\subsubsection{Erosionsschutzprogramme}

Als abschließende Maßnahme dieses Kapitels werden Erosionsschutzprogramme betrachtet. Sie stellen zwar keine direkte einzelbetriebliche Maßnahme dar, haben allerdings durch die Neuausrichtung der Agrarpolitik und Einführung des Modulationsgesetzes in der Vergangenheit an Bedeutung gewonnen. Erosionsschutzprogramme fördern die konservierende Bodenbearbeitung auf einzelbetrieblicher Ebene. Im Folgenden wird die Maßnahme ausführlich erläutert. Dabei wird die aktuelle Fördersituation und die Verbreitung in den einzelnen Bundesländern berücksichtigt. Abschließend erfolgt eine Beispielberechnung anhand derer abzusehen ist, wie sich die Förderung der konservierenden Bodenbearbeitung auf die KNV auswirkt. 


\subsection{Einleitende Erwägungen}

Die Landwirte können die monetären Schädigungen des Bodens durch die Bewirtschaftung nicht exakt quantifizieren. Dies mindert zunächst den Anreiz, an entsprechenden Programmen teilzunehmen. Auf der anderen Seite entstehen für einige Landwirte Mitnahmeeffekte, wenn die Durchführung der konservierenden Bodenbearbeitung bereits günstige KNV aufweist, wie bspw. in Kapitel 6.3.1.1.3 dargestellt wurde. Dies ist ein wesentlicher Kritikpunkt an Erosionsschutzprogrammen. Ein in der Hinsicht effizienteres Programm ist das Conservation Reserve Program der USA. Dieses beinhaltet u. a. ein Ausschreibungsverfahren, welches Mitnahmeeffekte begrenzt. ${ }^{426}$

Aus einzelbetrieblicher Sicht erhöht ein Erosionsschutzprogramm zunächst den Anreiz, Mitnahmeeffekte zu realisieren. Außerdem führen sie dazu, dass einige Landwirte zusätzlich bodenschonend wirtschaften und das Risiko der Umstellung abgemildert wird. In der folgenden Tabelle sind die Fördergegenstände und -höhen für die einzelnen Erosionsschutzprogramme der Bundesländer dargestellt. Bei einigen Programmen, wie z. B. die in Niedersachsen und Schleswig-Holstein, handelt es sich um Förderungen, die durch das Modulationsgesetz entstanden sind. Andere, wie z. B. die Nordrhein-Westfalens und Sachsens, bestehen bereits längere Zeit. Die Länge des Förderzeitraums beträgt i. d. R. fünf Jahre. Dabei ist zu berücksichtigen, dass einige Bundesländer wie bspw. Niedersachsen, aufgrund der Haushaltslage z. Zt. keine Neuanträge annehmen.

${ }^{426}$ Vgl. hierzu PlankL (1999) sowie LATACZ-LOHMANN u. BUCKWELl (1998) 
Tabelle 41: Fördermaßnahmen im Rahmen des Erosionsschutzes in den einzelnen Bundesländern für das Jahr 2003. Stand: Dezember $2003^{427}$

\begin{tabular}{|c|c|c|c|}
\hline $\begin{array}{l}\text { Bundes- } \\
\text { land }\end{array}$ & $\begin{array}{l}\text { Förderpro- } \\
\text { gramm }\end{array}$ & Fördergegenstand & $\begin{array}{c}\text { Förderbetrag } \\
(€ / \text { ha })\end{array}$ \\
\hline Bayern & KULAP A & Mulchsaat in Reihenkulturen mit ZF & 100 \\
\hline B-W & MEKA II & $\begin{array}{l}\text { Mulchsaat mit und ohne Saatbettberei- } \\
\text { tung mit ZF }\end{array}$ & 60 \\
\hline Hessen & HEKUL & Mulch- u. Direktsaat mit ZF & 60 \\
\hline Nds. & $\begin{array}{l}\text { NAU } \\
\text { AFP }\end{array}$ & $\begin{array}{c}\text { Mulch- u. Direktsaat mit ZF } \\
\text { Anschaffung von Mulchsaatgeräten }\end{array}$ & $\begin{array}{c}72 \\
\text { Zuschuss bis zu } \\
20 \%{ }^{428}\end{array}$ \\
\hline NRW & $\begin{array}{c}\text { RL „Förderung } \\
\text { von Erosions- } \\
\text { schutzmaß- } \\
\text { nahmen“ }\end{array}$ & $\begin{array}{l}\text { Mulch- u. Direktsaat bei Rüben-, Mais-, } \\
\text { Raps-, Leguminosen- und Kartoffelan- } \\
\text { bau mit ZF } \\
\text { - Förderung nach Gebietskulisse }\end{array}$ & $102 € / \mathrm{ha}$ \\
\hline R-P & FUL & $\begin{array}{c}\text { Mulchsaat bei Mais und Zuckerrüben } \\
- \text { mit ZF } \\
\text { - im Strohmulchverfahren }\end{array}$ & $\begin{array}{l}117,6 \\
46,02\end{array}$ \\
\hline S-A & $\begin{array}{l}\text { Umsetzung der } \\
\text { VO (EG) Nr. } \\
1257 / 99\end{array}$ & Mulch- und Direktsaat & 42 \\
\hline Sachsen & $\begin{array}{c}\text { UL } \\
\text { RL 21/2003 }\end{array}$ & $\begin{array}{c}\text { Mulchsaat } \\
\text { Zwischenfruchtanbau } \\
\text { Anbau von Untersaaten } \\
\text { Anschaffung von Mulchsaatgeräten }\end{array}$ & $\begin{array}{c}25 \\
66 \\
51 \\
\text { Zuschuss bis zu } \\
35 \%{ }^{429} \\
\end{array}$ \\
\hline $\mathrm{S}-\mathrm{H}^{430}$ & $\begin{array}{l}\text { VO (EG) Nr. } \\
1257 / 99\end{array}$ & Mulch- und Direktsaat & 60 \\
\hline
\end{tabular}

Quelle: Eigene Zusammenstellung

Für die Förderprogramme in Nordrhein-Westfalen und Sachsen liegen bereits mehrjährige Dokumentationen über die Akzeptanz in der Landwirtschaft vor. Bspw. wurden Ende 2002 in NRW 60.000 ha im Rahmen des Erosionsschutzprogramms gefördert. Dies entspricht 1.700 Förderanträgen. ${ }^{431}$ Die Entwicklung der im Rahmen des Förderprogramms bewirtschafteten Fläche ist in der Abbildung 24 dargestellt. Dabei ist eine kontinuierliche linear verlaufende Steigerung der bewirtschafteten Fläche festzustellen.

\footnotetext{
${ }^{427}$ Zum Stand der Umsetzung der Modulation siehe auch STANGE (2004).

${ }^{428}$ Bei der Bezuschussung ist das Investitionsvolumen entscheidend. Bei einem Investitionsvolumen von $10.000-100.000 €$ wird ein Zuschuss von bis zu $20 \%$ gewährt.

${ }^{429} \mathrm{Bis}$ zu einem Investitionsvolumen von $50.000 €$.

${ }^{430}$ Keine Neuanträge in 2004 möglich.

${ }^{431}$ Vgl. schriftliche Auskunft WEINS.
} 
Abbildung 24: Entwicklung der durch das Erosionsschutzprogramm NRW geförderten pfluglos bewirtschafteten Fläche für beide Kammergebiete

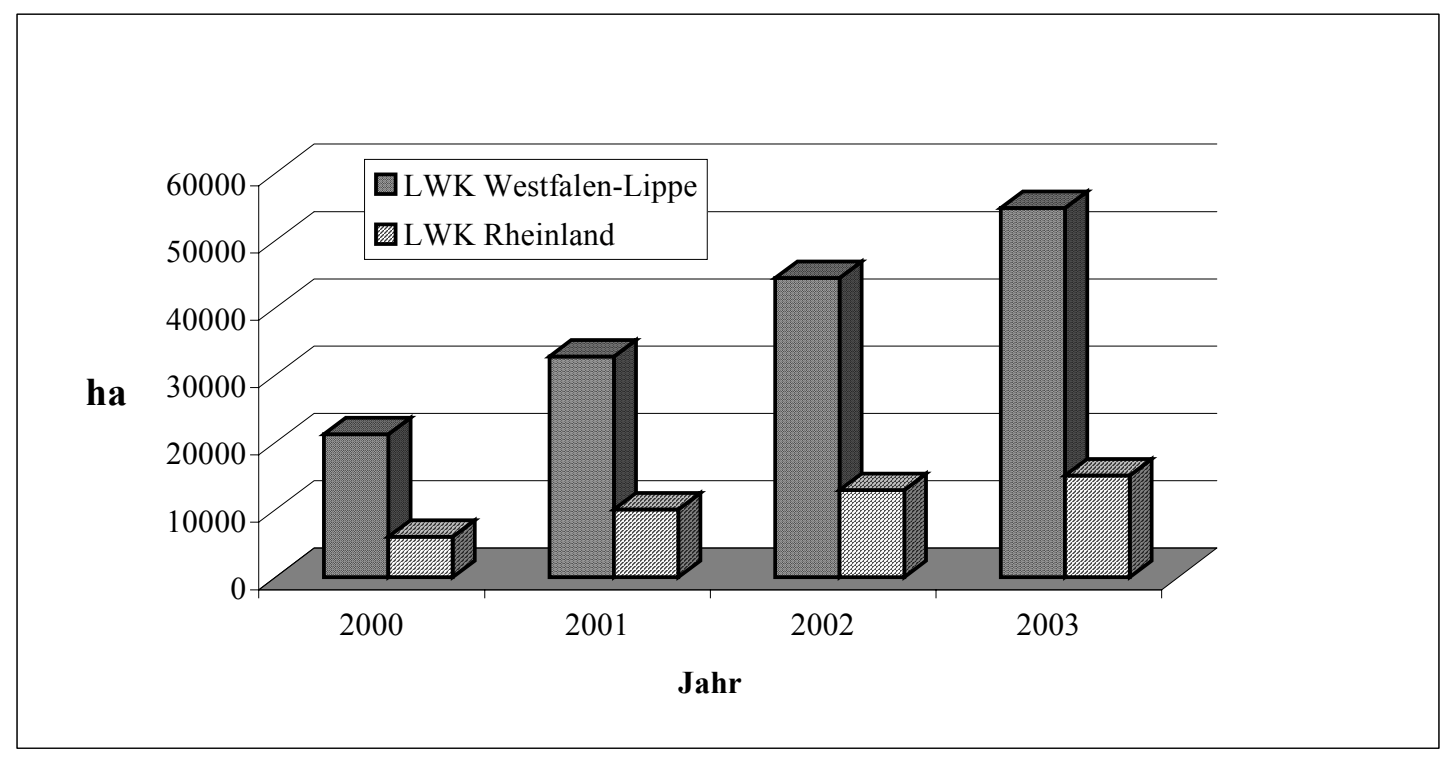

Quelle: NigGeschulze (2003), S. 22

Die Entwicklung der Verbreitung der geförderten Fläche im Rahmen des Programms „Umweltgerechte Landwirtschaft“ Sachsens ist in der Abbildung $25 \mathrm{zu}$ erkennen. Auch in diesem Programm sind die geförderten Flächen kontinuierlich gestiegen. In 2002/03 betrug der Anteil der geförderten Fläche 25,9 \% an der Ackerfläche. 


\section{Abbildung 25: Förderumfang der Maßnahme Konservierende Bodenbearbeitung in}

\section{Sachsen seit 1993}

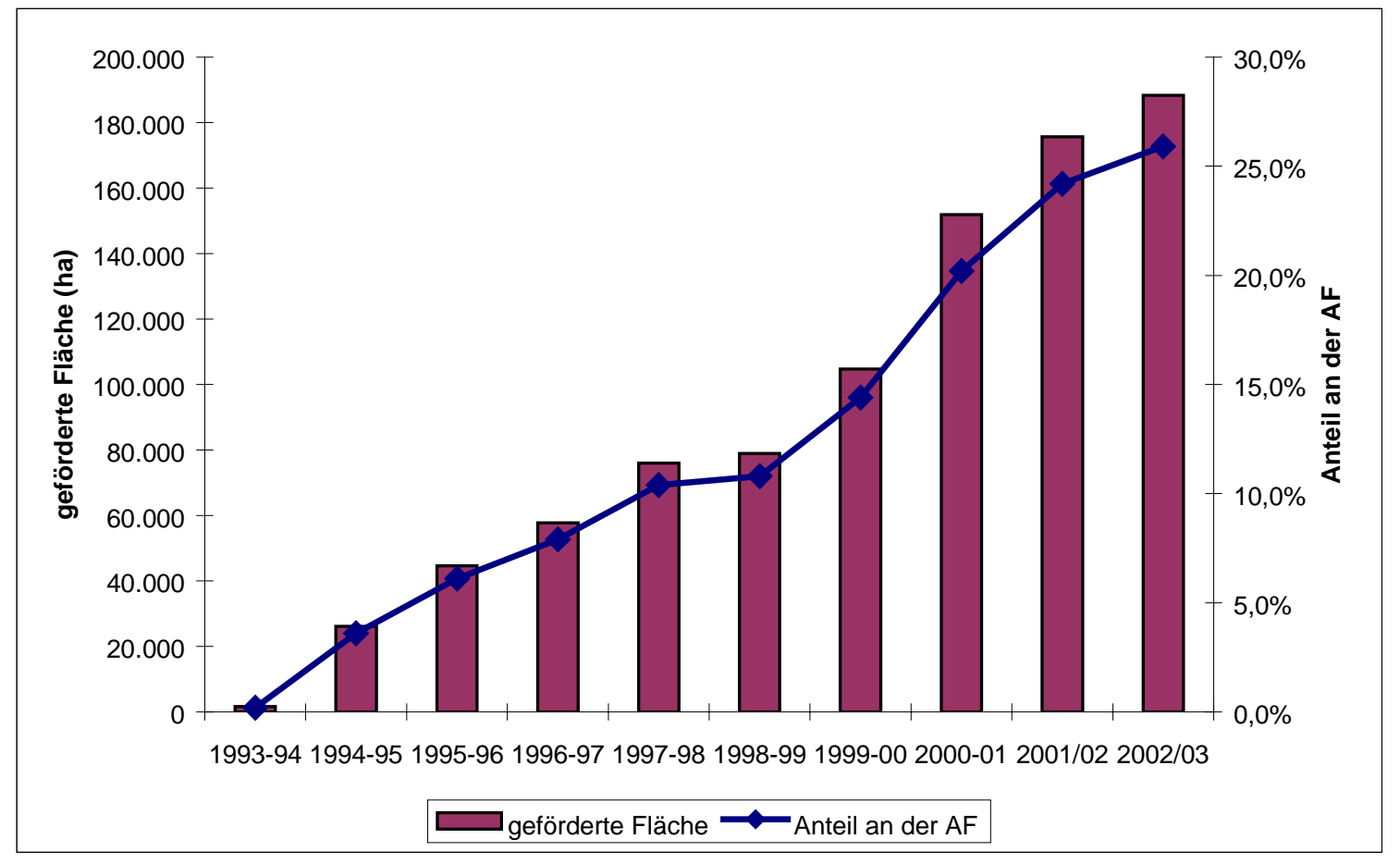

Quelle: SCHMIDT (2002), S. 114 u. SÄCHSISCHER AGRARBERICHT (2002)

\subsection{Betriebswirtschaftliche Analyse der Maßnahme}

Die einzelbetriebliche Betrachtung der Erosionsschutzprogramme zeigt, dass für konservierende Bodenbearbeitung Prämien in Höhe von 25-100 €/ha gezahlt werden. Diese Zahlungen sind für fünf Jahre garantiert, dann muss der Landwirt einen neuen Antrag stellen. Die Zahlung verbessert die Vorzüglichkeit der konservierenden Bodenbearbeitung. Dies wird an den Ergebnissen des Kapitels 6.3.1.1.3 deutlich. Dort wurde für die konservierende Bodenbearbeitung folgende KW in Tabelle 42 ohne Förderung errechnet. Diese Berechnung wird um die Förderung durch Erosionsschutzprogramme mit $25 € /$ ha erweitert:

Tabelle 42: KW (in $€ / t$ verhinderter Bodenabtrag) bei der konservierenden Bodenbearbeitung

\begin{tabular}{lcc}
\hline & Ohne Förderung & Förderung: $\mathbf{2 5} \boldsymbol{\epsilon} / \mathbf{h a}$ \\
\hline Mulchsaat & $-4,9--2,7$ & $-10,6--5,7$ \\
Direktsaat & $-12,5--6,7$ & $-18,2--9,8$ \\
\hline
\end{tabular}

Quelle: Eigene Berechnungen 
Die KW der konservierenden Bodenbearbeitung ist negativ. Durch eine zusätzliche Förderung würde eine Verbesserung der KW stattfinden.

Die Teilnahme an den Erosionsschutzprogrammen der Länder verbessert auch das KNV der Maßnahme. Angenommen wird, dass der Landwirt die Förderung für die ersten fünf Jahre seiner Umstellung erhält. Der Zinssatz beträgt $4 \%$ und der Betrachtungszeitraum 25 Jahre. Die Ergebnisse sind in der Tabelle 43 dargestellt. Die Berechnungen können der Tabelle A-51 und A-52 entnommen werden.

Tabelle 43: Einzelbetriebliches KNV der Mulch- und Direktsaat bei einem Zinssatz von $4 \%$ und mittleren Bodenabtragsraten ${ }^{432}$, inkl. Förderung (60 €/ha)

\begin{tabular}{lcccc}
\hline \multicolumn{1}{c}{ Bodenabtrag (t/ha) } & $\begin{array}{c}\text { Mulchsaat } \\
(4,6)\end{array}$ & $\begin{array}{c}\text { Mulchsaat } \\
(8,7)\end{array}$ & $\begin{array}{c}\text { Direktsaat } \\
(4,6)\end{array}$ & $\begin{array}{c}\text { Direktsaat } \\
(8,7)\end{array}$ \\
\hline KNV in \% & 0,26 & 0,22 & 0,22 & 0,19 \\
$\varnothing$ Änd. gegenüber Situation ohne & $-0,06$ & $-0,04$ & $-0,03$ & $-0,03$ \\
Förderung in \%: & & & & 102 \\
$\varnothing$ jährl. Vorteil (€/ha/a) & 69 & 88 & +18 & +16 \\
$\varnothing$ Änd. gegenüber Situation ohne & +17 & +17 & & \\
Förderung in $€ /$ ha/a: & & & & \\
\hline
\end{tabular}

Quelle: Eigene Berechnungen, Zinssatz $4 \%$

Die obige Tabelle zeigt für das in dieser Arbeit gewählte Beispiel die Höhe der Verbesserung des KNV durch eine Förderung in Höhe von $60 € /$ ha. Die KNV haben sich um $0,04 \%$ bis zu 0,12\% gegenüber der Ausgangssituation verbessert, die durchschnittlichen jährlichen Vorteile liegen um $17 € /$ ha höher.

\subsection{Schlussfolgerungen}

Erosionsschutzprogramme dienen als Katalysator, also zur Beschleunigung der Verbreitung konservierender Bodenbearbeitungsverfahren. Positive Ergebnisse sind aus den Bundesländern Sachsen und NRW bekannt.

Die obigen Berechnungen haben gezeigt, dass die Förderung von konservierenden Bodenbearbeitungsverfahren einen erheblichen Beitrag zur Verbesserung der Wirtschaftlichkeit dieser Maßnahmen leisten kann. In dem gewählten Beispiel wurden jedoch nur

\footnotetext{
${ }^{432}$ Die Werte in Klammern geben die mittleren Bodenabtragsraten des Standorts an.
} 
Mitnahmeeffekte geschaffen, da die Maßnahmen auch ohne Förderung wirtschaftlich sind. Dies ist zugleich ein wesentlicher Kritikpunkt an derartigen Fördermaßnahmen ist. Da jedoch die konservierende Bodenbearbeitung eine wichtige Schlüsselmaßnahme für den landwirtschaftlichen Bodenschutz ist und die Förderdauer jeweils nur fünf Jahre beträgt, sind sämtliche Erosionsschutzprogramme als Anschubfinanzierung zu sehen. Die Bundesländer können, wie derzeit teils geschehen, die Programme aussetzen. Hierdurch darf allerdings nicht eine „Politik nach Kassenlage“ entstehen, welche die Zukunftsperspektiven der Landwirte verklärt.

Eine Gebietskulisse, wie sie in NRW vorliegt, ist generell zu begrüßen, da sie gewährleistet, dass die Mittel in die akut gefährdeten Gebiete fließen, was ebenfalls die Mitnahmeeffekte senkt.

\subsubsection{Maßnahmen zur Verhinderung von Bodenverdichtungen}

Die landtechnische Entwicklung in der Vergangenheit hat dazu geführt, dass größere Maschinen und höhere Lasten auf den landwirtschaftlichen Nutzflächen zum Einsatz kommen. Außerdem bestehen zwischen der Landtechnikindustrie und wissenschaftlichen Instituten seit längerem Bemühungen, den Einsatz von leistungsfähigen Maschinen und den Schutz vor Bodenschadverdichtungen zu vereinen. ${ }^{433}$ In der folgenden Abbildung ist eine Übersicht von gängigen Fahrwerken dargestellt, die zum größten Teil Gegenstand der nachfolgenden betriebswirtschaftlichen Berechnungen sind.

\section{Abbildung 26: Schematische Darstellung der Fahrwerke}
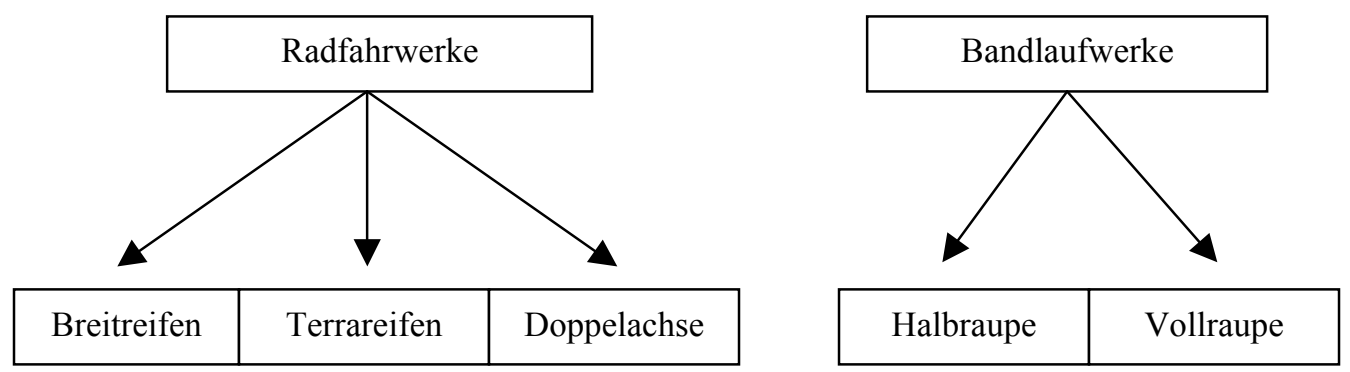

Quelle: QuAs, SchWIEGER u. WeIßBACH (1995), S. 16 
Im Folgenden wird v. a. der Einsatz von Breit- und Terrareifen sowie der Einsatz der Vollraupe behandelt, da sich diese Systeme am weitesten in der landwirtschaftlichen Praxis verbreitet haben.

Im Folgenden werden die obigen Systeme sowie Reifendruckregelanlagen zunächst dargestellt und dann anhand von Kostenvergleichen, KW-Berechnungen und KNA verglichen und analysiert.

\subsubsection{Reifenwahl}

Die Einflussmöglichkeiten des landwirtschaftlichen Betriebsleiters auf die Entstehung von Bodenschadverdichtungen bestehen neben dem Einsatz von Maschinen mit geringen Radlasten v. a. in der Reifenwahl und dem Luftdruck. Dabei ist die Beziehung zwischen der Breite des Reifens und dem zu wählenden Luftdruck zu berücksichtigen. Je breiter die Reifen sind, desto niedriger kann der Luftdruck gewählt werden. Obwohl diese Zusammenhänge schon längere Zeit bekannt sind, zeigen Ergebnisse einer Befragung von landwirtschaftlichen Betriebsleitern in Sachsen, dass nur ein geringer Teil der Traktoren und Mähdrescher mit Breit-, Terra- oder Zwillingsreifen ausgestattet sind und daher noch erhebliche Reserven auf den landwirtschaftlichen Betrieben vorhanden sind. ${ }^{434}$ Für die westlichen Bundesländer ist zwar davon auszugehen, dass die Maschinengewichte niedriger sind, dafür ist aber auch anzunehmen, dass der Anteil an Breit- Terra- oder Zwillingsreifen noch geringer ist und diese Reifen hauptsächlich nur beim überbetrieblichen Maschineneinsatz zum Einsatz kommen. Nachfolgend werden die Kosten von Standard-, Breit- und Terrareifen verglichen, bevor eine KWA und eine KNA durchgeführt wird.

\subsection{Einleitende Erwägungen}

Der Landwirt sieht sich bezogen auf die Reifenwahl für seine landwirtschaftlichen Maschinen einem zunehmenden Angebot an bodenschonenden Reifen gegenüber. Reifen müssen in den Betrieben zahlreiche Funktionen erfüllen. Auf der Straße sollten sie sich durch einen ruhigen Schnelllauf, Komfort und einen geringen Verschleiß auszeichnen. Auf dem Ackerboden sollten sie eine möglichst hohe Zugkraft, einen hohen Wirkungs-

\footnotetext{
${ }^{434}$ Vgl. StAhL, SCHMidT U. GierKe (2001), S. 108
} 
grad, einen geringen Bodendruck sowie eine Narbenschonung bei Grünlandbewirtschaftung gewährleisten.

Neben klassischen Lösungen, wie z. B. Zwillingsbereifung und einfachen Breitreifen, werden mittlerweile auch Niederquerschnittsreifen angeboten, die mit einem konstanten Luftdruck von etwa 1,0 bar sowohl auf der Straße als auch auf dem Acker gefahren werden können. Eine Reifendruckregelanlage ist hierfür folglich nicht notwendig. Die Kosten der auf der Agritechnica 2003 vorgestellten Reifen sind jedoch noch nicht bekannt.

Die Reifenwahl wird von den Vorgaben für die Benutzung öffentlicher Straßen eingegrenzt. Für land- und forstwirtschaftliche Fahrzeuge gilt nach $\S 32$ Abs. 1 StVZO generell eine maximale Gesamtbreite von $3 \mathrm{~m}$. Ausnahmeregelungen für höhere Breiten sind in den einzelnen Landkreisen gesondert zu erfragen. Die Beschränkung der Gesamtbreite bedeutet i. d. R., dass die maximale Reifenbreite von $900 \mathrm{~mm}$ nicht überschritten werden darf.

\subsection{Betriebswirtschaftliche Analyse der Maßnahme}

In der Übersicht 16 ist die Vorgehensweise bei der KNA für bodenschonende Reifen dargestellt. 
Übersicht 16: Vorgehensweise bei der KNA für verschiedene bodenschonende Reifen

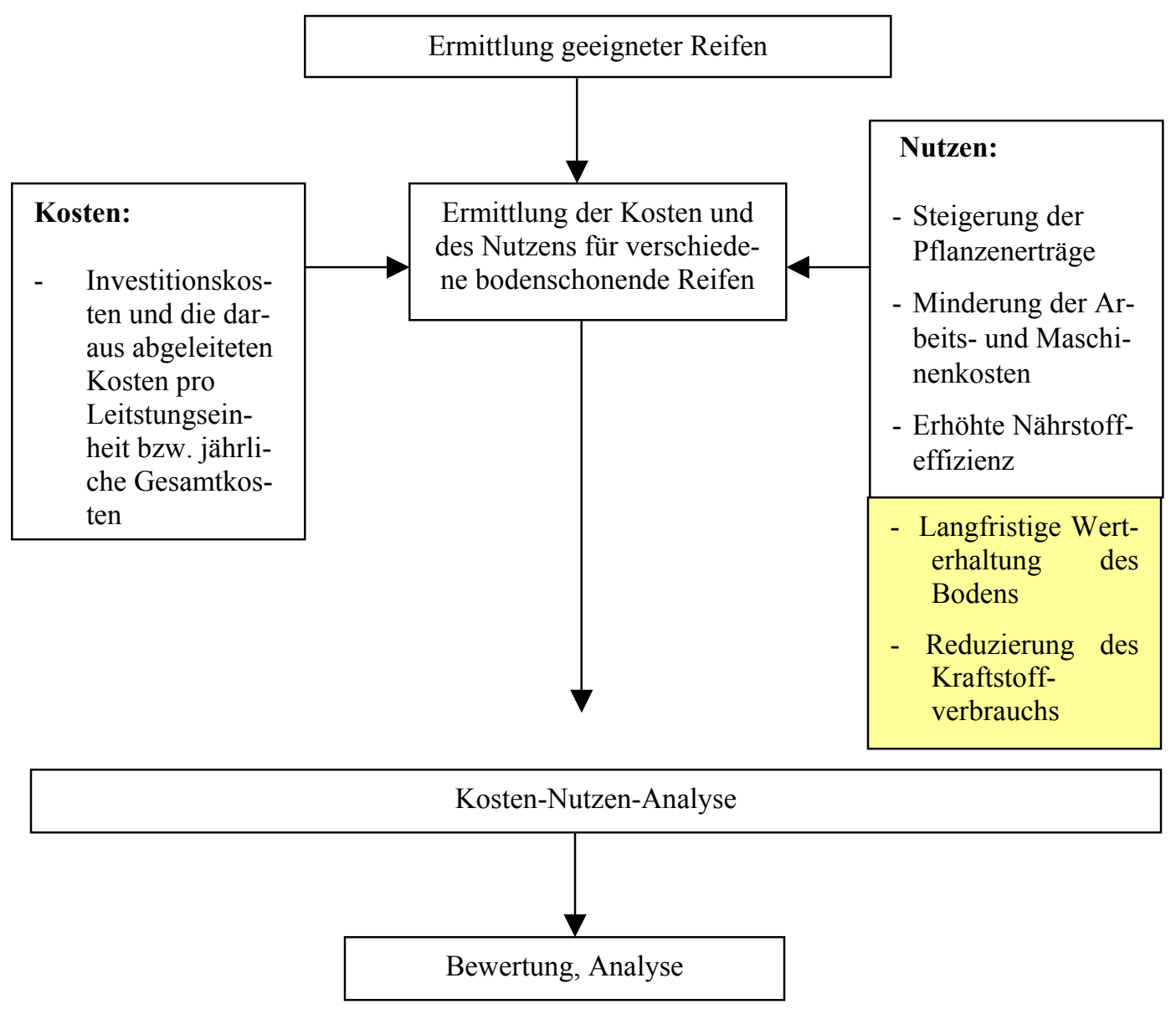

Quelle: Eigene Darstellung, Schraffierung: Positionen konnten aufgrund fehlender Daten in der Berechnung nicht berücksichtigt werden

Die Kosten verschiedener Reifen, darunter ein Standardreifen, ein Breit- sowie ein Terrareifen sind in der Tabelle 44 dargestellt. Zudem werden die Auswirkungen des Einsatzes der Reifen auf den Ober- und Unterboden eingeschätzt. 
Tabelle 44: Anschaffungskosten und Bodendruck unter drei verschiedenen Reifen bei einer Radlast von $6,8 \mathrm{t}$ und verschiedenen Reifeninnendrücken

\begin{tabular}{lccc}
\hline \multicolumn{1}{c}{ Radlast } & $\mathbf{6 , 8 ~}$ & $\mathbf{6 , 8 ~ t}$ & $\mathbf{6 , 8 ~ t}$ \\
\hline Reifenart & $23,1 \mathrm{R} 26 \mathrm{BIB}^{\prime} \mathrm{X}$ & $30,5 \mathrm{LR} 32 \mathrm{BIB}^{\prime} \mathrm{X}$ & $73 \times 44,0-32 \mathrm{NHS}$ \\
Anschaffungskosten ${ }^{435}$ & $1.778 €$ & $3.324-6.030 €$ & $7.902 €$ \\
Gesamtkosten $(€ / \mathrm{h})^{436}$ & 0,52 & 1,38 & 2,34 \\
Mehrkosten Breit- Terrareifen & & & \\
$(€ / \mathrm{h})$ & & 0,86 & 1,82 \\
Reifeninnendruck & $2,4 \mathrm{bar}$ & $1,3 \mathrm{bar}$ & $1,2 \mathrm{bar}$ \\
Bodendruck $(20 \mathrm{~cm})$ & $>2 \mathrm{bar}$ & $1,5 \mathrm{bar}$ & $1 \mathrm{bar}$ \\
Bodendruck $(40 \mathrm{~cm})$ & $1 \mathrm{bar}$ & $0,4 \mathrm{bar}$ & $0,3 \mathrm{bar}$ \\
\hline
\end{tabular}

Quelle: BMVEL (2001), S. 28

Es ist zu erkennen, dass die Bodendrücke mit zunehmender Tiefe spürbar abnehmen. Bei einem Terrareifen (73x44,0-32 NHS) sind die Bodendrücke, insbesondere auch unterhalb der Krumenbasis, am geringsten. Ein Vorteil dieser Bereifung ist neben der höheren Breite auch die Möglichkeit, mit geringeren Reifeninnendrücken $\mathrm{zu}$ fahren und somit den Kontaktflächendruck überproportional zu mindern. Ähnliche Effekte werden auch in im Kapitel 6.3.2.2.1 dargestellt.

Bei der Kostenbetrachtung ist zu beachten, dass die jährlichen Gesamtkosten landwirtschaftlicher Reifen maßgeblich von der Nutzungsdauer abhängen. Bei den in der folgenden Berechnung verwendeten Reifen wird von einer durchschnittliche Nutzungsdauer von $3.500 \mathrm{~h}$ ausgegangen. Bei der Nutzung von Breit- bzw. Terrareifen kann es bei zunehmenden Straßenfahrten zu einer höheren Abnutzung kommen. Dies wird in der nachfolgenden Berechnung allerdings nicht berücksichtigt. Bei jährlichen Nutzungsdauer von 800 h/a ergeben sich für die bodenschonenden Reifen im Vergleich zum Standardreifen höhere Gesamtkosten von $0,86 € / \mathrm{h}$ für den Breitreifen und $1,82 € / \mathrm{h}$ für die Terrabereifung. Diese Kosten gehen unter Bezugnahme der jährlichen Einsatzzeit je Hektar in die KNA ein da sie diejenigen sind, welche durch den monetären Nutzen der bodenschonende Wirtschaftsweise gedeckt werden müssen.

In der Literatur sind Vergleichswerte für die Kosten bodenschonender Reifen zu finden. Die Kostendatei „Preise für Leihmaschinen und Dienstleistungen“ des $\mathrm{KTBL}^{437}$ weist bspw. pauschale Werte aus. In dieser Datei werden Zuschläge für Breitreifen von

\footnotetext{
${ }^{435}$ Vgl. RHG-REIFENPREISLISTE 2002, für zwei Schlepperreifen.

${ }^{436}$ Zinssatz $4 \%$, Nutzungsdauer 3.500 h.

${ }^{437}$ Vgl. KTBL (2002b)
} 
5,1-7,7 €/h angegeben. ISENSEE geht beim Vergleich von 650er Reifen mit 900er Reifen von Mehrkosten für den kompletten Schlepper in Höhe von 1-2 €/h aus. ${ }^{438}$ Für Niederquerschnittsreifen liegen noch keine Preise des Herstellers, in diesem Fall Michelin, vor.

Aufgrund der eigenen Kostenberechnungen wird zunächst eine KWA für die o. g. Reifenarten durchgeführt. Dabei werden die zuvor ermittelten zusätzlichen jährlichen Kosten $^{439}$ der bodenschonenden Reifen auf die in Tabelle 44 dargestellte Minderung des Bodendrucks gegenüber Standardreifen bezogen. Es wird davon ausgegangen, dass im Fall der Breitreifen im Oberboden die Druckreduktion um 1 bar nach 2 Jahren und im Unterboden nach 2,5 Jahren erfolgt. ${ }^{440}$ Für die Terrareifen ergeben sich Werte von $1 \mathrm{Jahr}$ für den Ober- sowie 3,3 Jahre für den Unterboden. Hieraus ergeben sich folgende KW:

Tabelle 45: KW (€/bar Bodendruckabsenkung) beim Einsatz von Breit- und Terrareifen bezogen auf den Ober- und Unterboden im Vergleich zum Standardreifen

\begin{tabular}{lcc}
\hline & Oberboden (20 cm) & Unterboden $\mathbf{( 4 0} \mathbf{~ c m})$ \\
\hline Bezugsgröße & Kosten je bar Druckabsenkung & Kosten je bar Druckabsenkung \\
Breitreifen & $1.348,9$ & $1.130,9$ \\
Terrareifen & $1.574,8$ & $2.062,5$ \\
\hline
\end{tabular}

Quelle: Eigene Berechnungen

Die an dieser Stelle ermittelten KW werden in den folgenden Kapiteln mit den dort analysierten Maßnahmen verglichen. Bisher ist zu erkennen, dass die Breitreifen günstigerer KW im Ober- sowie v. a. im Unterboden aufweisen.

\section{Kosten-Nutzen-Analyse}

Für die folgende KNA werden die oben ermittelten jährlichen zusätzlichen Gesamtkosten für die beiden bodenschonenden Bereifungen in Höhe von $0,86 € / \mathrm{h}$ bzw. 1,82 €/h verwendet. Unter der Annahme, dass die Reifen durchschnittlich 9,3 h/ha/a auf den ldw. Flächen eingesetzt werden ergeben sich in die KNA einzubeziehende zusätzliche Kosten von $8,0 € /$ ha für die Breitreifen und $17,0 €$ /ha für die Terrareifen.

\footnotetext{
${ }^{438}$ Vgl. hierzu ISENSEE (2004), S. 31

${ }^{439}$ Berechnung: $800 \mathrm{~h} / \mathrm{a}$ * zusätzliche Gesamtkosten in $€ /$ h sowie Hinzurechnung der Zinskosten.

${ }^{440}$ Vgl. Tabelle 44
} 
Der Nutzen des Einsatzes bodenschonender Reifen besteht neben der Verminderung von Bodenverdichtungen auch in der Verminderung von Schlupf und des Zugkraftbedarfs, was mit einer Senkung des Dieselverbrauchs und Einsparungen von Arbeitszeit einhergeht. VOLK u. SCHNAPP ermitteln bspw. für einen 160 PS-Traktor bei Verwendung von Breit- gegenüber Standardreifen, beim Pflügen eine Arbeitszeitminderung von bis zu $20 \%$ und einer Dieseleinsparung von bis zu $10 \%{ }^{441}$ In diesem Fall würden sich bspw. bei einem 5-Schar-Pflug Einsparungen von 1,68-5,52 €/ha ${ }^{442}$ ergeben. Diese Einsparpotenziale gelten für alle durch Zugkraft angetriebenen Maschinen und Geräte und hängen von den Feuchteverhältnissen des Bodens ab. Für zapfwellenbetriebene Geräte, wie z. B. Kreiseleggen, kann mit $33 \%$ der obigen Einsparungen gerechnet werden. Für die in Tabelle A-13 dargestellte Beispielfruchtfolge WR-WW-WG würde dies ein Nutzen je nach Bodenfeuchte von 3,17 - 12,67 €/ha bedeuten. ${ }^{443}$ Für die Fruchtfolgen mit Zuckerrübenanteil werden Einsparpotenziale von 3,39-13,53 €/ha angenommen. Die Autoren geben außerdem an, dass beim Einsatz von Niederquerschnittsreifen u. ä., die mit einem Reifeninnendruck von 0,8 bar gefahren werden können, ertragsmindernde Bodenverdichtungen nicht entstehen. Da dies allerdings nicht für die in diesem Beispiel dargestellten Reifentypen zutrifft, können nur mögliche Ertragssteigerungen angenommen werden, um eine KNA durchzuführen.

Wie in Übersicht A-1 zu sehen ist, können durch die gänzliche Verminderung von Unterbodenschadverdichtungen Ertragsminderungen von 5-50 \% verhindert werden. Im Folgenden wird dieser Nutzenfaktor mit anderen Nutzenpositionen, die im Zusammenhang mit der Verminderung von Bodenschadverdichtungen zu erwarten sind, zusammengefasst. Analog zu der Ermittlung des Nutzens durch die Verminderung der Bodenerosion in Kapitel 6.3.1.1.3 wird im Folgenden eine Berechnung für die Bodenschadverdichtung durchgeführt. Diese Werte werden ebenfalls in den nachfolgenden Berechnungen verwendet.

\footnotetext{
${ }^{441}$ Vgl. VOLK und SCHNAPP (2003), S. 125

${ }^{442}$ Annahmen: 2,331 Diesel* 0,516 $€ / 1=1,2 €+1,44$ Akh * 0,2*15 $€ / \mathrm{h}$

${ }^{443}$ Der Mähdrusch wird an dieser Stelle nicht betrachtet, da die Untersuchungen von VOLK u. SCHNAPP nur mit Schlepperreifen durchgeführt wurden. Außerdem sind die bei der Getreideernte vorherrschenden Bodenfeuchten nicht mit denen der Frühjahrsbetellung zu vergleichen.
} 


\section{Ermittlung des Nutzens durch Verminderung oder Verhinderung von Bodenschad- verdichtungen:}

Die Literatur zeigt eindeutig, dass Ober- bzw. Unterbodenverdichtungen negative Auswirkungen auf den Stoffhaushalt des Bodens und somit auch auf den Pflanzenertrag haben. Die Höhe dieser negativen Auswirkungen ist allerdings nicht eindeutig zu quantifizieren und in Feldversuchen z. T. auch widersprüchlich.

Bei den oben erwähnten Ergebnissen von $\mathrm{HORN}^{444}$ werden im Speziellen die Ergebnisse von WIERMANN ${ }^{445}$ herangezogen, der bei unterschiedlichen Achslasten von 5 und $10 \mathrm{t}$ auf dem Reinshof ${ }^{446}$ Ertragsrückgänge gemessen hat. Sie betragen bei Achslasten von $5 \mathrm{t}$ im ersten Jahr $20 \%$ und in den drei Folgejahren $5 \%$ und bei Achslasten von $10 \mathrm{t}$ im ersten Jahr $35 \%$ und in den Folgejahren 15 \%. Diese Ergebnisse sind verglichen mit den anderen Forschungsergebnissen tendenziell pessimistisch und daher als Obergrenze anzusehen. Des Weiteren wird aufgrund fehlender Langzeituntersuchungen keine weitere Schadverdichtung in den Folgejahren unterstellt. Diese Annahmen führen dazu, dass der Nutzen je nach betrachteter Fruchtfolge anders ausfällt. Die Übertragung der Ergebnisse von Wiermann auf verschiedene Fruchtfolgen ist in Tabelle A-17 für verschiedene Induzierungsstärken (Achslasten von $5 \mathrm{t}$ und $10 \mathrm{t}$ ) dargestellt. Aus dieser Tabelle werden die Nutzenbeträge entnommen, die in der folgenden KNA verwendet werden. ${ }^{447}$

Als weiterer Nutzeneffekt der Verhinderung von Bodenschadverdichtungen sind die entfallenen Bearbeitungskosten zur Behebung der Verdichtung und zur Schaffung eines geeigneten Saatbettes zu nennen. Es existieren zwar in der Literatur keine derartigen Untersuchungsergebnisse, trotzdem wird in einer ergänzenden Variante von einem zusätzlichen Arbeitsgang mit dem Schwer- oder Flügelschargrubber ausgegangen. Die Kosten hierfür liegen bei etwa $46,0 €$ /ha.

Ein weiterer Nutzeneffekt geht durch die verhinderte Minderung der Nährstoffeffizienz aus. An dieser Stelle wird von einer Minderung der Nährstoffeffizienz von $20 \%$ ausgegangen. ${ }^{448}$ Für die einzelnen Fruchtfolgen lässt sich dieser Effekt aus den DB der

\footnotetext{
${ }^{444}$ Vgl. Übersicht A-1

${ }^{445}$ Vgl. ebd.

${ }^{446}$ Vgl. Kapitel 6.3.1.1.5

${ }^{447}$ siehe unten

${ }^{448}$ Analog zu den Ergebnissen von Helal et al. (1994), siehe Kap. 3.1.3.2.
} 
LWK Hannover ersehen. Für die Fruchtfolge WR-WW-WG ergibt sich demnach ein Wert inklusive variabler Arbeits- und Maschinenkosten ${ }^{449}$ von 41,08 €/ha, für WR-WWWW 41,82 €/ha, für ZR-WW-WG 46,8 €/ha und für ZR-WW-WW 47,4 €/ha. ${ }^{450}$

Die langfristige Werterhaltung des Bodens durch Maßnahmen zur Verhinderung von Bodenschadverdichtungen ist sicherlich gegeben. Im Verhältnis zur Beziehung zwischen Bodenerosion und Wertverlust ist bei der Bodenschadverdichtung keine derart lineare Beziehung feststellbar. Dafür sind die Schäden, z. B. Fahrspuren oder Wachstumsstörungen im Bestand visuell erkennbar und führen zu einer Pacht- oder Kaufwertminderung. Eine analoge Berechnung wie in Kapitel 6.3.1.1.3 ist jedoch nicht möglich. Daher bleibt dieser Nutzeneffekt in der KNA unberücksichtigt.

\section{Zusammenfassende Darstellung der KNA}

In der folgenden KNA werden die zusätzlichen jährlichen Gesamtkosten der bodenschonenden Bereifung in Höhe von $8 € /$ ha beim Breit- und $17 € /$ ha beim Terrareifen verwendet. Aufgrund der Vielzahl der Nutzenannahmen werden die für die KNA relevanten Annahmen kurz und übergreifend zusammengefasst.

- Steigerung der Erträge (Ausführliche Darstellung in Tabelle A-17)

- Verminderung des Schlupfs und Zugkraftbedarfs $(3,17 € /$ ha $-12,67 € /$ ha bei Rapsfruchtfolge; 3,39 €/ha - 13,53€/ha bei Zuckerrübenfruchtfolge)

- Verminderung der Bearbeitungskosten zur Beseitigung der Verdichtung (46 €/ha)

- Verhinderung der Reduzierung der Nährstoffeffizienz (41,08 €/ha - 47,4 €/ha)

Die jeweiligen gesamte Nutzenposition ergeben sich dementsprechend aus der Addition der obig angeführten Werte. Um sich an die in der Realität möglichen verschiedenen Nutzenwirksamkeiten anzunähern, wird angenommen, dass die jeweiligen Reifen nicht die gesamten obigen Nutzeneffekte realisieren können. Aufgrund der fehlenden Hinweise in der Literatur werden daher die Wirkungsniveaus variiert. Dabei wird unterstellt, dass die Nutzenwirksamkeit bei den Breitreifen $5 \%$ bzw. $25 \%$ und bei den Terrareifen $5 \%$

\footnotetext{
${ }^{449}$ Annahme: $3 *$ Schlepper (54 kW) mit Anbauschleuderstreuer (1t). 10,1 €/ha * 0,2=2,02 €/ha

${ }^{450} \mathrm{Zu}$ den Aufwendungen für Dünger siehe LWK Hannover (2003). Annahmen der Ertragsniveaus: WW = $80 \mathrm{dt} / \mathrm{ha}, \mathrm{WG}=80 \mathrm{dt} / \mathrm{h}, \mathrm{WR}=25 \mathrm{dt} / \mathrm{ha}$ und $\mathrm{ZR}=550 \mathrm{dt} / \mathrm{ha}$.
} 
bzw. $50 \%$ des oben beschriebenen Gesamtnutzens beträgt. Es wird jeweils von einer Bodenschadverdichtung ausgegangen. Weiterhin werden zwei Fruchtfolgen berücksichtigt. Bei der Fruchtfolge WR-WW-WG wird davon ausgegangen, dass die Bodenschadverdichtung durch eine Achslast von $5 \mathrm{t}$, und bei ZR-WW-WG durch eine Achslast von $10 \mathrm{t}$ induziert wurde. Bspw. ergibt sich daraus für den Terrareifen mit einer Wirksamkeit von $5 \%$ beim Einsatz in der Fruchtfolge ZR-WW-WG im ersten Jahr ein Betrag von 64,19 $€ /$ ha $^{451}$, im zweiten Jahr 25,03 €/ha, im dritten $23,7 € /$ ha, im vierten $37,91 € /$ ha und für die Folgejahre je 13,53 €/ha wegen der Verminderung des Zugkraftbedarfs. Der Betrachtungszeitraum beträgt 25 Jahre und der Zinssatz $4 \%$. Die umfassenden Berechnungen sind in der Tabelle A-53 bis Tabelle A-60 dargestellt.

Tabelle 46: KNV beim Einsatz von Breit- und Terrareifen, optimistische Annahmen $^{452}$

\begin{tabular}{lcc|cc}
\hline & \multicolumn{2}{c|}{ Breitreifen } & \multicolumn{2}{c}{ Terrareifen } \\
Verm. Auswirkungen BSV & $5 \%$ & $25 \%$ & $5 \%$ & $50 \%$ \\
\hline KNV in \% & $\mathbf{0 , 5 4}$ & $\mathbf{0 , 3 6}$ & $\mathbf{1 , 1 7}$ & $\mathbf{0 , 5 3}$ \\
\hline$\varnothing$ jährl. Vorteil $(€ / \mathrm{ha} / \mathrm{a})$ & 7 & 14 & -2 & 15 \\
\hline & \multicolumn{4}{c}{ ZR-WW-WG } \\
\hline KNV in \% & $\mathbf{0 , 4 1}$ & $\mathbf{0 , 1 9}$ & $\mathbf{0 , 8 8}$ & $\mathbf{0 , 2 4}$ \\
\hline$\varnothing$ jährl. Vorteil $(€ / \mathrm{ha} / \mathrm{a})$ & 11 & 34 & 2 & 54 \\
\hline
\end{tabular}

Quelle: Eigene Berechnungen

Die KNV sind in der Tabelle 46 dargestellt. Aufgrund der marginalen Kosten für die bodenschonende Bereifung war bereits zu erwarten, dass es sich beim Einsatz dieser Technik um eine wirtschaftliche und bodenschonende Maßnahme handelt. Dies wird bei den Breitreifen deutlich wenn berücksichtigt wird, dass den Kosten von $8 € /$ ha ein Nutzen durch Verringerung von Schlupf und Zugkraftbedarf von 12,67 €/ha gegenübersteht.

Durch eine einmalige Verhinderung einer Bodenschadverdichtung können in diesem Beispiel, mit Ausnahme der Terrareifen bei der Fruchtfolge WR-WW-WG und einer Wirkung von $5 \%$, sehr gute KNV erzielt werden. Hierbei muss allerdings berücksichtigt werden, dass bezüglich der Nutzeneffekte sehr optimistische Werte angenommen wur-

\footnotetext{
451 Exemplarischer Rechenweg: $(919,7 € / \mathrm{ha}+46 € / \mathrm{ha}+47,4 € / \mathrm{ha}) * 0,05+13,53 € / \mathrm{ha}=64,19 € / \mathrm{ha}$.

${ }^{452}$ Annahmen: Ertragssteigerung im ersten Jahr von $20 \%$ und in den drei Folgejahren von $5 \%$. Verbesserung der Nährstoffeffizienz von $20 \%$, alle anderen Annahmen sind im Text erläutert. Auslastung: 100 ha/a.
} 
den. Allerdings ist in der Praxis davon auszugehen, dass in einem Zeitraum von 25 Jahren wiederum mehr als eine Bodenschadverdichtung vermindert wird.

Bei den obigen Ergebnissen muss berücksichtigt werden, dass es sich um eine Schlagbetrachtung handelt die davon ausgeht, dass ein hohes Schadverdichtungspotenzial vorliegt, wie es bspw. auf dem Reinshof gemessen wurde. Auf der Ebene des Einzelbetriebes sind i. d. R. verschiedene Anteile derartiger Flächen mit unterschiedlichen Schadverdichtungspotenzialen, auch innerhalb eines Schlages, vorhanden. Diese Anteile bestimmen daher den Nutzen von Breit- und Terrareifen maßgeblich. Daher müssen diese, wenn eine KNA auf der Ebene des Einzelbetriebes durchgeführt werden soll, bekannt sein. Ansonsten kommt es zu einer Über- oder Unterschätzung des Nutzens.

Eine weitere Einschränkung des Nutzens wird durch die Radlasten der eingesetzten Maschinen ausgeübt. Wird bspw. in gleichem Maße das Fahrzeuggewicht wie die Aufstandsfläche erhöht, nimmt zwar der Kontaktflächendruck zu, dies führt an der Oberfläche nicht zu einer Zunahme des Bodendrucks. Dafür werden die Drücke aber stärker in den Unterboden verlagert und $\mathrm{u}$. U. durch Überschneidungen von Druckzwiebeln noch weiter verstärkt.

\subsection{Schlussfolgerungen}

Die Berechnungen der Kosten und des Nutzens verschiedener Reifenarten haben gezeigt, dass eine bodenschonende und gleichzeitig wirtschaftliche Reifenwahl für den Einzelbetrieb möglich ist. Aufgrund der notwendigen Auslastung der Reifen und der zunächst hohen Anschaffungskosten sind bodenschonende Reifen nicht für kleine Betriebe zu empfehlen, zumal die entsprechende Folgetechnik hierfür fehlt. Lohnunternehmen und Maschinenringen sind allerdings eine konsequente bodenschonende Bereifung zu empfehlen, da sie i. d. R. viele Arbeiten mit großen Maschinen ausführen. Die Landwirte sollten bei der Vergabe von Arbeiten an diese Dienstleister eine derartige Bereifung fordern. Inwieweit Breit- und Terrareifen auch an Erntemaschinen generell Bodenschadverdichtungen vermeiden, kann an dieser Stelle nicht geklärt werden. Hierzu müssen weitere, nach Bodenart und Feuchtezustände differenzierte Versuche gemacht werden, um spezielle Aussagen über die bodenschonende Wirkung geben zu können. Es darf jedoch nicht vernachlässigt werden, dass den Maßnahmen der Reifenwahl enge Grenzen gesetzt sind. Sie soll- 
te nicht als Argumentation dafür dienen, immer höhere Fahrzeuggewichte ohne eine entsprechende Begleitforschung auf dem Acker zuzulassen.

\subsubsection{Reifendruckregelsysteme}

In der Vergangenheit war in der deutschen Landwirtschaft ein stetiger Strukturwandel zu erkennen. Diese Tendenzen werden sich aufgrund der allgemeinen Preis- und Agrarpolitik der EU sowie der wachsenden Konkurrenz durch die Osterweiterungen fortsetzen. Der Strukturwandel hat zur Folge, dass die wachsenden Betriebe u. a. immer höhere HofFeldentfernung aufweisen. Um die zusätzlich hinzugepachteten oder erworbenen Flächen bewirtschaften zu können, erhöht sich der Anteil der Straßenfahrten. Hierdurch entstehen in den Betrieben aus Sicht des Bodenschutzes suboptimale Verhältnisse. Auf der einen Seite muss mit üblichen Reifen auf der Straße mit einem hohen Luftdruck von über 1,2 bar gefahren werden, um den Verschleiß gering zu halten. Auf der anderen Seite schadet dieser hohe Luftdruck dem Boden bei Ackerfahrten. Eine Lösung dieses Problems kann die Anschaffung einer Reifendruckregelanlage für Traktoren oder Transportgeräte, wie z. B. Güllewagen, sein. Nachfolgend wird diese Möglichkeit anhand der KWA und KNA analysiert.

\subsection{Einleitende Erwägungen}

Ein wesentlicher Einfluss auf die Bodenbelastung wird vom Luftinnendruck des Reifens ausgeübt. Mit zunehmenden Luftinnendruck steigt der Bodendruck, wie Abbildung 27 zeigt, in $10 \mathrm{~cm}$ und $40 \mathrm{~cm}$ Tiefe linear an. Hiernach muss es das Ziel der Landwirte sein, mit möglichst geringen Luftdrücken, bspw. mit 0,8 bar, den Acker zu befahren. Dies ist neben dem Einsatz von Niederquerschnittsreifen mit Reifendruckregelanlagen möglich. 


\section{Abbildung 27: Die Beziehung zwischen Luftdruck des Reifens und Bodendruck im} Ober- und Unterboden ${ }^{453}$

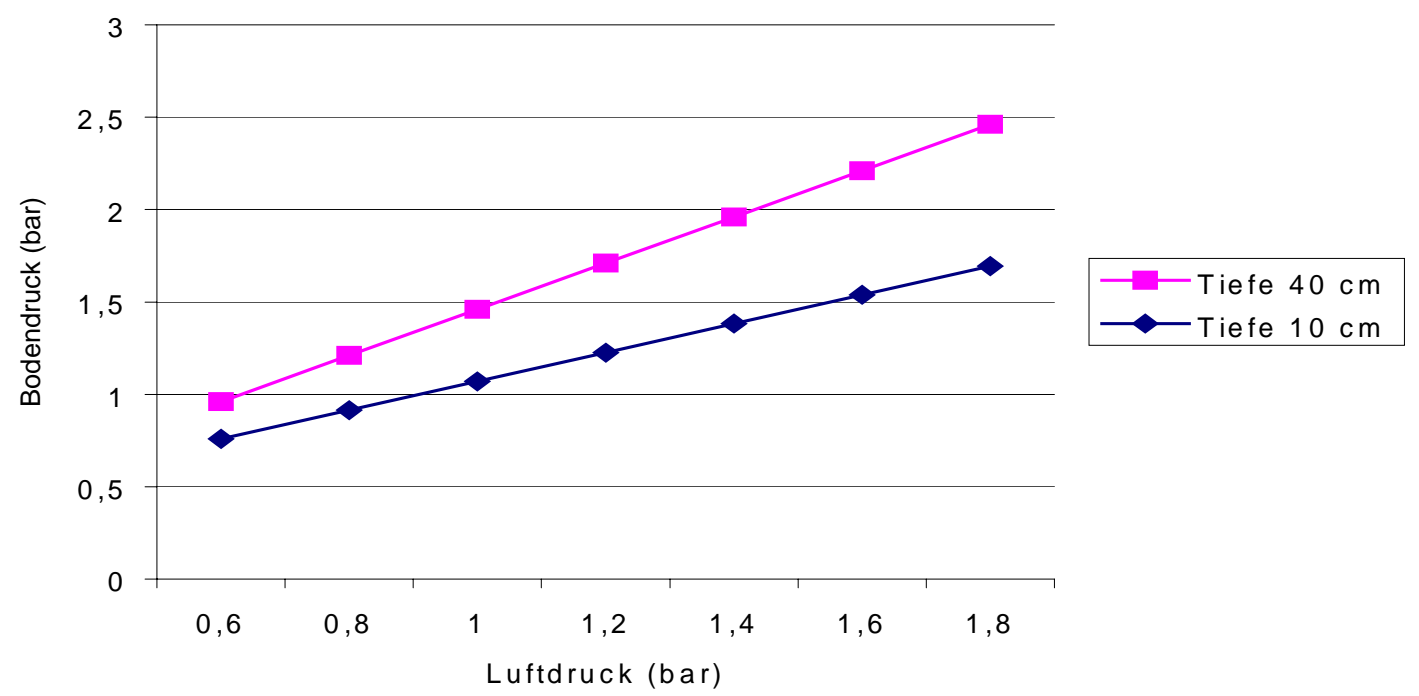

Quelle: WEIßBACH (2002)

Reifendruckregelanlagen lassen sich in stationäre und mobile Systeme einteilen. Die stationären Systeme senken den Luftdruck an einer fest installierten Basisstation, die sich i. d. R. an der Hofstelle befindet. Diese Systeme sind sehr kostengünstig ${ }^{454}$, eignen sich allerdings nur für arrondierte Betriebe. Die Kosten der mobilen Reifendruckregelsysteme sind zwar, wie das folgende Kapitel 6.3.2.2.2 zeigt, höher, dafür kann der Luftdruck während der Fahrt angeglichen werden. Dies hat den Vorteil, dass bei jeder Tätigkeit ein optimaler Luftdruck vorherrscht und somit Schadverdichtungen vorgebeugt wird. Dieser Vorteil ist v. a. bei Arbeiten, die abwechselnde Straßen- und Ackerfahrten erfordern, besonders hervorzuheben.

\subsection{Betriebswirtschaftliche Analyse der Maßnahme}

Die Vorgehensweise bei der KNA für Reifendruckregelanlagen ist in der Übersicht 17 zusammengefasst.

\footnotetext{
${ }^{453}$ Die Regressionsgleichung für Gerade in $10 \mathrm{~cm}$ Tiefe lautet $\mathrm{y}=0,78 \mathrm{x}+0,29, \mathrm{R}^{2}=0,97$ und die für die Gerade in $40 \mathrm{~cm}$ Tiefe lautet $\mathrm{y}=0,47 \mathrm{x}-0,08, \mathrm{R}^{2}=0,73$.

${ }^{454}$ Die Anschaffungskosten liegen zwischen 928 und $1827 €$.
} 


\section{Übersicht 17: Vorgehensweise bei der KNA für Reifendruckregelanlagen}

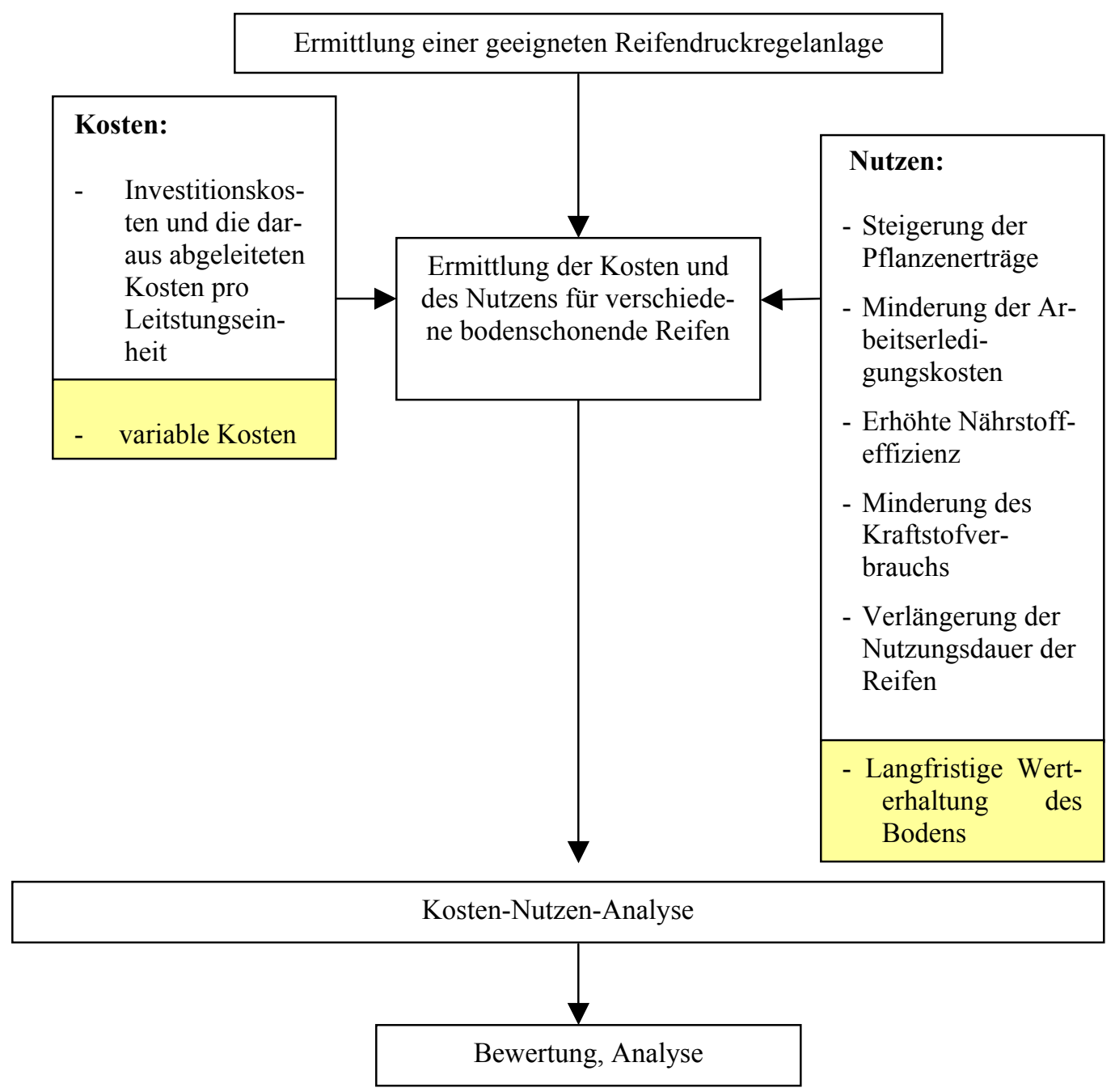

Quelle: Eigene Darstellung, Schraffierung: Positionen konnten aufgrund fehlender Daten in der Berechnung nicht berücksichtigt werden

Die Anschaffungskosten für Reifendruckregelanlagen ${ }^{455}$ zur Druckregelung während der Fahrt liegen für Traktoren je nach Achsart zwischen $2262 €$ und $3654 €$ (Hinterachse) bzw. $1044 €$ bis $2320 €$ (Vorderachse). Ferner werden Reifendruckregelanlagen für Güllefässer und Anhänger angeboten. In diesen Fällen betragen die Investitionskosten für Einachser $2.800 €$, für Zweiachser $3.500 €$ und für Dreiachser $4.400 €$. Alle genannten Preise sind ohne Montage zu verstehen. Da allen Modellen eine Anbauanleitung beiliegt ist es möglich, dass der Landwirt selbst den Umbau übernimmt. Ansonsten sind etwa

\footnotetext{
${ }^{455}$ Die Investitionskosten für Reifendruckregelanlagen wurden der Preisliste des Unternehmens PöSGES \& TIGGES GMBH, Neuss entnommen und verstehen sich ohne USt.
} 
$500 €$ für die Montage zu veranschlagen. Die Nutzungsdauer ist nach Herstellerangaben äquivalent zur Nutzungsdauer eines Schleppers. Danach bemisst sich auch der Abschreibungszeitraum. In diesem Fall soll die Gesamtnutzungsdauer $8800 \mathrm{~h}$ und die jährliche 800 h betragen, wodurch sich eine höhere Vergleichbarkeit mit der Verwendung von bodenschonenden Reifen in Kapitel 6.3.2.1 ergibt. Die variablen Kosten von Reifendruckregelanlagen sind bisher nicht vom KTBL ermittelt worden und auch sonst noch nicht erfasst und veröffentlicht worden. ${ }^{456}$ Die jährlichen Gesamtkosten von Reifendruckregelanlagen sind in der nachfolgenden Tabelle dargestellt.

Tabelle 47: Jährliche Gesamtkosten von Reifendruckregelanlagen für Schlepper und Anhänger

\begin{tabular}{ccc}
\hline & $\begin{array}{c}\text { Anschaffungskosten } \\
\text { incl. Montagekosten }(€) \text { sowie } \\
\text { Gesamtkosten pro } \mathrm{h}\end{array}$ & $\begin{array}{c}\text { Jährliche Gesamtkosten } \\
(\boldsymbol{\epsilon} / \mathbf{a})\end{array}$ \\
\hline Schlepper & $3.806-6.474$ & \\
Gesamtkosten/h & $0,44-0,76$ & $\mathbf{3 5 2 , 0 - 6 0 8 , 0}$ \\
Anhänger & $3.300-4.900$ & \\
Gesamtkosten $/ \mathrm{h}$ & $0,39-0,57$ & $\mathbf{3 1 2 , 0 - 4 5 6 , 0}$ \\
\hline
\end{tabular}

Quelle: Eigene Berechnungen

Zunächst wird eine KWA dieser Maßnahme durchgeführt. Die jährlichen Kosten in Tabelle 47 betragen für Schlepper und Anhänger somit $664 € / a$ bis maximal $1064 € / a$. Die Wirksamkeit von Reifendruckregelanlagen kann durch die Verminderung des Bodendrucks dargestellt werden. Diese Wirksamkeit des Absenkens des Luftdrucks wurde in Abbildung 27 bereits dargestellt und kann bei WEIßBACH ${ }^{458}$ in verschiedenen Forschungsarbeiten eingesehen werden. Ausgehend von den dort ermittelten Regressionsgleichungen ergibt sich bei einer typischen Absenkung des Luftdrucks von 1,2 bar für Straßenfahrten auf 0,8 bar für die Ackernutzung eine Absenkung des Bodendrucks von 0,312 bar im Oberboden $(10 \mathrm{~cm})$ und 0,188 bar im Unterboden $(40 \mathrm{~cm})$. Für die KWA wird daher angenommen, dass im Unterboden eine Druckabsenkung nach 5,3 Jahren und im Oberboden nach 3,2 Jahren erfolgt.

\footnotetext{
${ }^{456}$ Vgl. Persönliche Mitteilung Herr Prof. VOLK

${ }^{457}$ Durch Multiplikation der Gesamtkosten/h und der jährlichen Einsatzzeit von 800 h/a.

${ }^{458}$ Vgl. WEIßBACH (2002), S. 115
} 
Tabelle 48: KW beim Einsatz von Reifendruckregelsystemen bei Schleppern, Anhängern sowie deren Kombination bezogen auf den Ober- und Unterboden

\begin{tabular}{lcc}
\hline & Oberboden (10 cm) & Unterboden (40 cm) \\
\hline Bezugsgröße & Kosten in $€$ je bar Druckabsenkung & Kosten in $€$ je bar Druckabsenkung \\
Schlepper & $1.037,9-1.792,8$ & $1.651,6-2.852,8$ \\
Anhänger & $920,0-1.344,0$ & $1.463,9-2.139,6$ \\
Kombination & $1.957,9-3.137,5$ & $3.115,6-4.992,5$ \\
\hline
\end{tabular}

Quelle: Eigene Berechnungen

Die Ergebnisse der KWA zeigen im Vergleich zu denen in Kapitel 6.3.2.1.2, dass die KW zwischen bodenschonenden Reifen und Reifendruckregelanlagen an Schleppern vergleichbar ist.

Der Nutzen von Reifendruckregelanlagen wurde von UPPENKAMP ${ }^{459}$ näher untersucht. Die Ergebnisse finden sich in der Tabelle 49. Er verwendet eine einzelbetriebliche Betrachtung und stellt die durch Senkung der Arbeitserledigungskosten erzielbaren Einsparungen durch Reifendruckregelanlagen den Investitionskosten gegenüber. Beim 50-haBetrieb steigen die Kosten demnach um $150 € / \mathrm{a}$ an und beim 200 ha-Betrieb sinken sie um $596 € /$.

Tabelle 49: Veränderung der Kosten durch eine Reifendruckregelanlage am Schlepper und Güllefass im landwirtschaftlichen Betrieb

\begin{tabular}{|c|c|c|c|}
\hline & $\begin{array}{l}50 \text { ha- } \\
\text { Betrieb }\end{array}$ & $\begin{array}{l}200 \text { ha- } \\
\text { Betrieb }\end{array}$ & Effekt durch RR \\
\hline Grubbern $(€ / a)$ & -57 & -178 & Schlupf von $18 \%$ auf $10 \%$ \\
\hline Pflügen (€/a) & -136 & -536 & Schlupf von $25 \%$ auf $15 \%$ \\
\hline Gülle fahren $(€ / a)$ & -12 & -182 & $\begin{array}{l}\text { Geringerer Dieselverbrauch: } \\
50 \text { ha: } 1,5 \text { 1/h (110 PS- } \\
\text { Schlepper) } \\
200 \text { ha: } 2,4 \text { 1/h (160 PS- } \\
\text { Schlepper) }\end{array}$ \\
\hline Summe $(€ / \mathbf{a})$ & -205 & -896 & \\
\hline Kosten der Investition $(€ / a)$ & +360 & +300 & $\begin{array}{l}\text { Investition für RR: } \\
50 \text { ha: } 2400 € \\
200 \text { ha: } 2000 €\end{array}$ \\
\hline $\begin{array}{l}\text { Veränderung der Kosten } \\
\text { durch } \operatorname{RR}(€ / \mathbf{a})\end{array}$ & +150 & -596 & \\
\hline
\end{tabular}

Quelle: Verändert nach UPPENKAMP (2003), S. 130

${ }^{459}$ Vgl. UPPENKAMP (2003) 
Damit werden nur die Verminderungen der Arbeitserledigungskosten berücksichtigt. Des Weiteren kommt es durch den Einsatz von Reifendruckregelanlagen zu einer Verringerung des Kraftstoffverbrauchs von $3 \%$ bei Ackerarbeiten und bis zu $17 \%$ bei schweren Zugarbeiten, wie z. B. Gülleausbringung. ${ }^{460}$

Weiterhin werden zusätzliche Nutzeneffekte betrachtet. Hierzu gehört die Steigerung der Pflanzenerträge und die Erhöhung der Nährstoffeffizienz. Die Ertragseffekte von vermiedenen Bodenschadverdichtungen durch Reifendruckregelanlagen sind bisher nicht untersucht worden. Deshalb können hierüber keine Aussagen getroffen werden, obwohl nach Herstellerangaben eine Steigerung der Pflanzenerträge bis zu $6 \%$ erzielt werden kann. Hierzu fehlen jedoch wissenschaftlich fundierte Hinweise. In der folgenden KNA müssen diese Werte daher variiert, bzw. aus den Berechnungen in Kapitel 6.3.2.1.2 übernommen werden.

Außerdem erhöht sich durch die variable Gestaltung des Luftdrucks v. a. bei Straßenfahrten die Nutzungsdauer der Reifen. Diese kann sich nach Ergebnissen von $\operatorname{VoLK}^{461}$ von 3.500 Betriebsstunden auf 5.000 Betriebsstunden erhöhen. Dies entspricht einer Erhöhung der Nutzungsdauer von ca. 43 \%. Das Einsparpotenzial für einen Standardreifen (Kosten von 0,52€/h) wie in Kapitel 6.3.2.1.2 verwendet, beträgt bei einer Erhöhung der Nutzungsdauer $0,22 € / \mathrm{h}$.

\section{Zusammenfassende Kosten-Nutzen-Analyse}

Zunächst werden die gemittelten jährlichen Gesamtkosten der Reifendruckregelanlagen aus der Tabelle 47 verwendet. Analog zu der KNA bei bodenschonenden Reifen in Kapitel 6.3.2.1.2 wird davon ausgegangen, dass die jährliche Einsatzzeit auf den Nutzflächen 9,3 h/ha/a beträgt. Somit ergeben sich hektarbezogene Gesamtkosten für Reifendruckregelanlage am Schlepper von 5,58 €/ha. Die Gesamtkosten der Reifendruckregelanlage des Anhängers wird pauschal mit $10 \%$ der Einsatzzeit des Schleppers, also 0,93 h/ha/a, bewertet. Hieraus ergeben sich er KNA zu verwendende Gesamtkosten von 6,02 €/ha. Bezüglich der zahlreichen Nutzenannahmen werden im Folgenden verschiedene Varianten definiert, die im Rahmen der KNA berechnet werden.

\footnotetext{
${ }^{460}$ Vgl. hierzu WiLlMER (2001) u. UPPENKAMP (2000)

${ }^{461}$ Vgl. Persönliche Mitteilung Herr Prof. VoLK.
} 
Variante 1: Es wird die Fruchtfolge WR-WW-WG und daraus resultierenden Erlösminderungen durch Bodenschadverdichtungen (Tabelle A-18) angenommen. Die Kosten betragen 6,02 €/ha. Die Reduzierung der Bodenschadverdichtung und deren betriebswirtschaftlichen Folgen wird zwischen $5 \%$ und $50 \%$ variiert. Weiterhin wird die Reduzierung des Dieselverbrauchs in Höhe von 0,91 €/ha, die Reduzierung des Schlupfs beim Grubbern und Pflügen in Höhe von 3,57 €/ha aus den Ergebnissen von UPPENKAMP (200-ha-Betrieb) verwendet. $^{462}$ Die Erhöhung der Nährstoffeffizienz beträgt analog zu dem Kapitel 6.3.2.1.2 41,08 €/ha. Der Nutzen durch die Erhöhung der Nutzungsdauer der Reifen wird mit 2,1€/ha veranschlagt. ${ }^{463}$ Es wird wie im Kapitel 6.3.2.1.2 davon ausgegangen, dass bei einer vollständigen Verhinderung einer Bodenschadverdichtung ein Arbeitsgang in Höhe von $46 € /$ ha entfällt. Dementsprechend beträgt der Nutzen bei einer Wirksamkeit von $5 \%$ im ersten Jahr $19,75 € / \mathrm{ha}^{464}$, im zweiten Jahr 22,03€/ha, im dritten Jahr 21,59€/ha, im fünften Jahr 22,02 €/ha und für die Folgejahre 5,67 €/ha aufgrund der Schlupfverringerung und der Verlängerung der Nutzungsdauer. Es wird von einer Bodenschadverdichtung in dem Betrachtungszeitraum von 25 Jahren ausgegangen. Der Zinssatz beträgt $4 \%$.

Variante 2: In der Variante 2 ist eine Änderung der Fruchtfolge enthalten. Es wird die Fruchtfolge ZR-WW-WG (Belastung: 10 t) und die daraus resultierenden betriebswirtschaftlichen Folgen betrachtet. Eine ausführliche Darstellung dieser Fruchtfolge ist in Tabelle A-18 gegeben. Weiterhin sind alle Annahmen der Variante 1 maßgebend.

Variante 3: In dieser Variante wird die Fruchtfolge WR-WW-WG betrachtet. Es wird davon ausgegangen, dass keine Verminderung der Folgen einer Bodenschadverdichtung stattfindet. Ansonsten ergeben sich keine Änderungen im Vergleich zu Variante 1.

\footnotetext{
${ }^{462}$ Siehe Tabelle 49.

${ }^{463}$ Die Einsparungen von 0,22 $€ / \mathrm{h}$ werden mit der durchschnittlichen Einsatzzeit von 9,3 h/ha multipliziert. ${ }^{464}$ Exemplarischer Rechenweg: $(176,3 € / \mathrm{ha}+46 € / \mathrm{ha}+41,08 € / \mathrm{ha}) * 0,05+0,91 € / \mathrm{ha}+3,57 € / \mathrm{ha}+2,1 € / \mathrm{ha}=$ $19,75 € /$ ha.
} 
Die Ergebnisse der Kann sind in der Tabelle 50 dargestellt. Die hierzu gehörenden Berechnungen können der Tabelle A-61 bis Tabelle A-65 entnommen werden.

\section{Tabelle 50: KNV beim Einsatz von Reifenreglern und verschiedenen Varianten}

\begin{tabular}{cccc}
\hline Variante & $\begin{array}{c}\text { Wirksamkeit } \\
\text { gegenüber BSV }\end{array}$ & KNV in \% & $\begin{array}{c}\varnothing \text { jährl. Vorteil } \\
(€ / \text { ha/a) }\end{array}$ \\
\hline 1 & $5 \%$ & 0,65 & 3,3 \\
1 & $50 \%$ & 0,22 & 21,5 \\
2 & $5 \%$ & 0,46 & 7,1 \\
2 & $50 \%$ & 0,12 & 43,3 \\
3 & - & 0,91 & 0,6 \\
\hline
\end{tabular}

Quelle Eigene Berechnungen

Die Ergebnisse zeigen generell, dass die Anschaffung einer Reifendruckregelanlage unter den getätigten Annahmen lohnenswert ist. Selbst wenn dadurch keine Bodenschadverdichtung und deren Auswirkungen verhindert wird, reichen die positiven Wirkungen der Anlage, wie z. B. die Dieseleinsparung, Schlupfminderung oder die Erhöhung der Nutzungsdauer der Reifen aus, um einen Überschuss des Nutzens über die Kosten zu erreichen.

\subsection{Schlussfolgerungen}

Wie die KW und die KNA zeigen, können Reifendruckregelanlagen eine Alternative zur Minderung des Bodendrucks sein. Die Wirtschaftlichkeit hängt maßgeblich von den im Einzelbetrieb vorherrschenden Schadverdichtungsklassen und der Betriebsgröße ab. Um exaktere Aussagen zum KNV zu machen, müssen allerdings die Forschungsaktivitäten zur Ermittlung der Wirkungsbeziehung zwischen der Verminderung des Bodendrucks und der Steigerung der Pflanzenerträge intensiviert werden. Analog zu den Empfehlungen zum Einsatz von bodenschonenden Reifen ist auch für diese Maßnahme zu empfehlen, dass Reifendruckregelanlagen v. a. von Lohnunternehmen und Maschinenringen eingesetzt werden sollten.

Ob die von der Firma Michelin vorgestellten Niederdruckreifen eine wirtschaftliche Alternative sein können, wird sich zeigen, wenn die Kosten dieser Reifen bekannt sind. 


\subsubsection{Gleisbandfahrzeuge}

In diesem Kapitel wird die Wirtschaftlichkeit des Einsatzes von Raupenschleppern im Vergleich zu herkömmlichen Großschleppern und deren Zusammenhang zum Bodenschutz anhand eines Vergleichs der Verfahrenskosten sowie einer KWA untersucht.

\subsection{Einleitende Erwägungen}

Neben der bodenschonenden Bereifung und dem Einsatz von Reifendruckregelanlagen besteht die Möglichkeit, durch den Einsatz von Gleisbändern den Bodendruck zu senken. Bereits Anfang der 80er Jahre wurde in der damaligen DDR die bodenschonende Wirkung von Gleisbandfahrzeugen untersucht. Das aus der Militär- und Baumaschinentechnik übernommene Prinzip der Gleisbänder erhöht die Kontaktfläche und führt dadurch zu einer Senkung des Kontaktflächendrucks. Außerdem gewähren sie im Gegensatz zu den vor einigen Jahrzehnten bereits eingesetzten Kettenlaufwerken aus Stahl einen wesentlich geringeren Verschlei $\beta^{465}$. Durch den durch die Wiedervereinigung entstandenen Bedarf an leistungsfähigen Zugmaschinen stieg auch der Bedarf an Raupenfahrzeugen in Deutschland. Gegenwärtig ist dieser Trend ein wenig gebremst worden. Ein wesentlicher Grund hierfür ist die Weiterentwicklung kostengünstigerer Fahrzeuge, wie z. B. Knicklenker als alternative Großschlepper. Die Vorteile von Gleisbandfahrzeugen liegen in folgenden Sachverhalten begründet:

- Hohe Umsetzung der Motorleistung in Zugkraft,

- Geringer Schlupf $\left(3-5 \%{ }^{466}\right)$,

- Geringer Bodendruck $(<0,5-1 \text { bar })^{467}$ und

- Gute Handhabbarkeit bei schwierigen Bodenverhältnissen wie Hängen oder Minutenböden.

Den Vorteilen dieser Maschinen stehen mittlerweile kaum noch technische Nachteile gegenüber. Ein Hauptproblem der Gleisbandfahrzeuge ist jedoch immer noch die Tatsache, dass der o. g. reduzierte Bodendruck aufgrund der biegbaren Gleisbänder nicht gleichmä-

\footnotetext{
${ }^{465}$ Vgl. HERRMANN (1999), S. 81

${ }^{466}$ Vgl. ISENSEE u. WEIßBACH (1998), S. 48

${ }^{467}$ Vgl. HÖFFLINGER u. POKRIEFKE (1995), S. 126
} 
Big besteht, sondern dass unter den Stützrollen ein höherer Bodendruck herrscht. ${ }^{468}$ Typische Druckverläufe, sowohl statisch als auch unter Zug, zeigen, dass in Tiefen bis $10 \mathrm{~cm}$ der Bodendruck bis auf 1,0 bar ansteigen kann. Dieser Wert wird von ISENSEE und WEIßBACH noch durchaus als bodenschonend bezeichnet. ${ }^{469}$ Im Folgenden wird das Bandlaufwerk einer Raupe mit den anderen Bereifungsvarianten verglichen.

Ein direkter Vergleich zwischen Gleisbandfahrzeugen mit Standardtraktoren ist oft nicht möglich, da i. d. R. bei Gleisbandfahrzeugen eine höhere Leistung vorliegt. Ein kleines Gleisbandfahrzeug mit $175 \mathrm{~kW}$ weist allerdings rd. $22.000 €$ höhere Anschaffungskosten auf als ein vergleichbarer Standardschlepper. ${ }^{470}$ Die nähere Betrachtung der Kosten ist im nächsten Kapitel aufgeführt. Aufgrund der hohen Anschaffungskosten bedarf ein Gleisbandfahrzeug eine hohe Auslastung. Für kleine und mittlere Betriebe ist der Raupeneinsatz daher keine Alternative zum Schleppereinsatz.

Die Auslastung scheitert in vielen Betrieben an den geringen Einsatzmöglichkeiten von Gleisbandlaufwerken. Hierzu gehört bei einer derartigen Überlegung die Kenntnis über die konkreten Einsatzzeiten, die in der Tabelle 51 dargestellt sind.

Tabelle 51: Potenzielle Einsatzzeiten für Gleisbandfahrzeuge oder Großschlepper der 200 kW-Klasse bei klassischen Arbeitsgängen

\begin{tabular}{ccccc}
\hline Arbeitsverfahren & & Zahl Wochen & Std./Tag & Fläche ha/Jahr \\
\hline Pflügen, 12 Schar & $0,5 \mathrm{~h} /$ ha & 18 & 8 & 1500 \\
& & & 12 & 2200 \\
\hline Tiefgrubbern, 6 m & $0,33 \mathrm{~h} /$ ha & 14 & 8 & 1700 \\
& & & 12 & 2500 \\
\hline
\end{tabular}

Quelle: Verändert nach ISENSEE u. WEIßBACH (1998), S. 49

Die obige Tabelle zeigt, dass je nach Tagesleistung der maximale jährliche Einsatz 15002200 ha/a beim Pflügen und 1700-2500 h/a beim Tiefgrubbern, der eigentlichen Spezialität der Raupenschlepper mit Gummibandlaufwerken, betragen kann.

\footnotetext{
${ }^{468} \mathrm{Vgl}$. ISENSEE u. WeißBACH (1998), S. $45 \mathrm{f}$.

${ }^{469}$ Vgl. ebd., S. 46

${ }^{470}$ Vgl. KTBL (2002a), S. 12
} 


\subsection{Betriebswirtschaftliche Analyse der Maßnahme}

Die Vorgehensweise bei der Durchführung der folgenden Analyse ist in der Übersicht 18 dargestellt. Aufgrund der geringen Literaturergebnisse kann keine KNA für den Einsatz von Raupenschleppern durchgeführt werden. Stattdessen wird ein Kostenvergleich, der u. a. auf den Forschungsbedarf hinweist, und eine KWA durchgeführt.

\section{Übersicht 18: Vorgehensweise bei der Analyse für den Einsatz von Raupenschlep- pern}

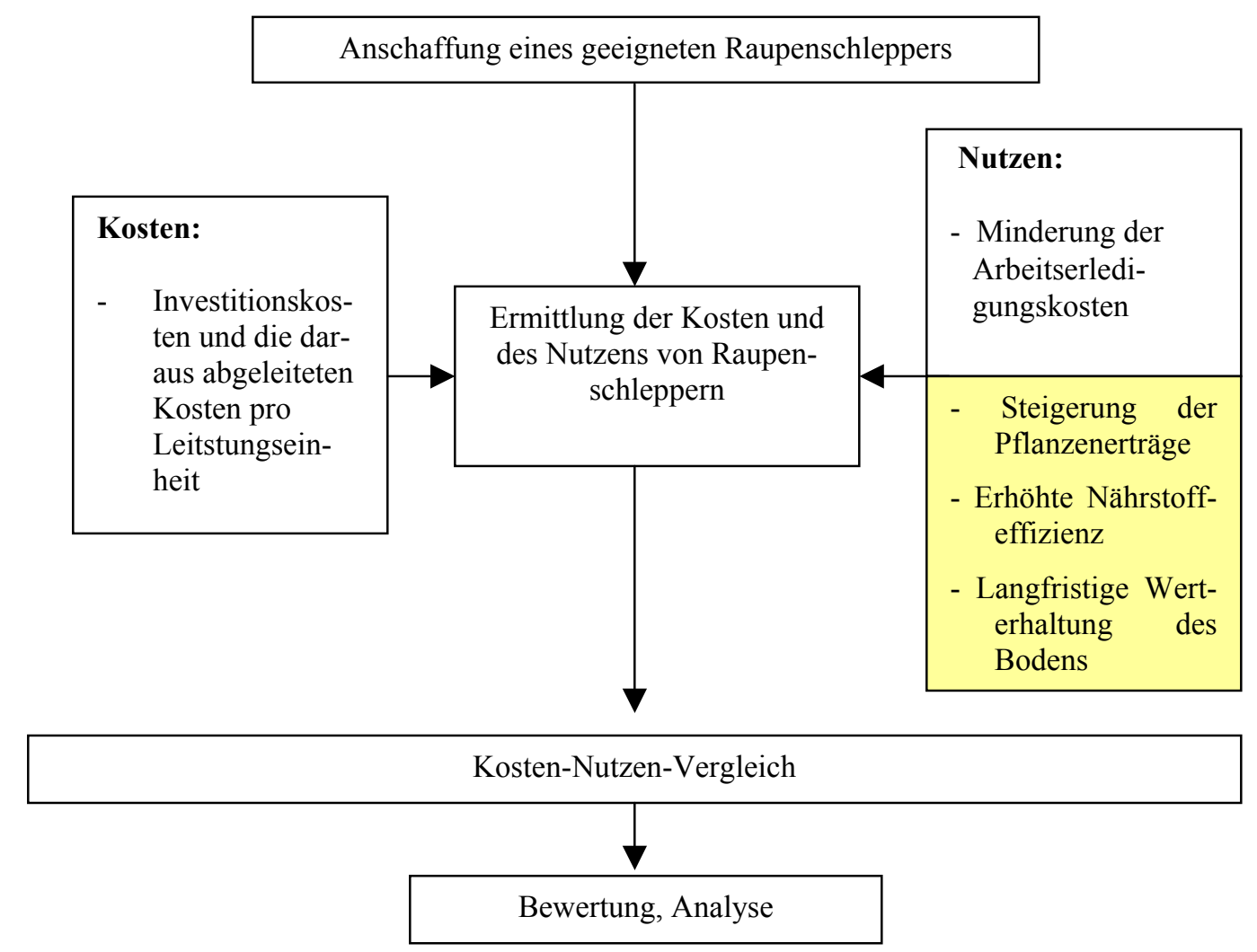

Quelle: Eigene Darstellung, Schraffierung: Positionen konnten aufgrund fehlender Daten in der Berechnung nicht berücksichtigt werden

Zunächst werden die Kosten des Einsatzes von Raupenschleppern analysiert. Wie bereits im obigen Kapitel erwähnt, weisen Gleisbandfahrzeuge wesentlich höhere Anschaffungskosten als Standardschlepper auf. Die Anschaffungskosten sowie die Verfahrenskosten sind in der Tabelle 52 dargestellt. 
Tabelle 52: Vergleich von Leistung und Kosten verschiedener Maschinen beim Pflügen bei einer durchschnittlichen Schlaggröße von 40 ha

\begin{tabular}{lc|ccc}
\hline & $\begin{array}{c}\text { Ein- } \\
\text { heit }\end{array}$ & $\begin{array}{c}\text { Traktor + 9-Schar- } \\
\text { Drehpflug }\end{array}$ & $\begin{array}{c}\text { Traktor + 12-Schar- } \\
\text { Drehpflug+Packer }\end{array}$ & $\begin{array}{c}\text { Raupe + 12-Schar- } \\
\text { Drehpflug }\end{array}$ \\
\hline $\begin{array}{l}\text { Anschaffung } \\
\text { skosten }\end{array}$ & $€$ & 93.000 & 108.000 & 155.000 \\
\hline Nennleistung & $\mathrm{kW}$ & 157 & 220 & 225 \\
\hline $\begin{array}{l}\text { Flächenleis- } \\
\text { tung }\end{array}$ & $\mathrm{ha} / \mathrm{h}$ & 1,69 & 2,25 & 2,25 \\
\hline $\begin{array}{l}\text { Verfahrens- } \\
\text { kosten }\end{array}$ & $\mathbf{\epsilon} / \mathbf{h a}$ & $\mathbf{6 2 , 7}$ & $\mathbf{7 1 , 3 5}$ & $\mathbf{6 9 , 4}$ \\
\hline
\end{tabular}

Quelle: Berechnungen nach KTBL (2002)

Der Anschaffungspreis ist bei der Raupe wesentlich höher, er liegt um $198 €$ €/kW höher als bei einem vergleichbaren Großschlepper. Werden für diesen allerdings Zwillingsräder mit Kosten von $6.000 €$ je Reifen angeschafft, verringert sich der Abstand auf $171 € / \mathrm{kW}$. Die Bänder der Raupe weisen ähnliche Kosten auf, sind jedoch in den ausgewiesenen Kosten für Raupenschlepper enthalten. Im Beispiel der obigen Tabelle ist zu sehen, dass keine Unterschiede in der Flächenleistung zwischen Großschlepper und Raupe bestehen. Die Verfahrenskosten sind ebenfalls ähnlich. Es ist zu berücksichtigen, dass aufgrund der KTBL-Daten kein direkter Vergleich beider Verfahren möglich war. Im Vergleich zum großen Standardtraktor ergibt sich ein Leistungsunterschied von $33 \%$.

Ergänzend zu diesem Vergleich kann ein Vergleich zwischen Raupe (410 PS) und Großschlepper (350 PS) auf einem Praxisbetrieb von DAMMER angeführt werden. Hierbei ging es in erster Linie um den Einsatz beider Maschinen bei der Stoppelbearbeitung. Die Raupe wies eine Flächenleistung von 10,2 ha/h auf, während der Großschlepper 8,2 ha/h schaffte. Die Arbeitszeit lag bei der Raupe 1,9 ha/h höher. ${ }^{471}$

Aufgrund der typischen Einsatzzeiten von Rad- und Raupenschleppern ergibt sich folgende Beziehung zwischen Investitionsbedarf und jährlicher Einsatzfläche.

\footnotetext{
${ }^{471}$ Vgl. hierzu DAMMER (2002)
} 
Abbildung 28: Kapitalbedarf eines Standardschleppers (157 kW), eines Großschleppers $(220 \mathrm{~kW})$ und einer Raupe $(225 \mathrm{~kW})$ bezogen auf die jährliche Einsatzfläche

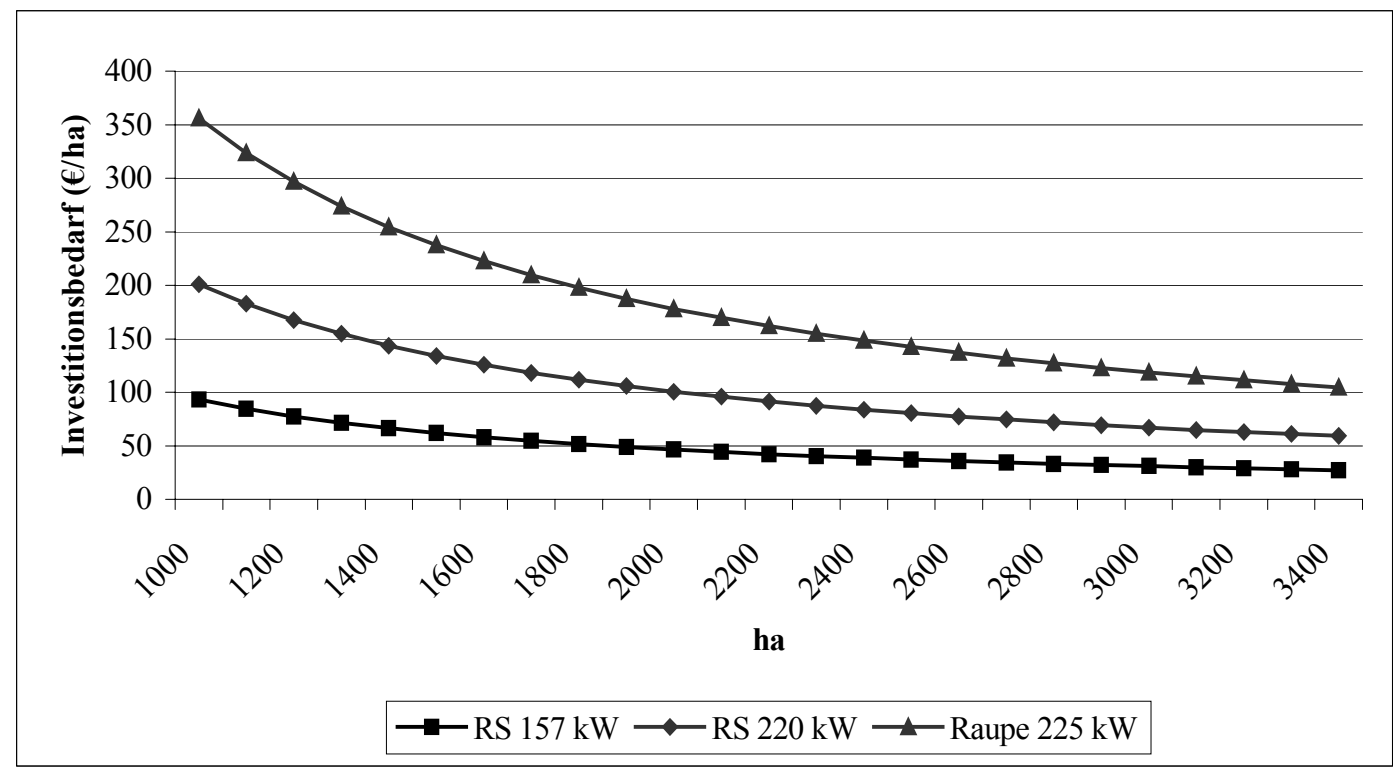

Quelle: Eigene Darstellung

Der Nutzen des Einsatzes von Raupenschleppern besteht zunächst in der Verminderung von Bodenschadverdichtungen. Diesbezüglich sind in der Literatur einstimmige Aussagen zu finden. ${ }^{472}$. Die Höhe dieses Nutzens ist jedoch nicht entsprechend eindeutig und bisher auch wenig untersucht. Im Folgenden werden Ergebnisse von WEIßBACH bezüglich der Bodendruckreduzierung verschiedener Bereifungsvarianten im Vergleich mit einem Bandlaufwerk dargestellt. ${ }^{473}$

\footnotetext{
${ }^{472}$ Vgl. HöFflinger U. POKRIEFKe (1995), ISENSEE U. WEIßBACH (1998) sowie HeRRMANN (1998)

${ }^{473}$ Vgl. WEIßBACH (1998), S. 72 ff.
} 
Tabelle 53: Bodendrücke (in bar) in Tiefen von $10-40 \mathrm{~cm}$ bei verschiedenen Bereifungsvarianten im Vergleich zum Bandlaufwerk

\begin{tabular}{c|ccccccc}
\hline & $10 \mathrm{~cm}$ & $15 \mathrm{~cm}$ & $20 \mathrm{~cm}$ & $25 \mathrm{~cm}$ & $30 \mathrm{~cm}$ & $35 \mathrm{~cm}$ & $40 \mathrm{~cm}$ \\
\hline $\begin{array}{c}\mathbf{6 2 0} / 70 \text { R 42 } \\
(1,6 \text { bar })\end{array}$ & 1,2 & 1,0 & 0,8 & 0,7 & 0,5 & 0,35 & 0,15 \\
$\begin{array}{c}\mathbf{7 1 0 / 7 0 ~ R ~ 3 4} \\
(1,0 \text { bar) } \\
\text { Raupe }\end{array}$ & 1,0 & 0,95 & 0,8 & 0,7 & 0,4 & 0,25 & 0,1 \\
\hline $\begin{array}{c}\text { Minderung } \\
\text { (bar) }\end{array}$ & 0,8 & 0,6 & 0,4 & 0,25 & 0,1 & 0,1 & 0,05 \\
\hline
\end{tabular}

Quelle: Verändert nach WeIßBACH (1998), S. 73

Die Bodendrücke liegen demnach deutlich unter denen, die von vergleichbaren Großschleppern induziert wurden. Es können Reduzierungen bis 0,45 bar verzeichnet werden. Da diese Ergebnisse die umfangreichsten und detailliertesten sind, die vorliegen, werden sie nachfolgend für die Berechnung der Kostenwirksamkeit sowie des KNV verwendet.

Weiterhin zeigen Untersuchungen von WEIßBACH, dass Raupenfahrzeuge durch die Verminderung des Schlupfs unter entsprechenden feuchten Bedingungen eine höhere Flächenleistung aufweisen können. Im Vergleich zwischen einem Großschlepper und einer Raupe beim Tiefgrubbern konnte gezeigt werden, dass die Raupe $5 \%$ Schlupf aufwies und der Großschlepper 15 \%. Die Raupe hatte in diesem Beispiel demnach eine höhere Flächenleistung von $10 \%$. Der Autor weist in diesem Zusammenhang darauf hin, dass dieser Nutzen nicht zwangsläufig gegeben sein muss, was die KTBL-Daten in Tabelle 52 ebenfalls zeigen. Der Schlupf unter Raupenlaufwerken steigt bspw. auch bei sehr ungünstigen Bedingungen wie bei aufliegendem Rübenblatt auf dem Oberboden stark an. ${ }^{474}$

Für die folgende KWA wird zunächst ein Vergleich der jährlichen Gesamtkosten von Raupenschleppern $(13.428,0 € / \mathrm{a})^{475}$ zu Großschleppern mit Terrareifen $(10.040,8 € / \mathrm{a})^{476}$ getätigt. Somit ergibt sich ein Unterschied in den jährlichen Gesamtkosten zwischen der Raupe und dem Traktor mit Terrabereifung (710/70 R 34) von 3387,2 €/a. Daher wird der Wirkungsunterschied bezüglich der Bodendruckreduzierung zwischen der Raupe und

\footnotetext{
${ }^{474}$ Vgl. WeIßBACH (1998), S. 72

475 Annahme: Anschaffungspreis $155.000 €$; Gesamtnutzungsdauer: 10.000 h; jährl. Nutzungsdauer 833 h; Zinssatz $4 \%$.

476 Annahmen: Anschaffungspreis Traktor: $108.000 €$, Anschaffungspreis Reifen $7.902 €$; Gesamtnutzungsdauer 10.000h; jährl. Nutzungsdauer 833 h; Zinssatz $4 \%$.
} 
dem Traktor mit Terrabereifung im Folgenden auf die zusätzlichen jährlichen Kosten von 3.387,2 €/a bezogen. Bezüglich der Wirksamkeit wird auf die Ergebnisse in Tabelle 53 zurückgegriffen. Um eine Vergleichbarkeit zu den anderen Maßnahmen zu gewärhleisten wird davon ausgegangen, dass bspw. bei einer mittleren Minderung von 0,3 bar in einer Tiefe von $10 \mathrm{~cm}$ es 3,3 Jahre dauert, bis eine die Druckminderung 1 bar beträgt. Aus diesen Annahmen ergeben sich folgende KW:

Tabelle 54: KW des Raupeneinsatzes mit Pflug und Tiefgrubber im Vergleich zum Großschlepper

\begin{tabular}{c|cc}
\hline Tiefe & $\begin{array}{c}\text { Verm.. des Bodendrucks } \\
\text { (Raupe vs. 710/70 R 34) }\end{array}$ & $\begin{array}{c}\text { KW in } € / \text { bar Boden- } \\
\text { druckabsenkung }\end{array}$ \\
\hline $10 \mathrm{~cm}$ & 0,2 & $15.079,2$ \\
$20 \mathrm{~cm}$ & 0,4 & $7.908,95$ \\
$30 \mathrm{~cm}$ & 0,3 & $10.377,56$ \\
$40 \mathrm{~cm}$ & 0,05 & $46.033,15$ \\
\hline \multicolumn{2}{l}{ Durchschnittliche KW } & $\mathbf{1 9 . 8 4 9 , 7 1}$ \\
\hline
\end{tabular}

Quelle: Eigene Berechnungen

Ein Vergleich der KW mit den bisher ermittelten Werten in Kapitel 6.3.2.2.2 und Kapitel 6.3.2.1.2 zeigt, dass bei der Verwendung von Raupen deutlich höhere Kosten pro Wirkungseinheit entstehen. Dies liegt in erster Linie an den höheren jährlichen Gesamtkosten im Vergleich zu Traktoren mit bodenschonender Bereifung.

An dieser Stelle ist keine KNA möglich, da es an fundierten Ergebnissen zum Nutzen von Gleisbandlaufwerken fehlt. Lediglich die Einsparungen von Arbeitserledigungskosten aufgrund des geringeren Schlupfs bei Raupenschleppern ist wissenschaftlich belegt. Außerdem ist die tatsächliche Reduzierung des Bodendrucks, durch die bereits beschriebenen Nachteile der Umlenkrollen, umstritten. Ferner sind weitere Nutzeneffekte, wie z. B. die möglichen Ertragssteigerungen durch die Reduzierung des Bodendrucks, nicht annähernd bekannt. Würden die vorhandenen Nutzeneffekte den Kosten von Gleisbandlaufwerken gegenübergestellt, ergäben sich aufgrund der hohen zusätzlichen Kosten der Raupenschlepper sehr schlechte KNV, die nur durch eine sehr hohe Auslastung und somit Fixkostendegression verbessert werden könnten. 


\subsection{Schlussfolgerungen}

Raupenschlepper eignen sich aufgrund der nötigen hohen Auslastung nur für große Betriebe oder für den überbetrieblichen Einsatz. Die Wirkung der Raupenschlepper auf den Boden ist umstritten und die Begleitforschung ist sehr eingeschränkt. Trotzdem konnten Ergebnisse, v. a. bei der Kostenwirksamkeit, erzielt werden. Diese zeigen, dass die KW gegenüber der Nutzung von Breit- und Terrareifen sowie der Nutzung von Reifendruckregelanlagen ungünstiger ist. Dieses Ergebnis wird sich auch bei einer Auslastungssteigerung nicht wesentlich ändern.

\subsubsection{Begrenzung der Radlasten}

Im Folgenden wird anhand der Zuckerrübenernte gezeigt, wie sich die Begrenzung der Radlasten auf einzelbetrieblicher Ebene auswirken kann. Dies geschieht anhand der KWA und der KNA.

\subsection{Einleitende Erwägungen}

Es gibt bereits seit einiger Zeit Vorschläge der Begrenzung von Radlasten und somit des Bodendrucks. RABOCHEV und BACHTIN schlugen z. B. 1978 vor, den Bodendruck v. a. im Frühjahr zu begrenzen. ${ }^{477}$ Auch PETELKAU et al. ${ }^{478}$ entwickelten 1986 Richtwerte für die maximale Bodenbelastung. Trotz dieser frühen Vorschläge hat sich eine Begrenzung der Radlasten bisher nicht durchsetzen können, dennoch ist eine sehr intensive Diskussion zwischen Verbänden, Wissenschaftlern, Politikern und Praktikern festzustellen, wie in Kapitel 4.4 .2 bereits deutlich wurde.

Ein großes Problem der Begrenzung der Radlasten ist die mangelnde Praktikabilität, was bereits bei der Frage der optimalen Bodenfeuchte deutlich wird. Die Tragfähigkeit des Bodens hängt in entscheidendem Maße von der jeweiligen Bodenfeuchte ab. Daher sind generelle Grenzwerte für alle Bodenarten und Feuchtezustände zunächst suboptimal. In Deutschland besteht zudem ein klimatisches Problem, was bedeutet, dass es nur wenige Feldtage gibt, an denen die Bodenfeuchte nicht zu hoch ist. So herrschen im Durchschnitt nur etwa für $60 \%$ der verfügbaren Feldarbeitstage belastbare Böden vor. ${ }^{479}$ Vor allem die

\footnotetext{
${ }^{477}$ Vgl. RABOCHEV u. BACHTIN (1978), zit. in DÜRR et al. (1995), S. 129

${ }^{478}$ Vgl. PetELKAU et al. (1986), zit. in DÜRR et al. (1995), S. 130

${ }^{479}$ Vgl. DürR et al. (1995), S. 131
} 
termingebundenen Arbeiten der Hackfruchternte fallen in die aus Sicht des Bodenschutzes ungünstigen Zeiträume. Deshalb fordern international tätige Bodenwissenschaftler eine Reduzierung der Achslast auf 2-6 t, um weitgehende Bodenschadverdichtungen zu vermeiden. ${ }^{480}$

Gerade bei der Zuckerrübenerntetechnik hat es in den letzten Jahren einige Innovationen gegeben, welche die Bodenbelastung reduzieren können. Hier sind v. a.

- das spurversetzte Fahren,

- die Ausstattung mit Gleisbandlaufwerken,

- die Veränderung der Blattablage und

- Befahrbarkeitssensoren.

zu nennen. Diese technischen Weiterentwicklungen können jedoch den vom beladenen Zuckerrübenroder ausgehenden Bodendruck nur bedingt vermindern. Im Gegensatz zu den Bemühungen, die Zuckerrübenproduktion bodenschonend und umweltgerecht zu gestalten, gibt es v. a. im europäischen Ausland z. T. entgegengesetzte Tendenzen. Bei einigen europäischen Landmaschinenherstellern wurden bereits 8- 9-reihige Zuckerrübenroder entwickelt, und selbst 12-reihige Zuckerrübenroder kommen im europäischen Ausland mittlerweile zum Einsatz. Aus Sicht des Bodenschutzes wäre es daher optimal, leichte Maschinen mit möglichst hohen Arbeitsbreiten einzusetzen. Diese Forderung von Wissenschaftlern kann allerdings in der landwirtschaftlichen Praxis aus betriebswirtschaftlichen Gesichtspunkten nicht immer erfüllt werden.

Neben den technischen Neuerungen können auch Änderungen in der Organisation und in den Anpassungen von Arbeitsverfahren Verbesserungen für den Bodenschutz hervorrufen. Hierzu zählt die geeignete Terminwahl, das häufigere Abbunkern etc.

\subsection{Betriebswirtschaftliche Analyse der Maßnahmen}

Im Folgenden wird die Vorgehensweise der KNA bei der Reduzierung von Radlasten in der Zuckerrübenernte dargestellt.

${ }^{480}$ Vgl. DÜRR et al. (1995), S. 132 


\section{Übersicht 19: Vorgehensweise bei der KNA für die Maßnahme der Reduzierung der Radlasten}

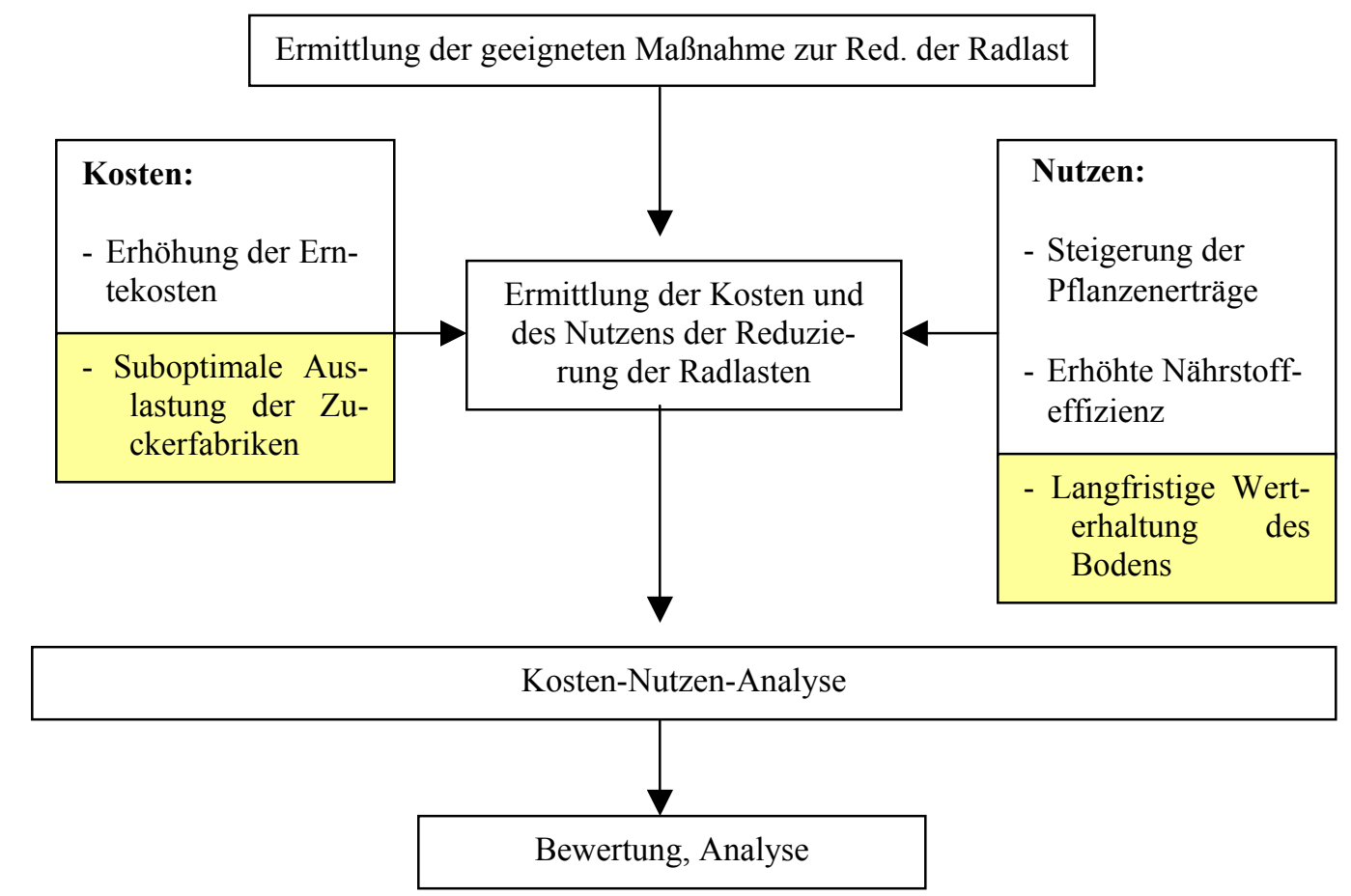

Quelle: Eigene Darstellung, Schraffierung: Positionen konnten aufgrund fehlender Daten in der Berechnung nicht berücksichtigt werden

Zunächst werden die einzelbetrieblichen Kosten einer derartigen Forderung der Wissenschaft auf einzelbetrieblicher Ebene betrachtet. Wie die Tabelle 4 zeigt, würde eine Begrenzung der Radlasten v. a. die Ernteverfahren betreffen. Bspw. könnte ein 6-reihiger Zuckerrübenroder entweder gar nicht oder nur mit unvollständig gefülltem Bunker über den Acker fahren, wodurch v. a. zusätzliche Arbeits- und Maschinenkosten entstehen würden, welche die Produktion verteuerten. Das Gleiche würde, wenn auch nicht in so hohem Maße, u. U. auch für die Getreideernte und die Ausbringung von Wirtschaftsdüngern gelten. In den folgenden Berechnungen werden drei Optionen eingebunden:

- Reduzierung der Einsatzfläche und somit der Auslastung,

- Mögliche zusätzliche Kosten durch mehrfaches Abbunkern und

- Verwendung eines kleineren Rübenroders

Zunächst wird ein Vergleich der Gesamtkosten von Zuckerrübenernteverfahren durchgeführt. Dabei werden der sechsreihige selbstfahrende Köpfrodebunker (SKRB 6) und der zweireihige gezogene Köpfrodebunker (GKRB 2) anhand von KTBL-Daten miteinander 
verglichen. Während der GKRB 2 Verfahrenskosten von 315,02 €/ha aufweist, liegen diese beim SKRB 6 je nach Bunkergröße bei 322,51 €/ha (15 t), 355,71 €/ha (20 t) und $389,79 € /$ ha $(25$ t). Die Leistung der selbstfahrenden Erntemaschinen ist im Durchschnitt 1,5 bis 1,6 mal höher als bei der gezogenen Erntemaschine. Hieraus und aus der Tatsache, dass die Gesamtkosten sehr stark von der Auslastung abhängen, lässt sich ableiten, dass es das Ziel der Maschinengemeinschaften, Lohnunternehmen oder Maschinenringe sein muss, die selbstfahrenden Zuckerrübenroder möglichst oberhalb der Abschreibungsschwelle der KTBL-Daten von 250 ha/a auszulasten. Dieser Zusammenhang ist für beide betrachteten Verfahren in der Abbildung 29 dargestellt.

\section{Abbildung 29: Verfahrenskosten von Zuckerrüben bei einem SKRB-6 und GKRB-2} in Abhängigkeit von der Auslastung

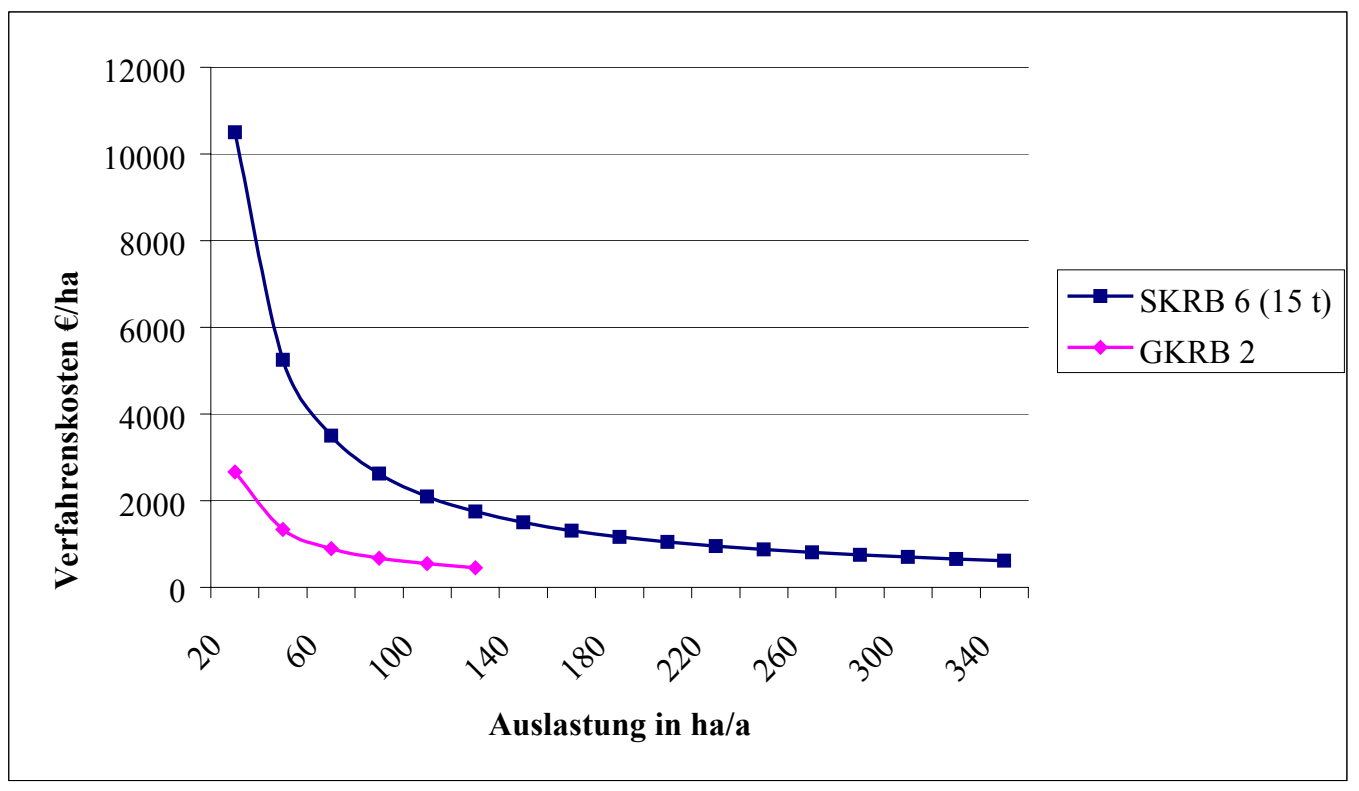

Quelle: Eigene Darstellung; Berechnungen nach KTBL (2002) und (2002a)

Eine hohe Auslastung führt allerdings zu einer aus Sicht des Bodenschutzes suboptimalen Nutzung, da sich die Gefährdung des Bodens erhöht.

Des Weiteren sind die zusätzlichen Kosten durch mehrfaches Abbunkern zu ermitteln. Die Art des Abbunkerns beeinflusst in wesentlichem Maße den Arbeitszeitbedarf. Dabei sind zwei verschiedene Varianten denkbar. Auf der einen Seite kann abgebunkert werden, wenn der Bunker voll ist, auf der anderen Seite ist es denkbar, nach jeder bzw. jeder zweiten oder dritten Durchfahrt abzubunkern. Ergebnisse von LUDER, STARK und AMMANN auf 2-ha-Schlägen zeigen, dass die zusätzliche Arbeitszeit nur 0,12 Akh/ha (1,8 
$€ /$ ha) beträgt und daher minimal ist. Die zusätzlichen Maschinenkosten müssten dementsprechend 26,9 $€ /$ ha betragen, was Gesamtkosten von ca. $28,7 € /$ ha bedeutet. ${ }^{481}$ Bei 5-haSchlägen beträgt dieser Wert ebenfalls nur $0,25 \mathrm{Akh} / \mathrm{ha}(4 € / \mathrm{ha})$, es ergeben sich allerdings Gesamtkosten von ca. 60,0 €/ha. ${ }^{482}$ Wird ein SKRB-6 immer dann abgebunkert, wenn der Bunker zur Hälfte voll ist, kann am Gesamtgewicht $25 \%$ eingespart werden. ${ }^{483}$ Dies bedeutet eine Verringerung der Radlast von ca. 2,3 t am Hinterrad und 2,1 t am Vorderrad. Es können sich demnach durch das geschickte Positionieren der Rübenmiete sehr gute KW ergeben, die leicht zu ersehen sind. Es ist in diesem Fall zu bedenken, dass dies zu einer Abfahrt der Rüben vom Feld führt, welche zusätzliche Bodenbelastungen bewirken kann.

Mit der Begrenzung der Radlasten könnten Bodenschadverdichtungen, eine effiziente Kontrolle des Staates vorausgesetzt, fast gänzlich vermieden werden. Es werden an dieser Stelle die Ergebnisse des Kapitels 6.3.2.1.2 herangezogen. Der sich hieraus ergebende Nutzenbeitrag besteht aus den Ertrags- und somit Erlössteigerungen. Bei den Fruchtfolgen ZR-WW-WG sowie ZR-WW-WW ergeben sich in Verbindung mit der Tabelle A17 und der Tabelle A-18 demnach folgende Nutzenbeiträge:

Tabelle 55: Erlösvorteil bei der Minderung der Radlasten von $10 \mathrm{t}$ auf $5 \mathrm{t}$ in $\mathrm{Zu}$ ckerrübenfruchtfolgen bei der Verhinderung einer Bodenschadverdichtung (BSV)

\begin{tabular}{c|c|c|c|c}
\hline & \multicolumn{2}{|c|}{ ZR-WW-WG } & \multicolumn{2}{c}{ ZR-WW-WW } \\
& $5 \mathrm{t}$ & $10 \mathrm{t}$ & $5 \mathrm{t}$ & $10 \mathrm{t}$ \\
\hline $\begin{array}{c}\text { Jahr 1 } \\
\text { (ZR) }\end{array}$ & 525,6 & 909,7 & 525,6 & 909,7 \\
\hline $\begin{array}{c}\text { Jahr 2 } \\
\text { (WW) }\end{array}$ & 45,5 & 136,6 & 45,5 & 136,6 \\
\hline $\begin{array}{c}\text { Jahr 3 } \\
\text { WG } \\
\text { WW }\end{array}$ & 36,6 & 109,9 & & \\
\hline $\begin{array}{c}\text { Jahr 4 } \\
\text { (ZR) }\end{array}$ & 131,2 & 394,2 & 131,2 & 394,2 \\
\hline Nutzen (€/ha) & $\mathbf{4 8 4}$ & $\mathbf{8 1 1 , 5}$ & \multicolumn{2}{c}{$\mathbf{8 2 9 , 3}$} \\
\hline
\end{tabular}

Quelle: Eigene Berechnungen

\footnotetext{
${ }^{481}$ Vgl. KTBL (2002), S. 332. Annahme: GKRB-6, 20 t, Parzellengröße 5 ha.

${ }^{482}$ Vgl. ebd.

${ }^{483}$ Vgl. LUder, StARK u. AMmann (2001)

${ }^{484}$ Der Nutzen ist an dieser Stelle als Differenz der jeweiligen Spalten zu verstehen, da von einer Reduzierung der Radlast von $5 \mathrm{t}$ ausgegangen wird.
} 
Die obige Tabelle zeigt den monetären Nutzen durch die Erlössteigerung der Verhinderung einer BSV, die von WIERMANN für beide Achslasten am Reinshof gemessen wurde. $^{485}$ Wenn also unter den gegebenen Bedingungen die Achslasten von $10 \mathrm{t}$ auf $5 \mathrm{t}$ gesenkt werden, ergibt sich ein monetärer Vorteil von $811,5 €$ bzw. 829,3€.

Die Reduzierung der Arbeits- und Maschinenkosten zur Behebung der Schadverdichtung werden analog zu Kapitel 6.3.2.1.2 in diesem Beispiel nicht angenommen, da es zu keiner vollständigen Verhinderung der Bodenschadverdichtung kommt und damit gerechnet werden muss, dass ein Arbeitsgang zur Lockerung des Bodens anfällt. Dasselbe gilt auch für die eventuell eintretende Erhöhung der Nährstoffeffizienz.

Die Kosten liegen bei dieser Maßnahme bei 26,9€/ha für 2 ha-Schläge und $60 € /$ ha auf 5 ha-Schlägen. Der Nutzen liegt in der Reduzierung der Radlast um $25 \%$ und somit von 2,3 $\mathrm{t}$ am Hinterrad und 2,1 $\mathrm{t}$ am Vorderrad des Fahrzeugs. ${ }^{486}$ Die Auswirkungen dieser Radlastenreduzierung lassen sich ohne entsprechende Daten aus Versuchsergebnissen o. ä. nicht auf eine Verminderung von Bodenschadverdichtungen übertragen. Es ist aber anzunehmen, dass der Nutzen höher ist und sich daher gute KNV ergeben.

Im Folgenden wird eine weitere Alternative der Reduzierung der Radlasten vorgestellt. Diese Alternative besteht darin, einen GKRB-2 einzusetzen. Dieser weist entsprechende Radlasten von ca. 4-5 t auf. ${ }^{487}$ Ausgehend von den Annahmen, dass der Einsatz dieses Gerätes ähnliche Nutzeneffekte wie bei WIERMANN et al. aufweist, ergeben sich in einem Vierjahreszeitraum Kosten bei der ZR-Ernte von 315,2 €/ha und ein nach Tabelle $55^{488}$ einen Nutzen von 811,5 €/ha (im ersten Jahr 384,1 €/ha, im zweiten Jahr 91,1 €/ha, im dritten Jahr 73,3 €/ha und im vierten Jahr $263 € /$ ha) bzw. 829,3 €/ha (im ersten Jahr $384,1 € /$ ha, im zweiten und im dritten Jahr 91,1 €/ha sowie im vierten Jahr $263 € /$ ha) innerhalb eines Vierjahreszeitraums. Diese Werte werden in den folgenden Berechnungen für drei weitere Jahre fortgeschrieben, um zu sehen, wann sich ein günstiges KNV ergibt. Die Berechnungen für die Fruchtfolge ZR-WW-WG können der Tabelle A-68 bis zur Tabelle A-70 entnommen werden.

\footnotetext{
${ }^{485}$ Vgl. Übersicht A-1

${ }^{486}$ Durch die Reduzierung der Gesamtmasse von 2,3 t/ha (Hinterrad) und 2,1 t/ha (Vorderrad).

${ }^{487}$ Vgl. BMVEL (2001), S. 19

${ }^{488}$ An dieser Stelle sind die Differenzen der Zeilen verwendet worden.
} 
Tabelle 56: KNV und durchschnittlicher jährlicher Vorteil bei der Reduzierung der Radlasten durch einen GKRB 2

\begin{tabular}{c|cc}
\hline $\begin{array}{c}\text { Verhinderung von } \\
\text {... BSV }\end{array}$ & \multicolumn{2}{|c}{ KNV (\%) und durchschnittlicher } \\
jährl. Vorteil (€/ha/a) \\
\hline & ZR-WW-WG & ZR-WW-WW \\
1 BSV & $2,40(-66)$ & $2,35(-65)$ \\
2 BSV & $1,27(-24)$ & $1,24(-22)$ \\
3 BSV & $0,90(13)$ & $0,88(16)$ \\
\hline
\end{tabular}

Quelle: Eigene Berechnungen

Wie in der obigen Tabelle zu sehen ist, ergeben sich erst günstige KNV, wenn mindestens drei BSV in Folge verhindert werden. Es lohnt sich demnach unter diesen gegebenen Bedingungen, den GKRB 2 einzusetzen. Als Einschränkung müssen jedoch die recht hohen Ertragsminderungen von WIERMANN genannt werden, die der Berechnung des Nutzens zu Grunde liegen. Außerdem ist zu prüfen, ob die Begrenzung der Radlasten durch den Einsatz des GKRB 2 tatsächlich zu den entsprechenden Verminderungen der Auswirkungen von Bodenschadverdichtungen führt.

\subsection{Schlussfolgerungen}

Es konnte gezeigt werden, dass der wirtschaftliche Einsatz von großen sechsreihigen Zuckerrübenrübenrodern durch eine hohe Auslastung „erkauft“ wird, somit zunächst nachteilig für den Bodenschutz ist. Auf der anderen Seite bestehen erhebliche Anstrengungen, die Zuckerrübenernte und Bodenschutzbelange zu vereinen. Viele dieser Neuerungen sind technischer Art und können noch nicht abschließend bewertet werden. Daher wurde am Beispiel kleiner Verfahrensänderungen bei der Zuckerrübenernte gezeigt, dass hiermit durchaus ein effektiver und wirtschaftlicher Bodenschutz möglich sein kann.

Insgesamt ist an dieser Stelle jedoch darauf hinzuweisen, dass die Forschungsintensität auf dem Gebiet des physikalischen Bodenschutzes erhöht werden muss. Es ist gerade bei den Diskussionen um Radlastbegrenzung in der Zuckerrübenernte zu festzustellen, dass die teilweise emotional geführten Diskussionen darauf zurückzuführen sind, dass seitens der Wissenschaft eine Begleitforschung dieser Entwicklungen noch nicht in hinreichendem Maße stattgefunden hat. 


\subsubsection{Weitere Maßnahmen zum physikalischen Schutz des Bodens}

Im folgenden Abschnitt wird anhand der Stilllegung von Minderertragsflächen eine weitere Maßnahmen zum Schutz des Bodens vor Bodenschadverdichtung und Bodenerosion dargestellt. Außerdem werden Maßnahmen zum Schutz des Bodens vor dem Flächenverbrauch diskutiert.

\subsubsection{Stilllegung von schlaginternen Minderertragsteilflächen}

In den folgenden Kapiteln wird die Maßnahme der Stilllegung von schlaginternen Minderertragsteilflächen behandelt, die für den physikalischen Bodenschutz zunächst untypisch erscheint, aber für bestimmte Gebiete Deutschlands eine hohe Bedeutung haben kann. Neben einer ausführlichen Vorstellung dieser Maßnahme wird die Wirtschaftlichkeit u. a. mit der KNA analysiert.

\subsection{Einleitende Erwägungen}

Die Standortheterogenität der Böden, die bis zum einzelnen Ackerschlag reicht, wurde bereits mehrfach betont. Durch ein heterogenes Relief oder verschiedene Ausgangssubstrate des Bodens kommt es zu unterschiedlich hohen Ertragspotenzialen auf einem Ackerschlag.

Minderertragsteilflächen können durch die langjährigen Beobachtungen des Landwirts kostengünstig spezifiziert werden. Zum großen Teil sind die Teilflächen mit geringen Ertragspotenzialen visuell zu erfassen, wenn es sich z. B. um nährstoffarme Sandlinsen, staunasse Senken, Überschwemmungsbereiche oder extreme Hangneigungen handelt. Der Nachteil hierbei ist, dass diese Methode der Erfassung sehr ungenau ist. ${ }^{489}$

Weitere Möglichkeiten bestehen in der Durchsicht von Bodenformen-, Hangneigungsund Meliorationsstandortkarten, Luftbildern sowie Daten der Reichsbodenschätzung. Eine wesentlich genauere aber auch kostenintensivere Möglichkeit bietet der Präzisionspflanzenbau (Precision Farming). Diese Technologie befindet sich größtenteils noch in der Entwicklung, Teilverfahren, wie z. B. die elektronische Ertragskartierung werden jedoch bereits in der Praxis eingesetzt.

\footnotetext{
${ }^{489}$ Vgl. hierzu SATTLER (2002), S. 1 f.
} 
Der Vorteil einer partiellen Stilllegung hängt v. a. von dem Minderertragspotenzial der stillzulegenden Fläche ab. Es ist dabei zu beachten, dass dieses im Einzelfall sehr hoch sein kann. Umfangreiche Ertragsmessungen auf staunassen Teilflächen haben Mindererträge von durchschnittlich $43 \%$ offenbart. ${ }^{490}$

Des Weiteren muss die Mindeststillegungsgröße beachtet werden. Sie muss zunächst als Stilllegungsfläche anerkannt werden. Nach Maßgabe der aktuellen Agrarpolitik muss sie eine Mindestgröße von 0,3 ha und eine Mindestbreite von $20 \mathrm{~m}$ umfassen. Ausnahmen können bei Schlägen gewährt werden, die von unveränderlichen Grenzen umschlossen sind (Mauern, Hecken oder Wasserläufe) oder die in Realteilungsgebieten liegen. ${ }^{491}$ Diese Einschränkung macht deutlich, dass die Maßnahme der Stilllegung von Minderertragsteilflächen unter momentanen agrarpolitischen Rahmenbedingungen tendenziell in den Neuen Bundesländern angewendet werden kann, da hier entsprechende Schlaggrößen vorhanden sind. Generell muss die Entwicklung der politischen Diskussion um die Flächenstillegung beobachtet werden.

\subsection{Betriebswirtschaftliche Analyse der Maßnahme}

In der Übersicht 20 ist die Vorgehensweise bei der Ermittlung des KNV bei der Stilllegung einer 1 ha großen Minderertragsteilfläche innerhalb eines Ackerschlages dargestellt.

\footnotetext{
${ }^{490}$ Vgl. Berger et al. (1999), zit. in SATTLER (2002), S. 1

${ }^{491}$ Vgl. BMVEL (2002), S. 12
} 


\section{Übersicht 20: Vorgehensweise bei der Analyse für die Stilllegung von Minderer- tragsteilflächen}

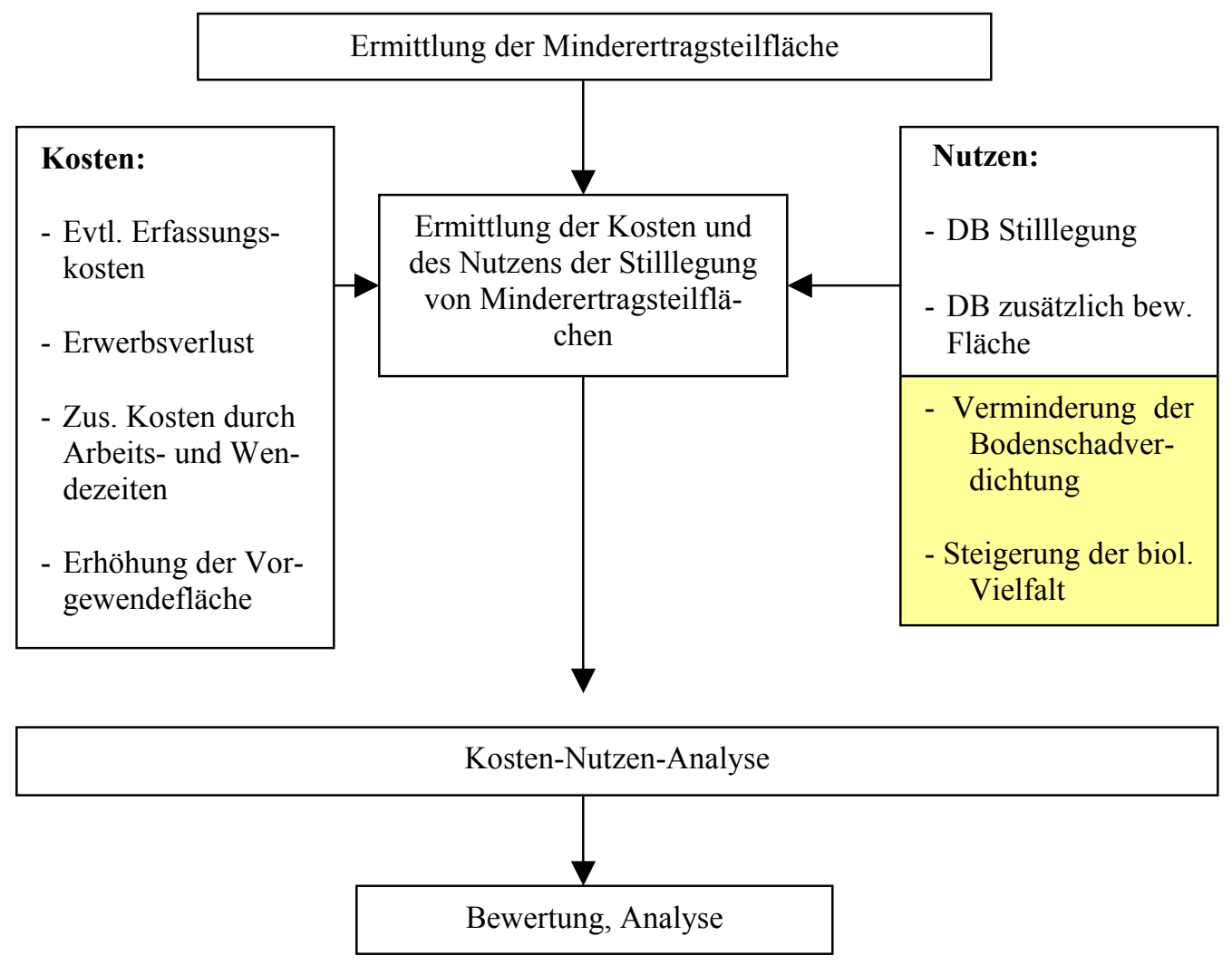

Quelle: Eigene Darstellung, Schraffierung: Positionen konnten aufgrund fehlender Daten in der Berechnung nicht berücksichtigt werden

Auf der Kostenseite ist zu nennen, dass dem Landwirt Kosten für die Ermittlung der Minderertragsteilflächen entstehen. Diese Kosten hängen davon ab, ob GPS-basierte Technik eingesetzt wird. Im Fall des Technikeinsatzes müssen obige Investitionskosten und weitere variable Kosten oder die Kosten der Durchführung durch einen Dienstleister berücksichtigt werden. Im Fall der Ermittlung durch den Betriebsleiter sind die entsprechenden Arbeitskosten anzusetzen. Die Arbeitskosten für die Beantragung der Stilllegungsfläche wird als marginal angesehen, da sie im Rahmen des allgemeinen Verwaltungsaufwandes in einem landwirtschaftlichen Betriebes nur sehr geringe Arbeitsaufwendungen verursacht.

Eine weitere Kostenkomponente ist zunächst der Verlust durch den Wegfall der landwirtschaftlichen Nutzung, der mit dem Erwerbsverlust bewertet wird. Bei der Beispielfruchtfolge WR-WW-WG beträgt der Erwerbsverlust $227 € /$ ha. Da der Erwerbsverlust der Minderertragsfläche jedoch von der Ertragsminderung abhängt, werden diese in der KNA dieses Kapitels zwischen $10 \%$ und 50 \% variiert. Da derzeit nicht davon ausgegan- 
gen werden kann, dass das Instrument der Flächenstillegung eine dauerhafte Maßnahme der Agrarpolitik sein wird, unterbleibt eine Kapitalisierung zum Dauerschaden. Stattdessen wird zunächst eine zehnjährige Stilllegung vorausgesetzt.

Durch eine Stilllegung kommt weiter hinzu, dass sich die Bewirtschaftung, z. B. durch Schaffung von Keilen, Durchschneidung des Schlages etc., erschweren kann. Hierbei kommt es zu höheren Arbeits- und Wendezeiten. Außerdem nimmt die Vorgewendefläche zu, wodurch es u. U. zu Ertragsminderungen kommen kann. ${ }^{492}$ Da die höheren Kosten durch Arbeits- und Wendezeiten sehr stark von der Lage der Minderertragsfläche abhängt, werden diese Kostenpositionen in den folgenden Berechnungen variiert. Bei der Berechnung der erhöhten Wendezeiten werden verschiedene Kantenlängen der Minderertragsfläche zugrunde gelegt. Es müssen sowohl die zusätzlichen Arbeitskosten als auch die zusätzlichen variablen Kosten des Schleppers bzw. Mähdreschers berücksichtigt werden. Die Berechnungen mit Hilfe der Datenbank des KTBL ${ }^{493}$ am Beispiel eines Getreideanbauverfahrens zeigen in Tabelle A-13, dass hierdurch je nach Kantenlänge der Minderertragsfläche zusätzliche Kosten von 12,4-63,4€ für die Minderertragsfläche entstehen können.

Die Kosten durch die Erhöhung der Vorgewendefläche werden im Folgenden für die obig verwendeten Kantenlängen ermittelt. Es wird eine Vorgewendebreite von $10 \mathrm{~m}$ unterstellt. Die möglichen Ertragsminderungen werden variiert. Wie in Tabelle A-15 im Anhang ersichtlich, können die Kosten durch den Minderertrag in Form eines geringeren Fruchtfolgedeckungsbeitrages je nach Höhe der Vorgewendefläche marginal zwischen 1,2-35,8 €/ha für die Minderertragsfläche liegen.

Der v. a. für den Einzelbetrieb wichtigste Nutzen besteht in dem Erhalt von Flächenzahlungen im Rahmen der Agenda 2000, die sich für $2002 \mathrm{im}$ Mittel um $342 €$ /ha bewegten. Im Folgenden wird von Ausgleichszahlungen von $348 € /$ ha (Niedersachsen, Region 1) ausgegangen. Außerdem kann der Landwirt Einsparungen bei Betriebsmitteln, den Maschinen- und Arbeitskosten realisieren. Weiterhin muss berücksichtigt werden, dass der Landwirt durch die Stilllegung einer Minderertragsfläche seine Pflichtstillegung um dieselbe Höhe reduzieren kann. Hierdurch gestaltet sich die Nutzenermittlung wie folgt:

\footnotetext{
${ }^{492}$ Vgl. hierzu Kapitel 6.3.1.3 ff.

${ }^{493}$ Vgl. KTBL (2000a)
} 


\section{- DB (Minderertragsfläche) \\ + DB Stilllegung (Minderertragsfläche) \\ + DB (zusätzlich bewirtschaftete Fläche) \\ = jährlicher Nutzen der Maßnahme}

Der Landwirt verliert zunächst den Deckungsbeitrag auf der Minderertragsfläche und bekommt für diese Fläche einen Stilllegungsausgleich. Durch die Reduzierung seiner Pflichtstillegung muss dieser Deckungsbeitrag hinzugerechnet werden.

Die Auswirkungen verschiedener Ertragsminderungen auf den Deckungsbeitrag ist in Abbildung 30 dargestellt. Außerdem ist der Deckungsbeitrag für eine Stilllegung mit Aussaat (Phacelia) in Höhe von 260,65 €/ha eingetragen. Einsparungen von variablen Maschinen- und Arbeitskosten gegenüber der Bewirtschaftung sind in diesem Betrag also indirekt enthalten. Mit Hilfe dieser Abbildung kann die Frage beantwortet werden, wann eine Stilllegung einer Minderertragsfläche vorteilhaft ist. In diesem Fall ist der Schnittpunkt der Fruchtfolge-Gerade mit der Stilllegungsgerade zu bestimmen. Dieser liegt bei 40,9 $9^{\mathbf{4 9 4}}$. Dies bedeutet, dass mindestens eine Ertragsminderung von 40,9\% vorliegen muss, damit eine Stilllegung gegenüber einer Bewirtschaftung vorteilhaft ist. Im Fall der obigen Versuchsergebnisse (43 \%-Ertragsminderung) wäre also eine Stilllegung nur marginal lohnenswert, da eine Prämie einen Vorteil von 17,68 €/ha hätte. Hieraus kann geschlossen werden, dass sich die Vorteilhaftigkeit dieser Maßnahme mit abnehmender Standortqualität erhöht. Hierbei ist zu beachten, dass bei einer Stilllegung mit Selbstbegrünung die Grenze der Vorteilhaftigkeit schon bei entsprechend geringeren Ertragsminderungen erreicht werden kann. Abschließend wäre noch zu prüfen, ob dieser Betrag durch die einzelnen Nachteile in der Bewirtschaftung aufgezehrt werden würde.

\footnotetext{
${ }^{494}$ Ermittelt durch das Gleichsetzen der Fruchtfolgegerade $(y=-8,26 x+598,15)$ und der Stilllegungsgeraden $(y=260,65)$.
} 


\section{Abbildung 30: Auswirkungen von verschiedenen Ertragsminderungen auf den De- ckungsbeitrag auf einer Minderertragsfläche}

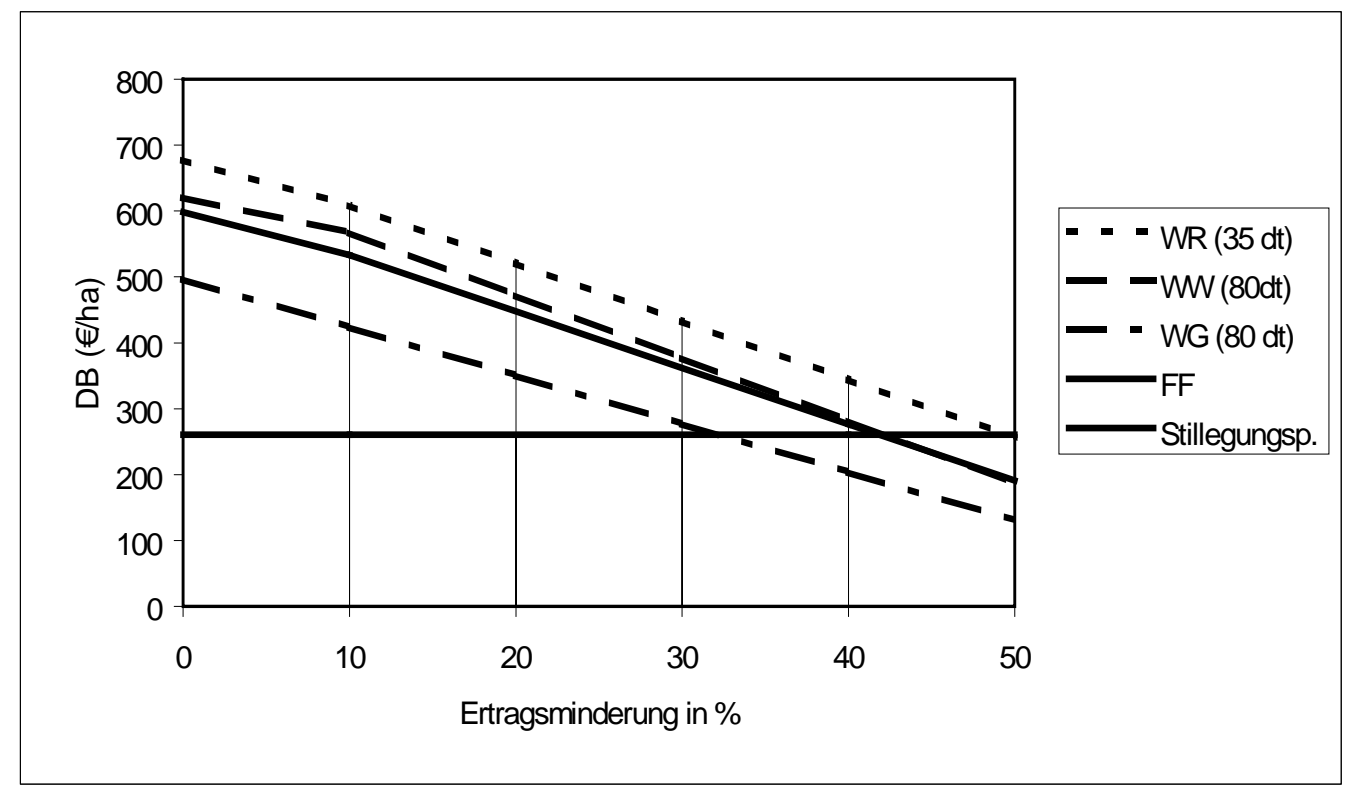

Quelle: Eigene Berechnungen nach LWK-HANNOVER (2002)

Aus Gesichtspunkten des Bodenschutzes ist es positiv zu bewerten, dass eine intensive Bearbeitung mit Landmaschinen weitestgehend unterbleibt und somit keine oder nur sehr geringe Bodenschadverdichtungen auf dieser Fläche entstehen. Pflegemaßnahmen der Stilllegungsfläche werden i. d. R. in Zeiträumen durchgeführt, in denen der Boden trocken ist.

Eine weitere Nutzenkomponente ist die Steigerung der biologischen Vielfalt durch die Schaffung von Lebensräumen auf der Stilllegungsfläche. Diese Komponente wird an dieser Stelle jedoch nicht weiter monetär bewertet, da es sich weitestgehend nicht um einen einzelbetrieblichen Nutzen, sondern um einen gesamtgesellschaftlichen Nutzen handelt. Positiv für den Einzelbetrieb könnte sich die Ansammlung von Nützlingen auswirken, die sich durch eine Senkung der Pflanzenschutzmittelaufwendungen bemerkbar macht.

\section{Kosten-Nutzen-Analyse}

In der folgenden KNA wird das KNV einer zehnjährigen Stilllegung einer Teilfläche von 1 ha berechnet. Der Betrachtungszeitraum beträgt 10 Jahre und der Zinssatz 4 \%. Es wird 
von zwei Standorten ausgegangen. Einem ertragreichen ${ }^{495}$, bei dem der Fruchtfolgedeckungsbeitrag wie im Kapitel 6.3.1.6 bei $564 € /$ ha und der Erwerbsverlust bei $427 € /$ ha liegt, und einem Standort mit einem Fruchtfolgedeckungsbeitrag ${ }^{496}$ von $356 € /$ ha und einem Erwerbsverlust von $227 € /$ ha. $^{497}$.

Die Kosten (Erwerbsverlust (384,3€/ha), zusätzliche Kosten durch Arbeits- und Wendezeiten (63,4€/ha), Erhöhung der Vorgewendekosten (35,8€/ha)) belaufen sich bspw. bei einer Ertragsminderung von $10 \%$ auf 483,5 €/ha und der Nutzen (Deckungsbeitrag der Stillegung $(260,65 € /$ ha) sowie zusätzlich bewirtschafteter Fläche (564 €/ha)) auf $824,65 € /$ ha, wenn der ertragreiche Standort vorausgesetzt wird. Beim ertragsschwachen Standort und einer Ertragsminderung von $10 \%$ ergeben sich Kosten von 303,5 €/ha (Erwerbsverlust 204,3 €/ha, zusätzliche Kosten durch Arbeits- und Wendezeiten (63,4 €/ha), Erhöhung der Vorgewendekosten $(35,8 € /$ ha)) und ein Nutzen von 625,65 €/ha (Deckungsbeitrag der Stillegung $(260,65 € /$ ha) sowie zusätzlich bewirtschafteter Fläche (365€/ha)). Die entsprechenden Berechnungen für diese beiden Fälle sind dem Anhang in Tabelle A-66 und Tabelle A-67 zu sehen.

Die KNV der einzelnen Varianten werden in der folgenden Tabelle zusammengefasst.

Tabelle 57: KNV und durchschnittlicher jährlicher Vorteil der Stilllegung einer Minderertragsfläche auf verschiedenen Standorten

\begin{tabular}{ccccc}
\hline Mindererträge & $\begin{array}{c}\text { Hoher Stand- } \\
\text { ortertrag }\end{array}$ & $\begin{array}{c}\varnothing \text { jährl. Vor- } \\
\text { teil (€/ha/a) }\end{array}$ & $\begin{array}{c}\text { Niedriger } \\
\text { Standortertrag }\end{array}$ & $\begin{array}{c}\varnothing_{\text {jährl. Vorteil }} \\
(\boldsymbol{\epsilon} / \mathbf{h a} / \mathbf{a})\end{array}$ \\
\hline$-10 \%$ & 0,59 & 341 & 0,49 & 322 \\
$-20 \%$ & 0,53 & 384 & 0,45 & 345 \\
$-30 \%$ & 0,48 & 426 & 0,41 & 368 \\
$-40 \%$ & 0,43 & 469 & 0,38 & 390 \\
$-50 \%$ & 0,38 & 512 & 0,34 & 413 \\
\hline
\end{tabular}

Quelle: Eigene Berechnungen

Die Ergebnisse der Tabelle 57 zeigen, dass bei allen Mindererträgen zwischen $10 \%$ und $50 \%$ unter den angenommenen Annahmen sehr gute KNV vorherrschen. Die KNV sind bei einem niedrigen Standortertrag noch günstiger als auf dem Hochertragsstandort.

\footnotetext{
${ }^{495}$ Ertragsniveau: $\mathrm{WR}=35 \mathrm{dt} ; \mathrm{WW}=80 \mathrm{dt}$ und $\mathrm{WG}=80 \mathrm{dt}$.

${ }^{496}$ Ertragsniveau: $\mathrm{WR}=25 \mathrm{dt} ; \mathrm{WW}=50 \mathrm{dt}$ und $\mathrm{WG}=50 \mathrm{dt}$.

${ }^{497}$ Vgl. Tabelle A-18
} 


\subsection{Schlussfolgerungen}

Es konnte gezeigt werden, dass die Stilllegung von Minderertragsteilflächen in das Kalkül des Landwirts, der über große Schläge verfügt, einbezogen werden sollte. Die KNV sind unter den Annahmen im vorigen Kapitel sehr gut. Letztlich entscheidet der Landwirt aufgrund seiner Erfahrung oder erhobenen Daten. Das Risiko dieser Maßnahme ist eher gering, da wenig Kapital gebunden wird und eine geringere und überschaubarere Bindungszeit als bei anderen Maßnahmen vorherrscht.

\subsubsection{Maßnahmen zur Verminderung des Flächenverbrauchs und deren ökonomische Bewertung}

Im folgenden Abschnitt werden verschiedene Maßnahmen der Verminderung des Flächenverbrauchs dargestellt.

\subsection{Einleitende Erwägungen}

Der Flächenverbrauch in Deutschland betrug in 2002 etwa 105 ha/Tag. Damit ist ein rückläufiger Trend zu beobachten, da der tägliche Flächenverbrauch 2001 noch 117 ha/Tag betragen hatte. ${ }^{498}$ Der Flächenverbrauch ist v. a. als Zunahme der Siedlungsund Verkehrsfläche zu sehen. Dabei ist zu beachten, dass die Begriffe Flächenverbrauch und Flächenversiegelung nicht gleichzusetzen sind. Die Versiegelung meint die konkrete Bebauung, durch die der Boden sämtliche natürliche Funktionen verliert. Bei der Ermittlung des Flächenverbrauch werden hingegen auch umbaute und somit nicht versiegelte Flächen berücksichtigt.

\subsection{Betriebswirtschaftliche Aspekte der Maßnahme}

In dem Schwerpunktpapier „Nationale Nachhaltigkeitsstrategie“ fordert die derzeitige Bundesregierung, den Flächenverbrauch bis zum Jahr 2020 auf 30 ha/Tag zu begrenzen. In der Expertenbefragung wurde die Frage gestellt, ob die Bundesregierung dieses Ziel der Nachhaltigkeitsstrategie erreichen könne. Hierzu gab es ein eindeutiges Ergebnis. 19 Befragte $(73,1 \%)$ waren der Meinung, dass das Ziel mit den geplanten Maßnahmen nicht

\footnotetext{
${ }^{498}$ Vgl. StAtistisches BundesAmt (2003)
} 
zu erreichen ist. Lediglich ein Experte war der Meinung, dass das Ziel zu erreichen sei, wenn der Bevölkerungsrückgang in Deutschland weiter anhalte.

Auf der einen Seite ist die Landwirtschaft nicht nur durch den Flächenverbrauch durch Überbauung und Abgrabung, sondern auch durch die damit zusammenhängenden Ausgleichs- und Ersatzmaßnahmen nach $\S 19$ BNatSchG belastet. Dadurch sinkt die landwirtschaftlich genutzte Fläche zum größten Teil unabhängig von ihrer Bonität und Qualität kontinuierlich und führt zu höheren Boden- und Pachtpreisen. Auf der anderen Seite wird durch landwirtschaftliche Investitionstätigkeiten in Gebäuden und Anlagen ebenfalls Boden versiegelt. Vor diesem Hintergrund können die Landwirte aktiven Bodenschutz praktizieren, indem sie durch Nutzung von Altgebäuden und -anlagen am Flächenrecycling teilnehmen.

Im Folgenden sind einige Vorschläge zur Reduzierung des Flächenverbrauchs aus der aktuellen Diskussion zusammengefasst:

- bessere Nutzungskonzepte für vorhandene Anlagen und Gebäudesubstanz (Flächenrecycling),

- Erweiterung und Ausschöpfung vorhandener Nutzungspotenziale (z. B. Ausbau von Altgebäuden, Überbauung von Verkehrsflächen etc.),

- Wiedernutzung von Industrie- und Baulandbrachen,

- Mobilisierung vorhandenen Baulandes (z. B. Baulücken etc.),

- Verhältnis von Innen- zu Außenentwicklung 3:199,

- Verkürzung der Entfernungspauschale,

- Einführung einer Flächennutzungssteuer und

- Differenzierung der Eigenheimpauschale (stärkere Förderung von „recycelten“ Gebäuden).

Aufgrund der geringen Bedeutung für landwirtschaftliche Betriebe werden nachfolgend nur bestimmte Aspekte erläutert. 
Um bessere Nutzungskonzepte für vorhandene Gebäude zu schaffen, ist es zunächst nötig, das Bauplanungsrecht zu reformieren. Bspw. müssen derartige Konzepte Eingang in Bebauungspläne u. ä. finden. Außerdem müssen Altgebäude und -anlagen besser genutzt werden. Die zunehmende Bebauung im Außenbereich führt dazu, dass viele Industrieund Wohnflächen in den Städten und Ballungsräumen brachliegen. Dieses Potenzial ist in der Vergangenheit kaum genutzt worden. Die Wirkung der Verkürzung oder Abschaffung der Entfernungspauschale auf den Flächenverbrauch ist umstritten. Außerdem handelt es sich hierbei politisch gesehen um ein sehr sensibles Thema, weshalb es nicht weiter betrachtet wird.

In der Vergangenheit sind von wissenschaftlicher Seite einige Modelle zur Besteuerung des Flächenverbrauchs entwickelt worden. Das am weitesten entwickelte ist das Modell von BIZER und EwRIGMANN ${ }^{500}$. Demnach sollen die Flächen in verschiedene Steuerklassen eingeteilt und besteuert werden. Das bisherige Aufkommen der Grundsteuer A soll bspw. den Steuerklassen I-V, also naturbelassenen Flächen, naturschonend genutzten Flächen, forstwirtschaftlich genutzten Flächen sowie sonstigen Freiflächen, wozu die ldw. genutzten Flächen gehören, sowie den versiegelten Flächen im Außenbereich zugeschlagen werden. Nach diesem Modell würden die naturschonend genutzten Flächen mit $0,19 € /$ ha, die forstwirtschaftlich genutzten Flächen mit 1,9 €/ha und 1dw. genutzte Flächen mit 19,4 €/ha belastet. Diese Belastung der landwirtschaftlichen Betriebe ist nach Meinung der Autoren äquivalent zur bisherigen, die zwischen 12,8 - 25,6€/ha liegt.

\subsection{Schlussfolgerungen}

Es ist unzweifelhaft, dass ein derart hoher Flächenverbrauch, wie z. Zt. besteht, nicht mit einer nachhaltigen Raumentwicklung vereinbar ist. Deshalb müssen zunächst alle vorliegenden Vorschläge zur Reduzierung des Flächenverbrauchs sorgfältig geprüft und volkswirtschaftlich untersucht werden. Die Landwirtschaft kann insofern mit einem guten Beispiel voran gehen, dass sie dafür sorgt, möglichst Altgebäuderecycling zu betreiben. Dies ist allerdings hinsichtlich der Vorgaben des Denkmalschutzes nicht immer möglich. Daher ist in diesem Zusammenhang über eine Lockerung der Regelungen nachzudenken,

\footnotetext{
${ }^{500}$ Vgl. BIZER u. EWRIGMANN (1999)
} 
wenn z. B. Umbaumaßnahmen an aufgrund von Denkmalschutzauflagen zu hohen Umbaukosten scheitern.

Zum dargestellten Flächennutzungssteuermodell ist anzumerken dass generell eine Prüfung des Bestands der Grundsteuer A in der jetzigen Form ansteht. Eine VerhältnismäBigkeit zwischen Erhebungskosten und Einnahmen scheint zweifelhaft. Bezüglich der Flächennutzungssteuer sollte ein gewisser Vorbehalt der Nutzung einer Steuer als Lenkungsinstrument vorherrschen. In diesem Zusammenhang sollte der Abgabe auf versiegelte Flächen Vorrang gegeben werden.

\subsection{Stofflicher Bodenschutz}

Analog zu den Maßnahmen des physikalischen Bodenschutzes werden im Folgenden Bodenschutzmaßnahmen zur Reduzierung von stofflichen Schädigungen des Bodens detailliert beschrieben und anhand der in Kapitel 6.2 dargestellten Maßnahmen auf deren betriebswirtschaftliche Vorzüglichkeit untersucht. Der stoffliche Bodenschutz befasst sich hauptsächlich mit der Verhinderung oder Reduzierung von schädlichen Einträgen wie Pflanzenschutz- und Düngemitteln, Klärschlämmen und Komposten. Die Analyse dieser Maßnahmen geschieht nicht in der Ausführlichkeit der oberen Kapitel, auf denen der Schwerpunkt dieser Arbeit liegt.

\subsubsection{Pflanzenschutzmitteleinsatz}

Die Probleme und der Stand des Einsatzes von PSM wurden bereits im Kapitel 3.1.3.2 dargestellt. Darauf aufbauend erfolgt exemplarisch eine allgemeine KNA für den Einsatz von Pflanzenschutzmitteln in erosionsgefährdeten Gebieten.

Die im Kapitel 3.1.3.2 dargestellten Probleme mit den landwirtschaftlichen Pflanzenschutzmitteleinsätzen wurden bisher kaum aus betriebswirtschaftlicher Sicht betrachtet. Dies ist ebenfalls in dem für den Gewässerschutz sehr wichtigen und wegweisenden Herausgeberwerk HANDBUCH DES GeWÄSSERSCHUTZES ${ }^{501} \mathrm{zu}$ erkennen. Dabei spielt die Problematik des Pflanzenschutzmitteleinsatzes für den (Grund-) Wasserschutz eine weitaus zentralere Rolle als für den Bodenschutz.

${ }^{501}$ Vgl. FredE u. DABBERT (1998) 
Die aus Sicht des Bodenschutzes und somit in einer rein ökologischen Betrachtung effizienteste Maßnahme zur Reduzierung von PSM-Einträgen ist der komplette Verzicht von PSM wie etwa im ökologischen Landbau. Daneben gibt es jedoch noch eine Zwischenform, die mit der Reduzierung des PSM-Einsatzes und dem Einsatz von Schadschwellen sowie mechanischen Unkrautbekämpfungsmittel im Integrierten Landbau praktiziert wird. Der Integrierte Landbau war bereits Gegenstand des Kapitels 6.3.1.1.5.

\subsubsection{Reduzierung des Einsatzes von Pflanzenschutzmitteln}

Im Folgenden wird die Reduzierung des Einsatzes von PSM durch die Anwendung des Schadschwellenprinzips betrachtet.

\subsection{Einleitende Erwägungen}

In der Übersicht 21 ist die Vorgehensweise bei der KNA für einen Teilbereich des Integrierten Landbaus, der Reduzierung der PSM-Ausbringung nach dem Schadschwellenprinzip, dargestellt. 


\section{Übersicht 21:Vorgehensweise bei der KNA für die Reduzierung des PSM-Einsatzes durch das Schadschwellenprinzip}

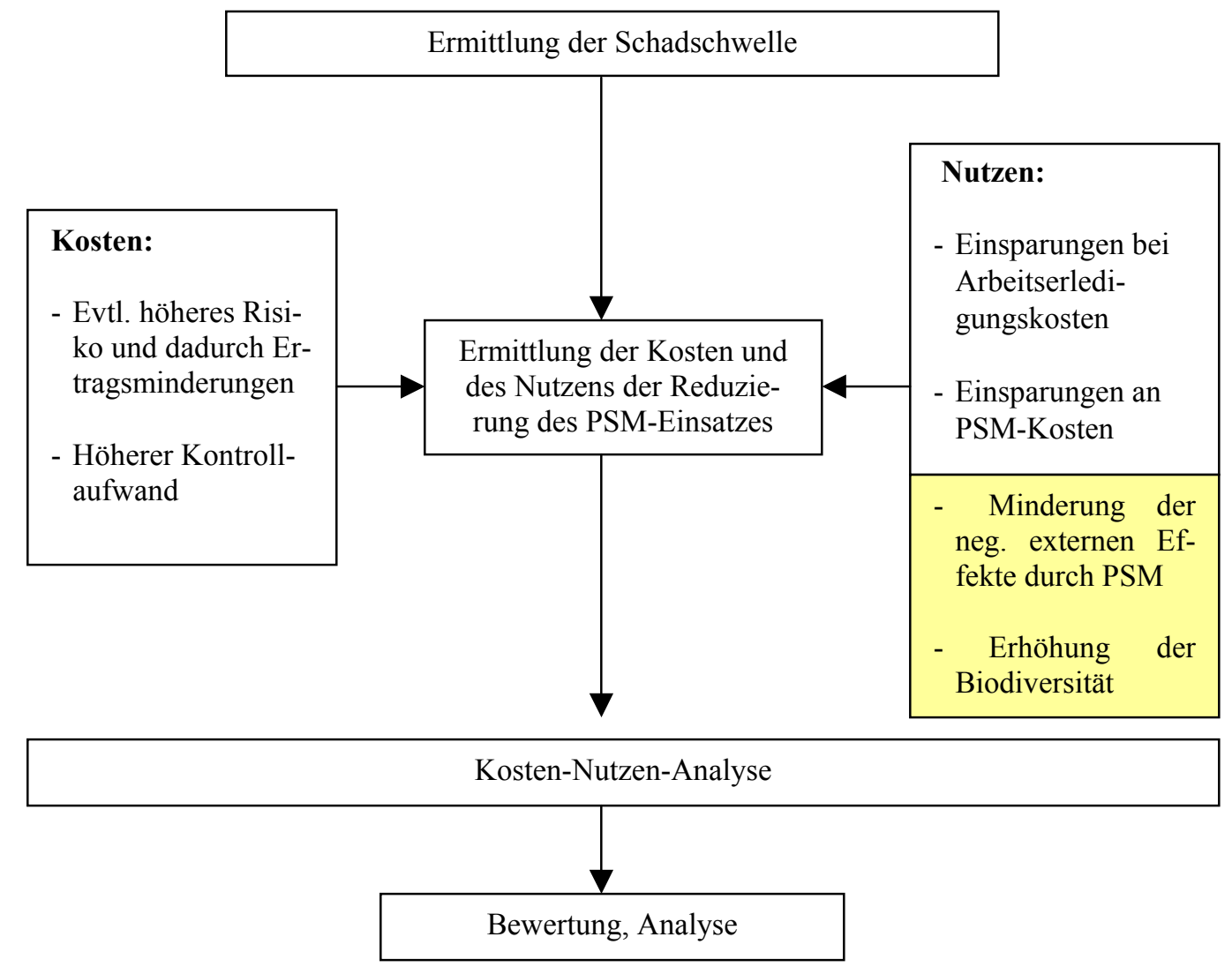

Quelle: Eigene Darstellung, Schraffierung: Positionen konnten aufgrund fehlender Daten in der Berechnung nicht berücksichtigt werden

Zunächst ist es notwendig, den integrierten Landbau von den anderen Wirtschaftsformen abzugrenzen. Derartige Definitionen finden sich bspw. bei DiERCKS U. HeITEFuss. Demnach umfasst der Integrierte Landbau ,standort- und umweltgerechte Systeme der Pflanzenproduktion, in denen unter Beachtung ökologischer und ökonomischer Anforderungen alle geeigneten und vertretbaren Verfahren ... in möglichst guter Abstimmung aufeinander ... eingesetzt werden, um langfristig sichere Erträge und betriebswirtschaftlichen Erfolg zu gewährleisten ${ }^{502}$. Für die weitere Betrachtungsweise ist jedoch das Schadschwellenprinzip des Integrierten Landbaus von elementarer Bedeutung. Bei wirtschaftlichen Schadschwellen handelt es sich um Richtwerte, die angeben, ab welcher Populationsdichte von Schadorganismen die hierdurch entstehenden monetären Schäden höher sind als die Kosten deren Bekämpfung. Durch die Anwendung der wirtschaftlichen Schadschwel- 
le können bspw. die folgende Tabelle 58 sowie die Ergebnisse des INTEX-Projektes in Kapitel 6.3.1.1.5 erklärt werden. Damit ist die konsequente Anwendung des Prinzips der wirtschaftlichen Schadschwelle das zentrale Instrument des Integrierten Landbaus, um u. a. Stoffeinträge zu reduzieren.

Tabelle 58: Ausgebrachte Wirkstoffmengen und Weizenerträge im langjährigen Anbauvergleich bei integrierter und konventioneller Bewirtschaftung

\begin{tabular}{ccccc}
\hline Zeitraum & \multicolumn{2}{c}{ Wirkstoffmengen (kg/ha) } & \multicolumn{2}{c}{ Weizenerträge (dt/ha) } \\
\hline & integriert & konventionell & integriert & konventionell \\
$1978-1981$ & 1,56 & 2,60 & 61,7 & 65,3 \\
$1982-1985$ & 2,57 & 3,38 & 65,5 & 66,1 \\
$1986-1989$ & 2,18 & 3,75 & 57,2 & 65,1 \\
Mittel & $\mathbf{2 , 1}$ & $\mathbf{3 , 2 4}$ & $\mathbf{6 1 , 4}$ & $\mathbf{6 5 , 5}$ \\
\hline
\end{tabular}

Quelle: EL TITI, zit. in FredE u. DABBERT (1998), S. 94

Die Tabelle 58 zeigt, dass im langjährigen Vergleich durch eine Reduzierung des PSMAufwandes bei Winterweizen um ca. 35 \% der Ertrag um 6,2 \% zurückgeht. Ein Vergleich dieser Ergebnisse mit denen des INTEX-Projektes kann durch den Vergleich mit Kapitel 6.3.1.1.5.1 gemacht werden.

\subsection{Betriebswirtschaftliche Analyse der Maßnahme}

Die zusätzlichen Kosten der Reduktion des PSM-Einsatzes setzen sich neben den evtl. entstehenden geringeren Erträgen aus den höheren Kontrollkosten zusammen. Diese könnten nur sehr pauschal angenommen werden und werden damit in der folgenden Berechnung nicht weiter berücksichtigt.

Bei der folgenden betriebswirtschaftlichen Analyse wird auf die in Kapitel 6.3.1.1.5.3 dargestellten Daten zurückgegriffen. Die Daten für die Betriebe Marienstein und Reinshof zeigen ein uneinheitliches Bild. Auf dem ertragreicheren Standort Reinshof konnten sowohl Pflanzenschutzmittelkosten (-40 bis -55 \%) als auch Maschinen- und Arbeitskosten $(0-23 \%)$ durch die integrierten Anbauverfahren eingespart werden. Dies bedeutet, dass die Maßnahme auf diesem Standort äußerst wirtschaftlich ist. Da davon auszugehen ist, dass es sich bei der Minderung von PSM ansonsten um reine externe Effekte handelt, entsteht für den Betrieb Reinshof ein Nettonutzen in Höhe der eingesparten Kosten. 
Beim Betrieb Marienstein gab es insofern keinen einzelbetrieblichen Nettonutzen des Integrierten Anbaus, als dass die Einsparungen bei den variablen Kosten die geringeren Markterlöse nicht kompensieren konnten. Aber auch hier müssen die externen Effekte durch die Verringerung der Ausbringungsmengen gesehen werden.

\section{Kosten-Nutzen-Analyse}

Ergänzend zu den Vergleichen der Betriebe des INTEX-Projekts in Kapitel 6.3.1.1.5.1 werden im Folgenden die Ergebnisse von EL TITI zu einer KNA herangezogen. Dabei werden allerdings aktuelle Input- wie auch Produktpreise verwendet. Der Zeitraum beträgt wie in der Untersuchung neun Jahre, die durchschnittliche Einsparung an PSM beträgt $35 \%$ und die durchschnittliche Ertragsminderung $6 \%$. Bei der Ermittlung der PSMKosten wird entsprechend dem Ertragsniveau und der Produktpreise des konventionellen Anbaus auf Daten der LWK-HANNOVER ${ }^{503}$ zurückgriffen. Die Erträge der betrachteten Standorte werden zwischen 50 und $100 \mathrm{dt} /$ ha variiert. Die KNV ergeben sich durch Gegenüberstellung der Erlösverluste und den eingesparten PSM-Kosten der jeweiligen Standorte. Der Zinssatz beträgt 4 \%. Die Berechnung ist in der Tabelle A-71 exemplarisch für den Standort mit einem Ertrag von $50 \mathrm{dt} / \mathrm{ha}$ dargestellt.

Tabelle 59: KNV bei Weizen im Integrierten Anbau auf verschiedenen Standorten mit Annahmen von EL TITI

\begin{tabular}{|c|c|c|c|c|}
\hline Standortertrag* (dt/ha) & 50 & 70 & 90 & 100 \\
\hline PSM-Kosten* $(€ / \text { ha })^{504}$ & 166,2 & 191,6 & 270,6 & 270,6 \\
\hline Ertrag (dt/ha) & 47,0 & 65,80 & 84,6 & 94,0 \\
\hline Erlösverluste $^{505}(€ / \mathrm{ha})$ & 32,58 & 47,46 & 60,0 & 65,16 \\
\hline $\begin{array}{l}\text { Eingesparte PSM-Kosten } \\
(€ / \text { ha })\end{array}$ & 58,17 & 67,06 & 94,71 & 94,71 \\
\hline KNV & $\mathbf{0 , 5 6}$ & 0,71 & 0,63 & 0,69 \\
\hline$\varnothing$ jährl. Vorteil (€/ha/a) & 25,6 & 19,46 & $\mathbf{3 4 , 7 0}$ & 29,54 \\
\hline
\end{tabular}

* In der Ausgangssituation (konventioneller Landbau)

Quelle: Eigene Berechnungen

\footnotetext{
${ }^{503}$ Vgl. LWK-HANNOVER (2002), S. 10 u. 18

${ }^{504}$ Variable Maschinenkosten: Anbauspritze, $15 \mathrm{~m}, 54 \mathrm{~kW}$.

${ }^{505}$ Angenommene Produktpreise: Standort $50 \mathrm{dt} / \mathrm{ha:}$ 10,86 €/dt; $70 \mathrm{dt} / \mathrm{ha}: 11,3 € / \mathrm{dt}, 90 \mathrm{dt} / \mathrm{ha}: 11,09 € / \mathrm{dt}$ und $100 \mathrm{dt} / \mathrm{ha}: 10,86 € / \mathrm{dt}$.
} 
Die Berechnungen zeigen, dass unter den angenommenen Bedingungen durchweg gute KNV erzielt werden. Alle Varianten weisen durchschnittliche jährliche Vorteile auf.

\subsection{Schlussfolgerungen}

Die Reduzierung des PSM-Einsatzes im Integrierten Landbau ist eine sehr gute Maßnahme, um die PSM-Einträge sinnvoll und wirtschaftlich zu reduzieren. Aber auch bei dieser Maßnahme ist dies unter dem Vorbehalt des Standortes zu sehen, wie die Ergebnisse der Betriebe Reinshof und Marienstein gezeigt haben. Der Integrierte Landbau verlangt allerdings von dem Landwirt ein erhöhtes Engagement bei der Bewirtschaftung. Dies äußert sich in den notwendigen höheren Kontrollaufwendungen, die anzusetzen sind. Die Ergebnisse zur Effizienz des Integrierten Landbaus werden in den folgenden Kapiteln mit den dort dargestellten Maßnahmen verglichen.

\subsubsection{Mechanische Unkrautbekämpfung}

Im folgenden Abschnitt wird eine weitere Maßnahme, u. a. des Integrierten Anbaus, die Mechanische Unkrautbekämpfung, als Maßnahme zur Reduzierung der PSM-Einträge durch die Landwirtschaft betrachtet und anhand einer KNA bewertet.

\subsection{Einleitende Erwägungen}

Bei der mechanischen Unkrautbekämpfung sind verschiedene Bekämpfungsvarianten zu unterscheiden. Es kann z. B. mit dem Striegel, der Hacke oder der Bürste gearbeitet werden. Außerdem besteht die Möglichkeit, das Unkraut mit thermischen Verfahren zu vernichten. Die Techniken der mechanischen Unkrautbekämpfung werden v. a. im Integrierten sowie im Ökologischen Landbau angewendet. Um einen ausreichenden Bekämpfungserfolg mit diesen Maßnahmen zu erzielen, sind einige Voraussetzungen zu erfüllen. Zunächst muss der Boden trocken sein, um Verschlämmungen etc. zu vermeiden. Deshalb eignen sich besonders gut leichte und sandige Böden für derartige Maßnahmen. Außerdem darf der Pflanzenbestand nicht durch die mechanische Maßnahme geschädigt werden. Hierzu müssen die Geräte optimal auf den Bestand eingestellt werden. 


\subsection{Betriebswirtschaftliche Analyse der Maßnahme}

In der Übersicht 22 ist die Vorgehensweise der Ermittlung des KNV dargestellt.

\section{Übersicht 22: Vorgehensweise bei der KNA für die Maßnahme der mechanischen Unkrautbekämpfung}

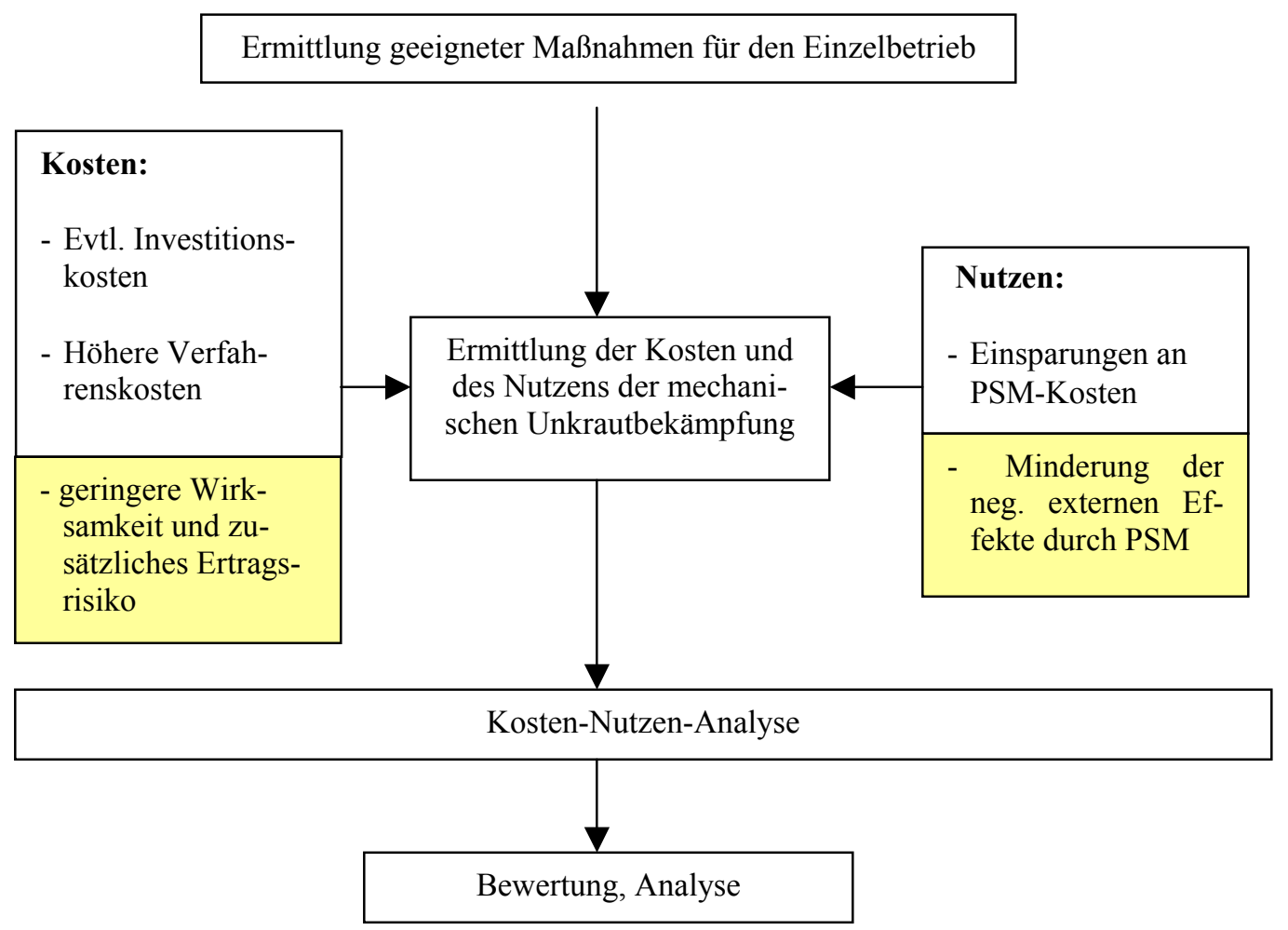

Quelle: Eigene Darstellung, Schraffierung: Positionen konnten aufgrund fehlender Daten in der Berechnung nicht berücksichtigt werden

Die Kosten dieser Verfahren sind in der Tabelle 60 in Abhängigkeit zur Arbeitsbreite, in Form von Verfahrenskosten aufbereitet.

Tabelle 60: Verfahrenskosten für unterschiedliche Unkrautbekämpfungsmaßnahmen

\begin{tabular}{|c|c|c|}
\hline Gerät & Arbeitsbreite (m) & Verfahrenskosten $(€ /$ ha) \\
\hline Hackstriegel & $3-24 \mathrm{~m}$ & $23,63-13,69$ \\
\hline Hackmaschine & 2- 12 reihig & $55,20-21,80$ \\
\hline Abflammgerät ${ }^{506}$ & 5- und 4-reihig & $81,53-65,33$ \\
\hline
\end{tabular}

Quelle: KTBL (2002) sowie eigene Berechnungen

${ }^{506}$ fünf- bzw. vierreihig 
Die obige Tabelle zeigt, dass es sich bei den mechanischen Unkrautbekämpfungsmaßnahmen um sehr kostenintensive Maßnahmen handelt. Zudem ist zu beachten, dass nur eine Überfahrt berücksichtigt wurde. In der Praxis ist allerdings nur bei mehreren Arbeitsgängen ein befriedigendes Ergebnis zu erzielen. Daher ist die mechanische Unkrautbekämpfung eher für den ökologischen Landbau, für Wasserschutzgebiete oder als Ergänzung zu chemischen Bekämpfungsmethoden geeignet.

Zunächst wird eine KWA des Striegelns der Fruchtfolge WR-WW-WG durchgeführt. Dabei ist die Wirksamkeit des Striegelns auf den Bekämpfungserfolg bezüglich Klettenlabkraut in der folgenden Tabelle dargestellt.

Tabelle 61: Prozentualer Bekämpfungserfolg und KW bei Klettenlabkraut bei chemischer und mechanischer Unkrautbekämpfung (Striegel) auf INTEX-Flächen

\begin{tabular}{ccccc}
\hline & $\begin{array}{c}\text { Chemische } \\
\text { Bekämpfung }\end{array}$ & $\begin{array}{c}\mathbf{K W}^{\mathbf{5 0 7}} \\
\mathbf{\epsilon} / \% \\
\text { Bekämp- } \\
\text { fungserfolg }\end{array}$ & $\begin{array}{c}\text { Mechanische } \\
\text { Bekämpfung }\end{array}$ & $\begin{array}{c}\text { KW } \\
\mathbf{\epsilon} / \mathbf{\%} \text { Bekämp- } \\
\text { fungserfolg }\end{array}$ \\
\hline Winterraps & $\mathbf{( \% )}$ & $0,35-0,42$ & 65 & $0,21-0,36$ \\
Winterweizen & 90 & $0,39-0,46$ & 64 & $0,21-0,37$ \\
Wintergerste & 100 & $0,35-0,41$ & 68 & $0,20-0,34$ \\
\hline
\end{tabular}

Quelle: NLÖ (2001), S. 62

In der obigen Tabelle ist zu sehen, dass der Wirkungsgrad, d. h. der prozentuale Bekämpfungserfolg der mechanischen Verfahren ca. $30 \%$ unter dem der chemischen liegt. Die KW liegen je nach Arbeitsbreite und PSM-Einsatz jedoch bei der mechanischen Variante unter denen der chemischen. Hieraus folgt, dass unter den i. d. R. praxisnahen Annahmen durch die Ergebnisse des INTEX-Projekts davon ausgegangen werden kann, dass der Striegeleinsatz durchaus wirtschaftlich sein kann. Für die anderen mechanisch arbeitenden Geräte ist eine derartige Aussage allerdings nicht zu treffen, da sie wesentlich höhere Verfahrenskosten aufweisen. Als Einschränkung sei an dieser Stelle jedoch angeführt, dass der obige Vergleich davon ausgeht, dass eine einmalige Behandlung mit dem Striegel ausreicht. Dies ist gerade bei starker Verunkrautung und ungünstiger Witterung in der Praxis nicht immer gegeben. Weitere Nachteile, v. a. die der ertragsschädigenden Wirkung der mechanischen Unkrautbekämpfung werden in der folgenden KNA berücksich-

\footnotetext{
${ }^{507}$ Bei der Berechnung der KW wird mit einer Herbizidanwendung und somit mit Verfahrenskosten in Höhe von 41,25 bzw. 35,04 €/ha (Ausbringung von 1,0 1 Starane bzw. 0,5 1 Starane und 1,0 1 Duplosan).
} 
tigt. Ein nicht direkt zu monetarisierender Nachteil des Striegels in überbetrieblichen Einsätzen ist die mögliche Verbreitung von Pflanzenkrankheiten und Unkräutern.

\section{Kosten-Nutzen-Analyse}

Bei Ersatz von Herbiziden durch mechanische Unkrautbekämpfung wurden Ertragsrückgänge von 0 bis $5 \mathrm{dt} / \mathrm{ha}$ bei Getreide gemessen. ${ }^{508}$ Daraus ergeben sich bei dem Striegelverfahren beim Winterweizen Leistungsverluste von bis zu 56,4€/ha und bei der Wintergerste von bis zu $45,8 € /$ ha. $^{509}$

Es ist zu berücksichtigen, dass die Wirkung der mechanischen Unkrautbekämpfung vom Reihenabstand abhängt. Je höher der Reihenabstand, desto wirkungsvoller sind mechanische Bekämpfungsmaßnahmen. Im Folgenden wird allerdings von einem üblichen Reihenabstand von $13 \mathrm{~cm}$ und als Ertragswirkung daher analog zu den Ergebnissen von IR$\mathrm{LA}^{510}$ von einer Ertragsveränderung bei Getreide von - 1,5 dt/ha ausgegangen werden. Die notwendigen Einsätze werden zwischen 1-3 Überfahrten variiert. Da keine entsprechenden Ergebnisse für die thermische Bekämpfung sowie die Hackmaschine vorliegen, wird im Folgenden nur eine KNA für den Striegeleinsatz in der Fruchtfolge WR-WW-WG durchgeführt, wobei für Winterraps keine Ertragsveränderung angenommen wird.

Die Verfahrenskosten des Striegeleinsatzes betragen 14,31 €/ha. ${ }^{511}$ Außerdem entstehen Kosten durch Ertragseinbußen in Höhe von $17 € /$ ha bei Winterweizen bzw. 13,7 €/ha bei Wintergerste für die entgangenen Erträge. Dementsprechend entstehen beim Weizen und einem Arbeitsgang Kosten von 31,31 €/ha. Dem gegenüber entstehen je nach Standort PSM-Einsparungen incl. Ausbringung zwischen 166,20 und 270,60 €/ha. In dem eben beschriebenen Fall beläuft sich der Nutzen daher auf 218,4 €/ha. Im Folgenden wird der Wert 218,4 €/ha verwendet. Der Betrachtungsraum beträgt 12 Jahre. Die Ergebnisse sind der Tabelle 62 zu entnehmen. Die Berechnungen für Weizen sind in der Tabelle A-72 bis Tabelle A-74 dargestellt.

\footnotetext{
${ }^{508}$ Vgl. IRLA (1991)

${ }^{509}$ bei einem Weizenpreis von $11,3 € / \mathrm{dt}$ und einem Gerstenpreis von 9,16€/dt.

${ }^{510}$ Vgl. IRLA (1991)

${ }^{511}$ Vgl. KTBL (2002), S. 322, Annahme: Striegel 12 m, 67 kW, Parzellengröße 5 ha.
} 
Tabelle 62: KNV (\%) und durchschnittlicher jährlicher Vorteil beim Striegeleinsatz in Winterweizen und -gerste

\begin{tabular}{lccc}
\hline & ein Arbeitsgang & vier Arbeitsgänge & sechs Arbeitsgänge \\
\hline Weizen & $\mathbf{0 , 1 4}$ & $\mathbf{0 , 3 4}$ & $\mathbf{0 , 4 7}$ \\
$\varnothing$ jährl. Vorteil $(€ / \mathrm{ha} / \mathrm{a})$ & 187,17 & 144,23 & 115,59 \\
Gerste & $\mathbf{0 , 1 3}$ & $\mathbf{0 , 3 2}$ & $\mathbf{0 , 4 6}$ \\
$\varnothing$ jährl. Vorteil $(€ / \mathrm{ha} / \mathrm{a})$ & 190,48 & 147,53 & 118,89 \\
\hline
\end{tabular}

Quelle: Eigene Berechnungen

$\mathrm{Zu}$ den Ergebnissen in Tabelle 62 ist anzumerken, dass optimistische Annahmen getroffen wurden. Wird ein Ertragsrückgang bei Getreide von $5 \mathrm{dt} / \mathrm{ha}$ durch den Striegeleinsatz vorausgesetzt, verschlechtern sich die KNV bei Winterweizen auf $0,32 \%, 0,52 \%$ und $0,65 \%$. Bei Gerste würden sie bei $0,28 \%, 0,47 \%$ und $0,6 \%$ liegen. Weiterhin ist zu bemerken, dass der Striegeleinsatz in Abhängigkeit von der Witterung (Durchwuchs etc.) zu höheren Ertragsschwankungen führen wird, da der Bekämpfungserfolg bei chemischen PSM höher ist.

\subsection{Schlussfolgerungen}

Es konnte für den Striegeleinsatz gezeigt werden, dass er unter den angenommen optimistischen Rahmenbedingungen eine günstige Maßnahme der Reduzierung des PSMEinsatzes ist. Anhand der KNV wird deutlich, dass selbst bei sechs Arbeitsgängen noch sehr gute KNV vorherrschen. Das Problem der geringen Verbreitung in der ldw. Praxis liegt zunächst in dem höheren Zeitbedarf, vor allem aber in dem geringeren Bekämpfungserfolg und somit in dem Risiko von Ertragseinbußen. Die anderen mechanischen Unkrautbekämpfungsmaßnahmen sind aufgrund ihrer höheren Kosten ungünstiger zu bewerten. Mechanische Unkrautbekämpfungsverfahren sind daher v. a. für Wasserschutzgebiete sowie für den Ökologischen Landbau zu empfehlen, da der Einsatz von PSM nicht oder nur sehr eingeschränkt erlaubt ist.

\subsubsection{Verzicht auf Pflanzenschutzmitteleinsatz}

Im Folgenden werden Ergebnisse zum Verzicht des PSM-Einsatzes auf Ackerflächen dargestellt und anhand einer KNA bewertet. 


\subsection{Einleitende Erwägungen}

Um sich dem Thema des Verbots der Ausbringung von PSM zu nähern, ist es zunächst nötig, die Auswirkungen auf den Pflanzenertrag zu ermitteln. Hierzu gibt es nur wenige spezifische Studien. Z. T. handelt es sich um umfangreiche, aber nicht auf die deutschen Verhältnisse ausgerichtete Arbeiten wie z. B. die von OERKE und STEINER ${ }^{512}$, die Ertragsverluste bei Nichtausbringung aus globaler Sicht betrachten. IRLA zeigt, dass der Ertrag von Getreide bei Nichtbehandlung mit Herbiziden um 3,5 dt/ha zurückgeht. ${ }^{513}$

Die kurz- und mittelfristigen Ertragseffekte bei einem Verbot des Einsatzes von PSM sind von SCHMITZ u. HARTMANN im Rahmen einer umfangreichen Expertenbefragung ermittelt worden und in der folgenden Tabelle zusammengetragen. Die Ertragsverluste errechnen sich mit den Preisen der Richtwert-Deckungsbeiträge der LWK-HANNOVER.

Tabelle 63: Kurz- und mittelfristige Ertragseffekte und -verluste bei verschiedenen Früchten bei einem Verbot von PSM ${ }^{514}$

\begin{tabular}{lccc}
\hline \multicolumn{1}{c}{ Anbaufrüchte } & $\begin{array}{c}\text { Ohne } \\
\text { Verbot } \\
\text { (dt/ha) }\end{array}$ & $\begin{array}{c}\text { Kurzfr. Wirkungen } \\
\text { (1. Jahr) }\end{array}$ & $\begin{array}{c}\text { Mittelfr. Wirkungen } \\
\text { (Rotation) }\end{array}$ \\
\hline WW (dt/ha) & 78,4 & $56,1(-28,4 \%)$ & $50,9(-35,1 \%)$ \\
Ertragsverluste (€/dt) & & 252,0 & 310,75 \\
\hline WG (dt/ha) & 72,3 & $51,6(-28,6 \%)$ & $46,0(-36,4 \%)$ \\
Ertragsverluste (€/dt) & & 189,6 & 240,9 \\
\hline WR (dt/ha) & 36,7 & $23,9(-34,9 \%)$ & $22,4(-39,0 \%)$ \\
Ertragsverluste (€/dt) & & 322,3 & 360,0 \\
\hline
\end{tabular}

Quelle: Verändert nach SCHMITZ u. HARTMANN (1993) sowie eigene Berechnungen

In der Tabelle 63 ist zu erkennen, dass kurzfristig die Erträge zwischen 28,4 bis zu 34,9 \% zurückgehen können. Mittelfristig gehen die Erträge bis zu 39 \% zurück, wobei der Winterraps in beiden Zeiträumen am stärksten betroffen ist. GUTSCHE hat den Effekt des Verbots von Herbiziden auf dieselbe Fruchtfolge angewandt. Er kommt zu kurzfristigen Ertragseinbußen von $11,7 \%$ bei WW, 9,4 \% bei WG und $6,2 \%$ bei WR. ${ }^{515}$

\footnotetext{
${ }^{512}$ Vgl. OERKE u. STEINER (1996)

${ }^{513}$ Vgl. IRLA (1991)

${ }^{514}$ Angenommene Produktpreise: Weizenpreis $=11,3 € / \mathrm{dt}$, Gerstenpreis $=9,16 € / \mathrm{dt}$ und Winterrapspreis $=$ $25,18 € / \mathrm{dt}$.

${ }^{515}$ Vgl. GUTSCHE (1995), zit. in NLÖ (2001), S. 42
} 


\subsection{Betriebswirtschaftliche Analyse der Maßnahme}

In der Übersicht 23 wird die Vorgehensweise bei der Ermittlung des KNV beim Verzicht auf PSM dargestellt.

Übersicht 23: Vorgehensweise bei der KNA für die Maßnahme des Verzichts auf PSM-Ausbringung

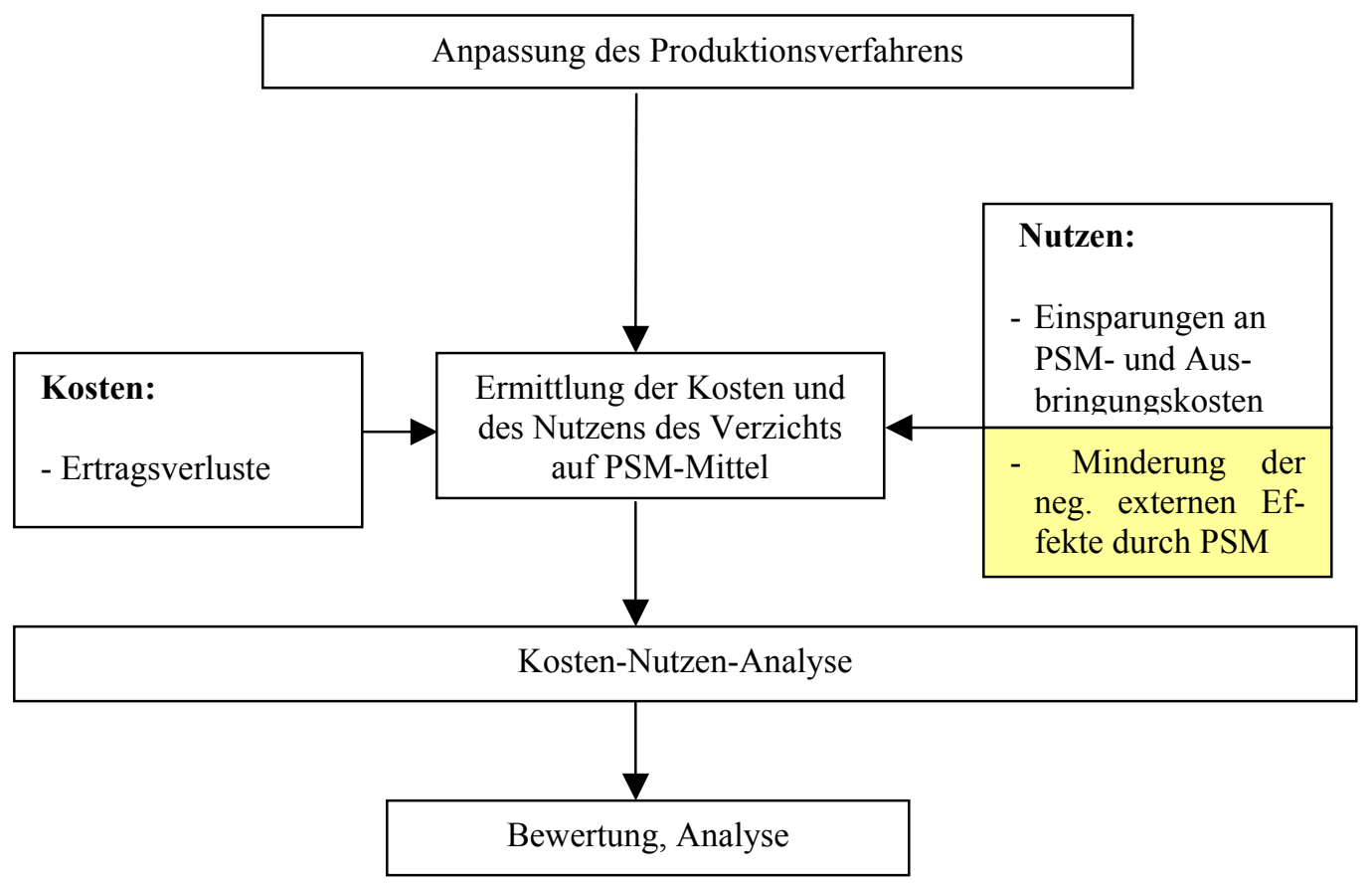

Quelle: Eigene Darstellung, Schraffierung: Positionen konnten aufgrund fehlender Daten in der Berechnung nicht berücksichtigt werden

Ausgehend von den in Tabelle 63 dargestellten Kosten eines Verbots der PSMAusbringung durch Ertragsverluste und der auf der anderen Seite anfallenden Nutzenposition durch die Einsparung an Pflanzenschutzmitteln und Ausbringungskosten, die in Tabelle 64 dargestellt sind, wird im Folgenden eine KNA durchgeführt. Dabei wird von den Aufwandmengen ausgegangen, die den Richtwert-Deckungsbeiträgen der LWK Hannover zugrunde liegen. Es wird dabei lediglich von einem 12-jährigen Zeitraum ausgegangen. Der Zinssatz beträgt 4 \%. Die Einsparungen durch das PSM-Verbot sowie die hieraus entstehende KNV sind für die Früchte WW, WG und WR in der Tabelle 64 dargestellt, die Berechnungen für WW sind der Tabelle A-75 und der Tabelle A-76 zu entnehmen. 
Tabelle 64: Einsparbare PSM-Kosten sowie das KNV bei einem Verbot von PSM für die Früchte WW, WG und $W^{516}$

\begin{tabular}{c|c|c|c}
\hline $\begin{array}{c}\text { Anbau- } \\
\text { früchte }\end{array}$ & $\begin{array}{c}\text { Einsparbare PSM- } \\
\text { Kosten } \\
\boldsymbol{\epsilon} / \mathbf{h a}\end{array}$ & KNV (\%) & $\begin{array}{c}\varnothing \text { jährl. Vorteil } \\
(\mathbf{\epsilon} / \mathbf{h a} / \mathbf{a})\end{array}$ \\
\hline WW & $166,20-270,60$ & $1,83-1,13$ & $-138,6$ bis $-34,1$ \\
WG & $100,20-190,30$ & $2,35-1,20$ & $-135,5$ bis $-40,0$ \\
WR & $132,80-253,70$ & $2,68-1,40$ & $-223,4$ bis $-102,5$ \\
\hline
\end{tabular}

Quelle: Eigene Berechnungen, Zinssatz $4 \%$

Die Ergebnisse der Tabelle 64 zeigen, dass ein PSM-Verbot erhebliche Auswirkungen auf die Wirtschaftlichkeit der einzelnen Anbaufrüchte hat. In allen Fällen reichen die Einsparmöglichkeiten nicht aus, um den Ertragsverlust auszugleichen. Lediglich bei WW und WG und einer zuvor hohen Intensität des PSM- Einsatzes besteht die Möglichkeit, ein ausgeglichenes KNV zu erzielen.

\subsection{Schlussfolgerungen}

Die Berechnungen zeigen, dass das KNV für den Verzicht auf PSM sehr ungünstig ist. Dies liegt v. a. an der fehlenden Berücksichtigung der externen Effekte. Bei den Ergebnissen konnte allerdings nur eine mittelfristige Wirkung der eingesparten PSM vorausgesetzt werden. Durch eine Kombination aus dem Verzicht des PSM- und Striegeleinsatzes konnten die KNV deutlich verbessert werden.

Einige mögliche Vermeidungsstrategie, die Gentechnologie, wurde bei den Berechnungen nicht angesprochen, da bisher keine Akzeptanz in der Bevölkerung und in der Politik vorhanden ist und mit einer konkreten und flächendeckenden Einführung der Gentechnologie kurz- und mittelfristig nicht zu rechnen ist. ${ }^{517}$ Es ist mit der Gentechnologie möglich, resistente Pflanzen $\mathrm{zu}$ entwickeln und somit die entsprechenden PSMAufwendungen zu reduzieren.

\subsubsection{Kompost- und Klärschlammeinsatz}

In den folgenden Kapiteln werden die Maßnahmen des Klärschlamm- und Komposteinsatzes auf ihre bodenschützende Wirkung untersucht. Hierbei handelt es sich nicht i. e. S.

\footnotetext{
${ }^{516}$ Unter einer ceteris-paribus-Bedingung.

${ }^{517}$ Der Entwurf eines Gentechnikgesetzes liegt derzeit vor, jedoch ist die Haftungsfrage noch nicht geklärt.
} 
um Bodenschutzmaßnahmen, da die Maßnahmen auch hohe Risiken für den Boden aufweisen können. Daher erfolgt zugleich eine kritische Betrachtung und Diskussion über die Klärschlamm- und Kompostausbringung.

\subsubsection{Klärschlammeinsatz}

Im Folgenden wird die Klärschlammausbringung behandelt. Dabei werden die Kosten und die möglichen Nutzenpositionen diskutiert. Eine KNA wird nicht vorgenommen.

\subsection{Einleitende Erwägungen}

Die Vor- und Nachteile des Klärschlammeinsatzes wurden bereits in Kapitel 3.1.3.3 diskutiert. Obwohl die Klärschlammverwertung in Deutschland intensiv diskutiert wird, fehlen im Gegensatz zur Kompostausbringung z. T. wissenschaftlich fundierte Kenntnisse außerhalb der fast ausschließlich rein naturwissenschaftlich geprägten Diskussion. Aus diesem Grund kann im nachfolgenden Kapitel nur eine verbale Kosten-NutzenBetrachtung vorgenommen werden.

\subsection{Betriebswirtschaftliche Analyse der Maßnahme}

Zunächst werden die Kosten der Klärschlammausbringung ermittelt. Die Kosten für Klärschlammproduzenten sind in der Übersicht A-3 dargestellt. Demnach betragen sie für die Verwertung inkl. Ausbringung etwa 40 - 50 €/t FM. In der Praxis werden diese Kosten i. d. R. auch vollständig vom Klärschlammproduzenten getragen. In einigen Fällen werden zusätzliche Zahlungen für die landwirtschaftliche Verwertung in Form eines Abnahmeentgelts getätigt. In der folgenden Kosten-Nutzen-Betrachtung wird allerdings vom zuerst genannten Fall ausgegangen. Da nach der AbfKlärV das Risiko des Landwirts bei der Bereitstellung von Ackerflächen finanziell durch den Klärschlammfonds abgesichert ist, können faktisch auch keine Kosten für Haftungsschäden etc. angesetzt werden.

Der Nutzen von Klärschlamm wird in erster Linie durch eventuelle Zahlungen der Klärschlammproduzenten sowie dem Nährstoffwert bestimmt. Daher zeigt die Tabelle 65 die mit aktuellen Nährstoffpreisen bewerteten Nährstoffgehalte einer typischen Ausbringungsmenge von $5 \mathrm{t} \mathrm{TM/ha.}$ 
Tabelle 65: Nährstoffwert einer Klärschlammgabe (5 t TM/ha)

\begin{tabular}{cccc}
\hline $\begin{array}{c}\text { Nähr- } \\
\text { stoff }\end{array}$ & $\begin{array}{c}\text { Nährstoff- u. Aus- } \\
\text { bringungskosten } \\
(\boldsymbol{\epsilon} / \mathbf{k g})\end{array}$ & $\begin{array}{c}\text { Nährstoffmengen* } \\
(\mathbf{k g} / \mathbf{h a})\end{array}$ & $\begin{array}{c}\text { Monetärer Nutzen } \\
(\boldsymbol{\epsilon} / \mathbf{h a})\end{array}$ \\
\hline $\mathrm{N}$ & 0,66 & 41 & 27,1 \\
$\mathrm{P}$ & 0,52 & 75 & 39,0 \\
$\mathrm{Mg}$ & 0,7 & 35 & 24,5 \\
$\mathrm{Ca}$ & 0,04 & 460 & 18,4 \\
\hline Gesamt & & & $\mathbf{1 0 9 , 0}$ \\
\hline
\end{tabular}

* Mineraldüngeräquivalent

Quelle: N.N. (1998), S. 53, FID (2002) und eigene Berechnungen

Wie die Tabelle 65 zeigt, liegt der Nährstoffwert einer Klärschlammgabe, unabhängig von der Pflanzenverfügbarkeit, bei ca. $109 € /$ ha. Dieser Wert muss zu den eventuell gezahlten Prämien für die Bereitstellung der Ackerfläche hinzugerechnet werden und ergibt den Gesamtnutzen der Maßnahme.

Der volkswirtschaftliche Nutzen besteht in der Differenz zwischen den niedrigeren Kosten der landwirtschaftlichen Klärschlammausbringung und den entsprechenden Alternativen, wie z. B. der thermischen Verwertung oder Deponierung. ${ }^{518}$

\subsection{Schlussfolgerungen}

Abschließend ist festzustellen, dass aus isoliert betriebswirtschaftlicher Sicht betrachtet die Klärschlammverwertung zu empfehlen ist, da sie direkt zum Einkommen des Landwirts beiträgt und mögliche Risiken durch den Klärschlammfonds abgesichert sind. Aus ökologischer Sicht ist der Nutzen der ldw. Klärschlammverwertung jedoch sehr unsicher und in der landwirtschaftlichen Praxis gibt es viele Vorbehalte gegenüber der Klärschlammausbringung. Bezüglich des Klärschlammfonds gibt es noch zu wenige praktische Anwendungsfälle, um die Wirksamkeit dieses Instrument zur Risikoabsicherung zu bewerten. Es muss weiterhin gewährleistet sein, dass die Grenzwerte der AbfKlärV nachhaltig eingehalten werden. Hierzu ist es nötig, nur hochwertigen und gering belasteten Klärschlamm auf ldw. Nutzflächen auszubringen. Gütesiegel für Klärschlamm können dazu beitragen, dass die Akzeptanz dieser Verwertungsmethode sowohl bei den Landwirten als auch bei der Bevölkerung ansteigt. Die Frage, ob es überhaupt sinnvoll ist, Klär-

\footnotetext{
${ }^{518}$ Vgl. hierzu u. a. QUICKER u. FAULSTICH (2002)
} 
schlamm auf Ackerflächen auszubringen, kann an dieser Stelle nicht abschließend geklärt werden, da es sich in erster Linie um ein volkswirtschaftliches Problem handelt. Aus einzelbetrieblicher Sicht ist der Einsatz von Klärschlämmen auf Ackerflächen jedoch sinnvoll.

\subsubsection{Komposteinsatz}

Im Folgenden wird der Komposteinsatz als Bodenschutzmaßnahme betrachtet und anhand einer KWA und einer KNA auf seine Effizienz hin untersucht.

\subsection{Einleitende Erwägungen}

Eine Diskussion um die Vor- und Nachteile des Komposteinsatzes wurde bereits in Kapitel 3.1.3.4 durchgeführt. Im Gegensatz zum Klärschlammeinsatz zeichnet sich der Komposteinsatz durch eine, wenn auch geringe, erosionsmindernde Wirkung aus. Daher kann in diesem Fall von einer Bodenschutzmaßnahme gesprochen werden. Im Gegensatz zur Klärschlammausbringung liegen für die Kompostausbringung mehrere Forschungsarbeiten vor, die den Nutzen dieser Maßnahme aufzeigen und sich als Grundlage einer KNA eignen.

\subsection{Betriebswirtschaftliche Analyse der Maßnahme}

Die Vorgehensweise für die KNA ist der Übersicht $24 \mathrm{zu}$ entnehmen. 


\section{Übersicht 24: Vorgehensweise bei der KNA für die Kompostausbringung}

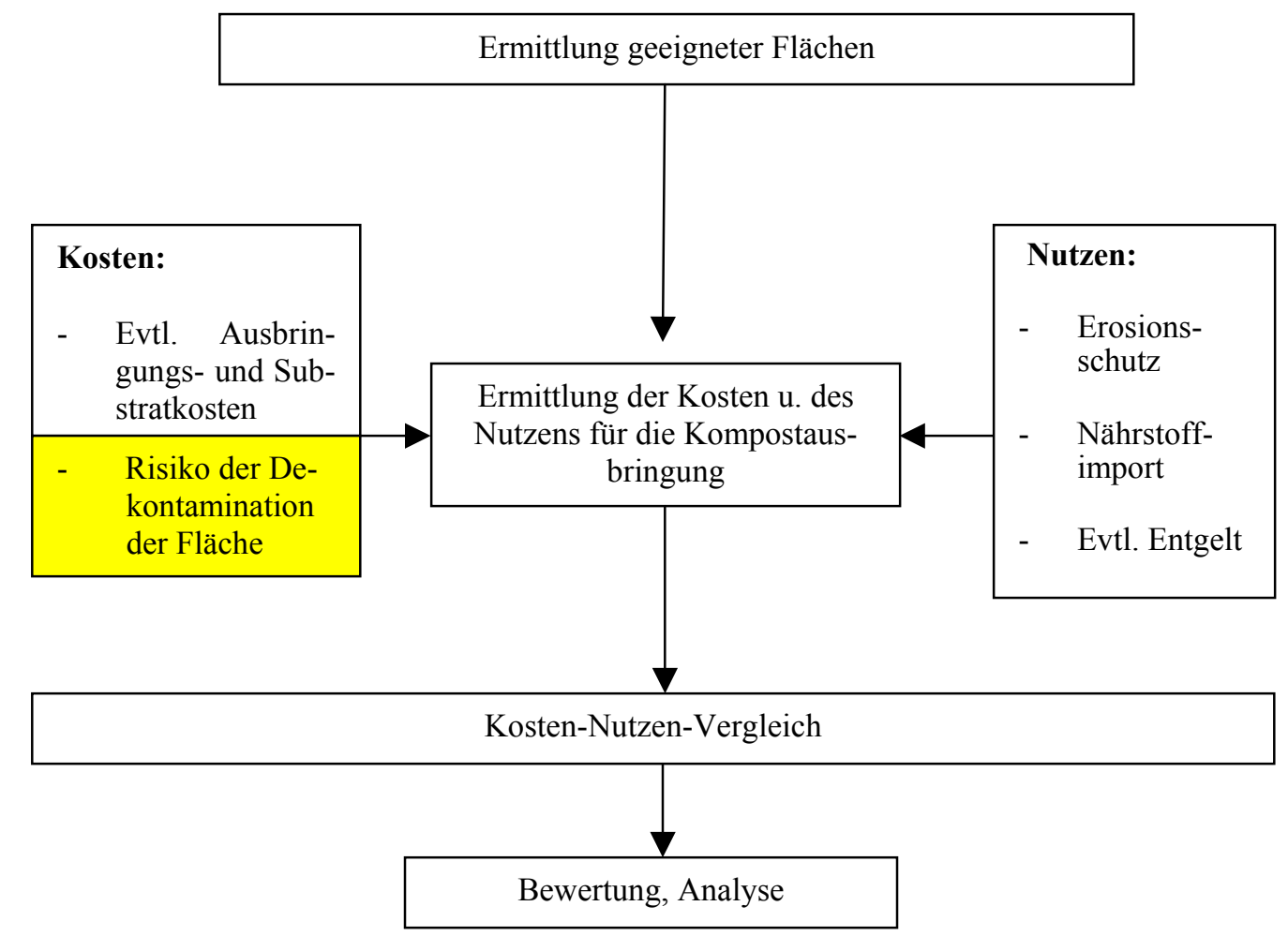

Quelle: Eigene Darstellung, Schraffierung: Positionen konnten aufgrund fehlender Daten in der Berechnung nicht berücksichtigt werden

Die Kosten der Kompostausbringung hängen von dem vom Landwirt gewählten Verfahren ab. Die Ausbringung kann zum einen durch eine komplette Fremdmechanisierung erfolgen, zum anderen kann der Landwirt den Kompost mit seinen eigenen Maschinen transportieren und ausbringen. Innerhalb dieser beiden Modelle gibt es eine Vielzahl von Mischformen. Im Folgenden sollen jedoch nur die gebräuchlichsten Formen der Kompostausbringung betrachtet werden. Da der größte Teil der Kosten beim Transport des Komposts anfällt, wird dieser Teil des Verfahrens in der Regel von Spediteuren durchgeführt und werden auch die Kosten von diesem getragen.

Als Ausbringungstechnik bieten sich Universalstreuer (Tellerbreitstreuer) an, da sich Biokompost hinsichtlich der Schüttdichte, der Korngröße sowie der Streubarkeit von Klärschlamm und Stallmist unterscheidet. Grundsätzlich ist zwar eine Ausbringung mit einem Stallmiststreuer möglich, jedoch wird mit diesem keine ausreichende Streubreite erreicht und ist daher im Rahmen einer umweltgerechten und nachhaltigen Landbewirtschaftung nicht zu empfehlen. Deshalb wird im Folgenden von einer überbetrieblichen Ausbringung ausgegangen. Es werden zwei Verfahrensvarianten unterschieden. Bei ge- 
ringen Transportentfernungen bieten sich einphasige Verfahren an. Mit zunehmender Entfernung sind jedoch mehrphasige Verfahren vorteilhafter.

\section{Abbildung 31: Maschinen- und Arbeitskosten bei der Ausbringung von Kompost (Hof-Feld)}

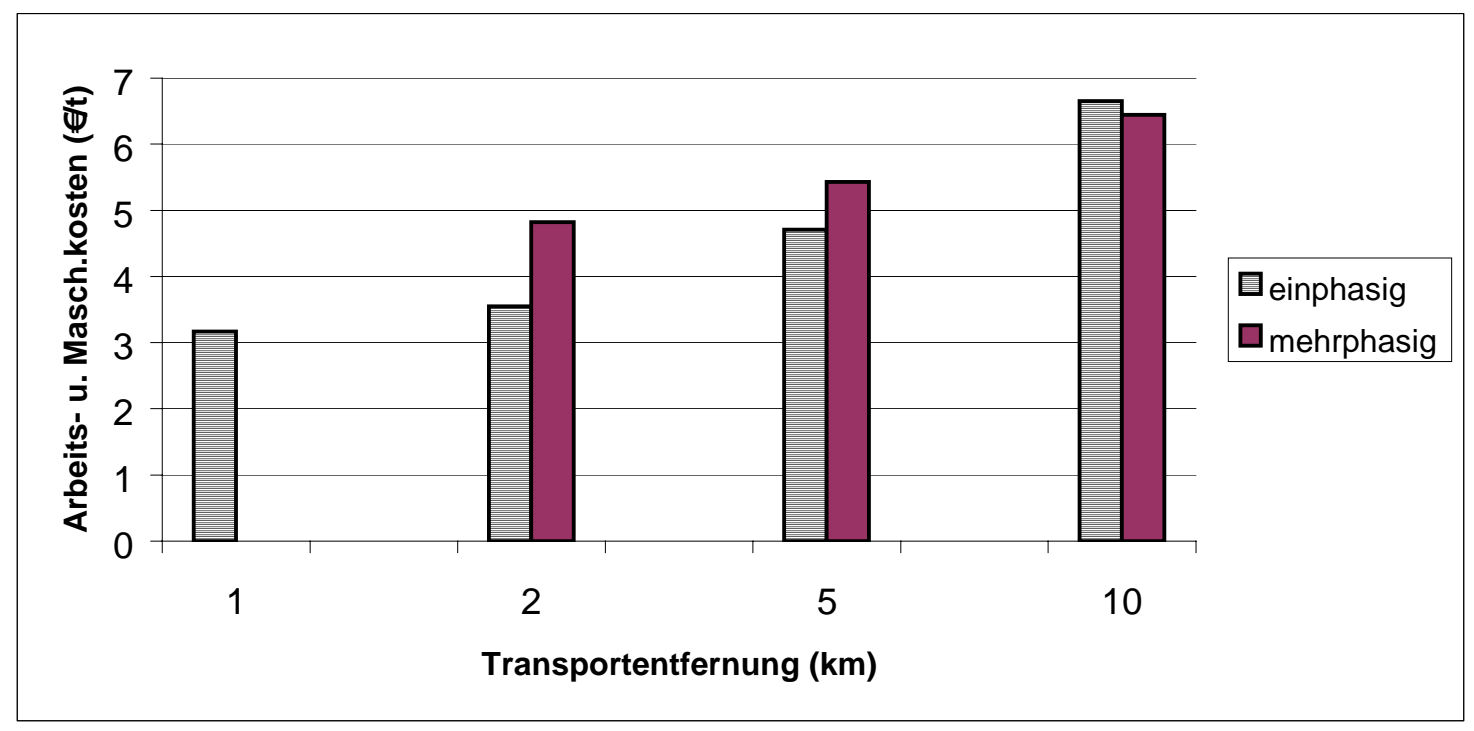

Quelle: KTBL (2002), entnommen aus AID (2003), S. 29

Dies hängt jedoch stark von der Schlagkraft des Komposttransports ab. Im obigen Beispiel sind zwei Anhänger mit je 12 t Nutzlast unterstellt. Abweichend dazu ermitteln KUHLMANN et al. ${ }^{519}$ bei einphasigen Verfahren bei der einzelbetrieblichen Kompostausbringung Vollkosten in Höhe von 6,2 €/t bzw. 5,6 €/t (bei einer auszubringenden Kompostmenge von $3000 \mathrm{t}$ bzw. $6000 \mathrm{t}$ und einer Transportentfernung von $5 \mathrm{~km}$ ). Die überbetriebliche Kompostausbringung führt bei 6000 t auszubringendem Kompost zu Vollkosten in Höhe von $4,8 € /$ t.

In der landwirtschaftlichen Praxis sind die Kosten des Komposts i. d. R. Gegenstand der Verhandlungen zwischen Landwirt und Komposthersteller. Dabei kommt es vor, dass der Komposthersteller sowohl die Kosten für den Kompost als auch für die Anlieferung und Ausbringung übernimmt. In einigen Regionen wird für den Kompost ein Preis von 1,5€/t TM bezahlt, die Anlieferungskosten übernimmt jedoch i. d. R. der Komposthersteller. ${ }^{520}$

\footnotetext{
${ }^{519}$ Vgl. KuHLMANN et al. (1995), S. 8 f.

${ }^{520}$ Vgl. mündliche Mitteilung FRAU SCHMIDT
} 
Vor diesem Hintergrund ist davon auszugehen, dass Kosten in Höhe von 0-7,7 €/t TM entstehen.

Der Nutzen der Kompostausbringung, d. h. der direkte Einfluss der Kompostdüngung, ist in einem Verbund-Forschungsprojekt der DEUTSCHEN BUNDESSTIFTUNG UMWELT untersucht worden. Dabei wurde über einem Zeitraum von sieben Jahren die Bodenerosion durch Wasser auf einer Fläche mit und ohne Kompostdüngung (10 t/ha/a) gemessen und miteinander verglichen. Die Ergebnisse sind der nachfolgenden Tabelle zu entnehmen.

Tabelle 66: Veränderung der Bodenerosion durch Wasser durch Kompostausbringung bei einer Ausbringungsmenge von 10 t/ha/a auf verschiedenen Standorten

\begin{tabular}{|c|c|c|c|c|c|c|c|c|}
\hline Jahr & 1 & 2 & 3 & 4 & 5 & 6 & 7 & $\varnothing$ \\
\hline Veränderung in \% & $-0,6$ & $-1,2$ & $-1,8$ & $-2,4$ & $-3,1$ & $-3,7$ & $-4,3$ & $-2,4$ \\
\hline Standort $A^{*}$ in $t$ & $-0,03$ & $-0,05$ & $-0,08$ & $-0,11$ & $-0,14$ & $-0,17$ & $-0,20$ & $-0,11$ \\
\hline $\begin{array}{l}\text { Monet. Vorteil } \\
(€ / \text { ha) }\end{array}$ & 0,14 & 0,24 & 0,38 & 0,52 & 0,67 & 0,82 & 0,96 & $\mathbf{0 , 5 3}$ \\
\hline Standort $B^{* *}$ in $t$ & $-0,05$ & $-0,10$ & $-0,16$ & $-0,21$ & $-0,27$ & $-0,32$ & $-0,37$ & $-0,21$ \\
\hline $\begin{array}{l}\text { Monet. Vorteil } \\
(€ / \text { ha) }\end{array}$ & 0,24 & 0,48 & 0,77 & 1,01 & 1,30 & 1,54 & 1,78 & 1,02 \\
\hline
\end{tabular}

* Standort A mit einem durchschnittlichen Bodenabtrag von 4,6 t/ha.

${ }^{* *}$ Standort B mit einem durchschnittlichen Bodenabtrag von $8,7 \mathrm{t} / \mathrm{ha}$.

Quelle: SCHREIBER (2002) und eigene Berechnungen

Es ist festzustellen, dass innerhalb der siebenjährigen Projektlaufzeit die Bodenerosion durch Wasser um durchschnittlich 2,4 \% pro Jahr gemindert werden konnte. Dies hängt v. a. mit der Wirkung der aufgebrachten organischen Substanz zusammen, die strukturstabilisierend wirkt. Bezogen auf einen Standort mit einem durchschnittlichen jährlichen Bodenabtrag von 4,6 t/ha bedeutet dies eine durchschnittliche Reduktion um 0,11 t/ha/a. Nach den in Kapitel 6.3.1.1.3 errechneten Werten ${ }^{522}$ für den nicht erodierten Boden ergibt sich ein durchschnittlicher Nutzen von $0,53 € /$ ha/a. Für den zweiten Standort mit einem durchschnittlichen Bodenabtrag von 8,7 t/ha ergibt sich eine höhere Reduktion des Bodenabtrages von $0,21 \mathrm{t} / \mathrm{ha}$ und einen höheren Nutzen, der mit 1,02 €/ha/a aber trotzdem als marginal anzusehen ist.

\footnotetext{
${ }^{521}$ An dieser Stelle wird analog zu den bisherigen Berechnungen von einem Nutzen von 4,8 €/t ausgegangen.

${ }_{522}$ An dieser Stelle wird von einem Nutzen von 5,6 €/ha ausgegangen.
} 
Bezüglich der Minderung der Winderosion liegen Versuchsergebnisse von HARTMANN ${ }^{523}$ vor. In den Versuchen wurden Windkanalexperimente zur Ermittlung der erosionsvermindernden Wirkung verschiedener Komposte mit Podsol-Bodenmaterial, welches typisch für die Wildeshauser Geest ist, im Vergleich zu ausschließlich mit Mineraldünger gedüngtem Boden durchgeführt. Nach diesen Ergebnissen ist bereits nach zweimaliger Kompostausbringung eine positive Wirkung durch die Aggregatstabilisierung des Bodens erkennbar. Die Reduzierung des erodierten Bodenmaterials beträgt gegenüber der Mineraldüngervariante etwa $39 \%$. Aus dem oben Dargestellten ergibt sich, dass Biokomposte auf Sandböden mit geringen Ernterückständen einen hohen Nutzen stiften.

Da die Reduzierung der Bodenerosion nicht das Ziel einer Kompostdüngung ist, wird an dieser Stelle keine entsprechende KWA für die Kompostanwendung durchgeführt.

Weiterhin ist bei der Kompostausbringung zu beachten, dass der Kompost durch den Gehalt an Nährstoffen einen Nährstoffwert aufweist. Dieser ist in Tabelle 67 für die folgenden Berechnungen ohne N dargestellt, um eine doppelte Berücksichtigung zu vermeiden. Um den pflanzenverfügbaren Nährstoffwert des eingebrachten Komposts quantifizieren zu können, wird der Marktwert einer wirkungsgleichen Menge eines zuzukaufenden Substituts angesetzt. Dies geschieht mittels durchschnittlicher Handelsdüngerpreise auf der Basis von Reinnährstoffen der Landwirtschafskammer Hannover ${ }^{524}$.

Tabelle 67: Nährstoffwert einer Kompostgabe (10 t TM/ha/a) ohne Berücksichtigung des Stickstoffs

\begin{tabular}{cccc}
\hline $\begin{array}{c}\text { Nähr- } \\
\text { stoff }\end{array}$ & $\begin{array}{c}\text { Nährstoff- und Aus- } \\
\text { bringungskosten } \\
(\boldsymbol{\epsilon} / \mathbf{k g})\end{array}$ & Nährstoffmengen & Monetärer Nutzen \\
\hline $\mathrm{P}$ & 0,52 & $\mathbf{( k g / \mathbf { h a } )}$ & $(\boldsymbol{\epsilon} / \mathbf{h a})$ \\
$\mathrm{K}$ & 0,24 & 110 & 36,4 \\
$\mathrm{Mg}$ & 0,7 & 70 & 26,4 \\
$\mathrm{Ca}$ & 0,04 & 400 & 49,0 \\
\hline Gesamt & & & 16,0 \\
\hline
\end{tabular}

Quelle:. AID (2003), S. 53, FID (2002) und eigene Berechnungen

\footnotetext{
${ }^{523}$ Vgl. HARTMANN (2003), S. 62 ff.

${ }^{524}$ Vgl. Tabelle A-19
} 
Der Berechnung liegt zu Grunde, dass die Mengen an Kalium und Phosphor im Kompost in der gesamten Höhe angerechnet sowie durch zugekaufte Mineraldünger vollständig ersetzt werden können.

Aus den Nährstoffgehalten der Tabelle 67 ergibt sich ein Wert von 12,78 €/t TM. Hierbei ist zu berücksichtigen, dass der Wert des Stickstoffs gesondert betrachtet wird. Dies ist darin begründet, dass der Stickstoff des Komposts nicht im Jahr der Ausbringung pflanzenverfügbar ist. Diesbezügliche pflanzenbauliche Versuche weisen erhebliche Streuungen in den Ergebnissen auf, so dass an dieser Stelle nur mit Modellannahmen nach DöHLER $^{525}$, der eine kontinuierliche Nachlieferung von $3 \%$ des mit Bioabfallkompost ausgebrachten Stickstoffs voraussetzt, gearbeitet werden kann. Wie bereits in Kapitel 3.1.3.4 dargestellt wurde, verfügen 30 t TM über $390 \mathrm{~kg} \mathrm{~N}$. Die hieraus resultierenden Nutzenbeträge, bei einer Freisetzung von 3 \%/a sind in der Tabelle A-20 zu entnehmen. Dabei wird ein $\mathrm{kg} \mathrm{N}$ mit $0,50 € / \mathrm{kg}$ bewertet. Diese Werte werden in der unten durchgeführten KNA berücksichtigt.

\section{Zusammenfassende Kosten-Nutzen-Analyse}

Die KNA wird im Folgenden mit den oben gewonnenen Daten durchgeführt. Dabei werden die Kosten zwischen 4,8 €/t TM (Variante 1) und 6,2 €/t TM (Variante 2) variiert. ${ }^{526}$ Der Landwirt übernimmt somit selbst die Kosten der Ausbringung. Als Nutzen ergibt sich zunächst die Verhinderung der Bodenerosion. Hierbei werden die Werte in Tabelle 66 hinzugezogen. Da nur eine Ausbringung (30 t TM) vorausgesetzt wird, fallen diese Nutzenpositionen dementsprechend nur in den ersten sieben Jahren an. Der wesentliche Nutzen der Kompostausbringung setzt sich aus den Nährstoffwerten (12,78 €/t TM) und v. a. aus der Stickstofffreisetzung der nachfolgenden Jahre zusammen. Der Stickstoffwert wird der Tabelle A-20 entnommen. Der Betrachtungszeitraum beträgt 25 Jahre und es wird ein Zinssatz von $4 \%$ unterstellt. Die Berechnungen für den Standort mit einem Bodenabtrag von 4,6 t/ha/a sind in der Tabelle A-77 und in der Tabelle A-78 dargestellt.

\footnotetext{
${ }^{525}$ Vgl. DÖHLER (1994), S. 22 ff.

${ }^{526}$ Vgl. hierzu Abbildung 31 und die in diesem Zusammenhang dargestellten Werte.
} 
Tabelle 68: KNV der Ausbringung von Kompost bei unterschiedlichen Ausbringungskosten

\begin{tabular}{|lcc|}
\hline Standort & $\mathbf{4 , 6}$ t/ha/a & $\mathbf{8 , 6}$ t/ha/a \\
\hline KNV in \% Var. 1 & 1,27 & 1,03 \\
$\varnothing$ jährl. Vorteil (€/ha/a) & $-1,9$ & $-0,2$ \\
\hline KNV in \% Var. 2 & 1,64 & 1,60 \\
$\varnothing$ jährl. Vorteil (€/ha/a) & $-4,5$ & $-4,3$ \\
\hline
\end{tabular}

Quelle: Eigene Berechnungen, Zinssatz 4 \%

In der Tabelle 68 ist zu erkennen, dass bei den angenommenen Ausbringungskosten von 4,8 €/t TM und 6,2 €/t TM keine Variante wirtschaftlich ist. Lediglich die Variante 1 bei einem Standort mit einem Bodenabtrag von 8,6 t/ha/a kommt annähernd in einen rentablen Bereich.

\subsection{Schlussfolgerungen}

Zusammenfassend ist darzustellen, dass die Kompostanwendung Vorteile für den landwirtschaftlichen Betrieb aufweisen kann. Diese hängen von den Kosten ab, die der Landwirt für die Ausbringung des Komposts tragen muss. Der erosionshemmende Faktor des Komposts ist gegenüber den monetären Vorteilen der Nährstoffe gering einzustufen. Daher ist diese Maßnahme nicht primär als Erosionsschutzmaßnahme zu empfehlen. Weiterhin ist zu beachten, dass bei der Kompostausbringung trotz gesetzlicher Vorschriften und Kontrollen und geringer Ausnutzungsgrade der Schwermetallgrenzwerte ein Restrisiko für den Boden und die Umwelt nicht ganz ausgeschlossen werden kann, obwohl dieses kontrollierbar scheint. Ein direkter NK-Vergleich zwischen den beiden Maßnahmen ist aufgrund des geringen Wissens über den Nutzen beim Klärschlamm nicht möglich. Da die Ausbringung von Klärschlamm definitiv keine vergleichbar hohen Nutzenkomponenten aufweist, ist davon auszugehen, dass das KNV deutlich schlechter ausfallen würde.

\subsubsection{Zusammenfassung und Diskussion der Ergebnisse}

In den folgenden Abschnitten werden die Ergebnisse der vorigen Kapitel zusammenfassend dargestellt und miteinander verglichen. Dabei erfolgt wiederum die Einteilung der Maßnahmen in Maßnahmen des physikalischen und stofflichen Bodenschutzes. 


\subsubsection{Zusammenfassende Darstellung der Ergebnisse der Maßnahmen des physikalischen Bodenschutzes gegen Erosion}

Zunächst werden die KW der Maßnahmen des physikalischen Bodenschutzes zum Schutz gegen Bodenerosion zusammengefasst. Dies geschieht anhand von Abbildungen, welche die KW und KNV grafisch darstellen. Eine direkte Vergleichbarkeit ist allerdings aufgrund der unterschiedlichen Annahmen in den einzelnen Berechnungen nur bedingt gegeben. Die im Folgenden getroffenen Vergleiche dürfen daher nur als grobe Abschätzung der Unterschiede der Ergebnisse interpretiert werden.

Die Abbildung 32 zeigt, dass sowohl die Anlage von Grünstreifen als auch die Tiefenlockerung die günstigsten KW aufweisen. Dafür ist die Spannbreite der Ergebnisse allerdings im Verhältnis zu derjenigen der konservierenden Bodenbearbeitung sehr hoch und die Ergebnisse sind unter einigen Vorbehalten zu sehen, die den entsprechenden Kapiteln zu entnehmen sind.

Der Zwischenfruchtanbau weist ebenfalls günstige KW auf, dafür verfügt diese Maßnahme über eine hohe Spannbreite, die in den unterschiedlichen Aussaatkosten begründet ist. Die konservierende Bodenbearbeitung weist zwar nicht die günstigsten $\mathrm{KW}$ auf, dafür aber geringe Spannbreiten. Die Direktsaat erzielt ein etwas besseres Ergebnis als die Mulchsaat. Die Spannbreiten der konservierenden Bodenbearbeitung hängen im Wesentlichen von den Bodenabträgen des Standorts ab. Der Verzicht des Anbaus an Hanglagen weist eine gute KW auf, um diese Maßnahme abschließend zu beurteilen ist allerdings das schlechte KNV zu berücksichtigen. Die Bewirtschaftung quer zum Hang weist die größte Spannweite aus und kann die schlechteste KW besitzen. Dabei ist allerdings zu beachten, dass es sich eher um eine Kombinationsmaßnahme handelt, so dass sich die KW in Kombination bspw. mit Grünstreifen wesentlich verbessern kann. 


\section{Abbildung 32: Zusammenfassung der Ergebnisse der KWA von Maßnahmen zum}

\section{Schutz vor Bodenerosion}

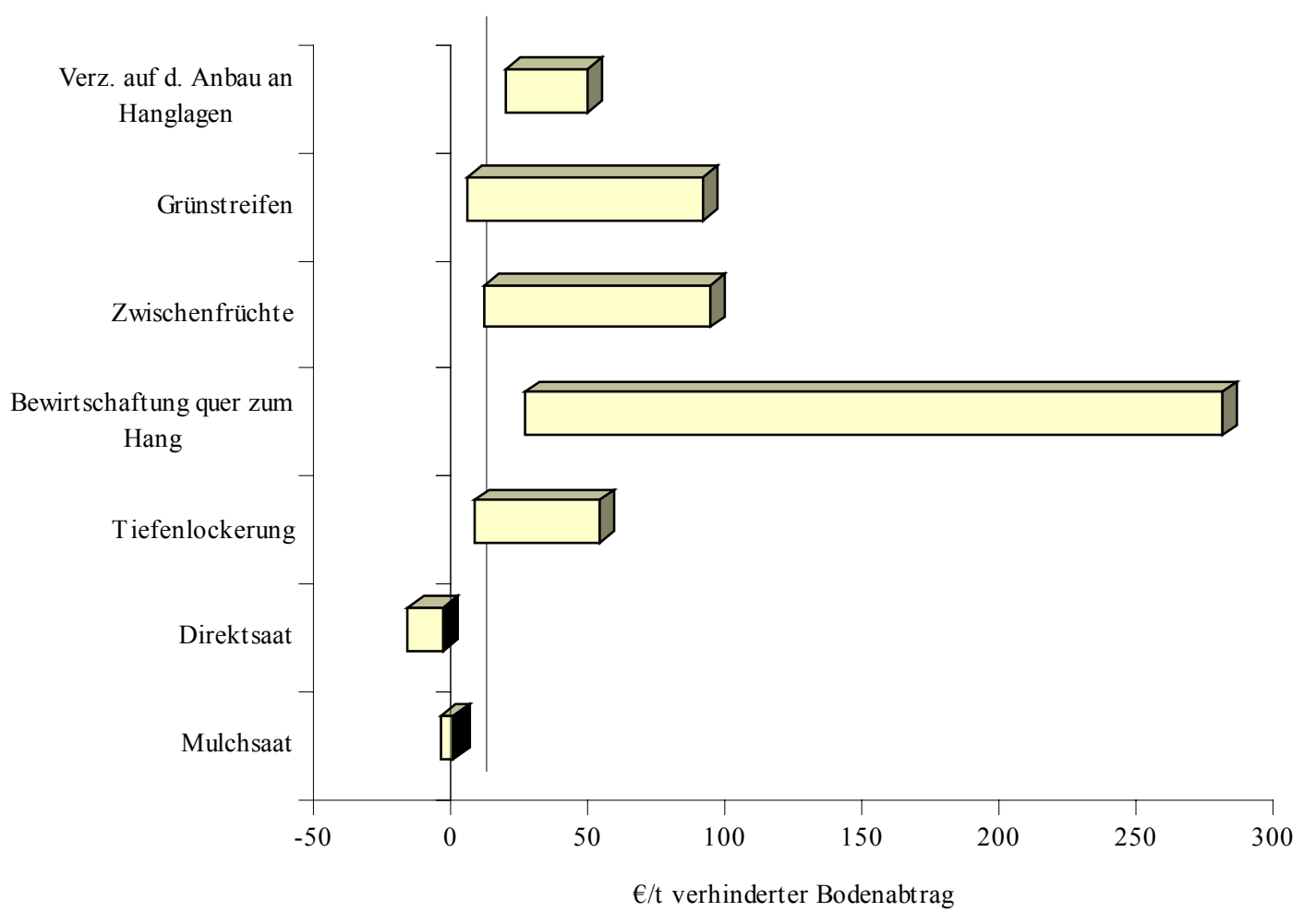

Quelle: Eigene Darstellung

In der Abbildung 33 werden die KW der Maßnahmen des Schutzes gegen Bodenerosion aufgezeigt. Unter Berücksichtigung der in dieser Arbeit gewählten Voraussetzungen und Einschränkungen bei den einzelnen Maßnahmen ist zu sehen, dass nicht alle Maßnahmen potenziell gute KW erreichen. Für die Maßnahmen der konservierenden Bodenbearbeitung ist eine negative KW zu erkennen die als positiv einzuordnen ist. Die Bewirtschaftung quer zum Hang weist eine sehr hohe Schwankungsbreite auf. 


\section{Abbildung 33: Zusammenfassung der Ergebnisse der KNA von Maßnahmen zum}

\section{Schutz vor Bodenerosion}

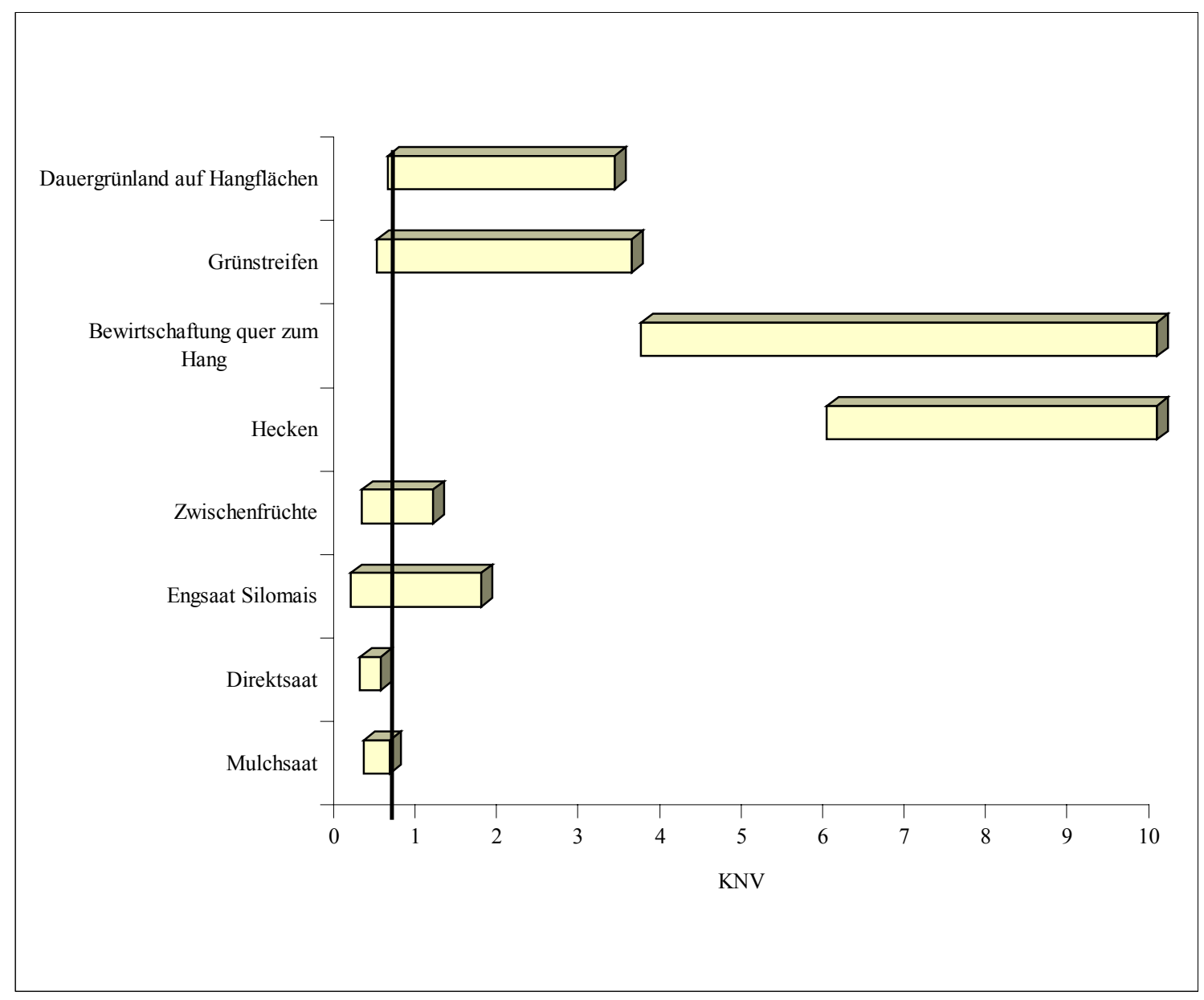

Quelle: Eigene Darstellung

Unter der Berücksichtigung der Schwankungsbreite weist die konservierende Bodenbearbeitung und der Zwischenfruchtanbau das günstigste KNV auf. Dann folgt die Engreihensaat von Silomais, welche bezogen auf das KNV eine deutlich höhere Schwankungsbreite hat. Die Umwandlung von Hangflächen in Dauergrünland, die Anlage von Hecken sowie die Anlage von Grünstreifen sowie die Bewirtschaftung quer zum Hang und Anlage von Hecken weisen mit hoher Wahrscheinlichkeit keine rentablen KNV auf. Aufgrund der besseren Übersichtlichkeit sind die Werte für die KNV auf der Abszisse nur bis $10 \%$ eingetragen. Die Werte für die Hecken und Bewirtschaftung quer zum Hang können jedoch ein Vielfaches betragen und daher an dieser Stelle nur bedingt angezeigt werden. 


\subsubsection{Zusammenfassende Darstellung der Ergebnisse der Maßnahmen des} physikalischen Bodenschutzes zum Schutz gegen Bodenschadverdichtung

In diesem Abschnitt werden die physikalischen Maßnahmen zum Schutz des Bodens anhand der errechneten KW und KNV miteinander verglichen. Die folgende Abbildung zeigt die KW der verschiedenen Maßnahmen. Die KW werden auf die Bereiche des Ober- und Unterbodens bezogen.

\section{Abbildung 34: KW bei verschiedenen Maßnahmen zur Reduzierung des Boden- drucks}

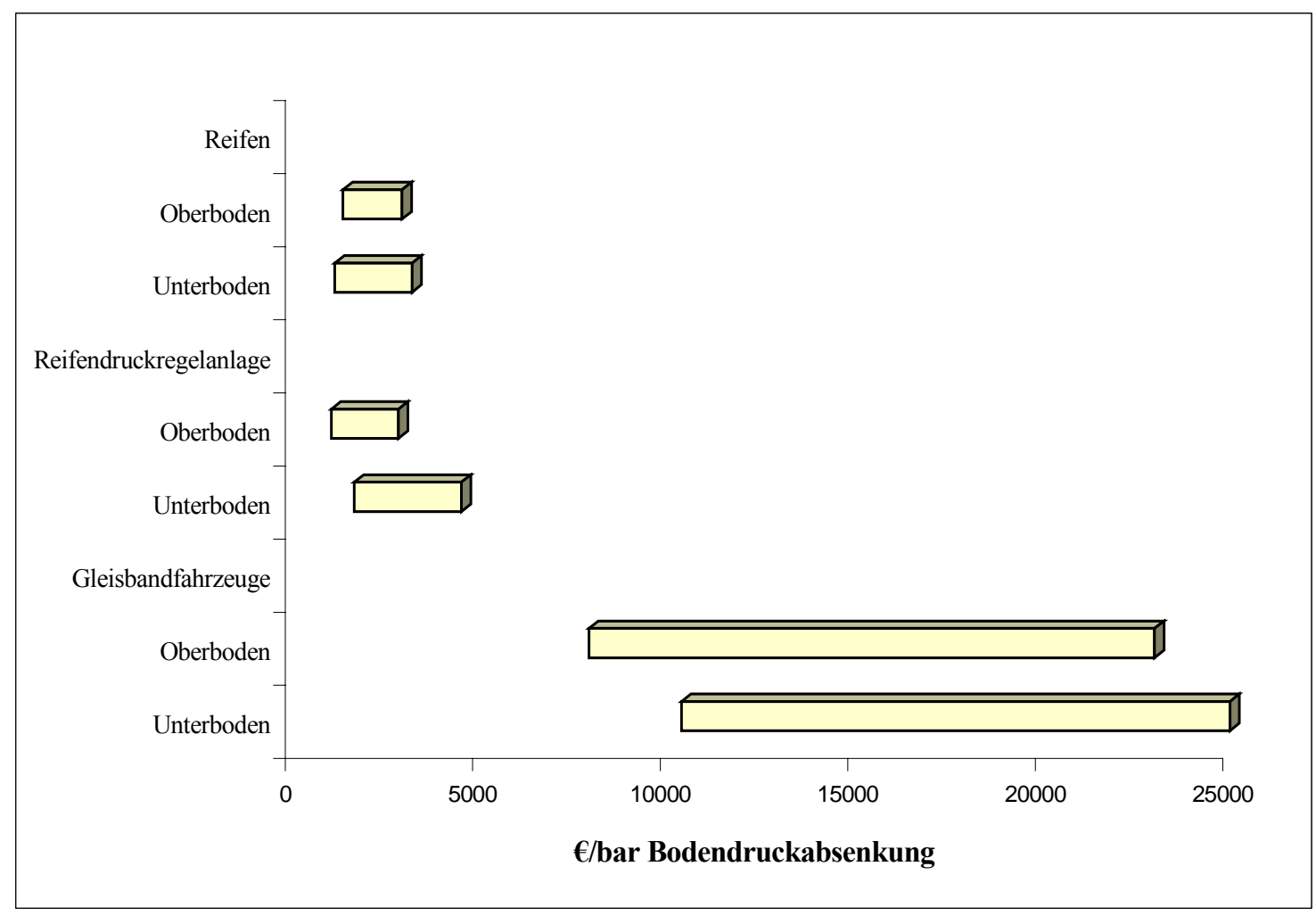

Quelle: Eigene Darstellung

Es ist zu erkennen, dass die KW für den Oberboden tendenziell besser ist als für den Unterboden. Dies liegt an der abnehmenden Wirksamkeit im Unterboden. Die Reifendruckregelanlage weist im Oberboden die beste KW auf. Danach folgt die Verwendung von bodenschonenden Reifen. Der Einsatz von Gleisbändern weist tendenziell schlechtere KW auf, was mit den jährlichen Gesamtkosten begründet ist. Hierbei ist zu berücksichtigen, dass aufgrund der Übersichtlichkeit die Skala nicht bis zu den nötigen darzustellen- 
den Kosten von bis zu 46.033,15 €/bar Bodendruckabsenkung im Unterboden aufgezeigt werden konnte.

In der Abbildung 35 werden die KNV der Maßnahmen des Schutzes gegen Bodenschadverdichtungen aufgezeigt. Es konnten allerdings nicht alle Maßnahmen berechnet werden. Unter Berücksichtigung der in dieser Arbeit gewählten Voraussetzungen und Einschränkungen bei den einzelnen Maßnahmen ist zu erkennen, dass alle Maßnahmen potenziell ein günstiges KNV erreichen können. Für die Stilllegung einer Minderertragsfläche gilt dies in jedem Fall.

\section{Abbildung 35: Zusammenfassung der Ergebnisse der KNA von Maßnahmen zum}

\section{Schutz vor Bodenschadverdichtungen}

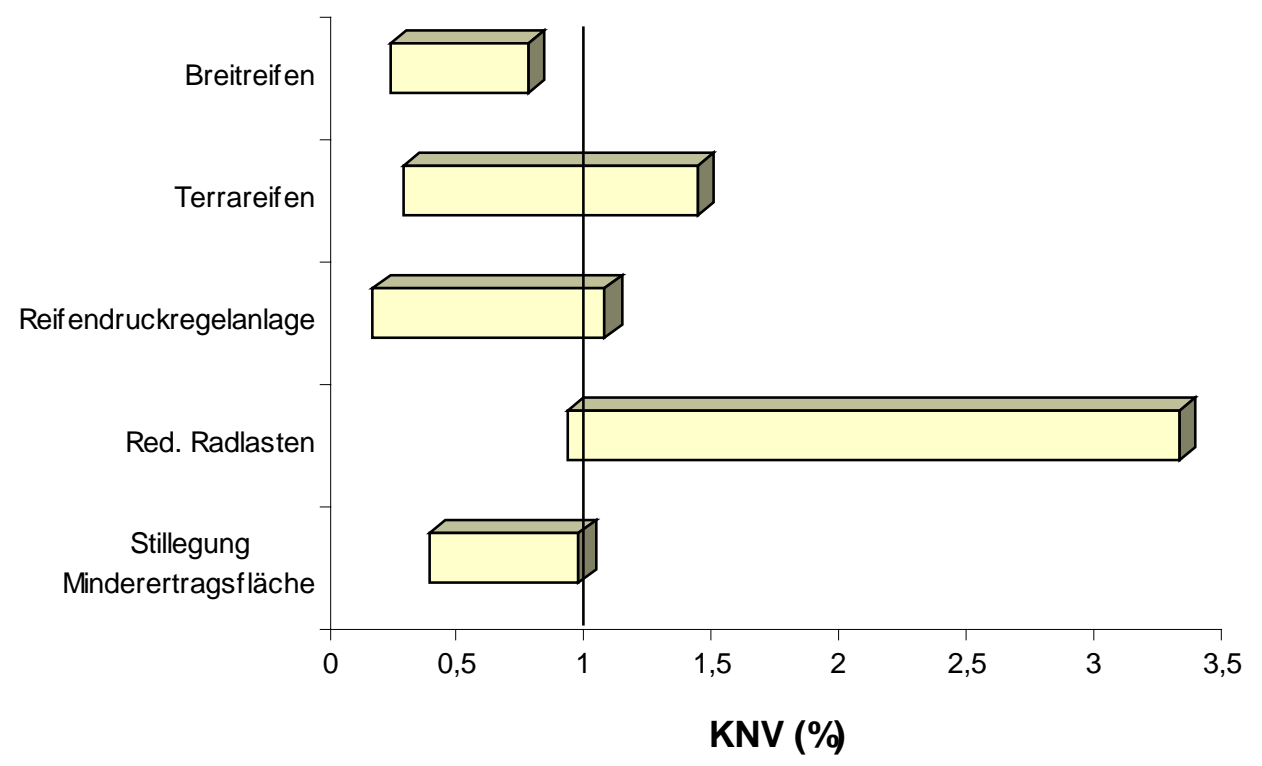

Quelle: Eigene Darstellung

Der Einsatz von Reifendruckregelanlagen sowie der Einsatz von bodenschonender Bereifung zeichnet sich durch die besten KNV aus. Dabei ist das Ergebnis für den Einsatz von Breitreifen günstiger als das für den Einsatz der Terrareifen, was mit den wesentlich höheren Anschaffungskosten für Terrareifen begründet werden kann. Die Reduzierung der Radlasten hat den größten Spielraum von allen Maßnahmen und weist die schlechtesten KNV aus. 


\subsubsection{Zusammenfassende Darstellung der Ergebnisse der Maßnahmen des stofflichen Bodenschutzes}

In diesem Kapitel werden die Ergebnisse der Berechnungen innerhalb des stofflichen Bodenschutzes zusammengefasst. Zunächst werden die verschiedenen Maßnahmen zur Reduzierung von PSM-Einträgen in den Naturhaushalt durch die Landwirtschaft anhand des KNV verglichen.

\section{Abbildung 36: KNV der Maßnahmen zur Reduzierung von PSM-Austrägen in der Landwirtschaft}

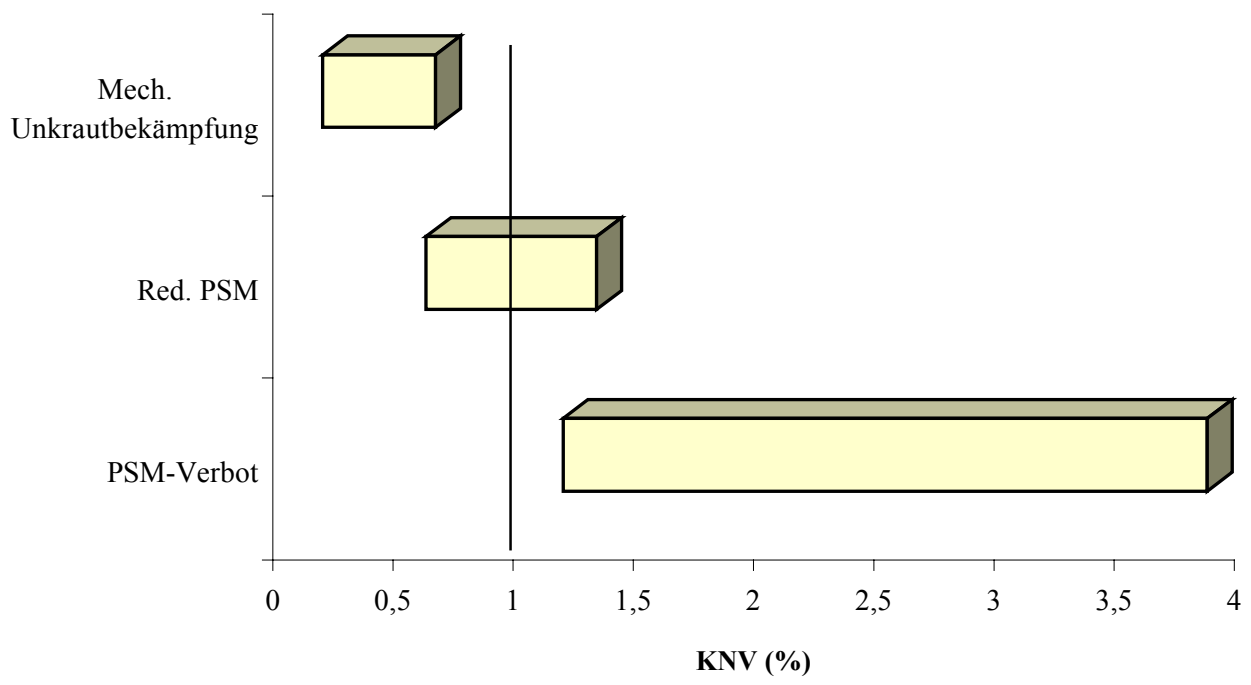

Quelle: Eigene Darstellung

Es ist in der Abbildung 36 zu sehen, dass die mechanische Unkrautbekämpfung, womit in diesem Fall der Striegeleinsatz gemeint ist, das beste KNV aufweist. Würde allerdings auf die thermische Unkrautbekämpfung oder auf Geräte mit Zapfwellenantrieb zurückgegriffen, wäre das KNV aufgrund der höheren Kosten deutlich schlechter einzustufen. Am schlechtesten schneidet das Verbot der PSM-Ausbringung ab, da die Ertragsverluste nicht durch die Einsparungen dieser Maßnahme kompensiert werden können. Die Reduzierung des PSM- Einsatzes nimmt eine Zwischenstellung ein. Es können mit dieser Maßnahme in Abhängigkeit von den Ertragsminderungen sowohl günstige als auch ungünstige KNV erreicht werden.

Die KNV für die Kompostausbringung liegen in den Berechnungen dieser Arbeit zwischen $1,03 \%$ und $1,64 \%$. Es ist zu berücksichtigen, dass in die Berechnungen verschie- 
dene Ausbringungskosten eingeflossen sind, die in der ldw. Praxis nicht immer anfallen. Ein Vergleich der KNV der Kompostausbringung mit der Klärschlammausbringung ist aufgrund fehlender Daten nicht möglich.

\subsection{Ergebnisse der Expertenbefragung}

Im Folgenden werden die Ergebnisse der Expertenbefragung zu den in Kapitel 6 behandelten Themen zusammengestellt. Diese sind nicht vollständig deckungsgleich, da zur Zeit der Befragung die zu analysierenden Maßnahmen noch nicht abschließend feststanden. Zunächst wurden die Experten zu Ihrer Einschätzung der Bedeutung verschiedener Bodenschutzmaßnahmen in Bezug auf die Schadensreduzierung befragt. Die Ergebnisse sind in der Abbildung 37 dargestellt.

\section{Abbildung 37: Beurteilung der Bedeutung der Maßnahmen für die Schadensredu- zierung mittels Expertenbefragung anhand des gewichteten Mittelwertes (n=22)}

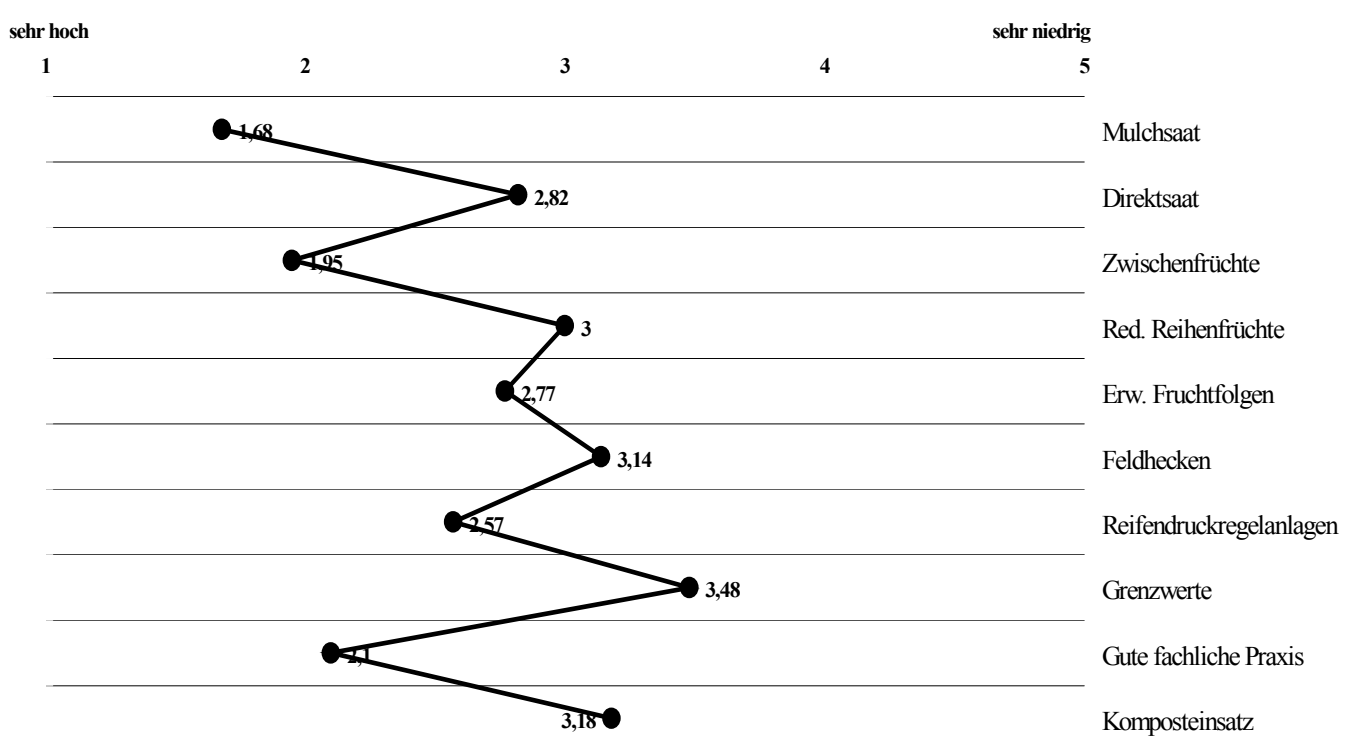

Quelle: Eigene Darstellung

Es ist zu erkennen, dass die Befragten v. a. der Mulchsaat, dem Anbau von Zwischenfrüchten sowie dem Instrument der guten fachlichen Praxis eine hohe Bedeutung bzgl. der Schadensreduzierung bescheinigen. Grenzwerten räumen sie hingegen die geringste Bedeutung ein. 
Die Ergebnisse der Expertenbefragung konnten in den Kapiteln 6.3 und 6.4 z. T. bestätigt werden. Allerdings hat die Literaturrecherche mehrmals ergeben, dass der Direktsaat ein höheres Reduktionspotenzial zuzumessen ist als der Mulchsaat. Ein Erklärungsansatz für diese Diskrepanz ist sicherlich die Tatsache, dass die Direktsaat auch für die Experten die weniger verbreitete und unbekanntere Maßnahme ist. Da in Deutschland weniger Erfahrungen vorliegen, ist es plausibel, dass hierdurch Vorbehalte bestehen.

Nachfolgend wurden die Experten nach ihrer Einschätzung zum einzelbetrieblichen KNV der dargestellten Bodenschutzmaßnahmen gefragt. Die Ergebnisse zeigt Abbildung 38.

\section{Abbildung 38: Beurteilung des einzelbetrieblichen KNV mittels Expertenbefragung anhand des gewichteten Mittelwertes $(n=19)$}

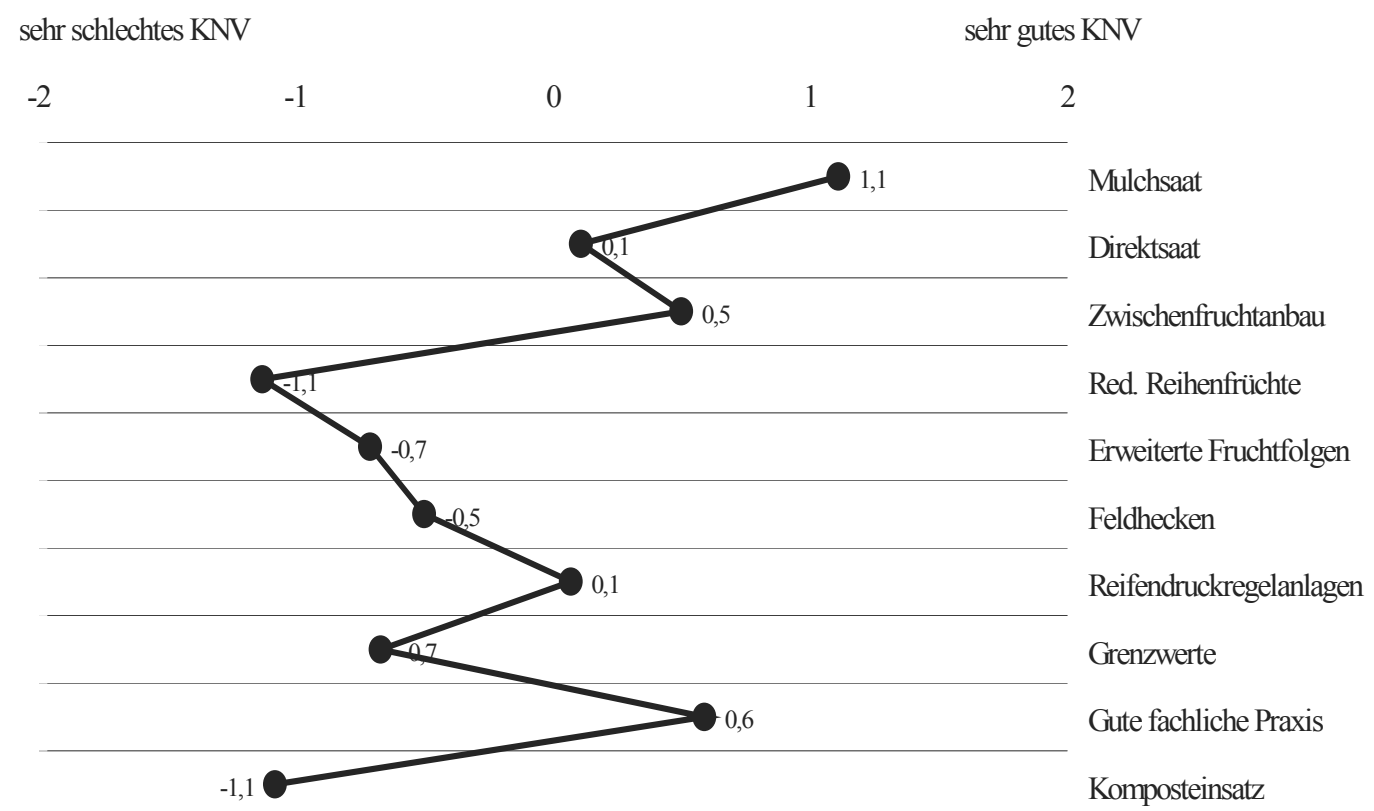

Quelle: Eigene Darstellung

Die Befragten sind der Meinung, dass die Mulchsaat das beste einzelbetriebliche KNV besitzt. Danach folgt die Einhaltung der guten fachlichen Praxis und der Zwischenfruchtanbau. Das schlechteste KNV wird beim Komposteinsatz und der Reduzierung von Reihenfrüchten vermutet. 
Die Berechnungen in dieser Arbeit führen prinzipiell zu ähnlichen Ergebnissen. Unterschiede liegen bspw. bei der Einschätzung und der Berechnung der KNA bei der konservierenden Bodenbearbeitung. Nach den Berechnungen dieser Arbeit ist das KNV der Direktsaat geringfügig besser als das der Mulchsaat. Der Komposteinsatz wird in der Expertenbefragung deutlich schlechter bewertet, als es das Ergebnis der Berechnungen gezeigt hat. 


\section{Schlussfolgerungen und Empfehlungen für Bodenschutz- konzepte landwirtschaftlicher Betriebe}

Die Ergebnisse dieser Arbeit dienen zur Unterstützung der Entwicklungen von Bodenschutzkonzepte auf Ebene der landwirtschaftlichen Betriebe. In den nachfolgenden Abschnitten werden die Ergebnisse zum Schutz vor Bodenerosion und die zum Schutz vor Bodenschadverdichtungen aufgegriffen und in bestehende Bodenschutzkonzepte integriert.

\subsection{Erweiterung von Bodenschutzkonzepte zum Schutz vor Boden- erosion}

In diesem Abschnitt werden die Ergebnisse des Kapitels 6 auf ein Beispiel eines bestehenden Schutzkonzeptes für Bodenerosion angewandt. Als Beispielkonzept wird das von FRIELINGHAUS und WINNIGE verwendet, welches ursprünglich für Brandenburg entwi-

ckelt wurde. ${ }^{527}$ Es geht zunächst von den einem größeren Gebiet, bspw. einer Gemarkung aus. 
Abbildung 39: Erweitertes Bodenschutzkonzept zur Ermittlung geeigneter einzelbetrieblicher Maßnahmen in Gebieten mit hoher Bodenerosionsgefährdung

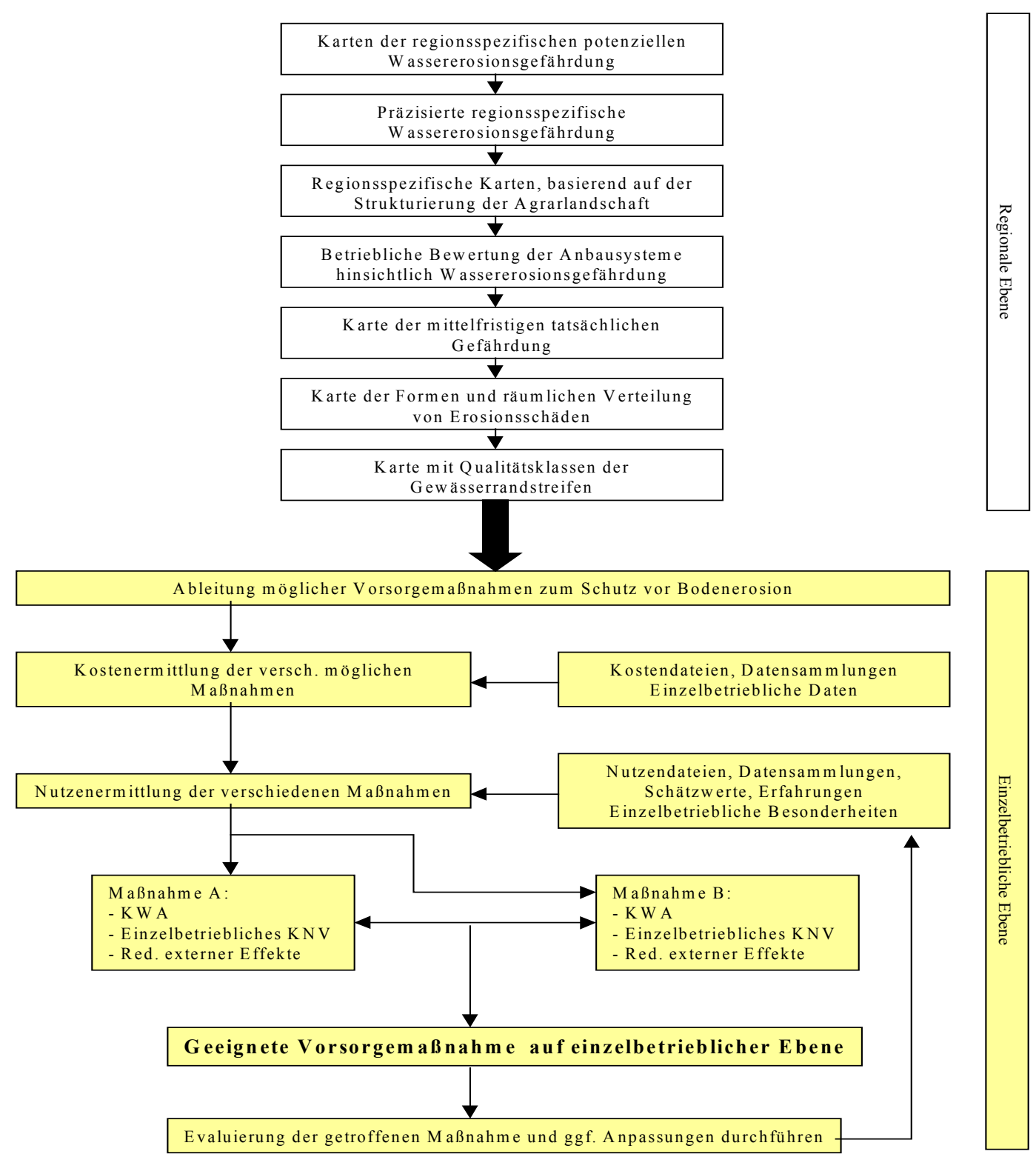

Quelle: Erweitert nach BMVEL (2001)

Zunächst werden, wenn vorhanden, die Karten der potenziellen Erosionsgefährdung herangezogen, die anschließend präzisiert werden. Daraufhin werden in diesem Konzept die Anbausysteme auf dem Betrieb erfasst und auf ihre Bodenerosionsgefährdung und bereits vorhandene Erosionsschäden untersucht. Darauffolgend können nach Abgleichung mit der Karte der Gewässerrandstreifen Aussagen über die tatsächliche Gefährdung des Bodens gemacht werden. Aus diesen Ergebnissen sollen nach dem Konzept Maßnahmen zur 
guten fachlichen Praxis abgeleitet werden. An diesem Punkt endet das vorliegende Konzept und wird entsprechend nach den betriebswirtschaftlichen Gesichtspunkten erweitert.

Der Wechsel zur einzelbetrieblichen Ebene geschieht durch den Vorschlag verschiedener Vorsorgemaßnahmen. Auf einzelbetrieblicher Ebene werden zunächst die Kosten der Maßnahmen erstellt und verglichen. Hierzu können KTBL-Daten, verschiedene Datensammlungen sowie einzelbetriebliche Daten, welche in diesem Fall die höchste Aussagekraft besitzen, herangezogen werden. Aber auch bei dem Einsatz von KTBL-Daten ist es durch Einbeziehung der einzelbetrieblichen Situation möglich, die Realitätsnähe und Genauigkeit der Berechnungen zu steigern. Die Frage besteht in der Praxis darin, wie hoch dieser zusätzliche Aufwand sein wird.

Die Nutzenermittlung sollte sich auf möglichst regional ermittelte Versuchsergebnisse stützen. Da diese, wie diese Arbeit mehrfach gezeigt hat, in vielen Bereichen noch nicht vorhanden sind, müssen Schätzwerte oder bestimmte Publikationen herangezogen werden. Bspw. kann diese Arbeit als erster Ansatz hierzu herangezogen werden, insgesamt ist jedoch dafür zu plädieren, dass entsprechende Datensammlungen ${ }^{528}$, wie es bspw. im Wasserschutz der Fall ist, aufgebaut werden, die Landwirten und Beratern die Nutzenermittlung der nötigen Maßnahmen deutlich erleichtern und eine möglichst hohe Genauigkeit und regionale Verwendbarkeit gewährleisten. Außerdem muss eine regelmäßige Aktualisierung vorgenommen werden.

Nach der Bewertung der Kosten und des Nutzens der vorgeschlagenen Alternativen muss diejenige gewählt werden, welche die beste KW bzw. das beste KNA aufweist und auf dem Einzelbetrieb die geringsten Umstellungsprobleme verursacht. Bei der Berücksichtigung des KNV in die Entscheidungsfindung muss bedacht werden, dass bei ähnlichen KNV diejenige Maßnahme gewählt werden sollte, welche die geringsten externen Effekte verursacht.

Bei Auswahl einer Bodenschutzmaßnahme sollte nachfolgend eine Evaluierung stattfinden, um den Erfolg abzuschätzen oder zu messen. Die gewonnenen Erfahrungen können

\footnotetext{
${ }^{528}$ Datensammlungen wie bspw. das Handbuch des Gewässerschutzes von FREDE U. DABBERT (1999) oder etwaige Handbücher für die Zusatzberatung im Wasserschutz. An dieser Stelle ist auf das Anwenderhandbuch für die Zusatzberatung Wasserschutz, NLÖ (2001), zu verweisen.
} 
u. U. wiederum in die Nutzendateien und Datensammlungen einfließen und für andere Gebiete zur Verfügung stehen.

Abschließend ist anzumerken, dass die Durchführung eines derartigen Konzeptes nur mit entsprechenden Beratern gelingen wird. Daher ist für erosionsgefährdete Gebiete zu überlegen, eine Bodenschutzberatung zu implementieren, sie zu kofinanzieren bzw. gewisse Zuschüsse für die Inanspruchnahme einer derartigen Beratung zu gewähren. Ob die gewählten Maßnahmen in erosionsgefährdeten Gebieten grundsätzlich gefördert werden sollten, kann hier nicht abschließend geklärt werden. Die Bereitstellung von Beratungswissen und -kompetenz neben der klassischen Förderung hat bspw. in den USA zu erheblichen Effizienzvorteilen geführt. Aufgrund leerer öffentlicher Haushaltkassen wird es in Deutschland jedoch keine explizite Bodenschutzberatung geben.

Die Ergebnisse dieser Arbeit können analog auch bei der Gefahrenabwehr auf einzelbetrieblicher Ebene Anwendung finden. Bei der Gefahrenabwehr von Bodenerosionsschäden können allerdings keine regionalen Karten der potenziellen Gefährdung zur Anwendung kommen, sondern es müssen für den einzelnen Schlag oder die einzelnen Schläge die Schadstellen und deren zukünftiges Schadpotenzial ermittelt werden. Daraufhin sind geeignete Maßnahmen aufzustellen. Die Ermittlung der geeigneten Maßnahmen kann der Abbildung 39 folgend durchgeführt werden. An dieser Stelle sollten demnach die Ergebnisse bzw. die Vorgehensweise in bisherigen Gefahrenabwehrkonzepte, wie etwa das des BUNDESVERBANDS BODEN ${ }^{529}$, berücksichtigt und somit auch betriebswirtschaftliche Aspekte Eingang finden. ${ }^{530}$

\subsection{Erweiterung von Bodenschutzkonzepte zum Schutz vor Boden- schadverdichtungen}

Zunächst wird in diesem Kapitel auf das allgemeine aber weit verbreitete Konzept für bodenschonendes Befahren von SOMMER ${ }^{531}$ eingegangen und um die Erkenntnisse dieser Arbeit erweitert. Dies wird in der Abbildung 40 dargestellt.

\footnotetext{
${ }^{529} \mathrm{Vgl}$. BundesVerband Boden (2003)

${ }^{530} \mathrm{Vgl}$. AGRA-EUROPE (2004), L $16 \mathrm{f}$.

${ }^{531}$ Vgl. SOMMER (1998)
} 


\section{Abbildung 40: Erweiterung des Konzeptes „Bodenschonendens Befahren“ von SOMMER um ökonomische Aspekte}

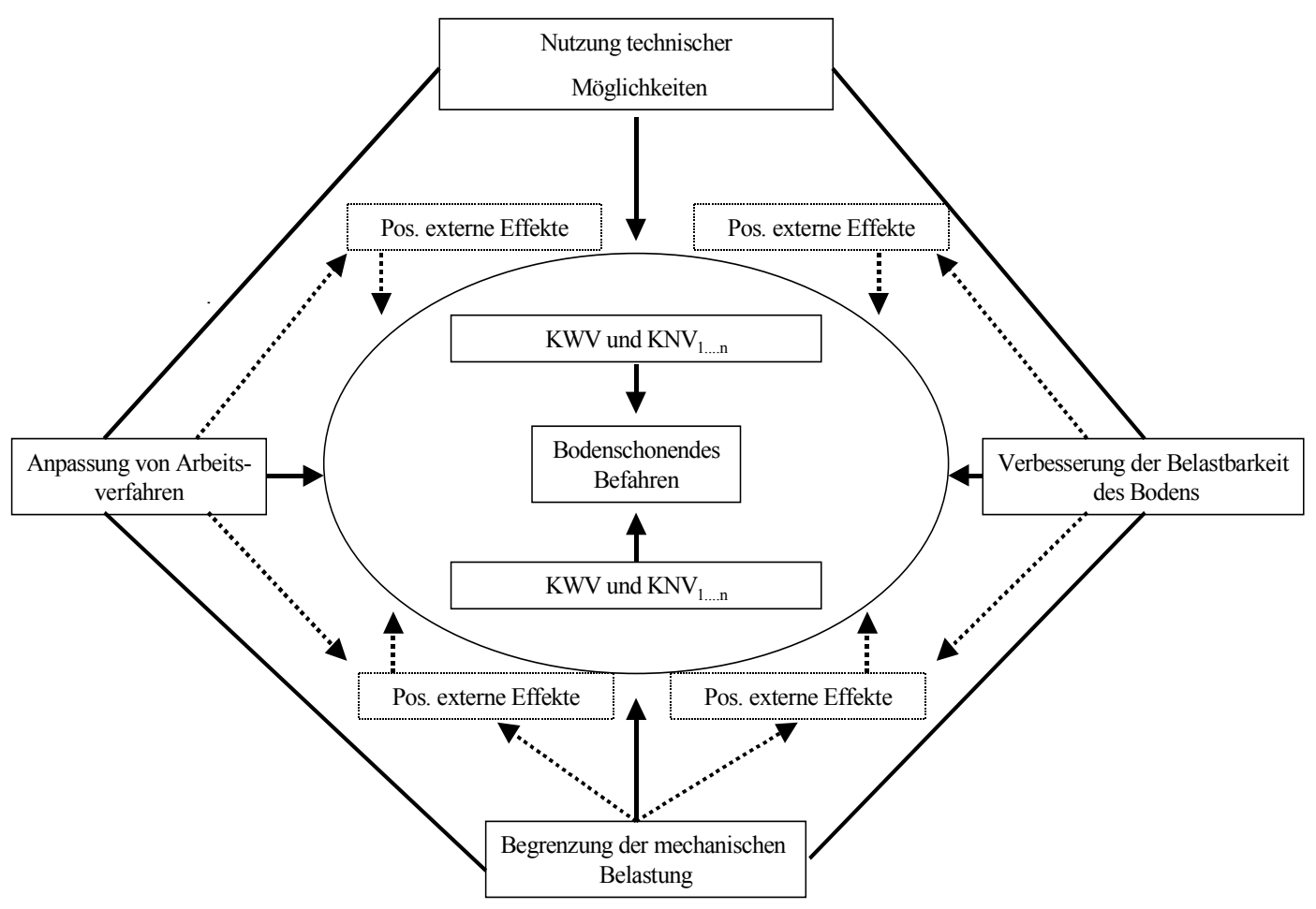

Quelle: Erweitert nach SOMMER (1998)

Das Konzept „Bodenschonendes Befahren“ geht von vier verschiedenen Möglichkeiten der Verbesserung der Situation des Bodens aus. Zum einen können technische Möglichkeiten, wie z. B. Reifenwahl etc. dazu führen, dass Bodenschadverdichtungen begrenzt werden. Auf der anderen Seite besteht die Möglichkeit, bspw. durch konsequent konservierende Bodenbearbeitung, eine bessere Belastbarkeit des Bodens zu erreichen. Weiterhin besteht die Möglichkeit, durch eine Begrenzung der mechanischen Belastung oder durch Anpassung von Arbeitsverfahren, bspw. durch Zusammenlegungen von Arbeitsgängen, den Bodendruck zu reduzieren.

Die Erweiterung besteht analog zu dieser Arbeit in der Einbeziehung der KW, des KNV und sowie der externen Effekte. Daher sollte jede Bodenschutzmaßnahme zunächst einer allgemeinen, bei Einführung jedoch einer einzelbetrieblichen Wirtschaftlichkeitsanalyse 
unterzogen werden. Dies dient zur Abschätzung der eventuellen zusätzlichen Belastung der Betriebe und zur Ableitung eines eventuellen Förderungsbedarfs.

Ein weiteres Bodenschutzkonzept für den Schutz des Bodens vor Bodenschadverdichtungen schlagen CHAMEN et al. vor. ${ }^{532}$ Sie konzentrieren sich hierbei auf den Einsatz einzelner Bodenschutzmaßnahmen auf Betriebsebene. Das Konzept ist in der Abbildung 41 dargestellt.

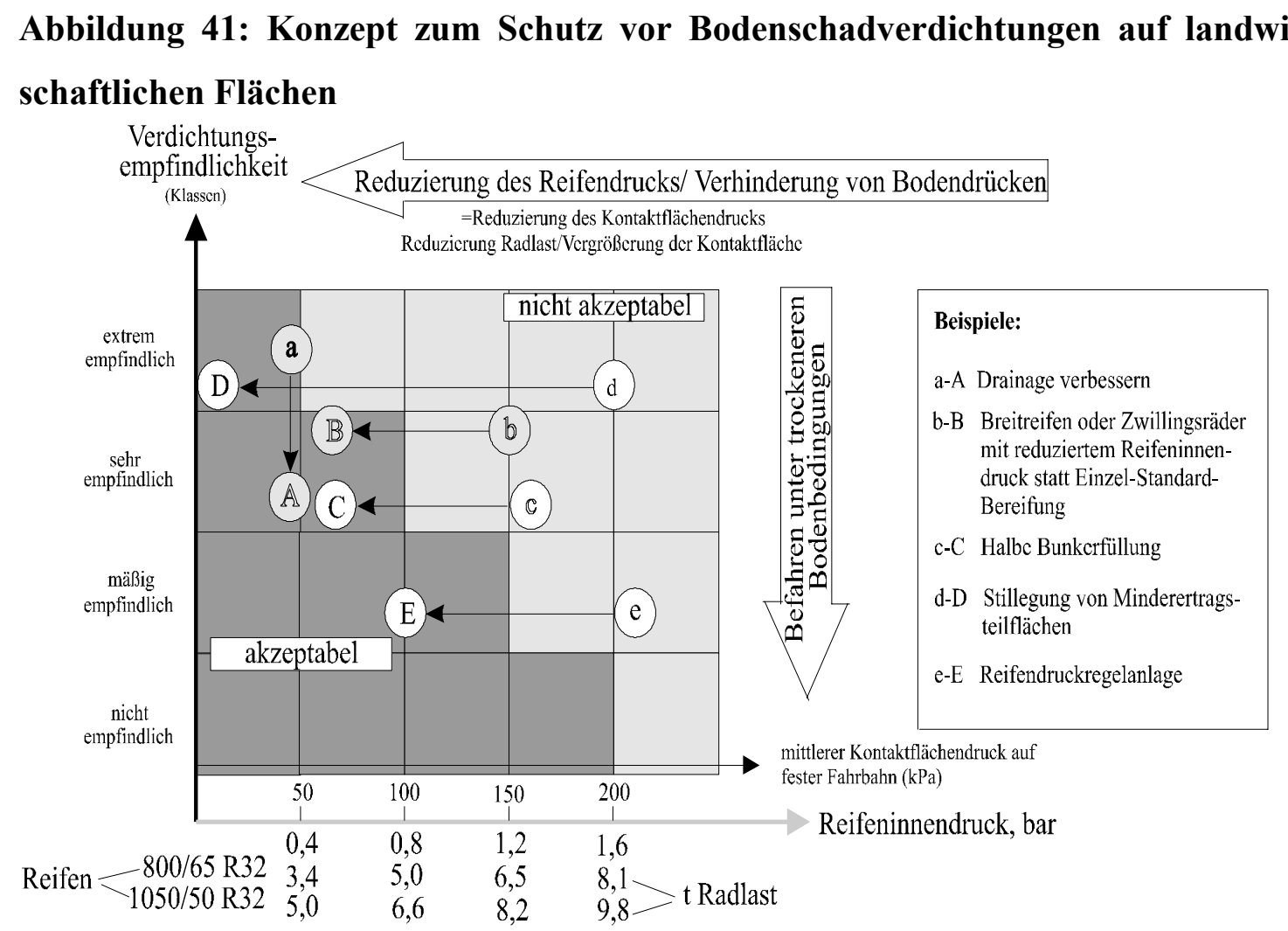

Quelle: Verändert nach CHAMEN et al., zitiert in BMVEL (2001)

Das Konzept geht von verschiedenen zuvor definierten Verdichtungsempfindlichkeitsklassen aus. Diese werden mit verschiedenen Radlasten und Reifeninnendrücken verknüpft, die zu einer Matrix führen. Hieraus lassen sich die akzeptablen oder nicht akzeptablen Bewirtschaftungsbedingungen ableiten. Auf der Basis dieser Matrix werden einige Ergebnisse dieser Arbeit bewertet. Die jeweilige Maßnahme kann demzufolge aufgrund der notwendigen Bodenschutzeffizienz vom Landwirt ausgewählt werden. 


\subsection{Weitere Empfehlungen für landwirtschaftliche Betriebe, Berater sowie Politik und Verwaltung}

Abschließend werden im Folgenden ergänzende Empfehlungen für einen effizienten Bodenschutz in Deutschland gemacht. Die Empfehlungen richten sich an Landwirte, die landwirtschaftliche Fachberatung sowie an die Politik und die Verwaltung.

\subsubsection{Empfehlungen für Landwirte}

Diese Arbeit hat gezeigt, dass es effektive Maßnahmen zum Schutz des Bodens gibt, die einen höheren einzelbetrieblichen Nutzen als dadurch anfallende Kosten haben. Dies sollte die Betriebsleiter dazu ermutigen, Vorurteile gegenüber Umweltauflagen abzubauen und sich, in ihrem eigenen Interesse, mit ihrem Boden und dessen Schutz zu beschäftigen. Hierzu gehört v. a. eine Auseinandersetzung mit den Empfehlungen der guten fachlichen Praxis, die in Zeitschriften, auf Tagungen oder Feldtagen vermittelt werden. Die Betriebsleiter sollten Innovationen, seien es neue Maschinen- oder Verfahrensentwicklungen im Bereich des Bodenschutzes, aufnehmen und sich mit diesen beschäftigen. Positive Beispiele für eine derartig erfolgreiche Innovation sind die Verfahren der konservierenden Bodenbearbeitung, die in vielen Regionen nach anfänglichen Vorbehalten seitens der Landwirte und deren Berater mittlerweile zu den Standardverfahren gehören. Um den Erfolg derartiger Innovationen zu erhöhen und zu beschleunigen, konnte in dieser Arbeit des Öfteren gezeigt werden, dass ein Zusammenschluss verschiedener Landwirte, z. B. zu Maschinengemeinschaften oder die Auslagerung von Arbeitsgängen, vorteilhaft für den Bodenschutz und für den wirtschaftlichen Erfolg des Betriebes ist. Daher ist den Landwirten, gerade bei kleinen und mittleren Betrieben mehr den je zu empfehlen, ihre Produktionskosten auf diesem Wege zu minimieren.

Doch auch der Flächentausch kann u. U. dazu führen, dass Bodenschutzmaßnahmen, wie z. B. die Bewirtschaftung quer zum Hang, wirtschaftlicher werden. Hierzu ist jedoch ein starker Dialog und ein entsprechendes Bewusstsein nötig.

Daher muss sich bei einigen Landwirten ein noch stärkeres „Bodenbewusstsein“ für ihren wichtigsten Produktionsfaktor herausbilden. Dies gilt nicht nur für den Boden, der sich im Eigentum befindet, sondern auch für den gepachteten Boden. An dieser Stelle sollte es zu einvernehmlichen Regelungen und Abstimmungen zwischen Verpächter und Pächter in den Verträgen kommen, damit der nachhaltige Schutz des Bodens gewahrt bleibt. Bo- 
denschutz muss in den landwirtschaftlichen Betrieben ferner als eine Generationenaufgabe wahrgenommen werden. Es muss in diesem Zusammenhang klar sein, dass der Boden ein „Langzeitgedächtnis“ besitzt und dies dazu führt, dass sich bestimmte Schädigungen wie z. B. stoffliche Schädigungen durch persistente Stoffe oder aber ein schleichender Bodenabtrag erst in der Zukunft auswirken bzw. erkannt werden.

\subsubsection{Empfehlungen an die Beratung}

Wie in der Expertenbefragung dieser Arbeit deutlich wurde, ist eine Ausweitung der ldw. Fachberatung um eine Bodenschutzberatung wünschenswert, stößt jedoch an finanzielle Grenzen der hierfür zuständigen Haushalte. Ein System wie in den USA, wo Bodenschutzberater und Landwirte auf einzelbetrieblicher Ebene zusammenarbeiten, wäre dagegen effizient. Auf kurz- und mittelfristige Sicht ist jedoch davon auszugehen, dass die Bodenschutzberatung lediglich als Querschnittsaufgabe der Offizialberatung und ähnlicher Beratungsformen (Kooperationen zwischen Bodenschutz- und Wasserschutzberatung sowie pflanzenbaulicher Beratung) durchgeführt wird. Daher kann von der landwirtschaftlichen Fachberatung nur ein begrenzter Wissenstransfer und eine eingeschränkte Betreuung erwartet werden. Diese sollte sich allerdings auf folgende Aufgaben konzentrieren:

- Vermittlung eines stärkeren Bodenbewusstseins bei den Landwirten

- Sensibilisierung für ein Kosten-Nutzen-Bewusstsein bei Bodenschutzmaßnahmen

- Erkennung der monetären Schäden durch Bodenschädigungen

- Anerkennung des direkten monetären Wertes des Bodens

- Unterstützung bei der Umsetzung von Bodenschutzmaßnahmen

-Unterstützung bei der Wahl der Maßnahmen (z. B. Einsatz von ABAG etc.),

-Hilfestellung bei der Umsetzung der Maßnahmen,

-Optimierung und Koordinierung der entsprechenden Fördermöglichkeiten und

-Evaluierung der Ergebnisse der Maßnahme.

- Heranführung der Landwirte an eigenständiges bodenschützendes Handeln

Um eine effiziente Bodenschutzberatung zu implementieren, sind einfache und leicht modifizierbare Datengrundlagen nötig. Im Bereich der Bodenerosion steht mit der ABAG 
eine solche bereits zur Verfügung. In erosionsgefährdeten Gebieten könnten des Weiteren noch physikalische Modelle zur Anwendung kommen. Bezüglich der Bodenschadverdichtung besteht allerdings noch erheblicher Forschungsbedarf bezüglich der Erstellung derartiger Modelle. Eine weitere Grundlage ist diese Arbeit. Sie muss allerdings auf die jeweilige einzelbetriebliche Situation abgestimmt werden. Daher ist, wie es im folgenden Unterkapitel geschieht, eine Empfehlung an die Verwaltungen zu richten, derartige Datengrundlagen für die Beratung zu erarbeiten.

\subsubsection{Empfehlungen an die Verwaltung und die Politik}

Die Bodenschutzverwaltungen und alle hierzu zählenden Institutionen gehören zu den jüngsten Administrationsstellen in Deutschland. Sie sind zum größten Teil aus verschiedenen Querschnittsbereichen, wie etwa den Wasser- oder Naturschutzverwaltungen hervorgegangen. Der Einfluss der Bodenschutzverwaltung auf den landwirtschaftlichen Bodenschutz ist aufgrund des gesetzlichen Rahmens sehr begrenzt. Ein zentraler Punkt betrifft jedoch nicht nur die Bodenschutzverwaltungen der Länder, sondern auch deren angeschlossene Institutionen, wie z. B. geologische Landesämter etc. Auf Basis der Erfahrungen mit diesen Institutionen bei der Erstellung dieser Arbeit ist eine bessere Zusammenarbeit und ein höherer Austausch von Daten zu empfehlen, um die Effizienz des Bodenschutzes zu erhöhen. Die Zersplitterung der Aufgabengebiete durch das föderale System trägt dazu bei, dass bundeseinheitliche Daten nicht oder nur sehr spät zusammengetragen werden können. Daher sind darauf basierende Vollzugsdefizite unausweichlich. Die Ministerien, in den meisten Bundesländern oberste Bodenschutzbehörden, müssten ein großes Interesse an der effektiven Zusammenarbeit in den Bundesländern haben. Gerade in Hinsicht auf eine zunehmende Ausrichtung der Umweltpolitik auf der Ebene der EU ist eine Effizienzsteigerung bedeutsam.

Eine weitere Empfehlung für die Bodenschutzverwaltung ist die Einbeziehung von ökonomischen Daten und Kennzahlen bei zu treffendenden Vorsorge- oder Gefahrenabwehrmaßnahmen. Hierbei sind die Ergebnisse dieser Arbeit als Orientierungsgrundlage hilfreich. Durch derartig abgestimmte Maßnahmen wird die Bereitschaft der Landwirte an der Teilnahme und eventuell an der Fortführung der verordneten Maßnahmen tendenziell erhöht. 
Die Politik sieht sich derzeit in einem Spannungsverhältnis zwischen der z. T. grundlegenden Kritik an dem geltenden Bodenschutzrecht und den daraus resultierenden Forderungen nach schärferen Regelungen und Gewährung von Ausgleichsansprüchen bzw. Fördergeldern und der Finanzprobleme der öffentlichen Haushalte.

Dieser Zusammenhang zeigt, dass der landwirtschaftliche Bodenschutz und dessen Maßnahmen, wie oben bereits erwähnt, auch nach ökonomischen Gesichtspunkten ausgerichtet werden müssen. Dies gilt v. a. bei Vorschriften von Maßnahmen wie bspw. beim Ackerbauverbot im geplanten Hochwasserschutzgebiet. Eine Auseinandersetzung mit den Ergebnissen dieser Arbeit oder eigens angefertigten Wirtschaftlichkeitsüberlegungen führen zu differenzierteren und effizienteren Maßnahmen, die von der Landwirtschaft eher akzeptiert und bereitwilliger umgesetzt werden. Daher sind, nach sorgfältiger und umfassender Prüfung, Extremlösungen, wie sie in der Vergangenheit gerade im Bereich der Umweltschutzpolitik favorisiert wurden, abzulehnen.

Des Weiteren ist der Politik zu empfehlen, ein EU-weites Bodenschutzrecht und damit möglichst einheitliche Umweltstandards zu erreichen. Zum einen gibt es in Europa sehr gefährdete Standorte, wie z. B. den Mittelmeerraum oder die landwirtschaftlichen Nutzflächen der neuen Beitrittsländer. Zum anderen führen ungleiche Umweltstandards in den Mitgliedsstaaten zu Wettbewerbsverzerrungen, die mit einem gemeinsamen Wirtschaftsraum und Binnenmarkt nicht vereinbar sind. Dies gilt allerdings nicht nur für den Bodenschutz im Speziellen, sondern auch für verwandte Rechtsbereiche wie das Dünge- und Pflanzenschutzmittelrecht. Exemplarisch kann hierfür auch die Umsetzung der CCRegelungen angeführt werden.

Die Förderung bodenschützender Maßnahmen ist generell zu begrüßen, jedoch auch bundeseinheitlich zu gestalten. Die Frage, ob ein Standort förderwürdig ist, sollte sich nicht nach Ländergrenzen, sondern nach der potenziellen Gefährdung richten. Die Ergebnisse dieser Arbeit geben für fast alle Maßnahmen Aufschluss darüber, wie hoch der Kostenbzw. Nutzenüberschuss ist. Hiervon lassen sich bedingt auch Förderbeträge ableiten. Die finanzielle Unterstützung von Bodenschutzmaßnahmen seitens des Staates sollte allerdings für einige Maßnahmen, z. B. für Mulch- und Direktsaatverfahren nur als Anschubfinanzierung gewährt werden, da sie i. d. R. einen Nutzenüberschuss aufweisen. 


\section{Zusammenfassung}

Der Boden gehört zu einem der wichtigsten Produktionsfunktionen für die Landwirtschaft und dient der Gesellschaft zur Versorgung mit lebenswichtigen Nahrungsmitteln.

Der landwirtschaftlich genutzte Boden ist allerdings durch verschiedene Beeinträchtigungen, die sowohl physikalischer als auch stofflicher Art sind, gefährdet. Zu den physikalischen Gefährdungen gehört die Bodenerosion, die Bodenschadverdichtung und die Bodenversiegelung. Die stofflichen Gefährdungen resultieren v. a. aus den Einträgen von PSM, Düngemitteln und Sekundärrohstoffdüngern. Die tatsächlichen Gefährdungen des Bodens lassen sich jedoch noch nicht für alle Gefahrenbereiche ausreichend quantifizieren. Die besten Datengrundlagen bestehen bei der Bodenversiegelung, den stofflichen Belastungen sowie z. T. bei der Bodenerosion, was im Fall der Bodenerosion an den einfachen Prognosemöglichkeiten, wie der ABAG liegt.

Bodenschädigungen führen sowohl auf einzelbetrieblicher als auch auf volkswirtschaftlicher Ebene zu verschiedenen monetären Schäden. Die einzelbetrieblichen OnsiteSchäden sind leichter zu ermitteln als die volkswirtschaftlichen Offsite-Schäden. Wichtig ist jedoch, dass sie vom Landwirt überhaupt wahrgenommen werden, was bspw. bei einer schleichenden Bodenerosion mit geringen Bodenabtragsraten schwierig ist.

Der Boden ist durch das im Jahr 1999 in Kraft getretene Bodenschutzrecht als letztes Umweltmedium erstmals durch ein Bundesgesetz unter Schutz gestellt worden. Grundsätzlich regelt das BBodSchG die Vorsorge, die Gefahrenabwehr und die Sanierung schädlicher Bodenveränderungen. Das BBodSchG enthält neben zahlreichen Regelungen zum Altlastenrecht Vorschriften für die landwirtschaftliche Bodennutzung. Hier spielt v. a. die gfP eine bedeutende Rolle. Bei Einhaltung verschiedener der ldw. Bodennutzung betreffender Grundsätze erfüllt der Landwirt sowohl die Vorsorge- als auch die Gefahrenabwehrpflichten. Aufgrund fehlender Anordnungs- oder Sanktionsmaßnahmen wird das Prinzip der gfP der ldw. Bodennutzung in der rechtswissenschaftlichen Diskussion oftmals kritisiert. Diese Kritik wird von den Experten in einer entsprechenden Befragung zum größten Teil jedoch nicht geteilt. Neben dem Prinzip der gfP werden weitere Inhalte des deutschen Bodenschutzrechts analysiert, hierzu gehört der Begriff des angemessenen Ausgleichs bei angeordneten Bodenschutzmaßnahmen sowie die flächenhafte Ausweisung von Bodenschutzgebieten nach $\S 21$ Abs. 3 BBodSchG. Eine weitere wichtige 
Rolle für den landwirtschaftlichen Bodenschutz stellen die Entwicklungen bezüglich der Cross-Compliance Regelungen dar. Durch die Kopplung von Umweltanforderungen an den Erhalt von Direktzahlen können auf der einen Seite Bodenschutzmaßnahmen an Bedeutung gewinnen. Auf der anderen Seite können diese Regelungen bei ungleichmäßiger Anwendung zu Wettbewerbsnachteilen führen.

Die Bereiche des Wasser- und Bodenschutzes sind sowohl seitens der naturwissenschaftlichen als auch seitens der rechtswissenschaftlichen Verknüpfungen eng miteinander verbunden und bedürfen einer Unterscheidung. Des Weiteren sind einige Rechtsvorschriften aus dem Bereich des Wasserschutzes auch für den Bodenschutz relevant. Zum einen ist dies die bereits z. T. umgesetzte WRRL, die auf Flusseinzugsgebietsebene Maßnahmen zur Erreichung eines guten Zustands der Oberflächengewässer vorsieht. Zum anderen ist das geplante Artikelgesetz zu einem vorsorgenden Hochwasserschutz maßgebend, welches v. a. den Ackerbau in bestimmten Überschwemmungsgebieten bis 2012 verbieten soll. An einem Beispiel konnte gezeigt werden, dass auf einzelbetrieblicher Ebene erhebliche Deckungsbeitragsverluste entstehen werden. Aus diesem Grund ist auch aus naturwissenschaftlicher Sicht daran zu appellieren, ein derartiges Verbot stärker zu differenzieren und abzustufen.

Die vorwiegend agrarwissenschaftlich geprägte Forschung hat bereits einige Bodenschutzmaßnahmen entwickelt und wissenschaftlich begleitet. Eine entsprechende agrarökonomische Begleitforschung hat jedoch nur in einigen Fällen stattgefunden. Basierend auf diesen und weiteren bodenkundlichen sowie pflanzenbaulichen Ergebnissen werden für verschiedene Maßnahmen des physikalischen und stofflichen Bodenschutzes Kostenvergleiche, KWA und KNA durchgeführt. Dabei wird folgende Reihenfolge beachtet: Wenn keine Daten über den Nutzen der Maßnahme vorhanden sind, wird ein Kostenvergleich durchgeführt. Gibt es weiterhin Ergebnisse, welche die Wirkung der Maßnahme auf den Bodenschutz hinreichend darlegen, werden die entsprechenden Kosten in Beziehung zur Wirkung gestellt. Das Ergebnis gibt an, wie hoch die Kosten bspw. für die Reduzierung des Bodenabtrags um eine Tonne Oberboden mit der betrachteten Maßnahme liegen. Wenn es möglich ist, sowohl die Kosten der Maßnahme zu erfassen als auch dessen Nutzen zumindest in wichtigen Teilbereichen zu monetarisieren, wird eine KNA durchgeführt. Dabei werden die diskontierten Kosten- und Nutzenströme gegenübergestellt und in Beziehung gesetzt. Das Ergebnis ist das KNV, welches als Verhältniszahl ei- 
ne wichtige Basis für den Vergleich der Maßnahmen stellt. Als weiteren Vergleichswert wird außerdem der durchschnittliche jährliche Vorteil der jeweiligen Maßnahme errechnet.

Durch diese Methodik erfolgt eine Differenzierung der Maßnahmen nach betriebswirtschaftlichen Gesichtspunkten und dient folglich verschiedenen Adressaten, wie z. B. Landwirten, Beratern und der Politik als Entscheidungs- bzw. Diskussionsgrundlage. Die Ergebnisse der Berechnungen und der Expertenbefragung bestätigen den Differenzierungsbedarf landwirtschaftlicher Bodenschutzmaßnahmen. Einige Maßnahmen, wie z. B. die konservierende Bodenbearbeitung und der Zwischenfruchtanbau weisen sehr gute KNV auf. Bei diesen Maßnahmen würde eine finanzielle Förderung zu Mitnahmeeffekten führen, da sie auf einigen Standorten sehr effizient sein können. Andere Maßnahmen, wie z. B. die Anlage von Feldhecken oder die Bewirtschaftung quer zum Hang weisen schlechte KNV auf und sind somit aus betriebswirtschaftlicher Sicht als unrentabel zu bezeichnen. Die z. T. hohen Schwankungsbreiten der Ergebnisse zeigen weiterhin, dass weitere Anstrengungen zur Verbesserung und Erweiterung der bodenkundlichen und naturwissenschaftlichen Datengrundlagen getätigt werden müssen, um exakte einzelschlagspezifische Ergebnisse zu erhalten.

Bei den Maßnahmen des physikalischen Bodenschutzes ist außerdem festzustellen, dass entsprechende Versuchsergebnisse über die Bodenschutzwirksamkeit fehlen, bzw. nur bedingt vorliegen. Ein erheblicher Entwicklungsbedarf besteht daher bei der weiteren Erforschung der Wirkungsbeziehung zwischen Bodendruck bzw. Bodenschadverdichtung und dem Ertrag. Diese Datengrundlagen fehlen wiederum bei den derzeit geführten Diskussionen um bodenschonende Radlasten bei Erntemaschinen. Bei den Maßnahmen zum Schutz vor Bodenschadverdichtungen handelt es sich oft um Investitionen des Einzelbetriebs (bspw. bodenschonende Bereifung oder Reifendruckregelanlagen), die ein hohes Risiko beinhalten. Die Ergebnisse zeigen, dass auch mit diesen Maßnahmen gute KNV erzielt werden können. V. a. die bodenschonende Bereifung und der Einsatz von Reifendruckregelanlagen sowie die Stilllegung von Minderertragsflächen gehören unter den angenommenen Bedingungen zu diesen Maßnahmen.

Die Maßnahmen zum Schutz vor stofflichen Belastungen werden ebenfalls, jedoch nicht in der hohen Intensität, behandelt. Hierbei zeigt sich, dass v. a. die Instrumente des Integrierten Landbaus, bspw. die mechanische Unkrautbekämpfung sowie die Anwendung 
des Schadschwellenprinzips, gute KNV aufweisen und somit rentabel sind. Dies gilt allerdings nicht für das gänzliche Verbot der Ausbringung von PSM.

Die Ergebnisse dieser Arbeit dienen als Ergänzung bestehender Bodenschutzkonzepte. Betriebswirtschaftliche Aussagen zu Maßnahmenprogrammen erhöhen u. U. die Akzeptanz und die Transparenz für alle Beteiligten. Daher sollten Berechungen wie die KWA oder KNA in entsprechende Konzepte integriert werden. Den Landwirten und deren Beratern ist zu empfehlen, das Bodenbewusstsein weiterzuentwickeln. Es muss klar sein, dass Boden nicht nur eine wichtige ökologische sondern auch ökonomische Komponente hat. Letztere kommt bereits dann zum Tragen, wenn der Boden, wenn auch nur schleichend, erodiert oder schadverdichtet wird. Für die Umsetzung von Bodenschutzmaßnahmen und deren Effizienz wäre es wünschenswert, wenn diese wie in den USA durch Bodenschutzberater unterstützt werden würden.

Die Verwaltung und die Politik sollten an einer möglichst engen Kooperationen interessiert sein. Dies gilt v. a. bei der interdisziplinären Zusammenarbeit mit geologischen Diensten etc. Eine umfassende, bundesweite Entwicklung und Bereitstellung von Daten darf nicht an den föderalen Strukturen scheitern. Die Politik sollte die Ergebnisse bzw. die Systematik dieser Arbeit dann aufnehmen, wenn es um Anordnungen zum Bodenund Wasserschutz geht. Hierdurch können Extremlösungen und dadurch entstehende Wettbewerbsnachteile, wie z. B. durch das Ackerbauverbot im Artikelgesetz zum vorsorgenden Hochwasserschutz, vermieden werden.

Bodenschädigungen machen nicht vor politischen Grenzen halt, sondern sind ein europäisches und auch weltweites Problem. Deshalb ist auf die Weiterentwicklung einer EUweiten Bodenschutzpolitik zu drängen, die auch die neuen Mitgliedsstaaten einbezieht. Weltweit müssen die bisherigen Absichtserklärungen dringend durch konkrete Maßnahmen erweitert werden. 


\section{Literaturverzeichnis}

Agra-Europe (1998): Gute fachliche Praxis kein unbestimmter Rechtsbegriff. Heft 26, S. L18

AGRA-Europe (2000): „Gute fachliche Praxis“ vernachlässigt Bodenschutz, Heft 7, S. L 11

AGRA-Europe (2003): Bodenverdichtungen durch Landmaschinen geraten ins Blickfeld, Heft 32, S. 24-25

Agra-EuROPE (2003a): Nährstoffmanagement im Blickfeld von Wissenschaft und Praxis, Heft 49, S. L6 ff.

AGRA-Europe (2004): DBV kritisiert Handlungsempfehlungen zum Bodenschutz, Heft 11, S. L $16 \mathrm{f}$.

Agra-Europe (2004a): Ministerin Höhn begrüßt EU-Vorschläge zum Bodenschutz, Heft 14, S. E 10 f.

AGRA-EuROPE (2004b): Schwermetallgrenzwerte für Düngemittel sollen nicht nach Bodenart differenziert werden, Heft 8, S.14 f.

AGRA-Europe (2004c): Bundeslandwirtschaftsministerium will Maßnahmen zur Erosionsvermeidung vorschreiben, Heft 19, S. L 1 f.

AID (2003): Kompost in der Landwirtschaft, Heft 1476, Hannover

Atteslander, P. (2000): Methoden der empirischen Sozialforschung, 9. Auflage, de Gruyter, Berlin

Auerswald, K. (2002): Bodenerosion. In: SchefFER, F. (2002): Lehrbuch der Bodenkunde, 15. Auflage, Spektrum Akademischer Verlag, Berlin, S. 416-425

AuerswaLd, K. U. SCHMidT (1986): Atlas der Erosionsgefährdung in Bayern - Karten zum flächenhaften Bodenabtrag durch Regen. GLA-Fachberichte, München

Auerswald, K. U. V. Perger, P. (1998): Bodenerosion durch Wasser. Ursachen, Schutzmaßnahmen und Prognose mit ${ }^{\mathrm{PC}} \mathrm{ABAG}, \mathrm{AID}$, Bonn

Auerswald, K., Schröder R. U. Fiener P. (2001): Landwirtschaft und Hochwasser. In: Deutsche Vereinigung FÜr WasSerwirtschaft AbWasser Und ABfall 
E. V: Hochwasser - Niedrigwasser - Risiken. DVWK-Landesverband Bayern, München

Bachmann, G., von Borries, D. U. DinkelberG, W. (1995): Bodenbelastungen in Land- und Forstwirtschaft - Abschätzung möglicher Ausgleichsforderungen der Land- und Forstwirtschaft nach $\S 14$ (2) E-BBodSchG, dargestellt anhand der Schwermetallbelastung der Böden. Zeitschrift für Kulturtechnik und Landentwicklung, Jg. 36, S. 1-5

BAHRS, E. (1999): Bewertung landwirtschaftlicher Betriebe für Substanzbesteuerungen. Dissertation, Schriftenreihe des HLBS, Heft 153, St. Augustin

BARUNKE, A. (2002): Die Stickstoffproblematik in der Landwirtschaft - Erfahrungen mit Stickstoffminderungspolitiken. Dissertation, Vauk-Verlag, Kiel

BANNIK, C. G. (2002): Grundsätze und Maßnahmen für eine vorsorgeorientierte Begrenzung von Schadstoffeinträgen in landbaulich genutzte Böden. In: KTBL (2002): Landwirtschaftliche Verwertung von Klärschlamm, Gülle und anderen Düngern unter Berücksichtigung des Umwelt- und Verbraucherschutzes, KTBL-Schrift 404, Darmstadt, S. 17-27

BeCKer, C., Miller, H. U. KoCH, H.-J. (1996): Pfluglose Bodenbearbeitung zu Zuckerrüben - pflanzenbauliche und ökonomische Ergebnisse einer Versuchsserie auf Großparzellen. Zuckerindustrie, Jg. 121, Heft 8, S. 609-615

BEHRENDT, H. et al. (1999): Nährstoffbilanzierung der Flußgebiete Deutschlands. Forschungsbericht 29625 515, UBA-FB 99-087, Umweltbundesamt, Berlin

Berekoven L., ECKert W. U. ElLENRIEder, P (1999): Marktforschung: Methodische Grundlagen und praktische Anwendung, Gabler, Wiesbaden

BERENDONK, C. (2003): Zwischenfrüchte: Allgemeine Anbauhinweise, Internet: www.riswick.de/pdf/futterbau/zfanbau_allg.pdf vom 13.10.2003

Bischoff, J. (2002): Weizen ohne Pflug. Neue Landwirtschaft, Heft 8, S. 26-28

Bizer, K. U. EWRigmanN, D. (1999): Steuerung der Flächennutzung. Informationen zur Raumentwicklung, Heft 8, S. 511-521 
BMU (2003): Entwurf: Gesetz zur Verbesserung des vorbeugenden Hochwasserschutzes. Internet: www.bmu.de/de/800/js/download/b_artikelgesetz_hochwasserschutz/, vom 08.08.2003

BMVEL (1995-2001): Jahresbericht über Flurbereinigung und agrarstrukturelle Vorplanung in Deutschland, Eigenverlag

BMVEL (1998): Gute fachliche Praxis im Pflanzenschutz, Bonn

BMVEL (2002): Agenda 2002. Pflanzlicher Bereich Agrarumweltmaßnahmen, Bonn

BMVEL (2002a): Neue Fördermöglichkeiten für umwelt- und tiergerechte Landwirtschaft, Sonderausgabe vom 23.12.2002, Bonn

BMVEL (2003): Ernährungs- und agrarpolitischer Bericht der Bundesregierung, Berlin

BöHrnsen, A. U. Tebrügge, F. (1993): Maschinenkosten senken - Direktsaat oder Pflug, Grubber oder Frässaat? Agrar-Übersicht, Heft 8, S. 18-21

BORCHERT, J. (1987): Hecken und Feldgehölze. Ihre Funktion im Natur- und Landschaftshaushalt. In: BUNDESANSTALT FÜR NATURSCHUTZ UND LANDSCHAFTSÖKOLOGIE (1988): Dokumentation für Umweltschutz und Landespflege. 28. Jahrgang, Sonderheft 9, Bibliographie Nr. 53, Deutscher Gemeindeverlag, Köln

Bosenius, U. (2001): Die Wasserrahmenrichtlinie. Wasser und Boden, Jg. 53, Heft $1 \mathrm{u}$. 2, S. $27-32$

BR-DRUCKS. 702/96, S.87

Brand-Sassen, H. U. Steinmann, H.-H. (2002): Betriebswirtschaftliche Charakterisierung der Anbausysteme. In: SteInMANN, H.-H. et al. (2002): Integrierte Ackerbausysteme in Versuch und Praxis. Ergebnisse aus dem Göttinger INTEXProjekt und seinen Demonstrationsflächen, Mecke-Druck, Duderstadt, S.82-96

Brodowski, N. U. Dörendahl, A. (2001): Untersaaten in Winterweizen in Abhängigkeit von Sortenwahl und Herbizideinsatz, Forschungsberichte des Fachbereichs Agrarwirtschaft Soest, Universität -Gesamthochschule- Paderborn, Nr. 12, Paderborn

BRUNOTTE, J. (2003): Handlungsempfehlungen zur guten fachlichen Praxis: Bodenerosion mindern, Bodenleben fördern. In: ARTMAnN, R. u. Bockisch, F.- J. :Nachhaltige Bodennutzung - aus technischer, pflanzenbaulicher, ökologischer 
und ökonomischer Sicht, Landbauforschung Völkenrode, Sonderheft 256, Braunschweig, S. 79-86

BRUnOtTE, J. et al. (1999): Bodenschonung und Kosteneinsparung. Einführung technischer Lösungskonzepte zur Minderung und Vorbeugung von Bodenschutzproblemen in der Pflanzenproduktion. FuE-Vorhaben 95 UM 03, KTBL, Darmstadt

BRUnOtTe, J. et al. (2000): Zur guten fachlichen Praxis beim Einsatz moderner Zuckerrüben-Erntetechnik. Zuckerrübe, 49. Jg., Heft 1, S. 34-40

Brunotte, J., Roth, C. H. U. Hollmann, P. (1994): Einzelbetrieblicher KostenNutzen-Vergleich. In: KTBL: Einführung von Verfahren der konservierenden Bodenbearbeitung in die Praxis, Darmstadt

BSTMLF (2001): Auswirkungen von landwirtschaftlichen Maschinen mit hohen Radlasten auf die Funktionsfähigkeit bayerischer Ackerböden, Gelbes Heft 74, München

BT- DRUCKS. 13/6701, S. 29

BT-DRUCKS. 13/7891

BT-DRUCKS. 13/7778, S. 85

BT-DRUCKS. 14/9852, S. 141

Buhse, J. (2003): Phosphordünger immer einarbeiten, Der Hessenbauer, Heft 15, S. 2123

BundeSREgIERUNG (2000): 2. Bericht gemäß Artikel 10 der Richtlinie 91/676/EWG des Rates vom 12. Dezember 1991 zum Schutz der Gewässer vor Verunreinigungen durch Nitrat aus landwirtschaftlichen Quellen. Internet: www.bmu.de/files/nitratrichtlinie.pdf am 11.8.03

BUNDESVERBAND BODEN (2003): Handlungsempfehlungen zur Gefahrenabwehr bei Bodenerosion, Internet: $\quad$ www.bvboden.de/downloads/BVB Gefahrenabwehr Erosion $13 \quad 10$ 03.pdf, vom 11.10.2003

CielejewsKi, H. (2003): Schweinegülle - Homogenisieren und Ausbringen. Internet: www.lk-wl.de/technik/schweineguelle.htm, am 6.6.03

Clark, C. et al. (1985): Eroding Soils. The off-farm impacts. The conservation foundation, Washington 
CzeratzKI, W. (1972): Die Ansprüche der Pflanzen an den physikalischen Bodenzustand. Landbauforschung Völkenrode, Heft 22, S. 29-36

DAMMER, S. (2002): Große Flächenleistung, aber hohe Auslastung nötig. Top agrar spezial, Heft 2, S. 12-14

DierCKS, R. u. HeitefuSS, R. (1990): Integrierter Landbau, BLV-Verlag, München

DIEz, T. (1990): Erosionsschäden vermeiden, AID-Heft 1108, Bonn

Diez, T. U. Hege, U. (1980): Pflanzenbauliche Maßnahmen zur Verminderung der Bodenerosion. Landwirtschaftliche Forschung, Sonderheft 37, Sauerländers Verlag, Frankfurt, S. 122-128

Doleschel, P. U. Heißenhuber, A. (1991): Externe Kosten der Bodenerosion. Bayerisches Landwirtschaftliches Jahrbuch, Jg. 68, S. 187-209, Verlag Kastner, Wolzach

DOMroese et al. (2001): Pflanzenschutzmittel im Grundwasser, Wasser und Boden, Heft 10, S. $35-38$

Dürr, H. J., Petelkau, H., Sommer, C. (1995): Literaturstudie „Bodenverdichtung““ Reihe: Texte des Umweltbundesamtes Nr. 55/95, Berlin

DVWK (1996): Bodenerosion durch Wasser - Kartieranleitung zur Erfassung aktueller Erosionsformen. Merkblätter zur Wasserwirtschaft 239, Verlag Paul Parey, Hamburg

Ehlers, W. (1996): Wasser in Boden und Pflanze. Dynamik des Wasserhaushalts als Grundlage von Pflanzenwachstum und Ertrag, Verlag Ulmer, Stuttgart

EHLERS, W. (1999): Breitreifen wirken nicht Wunder. Bauernzeitung, Heft 2, S. 21-23

Ehlers, W., SChMidTKe, K. U. RAUber, R. (2003): Änderung der Dichte und Gefügefunktion südniedersächsischer Lössböden unter Ackernutzung. Landnutzung und Landentwicklung, Heft 1, S. 9-18

EhLers, W., Werner, D. U. Fenner, S. (1994): Reconsolidation of a deep-loosened silt loam under plowing and conservation tillage, ISTRO-Conference, Proc. of $13^{\text {th }}$ International Conference, Aalborg, Denmark, S. 55-60 
EHRHARD, M. et al. (2002): Bundesweite Betrachtung der Zusammenhänge zwischen Agrarstatistikdaten und aktuellen Daten der Bodenschätzung. Umweltbundesamt, Forschungsbericht 20071 247, Berlin

EUROPÄISCHE KOMMISSION (2002): Towards a thematic strategy for soil protection. Internet: $\quad$ http://www.europa.eu.int/eur-lex/en/com/pdf/2002/com 2002 0179en01.pdf

FELDWISCH, N. (1999): Einflüsse land- und forstwirtschaftlicher Maßnahmen auf den Hochwasserabfluss - Wissensstand, Skalenprobleme, Modellansätze. Materialien des DVWK, 7/1999, Bonn

FELDWISCH, N. (2002): Einflüsse land- und forstwirtschaftlicher Maßnahmen auf den Hochwasserabfluss kleiner Einzugsgebiete. Wasserwirtschaft, Abwasser, Abfall, 49. Jg., Heft 3, S. 355-361

FeldWisCH, N. U. BARKOWSKI, D. (2003): Gebietsbezogener Bodenschutz. Reihe Bodenschutz und Altlasten, Band 13, Erich-Schmidt-Verlag, Berlin

FiSCHER-HüfTLE et al. (2003): Bundesnaturschutzgesetz. Kommentar. Verlag Kohlhammer, Stuttgart

FLEIGE, H. (1999): Ökologische und Ökonomische Bewertung der Bodenerosion am Beispiel einer Jungmoränenlandschaft Ostholsteins. Dissertation, Institut für Pflanzenernährung und Bodenkunde, Kiel

Fleige, H., Horn, R. U. WeißBACH, M. (1999): Bodenerosion in Fahrspuren und mögliche Erosionsschutzmaßnahmen. Wasser und Boden, Jg. 51, Heft 12, Blackwell, Berlin, S. 33-36

Frede, H. G. u. DABbert, S. (1998): Handbuch zum Gewässerschutz in der Landwirtschaft. Ecomed-Verlag, Landsberg

Frenz, W. (2000): Bundes- Bodenschutzgesetz. Kommentar, Verlag C. H. Beck, München

Friedrich, G. et al. (2001): Penetrometermessungen zur Erfassung von Bodenverdichtungen, Befragung über Bodenverdichtungen und Bodenschutz in ausgewählten Regionen in NRW. In: SchadVERDichtUNGEN IN ACKERBÖDEN - ENTSTEHUNG, Folgen, GegenmaßnAhmen. Tagungsband zur 14. Wissenschaftlichen Fachtagung der landwirtschaftlichen Fakultät der Universität Bonn, Teil 2, Bonn 
FRIELINGHAUS (1998a): Bodenbearbeitung und Bodenerosion. In: KTBL: Bodenbearbeitung und Bodenschutz. Schlussfolgerungen für die gute fachliche Praxis, Arbeitspapier 266, Darmstadt

Frielinghaus, M. (2003): Aufgabe der Wissenschaften für den Bodenschutz. Bodenschutz, Editorial, Heft 3, Erich-Schmidt-Verlag, Berlin

Frielinghaus, M. (1998): Bodenschutzprobleme in Ostdeutschland. In: Richter, G. (1998): Bodenerosion. Analyse und Bilanz eines Umweltproblems, Wissenschaftliche Buchgesellschaft, Darmstadt

Frielinghaus, M. (2002b): Bewertung der guten fachlichen Praxis im Bodenschutz. In: Studiengang Bodenwissenschaften DeR FH OsnabRÜCK (Hrsg.): Diskussionsforum Bodenwissenschaften: Vorsorgender Bodenschutz, Stand und Perspektiven der „guten fachlichen Praxis“ für die landwirtschaftliche Bodennutzung, Heft 3, Osnabrück

Frielinghaus, M. et al. (2001): Vorsorge gegen Bodenerosion. In: BMVEL (2001): Gute fachliche Praxis zur Vorsorge gegen Bodenschadverdichtungen und Bodenerosion, Bonn

Frielinghaus, M. ET AL. (2002a): Informationsheft zum landwirtschaftlichen Bodenschutz in Brandenburg. Teil Bodenerosion, Ministerium für Landwirtschaft, Umweltschutz und Raumordnung, Potsdam

Frielinghaus, M. U. SAttler, C. (2002): Bodenerosion vermeiden. Geeignete Schutzmaßnahmen zur Reduktion von Bodenverlusten durch Wind- $\mathrm{u}$. Wassererosion auf landwirtschaftlichen Nutzflächen, Infoblatt 3. GRANO-Projekt, ZALF und Ministerium für Landwirtschaft, Umweltschutz und Raumordnung Brandenburg, Frankfurt (Oder)

Frielinghaus, M.; Beese, F.; Ellerbrock, R. (1999): Risiken der Bodennutzung und Indikation von schädlichen Bodenveränderungen in der Gegenwart. In: BEESE, F. (1999): Schutz des Bodens, Economica-Verlag, Berlin

Fromм, O. (1997): Möglichkeiten und Grenzen einer ökonomischen Bewertung des Ökosystems Boden. Europäische Hochschulschriften, Verlag Peter Lang, Frankfurt 
Gall, H., Friesleben, R. u. Pesch, H. (1985): Schlagbezogene Pflanzbettvorbereitung - Fundament jeder Höchstertragskonzeption für Kartoffeln. Feldwirtschaft, Heft 26, S. $25 \mathrm{ff}$.

GIESKA et al. (2003): Physikalische Bodendegradierung in der Hildesheimer Börde und das Bundes-Bodenschutzgesetz. Berichte über Landwirtschaft 81, Heft 4, S. 485511

Grahlmann, G. (1986): Wirkungen von Gehölzstreifen auf den Ertrag landwirtschaftlicher Kulturpflanzen. VDLUFA-Kongressband 1986, VDLUFA-Verlag, Darmstadt

GunREBEN, M (2002): Erfahrungen der Klärschlammverwertung in Deutschland. Internet: www.uan.de/projekte/Gunreben_D.pdf vom 22.01.2004

GunReben, M. et al. (2003): Bodenqualitätskonzept Niedersachsen. Teil 1: Bodenerosion und Bodenversiegelung. Niedersächsisches Landesamt für Ökologie, Hannover

GuTSCHE, V. (2002): Vergleich des Risikopotenzials bei der Anwendung alter und neuer Pflanzenschutzmittel. Beitrag auf der DLG-Wintertagung 2002. Internet: http://www.dlg-frankfurt.de/pdf/wita2002/gutsche2002 .pdf

Gutser, R. u. Ebertseder, T. (2002): Definieren die Grundsätze der Düngeverordnung eine gute fachliche Düngepraxis ausreichend konkret? Defizite und Verbesserungsvorschläge aus Sicht der Wissenschaft. Beitrag auf der DLGWintertagung 2002. Internet: http://www.dlg-frankfurt.de/pdf/ wita2002/gutser2002.pdf

Hakansson, I. UND ReEDER, R. C. (1994): Subsoil compaction by vehicles with high axle load - extent, persistance and crop response. Soil \& Tillage Research 29, S. 277-304

HANuS, H. (1990): Bearbeitung und Verdichtung von Böden. In: Blume, H. P. (1990): Handbuch des Bodenschutzes, ecomed, Landsberg/Lech, S. 138-164

HanUSCH, H. (1994): Nutzen-Kosten-Analyse, 2. Auflage. Verlag Vahlen, München

HaRTGE, K. H. u. HoRn, R. (2002): Gedanken zum derzeitigen Verdichtungszustand von Ackerböden und zur Nachhaltigkeit ihrer Nutzung. Wasser und Boden, Heft 10, Blackwell-Verlag, Berlin, S. 34-38 
HaRtmann, R. (2003): Studien zur standortgerechten Kompostanwendung auf drei pedologisch unterschiedlichen, landwirtschaftlich genutzten Flächen der Wildeshauser Geest, Niedersachsen. Dissertation, Bremen

Hassenpflug, W. (1998). Bodenerosion durch Wind. In: Richter, G. (1998): Bodenerosion. Analyse und Bilanz eines Umweltproblems, Wissenschaftliche Buchgesellschaft, Darmstadt

Heißenhuber, A., u. Schmidtlein, E.-M. (1988): Ökologische und Ökonomische Probleme der Bodenerosion. Bayerisches Landwirtschaftliches Jahrbuch, Sonderheft 1, BLV-Verlag, München

Helal et al. (1994): Auswirkungen von Bodenverdichtungen auf die Wurzelfunktionen und den Nährstoffhaushalt. In: SOMMER et al. (1994a): Bodenverdichtung und Pflanzenertrag - ein Konzept für bodenschonendes Befahren, Landbauforschung Völkenrode, Sonderheft 147, Braunschweig-Völkenrode

HELMER, H. (2004): Wasserschutz bedroht intensive Viehhaltung. Landwirtschaftsblatt Westfalen-Lippe, Heft 7, S. 18-19

Herrmann, S. (1998): Immer wieder Raupen. Neue Landwirtschaft, Heft 9, S. 80-83

Höfflinger, W. u. PokriefKe, M. (1995): Weniger Bodenverdichtung durch Raupenfahrwerke? In: KTBL (1995): Bodenverdichtung, KTBL-Schrift 362, S. 124-128

Hollmans, F. (2003): Ökonomische Bewertung pflugloser Anbauverfahren im nationalen und internationalen Vergleich. In: ArtmanN, R. u. BockisCH, F.- J.: Nachhaltige Bodennutzung - aus technischer, pflanzenbaulicher, ökologischer und ökonomischer Sicht, Landbauforschung Völkenrode, Sonderheft 256, Braunschweig, S. 97-100

Holzwarth, F., Radtke, H., Hilger, B. (2000): Bundes- Bodenschutzgesetz/Bundes- Bodenschutz- und Altlastenverordnung. Handkommentar, Erich Schmidt Verlag, 2. Auflage, Berlin

HopPE, U. (1996): Pflanzenschutzmitteleinsatz in der Landwirtschaft aus ökonomischer und ökologischer Sicht: Ein Entscheidungsunterstützungs-Modell zur agrarpolitischen Ziel-Mittel-Analyse auf regionaler Ebene. Dissertation, Agrarwirtschaft, Sonderheft 157, Agri-Media, Holm 
HorN et al. (2000): Subsoil compaction. Advances in Geoecology 32, Catena Verlag, Reiskirchen

HoRN, R. (1999): Unterbodenverdichtungen - Gibt es gesicherte Hinweise auf Ertragseinbußen? Wasser u. Boden, Jg. 51, S. 15-18

HoRN, R. (2001): Die Verformung von Böden - Ursachen und Folgen für eine nachhaltige Landnutzung. In: BRÜMMER, G. W.: Schadverdichtungen in Ackerböden Entstehung, Folgen, Gegenmaßnahmen, Tagungsband zur 14. Wissenschaftlichen Fachtagung der landwirtschaftlichen Fakultät der Universität Bonn, Teil 2, Bonn

HORN, R. (2002): Mechanische Belastung und Bodenverformung. In: SchEFFER, F. (2002): Lehrbuch der Bodenkunde, 15. Auflage, Spektrum Akademischer Verlag, Berlin, S. 425-430

HoRN, R. (2003): Die Böden nehmen kein Wasser mehr auf. Frankfurter Allgemeine Zeitung vom 15.12.03, S. 9

HüBleR, K.- H., U. SChablitzKI, G. (1991): Volkswirtschaftliche Verluste durch Bodenbelastung in der BRD. Berichte 10/91 des Umweltbundesamtes, Erich Schmidt-Verlag Berlin

HundSDORFER, M. (1988): Kostendatei für Maßnahmen des Naturschutzes und der Landschaftspflege. Materialien 55, Bayerisches Staatsministerium für Landesentwicklung u. Umweltfragen, München

IEEP (2003): Background paper Developing cross-compliance in the EU. Internet: http://www.ieep.org.uk/research/Cross $\% 20$ Compliance/Project $\% 20$ outputs $\% 20$ and $\% 20$ available $\% 20$ documents.htm, vom 5.2.04

IRLA, E. (1991): Vergleich mechanischer und chemischer Unkrautbekämpfungsverfahren im Getreide. FAT-Bericht 398, Zürich

ISENSEE, E. (2003): Bodenschonung mit moderner Technik. In: ARTMANN, R. u. BoCKISCH, F.- J: Nachhaltige Bodennutzung - aus technischer, pflanzenbaulicher, ökologischer und ökonomischer Sicht, Landbauforschung Völkenrode, Sonderheft 256, Braunschweig, S. 61-72

ISENSEE, E. (2004): Volle Breitseite. Landwirtschaftliches Wochenblatt Weser-Ems, Nr. 7, S. $30-31$ 
ISENSEE, E. U. WEIßBACH, M. (1998): Gummiband-Laufwerk für Schlepper. Lohnt sich der Einsatz? Landwirtschaftsblatt Schleswig-Holstein, S. 44-50

JAgGard, K., Clark, C. U. Bell, S. (1984): An Analysis of yield from fields of sugar beet. Britisch Sugar Beet Review, 52, S. 67-69

JAROSCH, J. U. SCHWitTeK, U. (1991): Ökonomische Bewertung der Wiedereinführung landschaftsökologischer Strukturelemente in landwirtschaftlich genutzten Landschaften. Zeitschrift für Kulturtechnik und Landentwicklung, Verlag Paul Parey, Berlin

JAROSCH, J. U. ZEDDIES, J. (1994): Bodenerosion - Ökonomische Aspekte von Schäden und Schutzmaßnahmen. Berichte über Landwirtschaft, 205. Jg., Sonderheft Bodennutzung und Bodenfruchtbarkeit, S. 99-116

KirChNER, M. (2003): Passt Mulchsaat zu Ihnen? DLG-Mitteilungen, Heft 11, Frankfurt, S. 56-57

KLINGEBIEL, L. (2003): Wachstum und räumliche Ausbreitung der Tauben Trespe (Bromus sterilis L.) unter dem Einfluss von Unkrautbekämpfung und Bodenbearbeitung und Untersuchungen zur Anwendung von Satellitenortung bei der Unkrautkartierung. Masterarbeit, Göttingen

Klotz, F. U. HAAG, U. (2001): Systemvergleich Bodenbearbeitung, Versuchsbericht 1995-1999. Informationen für die Pflanzenproduktion, Sonderheft 2, Landesanstalt für Pflanzenbau Forchheim, Eigenverlag

KNAUER, N. (1990): Hecken sind besser als ihr Ruf. DLG-Mitteilungen/agrar inform, Heft 20, S. 52-54

KNOPP, L. U. LöHR, D. (2000): Bundes-Bodenschutzgesetz in der betrieblichen und steuerlichen Praxis, Heidelberg

KoBES, W. (1998): Das Bundes-Bodenschutzgesetz. Neue Zeitschrift für Verwaltungsrecht, Verlag Beck, München, S.786-797

KöHN, O. (2002): Die Ausweisung von Schutzgebieten und Ausgleichsregelungen für die Landwirtschaft - Erfahrungen im Wasserschutz und Empfehlungen für den Naturschutz. Dissertation, Cuvillier-Verlag, Göttingen 
KöHne, M. (2000): Landwirtschaftliche Taxationslehre, 3. Auflage, Parey-Buchverlag, Berlin

KöLleR, K. U. LiNKe, C. (2001): Erfolgreicher Ackerbau ohne Pflug. Wissenschaftliche Ergebnisse - Praktische Erfahrungen. 2. Auflage, DLG-Verlag, Frankfurt am Main

KöNIGER, S. U. SCHWAB, A. (2001): Einschätzung der Erosionsgefährdung mittels GIS. Schule und Beratung, Heft 13/01, S. IV 4-7

KowalewsKy, H.-H. (1996): Behandlung und Ausbringung von Flüssigmist. AID-Heft 1201, 2. Auflage, Bonn

KoWALEWSKY, H.-H. (2004): Breitverteiler dominieren den Markt. Land und Forst, Heft 4, S. 20-23

KTBL (1998): Datensammlung Landschaftspflege. 4. Auflage, Darmstadt

KTBL (1998a): Bodenbearbeitung und Bodenschutz. Schlussfolgerungen für die gute fachliche Praxis, Arbeitspapier 266, Darmstadt

KTBL (2000): Organische/mineralische Abfälle und Wirtschaftsdünger. Datenbank, CDROM, KTBL, Darmstadt

KTBL (2000a): Zur Kalkulation des Grundzeitbedarfs für unregelmässige Parzellenformen. Internet: http://www.ktbl.de/manag/arbeit/gz_help.htm vom 29.10.2003

KTBL (2000b): Definition und Einordnung der Einzelkornsaat. Arbeitsblatt 262, Landwirtschaftsverlag, Münster

KTBL (2002): Datensammlung Betriebsplanung Landwirtschaft 2002/2003. 18. Auflage, Darmstadt

KTBL (2002a): Taschenbuch Landwirtschaft 2002/2003. 21. Auflage, Darmstadt

KTBL (2002b): Preise für Leihmaschinen und Dienstleistungen. Internet: http://www.ktbl.de/manag/koop/umv_satz99.htm

Kuhlmann, F. ET AL. (1995): Einsatzmöglichkeiten und Absatzpotentiale von Biokompost in der Landwirtschaft - Modellberechnungen in vier Landkreisen Hessens. Abschlußbericht zum Projekt „Umweltverträgliche Anwendung von Bioabfallkompost in der Landwirtschaft", Betriebswirtschaftlicher Teil, unveröffentlicht 
LABO U. LAWA (2000): Abgrenzung zwischen Bundes- Bodenschutzgesetz und Wasserrecht. Internet: www.hamburg.de/Behoerden/Umweltbehoerde/ labo/dokument/abgrenz.pdf am 16.1.03

Landesanstalt für Pflanzenbau (2002): Umweltschonender Maisanbau. Merkblätter für die umweltgerechte Landbewirtschaftung, Nr. 23, Rheinstetten

LANDESANSTALT FÜR UMWELTSCHUTZ (2003): Prognose von Bodenerosion, Internet: http://www.xfaweb.baden-wuerttemberg.de/bofaweb/berichte/mzb04/ mzb04_c.htm

LANDWIRTSCHAFTSKAMMER HANNOVER (2002): Richtwertdeckungsbeiträge 2002 der Landwirtschaftskammer Hannover. Sonderheft B/2002, Hannover

LANDWIRTSCHAFTSKAMMER HANNOVER U. WESER-EMS (2003): Berechnungsgrundlagen für Ausgleichsleistungen in Wasserschutzgebieten gemäß $§ 51$ a NWG (sog. Blaubuch). Hannover u. Oldenburg

LANDWIRTSCHAFTSKAMMER WESER-EMS (2001): Jahresbericht 2001, Oldenburg

LATACZ-LOHMANN, U. (1993): Auswirkungen von Immissionsbelastungen in landwirtschaftlichen Betrieben (Methodik und Beispiele für Entschädigungsermittlungen). In: KlOKe, A., BirK, H.-J. u. LATACZ-LOHMANN (1993): Immissionen und Altlasten in der Landwirtschaft. HLBS-Schriftenreihe, Heft 137, Verlag Pflug u. Feder, St. Augustin

LataCZ-Lohmann, U. U. BUCKWEll, A. (1998): Einige ökonomische Überlegungen zu „Cross Compliance“. Agrarwirtschaft 47, Heft 11, S. 429-431

LATTEn, R. (1998): Landwirtschaft und Bodenschutz. In: BundESVERBAND Boden (1998). Bodenschutz und Bodenschutzrecht. Beiträge zur Jahrestagung des BVB 1998, Berlin

LAWA (2002): Gemeinsamer Bericht von LAWA und LABO zu Anforderungen an eine nachhaltige Landwirtschaft aus Sicht des Gewässer- und Bodenschutzes vor dem Hintergrund der Wasserrahmenrichtlinie, Hannover

LindstroM, M. J. u. Voorhees, W. B. (1994): Responses of Temperate Crops in North America to Soil Compaction. In: SoAne, B. D. u. VAn Ouwerkerk (1994): Soil Compaction in Crop Production, Elsevier, Amsterdam, S. 265-286 
Luder, W., Stark, R. U. Ammann, H. (2001): Zuckerrüben: Erntemanagement und kosten. Schwere Erntemaschinen gut auslasten, ohne die Böden zu verdichten, FAT-Berichte, Nr. 568, Ettenhausen, Schweiz

LÜTKe-EnTRUP, N. (2001): Zwischenfrüchte im umweltgerechten Pflanzenbau. AIDHeft 1060, Verlag Th. Mann, Gelsenkirchen

MANnheiM, T. (1996): Ammoniakemissionen von landwirtschaftlichen Nutzflächen Ursachen und Minderungsmaßnahmen. Dissertation, Stuttgart-Hohenheim

Melsa, A. (2002): Neubewertung der landwirtschaftlichen Klärschlammverwertung unter Berücksichtigung des Boden- und Verbraucherschutzes. Beitrag auf der DLG-Wintertagung 2002. Internet: http://www.dlgfrankfurt.de/pdf/wita2002/melsa2002.pdf

MeYeR, K. (2003): Viel Boden bewegt. Landwirtschaftliches Wochenblatt SchleswigHolstein, Heft 42, S. 21-23

Meyerhoff, J. U. DehnhardT, A. (2002): Nachhaltige Entwicklung der Elbe. In: Ökologisches Wirtschaften, Heft 5, S. 27-28

Meyer-RoschaU, J. (1994): An- und Durchschneidungsschäden auf Ackerflächen. Die Novellierung der Datengrundlage und Vorschläge zur Verbesserung der Entscheidungsmethodik. Schriftenreihe des HLBS, Heft 144, St. Augustin

Mishan, E. J. (1975). Elemente der Kosten-Nutzen-Analyse. Campus Verlag, Frankfurt, New York

MLUR BRANDEnBURG (2003): Ergebnisse aus dem Landespilotprojekt „Reduzierte Bodenbearbeitung“", Frankfurt/Oder

MönICKE, R. U. KÜHN, I. (2003): Klärschlamm und Bodenschutz - ein Widerspruch? Bauernzeitung Sachsen-Anhalt, Heft 34, Berlin

Mosimann, T. (1995): Schätzung der Bodenerosion in der Praxis und Beurteilung der Gefährdung der Bodenfruchtbarkeit durch Bodenabtrag. In: RosEnKRANZ et al. (1988): Bodenschutz. Ergänzbares Handbuch, Loseblattsammlung, ErichSchmidt-Verlag, Berlin

MüHLENKAMP, H. (1994): Kosten-Nutzen-Analyse. Oldenbourg Verlag, München 
MüLLER, C. (2002): Die gute fachliche Praxis der landwirtschaftlichen Bodennutzung im Bundes-Bodenschutzgesetz. Agrarrecht, 32. Jahrgang, Heft 8, Landwirtschaftsverlag, Münster-Hiltrup, S. 237-241

N. N. (1998): Klärschlammeinsatz mit Bedacht. Neue Landwirtschaft, Heft 8, S. 51-53

N. N. (2001): Bodenschutz in Niedersachsen. In: NIEDERSÄCHSISCHES UMWELTMINISTERIUM (2002): Bodenschutz in Niedersachsen, Hannover

Neubauer, W. U. HÄndel, K. (2002): Erfolgreicher Pflugverzicht. Bauernzeitung Sachsen-Anhalt, Heft 34, S. 18-21

NigGESCHULZE, W. (2003): Bodenbearbeitung im Umbruch? Landwirtschaftliches Wocheblatt Westfalen-Lippe, Heft 48, S. 22-24

NitSChE et al. (2000): Minderung des P-Abtrags von Ackerflächen durch konservierende Bodenbearbeitung. Mittlg. Bodenkdl. Gesellschaft, Heft 92, S. 178-181

Oerke, E. C. u. Steiner, V. (1996): Ertragsverluste u. Pflanzenschutz; die Anbausituation für die wichtigsten Kulturpflanzen. Mitteilungen aus der Biologischen Bundesanstalt, Heft 301, Blackwell-Verlag, Berlin

OLFE, G. U. SCHÖN, H. (1986): Bodenbelastung durch Schlepper- und Maschineneinsatz in der Landwirtschaft. KTBL-Schrift 308, Darmstadt

Peine, F.-J. (2002): Landwirtschaftliche Bodennutzung und Bundes-Bodenschutzgesetz. Natur und Recht, Heft 9, Blackwell, Berlin

Peine, F.-J. (2003): Die Kritik am Bundes-Bodenschutzgesetz - nach fünf Jahren revisited. Umwelt- u. Planungsrecht, Heft 11+12, Jehle Rehm, München

Peters, U. (2001): Ökonomische Beurteilung der pfluglosen Bodenbewirtschaftung. Bauernblatt Schleswig-Holstein und Hamburg, 24.11.01, S. 39-41

Peyker, W., Henkel, K. U. Hochberg, H. (2000): Umsetzung des Maisengreihenanbaus im Einzugsgebiet Trinkwassertalsperrensystems Weida-ZeulenrodaLössau. Thüringer Landesanstalt für Landwirtschaft, Jena

PfaHLER, K. (1986): Einfluss der Hangneigung auf die Verfahrenstechnik am Beispiel des Anbaus von Getreide und Körnermais - eine Grundlage für die Bewertung hängiger Flurstücke. Dissertation, Institut für Landtechnik Weihenstephan 
Pimentel, K. et al. (1995): Environmental and economic cost of soil erosion and conservation benefits. Science, Vol. 267, S. 1117-1123

PlankL, R. (1999): Honorierung ökologischer Leistungen - Erfahrungen mit dem USamerikanischen „Conservation Reserve Program“ (CRP). Landbauforschung Völkenrode, 201, S. 163-175

PretTy, J. et al. (2002): Externe Kosten in der Landwirtschaft - Herausforderungen für die Politik. Ökologie und Landbau, Jg. 122, Heft 2, S. 19-24

Quas, M., SChwieger, H. u. WeißBaCh, M. (1995): Bodenschonende Fahrwerke an Arbeitsmaschinen. Forschungsbericht Agrartechnik des Arbeitskreises Forschung und Lehre der Max-Eyth-Gesellschaft Agrartechnik im VDI, Nr. 279, Selbstverlag, Kiel

Quicker, P. u. Faulstich, M. (2002): Kosten der Klärschlammentsorgung. Wasser u. Boden, Heft 6, Blackwell Verlag, Berlin, S. 22-27

SRU (1996): Sondergutachten: Konzepte einer dauerhaft-umweltgerechten Nutzung ländlicher Räume. Metzler-Poeschel, Stuttgart

RATHE, A. (1998): Qualitätsziele und -standards für Bodenerosion in Niedersachsen Grundlagen für ein Bodenqualitätskonzept. Diplomarbeit Universität Hannover, unveröffentlicht

RHG (2000): Pflanzenschutzmittel-Preisliste, Hannover

RiChTER, G. (1965): Bodenerosion. Schäden und gefährdete Gebiete in der Bundesrepublik Deutschland. Text und Kartenband. Forschungen zur deutschen Landeskunde. Bd. 152, Bad Godesberg

Richter, G. (1998). Bodenerosion und Kulturlandschaft. In: Richter, G. (1998). Bodenerosion: Analyse und Bilanz eines Umweltproblems, Wissenschaftliche Buchgesellschaft, Darmstadt

Rode, M. (1995): Quantifizierung der Phosphorbelastung von Fließgewässern durch landwirtschaftliche Flächennutzung. In: INSTITUT FÜR LANDESKULTUR: Boden und Landschaft. Band 1, , Gießen 
Roth, D. U. BERGER, W. (1999): Kosten der Landschaftspflege im Agrarraum. In: KoNOLD, W., BöcKeR, R. U. HAMPICKE, U. (1999): Handbuch Naturschutz und Landschaftspflege, Ecomed, Landsberg

SÄCHSISCHE LANDESANSTALT FÜR LANDWIRTSCHAFT (2001): Umweltgerechte Landwirtschaft im Freistaat Sachsen. Ergebnisse der Begleitung und Bewertung in der Förderperiode 1994 bis 1999, Schriftenreihe der LfL, 6. Jg., Heft 1, Eigenverlag, Leipzig

SATTLER, C. (2002): Kleinräumige Stilllegung von Minderertragsflächen. Ein ökonomisch tragfähiges Konzept zur Entwicklung und zum Schutz von Lebensräumen innerhalb von Ackerflächen. Infoblatt 1 GRANO-Projekt, ZALF und Ministerium für Landwirtschaft, Umweltschutz und Raumordnung Brandenburg, Frankfurt (Oder)

SAUPE, G. (1992): Wirkung von Konturgrasstreifen zur Erosionsbekämpfung unter Praxisbedingungen. Zeitschrift für Kulturtechnik und Landentwicklung, 33, Blackwell-Verlag, Berlin, S. 150-163

SCHACH, P. (1987): Bewertung von Erosionsschutzmassnahmen. In: GEWISOLA: Schriften der Gesellschaft für Wirtschafts- und Sozialwissenschaften des Landbaues e. V., Band 23, Landwirtschaftsverlag Münster-Hiltrup, S. 317-325

SCHÄFER, B. (2004): Landwirtschaft nach 2006 in Deutschland. Handlungsfelder aus pflanzenbaulicher Sicht. Agrarforum der FH Soest. Internet: www.fhsoest.de/fb9/agrarforum/09.01.2004/Schaefer.pdf

SCHEFFER, P., Schachtschabel, P. u. Auerswald, K. (1998): Lehrbuch der Bodenkunde. Enke-Verlag, Stuttgart

SCHENKEL, W. (2000): Nachhaltige Bodennutzung in Deutschland - Herausforderungen und Perspektiven. In: BLuM W.E.H. ET AL (2000). Neue Wege zu nachhaltiger Bodennutzung. Eine Veranstaltung der Deutschen Bundesstiftung Umwelt zur EXPO 2000. Erich Schmidt Verlag, Berlin

SCHINDLER, M. (2003): Nur Kosten oder auch Gewinn? Land u. Forst, Heft 29, S. 26-29

SCHMidT, J. et al. (1999): Planung und Bemessung von Erosionsschutzmaßnahmen auf landwirtschaftlich genutzten Flächen. Wasser und Boden, 51. Jg., Heft 12, S. 19- 
SCHMIDT, R. (1991): Anthropogene Veränderung und Degradation landwirtschaftlich genutzter Böden in den Neuen Bundesländern Deutschlands. Zeitschrift für Kulturtechnik und Landesentwicklung, Heft 32, Blackwell, Berlin, S. 282-290

SCHMIDT, W.-A. (2002): Umsetzung der konservierenden Bodenbearbeitung in Sachsen. Abschlussbericht zum Forschungsvorhaben „Entwicklung von dauerhaft umweltgerechten Landbewirtschaftungsverfahren im sächsischen Einzugsgebiet der Elbe, LfL Sachsen, Leipzig

SCHMITT, K.-O. U. FiSCH, R. (2003): Wasser ist durch nichts zu ersetzen. Hessenbauer, Heft 50, S. 10-11

SChmitz, M. U. Hartmann, M. (1993): Landwirtschaft und Chemie. Simulationsstudie zu den Auswirkungen des Einsatzes von Mineraldünger und Pflanzenschutzmitteln aus ökonomischer Sicht. Wissenschaftsverlag Vauk, Kiel

SchmitZ, P. U. KISSLING, M. (1999): Diskussionsbeiträge: Nutzen-Kosten-Analyse des Pflanzenschutzmitteleinsatzes. Agrarwirtschaft 48, Heft 7, Deutscher Fachverlag, Frankfurt, S. 269-274

Schnug, E. U. Haneklaus,S. (2002): Landwirtschaftliche Produktionstechnik und Infiltration von Böden - Beitrag des ökologischen Landbaus zum vorsorgenden Hochwasserschutz. Landbauforschung Völkenrode 52, Heft 4, Braunschweig

SCHREIBER, A. (2002): Kompostverwertung in der Landwirtschaft. Handout zum gleichnamigen Verbund-Forschungsthema der DBU. Internet: http://www. unihohenheim.de/ apo420/publikationen/schreiber-handout .pdf am 19. 06.03

SChWERTMANN U. Vogl, W. (1985): Bodenerosion durch Wasser. VDLUFASchriftenreihe 16, Kongreßband 2, S. 65-68

Schwertmann, U., VogL, W. und Kainz, M. (1987): Die Vorausschätzung des Bodenabtrages durch Wasser in Bayern. Weihenstephan

SMEdDINCK, U. U. HogenMüLleR, D. (2000): Die „Landwirtschaftsklausel“ in der Entstehungsgeschichte des Bundes-Bodenschutzgesetzes. Zeitschrift für angewandte Umweltforschung, Jg. 13, Heft 3/4, S. 298-314

Sommer, C. (1974): Die Verdichtungsempfindlichkeit zweier Ackerböden. Ein Beitrag zum Verhalten von Böden unter vertikaler Belastung im Saugspannungsbereich 
bis pF 2,7. Dissertation TU Braunschweig, Landbauforschung Völkenrode, Sonderheft 24

SoMmer, C. (1998): Ein Konzept zur Vorbeugung von Bodenschadverdichtungen in der pflanzlichen Produktion, Bodenschutz, Heft 1, S. 12-16

Sommer, C. (2000): Ursachen und Folgen von Bodenverdichtungen sowie Möglichkeiten ihrer Verminderung. Landtechnik, 40. Jahrgang, Heft 9

Sommer, C. et al. (1994): Bodenverdichtung und Pflanzenertrag - ein Konzept für bodenschonendes Befahren. In: SOMMER, C. (1994): Belastung, Beanspruchung und Verdichtung von Böden durch landwirtschaftliche Maschinen und deren Auswirkungen auf Bodengefüge, Bodenorganismen und biologischen Prozesse sowie Pflanzenwachstum und Ertrag, Landbauforschung Völkenrode, Sonderheft 147, Braunschweig

SOMmer, C. et al. (1994): Einführung von Verfahren der konservierenden Bodenbearbeitung in die Praxis. FuE-Vorhaben 87 UM 01. Institutsbericht 222, FAL, Braunschweig

Sommer, C. et al. (1994a): Bodenverdichtung und Pflanzenertrag - ein Konzept für bodenschonendes Befahren. Landbauforschung Völkenrode, Sonderheft 147, Braunschweig-Völkenrode

Sommer, C., Brandhuber, R., Brunotte, J. U. BuChner, W. (2001): Vorsorge gegen Bodenschadverdichtungen. In: BMVEL (2001): Gute fachliche Praxis zur Vorsorge gegen Bodenschadverdichtungen und Bodenerosion, Eigenverlag, Bonn

Sommer, C., Lebert, M. U. BRUnotTe, J. (2003): Indikatoren zum physikalischen Bodenschutz - warum, wofür, wie?. In: DAV E. V. (2003): Umweltindikatoren Schlüssel für eine umweltverträgliche Land- und Forstwirtschaft, Schriftenreihe agrarspectrum, Band 36, DLG-Verlag, Frankfurt am Main, S. 175-186

SPAROVEK, G. (2002): A simple model to predict river floods: a contribution to quantify the significance of soil infiltration rates. Landbauforschung Völkenrode 52, Heft 3, Braunschweig

SRU (1996): Zur Umsetzung einer dauerhaft Entwicklung, Metzler-Poeschel, Stuttgart 
SRU (2004): Meeresumweltschutz für Nord- und Ostsee. Sondergutachten, NomosVerlag, Baden-Baden

Stahl, H., Schmidt, W. U. Gierke, U. (2001): Beratung zur guten fachlichen Praxis zum Schutz des Bodengefüges - Ansätze, Strategien, offene Fragen. In: BRÜMMER, G. W.: Schadverdichtungen in Ackerböden - Entstehung, Folgen, Gegenmaßnahmen, Tagungsband zur 14. Wissenschaftlichen Fachtagung der landwirtschaftlichen Fakultät der Universität Bonn, Teil 2, Bonn

STANGE, B. (2004): Das Geld hat oft nicht gereicht, DLG-Mitteilungen, Heft 4, S. 68-69

STATISTISCHES BUNDESAMT (2003): Umweltbeanspruchung rückläufig - Positive Signale jetzt auch bei der Flächennutzung, Pressemitteilung v. 6.11.2003, Wiesbaden

Steinmann, H.-H. (2002): Beschreibung der Standorte und Anbausysteme. In: STEINMANN, H.-H. et al. (2002): Integrierte Ackerbausysteme in Versuch und Praxis. Ergebnisse aus dem Göttinger INTEX-Projekt und seinen Demonstrationsflächen, Mecke-Druck, Duderstadt, S.8-14

STRotdReES, J. (2003): Landwirtschaftliche Nutzungskonzepte für Überschwemmungsgebiete im Kontext der Gewässerentwicklungsplanung Mittlere Leine. Landnutzung und Landentwicklung, Blackwell, Berlin, S. 129-133

TEBRÜGGE, F. (2003): Konservierende Bodenbearbeitung gestern, heute, morgen - von wendender über nicht wendende Bodenbearbeitung zu Direktsaat. In: ARTMANN, R. u. BocKISCH, F.- J. (Hrsg.): Nachhaltige Bodennutzung - aus technischer, pflanzenbaulicher, ökologischer und ökonomischer Sicht, Landbauforschung Völkenrode, Sonderheft 256, Braunschweig, S. 49-58

Teiwes, K. (1997): Standortbedingungen. In: GerowitT, B. U. Wildenhayn, M.: Ökologische und ökonomische Auswirkungen von Extensivierungsmaßnahmen im Ackerbau. Ergebnisse des Göttinger INTEX-Projekts 1990-94. Goltze, Göttingen

THÜRINGER LANDESANSTALT FÜR LANDWIRTSCHAFT (1999): Landwirtschaft und Landschaftspflege in Thüringen, Schriftenreihe, Heft 1, Jena

TiJink, F. G. J. (2003): Technical guidelines to avoid soil compaction in cropping systems with sugar beet. In: ARTMANN, R. u. BOKISCH, F.- J. (Hrsg.): Nachhaltige Bodennutzung - aus technischer, pflanzenbaulicher, ökologischer und ökonomi- 
scher Sicht, Landbauforschung Völkenrode, Sonderheft 256, Braunschweig, S. $39-49$

Tremel, S. (2000): Umweltcontrolling für landwirtschaftliche Unternehmen. Dissertation. Shaker-Verlag, Aachen

UMWELTBUNDESAMT (2002): Umweltdaten. Internet: www.envit.de/ umweltdaten, 10.6.03

UPPENKAMP, N. (2000): Dieselkosten: Wo können Ackerbauern sparen? Top agrar. Heft Nr. 11, S. 28-30

UPPENKAMP, N. (2003): Wann rechnet sich der Pflugverzicht? Top agrar, Heft 1, S.26-29

UPPENKAMP, N. (2003a): Angepasster Reifen. Geld sparen und Umwelt schonen. Mais, Heft 4, Verlag Th. Mann, Gelsenkirchen, S. 128-131

Versteyl, L.-A. U. Sondermann, W. D. (2002): Bundes- Bodenschutzgesetz. Kommentar, Verlag C. H. Beck, München

VolK, L. U. SCHNAPP, K. (2003): Richtige Reifenwahl ist praktizierter Bodenschutz. Mais, 31. Jg., Heft 4, S. 124-127

WAibel, H. U. Fleischer, W. (1998): Kosten und Nutzen des chemischen Pflanzenschutzes aus gesamtwirtschaftlicher Sicht, Verlag Vauk, Kiel

WeInS, C. (2001): Schritte zur Agrarwende: Die „gute fachliche Praxis“ konkretisieren. Natur \& Recht, Heft 4, Blackwell-Verlag, Berlin, S. 247-249

WeißBACH, M. (2002): Bodenschonung mit leistungsfähiger Technik. Zuckerrübe. 51. Jg., Heft 6, S. 306-308

Werbeck, N. U. Wink, R. (1994): Der Entwurf der Bundesregierung für ein BundesBodenschutzgesetz - Wegbereiter oder Hürde für einen effizienzorientierten Bodenschutz? Zeitschrift für angewandte Umweltforschung. Heft 3, Seite 403414

Werner, D., Reich, J. U. Degner, J. (1999): „Pflügen“ oder „Nicht Pflügen“. In: THÜRINGER MINISTERIUM FÜR LANDWIRTSCHAFT, NATURSCHUTZ UND UMWELT (Hrsg.): Einfluss der Großflächen-Landwirtschaft auf den Boden, Jena 
Werner, W. U. WODSAK (1994): Stickstoff- und Phosphateintrag über diffuse Quellen in Fließgewässer des Elbeeinzugsgebietes im Bereich der ehemaligen DDR. Schriftenreihe agrarspectrum, Band 19, Verlagsunion Agrar, Frankfurt

WiLhelM, J. (1999): Ökologische und ökonomische Bewertung von Umweltprogrammen. Reihe Europäische Hochschulschriften, Band 2542, Verlag Peter Lang, Frankfurt

Willmer, H. (2001): So einfach können Sie $10 \%$ Diesel sparen. Profi. Heft Nr. 12, S. 96-99

WoodrufF, et al. (1965): A wind erosion equation. Soil Sc. Proc., Vol. 29, new york

ZAPF, R. (1997): Mechanische Bodenbelastung durch die landwirtschaftliche Pflanzenproduktion in Bayern. In: Bodenkultur und Pflanzenbau, Heft 7, Bayerische Landesanstalt für Bodenkultur und Pflanzenbau, München

\section{Rechtsquellenverzeichnis:}

AMTSBLATT FÜR DEN LANDKREIS GOSLAR vom 27.08.2001, Nr. 13

BAUGESETZBUCH (BauGB) vom 23.06.1960 (BGBl. I, S. 341), zuletzt geändert am 23.07.2002 (BGB1. I, S. 2850)

BIOABFALlVERORDNUNG (BioAbfV): Verordnung über die Verwertung von Bioabfällen auf landwirtschaftlich, forstwirtschaftlich oder gärtnerisch genutzten Böden vom 21.09.1998 (BGB1. I 1998, S. 2955), zuletzt geändert am 26.11.2003 (BGB1. I, S. 2373)

Bundes-Bodenschutz- Und AltLastenverordnung (BBodSchV) vom 12.07. 1999 (BGBl. I S. 1554)

Bundes-Bodenschutzgesetz (BBodSchG): Gesetz zum Schutz vor schädlichen Bodenveränderungen und zur Sanierung von Altlasten vom 17.03.1998 (BGB1. I S. 502)

BUNDESIMMISSIONSSCHUTZGESETZ (BImSchG): Gesetz zum Schutz vor schädlichen Umwelteinwirkungen durch Luftverunreinigungen, Geräusche, Erschütterungen 
und ähnlichen Vorgängen vom 15.03.1974 (BGB1. I, S. 1193), zuletzt geändert am 6.01.2004 (BGB1. I, S. 2)

BunDESNATURSCHUTZGESETZ (BNatSchG): Gesetz über Naturschutz und Landespflege vom 25.03.2002 (BGB1. I, S. 1193), zuletzt geändert am 25.11.2003 (BGB1. I, S.2304)

Bundeswaldgesetz (BWaldG): Gesetz zur Erhaltung des Waldes und zur Förderung der Forstwirtschaft vom 2.05.1975 (BGB1. I, S. 1037), zuletzt geändert am 29.10.2001 (BGB1. I, S. 2785)

BÜrgERLICHES GESETZBUCH (BGB) vom 18.09.1896 (RGB1., S.195), zuletzt geändert am 27.06.2000 (BGB1. I, S. 897)

DÜNGEMITTELGESETZ (DMG) vom 15.11.1977 (BGBl. I, S. 2134), zuletzt geändert am 29.10.2001 (BGB1. I, S. 2785)

DÜNGEVERORDNUNG (DüngeVO): Verordnung über die Grundsätze der guten fachlichen Praxis beim Düngen vom 6.02.1996 (BGBl. I, S. 118), zuletzt geändert am 14.02.2003 (BGB1. I S. 235)

EU-WASSERRAHMENRICHTLINIE (WRRL): Richtlinie (EG) Nr. 2000/60 zur Schaffung eines Ordnungsrahmens für Maßnahmen der Gemeinschaft im Bereich der Wasserpolitik (AB1. EG Nr. L 327, S.1)

FLURBEREINIGUNGSGESETZ (FlurbG) vom 14.07.1953 (BGB1. I, S.593), zuletzt geändert am 20.12.2001 (BGB1. I, S. 3987)

GENTECHNIKGESETZ (GenTG): Gesetz zur Regelung der Gentechnik vom 20.07.1990 (BGBl. I, S. 1080), zuletzt geändert am 16.05.2002 (BGBl. I, S. 3220)

GRUNDBUCHORDNUNG (GBO) vom 24.03.1897, zuletzt geändert am 26.10.2001 (RGB1. 1897, S. 139)

KLÄRSCHLAMMVERORDNUNG (AbfKlärV) vom 15.04.1992 (BGBl. I, S. 912), zuletzt geändert am 26.11.2003 (BGB1. I, S. 2373)

KREISLAUfWIRTSCHAFTS- UND ABFALlGESETZ (KrW-/AbfG): Gesetz zur Förderung der Kreislaufwirtschaft und Sicherung der umweltverträglichen Beseitigung von Ab- 
fällen vom 27.09.1994 (BGBl. I, S. 2705), zuletzt geändert am 25.01.2004 (BGB1. I, S. 82)

NitRATRICHTLINIE: Richtlinie (EWG) Nr. 91/676 zum Schutz der Gewässer von Verunreinigungen durch Nitrat aus landwirtschaftlichen Quellen (ABl. EG Nr. L 375 S.1)

PflanzenschutZmittelgesetz (PflSchG): Gesetz zum Schutz der Kulturpflanzen vom 15.09.1986 (BGB1. I, S. 1505), zuletzt geändert am 25.11.2003 (BGB1. I, S. 2304) Wasserhaushaltsgesetz (WHG): Gesetz zur Ordnung des Wasserhaushalts vom 27.07.1957 (BGB1. I, S. 1110), zuletzt geändert am 6.01.2004 (BGB1. I, S. 2)

\section{Mündliche und schriftliche Auskünfte}

Herr NotTeR (Ministerium für Umwelt und Verkehr Baden-Württemberg, Referat Boden, Stuttgart), vom 31.01.2003

Herr WEINS (Ministerium für Umwelt- und Naturschutz, Landwirtschaft und Verbraucherschutz Nordrhein-Westfalen, Referat Agrarumweltförderung, Düsseldorf), vom 28.02.2003

Herr BIERWISCH (Amt für Agrarstruktur Göttingen), vom 08.08.2002

Herr STÖßER (Landwirtschaftskammer Schleswig-Holstein, Abt. Boden- und Grundwasserschutz, Itzehoe), vom 17.06.2003

Herr NeumanN (Bundesqualitätsgemeinschaft Sero-Dünger e. V., Bonn), vom 11.08 .2003

Herr Dr. NEIDHART (Niedersächsisches Umweltministerium, Hannover), vom 12.08.2003

Herr Dr. WEIERSHÄUSER (KTBL, Darmstadt), vom 20.08.2003

Herr JANSEN (Firma Pösges u. Tigges GmbH, Neuss), vom 26.11.2003

Herr Prof. VoLK (Fachhochschule Südwestfalen, Fachbereich Landtechnik, Soest), vom 12.01 .2004 
Frau SCHMIDT (GÜTEGEMEINSCHAFT KOMPOST E. V., Köln), vom 02.02.2004

Herr PAUlSEN (Firma Terrestris GbR, Bonn), vom 31.07.2003

Frau Dr. EPPERLEIN (Gesellschaft für konservierende Bodenbearbeitung, Berlin), vom 27.03.2003

Herr Dr. KowALEWSKY (Landwirtschaftskammer Weser-Ems, Oldenburg), vom 09.03 .2004 
Anhang

Teil I

Ergänzende Abbildung sowie Übersichten und Tabellen 


\section{Übersicht A-1: Auswirkungen von Unterbodenverdichtungen auf den Ertrag}

\begin{tabular}{|c|c|c|c|c|c|c|c|}
\hline Autoren & Jahr & Boden & Standort & $\begin{array}{l}\text { Achs- } \\
\text { last } \\
(\mathrm{t})\end{array}$ & $\begin{array}{l}\text { Ertra } \\
\text { 1.Jah } \\
\% \\
\end{array}$ & $\begin{array}{l}\text { sveränderungen } \\
\text { Red. } \\
\text { Folge- } \\
\text { jahre }\end{array}$ & Kosten \\
\hline VORHEES & 1999 & $\begin{array}{l}\mathrm{L} \\
\mathrm{L}\end{array}$ & $\begin{array}{l}\text { Quebec } \\
\text { Quebec }\end{array}$ & $\begin{array}{l}10,8 \\
18,0 \\
10,8 \\
18,0\end{array}$ & $\begin{array}{l}+3 \\
-9 \\
-51 \\
-55\end{array}$ & $\begin{array}{r}- \\
- \\
8 \% \text { f. } 6 \text { Jahre } \\
12 \% \text { f. } 6 \text { Jahre }\end{array}$ & \\
\hline VORHEES & 1999 & $\begin{array}{l}\mathrm{sL} \\
\mathrm{sL}\end{array}$ & $\begin{array}{l}\text { Wiscon- } \\
\sin \\
\text { Wiscon- } \\
\text { sin }\end{array}$ & $\begin{array}{l}7,2 \\
11,2 \\
7,2 \\
11,2\end{array}$ & $\begin{array}{l}-4 \\
-15 \\
-14 \\
-43\end{array}$ & $\begin{array}{l}2 \% \text { f. } 3 \text { Jahre } \\
3 \% \text { f. } 3 \text { Jahre } \\
6 \% \text { f. } 3 \text { Jahre } \\
5 \% \text { f. } 3 \text { Jahre }\end{array}$ & \\
\hline VORHEES & 1999 & $\begin{array}{l}\text { tL } \\
\text { (tro- } \\
\text { cken) } \\
\text { tL } \\
\text { (feucht) }\end{array}$ & $\begin{array}{l}\text { Minne- } \\
\text { sota }\end{array}$ & $\begin{array}{l}9 \\
18 \\
9 \\
18\end{array}$ & $\begin{array}{l}+1 \\
-7 \\
-9 \\
-30\end{array}$ & $\begin{array}{l}1 \% \text { f. } 4 \text { Jahre } \\
1 \% \text { f. } 4 \text { Jahre } \\
2 \% \text { f. } 4 \text { Jahre } \\
6 \% \text { f. } 11 \text { Jahre }\end{array}$ & \\
\hline $\begin{array}{l}\text { ARVIDS- } \\
\text { SON } \\
\text { HAKANS } \\
\text { SON } \\
\end{array}$ & 1991 & SL-1T & $\begin{array}{l}\text { Schwe- } \\
\text { den }\end{array}$ & 19 & \multicolumn{2}{|c|}{ 0,5/Jahr } & $\begin{array}{l}100 \\
\text { Mio. } \\
€ / a^{533}\end{array}$ \\
\hline $\begin{array}{l}\text { ALBAS et } \\
\text { al. }\end{array}$ & 1994 & & $\begin{array}{l}\text { Nieder- } \\
\text { lande }\end{array}$ & & \multicolumn{2}{|c|}{$7 \%$ bei Silomais } & $\begin{array}{l}21.000 \\
€ / \mathrm{a}\end{array}$ \\
\hline $\begin{array}{l}\text { STAN- } \\
\text { NERS, } \\
\text { BOUR- } \\
\text { DEAU } \\
\end{array}$ & 1995 & & Europa & & \multicolumn{2}{|l|}{$5-50$} & \\
\hline LIEBERT & 1995 & $\begin{array}{l}\text { nasse } \\
\text { Böden }\end{array}$ & $\begin{array}{l}\text { UdSSR } \\
\text { UdSSR } \\
\text { UdSSR }\end{array}$ & & \multicolumn{2}{|c|}{$\begin{array}{l}-7,5 \% \text { Getreide } \\
-3 \% \text { ZR } \\
-4 \% \text { Mais }\end{array}$} & \\
\hline $\begin{array}{l}\text { WIER- } \\
\text { MANN }\end{array}$ & 1998 & $\mathrm{~L}, \mathrm{~Pb}$ & $\begin{array}{l}\text { Reinshof } \\
\text { konv. } \\
\text { kons. }\end{array}$ & $\begin{array}{l}5 \\
\\
10 \\
5 \\
10 \\
\end{array}$ & $\begin{array}{c}-20 \\
-35 \\
0 \\
-15\end{array}$ & $\begin{array}{r}5 \% \text { f. } 3 \text { Jahre } \\
15 \% \text { f. } 3 \text { Jahre } \\
0 \% \text { f. } 3 \text { Jahre } \\
5 \% \text { f. } 3 \text { Jahre }\end{array}$ & \\
\hline
\end{tabular}

Quelle: Verändert nach HoRN (1999), S.16

${ }^{533}$ Durch die Ernte mit einem 38 t KRB wurde für schwedische Verhältnisse ein jährlicher Ertragsverlust von $0,5 \%$ ermittelt. Für eine Zuckerrübenanbaufläche von 500.000 ha bedeutet dies Ertragseinbußen von 100 Mio. $€ / J a h r$. 


\section{Übersicht A-2: Kosteneinsparungen durch konservierende Bodenbearbeitung}

\begin{tabular}{|c|c|c|c|c|c|}
\hline Quelle & $\begin{array}{l}\text { Produkti- } \\
\text { onsverfah- } \\
\text { ren }\end{array}$ & Zeitraum & $\begin{array}{l}\text { Frucht, - } \\
\text { folge }\end{array}$ & Kostenart & $\begin{array}{l}\text { Kostenverän- } \\
\text { derung (€/ha) }\end{array}$ \\
\hline $\begin{array}{l}\text { ACKERMANN } \\
(2002)\end{array}$ & $\begin{array}{l}\text { Streifenfräse } \\
\text { Zinkenrotor } \\
\text { Schlitzsaat }\end{array}$ & $\begin{array}{l}1991-1995 \\
1991-1995 \\
1991-1995\end{array}$ & $\begin{array}{l}\text { Silomais } \\
\text { Silomais } \\
\text { Silomais }\end{array}$ & v. M.kosten & $\begin{array}{l}+101,8^{534} \\
+86,1 \\
+90,5\end{array}$ \\
\hline $\begin{array}{l}\text { BECKER, } \\
\text { MILLER, } \\
\text { KOCH (1996) }\end{array}$ & $\begin{array}{l}\text { Locker }^{535} \\
\text { Mulchsaat } \\
\text { Direktsaat }\end{array}$ & $\begin{array}{l}1990-1995 \\
1990-1995 \\
1990-1995\end{array}$ & $\begin{array}{l}\text { Zuckerrüben } \\
\text { Zuckerrüben } \\
\text { Zuckerrüben }\end{array}$ & $\begin{array}{l}\text { v. Kosten, } \\
\text { Masch.k. u. } \\
\text { Arbeitskosten }\end{array}$ & $\begin{array}{l}-\quad 28,7 \\
-\quad 38,5 \\
-\quad 87,2\end{array}$ \\
\hline $\begin{array}{l}\text { WEGENER, } \\
\text { MILLER, } \\
\text { KOCH (2000) }\end{array}$ & $\begin{array}{l}\text { Locker } \\
\text { Mulchsaat } \\
\text { Direktsaat }\end{array}$ & $\begin{array}{l}1994-1999 \\
1994-1999 \\
1994-1999\end{array}$ & $\begin{array}{l}\text { Zuckerrüben } \\
\text { Zuckerrüben } \\
\text { Zuckerrüben }\end{array}$ & $\begin{array}{l}\text { v. Kosten, } \\
\text { Masch.k. u. } \\
\text { Arbeitskosten }\end{array}$ & $\begin{array}{ll}- & 20,4 \\
- & 40,9 \\
- & 10,7\end{array}$ \\
\hline $\begin{array}{l}\text { WEGENER, } \\
\text { MILLER, } \\
\text { KOCH (2000) }\end{array}$ & $\begin{array}{l}\text { Locker } \\
\text { Mulchsaat } \\
\text { Direktsaat }\end{array}$ & $\begin{array}{l}1994-1999 \\
1994-1999 \\
1994-1999\end{array}$ & $\begin{array}{l}\text { Winterweizen } \\
\text { Winterweizen } \\
\text { Winterweizen }\end{array}$ & $\begin{array}{l}\text { v. Kosten, } \\
\text { Masch.k. u. } \\
\text { Arbeitskosten }\end{array}$ & $\begin{array}{l}-56,7 \\
-76,7 \\
-114,5\end{array}$ \\
\hline $\begin{array}{l}\text { SCHINDLER } \\
(2002)\end{array}$ & $\begin{array}{l}\text { Mulchsaat } \\
\text { Direktsaat }\end{array}$ & $\begin{array}{l}\text { Kein Feld- } \\
\text { versuch }\end{array}$ & $\begin{array}{l}\text { Getreide } \\
\text { Getreide }\end{array}$ & $\begin{array}{l}\text { Arbeitserledi- } \\
\text { gungskosten } \\
\text { Lohn }(15 € / \mathrm{h})\end{array}$ & $\begin{array}{c}-35,4^{536} \\
-55,3\end{array}$ \\
\hline $\begin{array}{l}\text { PETERS } \\
(2001)\end{array}$ & Mulchsaat & $1992-2000$ & $\begin{array}{l}\text { FF W-raps- } \\
\text { W-Weizen- } \\
\text { W-Gerste }\end{array}$ & $\begin{array}{l}\text { Arbeitserledi- } \\
\text { gungskosten }\end{array}$ & 51,1 \\
\hline $\begin{array}{l}\text { BISCHOFF } \\
(2002)\end{array}$ & $\begin{array}{l}\text { Mulchsaat } \\
\mathrm{I}^{537} \\
\text { Mulchsaat } \\
\text { II }^{538} \\
\text { Direktsaat }\end{array}$ & $1998-2001$ & $\begin{array}{l}\text { Winterweizen } \\
\text { Winterweizen } \\
\text { Winterweizen }\end{array}$ & $\begin{array}{l}\text { Var. Kosten, } \\
\text { Lohn }(12,8 \\
€ / h)\end{array}$ & $\begin{array}{l}-33 \\
-9 \\
+6\end{array}$ \\
\hline
\end{tabular}

${ }^{534}$ Kosten: Kosten der Arbeitserledigung für Anbau und Zwischenfruchtvarianten, einschließlich Herbizide und mechanischer Unkrautbekämpfung. Zu beachten ist ferner, dass das vergleichbare konventionelle Anbausystem ohne Zwischenfruchtanbau arbeitet.

${ }^{535}$ Locker: ohne Pflug; Lockerung mit Schichtengrubber

${ }^{536}$ Auf Basis einer Betriebsgröße von 80 ha, Mittelwert der Schlaggrößen von 2, 5 bzw. 10 ha

${ }^{537}$ Mulchsaat I: Bodenbearbeitung mit Scheibenegge + Grubber (10-15 cm Arbeitstiefe)

${ }^{538}$ Mulchsaat II: Bodenbearbeitung mit Scheibenegge (4-6 cm Arbeitstiefe) 


\begin{tabular}{|c|c|c|c|c|c|}
\hline $\begin{array}{l}\text { NEUBAUER U. } \\
\text { HÄNDEL } \\
(2002)\end{array}$ & $\begin{array}{l}\text { Mulchsaat I } \\
\text { Mulchsaat II }\end{array}$ & $1994-2001$ & $\begin{array}{l}\text { Winterweizen } \\
\text { Wintergerste } \\
\text { Zuckerrüben }\end{array}$ & $\begin{array}{l}\text { Arbeitserledi- } \\
\text { gungskosten, } \\
\text { Lohn }(12,8 \\
€ / \mathrm{h})\end{array}$ & $\begin{array}{l}-53 \\
-41 \\
-41\end{array}$ \\
\hline $\begin{array}{l}\text { KLOTZ U. } \\
\text { HAAG (2001) }\end{array}$ & $\begin{array}{l}\text { Mulchsaat } \\
\text { Direktsaat }\end{array}$ & 1995-1999 & $\begin{array}{l}\text { Im Mittel al- } \\
\text { ler Versuche }\end{array}$ & $\begin{array}{l}\text { Arbeitserledi- } \\
\text { gungskosten }\end{array}$ & $\begin{array}{l}-25,6--61,3 \\
-25,6--81,8\end{array}$ \\
\hline $\begin{array}{l}\text { UPPENKAMP } \\
(2003)\end{array}$ & Mulchsaat & $\begin{array}{l}\text { Betriebsver- } \\
\text { gleich }\end{array}$ & $\begin{array}{l}\text { Verschiedene } \\
\text { Fruchtfolgen }\end{array}$ & $\begin{array}{l}\text { Gesamtkos- } \\
\text { ten }\end{array}$ & $\begin{array}{l}-8 \%- \\
-19 \% / \text { Betrieb }\end{array}$ \\
\hline $\begin{array}{l}\text { BRUNOTTE et } \\
\text { al. (1999) }\end{array}$ & Mulchsaat & $1995-1997$ & $\begin{array}{l}\text { Winterweizen } \\
\text { Kartoffeln } \\
\text { Kleegras }\end{array}$ & $\begin{array}{l}\text { Maschinen- } \\
\text { und Schlep- } \\
\text { perkosten }\end{array}$ & $\begin{array}{c}27,35 \\
34,2 \\
44,0\end{array}$ \\
\hline $\begin{array}{l}\text { SCHMIDT U. } \\
\text { NiTZSCHE } \\
(2001)\end{array}$ & 更 & $\begin{array}{l}\text { Umfrage un- } \\
\text { ter sächsi- } \\
\text { schen Land- } \\
\text { wirten }\end{array}$ & $\begin{array}{l}\text { Winterweizen } \\
\text { Wintergerste } \\
\text { Winterroggen } \\
\text { Triticale } \\
\text { Sommergers- } \\
\text { te } \\
\text { Winterraps } \\
\text { Zuckerrüben } \\
\text { Kartoffeln } \\
\text { Mais } \\
\text { Ackergras } \\
\text { Grasvermeh- } \\
\text { rung } \\
\text { Getreide }\end{array}$ & $\begin{array}{l}\text { Arbeitserledi- } \\
\text { gungskosten }\end{array}$ & $\begin{array}{c}51,1-61,3 \\
51,1-61,3 \\
56,2 \\
56,2 \\
56,2 \\
\\
51,1-61,3 \\
51,1 \\
127,8 \\
51,1-76,7 \\
61,3 \\
61,3 \\
44,5-55,2\end{array}$ \\
\hline $\begin{array}{l}\text { BRAND- } \\
\text { SASSEN u. } \\
\text { STEINMANN } \\
(2003)\end{array}$ & Mulchsaat & $1995-2002$ & $\begin{array}{l}\text { Winterraps } \\
\text { Winterweizen } \\
\text { Wintergerste }\end{array}$ & & \\
\hline
\end{tabular}

Quelle: Eigene Zusammenstellung 
Abbildung A-1: Geräteeinsatz bei Bodenbearbeitung mit Pflug und konservierender

Bodenbearbeitung zur Erzielung unterschiedlicher Bodenbedeckungsgrade

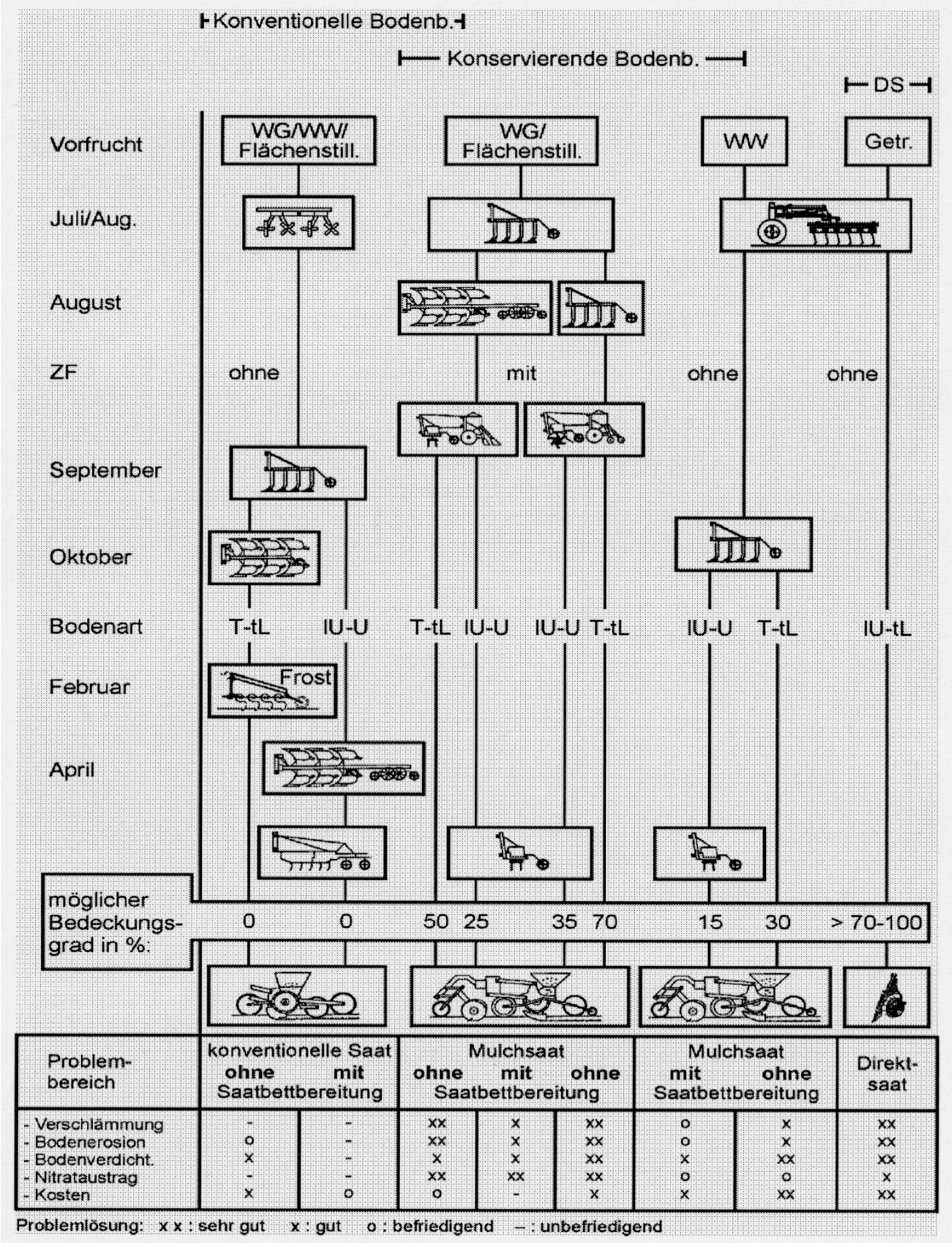

Quelle: BRUNOTTE (2003), S. 82 
Tabelle A-1: Ermittlung der Gesamtkosten für einen Umbruch bzw. eine Neueinsaat für die Fruchtfolge WR-WW-WG

a) Neuansaat ohne Umbruch, mit Herbizidmaßnahme ${ }^{539}$

\begin{tabular}{lcccc}
\hline$€ /$ ha & WR & WW & WG & $\varnothing$ \\
\hline Saatbettkombination (3 m) & 17,66 & 17,66 & 17,66 & \\
Drillmaschine (3 m) & 18,29 & 18,29 & 18,29 & \\
Saatgut $^{540}$ & 7,46 & 79,78 & 56,98 & \\
Pflanzenschutzmittelspritze (15 m) $^{541}$ & 5,74 & 5,74 & 5,74 & \\
Herbizid $^{54}$ & 53,65 & 29,53 & 32,00 & \\
Insgesamt & $\mathbf{1 0 2 , 8}$ & $\mathbf{1 5 1 , 0 9}$ & $\mathbf{1 3 0 , 6 7}$ & $\mathbf{1 2 8 , 1 8}$ \\
\hline
\end{tabular}

Quelle: Eigene Berechnungen nach KTBL (2002) u. LWK Hannover (2002)

b) Neuansaat mit Umbruch

\begin{tabular}{lcccc}
\hline$€ /$ ha & WR & WW & WG & $\varnothing$ \\
\hline Pflug (1,75) & 50,74 & 50,74 & 50,74 & \\
Saatbettkombination (3 m) & 17,66 & 17,66 & 17,66 & \\
Drillmaschine (3 m) $_{\text {Saatgut }^{542}}$ & 18,29 & 18,29 & 18,29 & \\
Insgesamt $^{\text {Ingesa }}$ & 7,46 & 79,78 & 56,98 & \\
\hline
\end{tabular}

Quelle: Eigene Berechnungen nach KTBL (2002) u. LWK Hannover (2002)

\footnotetext{
${ }^{539}$ Inkl. variablen und festen Maschinenkosten sowie den Arbeitskosten.

${ }^{540}$ Saatgutmenge Winterraps: 3,7 kg/ha, Winterweizen: $210 \mathrm{~kg} / \mathrm{ha}$, Wintergerste: $150 \mathrm{~kg} / \mathrm{ha}$. Annahme: Das Saatgut wird komplett zugekauft.

${ }^{541}$ Winterraps: 1,25 1 Butisan Top, Winterweizen: 0,5 1 Starane u. 1,0 1 Duplosan, Wintergerste: 0,75 1 Bacara.

${ }^{542}$ Saatgutmenge Winterraps: 3,7 kg/ha, Winterweizen: $210 \mathrm{~kg} / \mathrm{ha}$, Wintergerste: $150 \mathrm{~kg} / \mathrm{ha}$.
} 
Tabelle A-2: Einsparpotenziale für Pflanzenschutz- und Düngemittelkosten bei konservierender Bodenbearbeitung bei der Fruchtfolge WR-WW-WG

\section{Winterraps $^{543}$}

\begin{tabular}{|c|c|c|c|}
\hline \multirow{2}{*}{$\begin{array}{l}\text { \%-Abschwemmung } \\
\text { von Pflanzenschutz- } \\
\text { u. Düngemitteln }\end{array}$} & \multicolumn{2}{|c|}{ Verfahrenskosten Pflanzenschutz $(€ / \text { ha })^{544}$} & \multirow{2}{*}{$\begin{array}{l}\text { Var. Maschinenkosten Düngung } \\
(€ / \text { ha })^{545}\end{array}$} \\
\hline & Mittelkosten & Gesamt & \\
\hline 10 & 11,3 & 30,7 & 1,0 \\
\hline 25 & 28,4 & 33,2 & 2,5 \\
\hline 50 & 56,8 & 66,4 & 5,0 \\
\hline 75 & 85,1 & 99,0 & 7,5 \\
\hline 100 & 113,5 & 132,8 & 10,1 \\
\hline
\end{tabular}

\begin{tabular}{|c|c|c|c|}
\hline \multicolumn{4}{|c|}{ Winterweizen $^{546}$} \\
\hline \multirow{2}{*}{$\begin{array}{l}\text { \%-Abschwemmung } \\
\text { von Pflanzenschutz- } \\
\text { u. Düngemitteln }\end{array}$} & \multicolumn{2}{|c|}{ Verfahrenskosten Pflanzenschutz $(€ / \text { ha })^{547}$} & \multirow{2}{*}{$\begin{array}{l}\text { Var. Maschinenkosten Düngung } \\
(€ / \mathrm{ha})^{548}\end{array}$} \\
\hline & Mittelkosten & Gesamt & \\
\hline 10 & 16,4 & 45,6 & 1,0 \\
\hline 25 & 41,0 & 48,3 & 2,5 \\
\hline 50 & 82,0 & 96,6 & 5,0 \\
\hline 75 & 123,0 & 144,9 & 7,5 \\
\hline 100 & 164,0 & 193,2 & 10,1 \\
\hline \multicolumn{4}{|c|}{ Wintergerste $^{549}$} \\
\hline$\%$-Abschwemmung & \multicolumn{2}{|c|}{ Verfahrenskosten Pflanzenschutz $(€ / \text { ha })^{550}$} & Var. Maschinenkosten Düngung \\
\hline u. Düngemitteln & Mittelkosten & Gesamt & \\
\hline 10 & 13,2 & 15,4 & 1,0 \\
\hline 25 & 33,0 & 38,5 & 2,5 \\
\hline 50 & 66,0 & 77,0 & 5,0 \\
\hline 75 & 99,1 & 115,6 & 7,5 \\
\hline 100 & 132,1 & 154,1 & 10,1 \\
\hline
\end{tabular}

${ }^{543}$ Vgl. FID (2002), S. 21. Annahme: Alle Ertragsklassen, Aufwandstufe mittel.

${ }^{544}$ Anbauspritze $15 \mathrm{~m}, 54 \mathrm{~kW}$.

${ }^{545}$ FID (2002), S. 44. Annahme: Winterraps 35 dt, $3 *$ Anbauschleuderstreuer $1 \mathrm{t}, 54 \mathrm{~kW}$.

${ }^{546}$ Vgl. FID (2002), S. 18. Annahme: Windhalmstandort, Ertragsniveau $>60$ dt, Aufwandstufe mittel.

${ }^{547}$ Anbauspritze $15 \mathrm{~m}, 54 \mathrm{~kW}$.

${ }^{548}$ FID (2002), S. 44. Annahme: Winterraps 35 dt, $3 *$ Anbauschleuderstreuer $1 \mathrm{t}, 54 \mathrm{~kW}$.

${ }^{549}$ Vgl. FID (2002), S. 19. Annahme: Windhalmstandort, Ertragsniveau > 60 dt, Aufwandstufe mittel.

${ }^{550}$ Anbauspritze $15 \mathrm{~m}, 54 \mathrm{~kW}$.

${ }^{551}$ FID (2002), S. 44. Annahme: Winterraps 35 dt, $3 *$ Anbauschleuderstreuer 1 t, 54 kW. 


\section{Gesamtkosten im Mittel der Fruchtfolge}

\%-Abschwemmung von Pflanzen- Gesamtkosten

schutz- u. Düngemitteln $\quad(€ /$ ha $)$

\begin{tabular}{cc}
\hline 10 & $\mathbf{3 1 , 6}$ \\
25 & $\mathbf{4 2 , 5}$ \\
50 & $\mathbf{8 5 , 0}$ \\
75 & $\mathbf{1 2 7 , 3}$ \\
100 & $\mathbf{1 7 0 , 1}$ \\
\hline
\end{tabular}

Quelle: Eigene Berechnungen nach KTBL (2002) u. FID (2002)

Tabelle A-3: Zusätzliche Arbeitskosten (Akh/ha) bei der Bewirtschaftung quer zum Hang anhand eines typischen Anbauverfahrens für Raps-Getreide

\begin{tabular}{|c|c|c|c|c|c|}
\hline $\begin{array}{l}\text { Schlagform u.- } \\
\text { größe }\left(\mathbf{m}^{*} \mathbf{m}\right)\end{array}$ & $50 * 100$ & $60 * 100$ & $70 * 100$ & $80 * 100$ & $90 * 100$ \\
\hline \multicolumn{6}{|l|}{ Pflug (2,1 m) } \\
\hline längs & 1,515 & 1,492 & 1,461 & 1,45 & 1,442 \\
\hline quer & 2,004 & 1,814 & 1,679 & 1,57 & 1,498 \\
\hline Differenz & 0,489 & 0,322 & 0,218 & 0,12 & 0,056 \\
\hline \multicolumn{6}{|l|}{$\begin{array}{l}\text { Saatbettkom- } \\
\text { bination (3 m) }\end{array}$} \\
\hline längs & 0,547 & 0,537 & 0,53 & 0,531 & 0,526 \\
\hline quer & 0,71 & 0,648 & 0,603 & 0,569 & 0,543 \\
\hline Differenz & 0,163 & 0,111 & 0,073 & 0,038 & 0,017 \\
\hline \multicolumn{6}{|l|}{ Drillen (3m) } \\
\hline längs & 0,775 & 0,762 & 0,752 & 0,754 & 0,747 \\
\hline quer & 0,994 & 0,91 & 0,85 & 0,85 & 0,77 \\
\hline Differenz & 0,219 & 0,148 & 0,098 & 0,096 & 0,023 \\
\hline \multicolumn{6}{|l|}{$\begin{array}{l}\text { Mineraldünger } \\
\text { streuen }(15 \mathrm{~m}) \\
* 3\end{array}$} \\
\hline längs & 0,372 & 0,387 & 0,396 & 0,378 & 0,387 \\
\hline quer & 0,534 & 0,489 & 0,453 & 0,429 & 0,408 \\
\hline Differenz & 0,162 & 0,102 & 0,057 & 0,051 & 0,021 \\
\hline \multicolumn{6}{|l|}{ 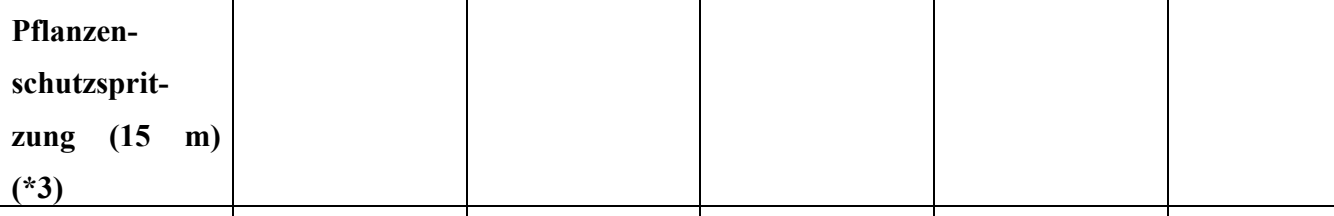 } \\
\hline längs & 0,438 & 0,456 & 0,468 & 0,444 & 0,456 \\
\hline
\end{tabular}




\begin{tabular}{|c|c|c|c|c|c|}
\hline $\begin{array}{l}\text { Schlagform u.- } \\
\text { größe }\left(m^{*} m\right)\end{array}$ & $50 * 100$ & $60 * 100$ & $70 * 100$ & $80 * 100$ & $90 * 100$ \\
\hline quer & 0,711 & 0,642 & 0,591 & 0,507 & 0,483 \\
\hline Differenz & 0,273 & 0,186 & 0,123 & 0,063 & 0,027 \\
\hline \multicolumn{6}{|l|}{$\begin{array}{l}\text { Mähdrusch (5 } \\
\text { m) }\end{array}$} \\
\hline längs & 0,625 & 0,622 & 0,62 & 0,618 & 0,616 \\
\hline quer & 1,221 & 1,156 & 1,11 & 1,076 & 1,049 \\
\hline Differenz & 0,596 & 0,534 & 0,49 & 0,458 & 0,433 \\
\hline $\begin{array}{l}\text { Arbeitskosten } \\
(15 € / h)\end{array}$ & $\begin{array}{c}1,911 * 15= \\
28,67 € \\
=57,34 € / \mathrm{ha}\end{array}$ & $\begin{array}{c}1,403 * 15= \\
21,04 € \\
=35,1 € / \mathrm{ha}\end{array}$ & $\begin{array}{c}1,059 * 15= \\
15,89 € \\
=22,7 € / h a\end{array}$ & $\begin{array}{c}0,826 * 15= \\
12,39 € \\
=15,49 € / h a\end{array}$ & $\begin{array}{c}0,577 * 15= \\
8,655 € \\
=9,61 € / h a\end{array}$ \\
\hline
\end{tabular}

Quelle: Eigene Berechnungen nach KTBL (2000a)

Tabelle A-4: Zusätzliche Arbeitskosten (Akh/ha) bei der Bewirtschaftung quer zum Hang anhand eines typischen Anbauverfahrens für Raps-Getreide bei pflugloser

\section{Bestellung}

\begin{tabular}{|c|c|c|c|c|c|}
\hline $\begin{array}{l}\text { Schlagform u. - } \\
\text { größe (m*m) }\end{array}$ & $50 * 100$ & $60 * 100$ & $70 * 100$ & $80 * 100$ & $90 * 100$ \\
\hline \multicolumn{6}{|l|}{ Pflug $(2,1 \mathrm{~m})$} \\
\hline längs & 0,889 & 0,877 & 0,87 & 0,864 & 0,859 \\
\hline quer & 1,148 & 1,048 & 0,977 & 0,923 & 0,882 \\
\hline Differenz & 0,259 & 0,171 & 0,107 & 0,059 & 0,023 \\
\hline \multicolumn{6}{|l|}{$\begin{array}{l}\text { Saatbettkombi- } \\
\text { nation }(3 \mathrm{~m})\end{array}$} \\
\hline längs & 0,547 & 0,537 & 0,53 & 0,531 & 0,526 \\
\hline quer & 0,71 & 0,648 & 0,603 & 0,569 & 0,543 \\
\hline Differenz & 0,163 & 0,111 & 0,073 & 0,038 & 0,017 \\
\hline \multicolumn{6}{|l|}{ Drillen $(3 \mathrm{~m})$} \\
\hline längs & 0,775 & 0,762 & 0,752 & 0,754 & 0,747 \\
\hline quer & 0,994 & 0,91 & 0,85 & 0,85 & 0,77 \\
\hline Differenz & 0,219 & 0,148 & 0,098 & 0,096 & 0,023 \\
\hline \multicolumn{6}{|l|}{$\begin{array}{l}\text { Mineraldünger } \\
\text { streuen }(15 \mathrm{~m}) \\
* 3\end{array}$} \\
\hline längs & 0,372 & 0,387 & 0,396 & 0,378 & 0,387 \\
\hline quer & 0,534 & 0,489 & 0,453 & 0,429 & 0,408 \\
\hline Differenz & 0,162 & 0,102 & 0,057 & 0,051 & 0,021 \\
\hline \multicolumn{6}{|l|}{$\begin{array}{l}\text { Pflanzenschutz- } \\
\text { spritzung }(15 \mathrm{~m}) \\
(* 3)\end{array}$} \\
\hline längs & 0,438 & 0,456 & 0,468 & 0,444 & 0,456 \\
\hline quer & 0,711 & 0,642 & 0,591 & 0,507 & 0,483 \\
\hline
\end{tabular}




\begin{tabular}{|c|c|c|c|c|c|}
\hline $\begin{array}{l}\text { Schlagform u. - } \\
\text { größe (m*m) }\end{array}$ & $50 * 100$ & $60 * 100$ & $70 * 100$ & $80 * 100$ & $90 * 100$ \\
\hline Differenz & 0,273 & 0,186 & 0,123 & 0,063 & 0,027 \\
\hline \multicolumn{6}{|l|}{ Mähdrusch (5 m) } \\
\hline längs & 0,625 & 0,622 & 0,62 & 0,618 & 0,616 \\
\hline quer & 1,221 & 1,156 & 1,11 & 1,076 & 1,049 \\
\hline Differenz & 0,596 & 0,534 & 0,49 & 0,458 & 0,433 \\
\hline $\begin{array}{l}\text { Arbeitskosten } \\
(15 € / \mathrm{h})\end{array}$ & $\begin{array}{c}1,672 * 15=25,08 \\
€ \\
=50,2 € / \mathrm{ha} \\
\end{array}$ & $\begin{array}{c}1,252 * 15= \\
18,78 € \\
=31,3 € / \mathrm{ha}\end{array}$ & $\begin{array}{c}0,948 * 15= \\
14,22 € \\
=20,3 € / \mathrm{ha}\end{array}$ & $\begin{array}{c}0,765 * 15= \\
11,47 € \\
=14,3 € / \mathrm{ha}\end{array}$ & $\begin{array}{c}0,544 * 15=8,16 \\
€ \\
=9,07 € / \mathrm{ha}\end{array}$ \\
\hline
\end{tabular}

Quelle: Eigene Berechnungen nach KTBL (2000a)

Tabelle A-5: Monetärer Nutzen durch die Anwendung der Maisengsaat bei verschiedenen Ertragssteigerungen

\begin{tabular}{cccc}
\hline $\begin{array}{c}\text { Ertragssteigerung (in } \\
\%)\end{array}$ & $\begin{array}{c}\text { Zusätzlicher Nettoer- } \\
\operatorname{trag}(\mathrm{dt} / \mathrm{ha})\end{array}$ & $\begin{array}{c}\text { Zusätzliche Energie } \\
(\text { MJ NEL) }\end{array}$ & $\begin{array}{c}\text { Zusätzlicher monetärer } \\
\text { Vorteil }(€ / \text { ha })\end{array}$ \\
\hline 2 & 9 & 929,7 & 19,5 \\
4 & 18 & 1859,4 & 39,0 \\
6 & 27 & 2789,1 & 58,6 \\
8 & 36 & 3718,8 & 78,1 \\
10 & 45 & 4648,5 & 97,6 \\
12 & 54 & 5578,2 & 117,1 \\
14 & 63 & 6507,9 & 136,7 \\
16 & 72 & 7437,6 & 156,2 \\
18 & 81 & 8367,3 & 175,7 \\
20 & 90 & 9297 & 195,2 \\
22 & 99 & 10226,7 & 214,8 \\
\hline
\end{tabular}

Quelle: Eigene Berechnungen nach FID (2002) 
Tabelle A-6: Einfluss der Verwendung von Grünstreifen und deren Einfluss auf den

Bodenabtrag bei verschiedenen Fruchtfolgen

Langfristiger Bodenabtrag in $\mathrm{t} / \mathrm{ha}$

Fruchtfolge

$8 \%^{1} \quad 200 \mathrm{~m}^{2} \quad 10 \% 200 \mathrm{~m} \quad 12 \% 200 \mathrm{~m}$

Bewirtschaftung quer zum Hang

WR-WW-WG

4,3

5,8

7,5

WW-WW-WW

WR-WW-WW

3,8

4,3

5,1

6,6

Bew. quer zum Hang u. Grünstreifen

WR-WW-WG

2,3

5,8

7,5

WW-WW-WW

2,0

3,4

4,7

WR-WW-WW

2,3

4,2

Bewirtschaftung in Gefällerichtung

WR-WW-WG

4,9

3,0

WW-WW-WW

4,9

3,4

4,7

WR-WW-WW

4,9

6,3

7,9

5,6

6,9

${ }^{1}$ Hangneigung

${ }^{2}$ Hanglänge

Quelle: Eigene Berechnungen mit ${ }^{\mathrm{PC}} \mathrm{ABAG}$ 
Tabelle A-7: Mögliche KW (€/t verhinderter Bodenabtrag) für verschiedene Grünstreifen und verschiedenen Standorten

\begin{tabular}{|c|c|c|c|c|}
\hline $\begin{array}{l}\text { Streifen- } \\
\text { breite }(\mathrm{m})\end{array}$ & $\begin{array}{l}\text { Reihenab- } \\
\text { stand (m) }\end{array}$ & $\begin{array}{l}\text { Verminde- } \\
\text { rung des } \\
\text { BA (in \%) }\end{array}$ & $\begin{array}{l}\mathrm{KW}(€ / \mathrm{t}) \\
\text { Standort: } \\
6 \mathrm{t} / \mathrm{ha}^{*} \mathrm{a}\end{array}$ & $\begin{array}{l}\mathrm{KW}(€ / \mathrm{t}) \\
\text { Standort: } \\
10 \mathrm{t} / \mathrm{ha}^{*} \mathrm{a}\end{array}$ \\
\hline \multirow{9}{*}{3} & \multirow{3}{*}{20} & 20 & 33,8 & 22,3 \\
\hline & & 87 & 7,8 & 4,7 \\
\hline & & 95 & 7,1 & 4,3 \\
\hline & \multirow{3}{*}{40} & 20 & 18,0 & 10,8 \\
\hline & & 87 & 4,1 & 2,5 \\
\hline & & 95 & 3,8 & 2,3 \\
\hline & \multirow{3}{*}{20} & 20 & 59,7 & 35,8 \\
\hline & & 87 & 13,7 & 8,2 \\
\hline & & 95 & 13,0 & 7,5 \\
\hline \multirow[t]{3}{*}{6} & \multirow{3}{*}{40} & 20 & 33,9 & 20,3 \\
\hline & & 87 & 7,8 & 4,7 \\
\hline & & 95 & 7,1 & 4,3 \\
\hline \multirow{7}{*}{9} & \multirow{3}{*}{20} & 20 & 80,2 & 48,1 \\
\hline & & 87 & 18,4 & 11,1 \\
\hline & & 95 & 17,0 & 10,1 \\
\hline & \multirow{3}{*}{40} & 20 & 48,0 & 28,5 \\
\hline & & 87 & 10,9 & 6,5 \\
\hline & & 95 & 10,0 & 6,0 \\
\hline & \multirow{4}{*}{20} & 20 & 86,2 & 51,7 \\
\hline \multirow{5}{*}{10} & & 87 & 19,8 & 11,9 \\
\hline & & 95 & 18,1 & 10,9 \\
\hline & & 20 & 51,7 & 31,0 \\
\hline & \multirow{2}{*}{40} & 87 & 11,9 & 7,1 \\
\hline & & 95 & 11,0 & 6,5 \\
\hline
\end{tabular}

Quelle: Eigene Berechnungen 
Tabelle A-8: Erwerbsverlust bei Verzicht des Anbaus an Hanglagen (WR-WWWW)

\begin{tabular}{lcccc}
\hline $\begin{array}{l}\text { Einzuschränkende Produktions- } \\
\text { oder Stilllegungsverfahren }\end{array}$ & $\begin{array}{c}\text { WR } \\
(25 \mathrm{dt} / \mathrm{ha})\end{array}$ & $\begin{array}{c}\text { WW } \\
(60 \mathrm{dt} / \mathrm{ha})\end{array}$ & $\begin{array}{c}\text { WW } \\
(60 \mathrm{dt} / \mathrm{ha})\end{array}$ & $\begin{array}{c}\text { Rotations- } \\
\text { brache }^{552}\end{array}$ \\
\hline Anteil (\%) & 30 & 30 & 30 & 10 \\
Deckungsbeitrag $(€ / \mathrm{ha})^{553}$ & 445 & 420 & 420 & 261 \\
Eingesparte Arbeitszeit (Akh/ha) & 8,4 & 9,1 & 9,1 & 6 \\
\hline Gewogener Durchschnitt des DB (€/ha) & & & & 412 \\
Einsparbare Gemeinkosten ( $(/ \mathrm{ha})$ & & & 0 \\
Vorteile aus der Arbeitsersparnis & & & 326 \\
(Akh*10 $€ / \mathrm{h})$ & & & 86 \\
Verbleibende Einkommensbeeinträchti- & & & \\
gung $(€ /$ ha) & & &
\end{tabular}

Quelle: Eigene Berechnungen nach KÖHNE (2000), S. 208 f.

Tabelle A-9: Max. Bodendruck und Druckabbau auf abgesetztem, feuchten Boden für verschiedene Fahrwerke

\begin{tabular}{|l|c|c|c|c|c|c|}
\hline $\begin{array}{l}\text { Fahrwerk } \\
\text { (Gesamtmas- } \\
\text { se) }\end{array}$ & KFD & \multicolumn{4}{|c|}{ Druck (in bar) in Tiefe. } & Druckabbau \\
\hline $\begin{array}{l}\text { Breitreifen } \\
(11,4 \mathrm{t})\end{array}$ & 1,13 & - & - & - & - & 54 \\
\hline $\begin{array}{l}\text { Terrareifen } \\
(11,7 \mathrm{t})\end{array}$ & 0,73 & 1,23 & 0,66 & 0,36 & 0,01 & 46 \\
\hline $\begin{array}{l}\text { Halbraupe } \\
(11,2 \mathrm{t})\end{array}$ & 0,82 & 1,45 & 0,79 & 0,40 & 0,02 & 65 \\
\hline $\begin{array}{l}\text { Bandlaufwerk } \\
(20 \mathrm{t})\end{array}$ & 0,44 & 1,08 & 0,60 & 0,19 & 0,08 & 56 \\
\hline
\end{tabular}

Quelle: QUAS, SCHWIEGERT U. WEIßBACH (1995), S. 55

${ }^{553}$ Richtwertdeckungsbeiträge der LWK HANNOVER (2002), niedriges Ertragsniveau, ohne USt, jedoch inkl. flächenbezogener öffentlicher Zuwendungen. 
Tabelle A-10: Max. Bodendruck und Druckabbau auf abgesetztem, nassen Boden für verschiedene Fahrwerke

\begin{tabular}{|c|c|c|c|c|c|c|}
\hline \multirow{2}{*}{$\begin{array}{l}\text { Fahrwerk } \\
\text { (Gesamtmas- } \\
\text { se) }\end{array}$} & \multirow[t]{2}{*}{ KFD } & \multicolumn{4}{|c|}{ Druck (in bar) in Tiefe. } & \multirow[t]{2}{*}{ Druckabbau } \\
\hline & & $10 \mathrm{~cm}$ & $15 \mathrm{~cm}$ & $25 \mathrm{~cm}$ & $40 \mathrm{~cm}$ & \\
\hline $\begin{array}{l}\text { Breitreifen } \\
(11,4 \mathrm{t})\end{array}$ & 1,13 & 1,35 & 1,11 & 0,70 & 0,30 & 43 \\
\hline $\begin{array}{l}\text { Terrareifen } \\
(11,7 \mathrm{t})\end{array}$ & 0,73 & 1,26 & 0,91 & 0,76 & 0,20 & 31 \\
\hline $\begin{array}{l}\text { Halbraupe } \\
(11,2 \mathrm{t})\end{array}$ & 0,30 & 1,03 & 0,25 & 0,14 & 0,13 & 52 \\
\hline $\begin{array}{l}\text { Bandlaufwerk } \\
(20 \mathrm{t})\end{array}$ & 0,30 & 0,63 & 0,30 & 0,23 & 0,15 & 23 \\
\hline
\end{tabular}

Quelle: QUAS, SCHWIEGERT U. WeIßBACH (1995), S. 56

Tabelle A-11: Max. Bodendruck und Druckabbau auf lockerem, feuchten Boden für verschiedene Fahrwerke

\begin{tabular}{|l|c|c|c|c|c|c|}
\hline $\begin{array}{l}\text { Fahrwerk } \\
\text { (Gesamtmas- } \\
\text { se) }\end{array}$ & KFD & \multicolumn{4}{|c|}{ Druck (in bar) in Tiefe. } & Druckabbau \\
\hline $\begin{array}{l}\text { Breitreifen } \\
(11,4 \mathrm{t})\end{array}$ & 1,13 & - & - & - & - & - \\
\hline $\begin{array}{l}\text { Terrareifen } \\
(11,7 \mathrm{t})\end{array}$ & 0,73 & 1,30 & 1,13 & 0,63 & 0,32 & 45 \\
\hline $\begin{array}{l}\text { Halbraupe } \\
(11,2 \mathrm{t})\end{array}$ & 0,82 & 1,01 & 0,78 & 0,41 & 0,16 & 39 \\
\hline $\begin{array}{l}\text { Bandlaufwerk } \\
(20 \mathrm{t})\end{array}$ & 0,44 & 0,80 & 0,45 & 0,20 & 0,23 & 57 \\
\hline
\end{tabular}

Quelle: QUAS, SCHWIEGERT U. WeIßBACH (1995), S. 58

Tabelle A-12: Max. Bodendruck und Druckabbau auf lockerem, nassem Boden für verschiedene Fahrwerke

\begin{tabular}{|l|c|c|c|c|c|c|}
\hline $\begin{array}{l}\text { Fahrwerk } \\
\text { (Gesamtmas- } \\
\text { se })\end{array}$ & KFD & \multicolumn{4}{|c|}{ Druck (in bar) in Tiefe. } & Druckabbau \\
\hline $\begin{array}{l}\text { Breitreifen } \\
(11,4 \mathrm{t})\end{array}$ & 1,13 & 1,64 & 1,52 & 1,22 & 0,47 & 28 \\
\hline $\begin{array}{l}\text { Terrareifen } \\
(11,7 \mathrm{t})\end{array}$ & 0,73 & 0,73 & 0,76 & 0,63 & 0,51 & 7 \\
\hline $\begin{array}{l}\text { Halbraupe } \\
(11,2 \mathrm{t})\end{array}$ & 0,30 & 0,71 & 0,36 & 0,27 & 0,16 & 26 \\
\hline $\begin{array}{l}\text { Bandlaufwerk } \\
(20 \mathrm{t})\end{array}$ & 0,30 & 0,52 & 0,28 & 0,19 & 0,02 & 20 \\
\hline
\end{tabular}

Quelle: QUAS, SCHWIEGERT U. WEIßBACH (1995), S. 59 
Tabelle A-13: Einsparungen (€/ha) durch Verminderung des Schlupfs bei verschiedenen Fruchtfolgen bei Verwendung von bodenschonender Bereifung gegenüber Standardreifen

\begin{tabular}{lcccc}
\hline \multicolumn{1}{c}{$\boldsymbol{\epsilon} / \mathbf{h a}$} & WR-WW-WG & ZR & WW & WG \\
\hline Pflug & $1,68-6,72$ & $1,68-6,72$ & $1,68-6,72$ & $1,68-6,72$ \\
Saatbett & $0,56-2,23$ & $0,56-2,23$ & $0,56-2,23$ & $0,56-2,23$ \\
Drillen & $0,64-2,6$ & $0,86-3,46$ & $0,64-2,6$ & $0,64-2,6$ \\
Min. dgg. & $0,11-0,42$ & $0,11-0,42$ & $0,11-0,42$ & $0,11-0,42$ \\
PSM & $0,18-0,7$ & $0,18-0,7$ & $0,18-0,7$ & $0,18-0,7$ \\
\hline Gesamt & $\mathbf{3 , 1 7 - 1 2 , 6 7}$ & $\mathbf{3 , 3 9 - 1 3 , 5 3}$ & $\mathbf{3 , 1 7 - 1 2 , 6 7}$ & $\mathbf{3 , 1 7 - 1 2 , 6 7}$ \\
\hline
\end{tabular}

Quelle: Eigene Berechnungen

Tabelle A-14:Kalkulation des Erwerbsverlustes bei der Stilllegung einer Minderertragsfläche bei der Fruchtfolge WR-WW-WG

\begin{tabular}{lcccc}
\hline $\begin{array}{l}\text { Einzuschränkende Produktions- } \\
\text { oder Stilllegungsverfahren }\end{array}$ & $\begin{array}{c}\text { WR } \\
(25 \mathrm{dt} / \mathrm{ha})\end{array}$ & $\begin{array}{c}\mathrm{WW} \\
(50 \mathrm{dt} / \mathrm{ha})\end{array}$ & $\begin{array}{c}\mathrm{WG} \\
(50 \mathrm{dt} / \mathrm{ha})\end{array}$ & $\begin{array}{c}\text { Rotations- } \\
\text { brache }^{554}\end{array}$ \\
\hline Anteil $(\%)$ & 30 & 30 & 30 & 10 \\
Deckungsbeitrag $(€ / \mathrm{ha})^{555}$ & 445 & 336 & 321 & 261 \\
Eingesparte Arbeitszeit (Akh/ha) & 8,4 & 8,5 & 8,0 & 6 \\
\hline Gewogener Durchschnitt des DB (€/ha) & & & 366 \\
Einsparbare Gemeinkosten (€/ha) & & & 0 \\
Vorteile aus der Arbeitsersparnis & & & 139 \\
$($ Akh*15 $€ /$ h) & & & \\
Verbleibende Einkommensbeeinträchti- & & & 227 \\
gung $(€ /$ ha) & & & \\
\hline
\end{tabular}

Quelle: Eigene Berechnungen nach KÖHNE (2000), S. 208 f.

${ }^{555}$ Richtwertdeckungsbeiträge der LWK HANNOVER (2002), niedriges Ertragsniveau, ohne USt, jedoch inkl. flächenbezogener öffentlicher Zuwendungen 
Tabelle A-15: Berechnung der Vorgewendekosten durch den Fruchtfolgedeckungsbeitragsverlust (WR-WW-WG) bei der Stilllegung einer Minderertragsfläche $(\mathrm{DB}=596,33 € / \mathrm{ha})$

\begin{tabular}{crrrrr}
\hline & \multicolumn{5}{c}{ Vorgewendebreite } \\
\hline $\begin{array}{l}\text { Ertragsver- } \\
\text { lust }\end{array}$ & $\mathbf{2 0 ~} \mathbf{m}$ & $\mathbf{4 0 ~} \mathbf{m}$ & $\mathbf{6 0} \mathbf{~ m}$ & $\mathbf{8 0} \mathbf{~ m}$ & $\mathbf{1 0 0} \mathbf{~ m}$ \\
\hline $5 \%$ & 1,19 & 2,39 & 3,58 & 4,77 & 5,96 \\
$10 \%$ & 2,39 & 4,77 & 7,16 & 9,54 & 11,93 \\
$15 \%$ & 3,58 & 7,16 & 10,73 & 14,31 & 17,89 \\
$20 \%$ & 4,77 & 9,54 & 14,31 & 19,08 & 23,85 \\
$25 \%$ & 5,96 & 11,93 & 17,89 & 23,85 & 29,82 \\
$30 \%$ & 7,16 & 14,31 & 21,47 & 28,62 & 35,78 \\
\hline
\end{tabular}

Quelle: Eigene Berechnungen

Tabelle A-16: Berechnung der zusätzlichen Arbeits- u. Maschinenkosten bei Stilllegung von Minderertragsteilflächen anhand eines typischen Anbauverfahrens von Getreide

\begin{tabular}{|c|c|c|c|c|c|}
\hline h,€/Kantenlänge & $20 \mathrm{~m}$ & $40 \mathrm{~m}$ & $60 \mathrm{~m}$ & $80 \mathrm{~m}$ & $100 \mathrm{~m}$ \\
\hline \multicolumn{6}{|l|}{ Pflug $(2,1 \mathrm{~m})$} \\
\hline Arbeitszeit & 0,19 & 0,38 & 0,57 & 0,76 & 0,95 \\
\hline Arbeitskosten & 2,8 & 5,7 & 8,5 & 11,4 & 14,2 \\
\hline Var. Masch.k. & 2,3 & 4,6 & 6,9 & 9,3 & 11,6 \\
\hline Insgesamt & 5,1 & 10,3 & 15,4 & 20,7 & 25,8 \\
\hline \multicolumn{6}{|l|}{$\begin{array}{l}\text { Saatbettkombinati- } \\
\text { on }(3 \mathrm{~m})\end{array}$} \\
\hline Arbeitszeit & 0,07 & 0,13 & 0,2 & 0,27 & 0,33 \\
\hline Arbeitskosten & 1,0 & 1,9 & 3 & 4 & 4,9 \\
\hline Var. Masch.k. & 0,6 & 1,2 & 1,8 & 2,4 & 3 \\
\hline Insgesamt & 1,6 & 3,1 & 4,8 & 6,4 & 7,9 \\
\hline \multicolumn{6}{|l|}{ Drillen (3m) } \\
\hline Arbeitszeit & 0,09 & 0,18 & 0,26 & 0,35 & 0,44 \\
\hline Arbeitskosten & 1,35 & 2,7 & 3,9 & 5,2 & 6,6 \\
\hline Var. Masch. k. & 0,6 & 1,2 & 1,8 & 2,4 & 3 \\
\hline Insgesamt & 1,95 & 3,9 & 5,7 & 7,6 & 9,6 \\
\hline \multicolumn{6}{|l|}{$\begin{array}{l}\text { Mineraldünger } \\
\text { streuen }(15 \mathrm{~m}) * 3\end{array}$} \\
\hline Arbeitszeit & 0,05 & 0,1 & 0,16 & 0,21 & 0,27 \\
\hline Arbeitskosten & 0,7 & 1,5 & 2,4 & 3,1 & 4 \\
\hline Var. Masch. k. & 0,4 & 0,8 & 1,2 & 1,6 & 2,1 \\
\hline Insgesamt & 1,1 & 2,3 & 3,6 & 4,7 & 6,1 \\
\hline \multicolumn{6}{|l|}{\begin{tabular}{l}
\multicolumn{3}{|l|}{ Pflanzenschutz- } \\
spritzung (15 \\
$(* 3)$
\end{tabular}} \\
\hline Arbeitszeit & 0,05 & 0,1 & 0,16 & 0,21 & 0,27 \\
\hline Arbeitskosten & 0,7 & 1,5 & 2,4 & 3,1 & 4 \\
\hline Var. Masch. k. & 0,4 & 0,8 & 1,2 & 1,6 & 2,1 \\
\hline Insgesamt & 1,1 & 2,3 & 3,6 & 4,7 & 6,1 \\
\hline Mähdrusch (5 m) & & & & & \\
\hline
\end{tabular}




\begin{tabular}{|l|c|c|c|c|c|}
\hline $\mathbf{h , € / K a n t e n l a ̈ n g e ~}$ & $\mathbf{2 0} \mathbf{~ m}$ & $\mathbf{4 0} \mathbf{~ m}$ & $\mathbf{6 0} \mathbf{~ m}$ & $\mathbf{8 0 ~} \mathbf{~}$ & $\mathbf{1 0 0} \mathbf{~ m}$ \\
\hline Arbeitskosten & 0,6 & 1,2 & 1,8 & 2,4 & 3 \\
\hline Var. Masch. k. & 1 & 2 & 2,9 & 3,9 & 4,9 \\
\hline Insgesamt & 1,6 & 1,4 & 4,7 & 6,3 & 7,9 \\
\hline Summe & $\mathbf{1 2 , 4 5}$ & $\mathbf{2 3 , 3}$ & $\mathbf{3 7 , 8}$ & $\mathbf{5 0 , 4}$ & $\mathbf{6 3 , 4}$ \\
\hline
\end{tabular}

Quelle: Eigene Berechnungen

Tabelle A-17: Berechnung der Erlösminderungen bei Induktion verschiedener Schadverdichtungen nach WIERMANN bei der Fruchtfolge WR-WW-WG

\section{Achslast $5 \mathrm{t}$}

\begin{tabular}{|c|c|c|c|c|c|}
\hline Jahr & Frucht & $\begin{array}{l}\text { Ertrag ohne } \\
\text { SV }(\mathrm{dt} / \mathrm{ha})^{556}\end{array}$ & $\begin{array}{l}\text { Ertrag bei ei- } \\
\text { ner SV im Jahr } \\
\text { (dt/ha) }\end{array}$ & $\begin{array}{l}\text { Ertragsverän- } \\
\text { derung durch } \\
\text { SV (\%) }\end{array}$ & $\begin{array}{l}\text { Erlösminde- } \\
\text { rung }(€ / \text { ha })^{557}\end{array}$ \\
\hline 1 & WR & 35 & 28 & -20 & 176,3 \\
\hline 2 & WW & 80 & 76 & -5 & 45,5 \\
\hline 3 & WG & 80 & 76 & -5 & 36,6 \\
\hline 4 & WR & 35 & 33,2 & -5 & 45,3 \\
\hline 5 & WW & 80 & 80 & 0 & 0 \\
\hline 6 & WG & 80 & 80 & 0 & 0 \\
\hline
\end{tabular}

Achslast $5 \mathrm{t}$

\begin{tabular}{|c|c|c|c|c|c|}
\hline Jahr & Frucht & $\begin{array}{l}\text { Ertrag ohne } \\
\text { SV (dt/ha) }\end{array}$ & $\begin{array}{l}\text { Ertrag bei ei- } \\
\text { ner SV im Jahr } \\
\text { (dt/ha) }\end{array}$ & $\begin{array}{l}\text { Ertragsverän- } \\
\text { derung durch } \\
\text { SV (\%) }\end{array}$ & $\begin{array}{l}\text { Erlösminde- } \\
\text { rung (€/ha) }\end{array}$ \\
\hline 1 & WR & 35 & 28 & -20 & 176,3 \\
\hline 2 & WW & 80 & 76 & -5 & 45,5 \\
\hline 3 & WW & 80 & 76 & -5 & 45,5 \\
\hline 4 & WR & 35 & 33,2 & -5 & 45,3 \\
\hline 5 & WW & 80 & 80 & 0 & 0 \\
\hline 6 & WW & 80 & 80 & 0 & 0 \\
\hline
\end{tabular}


Tabelle A-18: Berechnung der Erlösminderungen bei Induktion verschiedener Schadverdichtungen nach WIERMANN bei der Fruchtfolge ZR-WW-WG

Achslast $5 \mathrm{t}$

\begin{tabular}{|c|c|c|c|c|c|}
\hline Jahr & Frucht & $\begin{array}{l}\text { Ertrag ohne } \\
\text { SV (dt/ha) }\end{array}$ & $\begin{array}{l}\text { Ertrag bei ei- } \\
\text { ner SV im Jahr } \\
1(\mathrm{dt} / \mathrm{ha})\end{array}$ & $\begin{array}{l}\text { Ertragsverän- } \\
\text { derung durch } \\
\text { SV }(\%)\end{array}$ & $\begin{array}{l}\text { Erlösminde- } \\
\text { rung(€/ha })\end{array}$ \\
\hline 1 & ZR & 500 & 400 & -20 & 525,6 \\
\hline 2 & WW & 80 & 76 & -5 & 45,5 \\
\hline 3 & WG & 80 & 76 & -5 & 36,6 \\
\hline 4 & ZR & 500 & 475 & -5 & 131,25 \\
\hline 5 & WW & 80 & 80 & 0 & 0 \\
\hline 6 & WG & 80 & 80 & 0 & 0 \\
\hline
\end{tabular}

Achslast $10 \mathrm{t}$

\begin{tabular}{|c|c|c|c|c|c|}
\hline Jahr & Frucht & $\begin{array}{l}\text { Ertrag ohne } \\
\text { SV (dt/ha) }\end{array}$ & $\begin{array}{l}\text { Ertrag bei ei- } \\
\text { ner SV im Jahr } \\
1(\mathrm{dt} / \mathrm{ha})\end{array}$ & $\begin{array}{l}\text { Ertragsverän- } \\
\text { derung durch } \\
\text { SV (\%) }\end{array}$ & $\begin{array}{l}\text { Erlösminde- } \\
\text { rung (€/ha) }\end{array}$ \\
\hline 1 & ZR & 500 & 325 & -35 & 919,7 \\
\hline 2 & WW & 80 & 68 & -15 & 136,6 \\
\hline 3 & WG & 80 & 68 & -15 & 109,9 \\
\hline 4 & ZR & 500 & 425 & -15 & 394,2 \\
\hline 5 & WW & 80 & 80 & 0 & 0 \\
\hline 6 & WG & 80 & 80 & 0 & 0 \\
\hline
\end{tabular}

Quelle: Eigene Berechnungen 


\section{Übersicht A-3: Kosten der Verwertung von Klärschlamm für den Klärschlammab-} geber

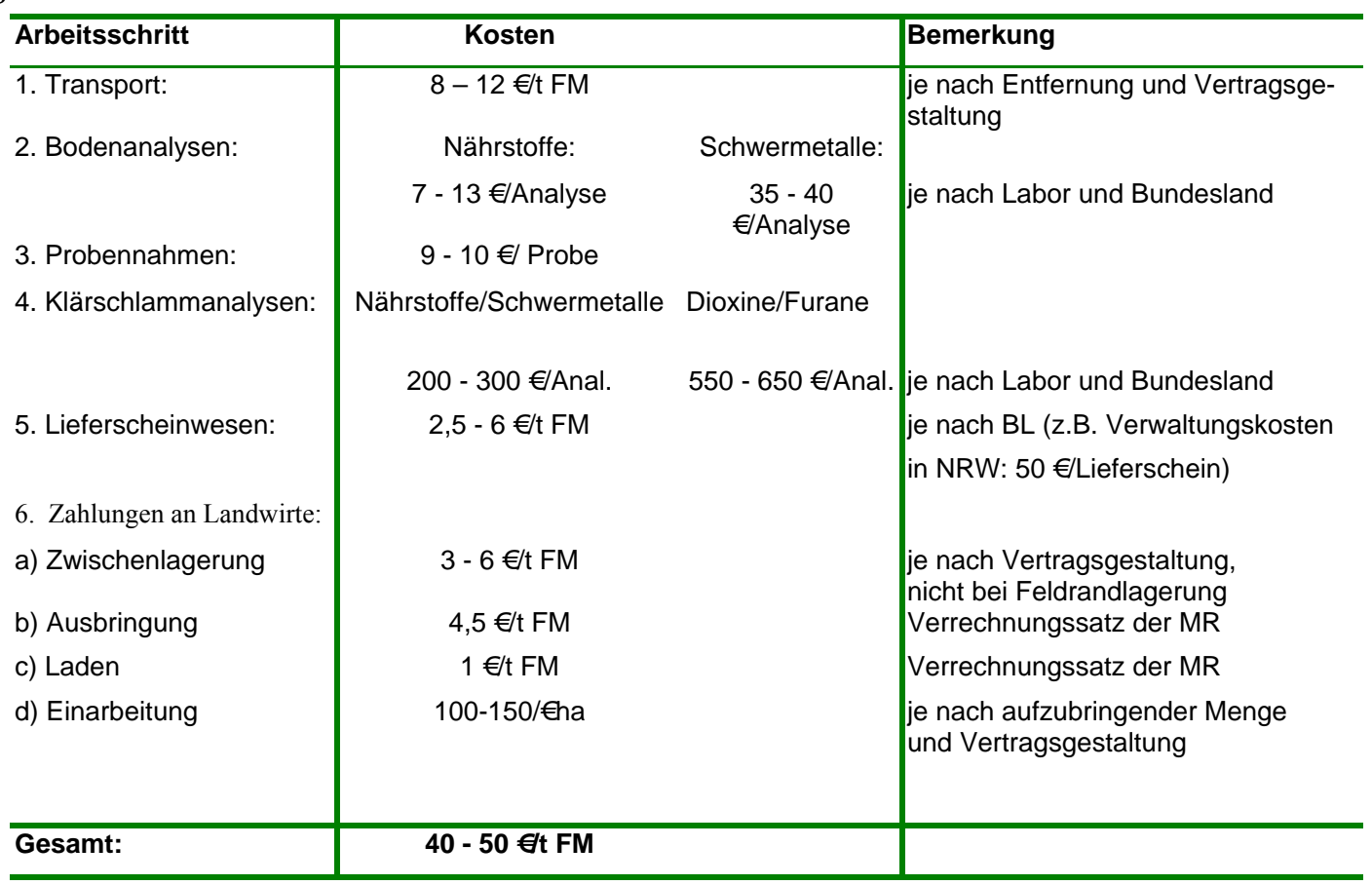

Quelle: schriftliche Mitteilung Herr Neumann (2003)

Tabelle A-19: Nährstoffgehalte und Marktpreise verschiedener Mineraldüngerarten im Kammergebiet Hannover

\begin{tabular}{|c|c|c|c|c|}
\hline Hauptnährstoff & Düngemittel & $\begin{array}{l}\text { Nährstoffgehalt } \\
(\%)\end{array}$ & $\begin{array}{l}\text { Brutto- } \\
\text { preis(€/dt) }\end{array}$ & $\begin{array}{l}\text { Bruttopreis je kg } \\
\text { Nährstoff }(€ / \mathrm{kg})\end{array}$ \\
\hline Stickstoff & KAS & 27 & 17,7 & 0,66 \\
\hline \multirow[t]{2}{*}{$(\mathrm{N})$} & Harnstoff & 46 & 21,9 & 0,47 \\
\hline & AHL & 28 & 15,4 & 0,55 \\
\hline Phosphor & Triple-Phosphat & 45 & 23,3 & 0,52 \\
\hline$\left(\mathrm{P}_{2} \mathrm{O}_{5}\right)$ & Diammonphosphat & 46 & 26,8 & 0,37 \\
\hline $\begin{array}{l}\text { Kalium } \\
\left(\mathrm{K}_{2} \mathrm{O}\right)\end{array}$ & Kornkali & 40 & 14,55 & 0,24 \\
\hline $\begin{array}{l}\text { Magnesium } \\
(\mathrm{MgO})\end{array}$ & Kieserit & 27 & 14,5 & \\
\hline \multirow[t]{2}{*}{$\begin{array}{l}\text { Kalzium } \\
(\mathrm{CaO})\end{array}$} & Carbo-Kalk & 30 & 0,75 & 0,025 \\
\hline & Granukal & 44,8 & 3,77 & 0,04 \\
\hline
\end{tabular}

Quelle: FID (2002), S.13 u. KTBL (2002) (Taschenbuch), S. 144 
Tabelle A-20: Nutzen einer Kompostgabe von 30 t TM/ha unter Berücksichtigung des N-Werts

\begin{tabular}{c|c|c|c}
\hline Jahr & Kompostgabe bzw. Rest-N (kg/ha) & N-Fixierung (kg/ha) & Nutzen (€/ha) \\
\hline 1 & 390 & 11,70 & 5,85 \\
2 & 378,30 & 11,35 & 5,67 \\
3 & 366,95 & 11,01 & 5,50 \\
4 & 355,94 & 10,68 & 5,34 \\
5 & 345,26 & 10,36 & 5,18 \\
6 & 334,91 & 10,05 & 5,02 \\
7 & 324,86 & 9,75 & 4,87 \\
8 & 315,11 & 9,45 & 4,73 \\
9 & 305,66 & 9,17 & 4,58 \\
10 & 296,49 & 8,89 & 4,45 \\
11 & 287,60 & 8,63 & 4,31 \\
12 & 278,97 & 8,37 & 4,18 \\
13 & 270,60 & 8,12 & 4,06 \\
14 & 262,48 & 7,87 & 3,94 \\
15 & 254,61 & 7,64 & 3,82 \\
16 & 246,97 & 7,41 & 3,70 \\
17 & 239,56 & 7,19 & 3,59 \\
18 & 232,37 & 6,97 & 3,49 \\
19 & 225,40 & 6,76 & 3,38 \\
20 & 218,64 & 6,56 & 3,28 \\
21 & 212,08 & 6,36 & 3,18 \\
22 & 205,72 & 6,17 & 3,09 \\
23 & 199,55 & 5,99 & 2,99 \\
24 & 193,56 & 5,81 & 2,90 \\
25 & 187,75 & 5,63 & 2,82 \\
\hline
\end{tabular}

Quelle: Eigene Berechnungen 
Teil II

\section{Fragebogen}




\section{Fragebogen}

\section{Zustand der landw. genutzten Böden in Deutschland}

1. Wie schätzen Sie den momentanen Zustand des landwirtschaftlich genutzten Bodens in

Deutschland aus ihrer Erfahrung heraus ein?

Zu dieser Frage möchte ich mich nicht äußern, da mir für eine hinreichende Beurteilung die Informationen fehlen.

\begin{tabular}{|c|c|c|c|c|c|c|}
\hline & sehr gut & gut & $\begin{array}{l}\text { befrie- } \\
\text { digend }\end{array}$ & schlecht & $\begin{array}{c}\text { sehr } \\
\text { schlecht }\end{array}$ & $\begin{array}{l}\text { weiß } \\
\text { nicht }\end{array}$ \\
\hline $\begin{array}{l}\text { Nicht-stoffliche Bodenbelastungen } \\
\text {... bez. Winderosion }\end{array}$ & $\square$ & $\square$ & $\square$ & $\square$ & $\square$ & $\square$ \\
\hline ... bez. Wassererosion & $\square$ & $\square$ & $\square$ & $\square$ & $\square$ & $\square$ \\
\hline ... bez. Bodenschadverdichtung & $\square$ & $\square$ & $\square$ & $\square$ & $\square$ & $\square$ \\
\hline ... bez. Bodenversiegelung & $\square$ & $\square$ & $\square$ & $\square$ & $\square$ & $\square$ \\
\hline ... sonstiges & $\square$ & $\square$ & $\square$ & $\square$ & $\square$ & $\square$ \\
\hline $\begin{array}{l}\text { Stoffl. Bodenbelastungen u. -einträge } \\
\text {... bez. Pflanzenschutzmittel }\end{array}$ & $\square$ & $\square$ & $\square$ & $\square$ & $\square$ & $\square$ \\
\hline ... bez. organischen Düngemitteln & $\square$ & $\square$ & $\square$ & $\square$ & $\square$ & $\square$ \\
\hline ... bez. anorganischen Düngemitteln & $\square$ & $\square$ & $\square$ & $\square$ & $\square$ & $\square$ \\
\hline ... bez. Kompostverwertung & $\square$ & $\square$ & $\square$ & $\square$ & $\square$ & $\square$ \\
\hline ... bez. Klärschlammverwertung & $\square$ & $\square$ & $\square$ & $\square$ & $\square$ & $\square$ \\
\hline ... sonstiges & $\square$ & $\square$ & $\square$ & $\square$ & $\square$ & $\square$ \\
\hline Sonstige Bodenbelastungen & $\square$ & $\square$ & $\square$ & $\square$ & $\square$ & $\square$ \\
\hline & $\square$ & $\square$ & $\square$ & $\square$ & $\square$ & $\square$ \\
\hline & $\square$ & $\square$ & $\square$ & $\square$ & $\square$ & $\square$ \\
\hline
\end{tabular}

Bemerkun-

gen/Hinweise:

2. Welche der o. g. Belastungen bzw. Einträge bilden Ihrer Meinung nach die größten zukünftigen Gefahrenpotenziale für den ldw. Boden? Bitte bilden Sie hierzu eine Rangfolge! (beginnend mit dem größten Gefahrenpotenzial)

1.

2.

3.

$\square$ Weiß nicht 
3. Welche der o. g. Belastungen bzw. Einträge sind Ihrer Meinung nach am leichtesten durch die landwirtschaftlichen Betriebe zu beheben bzw. zu kompensieren? Bitte bilden Sie ebenfalls eine Reihenfolge! (beginnend mit der leichtesten Behebung)

1.

2.

3.

Weiß nicht

4. Sehen Sie einen Unterschied in der Wirkung des ökologischen und konventionellen Landbaus auf den Boden?

$\mathrm{Ja}$

$\square$ Nein

$\square$ Weiß nicht

Nennen Sie bitte diese Unterschiede im einzelnen! 


\section{Beurteilung von Maßnahmen in der Idw. Bodennutzung zum Schutz des Bodens und deren ökonomische Bewertung}

Es haben sich z. T. durch intensive Forschungstätigkeiten zahlreiche Maßnahmen zur Reduzierung der Bodenbelastung entwickelt, die bereits in der ldw. Praxis ausgeführt und z. T. auch seitens des Staates gefördert werden. Einige gehören zur guten fachlichen Praxis in der Bodennutzung (nach $\S 17$ BBodSchG), andere gehen darüber hinaus. In der folgenden Tabelle sind die wichtigsten von ihnen dargestellt.

$\mathrm{Zu}$ diesem Themenbereich möchte ich mich nicht äußern, da mir für eine hinreichende Beurteilung die Informationen fehlen (bitte weiter mit Themenbereich III).

1. Beurteilen Sie bitte im Folgenden die Bedeutung der einzelnen Maßnahmen für den landwirtschaftlichen Bodenschutz in Bezug auf die Schadensreduzierung!

Zu dieser Frage möchte ich mich nicht äußern, da mir für eine hinreichende Beurteilung die Informationen fehlen.

\begin{tabular}{|c|c|c|c|c|c|c|}
\hline & $\begin{array}{l}\text { sehr hohe } \\
\text { Bedeutung }\end{array}$ & $\begin{array}{l}\text { hohe } \mathrm{Be}- \\
\text { deutung }\end{array}$ & $\begin{array}{l}\text { mittlere } \\
\text { Bedeutung }\end{array}$ & $\begin{array}{l}\text { geringe } \\
\text { Bedeu- } \\
\text { tung }\end{array}$ & $\begin{array}{c}\text { sehr ge- } \\
\text { ringe Be- } \\
\text { deutung }\end{array}$ & $\begin{array}{l}\text { weiß } \\
\text { nicht }\end{array}$ \\
\hline $\begin{array}{l}\text { Ldw. u. technische Maßnahmen } \\
\text { Mulchsaat }\end{array}$ & $\square$ & $\square$ & $\square$ & $\square$ & $\square$ & $\square$ \\
\hline Direktsaat & $\square$ & $\square$ & $\square$ & $\square$ & $\square$ & $\square$ \\
\hline Zwischenfruchtanbau & $\square$ & $\square$ & $\square$ & $\square$ & $\square$ & $\square$ \\
\hline Reduzierung von Reihenfrüchten & $\square$ & $\square$ & $\square$ & $\square$ & $\square$ & $\square$ \\
\hline Erweiterte Fruchtfolgen & $\square$ & $\square$ & $\square$ & $\square$ & $\square$ & $\square$ \\
\hline Anbau von Feldhecken & $\square$ & $\square$ & $\square$ & $\square$ & $\square$ & $\square$ \\
\hline Reifendruckregelanlagen & $\square$ & $\square$ & $\square$ & $\square$ & $\square$ & $\square$ \\
\hline Sonstige & $\square$ & $\square$ & $\square$ & $\square$ & $\square$ & $\square$ \\
\hline $\begin{array}{l}\text { Administratorische Maßnahmen } \\
\text { Grenzwerte für den Bodendruck }\end{array}$ & $\square$ & $\square$ & $\square$ & $\square$ & $\square$ & $\square$ \\
\hline Gute fachliche Praxis & $\square$ & $\square$ & $\square$ & $\square$ & $\square$ & $\square$ \\
\hline Verbot der ldw. Klärschlammentsorgung & $\square$ & $\square$ & $\square$ & $\square$ & $\square$ & $\square$ \\
\hline Verbot der ldw. Kompostausbringung & $\square$ & $\square$ & $\square$ & $\square$ & $\square$ & $\square$ \\
\hline Sonstige & $\square$ & $\square$ & $\square$ & $\square$ & $\square$ & $\square$ \\
\hline $\begin{array}{l}\text { Ökonomische Anreize } \\
\text { Investitionsförderprogramme }\end{array}$ & $\square$ & $\square$ & $\square$ & $\square$ & $\square$ & $\square$ \\
\hline $\begin{array}{l}\text { andere Förderprogramme (z. B. MEKA, } \\
\text { KULAP) }\end{array}$ & $\square$ & $\square$ & $\square$ & $\square$ & $\square$ & $\square$ \\
\hline Stickstoff-Abgabe & $\square$ & $\square$ & $\square$ & $\square$ & $\square$ & $\square$ \\
\hline Flächennutzungssteuer & $\square$ & $\square$ & $\square$ & $\square$ & $\square$ & $\square$ \\
\hline Sonstige Maßnahmen & $\square$ & $\square$ & $\square$ & $\square$ & $\square$ & $\square$ \\
\hline & $\square$ & $\square$ & $\square$ & $\square$ & $\square$ & $\square$ \\
\hline & $\square$ & $\square$ & $\square$ & $\square$ & $\square$ & $\square$ \\
\hline
\end{tabular}


Bemerkun-

gen/Hinweise:

Die Auswirkungen der Maßnahmen auf den Betriebserfolg des ldw. Betriebes sowie die Effektivität der Maßnahmen können anhand eines einzelbetrieblichen Kosten-NutzenVergleichs ermittelt werden. Hierzu werden die zusätzlichen Kosten zur Durchsetzung der Maßnahme und deren unmittelbaren Nutzen gegenübergestellt. Das Ergebnis ist das Kosten-Nutzen-Verhältnis (KNV).

2. Beurteilen Sie bitte das Kosten-Nutzen-Verhältnis folgender landwirtschaftlicher, technischer sowie administratorischer Maßnahmen in Bezug auf die Schadensminderung von Bodenbelastungen auf einzelbetrieblicher Ebene!

Zu dieser Frage möchte ich mich nicht äußern, da mir für eine hinreichende Beurteilung die Informationen fehlen

\begin{tabular}{|c|c|c|c|c|c|c|}
\hline & $\begin{array}{l}\text { sehr gu- } \\
\text { tes KNV }\end{array}$ & $\begin{array}{l}\text { gutes } \\
\text { KNV }\end{array}$ & $\begin{array}{c}\text { ausgegli- } \\
\text { chenes } \\
\text { KNV }\end{array}$ & $\begin{array}{l}\text { schlecht- } \\
\text { es KNV }\end{array}$ & $\begin{array}{c}\text { sehr } \\
\text { schlecht- } \\
\text { es KNV }\end{array}$ & $\begin{array}{l}\text { weiß } \\
\text { nicht }\end{array}$ \\
\hline \multicolumn{7}{|l|}{ Ldw.- technische Maßnahmen } \\
\hline Mulchsaat & $\square$ & $\square$ & $\square$ & $\square$ & $\square$ & $\square$ \\
\hline Direktsaat & $\square$ & $\square$ & $\square$ & $\square$ & $\square$ & $\square$ \\
\hline Zwischenfruchtanbau & $\square$ & $\square$ & $\square$ & $\square$ & $\square$ & $\square$ \\
\hline Reduzierung von Reihenfrüchten & $\square$ & $\square$ & $\square$ & $\square$ & $\square$ & $\square$ \\
\hline Erweiterte Fruchtfolgen & $\square$ & $\square$ & $\square$ & $\square$ & $\square$ & $\square$ \\
\hline Anbau von Feldhecken & $\square$ & $\square$ & $\square$ & $\square$ & $\square$ & $\square$ \\
\hline Reifendruckregelanlagen & $\square$ & $\square$ & $\square$ & $\square$ & $\square$ & $\square$ \\
\hline \multicolumn{7}{|l|}{ Administratorische Maßnahmen } \\
\hline Grenzwerte für den Bodendruck & $\square$ & $\square$ & $\square$ & $\square$ & $\square$ & $\square$ \\
\hline Gute fachliche Praxis & $\square$ & $\square$ & $\square$ & $\square$ & $\square$ & $\square$ \\
\hline Verbot der ldw. Klärschlammentsorgung & $\square$ & $\square$ & $\square$ & $\square$ & $\square$ & $\square$ \\
\hline Verbot der ldw. Kompostausbringung & $\square$ & $\square$ & $\square$ & $\square$ & $\square$ & $\square$ \\
\hline \multicolumn{7}{|l|}{ Ökonomische Anreize } \\
\hline Investitionsförderprogramme & $\square$ & $\square$ & $\square$ & $\square$ & $\square$ & $\square$ \\
\hline $\begin{array}{l}\text { andere Förderprogramme (z. B. MEKA, } \\
\text { KULAP) }\end{array}$ & $\square$ & $\square$ & $\square$ & $\square$ & $\square$ & $\square$ \\
\hline Stickstoff-Abgabe & $\square$ & $\square$ & $\square$ & $\square$ & $\square$ & $\square$ \\
\hline Flächennutzungssteuer & $\square$ & $\square$ & $\square$ & $\square$ & $\square$ & $\square$ \\
\hline \multicolumn{7}{|l|}{ Sonstige Maßnahmen } \\
\hline & $\square$ & $\square$ & $\square$ & $\square$ & $\square$ & $\square$ \\
\hline & $\square$ & $\square$ & $\square$ & $\square$ & $\square$ & $\square$ \\
\hline & $\square$ & $\square$ & $\square$ & $\square$ & $\square$ & $\square$ \\
\hline
\end{tabular}

Bemerkun-

gen/Hinweise: 
Es gibt in einigen Bundesländern verschiedene Förderprogramme, die eine bodenschonende Bewirtschaftung monetär unterstützen. Außerdem stehen den Landwirten in Zukunft Mittel durch die Modulation zur Verfügung. § 17 BBodSchG setzt in den Grundsätzen der guten fachlichen Praxis jedoch schon eine bodenschonende Bewirtschaftungsweise voraus.

3. Wie sollten diese Zusammenhänge Ihrer Meinung nach geregelt werden ?

Die Landwirte sollen weiterhin diese Fördermaßnahmen nutzen können

Eine Förderung ist nicht gerechtfertigt

Die Förderung sollte nur als Anschubfinanzierung dienen, sie sollte

Jahre betragen

Weiß nicht

Bemerkun-

gen/Hinweise:

\section{Das Bodenschutzrecht in Deutschland}

Seit März 1999 wird das Umweltmedium Boden durch ein bundeseinheitliches Gesetz (Bundes-Bodenschutzgesetz) mit dem entsprechenden untergesetzlichen Regelwerk (Bundes-Bodenschutzverordnung) unter Schutz gestellt.

Zu diesem Themenbereich möchte ich mich nicht äußern, da mir für eine hinreichende Beurteilung die Informationen fehlen (bitte weiter mit Themenbereich IV).

1. Wie sind Sie mit dem aktuellen Bodenschutzrecht in Deutschland im Allgemeinen zufrieden?
sehr zufrieden
zufrieden
unzufrieden
sehr unzufrieden
weiß nicht 
Begründung:

2. Sind Ihrer Meinung nach schon diesbezügliche Auswirkungen in der ldw. Praxis zu erkennen?

Ja, folgende

Nein

Weiß nicht

Bemerkun-

gen/Hinweise:

2. Wie beurteilen Sie die folgenden gesetzlichen und untergesetzlichen Regelwerke in Bezug auf die Bedeutung für den Schutz des landwirtschaftlich genutzten Bodens?

Zu dieser Frage möchte ich mich nicht äußern, da mir für eine hinreichende Beurteilung die Informationen fehlen 


\begin{tabular}{|l|c|c|c|c|c|}
\hline & $\begin{array}{c}\text { sehr hohe } \\
\text { Bedeu- } \\
\text { tung }\end{array}$ & $\begin{array}{c}\text { hohe Be- } \\
\text { deu-tung }\end{array}$ & $\begin{array}{c}\text { mittlere } \\
\text { Bedeu- } \\
\text { tung }\end{array}$ & $\begin{array}{c}\text { geringe } \\
\text { Bedeu- } \\
\text { tung }\end{array}$ & $\begin{array}{c}\text { sehr ge- } \\
\text { ringe } \\
\text { Bedeu- } \\
\text { tung }\end{array}$ \\
\hline Bundes-Bodenschutzgesetz & $\begin{array}{c}\text { weiß } \\
\text { nicht }\end{array}$ \\
Bundes-Bodenschutzverordnung & $\square$ & $\square$ & $\square$ & $\square$ & $\square$ \\
Bundes-Naturschutzgesetz & $\square$ & $\square$ & $\square$ & $\square$ & $\square$ \\
Düngemittelgesetz & $\square$ & $\square$ & $\square$ & $\square$ & $\square$ \\
Düngemittelverordnung & $\square$ & $\square$ & $\square$ & $\square$ & $\square$ \\
Kreislaufwirtschafts- u. Abfallgesetz & $\square$ & $\square$ & $\square$ & $\square$ & $\square$ \\
Klärschlammverordnung & $\square$ & $\square$ & $\square$ & $\square$ & $\square$ \\
Pflanzenschutzmittelverordnung & $\square$ & $\square$ & $\square$ & $\square$ & $\square$ \\
Wasserhaushaltsgesetz & $\square$ & $\square$ & $\square$ & $\square$ & $\square$ \\
Raumordnungsgesetz & $\square$ & $\square$ & $\square$ & $\square$ & $\square$ \\
Pflanzenschutzgesetz & $\square$ & $\square$ & $\square$ & $\square$ & $\square$ \\
Flurbereinigungsgesetz & $\square$ & $\square$ & $\square$ & $\square$ & $\square$ \\
Bundes-Immissionsschutzgesetz & $\square$ & $\square$ & $\square$ \\
Gentechnikgesetz & $\square$ & $\square$ & $\square$ & $\square$ \\
Bundes-Berggesetz & $\square$ & $\square$ & $\square$ & $\square$ & $\square$ \\
Sonstige: & $\square$ & $\square$ & $\square$ \\
\hline \multicolumn{1}{l|l}{} & $\square$ & $\square$ \\
\hline
\end{tabular}

Bemerkun-

gen/Hinweise:

4. Wie beurteilen Sie den Reformbedarf für die o. g. Regelwerke bez. der bodenschutzrelevanten Inhalte? Bitte bringen Sie diese in eine Reihenfolge! (beginnend mit dem reformbedürftigsten Regelwerk)

1.

2.

3.

Weiß nicht

Begründung:

5. Wie beurteilen Sie den bisherigen Erfolg für den ldw. Bodenschutz durch den Erlass des Bundes-Bodenschutzgesetzes und der-verordnung im Speziellen?

sehr hoch 

hoch
niedrig
sehr niedrig
weiß nicht

6. Bitte nennen Sie die drei wichtigsten Mängel der beiden gesetzlichen Regelwerke in Stichworten und bringen Sie diese in eine Rangfolge! (beginnend mit dem wichtigsten Mangel)

1.

2.

3.

weiß nicht

Die Landwirte erfüllen durch den Vollzug der guten fachlichen Praxis nach $\S 17$ BBodSchG die Vorsorgepflicht nach $\S 7$ BBodSchG und u. U. auch die Gefahrenabwehrpflichten nach $\S 4$ BBodSchG. Damit wird der $\S 17 \mathrm{zu}$ einem sehr zentralen Instrument des landwirtschaftlichen Bodenschutzes. Deshalb wird diesem Instrument auch im Folgenden eine große Bedeutung in dieser Befragung beigemessen. Bitte beantworten Sie hierzu folgende Fragen:

7. Wie schätzen Sie die Wirksamkeit der Grundsätze der guten fachlichen Praxis für einen vorsorgenden Bodenschutz ein?

sehr wirksam

wirksam

nicht wirksam

sehr unwirksam

weiß nicht

Begründung und Verbesserungsvorschläge: 
7. Reichen Ihrer Meinung nach die Konkretisierungen der guten fachlichen Praxis aus, um einen flächendeckenden und vorsorgenden Bodenschutz in der Landwirtschaft zu gewährleisten?

reichen völlig

reichen aus

reichen nicht aus

völlig unzurei-

weiß nicht

aus

chend

Begründung und Verbesserungsvorschläge:

Mit dem $\S 17$ BBodSchG setzt der Gesetzgeber auf das Kooperationsprinzip, d. h. dass die Grundsätze der guten fachlichen Praxis durch Beratung und nicht durch Anordnungen oder Setzung von Grenzwerten in die ldw. Praxis transferiert werden sollen.

9. Besitzt Ihrer Meinung nach dieses Prinzip eine hinreichende Wirksamkeit?

$\mathrm{Ja}$

Nein

Weiß nicht

Begründung und Verbesserungsvorschläge: 


\section{Boden- und Hochwasserschutz}

Nicht zuletzt durch die Hochwasserereignisse der letzten Jahre ist der Einfluss der Landwirtschaft auf einen vorsorgenden Hochwasserschutz geführt worden. Nehmen Sie bitte im Folgenden zu der Beziehung zwischen landwirtschaftlicher Bodennutzung und Hochwasserschutz Stellung!

Zu diesem Themenbereich möchte ich mich nicht äußern, da mir für eine hinreichende Beurteilung die Informationen fehlen (bitte weiter mit Themenbereich V).

1. Die landwirtschaftliche Bodennutzung hat einen direkten Effekt auf den Oberflächenabfluss und somit auf die Intensität von Hochwasserereignissen. Was sind Ihrer Meinung nach die effektivsten Maßnahmen zur Reduzierung des Oberflächenabflusses?

$\mathrm{Zu}$ dieser Frage möchte ich mich nicht äußern, da mir für eine hinreichende Beurteilung die Informationen fehlen

\begin{tabular}{|c|c|c|c|c|c|c|}
\hline & \begin{tabular}{|c|} 
sehr hohe \\
Effekti- \\
vität
\end{tabular} & $\begin{array}{l}\text { hohe Ef- } \\
\text { fektivität }\end{array}$ & $\begin{array}{l}\text { mittlere } \\
\text { Effekti- } \\
\text { vität }\end{array}$ & $\begin{array}{c}\text { geringe } \\
\text { Effekti- } \\
\text { vität }\end{array}$ & $\begin{array}{l}\text { Sehr ge- } \\
\text { ringe Ef- } \\
\text { fektivität }\end{array}$ & $\begin{array}{l}\text { Weiß } \\
\text { nicht }\end{array}$ \\
\hline $\begin{array}{l}\text { Ldw.- technische Maßnahmen } \\
\text { Mulchsaat }\end{array}$ & $\square$ & $\square$ & $\square$ & $\square$ & $\square$ & $\square$ \\
\hline Direktsaat & $\square$ & $\square$ & $\square$ & $\square$ & $\square$ & $\square$ \\
\hline Zwischenfruchtanbau & $\square$ & $\square$ & $\square$ & $\square$ & $\square$ & $\square$ \\
\hline Reduzierung von Reihenfrüchten & $\square$ & $\square$ & $\square$ & $\square$ & $\square$ & $\square$ \\
\hline Erweiterte Fruchtfolgen & $\square$ & $\square$ & $\square$ & $\square$ & $\square$ & $\square$ \\
\hline Anbau von Feldhecken & $\square$ & $\square$ & $\square$ & $\square$ & $\square$ & $\square$ \\
\hline Reifendruckregelanlagen & $\square$ & $\square$ & $\square$ & $\square$ & $\square$ & $\square$ \\
\hline Sonstige & $\square$ & $\square$ & $\square$ & $\square$ & $\square$ & $\square$ \\
\hline $\begin{array}{l}\text { Administratorische Maßnahmen } \\
\text { Grenzwerte für den Bodendruck }\end{array}$ & $\square$ & $\square$ & $\square$ & $\square$ & $\square$ & $\square$ \\
\hline Gute fachliche Praxis & $\square$ & $\square$ & $\square$ & $\square$ & $\square$ & $\square$ \\
\hline Ausweisung von Hochwasserschutzgebieten & $\square$ & $\square$ & $\square$ & $\square$ & $\square$ & $\square$ \\
\hline zielgerichtete Bauleitplanung & $\square$ & $\square$ & $\square$ & $\square$ & $\square$ & $\square$ \\
\hline Umbrechungsverbot für Grünland & $\square$ & $\square$ & $\square$ & $\square$ & $\square$ & $\square$ \\
\hline Sonstige & $\square$ & $\square$ & $\square$ & $\square$ & $\square$ & $\square$ \\
\hline $\begin{array}{l}\text { Ökonomische Anreize } \\
\text { Investitionsförderprogramme }\end{array}$ & $\square$ & $\square$ & $\square$ & $\square$ & $\square$ & $\square$ \\
\hline Förderprogramme (z. B. MEKA, KULAP) & $\square$ & $\square$ & $\square$ & $\square$ & $\square$ & $\square$ \\
\hline Flächennutzungssteuer & $\square$ & $\square$ & $\square$ & $\square$ & $\square$ & $\square$ \\
\hline \multicolumn{7}{|l|}{ Sonstige Maßnahmen } \\
\hline & $\square$ & $\square$ & $\square$ & $\square$ & $\square$ & $\square$ \\
\hline & $\square$ & $\square$ & $\square$ & $\square$ & $\square$ & $\square$ \\
\hline & $\square$ & $\square$ & $\square$ & $\square$ & $\square$ & $\square$ \\
\hline
\end{tabular}


Bemerkungen/Hinweise:

2. Sollten Ihrer Meinung nach Landwirte, die die obigen, bzw. von Ihnen genannten Maßnahmen ergreifen und in ihren Betrieben durchsetzen, finanzielle Unterstützung erhalten?

$\mathrm{Ja}$

Nein

Weiß nicht

Begründung und Verbesserungsvorschläge:

3. Welche der obigen, bzw. von Ihnen genannten Maßnahmen sind besonders förderwürdig? Bringen Sie die wichtigsten bitte in eine Reihenfolge (beginnend mit der förderwürdigsten Maßnahme)

1.

2.

3.

Weiß nicht

Begründung: 


\section{Zukünftige Ausrichtung des Bodenschutzes}

Beurteilen Sie bitte folgende Aussagen.

1. Es fehlt in Deutschland eine flächendeckende Bodenschutzberatung zur

Durchsetzung vorsorgender Bodenschutzkonzepte für die Landwirtschaft.

stimme völlig zu stimme zu stimme nicht $\mathrm{zu}$ lehne völlig ab weiß nicht

2. Es fehlen in einigen Bereichen Grenzwerte zur Bodenschutzvorsorge.

stimme völlig zu stimme zu stimme nicht zu lehne völlig ab weiß nicht

3. Die Ausgleichsregelung nach $\S 10$ Abs. 2 BBodSchG ist eine ungerechtfertigte Sonderregelung für die Landwirtschaft.

stimme völlig zu stimme zu stimme nicht zu lehne völlig ab weiß nicht

4. Die Ausgleichsregelung nach $\S 10$ Abs. 2 BBodSchG hat in der Vollzugspraxis keine Bedeutung, da es kaum Fälle gibt, in denen ein Härteausgleich für Landwirte gezahlt werden wird.

Stimme völlig zu stimme zu stimme nicht zu lehne völlig ab weiß nicht

5. Die Umsetzung der EG-Wasserrahmenrichtlinie wird zu einer Stärkung des landwirtschaftlichen Bodenschutzes führen.

Stimme völlig zu stimme zu stimme nicht zu lehne völlig ab weiß nicht 
Begründung:

6. Sollte Ihrer Meinung nach die landwirtschaftliche Klärschlammverwertung in Zukunft beibehalten werden?

$\mathrm{Ja}$

Nein

Unter der Bedingung, dass

Weiß nicht

Begründung

7. Sollte Ihrer Meinung nach die landwirtschaftliche Kompostverwertung in Zukunft beibehalten werden?

$\mathrm{Ja}$

Nein

Unter der Bedingung, dass

Weiß nicht

Begründung: 
8. Wird das Ziel der Bundesregierung, den Flächenverbrauch bis zum Jahr 2020 auf 30 ha/Tag zu begrenzen, mit den vorgeschlagenen Maßnahmen erreicht?

$\mathrm{Ja}$

Nein

Unter der Bedingung, dass

Weiß nicht

9. Könnte Ihrer Meinung nach die Einführung einer Flächennutzungssteuer eine langfristige Reduzierung des Flächenverbrauchs in Deutschland bewirken?

$\mathrm{Ja}$

Nein

Weiß nicht

Begründung:

10. Der momentane Anteil der konservierenden Bodenbearbeitung an der Ackerfläche in Deutschland wird auf etwa $4 \%$ geschätzt. Wie hoch schätzen Sie diesen Anteil in diesem Jahr, in fünf bzw. in zehn Jahren ein?

$\begin{array}{lr}\text { Heute } & \% \\ \text { In fünf Jahren } & \% \\ \text { In zehn Jahren } & \%\end{array}$

Weiß nicht 
Bemerkungen/Hinweise:

11. In einigen Bundesländern wird im Rahmen der Verordnung (EWG) Nr. 2078/92 u. a. die konservierende Bodenbearbeitung gefördert. Hierdurch konnte z. T. eine große Verbreitung dieser Anbauverfahren erreicht werden (z. B. in Sachsen $30 \%$ der AF). Bitte Schätzen Sie die Entwicklung der dauerhaft mit konservierender Bodenbearbeitung bestellter Ackerfläche bei einem Wegfall dieser Förderung ein!

\begin{tabular}{|c|c|c|}
\hline Kurzfristig (innerhalb eines Jahres) & $\begin{array}{l}\text { mittelfristig (innerhalb von } 5 \text { Jah- } \\
\text { ren) }\end{array}$ & $\begin{array}{c}\text { langfristig (innerhalb von } 10 \text { Jah- } \\
\text { ren) }\end{array}$ \\
\hline Zunahme um _ $\%$ & Zunahme um _ $\%$ & Zunahme um __ $\%$ \\
\hline$\square$ keine Veränderung & $\square$ keine Veränderung & $\square$ keine Veränderung \\
\hline Abnahme um __ $\%$ & Abnahme um __ $\%$ & Abnahme um \\
\hline$\square$ Weiß nicht & $\square$ Weiß nicht & $\square$ Weiß nicht \\
\hline
\end{tabular}

Begründung:

12. Nehmen Sie bitte kurz Stellung zur Wirkung des Modulationsgesetzes, dass am 1.1. 2003 in Kraft getreten ist, auf den ldw. Bodenschutz?

13. Wie hoch werden sich die zukünftigen jährlichen Zahlungen seitens der staatl. Verwaltungen im Rahmen der Ausgleichsregelung des $\S 10$ Abs. 2 BBodSchG für geschädigte Landwirte in den nächsten Jahren entwickeln? 
Die Zahlungen werden steigen (voraussichtlich um $\%)$

Die Zahlungen bleiben unverändert

$\square$ Die Zahlungen werden sinken (voraussichtlich um $\%)$

Es werden somit ca. $€ / J a h r$ gezahlt werden

$\square$ Weiß nicht

Bemerkungen/Hinweise:

\section{Allgemeine Fragen zur Person}

In welchen Arbeitsbereichen sind Sie tätig? (Mehrfachnennungen möglich)

\section{Wissenschaft}

Bodenkunde /-wissenschaft

Pflanzenbau

landwirtschaftliche Betriebswirtschaftslehre

Bodenschutzforschung

Bodenschutz- und Umweltrecht

Sonstiges

\section{Landwirtschaftliche Beratung}

allgemeine Betriebsberatung

Betriebswirtschaftliche Beratung

Pflanzenbauliche Beratung

Bodenkundliche Beratung

Sonstiges 


\title{
Fachbehörde
}

landwirtschaftliche Fachbehörde

geologische/geografische Fachbehörde

Umweltfachbehörde

Sonstiges

Verband, Interessenvertretung

Sonstiges

In welchen Teilbereichen des Bodenschutzes sind Sie tätig, bzw. womit setzen Sie sich in Ihrer Tätigkeit vorwiegend auseinander? (Mehrfachnennungen möglich)

\author{
Allgemeine Bereiche \\ Bodenschutzrecht \\ Administration \\ Beratung
}

\section{Stofflicher Bodenschutz}

Klärschlamm-/Kompostverwertung

Düngemittelproblematik

Pflanzenschutzmittelproblematik

Schwermetallbelastung von landw. Böden

Sonstiges

\section{Physikalischer Bodenschutz}

Bodenerosion 
$\square \quad$ Bodenschadverdichtung

$\square \quad$ Bodenversiegelung

$\square \quad$ Sonstiges

Allgemeine Hinweise zum Fragebogen und Bemerkungen:

Vielen Dank für Ihre Mithilfe! 
Teil III

Berechnungen 
Tabelle A-21: Mulchsaat mit 4,6 t/ha Bodenabtrag, Effizienz 95 \% (verhinderter Bodenabtrag 4,4 t/ha/a)

\begin{tabular}{|c|c|c|c|c|c|c|c|c|c|}
\hline Jahr & $\begin{array}{c}\text { Kosten } \\
\text { (€/ha) }\end{array}$ & $\begin{array}{c}\text { Nutzen } \\
(€ / \text { ha) }\end{array}$ & $\begin{array}{l}\text { NK-Diff. } \\
\text { (€/ha) }\end{array}$ & $\begin{array}{c}\text { DF } \\
\text { (Zins } 4 \% \text { ) }\end{array}$ & $\begin{array}{c}\text { disk. Kosten } \\
\text { (€/ha) }\end{array}$ & $\begin{array}{c}\text { disk. Nutzen } \\
(€ / \mathrm{ha})\end{array}$ & $\begin{array}{c}\text { disk. NK-Diff. } \\
(€ / h a)\end{array}$ & $\begin{array}{c}\text { WGF } \\
\text { (Zins } 4 \% \text { ) }\end{array}$ & $\begin{array}{c}\varnothing \text { jährl. Vorteil } \\
(€ / \text { ha/a) }\end{array}$ \\
\hline 0 & 0 & 0 & 0,00 & 0,00 & 0,00 & 0,00 & 0,00 & & \\
\hline 1 & 24,93 & 67,52 & 42,59 & 0,9615 & 23,97 & 64,92 & 40,95 & & \\
\hline 2 & 24,93 & 67,52 & 42,59 & 0,9246 & 23,05 & 62,43 & 39,38 & & \\
\hline 3 & 24,93 & 67,52 & 42,59 & 0,889 & 22,16 & 60,03 & 37,86 & & \\
\hline 4 & 24,93 & 67,52 & 42,59 & 0,8548 & 21,31 & 57,72 & 36,41 & & \\
\hline 5 & 24,93 & 67,52 & 42,59 & 0,8219 & 20,49 & 55,49 & 35,00 & & \\
\hline 6 & 24,93 & 67,52 & 42,59 & 0,7903 & 19,70 & 53,36 & 33,66 & & \\
\hline 7 & 24,93 & 67,52 & 42,59 & 0,7599 & 18,94 & 51,31 & 32,36 & & \\
\hline 8 & 24,93 & 67,52 & 42,59 & 0,7307 & 18,22 & 49,34 & 31,12 & & \\
\hline 9 & 24,93 & 67,52 & 42,59 & 0,7026 & 17,52 & 47,44 & 29,92 & & \\
\hline 10 & 24,93 & 202,52 & 177,59 & 0,6756 & 16,84 & 136,82 & 119,98 & & \\
\hline 11 & 24,93 & 67,52 & 42,59 & 0,6496 & 16,19 & 43,86 & 27,67 & & \\
\hline 12 & 24,93 & 67,52 & 42,59 & 0,6246 & 15,57 & 42,17 & 26,60 & & \\
\hline 13 & 24,93 & 67,52 & 42,59 & 0,6006 & 14,97 & 40,55 & 25,58 & & \\
\hline 14 & 24,93 & 67,52 & 42,59 & 0,5775 & 14,40 & 38,99 & 24,60 & & \\
\hline 15 & 24,93 & 67,52 & 42,59 & 0,5553 & 13,84 & 37,49 & 23,65 & & \\
\hline 16 & 24,93 & 67,52 & 42,59 & 0,5339 & 13,31 & 36,05 & 22,74 & & \\
\hline 17 & 24,93 & 67,52 & 42,59 & 0,5134 & 12,80 & 34,66 & 21,87 & & \\
\hline 18 & 24,93 & 67,52 & 42,59 & 0,4936 & 12,31 & 33,33 & 21,02 & & \\
\hline 19 & 24,93 & 67,52 & 42,59 & 0,4746 & 11,83 & 32,04 & 20,21 & & \\
\hline 20 & 24,93 & 202,52 & 177,59 & 0,4564 & 11,38 & 92,43 & 81,05 & & \\
\hline 21 & 24,93 & 67,52 & 42,59 & 0,4388 & 10,94 & 29,63 & 18,69 & & \\
\hline 22 & 24,93 & 67,52 & 42,59 & 0,422 & 10,52 & 28,49 & 17,97 & & \\
\hline 23 & 24,93 & 67,52 & 42,59 & 0,4057 & 10,11 & 27,39 & 17,28 & & \\
\hline 24 & 24,93 & 67,52 & 42,59 & 0,3901 & 9,73 & 26,34 & 16,61 & & \\
\hline \multirow[t]{3}{*}{25} & 24,93 & 67,52 & 42,59 & 0,3751 & 9,35 & 25,33 & 15,98 & & \\
\hline & & & & & 389,46 & 1207,62 & 818,17 & 0,0640 & 52,36 \\
\hline & & & & KNV & & 0,32 & & & \\
\hline
\end{tabular}


Tabelle A-22: Mulchsaat, Standort mit 8,7 t/ha Bodenabtrag, Effizienz 95 \% (verhinderter Bodenabtrag 8,265 t/ha/a)

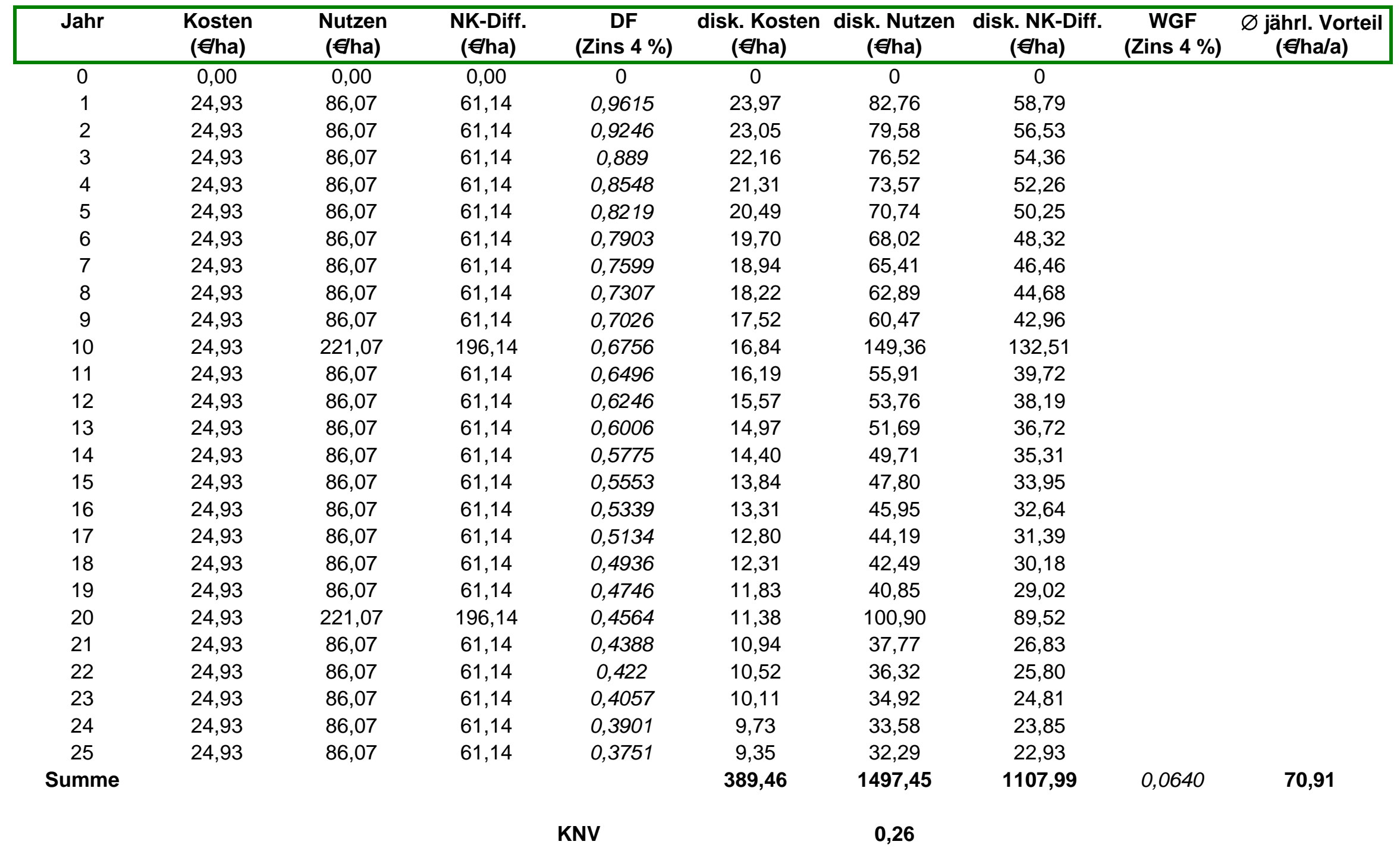


Tabelle A-23: Direktsaat, Standort mit 4,6 t/ha Bodenabtrag, Effizienz $98 \%$ (verhinderter Bodenabtrag 4,5 t/ha/a)

\begin{tabular}{|c|c|c|c|c|c|c|c|c|c|}
\hline Jahr & $\begin{array}{c}\text { Kosten } \\
\text { (€/ha) }\end{array}$ & $\begin{array}{c}\text { Nutzen } \\
\text { (€/ha) }\end{array}$ & $\begin{array}{c}\text { NK-Diff. } \\
\text { (€/ha) }\end{array}$ & $\begin{array}{c}\text { DF } \\
\text { (Zins } 4 \% \text { ) }\end{array}$ & $\begin{array}{c}\text { disk. Kosten } \\
(€ / \mathrm{ha})\end{array}$ & $\begin{array}{c}\text { disk. Nutzen } \\
\text { (€/ha) }\end{array}$ & $\begin{array}{c}\text { disk. NK-Diff. } \\
(€ / h a)\end{array}$ & $\begin{array}{c}\text { WGF } \\
\text { (Zins } 4 \% \text { ) }\end{array}$ & $\begin{array}{c}\varnothing \text { jährl. Vorteil } \\
(€ / \text { ha/a) }\end{array}$ \\
\hline 0 & 0 & 0 & 0,00 & 0,00 & 0,00 & 0,00 & 0,00 & & \\
\hline 1 & 29,45 & 106,20 & 76,75 & 0,9615 & 28,32 & 102,11 & 73,80 & & \\
\hline 2 & 29,45 & 106,20 & 76,75 & 0,9246 & 27,23 & 98,19 & 70,96 & & \\
\hline 3 & 29,45 & 106,20 & 76,75 & 0,889 & 26,18 & 94,41 & 68,23 & & \\
\hline 4 & 29,45 & 106,20 & 76,75 & 0,8548 & 25,17 & 90,78 & 65,61 & & \\
\hline 5 & 29,45 & 106,20 & 76,75 & 0,8219 & 24,20 & 87,29 & 63,08 & & \\
\hline 6 & 29,45 & 106,20 & 76,75 & 0,7903 & 23,27 & 83,93 & 60,66 & & \\
\hline 7 & 29,45 & 106,20 & 76,75 & 0,7599 & 22,38 & 80,70 & 58,32 & & \\
\hline 8 & 29,45 & 106,20 & 76,75 & 0,7307 & 21,52 & 77,60 & 56,08 & & \\
\hline 9 & 29,45 & 106,20 & 76,75 & 0,7026 & 20,69 & 74,62 & 53,92 & & \\
\hline 10 & 29,45 & 241,20 & 211,75 & 0,6756 & 19,90 & 162,95 & 143,06 & & \\
\hline 11 & 29,45 & 106,20 & 76,75 & 0,6496 & 19,13 & 68,99 & 49,86 & & \\
\hline 12 & 29,45 & 106,20 & 76,75 & 0,6246 & 18,39 & 66,33 & 47,94 & & \\
\hline 13 & 29,45 & 106,20 & 76,75 & 0,6006 & 17,69 & 63,78 & 46,10 & & \\
\hline 14 & 29,45 & 106,20 & 76,75 & 0,5775 & 17,01 & 61,33 & 44,32 & & \\
\hline 15 & 29,45 & 106,20 & 76,75 & 0,5553 & 16,35 & 58,97 & 42,62 & & \\
\hline 16 & 29,45 & 106,20 & 76,75 & 0,5339 & 15,72 & 56,70 & 40,98 & & \\
\hline 17 & 29,45 & 106,20 & 76,75 & 0,5134 & 15,12 & 54,52 & 39,40 & & \\
\hline 18 & 29,45 & 106,20 & 76,75 & 0,4936 & 14,54 & 52,42 & 37,88 & & \\
\hline 19 & 29,45 & 106,20 & 76,75 & 0,4746 & 13,98 & 50,40 & 36,43 & & \\
\hline 20 & 29,45 & 241,20 & 211,75 & 0,4564 & 13,44 & 110,08 & 96,64 & & \\
\hline 21 & 29,45 & 106,20 & 76,75 & 0,4388 & 12,92 & 46,60 & 33,68 & & \\
\hline 22 & 29,45 & 106,20 & 76,75 & 0,422 & 12,43 & 44,82 & 32,39 & & \\
\hline 23 & 29,45 & 106,20 & 76,75 & 0,4057 & 11,95 & 43,09 & 31,14 & & \\
\hline 24 & 29,45 & 106,20 & 76,75 & 0,3901 & 11,49 & 41,43 & 29,94 & & \\
\hline \multirow[t]{3}{*}{25} & 29,45 & 106,20 & 76,75 & 0,3751 & 11,05 & 39,84 & 28,79 & & \\
\hline & & & & & 460,07 & 1811,89 & 1351,82 & 0,0640 & 86,52 \\
\hline & & & & KNV & & 0,25 & & & \\
\hline
\end{tabular}


Tabelle A-24: Direktsaat, Standort mit 8,7t/ha Bodenabtrag, Effizienz $98 \%$ (verhinderter Bodenabtrag 8,5 t/ha/a)

\begin{tabular}{|c|c|c|c|c|c|c|c|c|c|}
\hline Jahr & $\begin{array}{c}\text { Kosten } \\
\text { (€/ha) }\end{array}$ & $\begin{array}{c}\text { Nutzen } \\
\text { (€/ha) }\end{array}$ & $\begin{array}{c}\text { NK-Diff. } \\
\text { (€/ha) }\end{array}$ & $\begin{array}{c}\text { DF } \\
\text { (Zins } 4 \% \text { ) }\end{array}$ & $\begin{array}{c}\text { disk. Kosten } \\
(€ / \text { ha) }\end{array}$ & $\begin{array}{c}\text { disk. Nutzen } \\
(€ / \text { ha) }\end{array}$ & $\begin{array}{c}\text { disk. NK-Diff. } \\
(€ / h a)\end{array}$ & $\begin{array}{c}\text { WGF } \\
\text { (Zins } 4 \% \text { ) }\end{array}$ & $\begin{array}{c}\varnothing \text { jährl. Vorteil } \\
(€ / \text { ha/a) }\end{array}$ \\
\hline 0 & 0,00 & 0,00 & 0,00 & 0,00 & 0,00 & 0,00 & 0,00 & & \\
\hline 1 & 29,45 & 125,40 & 95,95 & 0,9615 & 28,32 & 120,57 & 92,26 & & \\
\hline 2 & 29,45 & 125,40 & 95,95 & 0,9246 & 27,23 & 115,94 & 88,72 & & \\
\hline 3 & 29,45 & 125,40 & 95,95 & 0,889 & 26,18 & 111,48 & 85,30 & & \\
\hline 4 & 29,45 & 125,40 & 95,95 & 0,8548 & 25,17 & 107,19 & 82,02 & & \\
\hline 5 & 29,45 & 125,40 & 95,95 & 0,8219 & 24,20 & 103,07 & 78,86 & & \\
\hline 6 & 29,45 & 125,40 & 95,95 & 0,7903 & 23,27 & 99,10 & 75,83 & & \\
\hline 7 & 29,45 & 125,40 & 95,95 & 0,7599 & 22,38 & 95,29 & 72,91 & & \\
\hline 8 & 29,45 & 125,40 & 95,95 & 0,7307 & 21,52 & 91,63 & 70,11 & & \\
\hline 9 & 29,45 & 125,40 & 95,95 & 0,7026 & 20,69 & 88,11 & 67,41 & & \\
\hline 10 & 29,45 & 260,40 & 230,95 & 0,6756 & 19,90 & 175,93 & 156,03 & & \\
\hline 11 & 29,45 & 125,40 & 95,95 & 0,6496 & 19,13 & 81,46 & 62,33 & & \\
\hline 12 & 29,45 & 125,40 & 95,95 & 0,6246 & 18,39 & 78,32 & 59,93 & & \\
\hline 13 & 29,45 & 125,40 & 95,95 & 0,6006 & 17,69 & 75,32 & 57,63 & & \\
\hline 14 & 29,45 & 125,40 & 95,95 & 0,5775 & 17,01 & 72,42 & 55,41 & & \\
\hline 15 & 29,45 & 125,40 & 95,95 & 0,5553 & 16,35 & 69,63 & 53,28 & & \\
\hline 16 & 29,45 & 125,40 & 95,95 & 0,5339 & 15,72 & 66,95 & 51,23 & & \\
\hline 17 & 29,45 & 125,40 & 95,95 & 0,5134 & 15,12 & 64,38 & 49,26 & & \\
\hline 18 & 29,45 & 125,40 & 95,95 & 0,4936 & 14,54 & 61,90 & 47,36 & & \\
\hline 19 & 29,45 & 125,40 & 95,95 & 0,4746 & 13,98 & 59,51 & 45,54 & & \\
\hline 20 & 29,45 & 260,40 & 230,95 & 0,4564 & 13,44 & 118,85 & 105,41 & & \\
\hline 21 & 29,45 & 125,40 & 95,95 & 0,4388 & 12,92 & 55,03 & 42,10 & & \\
\hline 22 & 29,45 & 125,40 & 95,95 & 0,422 & 12,43 & 52,92 & 40,49 & & \\
\hline 23 & 29,45 & 125,40 & 95,95 & 0,4057 & 11,95 & 50,87 & 38,93 & & \\
\hline 24 & 29,45 & 125,40 & 95,95 & 0,3901 & 11,49 & 48,92 & 37,43 & & \\
\hline \multirow[t]{3}{*}{25} & 29,45 & 125,40 & 95,95 & 0,3751 & 11,05 & 47,04 & 35,99 & & \\
\hline & & & & & 460,07 & 2111,83 & 1651,76 & 0,0640 & 105,71 \\
\hline & & & & KNV & & 0,22 & & & \\
\hline
\end{tabular}


Tabelle A-25: Bewirtschaftung quer zum Hang, Kostenniveau niedrig; Nutzen in Höhe von 4,5 $€ /$ ha

\begin{tabular}{|c|c|c|c|c|c|c|c|c|c|}
\hline Jahr & $\begin{array}{l}\text { Kosten } \\
\text { (€/ha) }\end{array}$ & $\begin{array}{l}\text { Nutzen } \\
\text { (€/ha) }\end{array}$ & $\begin{array}{l}\text { NK-Diff. } \\
\text { (€/ha) }\end{array}$ & $\begin{array}{c}\text { DF } \\
\text { (Zins } 4 \% \text { ) }\end{array}$ & $\begin{array}{c}\text { disk. Kosten } \\
\text { (€/ha) }\end{array}$ & $\begin{array}{c}\text { disk. Nutzen } \\
\text { (€/ha) }\end{array}$ & $\begin{array}{c}\text { disk. NK-Diff. } \\
(€ / \mathrm{ha})\end{array}$ & $\begin{array}{c}\text { WGF } \\
\text { (Zins } 4 \% \text { ) }\end{array}$ & $\begin{array}{c}\varnothing \text { jährl. Vorteil } \\
\text { (€/ha/a) }\end{array}$ \\
\hline 0 & 0,00 & 0,00 & 0,00 & 0,00 & 0,00 & 0,00 & 0,00 & & \\
\hline 1 & 16,50 & 4,50 & $-12,00$ & 0,9615 & 15,86 & 4,33 & $-11,54$ & & \\
\hline 2 & 16,50 & 4,50 & $-12,00$ & 0,9246 & 15,26 & 4,16 & $-11,10$ & & \\
\hline 3 & 16,50 & 4,50 & $-12,00$ & 0,889 & 14,67 & 4,00 & $-10,67$ & & \\
\hline 4 & 16,50 & 4,50 & $-12,00$ & 0,8548 & 14,10 & 3,85 & $-10,26$ & & \\
\hline 5 & 16,50 & 4,50 & $-12,00$ & 0,8219 & 13,56 & 3,70 & $-9,86$ & & \\
\hline 6 & 16,50 & 4,50 & $-12,00$ & 0,7903 & 13,04 & 3,56 & $-9,48$ & & \\
\hline 7 & 16,50 & 4,50 & $-12,00$ & 0,7599 & 12,54 & 3,42 & $-9,12$ & & \\
\hline 8 & 16,50 & 4,50 & $-12,00$ & 0,7307 & 12,06 & 3,29 & $-8,77$ & & \\
\hline 9 & 16,50 & 4,50 & $-12,00$ & 0,7026 & 11,59 & 3,16 & $-8,43$ & & \\
\hline 10 & 16,50 & 4,50 & $-12,00$ & 0,6756 & 11,15 & 3,04 & $-8,11$ & & \\
\hline 11 & 16,50 & 4,50 & $-12,00$ & 0,6496 & 10,72 & 2,92 & $-7,80$ & & \\
\hline 12 & 16,50 & 4,50 & $-12,00$ & 0,6246 & 10,31 & 2,81 & $-7,50$ & & \\
\hline 13 & 16,50 & 4,50 & $-12,00$ & 0,6006 & 9,91 & 2,70 & $-7,21$ & & \\
\hline 14 & 16,50 & 4,50 & $-12,00$ & 0,5775 & 9,53 & 2,60 & $-6,93$ & & \\
\hline 15 & 16,50 & 4,50 & $-12,00$ & 0,5553 & 9,16 & 2,50 & $-6,66$ & & \\
\hline 16 & 16,50 & 4,50 & $-12,00$ & 0,5339 & 8,81 & 2,40 & $-6,41$ & & \\
\hline 17 & 16,50 & 4,50 & $-12,00$ & 0,5134 & 8,47 & 2,31 & $-6,16$ & & \\
\hline 18 & 16,50 & 4,50 & $-12,00$ & 0,4936 & 8,14 & 2,22 & $-5,92$ & & \\
\hline 19 & 16,50 & 4,50 & $-12,00$ & 0,4746 & 7,83 & 2,14 & $-5,70$ & & \\
\hline 20 & 16,50 & 4,50 & $-12,00$ & 0,4564 & 7,53 & 2,05 & $-5,48$ & & \\
\hline 21 & 16,50 & 4,50 & $-12,00$ & 0,4388 & 7,24 & 1,97 & $-5,27$ & & \\
\hline 22 & 16,50 & 4,50 & $-12,00$ & 0,422 & 6,96 & 1,90 & $-5,06$ & & \\
\hline 23 & 16,50 & 4,50 & $-12,00$ & 0,4057 & 6,69 & 1,83 & $-4,87$ & & \\
\hline 24 & 16,50 & 4,50 & $-12,00$ & 0,3901 & 6,44 & 1,76 & $-4,68$ & & \\
\hline \multirow[t]{3}{*}{25} & 16,50 & 4,50 & $-12,00$ & 0,3751 & 6,19 & 1,69 & $-4,50$ & & \\
\hline & & & & & 257,76 & 70,30 & $-187,47$ & 0,0640 & $-12,00$ \\
\hline & & & & KNV & & 3,67 & & & \\
\hline
\end{tabular}


Tabelle A-26: Bewirtschaftung quer zum Hang, Kostenniveau hoch; Nutzen in Höhe von 4,5 $€$ /ha

\begin{tabular}{|c|c|c|c|c|c|c|c|c|c|}
\hline Jahr & $\begin{array}{c}\text { Kosten } \\
\text { (€/ha) }\end{array}$ & $\begin{array}{c}\text { Nutzen } \\
\text { (€/ha) }\end{array}$ & $\begin{array}{l}\text { NK-Diff. } \\
\text { (€/ha) }\end{array}$ & $\begin{array}{c}\text { DF } \\
\text { (Zins } 4 \%)\end{array}$ & $\begin{array}{c}\text { disk. Kosten } \\
\text { (€/ha) }\end{array}$ & $\begin{array}{c}\text { disk. Nutzen } \\
\text { (€/ha) }\end{array}$ & $\begin{array}{l}\text { disk. NK-Diff. } \\
\qquad(€ / h a)\end{array}$ & $\begin{array}{c}\text { WGF } \\
\text { (Zins } 4 \% \text { ) }\end{array}$ & $\begin{array}{c}\varnothing \text { jährl. Vorteil } \\
\text { (€/ha/a) }\end{array}$ \\
\hline 0 & 0,00 & 0,00 & 0,00 & 0,00 & 0,00 & 0,00 & 0,00 & & \\
\hline 1 & 101,80 & 4,50 & $-97,30$ & 0,9615 & 97,88 & 4,33 & $-93,55$ & & \\
\hline 2 & 101,80 & 4,50 & $-97,30$ & 0,9246 & 94,12 & 4,16 & $-89,96$ & & \\
\hline 3 & 101,80 & 4,50 & $-97,30$ & 0,889 & 90,50 & 4,00 & $-86,50$ & & \\
\hline 4 & 101,80 & 4,50 & $-97,30$ & 0,8548 & 87,02 & 3,85 & $-83,17$ & & \\
\hline 5 & 101,80 & 4,50 & $-97,30$ & 0,8219 & 83,67 & 3,70 & $-79,97$ & & \\
\hline 6 & 101,80 & 4,50 & $-97,30$ & 0,7903 & 80,45 & 3,56 & $-76,90$ & & \\
\hline 7 & 101,80 & 4,50 & $-97,30$ & 0,7599 & 77,36 & 3,42 & $-73,94$ & & \\
\hline 8 & 101,80 & 4,50 & $-97,30$ & 0,7307 & 74,39 & 3,29 & $-71,10$ & & \\
\hline 9 & 101,80 & 4,50 & $-97,30$ & 0,7026 & 71,52 & 3,16 & $-68,36$ & & \\
\hline 10 & 101,80 & 4,50 & $-97,30$ & 0,6756 & 68,78 & 3,04 & $-65,74$ & & \\
\hline 11 & 101,80 & 4,50 & $-97,30$ & 0,6496 & 66,13 & 2,92 & $-63,21$ & & \\
\hline 12 & 101,80 & 4,50 & $-97,30$ & 0,6246 & 63,58 & 2,81 & $-60,77$ & & \\
\hline 13 & 101,80 & 4,50 & $-97,30$ & 0,6006 & 61,14 & 2,70 & $-58,44$ & & \\
\hline 15 & 101,80 & 4,50 & $-97,30$ & 0,5553 & 56,53 & 2,50 & $-54,03$ & & \\
\hline 16 & 101,80 & 4,50 & $-97,30$ & 0,5339 & 54,35 & 2,40 & $-51,95$ & & \\
\hline 17 & 101,80 & 4,50 & $-97,30$ & 0,5134 & 52,26 & 2,31 & $-49,95$ & & \\
\hline 18 & 101,80 & 4,50 & $-97,30$ & 0,4936 & 50,25 & 2,22 & $-48,03$ & & \\
\hline 19 & 101,80 & 4,50 & $-97,30$ & 0,4746 & 48,31 & 2,14 & $-46,18$ & & \\
\hline 20 & 101,80 & 4,50 & $-97,30$ & 0,4564 & 46,46 & 2,05 & $-44,41$ & & \\
\hline 21 & 101,80 & 4,50 & $-97,30$ & 0,4388 & 44,67 & 1,97 & $-42,70$ & & \\
\hline 22 & 101,80 & 4,50 & $-97,30$ & 0,422 & 42,96 & 1,90 & $-41,06$ & & \\
\hline 23 & 101,80 & 4,50 & $-97,30$ & 0,4057 & 41,30 & 1,83 & $-39,47$ & & \\
\hline 24 & 101,80 & 4,50 & $-97,30$ & 0,3901 & 39,71 & 1,76 & $-37,96$ & & \\
\hline \multirow[t]{3}{*}{25} & 101,80 & 4,50 & $-97,30$ & 0,3751 & 38,19 & 1,69 & $-36,50$ & & \\
\hline & & & & & 1590,33 & 70,30 & $-1520,03$ & 0,0640 & $-97,28$ \\
\hline & & & & KNV & & 22,62 & & & \\
\hline
\end{tabular}


Tabelle A-27:Bewirtschaftung quer zum Hang und Mulchsaat, Kostenniveau hoch

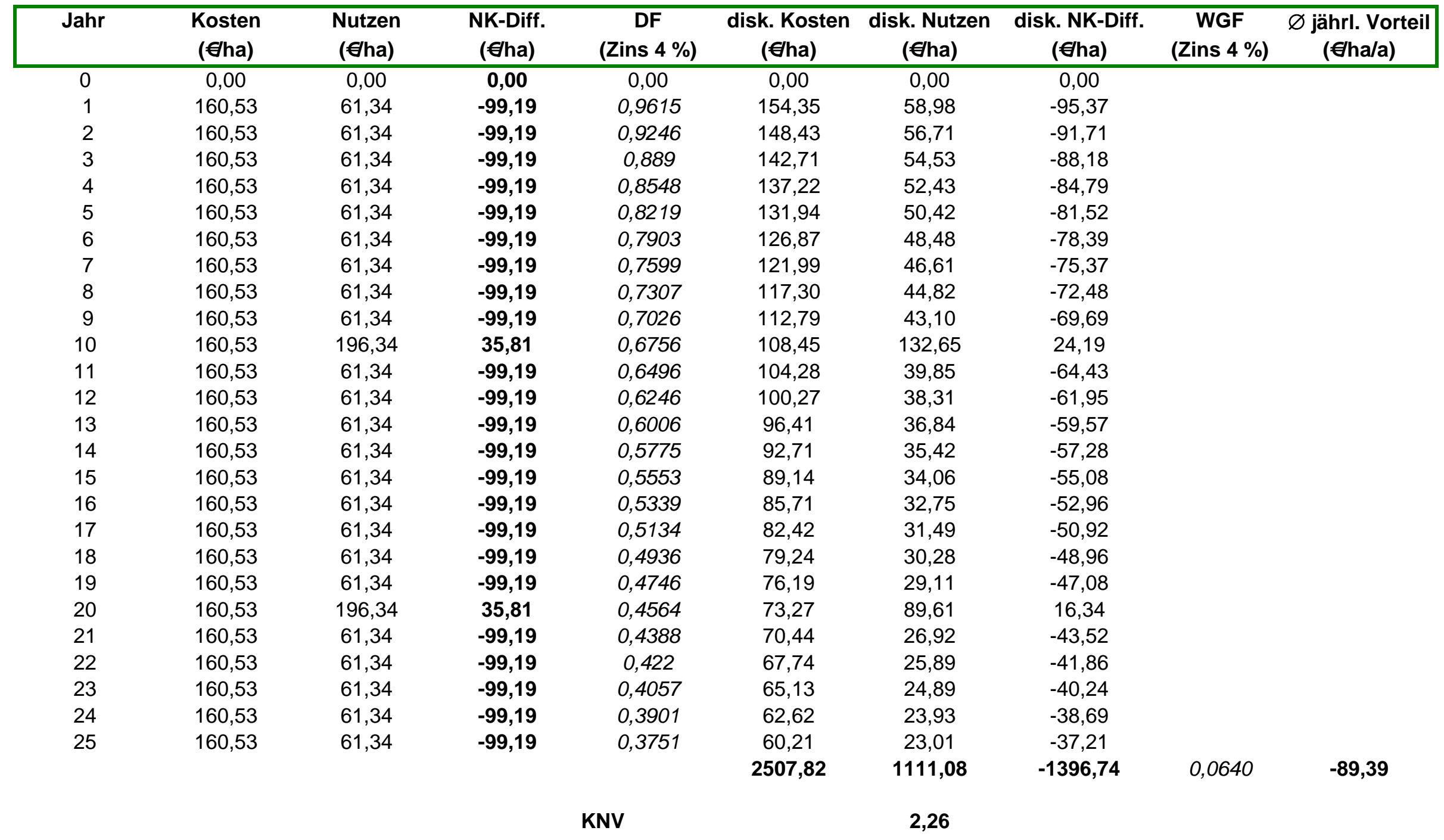


Tabelle A-28: Bewirtschaftung quer zum Hang und Mulchsaat, Kostenniveau niedrig

\begin{tabular}{|c|c|c|c|c|c|c|c|c|c|}
\hline Jahr & $\begin{array}{c}\text { Kosten } \\
(€ / \text { ha) }\end{array}$ & $\begin{array}{c}\text { Nutzen } \\
(€ / \text { ha) }\end{array}$ & $\begin{array}{c}\text { NK-Diff. } \\
\text { (€/ha) }\end{array}$ & $\begin{array}{c}\text { DF } \\
\text { (Zins } 4 \%)\end{array}$ & $\begin{array}{l}\text { disk. Kosten } \\
\text { (€/ha) }\end{array}$ & $\begin{array}{c}\text { disk. Nutzen } \\
(€ / \text { ha) }\end{array}$ & $\begin{array}{l}\text { disk. NK-Diff. } \\
(€ / \text { ha) }\end{array}$ & $\begin{array}{c}\text { WGF } \\
\text { (Zins } 4 \%)\end{array}$ & $\begin{array}{c}\varnothing \text { jährl. Vorteil } \\
(€ / \text { ha/a) }\end{array}$ \\
\hline 0 & 0,00 & 0,00 & 0,00 & 0,00 & 0,00 & 0,00 & 0,00 & & \\
\hline 1 & 75,23 & 61,34 & $-13,89$ & 0,9615 & 72,33 & 58,98 & $-13,36$ & & \\
\hline 2 & 75,23 & 61,34 & $-13,89$ & 0,9246 & 69,56 & 56,71 & $-12,84$ & & \\
\hline 3 & 75,23 & 61,34 & $-13,89$ & 0,889 & 66,88 & 54,53 & $-12,35$ & & \\
\hline 4 & 75,23 & 61,34 & $-13,89$ & 0,8548 & 64,31 & 52,43 & $-11,87$ & & \\
\hline 5 & 75,23 & 61,34 & $-13,89$ & 0,8219 & 61,83 & 50,42 & $-11,42$ & & \\
\hline 6 & 75,23 & 61,34 & $-13,89$ & 0,7903 & 59,45 & 48,48 & $-10,98$ & & \\
\hline 7 & 75,23 & 61,34 & $-13,89$ & 0,7599 & 57,17 & 46,61 & $-10,56$ & & \\
\hline 8 & 75,23 & 61,34 & $-13,89$ & 0,7307 & 54,97 & 44,82 & $-10,15$ & & \\
\hline 9 & 75,23 & 61,34 & $-13,89$ & 0,7026 & 52,86 & 43,10 & $-9,76$ & & \\
\hline 10 & 75,23 & 196,34 & 121,11 & 0,6756 & 50,83 & 132,65 & 81,82 & & \\
\hline 11 & 75,23 & 61,34 & $-13,89$ & 0,6496 & 48,87 & 39,85 & $-9,02$ & & \\
\hline 12 & 75,23 & 61,34 & $-13,89$ & 0,6246 & 46,99 & 38,31 & $-8,68$ & & \\
\hline 13 & 75,23 & 61,34 & $-13,89$ & 0,6006 & 45,18 & 36,84 & $-8,34$ & & \\
\hline 14 & 75,23 & 61,34 & $-13,89$ & 0,5775 & 43,45 & 35,42 & $-8,02$ & & \\
\hline 15 & 75,23 & 61,34 & $-13,89$ & 0,5553 & 41,78 & 34,06 & $-7,71$ & & \\
\hline 16 & 75,23 & 61,34 & $-13,89$ & 0,5339 & 40,17 & 32,75 & $-7,42$ & & \\
\hline 17 & 75,23 & 61,34 & $-13,89$ & 0,5134 & 38,62 & 31,49 & $-7,13$ & & \\
\hline 18 & 75,23 & 61,34 & $-13,89$ & 0,4936 & 37,13 & 30,28 & $-6,86$ & & \\
\hline 19 & 75,23 & 61,34 & $-13,89$ & 0,4746 & 35,70 & 29,11 & $-6,59$ & & \\
\hline 20 & 75,23 & 196,34 & 121,11 & 0,4564 & 34,33 & 89,61 & 55,27 & & \\
\hline 21 & 75,23 & 61,34 & $-13,89$ & 0,4388 & 33,01 & 26,92 & $-6,09$ & & \\
\hline 22 & 75,23 & 61,34 & $-13,89$ & 0,422 & 31,75 & 25,89 & $-5,86$ & & \\
\hline 23 & 75,23 & 61,34 & $-13,89$ & 0,4057 & 30,52 & 24,89 & $-5,64$ & & \\
\hline 24 & 75,23 & 61,34 & $-13,89$ & 0,3901 & 29,35 & 23,93 & $-5,42$ & & \\
\hline \multirow[t]{3}{*}{25} & 75,23 & 61,34 & $-13,89$ & 0,3751 & 28,22 & 23,01 & $-5,21$ & & \\
\hline & & & & & 1175,25 & 1111,08 & $-64,17$ & 0,0640 & $-4,11$ \\
\hline & & & & KNV & & 1,06 & & & \\
\hline
\end{tabular}


Tabelle A-29: Bewirtschaftung quer zum Hang und Direktsaat, Kostenniveau niedrig

\begin{tabular}{|c|c|c|c|c|c|c|c|c|c|}
\hline Jahr & $\begin{array}{c}\text { Kosten } \\
\text { (€/ha) }\end{array}$ & $\begin{array}{c}\text { Nutzen } \\
\text { (€/ha) }\end{array}$ & $\begin{array}{c}\text { NK-Diff. } \\
\text { (€/ha) }\end{array}$ & $\begin{array}{c}\text { DF } \\
\text { (Zins } 4 \%)\end{array}$ & $\begin{array}{c}\text { disk. Kosten } \\
(€ / \mathrm{ha})\end{array}$ & $\begin{array}{c}\text { disk. Nutzen } \\
(€ / \mathrm{ha})\end{array}$ & $\begin{array}{c}\text { disk. NK-Diff. } \\
(€ / \mathrm{ha})\end{array}$ & $\begin{array}{c}\text { WGF } \\
\text { (Zins } 4 \% \text { ) }\end{array}$ & $\begin{array}{c}\varnothing \text { jährl. Vorteil } \\
(€ / \text { ha/a) }\end{array}$ \\
\hline 0 & 0,00 & 0,00 & 0,00 & 0,00 & 0,00 & 0,00 & 0,00 & & \\
\hline 1 & 79,75 & 99,94 & 20,19 & 0,9615 & 76,68 & 96,09 & 19,41 & & \\
\hline 2 & 79,75 & 99,94 & 20,19 & 0,9246 & 73,74 & 92,40 & 18,67 & & \\
\hline 3 & 79,75 & 99,94 & 20,19 & 0,889 & 70,90 & 88,85 & 17,95 & & \\
\hline 4 & 79,75 & 99,94 & 20,19 & 0,8548 & 68,17 & 85,43 & 17,26 & & \\
\hline 5 & 79,75 & 99,94 & 20,19 & 0,8219 & 65,55 & 82,14 & 16,59 & & \\
\hline 6 & 79,75 & 99,94 & 20,19 & 0,7903 & 63,03 & 78,98 & 15,96 & & \\
\hline 7 & 79,75 & 99,94 & 20,19 & 0,7599 & 60,60 & 75,94 & 15,34 & & \\
\hline 8 & 79,75 & 99,94 & 20,19 & 0,7307 & 58,27 & 73,03 & 14,75 & & \\
\hline 9 & 79,75 & 99,94 & 20,19 & 0,7026 & 56,03 & 70,22 & 14,19 & & \\
\hline 10 & 79,75 & 234,94 & 155,19 & 0,6756 & 53,88 & 158,73 & 104,85 & & \\
\hline 11 & 79,75 & 99,94 & 20,19 & 0,6496 & 51,81 & 64,92 & 13,12 & & \\
\hline 12 & 79,75 & 99,94 & 20,19 & 0,6246 & 49,81 & 62,42 & 12,61 & & \\
\hline 13 & 79,75 & 99,94 & 20,19 & 0,6006 & 47,90 & 60,02 & 12,13 & & \\
\hline 14 & 79,75 & 99,94 & 20,19 & 0,5775 & 46,06 & 57,72 & 11,66 & & \\
\hline 15 & 79,75 & 99,94 & 20,19 & 0,5553 & 44,29 & 55,50 & 11,21 & & \\
\hline 16 & 79,75 & 99,94 & 20,19 & 0,5339 & 42,58 & 53,36 & 10,78 & & \\
\hline 17 & 79,75 & 99,94 & 20,19 & 0,5134 & 40,94 & 51,31 & 10,37 & & \\
\hline 18 & 79,75 & 99,94 & 20,19 & 0,4936 & 39,36 & 49,33 & 9,97 & & \\
\hline 19 & 79,75 & 99,94 & 20,19 & 0,4746 & 37,85 & 47,43 & 9,58 & & \\
\hline 20 & 79,75 & 234,94 & 155,19 & 0,4564 & 36,40 & 107,23 & 70,83 & & \\
\hline 21 & 79,75 & 99,94 & 20,19 & 0,4388 & 34,99 & 43,85 & 8,86 & & \\
\hline 22 & 79,75 & 99,94 & 20,19 & 0,422 & 33,65 & 42,17 & 8,52 & & \\
\hline 23 & 79,75 & 99,94 & 20,19 & 0,4057 & 32,35 & 40,55 & 8,19 & & \\
\hline 24 & 79,75 & 99,94 & 20,19 & 0,3901 & 31,11 & 38,99 & 7,88 & & \\
\hline \multirow[t]{2}{*}{25} & 79,75 & 99,94 & 20,19 & 0,3751 & 29,91 & 37,49 & 7,57 & & \\
\hline & & & & & 1245,86 & 1714,09 & 468,23 & 0,0640 & 29,97 \\
\hline
\end{tabular}


Tabelle A-30: Bewirtschaftung quer zum Hang und Direktsaat, Kostenniveau hoch

\begin{tabular}{|c|c|c|c|c|c|c|c|c|c|}
\hline Jahr & $\begin{array}{c}\text { Kosten } \\
\text { (€/ha) }\end{array}$ & $\begin{array}{l}\text { Nutzen } \\
\text { (€/ha) }\end{array}$ & $\begin{array}{l}\text { NK-Diff. } \\
\text { (€/ha) }\end{array}$ & $\begin{array}{c}\text { DF } \\
\text { (Zins } 4 \%)\end{array}$ & $\begin{array}{c}\text { disk. Kosten } \\
\text { (€/ha) }\end{array}$ & $\begin{array}{c}\text { disk. Nutzen } \\
\text { (€/ha) }\end{array}$ & $\begin{array}{c}\text { disk. NK-Diff. } \\
\text { (€/ha) }\end{array}$ & $\begin{array}{c}\text { WGF } \\
\text { (Zins } 4 \% \text { ) }\end{array}$ & $\begin{array}{c}\varnothing \text { jährl. Vorteil } \\
(€ / h a / a)\end{array}$ \\
\hline 0 & 0,00 & 0,00 & 0,00 & 0,00 & 0,00 & 0,00 & 0,00 & & \\
\hline 1 & 165,05 & 99,94 & $-65,11$ & 0,9615 & 158,70 & 96,09 & $-62,60$ & & \\
\hline 2 & 165,05 & 99,94 & $-65,11$ & 0,9246 & 152,61 & 92,40 & $-60,20$ & & \\
\hline 3 & 165,05 & 99,94 & $-65,11$ & 0,889 & 146,73 & 88,85 & $-57,88$ & & \\
\hline 4 & 165,05 & 99,94 & $-65,11$ & 0,8548 & 141,08 & 85,43 & $-55,66$ & & \\
\hline 5 & 165,05 & 99,94 & $-65,11$ & 0,8219 & 135,65 & 82,14 & $-53,51$ & & \\
\hline 6 & 165,05 & 99,94 & $-65,11$ & 0,7903 & 130,44 & 78,98 & $-51,46$ & & \\
\hline 7 & 165,05 & 99,94 & $-65,11$ & 0,7599 & 125,42 & 75,94 & $-49,48$ & & \\
\hline 8 & 165,05 & 99,94 & $-65,11$ & 0,7307 & 120,60 & 73,03 & $-47,58$ & & \\
\hline 9 & 165,05 & 99,94 & $-65,11$ & 0,7026 & 115,96 & 70,22 & $-45,75$ & & \\
\hline 10 & 165,05 & 234,94 & 69,89 & 0,6756 & 111,51 & 158,73 & 47,22 & & \\
\hline 11 & 165,05 & 99,94 & $-65,11$ & 0,6496 & 107,22 & 64,92 & $-42,30$ & & \\
\hline 12 & 165,05 & 99,94 & $-65,11$ & 0,6246 & 103,09 & 62,42 & $-40,67$ & & \\
\hline 13 & 165,05 & 99,94 & $-65,11$ & 0,6006 & 99,13 & 60,02 & $-39,11$ & & \\
\hline 14 & 165,05 & 99,94 & $-65,11$ & 0,5775 & 95,32 & 57,72 & $-37,60$ & & \\
\hline 15 & 165,05 & 99,94 & $-65,11$ & 0,5553 & 91,65 & 55,50 & $-36,16$ & & \\
\hline 16 & 165,05 & 99,94 & $-65,11$ & 0,5339 & 88,12 & 53,36 & $-34,76$ & & \\
\hline 17 & 165,05 & 99,94 & $-65,11$ & 0,5134 & 84,74 & 51,31 & $-33,43$ & & \\
\hline 18 & 165,05 & 99,94 & $-65,11$ & 0,4936 & 81,47 & 49,33 & $-32,14$ & & \\
\hline 19 & 165,05 & 99,94 & $-65,11$ & 0,4746 & 78,33 & 47,43 & $-30,90$ & & \\
\hline 20 & 165,05 & 234,94 & 69,89 & 0,4564 & 75,33 & 107,23 & 31,90 & & \\
\hline 21 & 165,05 & 99,94 & $-65,11$ & 0,4388 & 72,42 & 43,85 & $-28,57$ & & \\
\hline 22 & 165,05 & 99,94 & $-65,11$ & 0,422 & 69,65 & 42,17 & $-27,48$ & & \\
\hline 23 & 165,05 & 99,94 & $-65,11$ & 0,4057 & 66,96 & 40,55 & $-26,42$ & & \\
\hline 24 & 165,05 & 99,94 & $-65,11$ & 0,3901 & 64,39 & 38,99 & $-25,40$ & & \\
\hline \multirow[t]{2}{*}{25} & 165,05 & 99,94 & $-65,11$ & 0,3751 & 61,91 & 37,49 & $-24,42$ & & \\
\hline & & & & & 2578,43 & 1714,09 & $-864,33$ & 0,0640 & $-55,32$ \\
\hline
\end{tabular}


Tabelle A-31: Engreihensaat von Silomais, Ertragssteigerung von $2 \%$

\begin{tabular}{|c|c|c|c|c|c|c|c|c|c|}
\hline Jahr & $\begin{array}{c}\text { Kosten } \\
\text { (€/ha) }\end{array}$ & $\begin{array}{c}\text { Nutzen } \\
\text { (€/ha) }\end{array}$ & $\begin{array}{c}\text { NK-Diff. } \\
\text { (€/ha) }\end{array}$ & $\begin{array}{c}\text { DF } \\
\text { (Zins } 4 \% \text { ) }\end{array}$ & $\begin{array}{c}\text { disk. Kosten } \\
(€ / \text { ha })\end{array}$ & $\begin{array}{c}\text { disk. Nutzen } \\
\text { (€/ha) }\end{array}$ & $\begin{array}{c}\text { disk. NK-Diff. } \\
(€ / h a)\end{array}$ & $\begin{array}{c}\text { WGF } \\
\text { (Zins } 4 \% \text { ) }\end{array}$ & $\begin{array}{c}\varnothing \text { jährl. Vorteil } \\
(€ / h a / a)\end{array}$ \\
\hline 0 & 0,00 & 0,00 & 0,00 & 0,00 & 0,00 & 0,00 & 0,00 & & \\
\hline 1 & 20,62 & 19,50 & $-1,12$ & 0,9615 & 19,83 & 18,75 & $-1,08$ & & \\
\hline 2 & 20,62 & 19,50 & $-1,12$ & 0,9246 & 19,07 & 18,03 & $-1,04$ & & \\
\hline 3 & 20,62 & 19,50 & $-1,12$ & 0,889 & 18,33 & 17,34 & $-1,00$ & & \\
\hline 4 & 20,62 & 19,50 & $-1,12$ & 0,8548 & 17,63 & 16,67 & $-0,96$ & & \\
\hline 5 & 20,62 & 19,50 & $-1,12$ & 0,8219 & 16,95 & 16,03 & $-0,92$ & & \\
\hline 6 & 20,62 & 19,50 & $-1,12$ & 0,7903 & 16,30 & 15,41 & $-0,89$ & & \\
\hline 7 & 20,62 & 19,50 & $-1,12$ & 0,7599 & 15,67 & 14,82 & $-0,85$ & & \\
\hline 8 & 20,62 & 19,50 & $-1,12$ & 0,7307 & 15,07 & 14,25 & $-0,82$ & & \\
\hline 9 & 20,62 & 19,50 & $-1,12$ & 0,7026 & 14,49 & 13,70 & $-0,79$ & & \\
\hline 10 & 20,62 & 19,50 & $-1,12$ & 0,6756 & 13,93 & 13,17 & $-0,76$ & & \\
\hline 11 & 20,62 & 19,50 & $-1,12$ & 0,6496 & 13,39 & 12,67 & $-0,73$ & & \\
\hline 12 & 20,62 & 19,50 & $-1,12$ & 0,6246 & 12,88 & 12,18 & $-0,70$ & & \\
\hline 13 & 20,62 & 19,50 & $-1,12$ & 0,6006 & 12,38 & 11,71 & $-0,67$ & & \\
\hline 14 & 20,62 & 19,50 & $-1,12$ & 0,5775 & 11,91 & 11,26 & $-0,65$ & & \\
\hline 15 & 20,62 & 19,50 & $-1,12$ & 0,5553 & 11,45 & 10,83 & $-0,62$ & & \\
\hline 16 & 20,62 & 19,50 & $-1,12$ & 0,5339 & 11,01 & 10,41 & $-0,60$ & & \\
\hline 17 & 20,62 & 19,50 & $-1,12$ & 0,5134 & 10,59 & 10,01 & $-0,58$ & & \\
\hline 18 & 20,62 & 19,50 & $-1,12$ & 0,4936 & 10,18 & 9,63 & $-0,55$ & & \\
\hline 19 & 20,62 & 19,50 & $-1,12$ & 0,4746 & 9,79 & 9,25 & $-0,53$ & & \\
\hline 20 & 20,62 & 19,50 & $-1,12$ & 0,4564 & 9,41 & 8,90 & $-0,51$ & & \\
\hline 21 & 20,62 & 19,50 & $-1,12$ & 0,4388 & 9,05 & 8,56 & $-0,49$ & & \\
\hline 22 & 20,62 & 19,50 & $-1,12$ & 0,422 & 8,70 & 8,23 & $-0,47$ & & \\
\hline 23 & 20,62 & 19,50 & $-1,12$ & 0,4057 & 8,37 & 7,91 & $-0,45$ & & \\
\hline 24 & 20,62 & 19,50 & $-1,12$ & 0,3901 & 8,04 & 7,61 & $-0,44$ & & \\
\hline \multirow[t]{3}{*}{25} & 20,62 & 19,50 & $-1,12$ & 0,3751 & 7,73 & 7,31 & $-0,42$ & & \\
\hline & & & & & 322,13 & 304,63 & $-17,50$ & 0,0640 & $-1,12$ \\
\hline & & & & KNV & & 1,06 & & & \\
\hline
\end{tabular}


Tabelle A-32: Engreihensaat von Silomais, Ertragssteigerung von $22 \%$

\begin{tabular}{|c|c|c|c|c|c|c|c|c|c|}
\hline Jahr & $\begin{array}{c}\text { Kosten } \\
\text { (€/ha) }\end{array}$ & $\begin{array}{c}\text { Nutzen } \\
\text { (€/ha) }\end{array}$ & $\begin{array}{c}\text { NK-Diff. } \\
\text { (€/ha) }\end{array}$ & $\begin{array}{c}\text { DF } \\
\text { (Zins } 4 \%)\end{array}$ & $\begin{array}{c}\text { disk. Kosten } \\
(€ / \mathrm{ha})\end{array}$ & $\begin{array}{c}\text { disk. Nutzen } \\
(€ / h a)\end{array}$ & $\begin{array}{c}\text { disk. NK-Diff. } \\
(€ / h a)\end{array}$ & $\begin{array}{c}\text { WGF } \\
\text { (Zins } 4 \% \text { ) }\end{array}$ & $\begin{array}{c}\varnothing \text { jährl. Vorteil } \\
\text { (€/ha/a) }\end{array}$ \\
\hline 0 & 0 & 0 & 0,00 & 0,00 & 0,00 & 0,00 & 0,00 & & \\
\hline 1 & 20,62 & 215 & 194,18 & 0,9615 & 19,83 & 206,53 & 186,70 & & \\
\hline 2 & 20,62 & 215 & 194,18 & 0,9246 & 19,07 & 198,60 & 179,54 & & \\
\hline 3 & 20,62 & 215 & 194,18 & 0,889 & 18,33 & 190,96 & 172,63 & & \\
\hline 4 & 20,62 & 215 & 194,18 & 0,8548 & 17,63 & 183,61 & 165,99 & & \\
\hline 5 & 20,62 & 215 & 194,18 & 0,8219 & 16,95 & 176,54 & 159,60 & & \\
\hline 6 & 20,62 & 215 & 194,18 & 0,7903 & 16,30 & 169,76 & 153,46 & & \\
\hline 7 & 20,62 & 215 & 194,18 & 0,7599 & 15,67 & 163,23 & 147,56 & & \\
\hline 8 & 20,62 & 215 & 194,18 & 0,7307 & 15,07 & 156,95 & 141,89 & & \\
\hline 9 & 20,62 & 215 & 194,18 & 0,7026 & 14,49 & 150,92 & 136,43 & & \\
\hline 10 & 20,62 & 215 & 194,18 & 0,6756 & 13,93 & 145,12 & 131,19 & & \\
\hline 11 & 20,62 & 215 & 194,18 & 0,6496 & 13,39 & 139,53 & 126,14 & & \\
\hline 12 & 20,62 & 215 & 194,18 & 0,6246 & 12,88 & 134,16 & 121,28 & & \\
\hline 13 & 20,62 & 215 & 194,18 & 0,6006 & 12,38 & 129,01 & 116,62 & & \\
\hline 14 & 20,62 & 215 & 194,18 & 0,5775 & 11,91 & 124,05 & 112,14 & & \\
\hline 15 & 20,62 & 215 & 194,18 & 0,5553 & 11,45 & 119,28 & 107,83 & & \\
\hline 16 & 20,62 & 215 & 194,18 & 0,5339 & 11,01 & 114,68 & 103,67 & & \\
\hline 17 & 20,62 & 215 & 194,18 & 0,5134 & 10,59 & 110,28 & 99,69 & & \\
\hline 18 & 20,62 & 215 & 194,18 & 0,4936 & 10,18 & 106,03 & 95,85 & & \\
\hline 19 & 20,62 & 215 & 194,18 & 0,4746 & 9,79 & 101,94 & 92,16 & & \\
\hline 20 & 20,62 & 215 & 194,18 & 0,4564 & 9,41 & 98,03 & 88,62 & & \\
\hline 21 & 20,62 & 215 & 194,18 & 0,4388 & 9,05 & 94,25 & 85,21 & & \\
\hline 22 & 20,62 & 215 & 194,18 & 0,422 & 8,70 & 90,65 & 81,94 & & \\
\hline 23 & 20,62 & 215 & 194,18 & 0,4057 & 8,37 & 87,14 & 78,78 & & \\
\hline 24 & 20,62 & 215 & 194,18 & 0,3901 & 8,04 & 83,79 & 75,75 & & \\
\hline \multirow[t]{3}{*}{25} & 20,62 & 215 & 194,18 & 0,3751 & 7,73 & 80,57 & 72,84 & & \\
\hline & & & & & 322,13 & 3355,63 & 3033,50 & 0,0640 & 194,14 \\
\hline & & & & KNV & & 0,10 & & & \\
\hline
\end{tabular}


Tabelle A-33: ZUA, Variante 1

\begin{tabular}{|c|c|c|c|c|c|c|c|c|c|}
\hline Jahr & $\begin{array}{c}\text { Kosten } \\
\text { (€/ha) }\end{array}$ & $\begin{array}{l}\text { Nutzen } \\
\text { (€/ha) }\end{array}$ & $\begin{array}{c}\text { NK-Diff. } \\
\text { (€/ha) }\end{array}$ & $\begin{array}{c}\text { DF } \\
\text { (Zins } 4 \% \text { ) }\end{array}$ & $\begin{array}{c}\text { disk. Kosten } \\
(€ / h \mathrm{~h})\end{array}$ & $\begin{array}{c}\text { disk. Nutzen } \\
\text { (€/ha) }\end{array}$ & $\begin{array}{c}\text { disk. NK-Diff. } \\
(€ / h a)\end{array}$ & $\begin{array}{c}\text { WGF } \\
\text { (Zins } 4 \% \text { ) }\end{array}$ & $\begin{array}{c}\varnothing \text { jährl. Vorteil } \\
(€ / \text { ha/a) }\end{array}$ \\
\hline 0 & 0,00 & 0,00 & 0,00 & 0,00 & 0,00 & 0,00 & 0,00 & & \\
\hline 1 & 43,00 & 49,30 & 6,30 & 0,9615 & 41,34 & 47,40 & 6,06 & & \\
\hline 2 & 43,00 & 49,30 & 6,30 & 0,9246 & 39,76 & 45,58 & 5,82 & & \\
\hline 3 & 43,00 & 49,30 & 6,30 & 0,889 & 38,23 & 43,83 & 5,60 & & \\
\hline 4 & 43,00 & 49,30 & 6,30 & 0,8548 & 36,76 & 42,14 & 5,39 & & \\
\hline 5 & 43,00 & 49,30 & 6,30 & 0,8219 & 35,34 & 40,52 & 5,18 & & \\
\hline 6 & 43,00 & 49,30 & 6,30 & 0,7903 & 33,98 & 38,96 & 4,98 & & \\
\hline 7 & 43,00 & 49,30 & 6,30 & 0,7599 & 32,68 & 37,46 & 4,79 & & \\
\hline 8 & 43,00 & 49,30 & 6,30 & 0,7307 & 31,42 & 36,02 & 4,60 & & \\
\hline 9 & 43,00 & 49,30 & 6,30 & 0,7026 & 30,21 & 34,64 & 4,43 & & \\
\hline 10 & 43,00 & 49,30 & 6,30 & 0,6756 & 29,05 & 33,31 & 4,26 & & \\
\hline 11 & 43,00 & 49,30 & 6,30 & 0,6496 & 27,93 & 32,03 & 4,09 & & \\
\hline 12 & 43,00 & 49,30 & 6,30 & 0,6246 & 26,86 & 30,79 & 3,93 & & \\
\hline 13 & 43,00 & 49,30 & 6,30 & 0,6006 & 25,83 & 29,61 & 3,78 & & \\
\hline 14 & 43,00 & 49,30 & 6,30 & 0,5775 & 24,83 & 28,47 & 3,64 & & \\
\hline 15 & 43,00 & 49,30 & 6,30 & 0,5553 & 23,88 & 27,38 & 3,50 & & \\
\hline 16 & 43,00 & 49,30 & 6,30 & 0,5339 & 22,96 & 26,32 & 3,36 & & \\
\hline 17 & 43,00 & 49,30 & 6,30 & 0,5134 & 22,08 & 25,31 & 3,23 & & \\
\hline 18 & 43,00 & 49,30 & 6,30 & 0,4936 & 21,22 & 24,33 & 3,11 & & \\
\hline 19 & 43,00 & 49,30 & 6,30 & 0,4746 & 20,41 & 23,40 & 2,99 & & \\
\hline 20 & 43,00 & 49,30 & 6,30 & 0,4564 & 19,63 & 22,50 & 2,88 & & \\
\hline 21 & 43,00 & 49,30 & 6,30 & 0,4388 & 18,87 & 21,63 & 2,76 & & \\
\hline 22 & 43,00 & 49,30 & 6,30 & 0,422 & 18,15 & 20,80 & 2,66 & & \\
\hline 23 & 43,00 & 49,30 & 6,30 & 0,4057 & 17,45 & 20,00 & 2,56 & & \\
\hline 24 & 43,00 & 49,30 & 6,30 & 0,3901 & 16,77 & 19,23 & 2,46 & & \\
\hline \multirow[t]{3}{*}{25} & 43,00 & 49,30 & 6,30 & 0,3751 & 16,13 & 18,49 & 2,36 & & \\
\hline & & & & & 671,75 & 770,17 & 98,42 & 0,0640 & 6,30 \\
\hline & & & & KNV & & 0,87 & & & \\
\hline
\end{tabular}


Tabelle A-34: ZUA, Variante 2

\begin{tabular}{|c|c|c|c|c|c|c|c|c|c|}
\hline Jahr & $\begin{array}{c}\text { Kosten } \\
(€ / \text { ha) }\end{array}$ & $\begin{array}{c}\text { Nutzen } \\
\text { (€/ha) }\end{array}$ & $\begin{array}{c}\text { NK-Diff. } \\
\text { (€/ha) }\end{array}$ & $\begin{array}{c}\text { DF } \\
\text { (Zins } 4 \%) \\
\end{array}$ & $\begin{array}{c}\text { disk. Kosten } \\
\text { (€/ha) }\end{array}$ & $\begin{array}{c}\text { disk. Nutzen } \\
\text { (€/ha) }\end{array}$ & $\begin{array}{c}\text { disk. NK-Diff. } \\
(€ / h a)\end{array}$ & $\begin{array}{c}\text { WGF } \\
\text { (Zins } 4 \% \text { ) }\end{array}$ & $\begin{array}{c}\varnothing \text { jährl. Vorteil } \\
(€ / h a / a)\end{array}$ \\
\hline 0 & 0,00 & 0,00 & 0,00 & 0,00 & 0,00 & 0,00 & 0,00 & & \\
\hline 1 & 127,00 & 49,30 & $-77,70$ & 0,9615 & 122,11 & 47,40 & $-74,71$ & & \\
\hline 2 & 127,00 & 49,30 & $-77,70$ & 0,9246 & 117,42 & 45,58 & $-71,84$ & & \\
\hline 3 & 127,00 & 49,30 & $-77,70$ & 0,889 & 112,90 & 43,83 & $-69,08$ & & \\
\hline 4 & 127,00 & 49,30 & $-77,70$ & 0,8548 & 108,56 & 42,14 & $-66,42$ & & \\
\hline 5 & 127,00 & 49,30 & $-77,70$ & 0,8219 & 104,38 & 40,52 & $-63,86$ & & \\
\hline 6 & 127,00 & 49,30 & $-77,70$ & 0,7903 & 100,37 & 38,96 & $-61,41$ & & \\
\hline 7 & 127,00 & 49,30 & $-77,70$ & 0,7599 & 96,51 & 37,46 & $-59,04$ & & \\
\hline 8 & 127,00 & 49,30 & $-77,70$ & 0,7307 & 92,80 & 36,02 & $-56,78$ & & \\
\hline 9 & 127,00 & 49,30 & $-77,70$ & 0,7026 & 89,23 & 34,64 & $-54,59$ & & \\
\hline 10 & 127,00 & 49,30 & $-77,70$ & 0,6756 & 85,80 & 33,31 & $-52,49$ & & \\
\hline 11 & 127,00 & 49,30 & $-77,70$ & 0,6496 & 82,50 & 32,03 & $-50,47$ & & \\
\hline 12 & 127,00 & 49,30 & $-77,70$ & 0,6246 & 79,32 & 30,79 & $-48,53$ & & \\
\hline 13 & 127,00 & 49,30 & $-77,70$ & 0,6006 & 76,28 & 29,61 & $-46,67$ & & \\
\hline 14 & 127,00 & 49,30 & $-77,70$ & 0,5775 & 73,34 & 28,47 & $-44,87$ & & \\
\hline 15 & 127,00 & 49,30 & $-77,70$ & 0,5553 & 70,52 & 27,38 & $-43,15$ & & \\
\hline 16 & 127,00 & 49,30 & $-77,70$ & 0,5339 & 67,81 & 26,32 & $-41,48$ & & \\
\hline 17 & 127,00 & 49,30 & $-77,70$ & 0,5134 & 65,20 & 25,31 & $-39,89$ & & \\
\hline 18 & 127,00 & 49,30 & $-77,70$ & 0,4936 & 62,69 & 24,33 & $-38,35$ & & \\
\hline 19 & 127,00 & 49,30 & $-77,70$ & 0,4746 & 60,27 & 23,40 & $-36,88$ & & \\
\hline 20 & 127,00 & 49,30 & $-77,70$ & 0,4564 & 57,96 & 22,50 & $-35,46$ & & \\
\hline 21 & 127,00 & 49,30 & $-77,70$ & 0,4388 & 55,73 & 21,63 & $-34,09$ & & \\
\hline 22 & 127,00 & 49,30 & $-77,70$ & 0,422 & 53,59 & 20,80 & $-32,79$ & & \\
\hline 23 & 127,00 & 49,30 & $-77,70$ & 0,4057 & 51,52 & 20,00 & $-31,52$ & & \\
\hline 24 & 127,00 & 49,30 & $-77,70$ & 0,3901 & 49,54 & 19,23 & $-30,31$ & & \\
\hline \multirow[t]{2}{*}{25} & 127,00 & 49,30 & $-77,70$ & 0,3751 & 47,64 & 18,49 & $-29,15$ & & \\
\hline & & & & & 1984,01 & 770,17 & $-1213,84$ & 0,0640 & $-77,69$ \\
\hline
\end{tabular}


Tabelle A-35: ZUA, Variante 3

\begin{tabular}{|c|c|c|c|c|c|c|c|c|c|}
\hline Jahr & $\begin{array}{c}\text { Kosten } \\
(€ / \text { ha) }\end{array}$ & $\begin{array}{c}\text { Nutzen } \\
\text { (€/ha) }\end{array}$ & $\begin{array}{c}\text { NK-Diff. } \\
\text { (€/ha) }\end{array}$ & $\begin{array}{c}\text { DF } \\
\text { (Zins } 4 \%) \\
\end{array}$ & $\begin{array}{c}\text { disk. Kosten } \\
\text { (€/ha) }\end{array}$ & $\begin{array}{c}\text { disk. Nutzen } \\
\text { (€/ha) }\end{array}$ & $\begin{array}{c}\text { disk. NK-Diff. } \\
(€ / h a)\end{array}$ & $\begin{array}{c}\text { WGF } \\
\text { (Zins } 4 \% \text { ) }\end{array}$ & $\begin{array}{c}\varnothing \text { jährl. Vorteil } \\
(€ / h a / a)\end{array}$ \\
\hline 0 & 0 & 0,00 & 0,00 & 0,00 & 0,00 & 0,00 & 0,00 & & \\
\hline 1 & 127,00 & 173,20 & 46,20 & 0,9615 & 122,11 & 166,53 & 44,42 & & \\
\hline 2 & 127,00 & 173,20 & 46,20 & 0,9246 & 117,42 & 160,14 & 42,72 & & \\
\hline 3 & 127,00 & 173,20 & 46,20 & 0,889 & 112,90 & 153,97 & 41,07 & & \\
\hline 4 & 127,00 & 173,20 & 46,20 & 0,8548 & 108,56 & 148,05 & 39,49 & & \\
\hline 5 & 127,00 & 173,20 & 46,20 & 0,8219 & 104,38 & 142,35 & 37,97 & & \\
\hline 6 & 127,00 & 173,20 & 46,20 & 0,7903 & 100,37 & 136,88 & 36,51 & & \\
\hline 7 & 127,00 & 173,20 & 46,20 & 0,7599 & 96,51 & 131,61 & 35,11 & & \\
\hline 8 & 127,00 & 173,20 & 46,20 & 0,7307 & 92,80 & 126,56 & 33,76 & & \\
\hline 9 & 127,00 & 173,20 & 46,20 & 0,7026 & 89,23 & 121,69 & 32,46 & & \\
\hline 10 & 127,00 & 173,20 & 46,20 & 0,6756 & 85,80 & 117,01 & 31,21 & & \\
\hline 11 & 127,00 & 173,20 & 46,20 & 0,6496 & 82,50 & 112,51 & 30,01 & & \\
\hline 12 & 127,00 & 173,20 & 46,20 & 0,6246 & 79,32 & 108,18 & 28,86 & & \\
\hline 13 & 127,00 & 173,20 & 46,20 & 0,6006 & 76,28 & 104,02 & 27,75 & & \\
\hline 14 & 127,00 & 173,20 & 46,20 & 0,5775 & 73,34 & 100,02 & 26,68 & & \\
\hline 15 & 127,00 & 173,20 & 46,20 & 0,5553 & 70,52 & 96,18 & 25,65 & & \\
\hline 16 & 127,00 & 173,20 & 46,20 & 0,5339 & 67,81 & 92,47 & 24,67 & & \\
\hline 17 & 127,00 & 173,20 & 46,20 & 0,5134 & 65,20 & 88,92 & 23,72 & & \\
\hline 18 & 127,00 & 173,20 & 46,20 & 0,4936 & 62,69 & 85,49 & 22,80 & & \\
\hline 19 & 127,00 & 173,20 & 46,20 & 0,4746 & 60,27 & 82,20 & 21,93 & & \\
\hline 20 & 127,00 & 173,20 & 46,20 & 0,4564 & 57,96 & 79,05 & 21,09 & & \\
\hline 21 & 127,00 & 173,20 & 46,20 & 0,4388 & 55,73 & 76,00 & 20,27 & & \\
\hline 22 & 127,00 & 173,20 & 46,20 & 0,422 & 53,59 & 73,09 & 19,50 & & \\
\hline 23 & 127,00 & 173,20 & 46,20 & 0,4057 & 51,52 & 70,27 & 18,74 & & \\
\hline 24 & 127,00 & 173,20 & 46,20 & 0,3901 & 49,54 & 67,57 & 18,02 & & \\
\hline \multirow[t]{2}{*}{25} & 127,00 & 173,20 & 46,20 & 0,3751 & 47,64 & 64,97 & 17,33 & & \\
\hline & & & & & 1984,01 & 2705,75 & 721,74 & 0,0640 & 46,19 \\
\hline
\end{tabular}


Tabelle A-36: ZUA, Variante 4

\begin{tabular}{|c|c|c|c|c|c|c|c|c|c|}
\hline Jahr & $\begin{array}{l}\text { Kosten } \\
\text { (€/ha) }\end{array}$ & $\begin{array}{l}\text { Nutzen } \\
\text { (€/ha) }\end{array}$ & $\begin{array}{c}\text { NK-Diff. } \\
\text { (€/ha) }\end{array}$ & $\begin{array}{c}\text { DF } \\
\text { (Zins } 4 \% \text { ) }\end{array}$ & $\begin{array}{c}\text { disk. Kosten } \\
\qquad(€ / \mathrm{ha})\end{array}$ & $\begin{array}{c}\text { disk. Nutzen } \\
\text { (€/ha) }\end{array}$ & $\begin{array}{c}\text { disk. NK-Diff. } \\
(€ / \mathrm{ha})\end{array}$ & $\begin{array}{c}\text { WGF } \\
\text { (Zins } 4 \% \text { ) }\end{array}$ & $\begin{array}{c}\varnothing \text { jährl. Vorteil } \\
\text { (€/ha/a) }\end{array}$ \\
\hline 0 & 0 & 0,00 & 0,00 & 0,00 & 0,00 & 0,00 & 0,00 & & \\
\hline 1 & 43,00 & 173,20 & 130,20 & 0,9615 & 41,34 & 166,53 & 125,19 & & \\
\hline 2 & 43,00 & 173,20 & 130,20 & 0,9246 & 39,76 & 160,14 & 120,38 & & \\
\hline 3 & 43,00 & 173,20 & 130,20 & 0,889 & 38,23 & 153,97 & 115,75 & & \\
\hline 4 & 43,00 & 173,20 & 130,20 & 0,8548 & 36,76 & 148,05 & 111,29 & & \\
\hline 5 & 43,00 & 173,20 & 130,20 & 0,8219 & 35,34 & 142,35 & 107,01 & & \\
\hline 6 & 43,00 & 173,20 & 130,20 & 0,7903 & 33,98 & 136,88 & 102,90 & & \\
\hline 7 & 43,00 & 173,20 & 130,20 & 0,7599 & 32,68 & 131,61 & 98,94 & & \\
\hline 8 & 43,00 & 173,20 & 130,20 & 0,7307 & 31,42 & 126,56 & 95,14 & & \\
\hline 9 & 43,00 & 173,20 & 130,20 & 0,7026 & 30,21 & 121,69 & 91,48 & & \\
\hline 10 & 43,00 & 173,20 & 130,20 & 0,6756 & 29,05 & 117,01 & 87,96 & & \\
\hline 11 & 43,00 & 173,20 & 130,20 & 0,6496 & 27,93 & 112,51 & 84,58 & & \\
\hline 12 & 43,00 & 173,20 & 130,20 & 0,6246 & 26,86 & 108,18 & 81,32 & & \\
\hline 13 & 43,00 & 173,20 & 130,20 & 0,6006 & 25,83 & 104,02 & 78,20 & & \\
\hline 14 & 43,00 & 173,20 & 130,20 & 0,5775 & 24,83 & 100,02 & 75,19 & & \\
\hline 15 & 43,00 & 173,20 & 130,20 & 0,5553 & 23,88 & 96,18 & 72,30 & & \\
\hline 16 & 43,00 & 173,20 & 130,20 & 0,5339 & 22,96 & 92,47 & 69,51 & & \\
\hline 17 & 43,00 & 173,20 & 130,20 & 0,5134 & 22,08 & 88,92 & 66,84 & & \\
\hline 18 & 43,00 & 173,20 & 130,20 & 0,4936 & 21,22 & 85,49 & 64,27 & & \\
\hline 19 & 43,00 & 173,20 & 130,20 & 0,4746 & 20,41 & 82,20 & 61,79 & & \\
\hline 20 & 43,00 & 173,20 & 130,20 & 0,4564 & 19,63 & 79,05 & 59,42 & & \\
\hline 21 & 43,00 & 173,20 & 130,20 & 0,4388 & 18,87 & 76,00 & 57,13 & & \\
\hline 22 & 43,00 & 173,20 & 130,20 & 0,422 & 18,15 & 73,09 & 54,94 & & \\
\hline 23 & 43,00 & 173,20 & 130,20 & 0,4057 & 17,45 & 70,27 & 52,82 & & \\
\hline 24 & 43,00 & 173,20 & 130,20 & 0,3901 & 16,77 & 67,57 & 50,79 & & \\
\hline \multirow[t]{3}{*}{25} & 43,00 & 173,20 & 130,20 & 0,3751 & 16,13 & 64,97 & 48,84 & & \\
\hline & & & & & 671,75 & 2705,75 & 2034,00 & 0,0640 & 130,18 \\
\hline & & & & KNV & & 0,25 & & & \\
\hline
\end{tabular}


Tabelle A-37: Anlage von Windschutzhecken bei 10\% Ertragssteigerung und 0 \% Förderung

\begin{tabular}{|c|c|c|c|c|c|c|c|c|c|}
\hline Jahr & $\begin{array}{c}\text { Kosten } \\
\text { (€/ha) }\end{array}$ & $\begin{array}{c}\text { Nutzen } \\
\text { (€/ha) }\end{array}$ & $\begin{array}{c}\text { NK-Diff. } \\
\text { (€/ha) }\end{array}$ & $\begin{array}{c}\text { DF } \\
\text { (Zins } 4 \%)\end{array}$ & $\begin{array}{c}\text { disk. Kosten } \\
(€ / \mathrm{ha})\end{array}$ & $\begin{array}{c}\text { disk. Nutzen } \\
(€ / h a)\end{array}$ & $\begin{array}{c}\text { disk. NK-Diff. } \\
(€ / h a)\end{array}$ & $\begin{array}{c}\text { WGF } \\
\text { (Zins } 4 \% \text { ) }\end{array}$ & $\begin{array}{c}\varnothing \text { jährl. Vorteil } \\
\text { (€/ha/a) }\end{array}$ \\
\hline 0 & 2195 & 0 & $-2195,00$ & 0,00 & 2195,00 & 0,00 & $-2195,00$ & & \\
\hline 1 & 265,00 & 6 & $-259,02$ & 0,9615 & 254,80 & 5,75 & $-249,05$ & & \\
\hline 2 & 265,00 & 12 & $-253,04$ & 0,9246 & 245,02 & 11,06 & $-233,96$ & & \\
\hline 3 & 265,00 & 18 & $-247,06$ & 0,889 & 235,59 & 15,95 & $-219,64$ & & \\
\hline 4 & 265,00 & 24 & $-241,08$ & 0,8548 & 226,52 & 20,45 & $-206,08$ & & \\
\hline 5 & 275,00 & 30 & $-245,10$ & 0,8219 & 226,02 & 24,57 & $-201,45$ & & \\
\hline 6 & 265,00 & 36 & $-229,12$ & 0,7903 & 209,43 & 28,36 & $-181,07$ & & \\
\hline 7 & 265,00 & 42 & $-223,14$ & 0,7599 & 201,37 & 31,81 & $-169,56$ & & \\
\hline 8 & 265,00 & 48 & $-217,16$ & 0,7307 & 193,64 & 34,96 & $-158,68$ & & \\
\hline 9 & 265,00 & 54 & $-211,18$ & 0,7026 & 186,19 & 37,81 & $-148,38$ & & \\
\hline 10 & 275,00 & 60 & $-215,20$ & 0,6756 & 185,79 & 40,40 & $-145,39$ & & \\
\hline 11 & 265,00 & 60 & $-205,20$ & 0,6496 & 172,14 & 38,85 & $-133,30$ & & \\
\hline 12 & 265,00 & 60 & $-205,20$ & 0,6246 & 165,52 & 37,35 & $-128,17$ & & \\
\hline 13 & 265,00 & 60 & $-205,20$ & 0,6006 & 159,16 & 35,92 & $-123,24$ & & \\
\hline 14 & 265,00 & 60 & $-205,20$ & 0,5775 & 153,04 & 34,53 & $-118,50$ & & \\
\hline 15 & 275,00 & 60 & $-215,20$ & 0,5553 & 152,71 & 33,21 & $-119,50$ & & \\
\hline 16 & 265,00 & 60 & $-205,20$ & 0,5339 & 141,48 & 31,93 & $-109,56$ & & \\
\hline 17 & 265,00 & 60 & $-205,20$ & 0,5134 & 136,05 & 30,70 & $-105,35$ & & \\
\hline 18 & 265,00 & 60 & $-205,20$ & 0,4936 & 130,80 & 29,52 & $-101,29$ & & \\
\hline 19 & 265,00 & 60 & $-205,20$ & 0,4746 & 125,77 & 28,38 & $-97,39$ & & \\
\hline 20 & 275,00 & 60 & $-215,20$ & 0,4564 & 125,51 & 27,29 & $-98,22$ & & \\
\hline 21 & 265,00 & 60 & $-205,20$ & 0,4388 & 116,28 & 26,24 & $-90,04$ & & \\
\hline 22 & 265,00 & 60 & $-205,20$ & 0,422 & 111,83 & 25,24 & $-86,59$ & & \\
\hline 23 & 265,00 & 60 & $-205,20$ & 0,4057 & 107,51 & 24,26 & $-83,25$ & & \\
\hline 24 & 265,00 & 60 & $-205,20$ & 0,3901 & 103,38 & 23,33 & $-80,05$ & & \\
\hline \multirow[t]{3}{*}{25} & 275,00 & 60 & $-215,20$ & 0,3751 & 103,15 & 22,43 & $-80,72$ & & \\
\hline & & & & & 6363,70 & 700,28 & $-5663,41$ & 0,0640 & $-362,46$ \\
\hline & & & & KNV & & 9,09 & & & \\
\hline
\end{tabular}


Tabelle A-38: Anlage von Windschutzhecken bei 10\% Ertragssteigerung und 50 \% Förderung

\begin{tabular}{|c|c|c|c|c|c|c|c|c|c|}
\hline Jahr & $\begin{array}{l}\text { Kosten } \\
\text { (€/ha) }\end{array}$ & $\begin{array}{c}\text { Nutzen } \\
\text { (€/ha) }\end{array}$ & $\begin{array}{c}\text { NK-Diff. } \\
\text { (€/ha) }\end{array}$ & $\begin{array}{c}\text { DF } \\
\text { (Zins } 4 \%) \\
\end{array}$ & $\begin{array}{c}\text { disk. Kosten } \\
(€ / h a)\end{array}$ & $\begin{array}{c}\text { disk. Nutzen } \\
\text { (€/ha) }\end{array}$ & $\begin{array}{c}\text { disk. NK-Diff. } \\
(€ / h a)\end{array}$ & $\begin{array}{c}\text { WGF } \\
\text { (Zins } 4 \% \text { ) }\end{array}$ & $\begin{array}{c}\varnothing \text { jährl. Vorteil } \\
(€ / h a / a)\end{array}$ \\
\hline 0 & 1098 & 0 & $-1097,50$ & 0,00 & 1097,50 & 0,00 & $-1097,50$ & & \\
\hline 1 & 265,00 & 6 & $-259,02$ & 0,9615 & 254,80 & 5,75 & $-249,05$ & & \\
\hline 2 & 265,00 & 12 & $-253,04$ & 0,9246 & 245,02 & 11,06 & $-233,96$ & & \\
\hline 3 & 265,00 & 18 & $-247,06$ & 0,889 & 235,59 & 15,95 & $-219,64$ & & \\
\hline 4 & 265,00 & 24 & $-241,08$ & 0,8548 & 226,52 & 20,45 & $-206,08$ & & \\
\hline 5 & 275,00 & 30 & $-245,10$ & 0,8219 & 226,02 & 24,57 & $-201,45$ & & \\
\hline 6 & 265,00 & 36 & $-229,12$ & 0,7903 & 209,43 & 28,36 & $-181,07$ & & \\
\hline 7 & 265,00 & 42 & $-223,14$ & 0,7599 & 201,37 & 31,81 & $-169,56$ & & \\
\hline 8 & 265,00 & 48 & $-217,16$ & 0,7307 & 193,64 & 34,96 & $-158,68$ & & \\
\hline 9 & 265,00 & 54 & $-211,18$ & 0,7026 & 186,19 & 37,81 & $-148,38$ & & \\
\hline 10 & 275,00 & 60 & $-215,20$ & 0,6756 & 185,79 & 40,40 & $-145,39$ & & \\
\hline 11 & 265,00 & 60 & $-205,20$ & 0,6496 & 172,14 & 38,85 & $-133,30$ & & \\
\hline 12 & 265,00 & 60 & $-205,20$ & 0,6246 & 165,52 & 37,35 & $-128,17$ & & \\
\hline 13 & 265,00 & 60 & $-205,20$ & 0,6006 & 159,16 & 35,92 & $-123,24$ & & \\
\hline 14 & 265,00 & 60 & $-205,20$ & 0,5775 & 153,04 & 34,53 & $-118,50$ & & \\
\hline 15 & 275,00 & 60 & $-215,20$ & 0,5553 & 152,71 & 33,21 & $-119,50$ & & \\
\hline 16 & 265,00 & 60 & $-205,20$ & 0,5339 & 141,48 & 31,93 & $-109,56$ & & \\
\hline 17 & 265,00 & 60 & $-205,20$ & 0,5134 & 136,05 & 30,70 & $-105,35$ & & \\
\hline 18 & 265,00 & 60 & $-205,20$ & 0,4936 & 130,80 & 29,52 & $-101,29$ & & \\
\hline 19 & 265,00 & 60 & $-205,20$ & 0,4746 & 125,77 & 28,38 & $-97,39$ & & \\
\hline 20 & 275,00 & 60 & $-215,20$ & 0,4564 & 125,51 & 27,29 & $-98,22$ & & \\
\hline 21 & 265,00 & 60 & $-205,20$ & 0,4388 & 116,28 & 26,24 & $-90,04$ & & \\
\hline 22 & 265,00 & 60 & $-205,20$ & 0,422 & 111,83 & 25,24 & $-86,59$ & & \\
\hline 23 & 265,00 & 60 & $-205,20$ & 0,4057 & 107,51 & 24,26 & $-83,25$ & & \\
\hline 24 & 265,00 & 60 & $-205,20$ & 0,3901 & 103,38 & 23,33 & $-80,05$ & & \\
\hline \multirow[t]{2}{*}{25} & 275,00 & 60 & $-215,20$ & 0,3751 & 103,15 & 22,43 & $-80,72$ & & \\
\hline & & & & & 5266,20 & 700,28 & $-4565,91$ & 0,0640 & $-292,22$ \\
\hline
\end{tabular}


Tabelle A-39: Anlage von Windschutzhecken bei 0 \% Ertragssteigerung und 80 \% Förderung

\begin{tabular}{|c|c|c|c|c|c|c|c|c|c|}
\hline Jahr & $\begin{array}{c}\text { Kosten } \\
\text { (€/ha) }\end{array}$ & $\begin{array}{c}\text { Nutzen } \\
\text { (€/ha) }\end{array}$ & $\begin{array}{c}\text { NK-Diff. } \\
\text { (€/ha) }\end{array}$ & $\begin{array}{c}\text { DF } \\
\text { (Zins } 4 \%)\end{array}$ & $\begin{array}{c}\text { disk. Kosten } \\
\text { (€/ha) }\end{array}$ & $\begin{array}{c}\text { disk. Nutzen } \\
\text { (€/ha) }\end{array}$ & $\begin{array}{c}\text { disk. NK-Diff. } \\
(€ / h a)\end{array}$ & $\begin{array}{c}\text { WGF } \\
\text { (Zins } 4 \% \text { ) }\end{array}$ & $\begin{array}{c}\varnothing \text { jährl. Vorteil } \\
(€ / h a / a)\end{array}$ \\
\hline 0 & 439,00 & 0,00 & $-439,00$ & 0,00 & 439,00 & 0,00 & $-439,00$ & & \\
\hline 1 & 265,00 & 0,00 & $-265,00$ & 0,9615 & 254,80 & 0,00 & $-254,80$ & & \\
\hline 2 & 265,00 & 0,00 & $-265,00$ & 0,9246 & 245,02 & 0,00 & $-245,02$ & & \\
\hline 3 & 265,00 & 0,00 & $-265,00$ & 0,889 & 235,59 & 0,00 & $-235,59$ & & \\
\hline 4 & 265,00 & 0,00 & $-265,00$ & 0,8548 & 226,52 & 0,00 & $-226,52$ & & \\
\hline 5 & 275,00 & 0,00 & $-275,00$ & 0,8219 & 226,02 & 0,00 & $-226,02$ & & \\
\hline 6 & 265,00 & 0,00 & $-265,00$ & 0,7903 & 209,43 & 0,00 & $-209,43$ & & \\
\hline 7 & 265,00 & 0,00 & $-265,00$ & 0,7599 & 201,37 & 0,00 & $-201,37$ & & \\
\hline 8 & 265,00 & 0,00 & $-265,00$ & 0,7307 & 193,64 & 0,00 & $-193,64$ & & \\
\hline 9 & 265,00 & 0,00 & $-265,00$ & 0,7026 & 186,19 & 0,00 & $-186,19$ & & \\
\hline 10 & 275,00 & 0,00 & $-275,00$ & 0,6756 & 185,79 & 0,00 & $-185,79$ & & \\
\hline 11 & 265,00 & 0,00 & $-265,00$ & 0,6496 & 172,14 & 0,00 & $-172,14$ & & \\
\hline 12 & 265,00 & 0,00 & $-265,00$ & 0,6246 & 165,52 & 0,00 & $-165,52$ & & \\
\hline 13 & 265,00 & 0,00 & $-265,00$ & 0,6006 & 159,16 & 0,00 & $-159,16$ & & \\
\hline 14 & 265,00 & 0,00 & $-265,00$ & 0,5775 & 153,04 & 0,00 & $-153,04$ & & \\
\hline 15 & 275,00 & 0,00 & $-275,00$ & 0,5553 & 152,71 & 0,00 & $-152,71$ & & \\
\hline 16 & 265,00 & 0,00 & $-265,00$ & 0,5339 & 141,48 & 0,00 & $-141,48$ & & \\
\hline 17 & 265,00 & 0,00 & $-265,00$ & 0,5134 & 136,05 & 0,00 & $-136,05$ & & \\
\hline 18 & 265,00 & 0,00 & $-265,00$ & 0,4936 & 130,80 & 0,00 & $-130,80$ & & \\
\hline 19 & 265,00 & 0,00 & $-265,00$ & 0,4746 & 125,77 & 0,00 & $-125,77$ & & \\
\hline 20 & 275,00 & 0,00 & $-275,00$ & 0,4564 & 125,51 & 0,00 & $-125,51$ & & \\
\hline 21 & 265,00 & 0,00 & $-265,00$ & 0,4388 & 116,28 & 0,00 & $-116,28$ & & \\
\hline 22 & 265,00 & 0,00 & $-265,00$ & 0,422 & 111,83 & 0,00 & $-111,83$ & & \\
\hline 23 & 265,00 & 0,00 & $-265,00$ & 0,4057 & 107,51 & 0,00 & $-107,51$ & & \\
\hline 24 & 265,00 & 0,00 & $-265,00$ & 0,3901 & 103,38 & 0,00 & $-103,38$ & & \\
\hline \multirow[t]{2}{*}{25} & 275,00 & 0,00 & $-275,00$ & 0,3751 & 103,15 & 0,00 & $-103,15$ & & \\
\hline & & & & & 4607,70 & 0,00 & $-4607,70$ & 0,0640 & $-294,89$ \\
\hline
\end{tabular}


Tabelle A-40: Anlage von Windschutzhecken bei 5 \% Ertragssteigerung und 80 \% Förderung

\begin{tabular}{|c|c|c|c|c|c|c|c|c|c|}
\hline Jahr & $\begin{array}{l}\text { Kosten } \\
\text { (€/ha) }\end{array}$ & $\begin{array}{c}\text { Nutzen } \\
\text { (€/ha) }\end{array}$ & $\begin{array}{c}\text { NK-Diff. } \\
\text { (€/ha) }\end{array}$ & $\begin{array}{c}\text { DF } \\
\text { (Zins } 4 \%)\end{array}$ & $\begin{array}{c}\text { disk. Kosten } \\
\text { (€/ha) }\end{array}$ & $\begin{array}{c}\text { disk. Nutzen } \\
\text { (€/ha) }\end{array}$ & $\begin{array}{c}\text { disk. NK-Diff. } \\
(€ / h a)\end{array}$ & $\begin{array}{c}\text { WGF } \\
\text { (Zins } 4 \% \text { ) }\end{array}$ & $\begin{array}{c}\varnothing \text { jährl. Vorteil } \\
(€ / h a / a)\end{array}$ \\
\hline 0 & 439,00 & 0,00 & $-439,00$ & 0,00 & 439,00 & 0,00 & $-439,00$ & & \\
\hline 1 & 265,00 & 2,99 & $-262,01$ & 0,9615 & 254,80 & 2,87 & $-251,92$ & & \\
\hline 2 & 265,00 & 5,98 & $-259,02$ & 0,9246 & 245,02 & 5,53 & $-239,49$ & & \\
\hline 3 & 265,00 & 8,97 & $-256,03$ & 0,889 & 235,59 & 7,97 & $-227,61$ & & \\
\hline 4 & 265,00 & 11,96 & $-253,04$ & 0,8548 & 226,52 & 10,22 & $-216,30$ & & \\
\hline 5 & 275,00 & 14,95 & $-260,05$ & 0,8219 & 226,02 & 12,29 & $-213,74$ & & \\
\hline 6 & 265,00 & 17,94 & $-247,06$ & 0,7903 & 209,43 & 14,18 & $-195,25$ & & \\
\hline 7 & 265,00 & 20,93 & $-244,07$ & 0,7599 & 201,37 & 15,90 & $-185,47$ & & \\
\hline 8 & 265,00 & 23,92 & $-241,08$ & 0,7307 & 193,64 & 17,48 & $-176,16$ & & \\
\hline 9 & 265,00 & 26,91 & $-238,09$ & 0,7026 & 186,19 & 18,91 & $-167,28$ & & \\
\hline 10 & 275,00 & 29,90 & $-245,10$ & 0,6756 & 185,79 & 20,20 & $-165,59$ & & \\
\hline 11 & 265,00 & 29,90 & $-235,10$ & 0,6496 & 172,14 & 19,42 & $-152,72$ & & \\
\hline 12 & 265,00 & 29,90 & $-235,10$ & 0,6246 & 165,52 & 18,68 & $-146,84$ & & \\
\hline 13 & 265,00 & 29,90 & $-235,10$ & 0,6006 & 159,16 & 17,96 & $-141,20$ & & \\
\hline 14 & 265,00 & 29,90 & $-235,10$ & 0,5775 & 153,04 & 17,27 & $-135,77$ & & \\
\hline 15 & 275,00 & 29,90 & $-245,10$ & 0,5553 & 152,71 & 16,60 & $-136,10$ & & \\
\hline 16 & 265,00 & 29,90 & $-235,10$ & 0,5339 & 141,48 & 15,96 & $-125,52$ & & \\
\hline 17 & 265,00 & 29,90 & $-235,10$ & 0,5134 & 136,05 & 15,35 & $-120,70$ & & \\
\hline 18 & 265,00 & 29,90 & $-235,10$ & 0,4936 & 130,80 & 14,76 & $-116,05$ & & \\
\hline 19 & 265,00 & 29,90 & $-235,10$ & 0,4746 & 125,77 & 14,19 & $-111,58$ & & \\
\hline 20 & 275,00 & 29,90 & $-245,10$ & 0,4564 & 125,51 & 13,65 & $-111,86$ & & \\
\hline 21 & 265,00 & 29,90 & $-235,10$ & 0,4388 & 116,28 & 13,12 & $-103,16$ & & \\
\hline 22 & 265,00 & 29,90 & $-235,10$ & 0,422 & 111,83 & 12,62 & $-99,21$ & & \\
\hline 23 & 265,00 & 29,90 & $-235,10$ & 0,4057 & 107,51 & 12,13 & $-95,38$ & & \\
\hline 24 & 265,00 & 29,90 & $-235,10$ & 0,3901 & 103,38 & 11,66 & $-91,71$ & & \\
\hline \multirow[t]{2}{*}{25} & 275,00 & 29,90 & $-245,10$ & 0,3751 & 103,15 & 11,22 & $-91,94$ & & \\
\hline & & & & & 4607,70 & 350,14 & $-4257,56$ & 0,0640 & $-272,48$ \\
\hline
\end{tabular}


Tabelle A-41: Anlage von Windschutzhecken bei 10 \% Ertragssteigerung und 80 \% Förderung

\begin{tabular}{|c|c|c|c|c|c|c|c|c|c|}
\hline Jahr & $\begin{array}{l}\text { Kosten } \\
\text { (€/ha) }\end{array}$ & $\begin{array}{l}\text { Nutzen } \\
\text { (€/ha) }\end{array}$ & $\begin{array}{c}\text { NK-Diff. } \\
\text { (€/ha) }\end{array}$ & $\begin{array}{c}\text { DF } \\
\text { (Zins } 4 \%)\end{array}$ & $\begin{array}{c}\text { disk. Kosten } \\
\text { (€/ha) }\end{array}$ & $\begin{array}{c}\text { disk. Nutzen } \\
(€ / \mathrm{ha})\end{array}$ & $\begin{array}{c}\text { disk. NK-Diff. } \\
\text { (€/ha) }\end{array}$ & $\begin{array}{c}\text { WGF } \\
\text { (Zins } 4 \% \text { ) }\end{array}$ & $\begin{array}{c}\varnothing \text { jährl. Vorteil } \\
(€ / h a / a)\end{array}$ \\
\hline 0 & 439 & 0,00 & $-439,00$ & 0,00 & 439,00 & 0,00 & $-439,00$ & & \\
\hline 1 & 265,00 & 5,98 & $-259,02$ & 0,9615 & 254,80 & 5,75 & $-249,05$ & & \\
\hline 2 & 265,00 & 11,96 & $-253,04$ & 0,9246 & 245,02 & 11,06 & $-233,96$ & & \\
\hline 3 & 265,00 & 17,94 & $-247,06$ & 0,889 & 235,59 & 15,95 & $-219,64$ & & \\
\hline 4 & 265,00 & 23,92 & $-241,08$ & 0,8548 & 226,52 & 20,45 & $-206,08$ & & \\
\hline 5 & 275,00 & 29,90 & $-245,10$ & 0,8219 & 226,02 & 24,57 & $-201,45$ & & \\
\hline 6 & 265,00 & 35,88 & $-229,12$ & 0,7903 & 209,43 & 28,36 & $-181,07$ & & \\
\hline 7 & 265,00 & 41,86 & $-223,14$ & 0,7599 & 201,37 & 31,81 & $-169,56$ & & \\
\hline 8 & 265,00 & 47,84 & $-217,16$ & 0,7307 & 193,64 & 34,96 & $-158,68$ & & \\
\hline 9 & 265,00 & 53,82 & $-211,18$ & 0,7026 & 186,19 & 37,81 & $-148,38$ & & \\
\hline 10 & 275,00 & 59,80 & $-215,20$ & 0,6756 & 185,79 & 40,40 & $-145,39$ & & \\
\hline 11 & 265,00 & 59,80 & $-205,20$ & 0,6496 & 172,14 & 38,85 & $-133,30$ & & \\
\hline 12 & 265,00 & 59,80 & $-205,20$ & 0,6246 & 165,52 & 37,35 & $-128,17$ & & \\
\hline 13 & 265,00 & 59,80 & $-205,20$ & 0,6006 & 159,16 & 35,92 & $-123,24$ & & \\
\hline 14 & 265,00 & 59,80 & $-205,20$ & 0,5775 & 153,04 & 34,53 & $-118,50$ & & \\
\hline 15 & 275,00 & 59,80 & $-215,20$ & 0,5553 & 152,71 & 33,21 & $-119,50$ & & \\
\hline 16 & 265,00 & 59,80 & $-205,20$ & 0,5339 & 141,48 & 31,93 & $-109,56$ & & \\
\hline 17 & 265,00 & 59,80 & $-205,20$ & 0,5134 & 136,05 & 30,70 & $-105,35$ & & \\
\hline 18 & 265,00 & 59,80 & $-205,20$ & 0,4936 & 130,80 & 29,52 & $-101,29$ & & \\
\hline 19 & 265,00 & 59,80 & $-205,20$ & 0,4746 & 125,77 & 28,38 & $-97,39$ & & \\
\hline 20 & 275,00 & 59,80 & $-215,20$ & 0,4564 & 125,51 & 27,29 & $-98,22$ & & \\
\hline 21 & 265,00 & 59,80 & $-205,20$ & 0,4388 & 116,28 & 26,24 & $-90,04$ & & \\
\hline 22 & 265,00 & 59,80 & $-205,20$ & 0,422 & 111,83 & 25,24 & $-86,59$ & & \\
\hline 23 & 265,00 & 59,80 & $-205,20$ & 0,4057 & 107,51 & 24,26 & $-83,25$ & & \\
\hline 24 & 265,00 & 59,80 & $-205,20$ & 0,3901 & 103,38 & 23,33 & $-80,05$ & & \\
\hline \multirow[t]{2}{*}{25} & 275,00 & 59,80 & $-215,20$ & 0,3751 & 103,15 & 22,43 & $-80,72$ & & \\
\hline & & & & & 4607,70 & 700,28 & $-3907,41$ & 0,0640 & $-250,07$ \\
\hline
\end{tabular}


Tabelle A-42: Anlage von Windschutzhecken bei 0 \% Ertragssteigerung und $100 \%$ Förderung

\begin{tabular}{|c|c|c|c|c|c|c|c|c|c|}
\hline Jahr & $\begin{array}{c}\text { Kosten } \\
\text { (€/ha) }\end{array}$ & $\begin{array}{c}\text { Nutzen } \\
\text { (€/ha) }\end{array}$ & $\begin{array}{c}\text { NK-Diff. } \\
\text { (€/ha) }\end{array}$ & $\begin{array}{c}\text { DF } \\
\text { (Zins } 4 \%)\end{array}$ & $\begin{array}{c}\text { disk. Kosten } \\
\text { (€/ha) }\end{array}$ & $\begin{array}{c}\text { disk. Nutzen } \\
\text { (€/ha) }\end{array}$ & $\begin{array}{c}\text { disk. NK-Diff. } \\
(€ / h a)\end{array}$ & $\begin{array}{c}\text { WGF } \\
\text { (Zins } 4 \% \text { ) }\end{array}$ & $\begin{array}{c}\varnothing \text { jährl. Vorteil } \\
(€ / h a / a)\end{array}$ \\
\hline 0 & 0,00 & 0,00 & 0,00 & 0,00 & 0,00 & 0,00 & 0,00 & & \\
\hline 1 & 265,00 & 0,00 & $-265,00$ & 0,9615 & 254,80 & 0,00 & $-254,80$ & & \\
\hline 2 & 265,00 & 0,00 & $-265,00$ & 0,9246 & 245,02 & 0,00 & $-245,02$ & & \\
\hline 3 & 265,00 & 0,00 & $-265,00$ & 0,889 & 235,59 & 0,00 & $-235,59$ & & \\
\hline 4 & 265,00 & 0,00 & $-265,00$ & 0,8548 & 226,52 & 0,00 & $-226,52$ & & \\
\hline 5 & 275,00 & 0,00 & $-275,00$ & 0,8219 & 226,02 & 0,00 & $-226,02$ & & \\
\hline 6 & 265,00 & 0,00 & $-265,00$ & 0,7903 & 209,43 & 0,00 & $-209,43$ & & \\
\hline 7 & 265,00 & 0,00 & $-265,00$ & 0,7599 & 201,37 & 0,00 & $-201,37$ & & \\
\hline 8 & 265,00 & 0,00 & $-265,00$ & 0,7307 & 193,64 & 0,00 & $-193,64$ & & \\
\hline 9 & 265,00 & 0,00 & $-265,00$ & 0,7026 & 186,19 & 0,00 & $-186,19$ & & \\
\hline 10 & 275,00 & 0,00 & $-275,00$ & 0,6756 & 185,79 & 0,00 & $-185,79$ & & \\
\hline 11 & 265,00 & 0,00 & $-265,00$ & 0,6496 & 172,14 & 0,00 & $-172,14$ & & \\
\hline 12 & 265,00 & 0,00 & $-265,00$ & 0,6246 & 165,52 & 0,00 & $-165,52$ & & \\
\hline 13 & 265,00 & 0,00 & $-265,00$ & 0,6006 & 159,16 & 0,00 & $-159,16$ & & \\
\hline 14 & 265,00 & 0,00 & $-265,00$ & 0,5775 & 153,04 & 0,00 & $-153,04$ & & \\
\hline 15 & 275,00 & 0,00 & $-275,00$ & 0,5553 & 152,71 & 0,00 & $-152,71$ & & \\
\hline 16 & 265,00 & 0,00 & $-265,00$ & 0,5339 & 141,48 & 0,00 & $-141,48$ & & \\
\hline 17 & 265,00 & 0,00 & $-265,00$ & 0,5134 & 136,05 & 0,00 & $-136,05$ & & \\
\hline 18 & 265,00 & 0,00 & $-265,00$ & 0,4936 & 130,80 & 0,00 & $-130,80$ & & \\
\hline 19 & 265,00 & 0,00 & $-265,00$ & 0,4746 & 125,77 & 0,00 & $-125,77$ & & \\
\hline 20 & 275,00 & 0,00 & $-275,00$ & 0,4564 & 125,51 & 0,00 & $-125,51$ & & \\
\hline 21 & 265,00 & 0,00 & $-265,00$ & 0,4388 & 116,28 & 0,00 & $-116,28$ & & \\
\hline 22 & 265,00 & 0,00 & $-265,00$ & 0,422 & 111,83 & 0,00 & $-111,83$ & & \\
\hline 23 & 265,00 & 0,00 & $-265,00$ & 0,4057 & 107,51 & 0,00 & $-107,51$ & & \\
\hline 24 & 265,00 & 0,00 & $-265,00$ & 0,3901 & 103,38 & 0,00 & $-103,38$ & & \\
\hline \multirow{2}{*}{25} & 275,00 & 0,00 & $-275,00$ & 0,3751 & 103,15 & 0,00 & $-103,15$ & & \\
\hline & & & & & 4168,70 & 0,00 & $-4168,70$ & 0,0640 & $-266,80$ \\
\hline
\end{tabular}


Tabelle A-43: Anlage von Windschutzhecken bei 10 \% Ertragssteigerung und 100 \% Förderung

\begin{tabular}{|c|c|c|c|c|c|c|c|c|c|}
\hline Jahr & $\begin{array}{c}\text { Kosten } \\
\text { (€/ha) }\end{array}$ & $\begin{array}{c}\text { Nutzen } \\
\text { (€/ha) }\end{array}$ & $\begin{array}{c}\text { NK-Diff. } \\
\text { (€/ha) }\end{array}$ & $\begin{array}{c}\text { DF } \\
\text { (Zins } 4 \%)\end{array}$ & $\begin{array}{c}\text { disk. Kosten } \\
(€ / \mathrm{ha})\end{array}$ & $\begin{array}{c}\text { disk. Nutzen } \\
\text { (€/ha) }\end{array}$ & $\begin{array}{c}\text { disk. NK-Diff. } \\
(€ / h a)\end{array}$ & $\begin{array}{c}\text { WGF } \\
\text { (Zins } 4 \% \text { ) }\end{array}$ & $\begin{array}{c}\varnothing \text { jährl. Vorteil } \\
(€ / h a / a)\end{array}$ \\
\hline 0 & 0 & 0,00 & 0,00 & 0,00 & 0,00 & 0,00 & 0,00 & & \\
\hline 1 & 265,00 & 5,98 & $-259,02$ & 0,9615 & 254,80 & 5,75 & $-249,05$ & & \\
\hline 2 & 265,00 & 11,96 & $-253,04$ & 0,9246 & 245,02 & 11,06 & $-233,96$ & & \\
\hline 3 & 265,00 & 17,94 & $-247,06$ & 0,889 & 235,59 & 15,95 & $-219,64$ & & \\
\hline 4 & 265,00 & 23,92 & $-241,08$ & 0,8548 & 226,52 & 20,45 & $-206,08$ & & \\
\hline 5 & 275,00 & 29,90 & $-245,10$ & 0,8219 & 226,02 & 24,57 & $-201,45$ & & \\
\hline 6 & 265,00 & 35,88 & $-229,12$ & 0,7903 & 209,43 & 28,36 & $-181,07$ & & \\
\hline 7 & 265,00 & 41,86 & $-223,14$ & 0,7599 & 201,37 & 31,81 & $-169,56$ & & \\
\hline 8 & 265,00 & 47,84 & $-217,16$ & 0,7307 & 193,64 & 34,96 & $-158,68$ & & \\
\hline 9 & 265,00 & 53,82 & $-211,18$ & 0,7026 & 186,19 & 37,81 & $-148,38$ & & \\
\hline 10 & 275,00 & 59,80 & $-215,20$ & 0,6756 & 185,79 & 40,40 & $-145,39$ & & \\
\hline 11 & 265,00 & 59,80 & $-205,20$ & 0,6496 & 172,14 & 38,85 & $-133,30$ & & \\
\hline 12 & 265,00 & 59,80 & $-205,20$ & 0,6246 & 165,52 & 37,35 & $-128,17$ & & \\
\hline 13 & 265,00 & 59,80 & $-205,20$ & 0,6006 & 159,16 & 35,92 & $-123,24$ & & \\
\hline 14 & 265,00 & 59,80 & $-205,20$ & 0,5775 & 153,04 & 34,53 & $-118,50$ & & \\
\hline 15 & 275,00 & 59,80 & $-215,20$ & 0,5553 & 152,71 & 33,21 & $-119,50$ & & \\
\hline 16 & 265,00 & 59,80 & $-205,20$ & 0,5339 & 141,48 & 31,93 & $-109,56$ & & \\
\hline 17 & 265,00 & 59,80 & $-205,20$ & 0,5134 & 136,05 & 30,70 & $-105,35$ & & \\
\hline 18 & 265,00 & 59,80 & $-205,20$ & 0,4936 & 130,80 & 29,52 & $-101,29$ & & \\
\hline 19 & 265,00 & 59,80 & $-205,20$ & 0,4746 & 125,77 & 28,38 & $-97,39$ & & \\
\hline 20 & 275,00 & 59,80 & $-215,20$ & 0,4564 & 125,51 & 27,29 & $-98,22$ & & \\
\hline 21 & 265,00 & 59,80 & $-205,20$ & 0,4388 & 116,28 & 26,24 & $-90,04$ & & \\
\hline 22 & 265,00 & 59,80 & $-205,20$ & 0,422 & 111,83 & 25,24 & $-86,59$ & & \\
\hline 23 & 265,00 & 59,80 & $-205,20$ & 0,4057 & 107,51 & 24,26 & $-83,25$ & & \\
\hline 24 & 265,00 & 59,80 & $-205,20$ & 0,3901 & 103,38 & 23,33 & $-80,05$ & & \\
\hline \multirow[t]{2}{*}{25} & 275,00 & 59,80 & $-215,20$ & 0,3751 & 103,15 & 22,43 & $-80,72$ & & \\
\hline & & & & & 4168,70 & 700,28 & $-3468,41$ & 0,0640 & $-221,98$ \\
\hline
\end{tabular}


Tabelle A-44: Anlage von Grünstreifen, Bodenabtrag 6 t/ha/a, Streifenbreite 3m; Reihenabstand 20 m

\begin{tabular}{|c|c|c|c|c|c|c|c|c|c|}
\hline Jahr & $\begin{array}{c}\text { Kosten } \\
\text { (€/ha) }\end{array}$ & $\begin{array}{c}\text { Nutzen } \\
\text { (€/ha) }\end{array}$ & $\begin{array}{c}\text { NK-Diff. } \\
\text { (€/ha) }\end{array}$ & $\begin{array}{c}\text { DF } \\
\text { (Zins } 4 \%) \\
\end{array}$ & $\begin{array}{c}\text { disk. Kosten } \\
\text { (€/ha) }\end{array}$ & $\begin{array}{c}\text { disk. Nutzen } \\
\text { (€/ha) }\end{array}$ & $\begin{array}{c}\text { disk. NK-Diff. } \\
(€ / h a)\end{array}$ & $\begin{array}{c}\text { WGF } \\
\text { (Zins } 4 \% \text { ) }\end{array}$ & $\begin{array}{c}\varnothing \text { jährl. Vorteil } \\
(€ / h a / a)\end{array}$ \\
\hline 0 & 0,00 & 0,00 & 0,00 & 0,00 & 0,00 & 0,00 & 0,00 & & \\
\hline 1 & 39,00 & 27,40 & $-11,60$ & 0,9615 & 37,50 & 26,35 & $-11,15$ & & \\
\hline 2 & 39,00 & 27,40 & $-11,60$ & 0,9246 & 36,06 & 25,33 & $-10,73$ & & \\
\hline 3 & 39,00 & 27,40 & $-11,60$ & 0,889 & 34,67 & 24,36 & $-10,31$ & & \\
\hline 4 & 39,00 & 27,40 & $-11,60$ & 0,8548 & 33,34 & 23,42 & $-9,92$ & & \\
\hline 5 & 39,00 & 27,40 & $-11,60$ & 0,8219 & 32,05 & 22,52 & $-9,53$ & & \\
\hline 6 & 39,00 & 27,40 & $-11,60$ & 0,7903 & 30,82 & 21,65 & $-9,17$ & & \\
\hline 7 & 39,00 & 27,40 & $-11,60$ & 0,7599 & 29,64 & 20,82 & $-8,81$ & & \\
\hline 8 & 39,00 & 27,40 & $-11,60$ & 0,7307 & 28,50 & 20,02 & $-8,48$ & & \\
\hline 9 & 39,00 & 27,40 & $-11,60$ & 0,7026 & 27,40 & 19,25 & $-8,15$ & & \\
\hline 10 & 39,00 & 27,40 & $-11,60$ & 0,6756 & 26,35 & 18,51 & $-7,84$ & & \\
\hline 11 & 39,00 & 27,40 & $-11,60$ & 0,6496 & 25,33 & 17,80 & $-7,54$ & & \\
\hline 12 & 39,00 & 27,40 & $-11,60$ & 0,6246 & 24,36 & 17,11 & $-7,25$ & & \\
\hline 13 & 39,00 & 27,40 & $-11,60$ & 0,6006 & 23,42 & 16,46 & $-6,97$ & & \\
\hline 14 & 39,00 & 27,40 & $-11,60$ & 0,5775 & 22,52 & 15,82 & $-6,70$ & & \\
\hline 15 & 39,00 & 27,40 & $-11,60$ & 0,5553 & 21,66 & 15,22 & $-6,44$ & & \\
\hline 16 & 39,00 & 27,40 & $-11,60$ & 0,5339 & 20,82 & 14,63 & $-6,19$ & & \\
\hline 17 & 39,00 & 27,40 & $-11,60$ & 0,5134 & 20,02 & 14,07 & $-5,96$ & & \\
\hline 18 & 39,00 & 27,40 & $-11,60$ & 0,4936 & 19,25 & 13,52 & $-5,73$ & & \\
\hline 19 & 39,00 & 27,40 & $-11,60$ & 0,4746 & 18,51 & 13,00 & $-5,51$ & & \\
\hline 20 & 39,00 & 27,40 & $-11,60$ & 0,4564 & 17,80 & 12,51 & $-5,29$ & & \\
\hline 21 & 39,00 & 27,40 & $-11,60$ & 0,4388 & 17,11 & 12,02 & $-5,09$ & & \\
\hline 22 & 39,00 & 27,40 & $-11,60$ & 0,422 & 16,46 & 11,56 & $-4,90$ & & \\
\hline 23 & 39,00 & 27,40 & $-11,60$ & 0,4057 & 15,82 & 11,12 & $-4,71$ & & \\
\hline 24 & 39,00 & 27,40 & $-11,60$ & 0,3901 & 15,21 & 10,69 & $-4,53$ & & \\
\hline \multirow[t]{2}{*}{25} & 39,00 & 27,40 & $-11,60$ & 0,3751 & 14,63 & 10,28 & $-4,35$ & & \\
\hline & & & & & 609,26 & 428,05 & $-181,22$ & 0,0640 & $-11,60$ \\
\hline
\end{tabular}


Tabelle A-45: Anlage von Grünstreifen, Bodenabtrag 6 t/ha/a, Streifenbreite 3m; Reihenabstand 20 m

\begin{tabular}{|c|c|c|c|c|c|c|c|c|c|}
\hline Jahr & $\begin{array}{c}\text { Kosten } \\
\text { (€/ha) }\end{array}$ & $\begin{array}{c}\text { Nutzen } \\
\text { (€/ha) }\end{array}$ & $\begin{array}{c}\text { NK-Diff. } \\
\text { (€/ha) }\end{array}$ & $\begin{array}{c}\text { DF } \\
\text { (Zins } 4 \%)\end{array}$ & $\begin{array}{c}\text { disk. Kosten } \\
(€ / \mathrm{ha})\end{array}$ & $\begin{array}{c}\text { disk. Nutzen } \\
(€ / h a)\end{array}$ & $\begin{array}{c}\text { disk. NK-Diff. } \\
(€ / h a)\end{array}$ & $\begin{array}{c}\text { WGF } \\
\text { (Zins } 4 \% \text { ) }\end{array}$ & $\begin{array}{c}\varnothing \text { jährl. Vorteil } \\
\text { (€/ha/a) }\end{array}$ \\
\hline 0 & 0,00 & 0,00 & 0,00 & 0,00 & 0,00 & 0,00 & 0,00 & & \\
\hline 1 & 20,80 & 25,00 & 4,20 & 0,9615 & 20,00 & 24,04 & 4,04 & & \\
\hline 2 & 20,80 & 25,00 & 4,20 & 0,9246 & 19,23 & 23,12 & 3,88 & & \\
\hline 3 & 20,80 & 25,00 & 4,20 & 0,889 & 18,49 & 22,23 & 3,73 & & \\
\hline 4 & 20,80 & 25,00 & 4,20 & 0,8548 & 17,78 & 21,37 & 3,59 & & \\
\hline 5 & 20,80 & 25,00 & 4,20 & 0,8219 & 17,10 & 20,55 & 3,45 & & \\
\hline 6 & 20,80 & 25,00 & 4,20 & 0,7903 & 16,44 & 19,76 & 3,32 & & \\
\hline 7 & 20,80 & 25,00 & 4,20 & 0,7599 & 15,81 & 19,00 & 3,19 & & \\
\hline 8 & 20,80 & 25,00 & 4,20 & 0,7307 & 15,20 & 18,27 & 3,07 & & \\
\hline 9 & 20,80 & 25,00 & 4,20 & 0,7026 & 14,61 & 17,57 & 2,95 & & \\
\hline 10 & 20,80 & 25,00 & 4,20 & 0,6756 & 14,05 & 16,89 & 2,84 & & \\
\hline 11 & 20,80 & 25,00 & 4,20 & 0,6496 & 13,51 & 16,24 & 2,73 & & \\
\hline 12 & 20,80 & 25,00 & 4,20 & 0,6246 & 12,99 & 15,62 & 2,62 & & \\
\hline 13 & 20,80 & 25,00 & 4,20 & 0,6006 & 12,49 & 15,02 & 2,52 & & \\
\hline 14 & 20,80 & 25,00 & 4,20 & 0,5775 & 12,01 & 14,44 & 2,43 & & \\
\hline 15 & 20,80 & 25,00 & 4,20 & 0,5553 & 11,55 & 13,88 & 2,33 & & \\
\hline 16 & 20,80 & 25,00 & 4,20 & 0,5339 & 11,11 & 13,35 & 2,24 & & \\
\hline 17 & 20,80 & 25,00 & 4,20 & 0,5134 & 10,68 & 12,84 & 2,16 & & \\
\hline 18 & 20,80 & 25,00 & 4,20 & 0,4936 & 10,27 & 12,34 & 2,07 & & \\
\hline 19 & 20,80 & 25,00 & 4,20 & 0,4746 & 9,87 & 11,87 & 1,99 & & \\
\hline 20 & 20,80 & 25,00 & 4,20 & 0,4564 & 9,49 & 11,41 & 1,92 & & \\
\hline 21 & 20,80 & 25,00 & 4,20 & 0,4388 & 9,13 & 10,97 & 1,84 & & \\
\hline 22 & 20,80 & 25,00 & 4,20 & 0,422 & 8,78 & 10,55 & 1,77 & & \\
\hline 23 & 20,80 & 25,00 & 4,20 & 0,4057 & 8,44 & 10,14 & 1,70 & & \\
\hline 24 & 20,80 & 25,00 & 4,20 & 0,3901 & 8,11 & 9,75 & 1,64 & & \\
\hline \multirow[t]{3}{*}{25} & 20,80 & 25,00 & 4,20 & 0,3751 & 7,80 & 9,38 & 1,58 & & \\
\hline & & & & & 324,94 & 390,55 & 65,61 & 0,0640 & 4,20 \\
\hline & & & & KNV & & 0,83 & & & \\
\hline
\end{tabular}


Tabelle A-46: Anlage von Grünstreifen, Bodenabtrag 6 t/ha/a, Streifenbreite 3m; Reihenabstand 20 m

\begin{tabular}{|c|c|c|c|c|c|c|c|c|c|}
\hline Jahr & $\begin{array}{c}\text { Kosten } \\
\text { (€/ha) }\end{array}$ & $\begin{array}{l}\text { Nutzen } \\
\text { (€/ha) }\end{array}$ & $\begin{array}{c}\text { NK-Diff. } \\
\text { (€/ha) }\end{array}$ & $\begin{array}{c}\text { DF } \\
\text { (Zins } 4 \%) \\
\end{array}$ & $\begin{array}{c}\text { disk. Kosten } \\
\text { (€/ha) }\end{array}$ & $\begin{array}{c}\text { disk. Nutzen } \\
\text { (€/ha) }\end{array}$ & $\begin{array}{c}\text { disk. NK-Diff. } \\
(€ / h a)\end{array}$ & $\begin{array}{c}\text { WGF } \\
\text { (Zins } 4 \% \text { ) }\end{array}$ & $\begin{array}{c}\varnothing \text { jährl. Vorteil } \\
(€ / h a / a)\end{array}$ \\
\hline 0 & 0,00 & 0,00 & 0,00 & 0,00 & 0,00 & 0,00 & 0,00 & & \\
\hline 1 & 39,00 & 45,60 & 6,60 & 0,9615 & 37,50 & 43,84 & 6,35 & & \\
\hline 2 & 39,00 & 45,60 & 6,60 & 0,9246 & 36,06 & 42,16 & 6,10 & & \\
\hline 3 & 39,00 & 45,60 & 6,60 & 0,889 & 34,67 & 40,54 & 5,87 & & \\
\hline 4 & 39,00 & 45,60 & 6,60 & 0,8548 & 33,34 & 38,98 & 5,64 & & \\
\hline 5 & 39,00 & 45,60 & 6,60 & 0,8219 & 32,05 & 37,48 & 5,42 & & \\
\hline 6 & 39,00 & 45,60 & 6,60 & 0,7903 & 30,82 & 36,04 & 5,22 & & \\
\hline 7 & 39,00 & 45,60 & 6,60 & 0,7599 & 29,64 & 34,65 & 5,02 & & \\
\hline 8 & 39,00 & 45,60 & 6,60 & 0,7307 & 28,50 & 33,32 & 4,82 & & \\
\hline 9 & 39,00 & 45,60 & 6,60 & 0,7026 & 27,40 & 32,04 & 4,64 & & \\
\hline 10 & 39,00 & 45,60 & 6,60 & 0,6756 & 26,35 & 30,81 & 4,46 & & \\
\hline 11 & 39,00 & 45,60 & 6,60 & 0,6496 & 25,33 & 29,62 & 4,29 & & \\
\hline 12 & 39,00 & 45,60 & 6,60 & 0,6246 & 24,36 & 28,48 & 4,12 & & \\
\hline 13 & 39,00 & 45,60 & 6,60 & 0,6006 & 23,42 & 27,39 & 3,96 & & \\
\hline 14 & 39,00 & 45,60 & 6,60 & 0,5775 & 22,52 & 26,33 & 3,81 & & \\
\hline 15 & 39,00 & 45,60 & 6,60 & 0,5553 & 21,66 & 25,32 & 3,66 & & \\
\hline 16 & 39,00 & 45,60 & 6,60 & 0,5339 & 20,82 & 24,35 & 3,52 & & \\
\hline 17 & 39,00 & 45,60 & 6,60 & 0,5134 & 20,02 & 23,41 & 3,39 & & \\
\hline 18 & 39,00 & 45,60 & 6,60 & 0,4936 & 19,25 & 22,51 & 3,26 & & \\
\hline 19 & 39,00 & 45,60 & 6,60 & 0,4746 & 18,51 & 21,64 & 3,13 & & \\
\hline 20 & 39,00 & 45,60 & 6,60 & 0,4564 & 17,80 & 20,81 & 3,01 & & \\
\hline 21 & 39,00 & 45,60 & 6,60 & 0,4388 & 17,11 & 20,01 & 2,90 & & \\
\hline 22 & 39,00 & 45,60 & 6,60 & 0,422 & 16,46 & 19,24 & 2,79 & & \\
\hline 23 & 39,00 & 45,60 & 6,60 & 0,4057 & 15,82 & 18,50 & 2,68 & & \\
\hline 24 & 39,00 & 45,60 & 6,60 & 0,3901 & 15,21 & 17,79 & 2,57 & & \\
\hline \multirow[t]{2}{*}{25} & 39,00 & 45,60 & 6,60 & 0,3751 & 14,63 & 17,10 & 2,48 & & \\
\hline & & & & & 609,26 & 712,37 & 103,11 & 0,0640 & 6,60 \\
\hline
\end{tabular}


Tabelle A-47: Anlage von Grünstreifen, Bodenabtrag 6 t/ha/a, Streifenbreite 3m; Reihenabstand 20 m

\begin{tabular}{|c|c|c|c|c|c|c|c|c|c|}
\hline Jahr & $\begin{array}{c}\text { Kosten } \\
\text { (€/ha) }\end{array}$ & $\begin{array}{c}\text { Nutzen } \\
\text { (€/ha) }\end{array}$ & $\begin{array}{c}\text { NK-Diff. } \\
\text { (€/ha) }\end{array}$ & $\begin{array}{c}\text { DF } \\
\text { (Zins } 4 \%) \\
\end{array}$ & $\begin{array}{c}\text { disk. Kosten } \\
\text { (€/ha) }\end{array}$ & $\begin{array}{c}\text { disk. Nutzen } \\
\text { (€/ha) }\end{array}$ & $\begin{array}{c}\text { disk. NK-Diff. } \\
(€ / h a)\end{array}$ & $\begin{array}{c}\text { WGF } \\
\text { (Zins } 4 \%) \\
\end{array}$ & $\begin{array}{c}\varnothing \text { jährl. Vorteil } \\
(€ / h a / a)\end{array}$ \\
\hline 0 & 0,00 & 0,00 & 0,00 & 0,00 & 0,00 & 0,00 & 0,00 & & \\
\hline 1 & 20,80 & 41,80 & 21,00 & 0,9615 & 20,00 & 40,19 & 20,19 & & \\
\hline 2 & 20,80 & 41,80 & 21,00 & 0,9246 & 19,23 & 38,65 & 19,42 & & \\
\hline 3 & 20,80 & 41,80 & 21,00 & 0,889 & 18,49 & 37,16 & 18,67 & & \\
\hline 4 & 20,80 & 41,80 & 21,00 & 0,8548 & 17,78 & 35,73 & 17,95 & & \\
\hline 5 & 20,80 & 41,80 & 21,00 & 0,8219 & 17,10 & 34,36 & 17,26 & & \\
\hline 6 & 20,80 & 41,80 & 21,00 & 0,7903 & 16,44 & 33,03 & 16,60 & & \\
\hline 7 & 20,80 & 41,80 & 21,00 & 0,7599 & 15,81 & 31,76 & 15,96 & & \\
\hline 8 & 20,80 & 41,80 & 21,00 & 0,7307 & 15,20 & 30,54 & 15,34 & & \\
\hline 9 & 20,80 & 41,80 & 21,00 & 0,7026 & 14,61 & 29,37 & 14,75 & & \\
\hline 10 & 20,80 & 41,80 & 21,00 & 0,6756 & 14,05 & 28,24 & 14,19 & & \\
\hline 11 & 20,80 & 41,80 & 21,00 & 0,6496 & 13,51 & 27,15 & 13,64 & & \\
\hline 12 & 20,80 & 41,80 & 21,00 & 0,6246 & 12,99 & 26,11 & 13,12 & & \\
\hline 13 & 20,80 & 41,80 & 21,00 & 0,6006 & 12,49 & 25,11 & 12,61 & & \\
\hline 14 & 20,80 & 41,80 & 21,00 & 0,5775 & 12,01 & 24,14 & 12,13 & & \\
\hline 15 & 20,80 & 41,80 & 21,00 & 0,5553 & 11,55 & 23,21 & 11,66 & & \\
\hline 16 & 20,80 & 41,80 & 21,00 & 0,5339 & 11,11 & 22,32 & 11,21 & & \\
\hline 17 & 20,80 & 41,80 & 21,00 & 0,5134 & 10,68 & 21,46 & 10,78 & & \\
\hline 18 & 20,80 & 41,80 & 21,00 & 0,4936 & 10,27 & 20,63 & 10,37 & & \\
\hline 19 & 20,80 & 41,80 & 21,00 & 0,4746 & 9,87 & 19,84 & 9,97 & & \\
\hline 20 & 20,80 & 41,80 & 21,00 & 0,4564 & 9,49 & 19,08 & 9,58 & & \\
\hline 21 & 20,80 & 41,80 & 21,00 & 0,4388 & 9,13 & 18,34 & 9,21 & & \\
\hline 22 & 20,80 & 41,80 & 21,00 & 0,422 & 8,78 & 17,64 & 8,86 & & \\
\hline 23 & 20,80 & 41,80 & 21,00 & 0,4057 & 8,44 & 16,96 & 8,52 & & \\
\hline 24 & 20,80 & 41,80 & 21,00 & 0,3901 & 8,11 & 16,31 & 8,19 & & \\
\hline \multirow[t]{2}{*}{25} & 20,80 & 41,80 & 21,00 & 0,3751 & 7,80 & 15,68 & 7,88 & & \\
\hline & & & & & 324,94 & 653,00 & 328,06 & 0,0640 & 21,00 \\
\hline
\end{tabular}


Tabelle A-48: Verzicht des Anbaus an Hanglagen; Stillegung, WR-WW-WG, Bewirtschaftung quer zum Hang

\begin{tabular}{|c|c|c|c|c|c|c|c|c|c|}
\hline Jahr & $\begin{array}{c}\text { Kosten } \\
\text { (€/ha) }\end{array}$ & $\begin{array}{c}\text { Nutzen } \\
\text { (€/ha) }\end{array}$ & $\begin{array}{c}\text { NK-Diff. } \\
\text { (€/ha) }\end{array}$ & $\begin{array}{c}\text { DF } \\
\text { (Zins } 4 \%) \\
\end{array}$ & $\begin{array}{c}\text { disk. Kosten } \\
(€ / \mathrm{ha}) \\
\end{array}$ & $\begin{array}{c}\text { disk. Nutzen } \\
\text { (€/ha) }\end{array}$ & $\begin{array}{c}\text { disk. NK-Diff. } \\
(€ / h a)\end{array}$ & $\begin{array}{c}\text { WGF } \\
\text { (Zins } 4 \% \text { ) }\end{array}$ & $\begin{array}{c}\varnothing \text { jährl. Vorteil } \\
(€ / h a / a)\end{array}$ \\
\hline 0 & 0,00 & 0,00 & 0,00 & 0,00 & 0,00 & 0,00 & 0,00 & & \\
\hline 1 & 82,00 & 289,40 & 207,40 & 0,9615 & 78,84 & 278,26 & 199,42 & & \\
\hline 2 & 82,00 & 289,40 & 207,40 & 0,9246 & 75,82 & 267,58 & 191,76 & & \\
\hline 3 & 82,00 & 289,40 & 207,40 & 0,889 & 72,90 & 257,28 & 184,38 & & \\
\hline 4 & 82,00 & 289,40 & 207,40 & 0,8548 & 70,09 & 247,38 & 177,29 & & \\
\hline 5 & 82,00 & 289,40 & 207,40 & 0,8219 & 67,40 & 237,86 & 170,46 & & \\
\hline 6 & 82,00 & 289,40 & 207,40 & 0,7903 & 64,80 & 228,71 & 163,91 & & \\
\hline 7 & 82,00 & 289,40 & 207,40 & 0,7599 & 62,31 & 219,92 & 157,60 & & \\
\hline 8 & 82,00 & 289,40 & 207,40 & 0,7307 & 59,92 & 211,46 & 151,55 & & \\
\hline 9 & 82,00 & 289,40 & 207,40 & 0,7026 & 57,61 & 203,33 & 145,72 & & \\
\hline 10 & 82,00 & 289,40 & 207,40 & 0,6756 & 55,40 & 195,52 & 140,12 & & \\
\hline 11 & 82,00 & 289,40 & 207,40 & 0,6496 & 53,27 & 187,99 & 134,73 & & \\
\hline 12 & 82,00 & 289,40 & 207,40 & 0,6246 & 51,22 & 180,76 & 129,54 & & \\
\hline 13 & 82,00 & 289,40 & 207,40 & 0,6006 & 49,25 & 173,81 & 124,56 & & \\
\hline 14 & 82,00 & 289,40 & 207,40 & 0,5775 & 47,36 & 167,13 & 119,77 & & \\
\hline 15 & 82,00 & 289,40 & 207,40 & 0,5553 & 45,53 & 160,70 & 115,17 & & \\
\hline 16 & 82,00 & 289,40 & 207,40 & 0,5339 & 43,78 & 154,51 & 110,73 & & \\
\hline 17 & 82,00 & 289,40 & 207,40 & 0,5134 & 42,10 & 148,58 & 106,48 & & \\
\hline 18 & 82,00 & 289,40 & 207,40 & 0,4936 & 40,48 & 142,85 & 102,37 & & \\
\hline 19 & 82,00 & 289,40 & 207,40 & 0,4746 & 38,92 & 137,35 & 98,43 & & \\
\hline 20 & 82,00 & 289,40 & 207,40 & 0,4564 & 37,42 & 132,08 & 94,66 & & \\
\hline 21 & 82,00 & 289,40 & 207,40 & 0,4388 & 35,98 & 126,99 & 91,01 & & \\
\hline 22 & 82,00 & 289,40 & 207,40 & 0,422 & 34,60 & 122,13 & 87,52 & & \\
\hline 23 & 82,00 & 289,40 & 207,40 & 0,4057 & 33,27 & 117,41 & 84,14 & & \\
\hline 24 & 82,00 & 289,40 & 207,40 & 0,3901 & 31,99 & 112,89 & 80,91 & & \\
\hline \multirow[t]{2}{*}{25} & 82,00 & 289,40 & 207,40 & 0,3751 & 30,76 & 108,55 & 77,80 & & \\
\hline & & & & & 1281,01 & 4521,04 & 3240,02 & 0,0640 & 207,4 \\
\hline
\end{tabular}


Tabelle A-49: Verzicht des Anbaus an Hanglagen; Mutterkuhhaltung, WR-WW-WG, Bewirtschaftung in Gefällerichtung

\begin{tabular}{|c|c|c|c|c|c|c|c|c|c|}
\hline Jahr & $\begin{array}{c}\text { Kosten } \\
\text { (€/ha) }\end{array}$ & $\begin{array}{c}\text { Nutzen } \\
\text { (€/ha) }\end{array}$ & $\begin{array}{c}\text { NK-Diff. } \\
\text { (€/ha) }\end{array}$ & $\begin{array}{c}\text { DF } \\
\text { (Zins } 4 \%)\end{array}$ & $\begin{array}{c}\text { disk. Kosten } \\
(€ / \mathrm{ha})\end{array}$ & $\begin{array}{c}\text { disk. Nutzen } \\
(€ / h a)\end{array}$ & $\begin{array}{c}\text { disk. NK-Diff. } \\
(€ / h a)\end{array}$ & $\begin{array}{c}\text { WGF } \\
\text { (Zins } 4 \% \text { ) }\end{array}$ & $\begin{array}{c}\varnothing \text { jährl. Vorteil } \\
\text { (€/ha/a) }\end{array}$ \\
\hline 0 & 0,00 & 0,00 & 0,00 & 0,00 & 0,00 & 0,00 & 0,00 & & \\
\hline 1 & 378,20 & 81,80 & $-296,40$ & 0,9615 & 363,64 & 78,65 & $-284,99$ & & \\
\hline 2 & 82,00 & 81,80 & $-0,20$ & 0,9246 & 75,82 & 75,63 & $-0,18$ & & \\
\hline 3 & 82,00 & 81,80 & $-0,20$ & 0,889 & 72,90 & 72,72 & $-0,18$ & & \\
\hline 4 & 82,00 & 81,80 & $-0,20$ & 0,8548 & 70,09 & 69,92 & $-0,17$ & & \\
\hline 5 & 212,00 & 81,80 & $-130,20$ & 0,8219 & 174,24 & 67,23 & $-107,01$ & & \\
\hline 6 & 82,00 & 81,80 & $-0,20$ & 0,7903 & 64,80 & 64,65 & $-0,16$ & & \\
\hline 7 & 82,00 & 81,80 & $-0,20$ & 0,7599 & 62,31 & 62,16 & $-0,15$ & & \\
\hline 8 & 82,00 & 81,80 & $-0,20$ & 0,7307 & 59,92 & 59,77 & $-0,15$ & & \\
\hline 9 & 82,00 & 81,80 & $-0,20$ & 0,7026 & 57,61 & 57,47 & $-0,14$ & & \\
\hline 10 & 212,00 & 81,80 & $-130,20$ & 0,6756 & 143,23 & 55,26 & $-87,96$ & & \\
\hline 11 & 82,00 & 81,80 & $-0,20$ & 0,6496 & 53,27 & 53,14 & $-0,13$ & & \\
\hline 12 & 82,00 & 81,80 & $-0,20$ & 0,6246 & 51,22 & 51,09 & $-0,12$ & & \\
\hline 13 & 82,00 & 81,80 & $-0,20$ & 0,6006 & 49,25 & 49,13 & $-0,12$ & & \\
\hline 14 & 82,00 & 81,80 & $-0,20$ & 0,5775 & 47,36 & 47,24 & $-0,12$ & & \\
\hline 15 & 212,00 & 81,80 & $-130,20$ & 0,5553 & 117,72 & 45,42 & $-72,30$ & & \\
\hline 16 & 82,00 & 81,80 & $-0,20$ & 0,5339 & 43,78 & 43,67 & $-0,11$ & & \\
\hline 17 & 82,00 & 81,80 & $-0,20$ & 0,5134 & 42,10 & 42,00 & $-0,10$ & & \\
\hline 18 & 82,00 & 81,80 & $-0,20$ & 0,4936 & 40,48 & 40,38 & $-0,10$ & & \\
\hline 19 & 82,00 & 81,80 & $-0,20$ & 0,4746 & 38,92 & 38,82 & $-0,09$ & & \\
\hline 20 & 212,00 & 81,80 & $-130,20$ & 0,4564 & 96,76 & 37,33 & $-59,42$ & & \\
\hline 21 & 82,00 & 81,80 & $-0,20$ & 0,4388 & 35,98 & 35,89 & $-0,09$ & & \\
\hline 22 & 82,00 & 81,80 & $-0,20$ & 0,422 & 34,60 & 34,52 & $-0,08$ & & \\
\hline 23 & 82,00 & 81,80 & $-0,20$ & 0,4057 & 33,27 & 33,19 & $-0,08$ & & \\
\hline 24 & 82,00 & 81,80 & $-0,20$ & 0,3901 & 31,99 & 31,91 & $-0,08$ & & \\
\hline \multirow[t]{3}{*}{25} & 212,00 & 81,80 & $-130,20$ & 0,3751 & 79,52 & 30,68 & $-48,84$ & & \\
\hline & & & & & 1940,77 & 1277,89 & $-662,88$ & 0,0640 & $-42,42$ \\
\hline & & & & KNV & & 1,52 & & & \\
\hline
\end{tabular}


Tabelle A-50: Verzicht des Anbaus an Hanglagen; Verpachtung, WR-WW-WG, Bewirtschaftung quer zum Hang

\begin{tabular}{|c|c|c|c|c|c|c|c|c|c|}
\hline Jahr & $\begin{array}{c}\text { Kosten } \\
\text { (€/ha) }\end{array}$ & $\begin{array}{c}\text { Nutzen } \\
\text { (€/ha) }\end{array}$ & $\begin{array}{r}\text { NK-Diff. } \\
\text { (€/ha) }\end{array}$ & $\begin{array}{c}\text { DF } \\
\text { (Zins } 4 \% \text { ) }\end{array}$ & $\begin{array}{c}\text { disk. Kosten } \\
\text { (€/ha) }\end{array}$ & $\begin{array}{c}\text { disk. Nutzen } \\
\text { (€/ha) }\end{array}$ & $\begin{array}{c}\text { disk. NK-Diff. } \\
(€ / h a)\end{array}$ & $\begin{array}{c}\text { WGF } \\
\text { (Zins } 4 \% \text { ) }\end{array}$ & $\begin{array}{c}\varnothing \text { jährl. Vorteil } \\
(€ / h a / a)\end{array}$ \\
\hline 0 & 0,00 & 0,00 & 0,00 & 0,00 & 0,00 & 0,00 & 0,00 & & \\
\hline 1 & 82,00 & 81,80 & $-0,20$ & 0,9615 & 78,84 & 78,65 & $-0,19$ & & \\
\hline 2 & 82,00 & 81,80 & $-0,20$ & 0,9246 & 75,82 & 75,63 & $-0,18$ & & \\
\hline 3 & 82,00 & 81,80 & $-0,20$ & 0,889 & 72,90 & 72,72 & $-0,18$ & & \\
\hline 4 & 82,00 & 81,80 & $-0,20$ & 0,8548 & 70,09 & 69,92 & $-0,17$ & & \\
\hline 5 & 82,00 & 81,80 & $-0,20$ & 0,8219 & 67,40 & 67,23 & $-0,16$ & & \\
\hline 6 & 82,00 & 81,80 & $-0,20$ & 0,7903 & 64,80 & 64,65 & $-0,16$ & & \\
\hline 7 & 82,00 & 81,80 & $-0,20$ & 0,7599 & 62,31 & 62,16 & $-0,15$ & & \\
\hline 8 & 82,00 & 81,80 & $-0,20$ & 0,7307 & 59,92 & 59,77 & $-0,15$ & & \\
\hline 9 & 82,00 & 81,80 & $-0,20$ & 0,7026 & 57,61 & 57,47 & $-0,14$ & & \\
\hline 10 & 82,00 & 81,80 & $-0,20$ & 0,6756 & 55,40 & 55,26 & $-0,14$ & & \\
\hline 11 & 82,00 & 81,80 & $-0,20$ & 0,6496 & 53,27 & 53,14 & $-0,13$ & & \\
\hline 12 & 82,00 & 81,80 & $-0,20$ & 0,6246 & 51,22 & 51,09 & $-0,12$ & & \\
\hline 13 & 82,00 & 81,80 & $-0,20$ & 0,6006 & 49,25 & 49,13 & $-0,12$ & & \\
\hline 14 & 82,00 & 81,80 & $-0,20$ & 0,5775 & 47,36 & 47,24 & $-0,12$ & & \\
\hline 15 & 82,00 & 81,80 & $-0,20$ & 0,5553 & 45,53 & 45,42 & $-0,11$ & & \\
\hline 16 & 82,00 & 81,80 & $-0,20$ & 0,5339 & 43,78 & 43,67 & $-0,11$ & & \\
\hline 17 & 82,00 & 81,80 & $-0,20$ & 0,5134 & 42,10 & 42,00 & $-0,10$ & & \\
\hline 18 & 82,00 & 81,80 & $-0,20$ & 0,4936 & 40,48 & 40,38 & $-0,10$ & & \\
\hline 19 & 82,00 & 81,80 & $-0,20$ & 0,4746 & 38,92 & 38,82 & $-0,09$ & & \\
\hline 20 & 82,00 & 81,80 & $-0,20$ & 0,4564 & 37,42 & 37,33 & $-0,09$ & & \\
\hline 21 & 82,00 & 81,80 & $-0,20$ & 0,4388 & 35,98 & 35,89 & $-0,09$ & & \\
\hline 22 & 82,00 & 81,80 & $-0,20$ & 0,422 & 34,60 & 34,52 & $-0,08$ & & \\
\hline 23 & 82,00 & 81,80 & $-0,20$ & 0,4057 & 33,27 & 33,19 & $-0,08$ & & \\
\hline 24 & 82,00 & 81,80 & $-0,20$ & 0,3901 & 31,99 & 31,91 & $-0,08$ & & \\
\hline \multirow[t]{2}{*}{25} & 82,00 & 81,80 & $-0,20$ & 0,3751 & 30,76 & 30,68 & $-0,08$ & & \\
\hline & & & & & 1281,01 & 1277,89 & $-3,12$ & 0,0640 & $-0,20$ \\
\hline
\end{tabular}


Tabelle A-51: Mulchsaat, Standort mit 8,7 t/ha, Effizienz $95 \%$. (verh. BA 8,265 t/ha/a), inkl. Förderung 60 €/ha/a über fünf Jahre

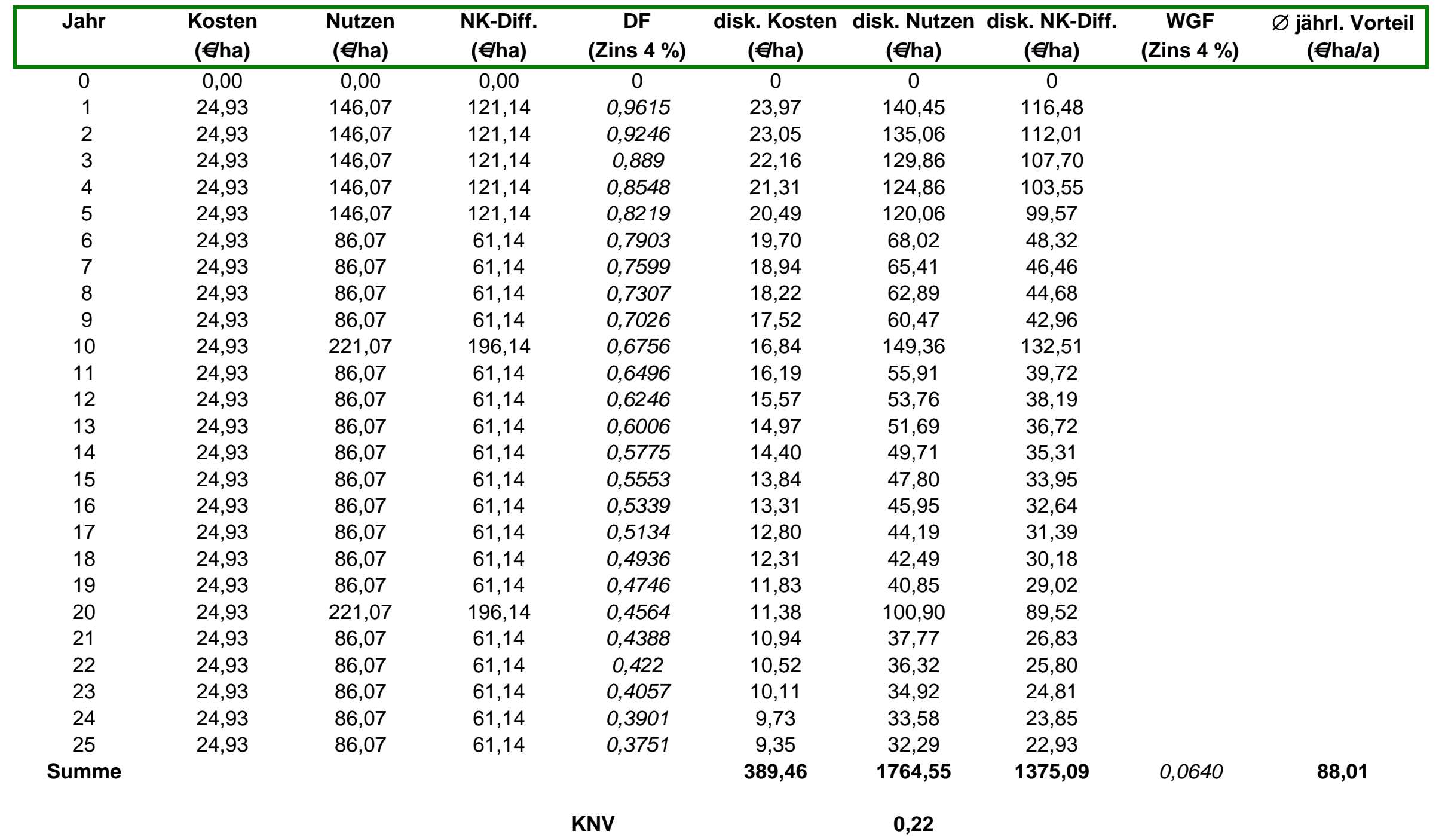


Tabelle A-52: Direktsaat Standort mit 8,7t/ha, Effizienz $98 \%$ (verh. BA 8,5 t/ha/a), inkl. Förderung $60 € /$ ha/a über fünf Jahre

\begin{tabular}{|c|c|c|c|c|c|c|c|c|c|}
\hline Jahr & $\begin{array}{c}\text { Kosten } \\
\text { (€/ha) }\end{array}$ & $\begin{array}{c}\text { Nutzen } \\
\text { (€/ha) }\end{array}$ & $\begin{array}{c}\text { NK-Diff. } \\
\text { (€/ha) }\end{array}$ & $\begin{array}{c}\text { DF } \\
\text { (Zins } 4 \%)\end{array}$ & $\begin{array}{c}\text { disk. Kosten } \\
\text { (€/ha) }\end{array}$ & $\begin{array}{c}\text { disk. Nutzen } \\
\text { (€/ha) }\end{array}$ & $\begin{array}{c}\text { disk. NK-Diff. } \\
(€ / h a)\end{array}$ & $\begin{array}{c}\text { WGF } \\
\text { (Zins } 4 \% \text { ) }\end{array}$ & $\begin{array}{c}\varnothing \text { jährl. Vorteil } \\
\text { (€/ha/a) }\end{array}$ \\
\hline 0 & 0,00 & 0,00 & 0,00 & 0,00 & 0,00 & 0,00 & 0,00 & & \\
\hline 1 & 29,45 & 185,40 & 155,95 & 0,9615 & 28,32 & 178,26 & 149,95 & & \\
\hline 2 & 29,45 & 185,40 & 155,95 & 0,9246 & 27,23 & 171,42 & 144,19 & & \\
\hline 3 & 29,45 & 185,40 & 155,95 & 0,889 & 26,18 & 164,82 & 138,64 & & \\
\hline 4 & 29,45 & 185,40 & 155,95 & 0,8548 & 25,17 & 158,48 & 133,31 & & \\
\hline 5 & 29,45 & 185,40 & 155,95 & 0,8219 & 24,20 & 152,38 & 128,18 & & \\
\hline 6 & 29,45 & 125,40 & 95,95 & 0,7903 & 23,27 & 99,10 & 75,83 & & \\
\hline 7 & 29,45 & 125,40 & 95,95 & 0,7599 & 22,38 & 95,29 & 72,91 & & \\
\hline 8 & 29,45 & 125,40 & 95,95 & 0,7307 & 21,52 & 91,63 & 70,11 & & \\
\hline 9 & 29,45 & 125,40 & 95,95 & 0,7026 & 20,69 & 88,11 & 67,41 & & \\
\hline 10 & 29,45 & 260,40 & 230,95 & 0,6756 & 19,90 & 175,93 & 156,03 & & \\
\hline 11 & 29,45 & 125,40 & 95,95 & 0,6496 & 19,13 & 81,46 & 62,33 & & \\
\hline 12 & 29,45 & 125,40 & 95,95 & 0,6246 & 18,39 & 78,32 & 59,93 & & \\
\hline 13 & 29,45 & 125,40 & 95,95 & 0,6006 & 17,69 & 75,32 & 57,63 & & \\
\hline 14 & 29,45 & 125,40 & 95,95 & 0,5775 & 17,01 & 72,42 & 55,41 & & \\
\hline 15 & 29,45 & 125,40 & 95,95 & 0,5553 & 16,35 & 69,63 & 53,28 & & \\
\hline 16 & 29,45 & 125,40 & 95,95 & 0,5339 & 15,72 & 66,95 & 51,23 & & \\
\hline 17 & 29,45 & 125,40 & 95,95 & 0,5134 & 15,12 & 64,38 & 49,26 & & \\
\hline 18 & 29,45 & 125,40 & 95,95 & 0,4936 & 14,54 & 61,90 & 47,36 & & \\
\hline 19 & 29,45 & 125,40 & 95,95 & 0,4746 & 13,98 & 59,51 & 45,54 & & \\
\hline 20 & 29,45 & 260,40 & 230,95 & 0,4564 & 13,44 & 118,85 & 105,41 & & \\
\hline 21 & 29,45 & 125,40 & 95,95 & 0,4388 & 12,92 & 55,03 & 42,10 & & \\
\hline 22 & 29,45 & 125,40 & 95,95 & 0,422 & 12,43 & 52,92 & 40,49 & & \\
\hline 23 & 29,45 & 125,40 & 95,95 & 0,4057 & 11,95 & 50,87 & 38,93 & & \\
\hline 24 & 29,45 & 125,40 & 95,95 & 0,3901 & 11,49 & 48,92 & 37,43 & & \\
\hline \multirow[t]{2}{*}{25} & 29,45 & 125,40 & 95,95 & 0,3751 & 11,05 & 47,04 & 35,99 & & \\
\hline & & & & & 460,07 & 2378,94 & 1918,87 & 0,0640 & 122,81 \\
\hline
\end{tabular}


Tabelle A-53: Breitreifen, WR-WW-WG mit einer Bodenschadverdichtungsverminderung und einer Wirksamkeit von $5 \%$

\begin{tabular}{|c|c|c|c|c|c|c|c|c|c|}
\hline Jahr & $\begin{array}{c}\text { Kosten } \\
\text { (€/ha) }\end{array}$ & $\begin{array}{c}\text { Nutzen } \\
\text { (€/ha) }\end{array}$ & $\begin{array}{c}\text { NK-Diff. } \\
\text { (€/ha) }\end{array}$ & $\begin{array}{c}\text { DF } \\
\text { (Zins } 4 \%)\end{array}$ & $\begin{array}{c}\text { disk. Kosten } \\
\text { (€/ha) }\end{array}$ & $\begin{array}{c}\text { disk. Nutzen } \\
(€ / h a)\end{array}$ & $\begin{array}{c}\text { disk. NK-Diff. } \\
(€ / h a)\end{array}$ & $\begin{array}{c}\text { WGF } \\
\text { (Zins } 4 \% \text { ) }\end{array}$ & $\begin{array}{c}\varnothing \text { jährl. Vorteil } \\
\text { (€/ha/a) }\end{array}$ \\
\hline 0 & 0,00 & 0,00 & 0,00 & 0,00 & 0,00 & 0,00 & 0,00 & & \\
\hline 1 & 8,00 & 26,47 & 18,47 & 0,9615 & 7,69 & 25,45 & 17,76 & & \\
\hline 2 & 8,00 & 19,93 & 11,93 & 0,9246 & 7,40 & 18,43 & 11,03 & & \\
\hline 3 & 8,00 & 19,49 & 11,49 & 0,889 & 7,11 & 17,32 & 10,21 & & \\
\hline 4 & 8,00 & 19,92 & 11,92 & 0,8548 & 6,84 & 17,03 & 10,19 & & \\
\hline 5 & 8,00 & 12,67 & 4,67 & 0,8219 & 6,58 & 10,41 & 3,84 & & \\
\hline 6 & 8,00 & 12,67 & 4,67 & 0,7903 & 6,32 & 10,01 & 3,69 & & \\
\hline 7 & 8,00 & 12,67 & 4,67 & 0,7599 & 6,08 & 9,63 & 3,55 & & \\
\hline 8 & 8,00 & 12,67 & 4,67 & 0,7307 & 5,85 & 9,26 & 3,41 & & \\
\hline 9 & 8,00 & 12,67 & 4,67 & 0,7026 & 5,62 & 8,90 & 3,28 & & \\
\hline 10 & 8,00 & 12,67 & 4,67 & 0,6756 & 5,40 & 8,56 & 3,16 & & \\
\hline 11 & 8,00 & 12,67 & 4,67 & 0,6496 & 5,20 & 8,23 & 3,03 & & \\
\hline 12 & 8,00 & 12,67 & 4,67 & 0,6246 & 5,00 & 7,91 & 2,92 & & \\
\hline 13 & 8,00 & 12,67 & 4,67 & 0,6006 & 4,80 & 7,61 & 2,80 & & \\
\hline 14 & 8,00 & 12,67 & 4,67 & 0,5775 & 4,62 & 7,32 & 2,70 & & \\
\hline 15 & 8,00 & 12,67 & 4,67 & 0,5553 & 4,44 & 7,04 & 2,59 & & \\
\hline 16 & 8,00 & 12,67 & 4,67 & 0,5339 & 4,27 & 6,76 & 2,49 & & \\
\hline 17 & 8,00 & 12,67 & 4,67 & 0,5134 & 4,11 & 6,50 & 2,40 & & \\
\hline 18 & 8,00 & 12,67 & 4,67 & 0,4936 & 3,95 & 6,25 & 2,31 & & \\
\hline 19 & 8,00 & 12,67 & 4,67 & 0,4746 & 3,80 & 6,01 & 2,22 & & \\
\hline 20 & 8,00 & 12,67 & 4,67 & 0,4564 & 3,65 & 5,78 & 2,13 & & \\
\hline 21 & 8,00 & 12,67 & 4,67 & 0,4388 & 3,51 & 5,56 & 2,05 & & \\
\hline 22 & 8,00 & 12,67 & 4,67 & 0,422 & 3,38 & 5,35 & 1,97 & & \\
\hline 23 & 8,00 & 12,67 & 4,67 & 0,4057 & 3,25 & 5,14 & 1,89 & & \\
\hline 24 & 8,00 & 12,67 & 4,67 & 0,3901 & 3,12 & 4,94 & 1,82 & & \\
\hline \multirow[t]{3}{*}{25} & 8,00 & 12,67 & 4,67 & 0,3751 & 3,00 & 4,75 & 1,75 & & \\
\hline & & & & & 124,98 & 230,18 & 105,20 & 0,0640 & 6,73 \\
\hline & & & & KNV & & 0,54 & & & \\
\hline
\end{tabular}


Tabelle A-54: Breitreifen, WR-WW-WG mit einer Bodenschadverdichtungsverminderung und einer Wirksamkeit von $25 \%$

\begin{tabular}{|c|c|c|c|c|c|c|c|c|c|}
\hline Jahr & $\begin{array}{l}\text { Kosten } \\
\text { (€/ha) }\end{array}$ & $\begin{array}{l}\text { Nutzen } \\
\text { (€/ha) }\end{array}$ & $\begin{array}{c}\text { NK-Diff. } \\
\text { (€/ha) }\end{array}$ & $\begin{array}{c}\text { DF } \\
\text { (Zins } 4 \%)\end{array}$ & $\begin{array}{c}\text { disk. Kosten } \\
\text { (€/ha) }\end{array}$ & $\begin{array}{c}\text { disk. Nutzen } \\
\text { (€/ha) }\end{array}$ & $\begin{array}{c}\text { disk. NK-Diff. } \\
(€ / h a)\end{array}$ & $\begin{array}{c}\text { WGF } \\
\text { (Zins } 4 \% \text { ) }\end{array}$ & $\begin{array}{c}\varnothing \text { jährl. Vor- } \\
\text { teil } \\
\text { (€/ha/a) }\end{array}$ \\
\hline 0 & 0,00 & 0,00 & 0,00 & 0,00 & 0,00 & 0,00 & 0,00 & & \\
\hline 1 & 8,00 & 78,52 & 70,52 & 0,9615 & 7,69 & 75,49 & 67,80 & & \\
\hline 2 & 8,00 & 45,82 & 37,82 & 0,9246 & 7,40 & 42,36 & 34,96 & & \\
\hline 3 & 8,00 & 43,59 & 35,59 & 0,889 & 7,11 & 38,75 & 31,64 & & \\
\hline 4 & 8,00 & 45,77 & 37,77 & 0,8548 & 6,84 & 39,12 & 32,28 & & \\
\hline 5 & 8,00 & 12,67 & 4,67 & 0,8219 & 6,58 & 10,41 & 3,84 & & \\
\hline 6 & 8,00 & 12,67 & 4,67 & 0,7903 & 6,32 & 10,01 & 3,69 & & \\
\hline 7 & 8,00 & 12,67 & 4,67 & 0,7599 & 6,08 & 9,63 & 3,55 & & \\
\hline 8 & 8,00 & 12,67 & 4,67 & 0,7307 & 5,85 & 9,26 & 3,41 & & \\
\hline 9 & 8,00 & 12,67 & 4,67 & 0,7026 & 5,62 & 8,90 & 3,28 & & \\
\hline 10 & 8,00 & 12,67 & 4,67 & 0,6756 & 5,40 & 8,56 & 3,16 & & \\
\hline 11 & 8,00 & 12,67 & 4,67 & 0,6496 & 5,20 & 8,23 & 3,03 & & \\
\hline 12 & 8,00 & 12,67 & 4,67 & 0,6246 & 5,00 & 7,91 & 2,92 & & \\
\hline 13 & 8,00 & 12,67 & 4,67 & 0,6006 & 4,80 & 7,61 & 2,80 & & \\
\hline 14 & 8,00 & 12,67 & 4,67 & 0,5775 & 4,62 & 7,32 & 2,70 & & \\
\hline 15 & 8,00 & 12,67 & 4,67 & 0,5553 & 4,44 & 7,04 & 2,59 & & \\
\hline 16 & 8,00 & 12,67 & 4,67 & 0,5339 & 4,27 & 6,76 & 2,49 & & \\
\hline 17 & 8,00 & 12,67 & 4,67 & 0,5134 & 4,11 & 6,50 & 2,40 & & \\
\hline 18 & 8,00 & 12,67 & 4,67 & 0,4936 & 3,95 & 6,25 & 2,31 & & \\
\hline 19 & 8,00 & 12,67 & 4,67 & 0,4746 & 3,80 & 6,01 & 2,22 & & \\
\hline 20 & 8,00 & 12,67 & 4,67 & 0,4564 & 3,65 & 5,78 & 2,13 & & \\
\hline 21 & 8,00 & 12,67 & 4,67 & 0,4388 & 3,51 & 5,56 & 2,05 & & \\
\hline 22 & 8,00 & 12,67 & 4,67 & 0,422 & 3,38 & 5,35 & 1,97 & & \\
\hline 23 & 8,00 & 12,67 & 4,67 & 0,4057 & 3,25 & 5,14 & 1,89 & & \\
\hline 24 & 8,00 & 12,67 & 4,67 & 0,3901 & 3,12 & 4,94 & 1,82 & & \\
\hline \multirow[t]{3}{*}{25} & 8,00 & 12,67 & 4,67 & 0,3751 & 3,00 & 4,75 & 1,75 & & \\
\hline & & & & & 124,98 & 347,67 & 222,69 & 0,0640 & 14,25 \\
\hline & & & & KNV & & 0,36 & & & \\
\hline
\end{tabular}


Tabelle A-55: Terrareifen, WR-WW-WG mit einer Bodenschadverdichtungsverminderung und einer Wirksamkeit von 5 \%

\begin{tabular}{|c|c|c|c|c|c|c|c|c|c|}
\hline Jahr & $\begin{array}{c}\text { Kosten } \\
(€ / \text { ha) }\end{array}$ & $\begin{array}{c}\text { Nutzen } \\
(€ / \text { ha) }\end{array}$ & $\begin{array}{l}\text { NK-Diff. } \\
\text { (€/ha) }\end{array}$ & $\begin{array}{c}\text { DF } \\
\text { (Zins } 4 \% \text { ) }\end{array}$ & $\begin{array}{c}\text { disk. Kosten } \\
(€ / \mathrm{ha})\end{array}$ & $\begin{array}{c}\text { disk. Nutzen } \\
(€ / \text { ha) }\end{array}$ & $\begin{array}{c}\text { disk. NK-Diff. } \\
(€ / \mathrm{ha})\end{array}$ & $\begin{array}{c}\text { WGF } \\
\text { (Zins } 4 \% \text { ) }\end{array}$ & $\begin{array}{c}\varnothing \text { jährl. Vorteil } \\
(€ / \text { ha/a) }\end{array}$ \\
\hline 0 & 0,00 & 0,00 & 0,00 & 0,00 & 0,00 & 0,00 & 0,00 & & \\
\hline 1 & 17,00 & 25,84 & 8,84 & 0,9615 & 16,35 & 24,84 & 8,50 & & \\
\hline 2 & 17,00 & 19,30 & 2,30 & 0,9246 & 15,72 & 17,84 & 2,13 & & \\
\hline 3 & 17,00 & 18,85 & 1,85 & 0,889 & 15,11 & 16,76 & 1,65 & & \\
\hline 4 & 17,00 & 19,29 & 2,29 & 0,8548 & 14,53 & 16,49 & 1,96 & & \\
\hline 5 & 17,00 & 12,67 & $-4,33$ & 0,8219 & 13,97 & 10,41 & $-3,56$ & & \\
\hline 6 & 17,00 & 12,67 & $-4,33$ & 0,7903 & 13,44 & 10,01 & $-3,42$ & & \\
\hline 7 & 17,00 & 12,67 & $-4,33$ & 0,7599 & 12,92 & 9,63 & $-3,29$ & & \\
\hline 8 & 17,00 & 12,67 & $-4,33$ & 0,7307 & 12,42 & 9,26 & $-3,16$ & & \\
\hline 9 & 17,00 & 12,67 & $-4,33$ & 0,7026 & 11,94 & 8,90 & $-3,04$ & & \\
\hline 10 & 17,00 & 12,67 & $-4,33$ & 0,6756 & 11,49 & 8,56 & $-2,93$ & & \\
\hline 11 & 17,00 & 12,67 & $-4,33$ & 0,6496 & 11,04 & 8,23 & $-2,81$ & & \\
\hline 12 & 17,00 & 12,67 & $-4,33$ & 0,6246 & 10,62 & 7,91 & $-2,70$ & & \\
\hline 13 & 17,00 & 12,67 & $-4,33$ & 0,6006 & 10,21 & 7,61 & $-2,60$ & & \\
\hline 14 & 17,00 & 12,67 & $-4,33$ & 0,5775 & 9,82 & 7,32 & $-2,50$ & & \\
\hline 15 & 17,00 & 12,67 & $-4,33$ & 0,5553 & 9,44 & 7,04 & $-2,40$ & & \\
\hline 16 & 17,00 & 12,67 & $-4,33$ & 0,5339 & 9,08 & 6,76 & $-2,31$ & & \\
\hline 17 & 17,00 & 12,67 & $-4,33$ & 0,5134 & 8,73 & 6,50 & $-2,22$ & & \\
\hline 18 & 17,00 & 12,67 & $-4,33$ & 0,4936 & 8,39 & 6,25 & $-2,14$ & & \\
\hline 19 & 17,00 & 12,67 & $-4,33$ & 0,4746 & 8,07 & 6,01 & $-2,06$ & & \\
\hline 20 & 17,00 & 12,67 & $-4,33$ & 0,4564 & 7,76 & 5,78 & $-1,98$ & & \\
\hline 21 & 17,00 & 12,67 & $-4,33$ & 0,4388 & 7,46 & 5,56 & $-1,90$ & & \\
\hline 22 & 17,00 & 12,67 & $-4,33$ & 0,422 & 7,17 & 5,35 & $-1,83$ & & \\
\hline 23 & 17,00 & 12,67 & $-4,33$ & 0,4057 & 6,90 & 5,14 & $-1,76$ & & \\
\hline 24 & 17,00 & 12,67 & $-4,33$ & 0,3901 & 6,63 & 4,94 & $-1,69$ & & \\
\hline \multirow[t]{2}{*}{25} & 17,00 & 12,67 & $-4,33$ & 0,3751 & 6,38 & 4,75 & $-1,62$ & & \\
\hline & & & & & 265,58 & 227,88 & $-37,70$ & 0,0640 & $-2,41$ \\
\hline
\end{tabular}


Tabelle A-56: Terrareifen, WR-WW-WG mit einer Bodenschadverdichtungsverminderung und einer Wirksamkeit von 50 \%

\begin{tabular}{|c|c|c|c|c|c|c|c|c|c|}
\hline Jahr & $\begin{array}{l}\text { Kosten } \\
\text { (€/ha) }\end{array}$ & $\begin{array}{c}\text { Nutzen } \\
\text { (€/ha) }\end{array}$ & $\begin{array}{c}\text { NK-Diff. } \\
\text { (€/ha) }\end{array}$ & $\begin{array}{c}\text { DF } \\
\text { (Zins } 4 \%)\end{array}$ & $\begin{array}{c}\text { disk. Kosten } \\
\text { (€/ha) }\end{array}$ & $\begin{array}{c}\text { disk. Nutzen } \\
\text { (€/ha) }\end{array}$ & $\begin{array}{l}\text { disk. NK-Diff. } \\
(€ / h a)\end{array}$ & $\begin{array}{c}\text { WGF } \\
\text { (Zins } 4 \% \text { ) }\end{array}$ & $\begin{array}{c}\varnothing \text { jährl. Vorteil } \\
(€ / h a / a)\end{array}$ \\
\hline 0 & 0,00 & 0,00 & 0,00 & 0,00 & 0,00 & 0,00 & 0,00 & & \\
\hline 1 & 17,00 & 144,36 & 127,36 & 0,9615 & 16,35 & 138,80 & 122,46 & & \\
\hline 2 & 17,00 & 78,96 & 61,96 & 0,9246 & 15,72 & 73,01 & 57,29 & & \\
\hline 3 & 17,00 & 74,51 & 57,51 & 0,889 & 15,11 & 66,24 & 51,13 & & \\
\hline 4 & 17,00 & 78,86 & 61,86 & 0,8548 & 14,53 & 67,41 & 52,88 & & \\
\hline 5 & 17,00 & 12,67 & $-4,33$ & 0,8219 & 13,97 & 10,41 & $-3,56$ & & \\
\hline 6 & 17,00 & 12,67 & $-4,33$ & 0,7903 & 13,44 & 10,01 & $-3,42$ & & \\
\hline 7 & 17,00 & 12,67 & $-4,33$ & 0,7599 & 12,92 & 9,63 & $-3,29$ & & \\
\hline 8 & 17,00 & 12,67 & $-4,33$ & 0,7307 & 12,42 & 9,26 & $-3,16$ & & \\
\hline 9 & 17,00 & 12,67 & $-4,33$ & 0,7026 & 11,94 & 8,90 & $-3,04$ & & \\
\hline 10 & 17,00 & 12,67 & $-4,33$ & 0,6756 & 11,49 & 8,56 & $-2,93$ & & \\
\hline 11 & 17,00 & 12,67 & $-4,33$ & 0,6496 & 11,04 & 8,23 & $-2,81$ & & \\
\hline 12 & 17,00 & 12,67 & $-4,33$ & 0,6246 & 10,62 & 7,91 & $-2,70$ & & \\
\hline 13 & 17,00 & 12,67 & $-4,33$ & 0,6006 & 10,21 & 7,61 & $-2,60$ & & \\
\hline 14 & 17,00 & 12,67 & $-4,33$ & 0,5775 & 9,82 & 7,32 & $-2,50$ & & \\
\hline 15 & 17,00 & 12,67 & $-4,33$ & 0,5553 & 9,44 & 7,04 & $-2,40$ & & \\
\hline 16 & 17,00 & 12,67 & $-4,33$ & 0,5339 & 9,08 & 6,76 & $-2,31$ & & \\
\hline 17 & 17,00 & 12,67 & $-4,33$ & 0,5134 & 8,73 & 6,50 & $-2,22$ & & \\
\hline 18 & 17,00 & 12,67 & $-4,33$ & 0,4936 & 8,39 & 6,25 & $-2,14$ & & \\
\hline 19 & 17,00 & 12,67 & $-4,33$ & 0,4746 & 8,07 & 6,01 & $-2,06$ & & \\
\hline 20 & 17,00 & 12,67 & $-4,33$ & 0,4564 & 7,76 & 5,78 & $-1,98$ & & \\
\hline 21 & 17,00 & 12,67 & $-4,33$ & 0,4388 & 7,46 & 5,56 & $-1,90$ & & \\
\hline 22 & 17,00 & 12,67 & $-4,33$ & 0,422 & 7,17 & 5,35 & $-1,83$ & & \\
\hline 23 & 17,00 & 12,67 & $-4,33$ & 0,4057 & 6,90 & 5,14 & $-1,76$ & & \\
\hline 24 & 17,00 & 12,67 & $-4,33$ & 0,3901 & 6,63 & 4,94 & $-1,69$ & & \\
\hline \multirow[t]{3}{*}{25} & 17,00 & 12,67 & $-4,33$ & 0,3751 & 6,38 & 4,75 & $-1,62$ & & \\
\hline & & & & & 265,58 & 497,40 & 231,82 & 0,0640 & 14,84 \\
\hline & & & & KNV & & 0,53 & & & \\
\hline
\end{tabular}


Tabelle A-57: Breitreifen, ZR-WW-WG mit einer Bodenschadverdichtungsverminderung und einer Wirksamkeit von $5 \%$

\begin{tabular}{|c|c|c|c|c|c|c|c|c|c|}
\hline Jahr & $\begin{array}{c}\text { Kosten } \\
(€ / \text { ha) }\end{array}$ & $\begin{array}{c}\text { Nutzen } \\
\text { (€/ha) }\end{array}$ & $\begin{array}{l}\text { NK-Diff. } \\
\text { (€/ha) }\end{array}$ & $\begin{array}{c}\text { DF } \\
\text { (Zins } 4 \% \text { ) }\end{array}$ & $\begin{array}{c}\text { disk. Kosten } \\
(€ / \mathrm{ha})\end{array}$ & $\begin{array}{c}\text { disk. Nutzen } \\
(€ / \mathrm{ha})\end{array}$ & $\begin{array}{c}\text { disk. NK-Diff. } \\
(€ / \text { ha) }\end{array}$ & $\begin{array}{c}\text { WGF } \\
\text { (Zins } 4 \% \text { ) }\end{array}$ & $\begin{array}{c}\varnothing \text { jährl. Vorteil } \\
(€ / \text { ha/a) }\end{array}$ \\
\hline 0 & 0,00 & 0,00 & 0,00 & 0,00 & 0,00 & 0,00 & 0,00 & & \\
\hline 1 & 8,00 & 64,19 & 56,19 & 0,9615 & 7,69 & 61,71 & 54,02 & & \\
\hline 2 & 8,00 & 25,03 & 17,03 & 0,9246 & 7,40 & 23,14 & 15,75 & & \\
\hline 3 & 8,00 & 23,70 & 15,70 & 0,889 & 7,11 & 21,06 & 13,95 & & \\
\hline 4 & 8,00 & 38,59 & 30,59 & 0,8548 & 6,84 & 32,98 & 26,15 & & \\
\hline 5 & 8,00 & 13,53 & 5,53 & 0,8219 & 6,58 & 11,12 & 4,55 & & \\
\hline 6 & 8,00 & 13,53 & 5,53 & 0,7903 & 6,32 & 10,69 & 4,37 & & \\
\hline 7 & 8,00 & 13,53 & 5,53 & 0,7599 & 6,08 & 10,28 & 4,20 & & \\
\hline 8 & 8,00 & 13,53 & 5,53 & 0,7307 & 5,85 & 9,89 & 4,04 & & \\
\hline 9 & 8,00 & 13,53 & 5,53 & 0,7026 & 5,62 & 9,51 & 3,89 & & \\
\hline 10 & 8,00 & 13,53 & 5,53 & 0,6756 & 5,40 & 9,14 & 3,74 & & \\
\hline 11 & 8,00 & 13,53 & 5,53 & 0,6496 & 5,20 & 8,79 & 3,59 & & \\
\hline 12 & 8,00 & 13,53 & 5,53 & 0,6246 & 5,00 & 8,45 & 3,45 & & \\
\hline 13 & 8,00 & 13,53 & 5,53 & 0,6006 & 4,80 & 8,13 & 3,32 & & \\
\hline 14 & 8,00 & 13,53 & 5,53 & 0,5775 & 4,62 & 7,81 & 3,19 & & \\
\hline 15 & 8,00 & 13,53 & 5,53 & 0,5553 & 4,44 & 7,51 & 3,07 & & \\
\hline 16 & 8,00 & 13,53 & 5,53 & 0,5339 & 4,27 & 7,22 & 2,95 & & \\
\hline 17 & 8,00 & 13,53 & 5,53 & 0,5134 & 4,11 & 6,95 & 2,84 & & \\
\hline 18 & 8,00 & 13,53 & 5,53 & 0,4936 & 3,95 & 6,68 & 2,73 & & \\
\hline 19 & 8,00 & 13,53 & 5,53 & 0,4746 & 3,80 & 6,42 & 2,62 & & \\
\hline 20 & 8,00 & 13,53 & 5,53 & 0,4564 & 3,65 & 6,18 & 2,52 & & \\
\hline 21 & 8,00 & 13,53 & 5,53 & 0,4388 & 3,51 & 5,94 & 2,43 & & \\
\hline 22 & 8,00 & 13,53 & 5,53 & 0,422 & 3,38 & 5,71 & 2,33 & & \\
\hline 23 & 8,00 & 13,53 & 5,53 & 0,4057 & 3,25 & 5,49 & 2,24 & & \\
\hline 24 & 8,00 & 13,53 & 5,53 & 0,3901 & 3,12 & 5,28 & 2,16 & & \\
\hline \multirow[t]{2}{*}{25} & 8,00 & 13,53 & 5,53 & 0,3751 & 3,00 & 5,08 & 2,07 & & \\
\hline & & & & & 124,98 & 301,16 & 176,18 & 0,0640 & 11,28 \\
\hline
\end{tabular}


Tabelle A-58: Breitreifen, ZR-WW-WG mit einer Bodenschadverdichtungsverminderung und einer Wirksamkeit von 25 \%

\begin{tabular}{|c|c|c|c|c|c|c|c|c|c|}
\hline Jahr & $\begin{array}{l}\text { Kosten } \\
\text { (€/ha) }\end{array}$ & $\begin{array}{l}\text { Nutzen } \\
\text { (€/ha) }\end{array}$ & $\begin{array}{l}\text { NK-Diff. } \\
\text { (€/ha) }\end{array}$ & $\begin{array}{c}\text { DF } \\
\text { (Zins } 4 \%)\end{array}$ & $\begin{array}{c}\text { disk. Kosten } \\
\text { (€/ha) }\end{array}$ & $\begin{array}{c}\text { disk. Nutzen } \\
\text { (€/ha) }\end{array}$ & $\begin{array}{l}\text { disk. NK-Diff. } \\
(€ / h a)\end{array}$ & $\begin{array}{c}\text { WGF } \\
\text { (Zins } 4 \% \text { ) }\end{array}$ & $\begin{array}{c}\varnothing \text { jährl. Vorteil } \\
(€ / h a / a)\end{array}$ \\
\hline 0 & 0,00 & 0,00 & 0,00 & 0,00 & 0,00 & 0,00 & 0,00 & & \\
\hline 1 & 8,00 & 266,81 & 258,81 & 0,9615 & 7,69 & 256,53 & 248,84 & & \\
\hline 2 & 8,00 & 71,03 & 63,03 & 0,9246 & 7,40 & 65,67 & 58,28 & & \\
\hline 3 & 8,00 & 64,36 & 56,36 & 0,889 & 7,11 & 57,21 & 50,10 & & \\
\hline 4 & 8,00 & 135,43 & 127,43 & 0,8548 & 6,84 & 115,77 & 108,93 & & \\
\hline 5 & 8,00 & 13,53 & 5,53 & 0,8219 & 6,58 & 11,12 & 4,55 & & \\
\hline 6 & 8,00 & 13,53 & 5,53 & 0,7903 & 6,32 & 10,69 & 4,37 & & \\
\hline 7 & 8,00 & 13,53 & 5,53 & 0,7599 & 6,08 & 10,28 & 4,20 & & \\
\hline 8 & 8,00 & 13,53 & 5,53 & 0,7307 & 5,85 & 9,89 & 4,04 & & \\
\hline 9 & 8,00 & 13,53 & 5,53 & 0,7026 & 5,62 & 9,51 & 3,89 & & \\
\hline 10 & 8,00 & 13,53 & 5,53 & 0,6756 & 5,40 & 9,14 & 3,74 & & \\
\hline 11 & 8,00 & 13,53 & 5,53 & 0,6496 & 5,20 & 8,79 & 3,59 & & \\
\hline 12 & 8,00 & 13,53 & 5,53 & 0,6246 & 5,00 & 8,45 & 3,45 & & \\
\hline 13 & 8,00 & 13,53 & 5,53 & 0,6006 & 4,80 & 8,13 & 3,32 & & \\
\hline 14 & 8,00 & 13,53 & 5,53 & 0,5775 & 4,62 & 7,81 & 3,19 & & \\
\hline 15 & 8,00 & 13,53 & 5,53 & 0,5553 & 4,44 & 7,51 & 3,07 & & \\
\hline 16 & 8,00 & 13,53 & 5,53 & 0,5339 & 4,27 & 7,22 & 2,95 & & \\
\hline 17 & 8,00 & 13,53 & 5,53 & 0,5134 & 4,11 & 6,95 & 2,84 & & \\
\hline 18 & 8,00 & 13,53 & 5,53 & 0,4936 & 3,95 & 6,68 & 2,73 & & \\
\hline 19 & 8,00 & 13,53 & 5,53 & 0,4746 & 3,80 & 6,42 & 2,62 & & \\
\hline 20 & 8,00 & 13,53 & 5,53 & 0,4564 & 3,65 & 6,18 & 2,52 & & \\
\hline 21 & 8,00 & 13,53 & 5,53 & 0,4388 & 3,51 & 5,94 & 2,43 & & \\
\hline 22 & 8,00 & 13,53 & 5,53 & 0,422 & 3,38 & 5,71 & 2,33 & & \\
\hline 23 & 8,00 & 13,53 & 5,53 & 0,4057 & 3,25 & 5,49 & 2,24 & & \\
\hline 24 & 8,00 & 13,53 & 5,53 & 0,3901 & 3,12 & 5,28 & 2,16 & & \\
\hline \multirow[t]{2}{*}{25} & 8,00 & 13,53 & 5,53 & 0,3751 & 3,00 & 5,08 & 2,07 & & \\
\hline & & & & & 124,98 & 657,44 & 532,46 & 0,0640 & 34,08 \\
\hline
\end{tabular}


Tabelle A-59: Terrareifen, ZR-WW-WG mit einer Bodenschadverdichtungsverminderung und einer Wirksamkeit von $5 \%$

\begin{tabular}{|c|c|c|c|c|c|c|c|c|c|}
\hline Jahr & $\begin{array}{l}\text { Kosten } \\
\text { (€/ha) }\end{array}$ & $\begin{array}{c}\text { Nutzen } \\
\text { (€/ha) }\end{array}$ & $\begin{array}{c}\text { NK-Diff. } \\
\text { (€/ha) }\end{array}$ & $\begin{array}{c}\text { DF } \\
\text { (Zins } 4 \% \text { ) }\end{array}$ & $\begin{array}{c}\text { disk. Kosten } \\
\text { (€/ha) }\end{array}$ & $\begin{array}{c}\text { disk. Nutzen } \\
\text { (€/ha) }\end{array}$ & $\begin{array}{l}\text { disk. NK-Diff. } \\
(€ / h a)\end{array}$ & $\begin{array}{c}\text { WGF } \\
\text { (Zins } 4 \% \text { ) }\end{array}$ & $\begin{array}{c}\varnothing \text { jährl. Vorteil } \\
\text { (€/ha/a) }\end{array}$ \\
\hline 0 & 0,00 & 0,00 & 0,00 & 0,00 & 0,00 & 0,00 & 0,00 & & \\
\hline 1 & 17,00 & 64,19 & 47,19 & 0,9615 & 16,35 & 61,71 & 45,37 & & \\
\hline 2 & 17,00 & 25,03 & 8,03 & 0,9246 & 15,72 & 23,14 & 7,42 & & \\
\hline 3 & 17,00 & 23,70 & 6,70 & 0,889 & 15,11 & 21,06 & 5,95 & & \\
\hline 4 & 17,00 & 37,91 & 20,91 & 0,8548 & 14,53 & 32,41 & 17,87 & & \\
\hline 5 & 17,00 & 13,53 & $-3,47$ & 0,8219 & 13,97 & 11,12 & $-2,85$ & & \\
\hline 6 & 17,00 & 13,53 & $-3,47$ & 0,7903 & 13,44 & 10,69 & $-2,74$ & & \\
\hline 7 & 17,00 & 13,53 & $-3,47$ & 0,7599 & 12,92 & 10,28 & $-2,64$ & & \\
\hline 8 & 17,00 & 13,53 & $-3,47$ & 0,7307 & 12,42 & 9,89 & $-2,54$ & & \\
\hline 9 & 17,00 & 13,53 & $-3,47$ & 0,7026 & 11,94 & 9,51 & $-2,44$ & & \\
\hline 10 & 17,00 & 13,53 & $-3,47$ & 0,6756 & 11,49 & 9,14 & $-2,34$ & & \\
\hline 11 & 17,00 & 13,53 & $-3,47$ & 0,6496 & 11,04 & 8,79 & $-2,25$ & & \\
\hline 12 & 17,00 & 13,53 & $-3,47$ & 0,6246 & 10,62 & 8,45 & $-2,17$ & & \\
\hline 13 & 17,00 & 13,53 & $-3,47$ & 0,6006 & 10,21 & 8,13 & $-2,08$ & & \\
\hline 14 & 17,00 & 13,53 & $-3,47$ & 0,5775 & 9,82 & 7,81 & $-2,00$ & & \\
\hline 15 & 17,00 & 13,53 & $-3,47$ & 0,5553 & 9,44 & 7,51 & $-1,93$ & & \\
\hline 16 & 17,00 & 13,53 & $-3,47$ & 0,5339 & 9,08 & 7,22 & $-1,85$ & & \\
\hline 17 & 17,00 & 13,53 & $-3,47$ & 0,5134 & 8,73 & 6,95 & $-1,78$ & & \\
\hline 18 & 17,00 & 13,53 & $-3,47$ & 0,4936 & 8,39 & 6,68 & $-1,71$ & & \\
\hline 19 & 17,00 & 13,53 & $-3,47$ & 0,4746 & 8,07 & 6,42 & $-1,65$ & & \\
\hline 20 & 17,00 & 13,53 & $-3,47$ & 0,4564 & 7,76 & 6,18 & $-1,58$ & & \\
\hline 21 & 17,00 & 13,53 & $-3,47$ & 0,4388 & 7,46 & 5,94 & $-1,52$ & & \\
\hline 22 & 17,00 & 13,53 & $-3,47$ & 0,422 & 7,17 & 5,71 & $-1,46$ & & \\
\hline 23 & 17,00 & 13,53 & $-3,47$ & 0,4057 & 6,90 & 5,49 & $-1,41$ & & \\
\hline 24 & 17,00 & 13,53 & $-3,47$ & 0,3901 & 6,63 & 5,28 & $-1,35$ & & \\
\hline \multirow[t]{3}{*}{25} & 17,00 & 13,53 & $-3,47$ & 0,3751 & 6,38 & 5,08 & $-1,30$ & & \\
\hline & & & & & 265,58 & 300,58 & 35,01 & 0,0640 & 2,24 \\
\hline & & & & KNV & & 0,88 & & & \\
\hline
\end{tabular}


Tabelle A-60: Terrareifen, ZR-WW-WG mit einer Bodenschadverdichtungsverminderung und einer Wirksamkeit von 50 \%

\begin{tabular}{|c|c|c|c|c|c|c|c|c|c|}
\hline Jahr & $\begin{array}{l}\text { Kosten } \\
\text { (€/ha) }\end{array}$ & $\begin{array}{c}\text { Nutzen } \\
\text { (€/ha) }\end{array}$ & $\begin{array}{c}\text { NK-Diff. } \\
\text { (€/ha) }\end{array}$ & $\begin{array}{c}\text { DF } \\
\text { (Zins } 4 \%)\end{array}$ & $\begin{array}{c}\text { disk. Kosten } \\
\text { (€/ha) }\end{array}$ & $\begin{array}{c}\text { disk. Nutzen } \\
\text { (€/ha) }\end{array}$ & $\begin{array}{l}\text { disk. NK-Diff. } \\
(€ / h a)\end{array}$ & $\begin{array}{c}\text { WGF } \\
\text { (Zins } 4 \% \text { ) }\end{array}$ & $\begin{array}{c}\varnothing \text { jährl. Vorteil } \\
(€ / h a / a)\end{array}$ \\
\hline 0 & 0,00 & 0,00 & 0,00 & 0,00 & 0,00 & 0,00 & 0,00 & & \\
\hline 1 & 17,00 & 520,08 & 503,08 & 0,9615 & 16,35 & 500,06 & 483,71 & & \\
\hline 2 & 17,00 & 128,53 & 111,53 & 0,9246 & 15,72 & 118,84 & 103,12 & & \\
\hline 3 & 17,00 & 115,18 & 98,18 & 0,889 & 15,11 & 102,40 & 87,28 & & \\
\hline 4 & 17,00 & 257,33 & 240,33 & 0,8548 & 14,53 & 219,97 & 205,43 & & \\
\hline 5 & 17,00 & 13,53 & $-3,47$ & 0,8219 & 13,97 & 11,12 & $-2,85$ & & \\
\hline 6 & 17,00 & 13,53 & $-3,47$ & 0,7903 & 13,44 & 10,69 & $-2,74$ & & \\
\hline 7 & 17,00 & 13,53 & $-3,47$ & 0,7599 & 12,92 & 10,28 & $-2,64$ & & \\
\hline 8 & 17,00 & 13,53 & $-3,47$ & 0,7307 & 12,42 & 9,89 & $-2,54$ & & \\
\hline 9 & 17,00 & 13,53 & $-3,47$ & 0,7026 & 11,94 & 9,51 & $-2,44$ & & \\
\hline 10 & 17,00 & 13,53 & $-3,47$ & 0,6756 & 11,49 & 9,14 & $-2,34$ & & \\
\hline 11 & 17,00 & 13,53 & $-3,47$ & 0,6496 & 11,04 & 8,79 & $-2,25$ & & \\
\hline 12 & 17,00 & 13,53 & $-3,47$ & 0,6246 & 10,62 & 8,45 & $-2,17$ & & \\
\hline 13 & 17,00 & 13,53 & $-3,47$ & 0,6006 & 10,21 & 8,13 & $-2,08$ & & \\
\hline 14 & 17,00 & 13,53 & $-3,47$ & 0,5775 & 9,82 & 7,81 & $-2,00$ & & \\
\hline 15 & 17,00 & 13,53 & $-3,47$ & 0,5553 & 9,44 & 7,51 & $-1,93$ & & \\
\hline 16 & 17,00 & 13,53 & $-3,47$ & 0,5339 & 9,08 & 7,22 & $-1,85$ & & \\
\hline 17 & 17,00 & 13,53 & $-3,47$ & 0,5134 & 8,73 & 6,95 & $-1,78$ & & \\
\hline 18 & 17,00 & 13,53 & $-3,47$ & 0,4936 & 8,39 & 6,68 & $-1,71$ & & \\
\hline 19 & 17,00 & 13,53 & $-3,47$ & 0,4746 & 8,07 & 6,42 & $-1,65$ & & \\
\hline 20 & 17,00 & 13,53 & $-3,47$ & 0,4564 & 7,76 & 6,18 & $-1,58$ & & \\
\hline 21 & 17,00 & 13,53 & $-3,47$ & 0,4388 & 7,46 & 5,94 & $-1,52$ & & \\
\hline 22 & 17,00 & 13,53 & $-3,47$ & 0,422 & 7,17 & 5,71 & $-1,46$ & & \\
\hline 23 & 17,00 & 13,53 & $-3,47$ & 0,4057 & 6,90 & 5,49 & $-1,41$ & & \\
\hline 24 & 17,00 & 13,53 & $-3,47$ & 0,3901 & 6,63 & 5,28 & $-1,35$ & & \\
\hline \multirow[t]{3}{*}{25} & 17,00 & 13,53 & $-3,47$ & 0,3751 & 6,38 & 5,08 & $-1,30$ & & \\
\hline & & & & & 265,58 & 1103,51 & 837,94 & 0,0640 & 53,63 \\
\hline & & & & KNV & & 0,24 & & & \\
\hline
\end{tabular}


Tabelle A-61: Einsatz von Reifendruckregelanlagen, Variante 1, Wirksamkeit $5 \%$

\begin{tabular}{|c|c|c|c|c|c|c|c|c|c|}
\hline Jahr & $\begin{array}{c}\text { Kosten } \\
\text { (€/ha) }\end{array}$ & $\begin{array}{l}\text { Nutzen } \\
\text { (€/ha) }\end{array}$ & $\begin{array}{c}\text { NK-Diff. } \\
\text { (€/ha) }\end{array}$ & $\begin{array}{c}\text { DF } \\
\text { (Zins } 4 \% \text { ) }\end{array}$ & $\begin{array}{c}\text { disk. Kosten } \\
(€ / \mathrm{ha})\end{array}$ & $\begin{array}{c}\text { disk. Nutzen } \\
\text { (€/ha) }\end{array}$ & $\begin{array}{c}\text { disk. NK-Diff. } \\
(€ / h a)\end{array}$ & $\begin{array}{c}\text { WGF } \\
\text { (Zins } 4 \% \text { ) }\end{array}$ & $\begin{array}{c}\varnothing \text { jährl. Vorteil } \\
(€ / h a / a)\end{array}$ \\
\hline 0 & 0,00 & 0,00 & 0,00 & 0,00 & 0,00 & 0,00 & 0,00 & & \\
\hline 1 & 6,02 & 19,75 & 13,73 & 0,9615 & 5,79 & 18,99 & 13,20 & & \\
\hline 2 & 6,02 & 22,03 & 16,01 & 0,9246 & 5,57 & 20,37 & 14,81 & & \\
\hline 3 & 6,02 & 21,59 & 15,57 & 0,889 & 5,35 & 19,19 & 13,84 & & \\
\hline 4 & 6,02 & 22,02 & 16,00 & 0,8548 & 5,15 & 18,82 & 13,68 & & \\
\hline 5 & 6,02 & 5,67 & $-0,35$ & 0,8219 & 4,95 & 4,66 & $-0,29$ & & \\
\hline 6 & 6,02 & 5,67 & $-0,35$ & 0,7903 & 4,76 & 4,48 & $-0,28$ & & \\
\hline 7 & 6,02 & 5,67 & $-0,35$ & 0,7599 & 4,57 & 4,31 & $-0,27$ & & \\
\hline 8 & 6,02 & 5,67 & $-0,35$ & 0,7307 & 4,40 & 4,14 & $-0,26$ & & \\
\hline 9 & 6,02 & 5,67 & $-0,35$ & 0,7026 & 4,23 & 3,98 & $-0,25$ & & \\
\hline 10 & 6,02 & 5,67 & $-0,35$ & 0,6756 & 4,07 & 3,83 & $-0,24$ & & \\
\hline 11 & 6,02 & 5,67 & $-0,35$ & 0,6496 & 3,91 & 3,68 & $-0,23$ & & \\
\hline 12 & 6,02 & 5,67 & $-0,35$ & 0,6246 & 3,76 & 3,54 & $-0,22$ & & \\
\hline 13 & 6,02 & 5,67 & $-0,35$ & 0,6006 & 3,62 & 3,41 & $-0,21$ & & \\
\hline 14 & 6,02 & 5,67 & $-0,35$ & 0,5775 & 3,48 & 3,27 & $-0,20$ & & \\
\hline 15 & 6,02 & 5,67 & $-0,35$ & 0,5553 & 3,34 & 3,15 & $-0,19$ & & \\
\hline 16 & 6,02 & 5,67 & $-0,35$ & 0,5339 & 3,21 & 3,03 & $-0,19$ & & \\
\hline 17 & 6,02 & 5,67 & $-0,35$ & 0,5134 & 3,09 & 2,91 & $-0,18$ & & \\
\hline 18 & 6,02 & 5,67 & $-0,35$ & 0,4936 & 2,97 & 2,80 & $-0,17$ & & \\
\hline 19 & 6,02 & 5,67 & $-0,35$ & 0,4746 & 2,86 & 2,69 & $-0,17$ & & \\
\hline 20 & 6,02 & 5,67 & $-0,35$ & 0,4564 & 2,75 & 2,59 & $-0,16$ & & \\
\hline 21 & 6,02 & 5,67 & $-0,35$ & 0,4388 & 2,64 & 2,49 & $-0,15$ & & \\
\hline 22 & 6,02 & 5,67 & $-0,35$ & 0,422 & 2,54 & 2,39 & $-0,15$ & & \\
\hline 23 & 6,02 & 5,67 & $-0,35$ & 0,4057 & 2,44 & 2,30 & $-0,14$ & & \\
\hline 24 & 6,02 & 5,67 & $-0,35$ & 0,3901 & 2,35 & 2,21 & $-0,14$ & & \\
\hline \multirow[t]{3}{*}{25} & 6,02 & 5,67 & $-0,35$ & 0,3751 & 2,26 & 2,13 & $-0,13$ & & \\
\hline & & & & & 94,05 & 145,37 & 51,33 & 0,0640 & 3,28 \\
\hline & & & & KNV & & 0,65 & & & \\
\hline
\end{tabular}


Tabelle A-62: Einsatz von Reifendruckregelanlagen, Variante 1, Wirksamkeit $50 \%$

\begin{tabular}{|c|c|c|c|c|c|c|c|c|c|}
\hline Jahr & $\begin{array}{c}\text { Kosten } \\
\text { (€/ha) }\end{array}$ & $\begin{array}{l}\text { Nutzen } \\
\text { (€/ha) }\end{array}$ & $\begin{array}{c}\text { NK-Diff. } \\
\text { (€/ha) }\end{array}$ & $\begin{array}{c}\text { DF } \\
\text { (Zins } 4 \%) \\
\end{array}$ & $\begin{array}{c}\text { disk. Kosten } \\
(€ / \mathrm{ha}) \\
\end{array}$ & $\begin{array}{c}\text { disk. Nutzen } \\
\text { (€/ha) }\end{array}$ & $\begin{array}{c}\text { disk. NK-Diff. } \\
(€ / h a)\end{array}$ & $\begin{array}{c}\text { WGF } \\
\text { (Zins } 4 \% \text { ) }\end{array}$ & $\begin{array}{c}\varnothing \text { jährl. Vorteil } \\
\text { (€/ha/a) }\end{array}$ \\
\hline 0 & 0,00 & 0,00 & 0,00 & 0,00 & 0,00 & 0,00 & 0,00 & & \\
\hline 1 & 6,02 & 138,27 & 132,25 & 0,9615 & 5,79 & 132,95 & 127,16 & & \\
\hline 2 & 6,02 & 87,40 & 81,38 & 0,9246 & 5,57 & 80,81 & 75,24 & & \\
\hline 3 & 6,02 & 82,95 & 76,93 & 0,889 & 5,35 & 73,74 & 68,39 & & \\
\hline 4 & 6,02 & 87,30 & 81,28 & 0,8548 & 5,15 & 74,62 & 69,47 & & \\
\hline 5 & 6,02 & 5,67 & $-0,35$ & 0,8219 & 4,95 & 4,66 & $-0,29$ & & \\
\hline 6 & 6,02 & 5,67 & $-0,35$ & 0,7903 & 4,76 & 4,48 & $-0,28$ & & \\
\hline 7 & 6,02 & 5,67 & $-0,35$ & 0,7599 & 4,57 & 4,31 & $-0,27$ & & \\
\hline 8 & 6,02 & 5,67 & $-0,35$ & 0,7307 & 4,40 & 4,14 & $-0,26$ & & \\
\hline 9 & 6,02 & 5,67 & $-0,35$ & 0,7026 & 4,23 & 3,98 & $-0,25$ & & \\
\hline 10 & 6,02 & 5,67 & $-0,35$ & 0,6756 & 4,07 & 3,83 & $-0,24$ & & \\
\hline 11 & 6,02 & 5,67 & $-0,35$ & 0,6496 & 3,91 & 3,68 & $-0,23$ & & \\
\hline 12 & 6,02 & 5,67 & $-0,35$ & 0,6246 & 3,76 & 3,54 & $-0,22$ & & \\
\hline 13 & 6,02 & 5,67 & $-0,35$ & 0,6006 & 3,62 & 3,41 & $-0,21$ & & \\
\hline 14 & 6,02 & 5,67 & $-0,35$ & 0,5775 & 3,48 & 3,27 & $-0,20$ & & \\
\hline 15 & 6,02 & 5,67 & $-0,35$ & 0,5553 & 3,34 & 3,15 & $-0,19$ & & \\
\hline 16 & 6,02 & 5,67 & $-0,35$ & 0,5339 & 3,21 & 3,03 & $-0,19$ & & \\
\hline 17 & 6,02 & 5,67 & $-0,35$ & 0,5134 & 3,09 & 2,91 & $-0,18$ & & \\
\hline 18 & 6,02 & 5,67 & $-0,35$ & 0,4936 & 2,97 & 2,80 & $-0,17$ & & \\
\hline 19 & 6,02 & 5,67 & $-0,35$ & 0,4746 & 2,86 & 2,69 & $-0,17$ & & \\
\hline 20 & 6,02 & 5,67 & $-0,35$ & 0,4564 & 2,75 & 2,59 & $-0,16$ & & \\
\hline 21 & 6,02 & 5,67 & $-0,35$ & 0,4388 & 2,64 & 2,49 & $-0,15$ & & \\
\hline 22 & 6,02 & 5,67 & $-0,35$ & 0,422 & 2,54 & 2,39 & $-0,15$ & & \\
\hline 23 & 6,02 & 5,67 & $-0,35$ & 0,4057 & 2,44 & 2,30 & $-0,14$ & & \\
\hline 24 & 6,02 & 5,67 & $-0,35$ & 0,3901 & 2,35 & 2,21 & $-0,14$ & & \\
\hline \multirow[t]{2}{*}{25} & 6,02 & 5,67 & $-0,35$ & 0,3751 & 2,26 & 2,13 & $-0,13$ & & \\
\hline & & & & & 94,05 & 430,11 & 336,06 & 0,0640 & 21,51 \\
\hline
\end{tabular}


Tabelle A-63: Einsatz von Reifendruckregelanlagen, Variante 2, Wirksamkeit $5 \%$

\begin{tabular}{|c|c|c|c|c|c|c|c|c|c|}
\hline Jahr & $\begin{array}{c}\text { Kosten } \\
\text { (€/ha) }\end{array}$ & $\begin{array}{l}\text { Nutzen } \\
\text { (€/ha) }\end{array}$ & $\begin{array}{c}\text { NK-Diff. } \\
\text { (€/ha) }\end{array}$ & $\begin{array}{c}\text { DF } \\
\text { (Zins } 4 \% \text { ) }\end{array}$ & $\begin{array}{c}\text { disk. Kosten } \\
(€ / \text { ha) }\end{array}$ & $\begin{array}{l}\text { disk. Nutzen } \\
(€ / h a)\end{array}$ & $\begin{array}{c}\text { disk. NK-Diff. } \\
(€ / h a)\end{array}$ & $\begin{array}{c}\text { WGF } \\
\text { (Zins } 4 \% \text { ) }\end{array}$ & $\begin{array}{c}\varnothing \text { jährl. Vorteil } \\
(€ / h / a)\end{array}$ \\
\hline 0 & 0,00 & 0,00 & 0,00 & 0,00 & 0,00 & 0,00 & 0,00 & & \\
\hline 1 & 6,02 & 57,24 & 51,22 & 0,9615 & 5,79 & 55,03 & 49,24 & & \\
\hline 2 & 6,02 & 26,90 & 20,88 & 0,9246 & 5,57 & 24,87 & 19,31 & & \\
\hline 3 & 6,02 & 25,57 & 19,55 & 0,889 & 5,35 & 22,73 & 17,38 & & \\
\hline 4 & 6,02 & 39,78 & 33,76 & 0,8548 & 5,15 & 34,01 & 28,86 & & \\
\hline 5 & 6,02 & 5,67 & $-0,35$ & 0,8219 & 4,95 & 4,66 & $-0,29$ & & \\
\hline 6 & 6,02 & 5,67 & $-0,35$ & 0,7903 & 4,76 & 4,48 & $-0,28$ & & \\
\hline 7 & 6,02 & 5,67 & $-0,35$ & 0,7599 & 4,57 & 4,31 & $-0,27$ & & \\
\hline 8 & 6,02 & 5,67 & $-0,35$ & 0,7307 & 4,40 & 4,14 & $-0,26$ & & \\
\hline 9 & 6,02 & 5,67 & $-0,35$ & 0,7026 & 4,23 & 3,98 & $-0,25$ & & \\
\hline 10 & 6,02 & 5,67 & $-0,35$ & 0,6756 & 4,07 & 3,83 & $-0,24$ & & \\
\hline 11 & 6,02 & 5,67 & $-0,35$ & 0,6496 & 3,91 & 3,68 & $-0,23$ & & \\
\hline 12 & 6,02 & 5,67 & $-0,35$ & 0,6246 & 3,76 & 3,54 & $-0,22$ & & \\
\hline 13 & 6,02 & 5,67 & $-0,35$ & 0,6006 & 3,62 & 3,41 & $-0,21$ & & \\
\hline 14 & 6,02 & 5,67 & $-0,35$ & 0,5775 & 3,48 & 3,27 & $-0,20$ & & \\
\hline 15 & 6,02 & 5,67 & $-0,35$ & 0,5553 & 3,34 & 3,15 & $-0,19$ & & \\
\hline 16 & 6,02 & 5,67 & $-0,35$ & 0,5339 & 3,21 & 3,03 & $-0,19$ & & \\
\hline 17 & 6,02 & 5,67 & $-0,35$ & 0,5134 & 3,09 & 2,91 & $-0,18$ & & \\
\hline 18 & 6,02 & 5,67 & $-0,35$ & 0,4936 & 2,97 & 2,80 & $-0,17$ & & \\
\hline 19 & 6,02 & 5,67 & $-0,35$ & 0,4746 & 2,86 & 2,69 & $-0,17$ & & \\
\hline 20 & 6,02 & 5,67 & $-0,35$ & 0,4564 & 2,75 & 2,59 & $-0,16$ & & \\
\hline 21 & 6,02 & 5,67 & $-0,35$ & 0,4388 & 2,64 & 2,49 & $-0,15$ & & \\
\hline 22 & 6,02 & 5,67 & $-0,35$ & 0,422 & 2,54 & 2,39 & $-0,15$ & & \\
\hline 23 & 6,02 & 5,67 & $-0,35$ & 0,4057 & 2,44 & 2,30 & $-0,14$ & & \\
\hline 24 & 6,02 & 5,67 & $-0,35$ & 0,3901 & 2,35 & 2,21 & $-0,14$ & & \\
\hline \multirow[t]{3}{*}{25} & 6,02 & 5,67 & $-0,35$ & 0,3751 & 2,26 & 2,13 & $-0,13$ & & \\
\hline & & & & & 94,05 & 204,64 & 110,59 & 0,0640 & 7,08 \\
\hline & & & & KNV & & 0,46 & & & \\
\hline
\end{tabular}


Tabelle A-64: Einsatz von Reifendruckregelanlagen, Variante 2, Wirksamkeit 50 \%

\begin{tabular}{|c|c|c|c|c|c|c|c|c|c|}
\hline Jahr & $\begin{array}{c}\text { Kosten } \\
\text { (€/ha) }\end{array}$ & $\begin{array}{c}\text { Nutzen } \\
\text { (€/ha) }\end{array}$ & $\begin{array}{c}\text { NK-Diff. } \\
\text { (€/ha) }\end{array}$ & $\begin{array}{c}\text { DF } \\
\text { (Zins } 4 \% \text { ) }\end{array}$ & $\begin{array}{c}\text { disk. Kosten } \\
\text { (€/ha) }\end{array}$ & $\begin{array}{c}\text { disk. Nutzen } \\
\text { (€/ha) }\end{array}$ & $\begin{array}{c}\text { disk. NK-Diff. } \\
(€ / \mathrm{ha})\end{array}$ & $\begin{array}{c}\text { WGF } \\
\text { (Zins } 4 \% \text { ) }\end{array}$ & $\begin{array}{c}\varnothing \text { jährl. Vorteil } \\
(€ / \text { ha/a) }\end{array}$ \\
\hline 0 & 0,00 & 0,00 & 0,00 & 0,00 & 0,00 & 0,00 & 0,00 & & \\
\hline 1 & 6,02 & 250,38 & 244,36 & 0,9615 & 5,79 & 240,74 & 234,95 & & \\
\hline 2 & 6,02 & 136,11 & 130,09 & 0,9246 & 5,57 & 125,84 & 120,28 & & \\
\hline 3 & 6,02 & 122,76 & 116,74 & 0,889 & 5,35 & 109,13 & 103,78 & & \\
\hline 4 & 6,02 & 264,91 & 258,89 & 0,8548 & 5,15 & 226,44 & 221,29 & & \\
\hline 5 & 6,02 & 5,67 & $-0,35$ & 0,8219 & 4,95 & 4,66 & $-0,29$ & & \\
\hline 6 & 6,02 & 5,67 & $-0,35$ & 0,7903 & 4,76 & 4,48 & $-0,28$ & & \\
\hline 7 & 6,02 & 5,67 & $-0,35$ & 0,7599 & 4,57 & 4,31 & $-0,27$ & & \\
\hline 8 & 6,02 & 5,67 & $-0,35$ & 0,7307 & 4,40 & 4,14 & $-0,26$ & & \\
\hline 9 & 6,02 & 5,67 & $-0,35$ & 0,7026 & 4,23 & 3,98 & $-0,25$ & & \\
\hline 10 & 6,02 & 5,67 & $-0,35$ & 0,6756 & 4,07 & 3,83 & $-0,24$ & & \\
\hline 11 & 6,02 & 5,67 & $-0,35$ & 0,6496 & 3,91 & 3,68 & $-0,23$ & & \\
\hline 12 & 6,02 & 5,67 & $-0,35$ & 0,6246 & 3,76 & 3,54 & $-0,22$ & & \\
\hline 13 & 6,02 & 5,67 & $-0,35$ & 0,6006 & 3,62 & 3,41 & $-0,21$ & & \\
\hline 14 & 6,02 & 5,67 & $-0,35$ & 0,5775 & 3,48 & 3,27 & $-0,20$ & & \\
\hline 15 & 6,02 & 5,67 & $-0,35$ & 0,5553 & 3,34 & 3,15 & $-0,19$ & & \\
\hline 16 & 6,02 & 5,67 & $-0,35$ & 0,5339 & 3,21 & 3,03 & $-0,19$ & & \\
\hline 17 & 6,02 & 5,67 & $-0,35$ & 0,5134 & 3,09 & 2,91 & $-0,18$ & & \\
\hline 18 & 6,02 & 5,67 & $-0,35$ & 0,4936 & 2,97 & 2,80 & $-0,17$ & & \\
\hline 19 & 6,02 & 5,67 & $-0,35$ & 0,4746 & 2,86 & 2,69 & $-0,17$ & & \\
\hline 20 & 6,02 & 5,67 & $-0,35$ & 0,4564 & 2,75 & 2,59 & $-0,16$ & & \\
\hline 21 & 6,02 & 5,67 & $-0,35$ & 0,4388 & 2,64 & 2,49 & $-0,15$ & & \\
\hline 22 & 6,02 & 5,67 & $-0,35$ & 0,422 & 2,54 & 2,39 & $-0,15$ & & \\
\hline 23 & 6,02 & 5,67 & $-0,35$ & 0,4057 & 2,44 & 2,30 & $-0,14$ & & \\
\hline 24 & 6,02 & 5,67 & $-0,35$ & 0,3901 & 2,35 & 2,21 & $-0,14$ & & \\
\hline \multirow[t]{3}{*}{25} & 6,02 & 5,67 & $-0,35$ & 0,3751 & 2,26 & 2,13 & $-0,13$ & & \\
\hline & & & & & 94,05 & 770,15 & 676,10 & 0,0640 & 43,27 \\
\hline & & & & KNV & & 0,12 & & & \\
\hline
\end{tabular}


Tabelle A-65: Einsatz von Reifendruckregelanlagen, Variante 3, 0 \% Wirkung

\begin{tabular}{|c|c|c|c|c|c|c|c|c|c|}
\hline Jahr & $\begin{array}{c}\text { Kosten } \\
\text { (€/ha) }\end{array}$ & $\begin{array}{c}\text { Nutzen } \\
\text { (€/ha) }\end{array}$ & $\begin{array}{c}\text { NK-Diff. } \\
\text { (€/ha) }\end{array}$ & $\begin{array}{c}\text { DF } \\
\text { (Zins } 4 \%)\end{array}$ & $\begin{array}{c}\text { disk. Kosten } \\
\text { (€/ha) }\end{array}$ & $\begin{array}{c}\text { disk. Nutzen } \\
(€ / h a)\end{array}$ & $\begin{array}{c}\text { disk. NK-Diff. } \\
(€ / h a)\end{array}$ & $\begin{array}{c}\text { WGF } \\
\text { (Zins } 4 \% \text { ) }\end{array}$ & $\begin{array}{c}\varnothing \text { jährl. Vorteil } \\
\text { (€/ha/a) }\end{array}$ \\
\hline 0 & 0,00 & 0,00 & 0,00 & 0,00 & 0,00 & 0,00 & 0,00 & & \\
\hline 1 & 6,02 & 6,58 & 0,56 & 0,9615 & 5,79 & 6,33 & 0,54 & & \\
\hline 2 & 6,02 & 6,58 & 0,56 & 0,9246 & 5,57 & 6,08 & 0,52 & & \\
\hline 3 & 6,02 & 6,58 & 0,56 & 0,889 & 5,35 & 5,85 & 0,50 & & \\
\hline 4 & 6,02 & 6,58 & 0,56 & 0,8548 & 5,15 & 5,62 & 0,48 & & \\
\hline 5 & 6,02 & 6,58 & 0,56 & 0,8219 & 4,95 & 5,41 & 0,46 & & \\
\hline 6 & 6,02 & 6,58 & 0,56 & 0,7903 & 4,76 & 5,20 & 0,44 & & \\
\hline 7 & 6,02 & 6,58 & 0,56 & 0,7599 & 4,57 & 5,00 & 0,43 & & \\
\hline 8 & 6,02 & 6,58 & 0,56 & 0,7307 & 4,40 & 4,81 & 0,41 & & \\
\hline 9 & 6,02 & 6,58 & 0,56 & 0,7026 & 4,23 & 4,62 & 0,39 & & \\
\hline 10 & 6,02 & 6,58 & 0,56 & 0,6756 & 4,07 & 4,45 & 0,38 & & \\
\hline 11 & 6,02 & 6,58 & 0,56 & 0,6496 & 3,91 & 4,27 & 0,36 & & \\
\hline 12 & 6,02 & 6,58 & 0,56 & 0,6246 & 3,76 & 4,11 & 0,35 & & \\
\hline 13 & 6,02 & 6,58 & 0,56 & 0,6006 & 3,62 & 3,95 & 0,34 & & \\
\hline 14 & 6,02 & 6,58 & 0,56 & 0,5775 & 3,48 & 3,80 & 0,32 & & \\
\hline 15 & 6,02 & 6,58 & 0,56 & 0,5553 & 3,34 & 3,65 & 0,31 & & \\
\hline 16 & 6,02 & 6,58 & 0,56 & 0,5339 & 3,21 & 3,51 & 0,30 & & \\
\hline 17 & 6,02 & 6,58 & 0,56 & 0,5134 & 3,09 & 3,38 & 0,29 & & \\
\hline 18 & 6,02 & 6,58 & 0,56 & 0,4936 & 2,97 & 3,25 & 0,28 & & \\
\hline 19 & 6,02 & 6,58 & 0,56 & 0,4746 & 2,86 & 3,12 & 0,27 & & \\
\hline 20 & 6,02 & 6,58 & 0,56 & 0,4564 & 2,75 & 3,00 & 0,26 & & \\
\hline 21 & 6,02 & 6,58 & 0,56 & 0,4388 & 2,64 & 2,89 & 0,25 & & \\
\hline 22 & 6,02 & 6,58 & 0,56 & 0,422 & 2,54 & 2,78 & 0,24 & & \\
\hline 23 & 6,02 & 6,58 & 0,56 & 0,4057 & 2,44 & 2,67 & 0,23 & & \\
\hline 24 & 6,02 & 6,58 & 0,56 & 0,3901 & 2,35 & 2,57 & 0,22 & & \\
\hline \multirow[t]{3}{*}{25} & 6,02 & 6,58 & 0,56 & 0,3751 & 2,26 & 2,47 & 0,21 & & \\
\hline & & & & & 94,05 & 102,79 & 8,75 & 0,0640 & 0,56 \\
\hline & & & & KNV & & 0,91 & & & \\
\hline
\end{tabular}


Tabelle A-66: Stilllegung von Minderertragsteilflächen, Ertragsminderung 10 \%, ertragreicher Standort

\begin{tabular}{|c|c|c|c|c|c|c|c|c|c|}
\hline Jahr & $\begin{array}{c}\text { Kosten } \\
\text { (€/ha) }\end{array}$ & $\begin{array}{c}\text { Nutzen } \\
\text { (€/ha) }\end{array}$ & $\begin{array}{c}\text { NK-Diff. } \\
\text { (€/ha) }\end{array}$ & $\begin{array}{c}\text { DF } \\
\text { (Zins } 4 \% \text { ) }\end{array}$ & $\begin{array}{c}\text { disk. Kosten } \\
\text { (€/ha) }\end{array}$ & $\begin{array}{c}\text { disk. Nutzen } \\
(€ / \mathrm{ha})\end{array}$ & $\begin{array}{c}\text { disk. NK-Diff. } \\
(€ / \mathrm{ha})\end{array}$ & $\begin{array}{c}\text { WGF } \\
\text { (Zins } 4 \% \text { ) }\end{array}$ & $\begin{array}{c}\varnothing \text { jährl. Vorteil } \\
(€ / h a / a)\end{array}$ \\
\hline 0 & 0,00 & 0,00 & 0,00 & 0,00 & 0,00 & 0,00 & 0,00 & & \\
\hline 1 & 483,50 & 824,65 & 341,15 & 0,9615 & 464,89 & 792,90 & 328,02 & & \\
\hline 2 & 483,50 & 824,65 & 341,15 & 0,9246 & 447,04 & 762,47 & 315,43 & & \\
\hline 3 & 483,50 & 824,65 & 341,15 & 0,889 & 429,83 & 733,11 & 303,28 & & \\
\hline 4 & 483,50 & 824,65 & 341,15 & 0,8548 & 413,30 & 704,91 & 291,62 & & \\
\hline 5 & 483,50 & 824,65 & 341,15 & 0,8219 & 397,39 & 677,78 & 280,39 & & \\
\hline 6 & 483,50 & 824,65 & 341,15 & 0,7903 & 382,11 & 651,72 & 269,61 & & \\
\hline 7 & 483,50 & 824,65 & 341,15 & 0,7599 & 367,41 & 626,65 & 259,24 & & \\
\hline 8 & 483,50 & 824,65 & 341,15 & 0,7307 & 353,29 & 602,57 & 249,28 & & \\
\hline 9 & 483,50 & 824,65 & 341,15 & 0,7026 & 339,71 & 579,40 & 239,69 & & \\
\hline \multirow[t]{2}{*}{10} & 483,50 & 824,65 & 341,15 & 0,6756 & 326,65 & 557,13 & 230,48 & & \\
\hline & & & & & 3921,62 & 6688,65 & 2767,03 & 0,1233 & 341,18 \\
\hline
\end{tabular}


Tabelle A-67: Stilllegung von Minderertragsteilflächen, Ertragsminderung 10 \%, ertragsschwacher Standort

\begin{tabular}{|c|c|c|c|c|c|c|c|c|c|}
\hline Jahr & $\begin{array}{c}\text { Kosten } \\
\text { (€/ha) }\end{array}$ & $\begin{array}{l}\text { Nutzen } \\
\text { (€/ha) }\end{array}$ & $\begin{array}{l}\text { NK-Diff. } \\
\text { (€/ha) }\end{array}$ & $\begin{array}{c}\text { DF } \\
\text { (Zins } 4 \% \text { ) }\end{array}$ & $\begin{array}{c}\text { disk. Kosten } \\
\text { (€/ha) }\end{array}$ & $\begin{array}{l}\text { disk. Nutzen } \\
\text { (€/ha) }\end{array}$ & $\begin{array}{c}\text { disk. NK-Diff. } \\
(€ / h a)\end{array}$ & $\begin{array}{c}\text { WGF } \\
\text { (Zins } 4 \% \text { ) }\end{array}$ & $\begin{array}{c}\varnothing \text { jährl. Vorteil } \\
\text { (€/ha/a) }\end{array}$ \\
\hline 0 & 0,00 & 0,00 & 0,00 & 0,00 & 0,00 & 0,00 & 0,00 & & \\
\hline 1 & 303,50 & 625,65 & 322,15 & 0,9615 & 291,82 & 601,56 & 309,75 & & \\
\hline 2 & 303,50 & 625,65 & 322,15 & 0,9246 & 280,62 & 578,48 & 297,86 & & \\
\hline 3 & 303,50 & 625,65 & 322,15 & 0,889 & 269,81 & 556,20 & 286,39 & & \\
\hline 4 & 303,50 & 625,65 & 322,15 & 0,8548 & 259,43 & 534,81 & 275,37 & & \\
\hline 5 & 303,50 & 625,65 & 322,15 & 0,8219 & 249,45 & 514,22 & 264,78 & & \\
\hline 6 & 303,50 & 625,65 & 322,15 & 0,7903 & 239,86 & 494,45 & 254,60 & & \\
\hline 7 & 303,50 & 625,65 & 322,15 & 0,7599 & 230,63 & 475,43 & 244,80 & & \\
\hline 8 & 303,50 & 625,65 & 322,15 & 0,7307 & 221,77 & 457,16 & 235,40 & & \\
\hline 9 & 303,50 & 625,65 & 322,15 & 0,7026 & 213,24 & 439,58 & 226,34 & & \\
\hline \multirow[t]{3}{*}{10} & 303,50 & 625,65 & 322,15 & 0,6756 & 205,04 & 422,69 & 217,64 & & \\
\hline & & & & & 2461,66 & 5074,58 & 2612,93 & 0,1233 & 322,17 \\
\hline & & & & KNV & & 0,49 & & & \\
\hline
\end{tabular}


Tabelle A-68: GKRB-2; ZR-WW-WG (eine Verminderung von BSV)

\begin{tabular}{|c|c|c|c|c|c|c|c|c|c|}
\hline Jahr & $\begin{array}{c}\text { Kosten } \\
\text { (€/ha) }\end{array}$ & $\begin{array}{l}\text { Nutzen } \\
\text { (€/ha) }\end{array}$ & $\begin{array}{c}\text { NK-Diff. } \\
\text { (€/ha) }\end{array}$ & $\begin{array}{c}\text { DF } \\
\text { (Zins } 4 \%)\end{array}$ & $\begin{array}{c}\text { disk. Kosten } \\
\text { (€/ha) }\end{array}$ & $\begin{array}{c}\text { disk. Nutzen } \\
\text { (€/ha) }\end{array}$ & $\begin{array}{l}\text { disk. NK-Diff. } \\
(€ / h a)\end{array}$ & $\begin{array}{c}\text { WGF } \\
\text { (Zins } 4 \% \text { ) }\end{array}$ & $\begin{array}{c}\varnothing \text { jährl. Vorteil } \\
(€ / h a / a)\end{array}$ \\
\hline 0 & 0,00 & 0,00 & 0,00 & 0,00 & 0,00 & 0,00 & 0,00 & & \\
\hline 1 & 315,20 & 384,10 & 68,90 & 0,9615 & 303,06 & 369,31 & 66,25 & & \\
\hline 2 & 0,00 & 91,10 & 91,10 & 0,9246 & 0,00 & 84,23 & 84,23 & & \\
\hline 3 & 0,00 & 73,30 & 73,30 & 0,889 & 0,00 & 65,16 & 65,16 & & \\
\hline 4 & 315,20 & 263,00 & $-52,20$ & 0,8548 & 269,43 & 224,81 & $-44,62$ & & \\
\hline 5 & 0,00 & 0,00 & 0,00 & 0,8219 & 0,00 & 0,00 & 0,00 & & \\
\hline 6 & 0,00 & 0,00 & 0,00 & 0,7903 & 0,00 & 0,00 & 0,00 & & \\
\hline 7 & 315,20 & 0,00 & $-315,20$ & 0,7599 & 239,52 & 0,00 & $-239,52$ & & \\
\hline 8 & 0,00 & 0,00 & 0,00 & 0,7307 & 0,00 & 0,00 & 0,00 & & \\
\hline 9 & 0,00 & 0,00 & 0,00 & 0,7026 & 0,00 & 0,00 & 0,00 & & \\
\hline 10 & 315,20 & 0,00 & $-315,20$ & 0,6756 & 212,95 & 0,00 & $-212,95$ & & \\
\hline 11 & 0,00 & 0,00 & 0,00 & 0,6496 & 0,00 & 0,00 & 0,00 & & \\
\hline 12 & 0,00 & 0,00 & 0,00 & 0,6246 & 0,00 & 0,00 & 0,00 & & \\
\hline 13 & 315,20 & 0,00 & $-315,20$ & 0,6006 & 189,31 & 0,00 & $-189,31$ & & \\
\hline 14 & 0,00 & 0,00 & 0,00 & 0,5775 & 0,00 & 0,00 & 0,00 & & \\
\hline 15 & 0,00 & 0,00 & 0,00 & 0,5553 & 0,00 & 0,00 & 0,00 & & \\
\hline 16 & 315,20 & 0,00 & $-315,20$ & 0,5339 & 168,29 & 0,00 & $-168,29$ & & \\
\hline 17 & 0,00 & 0,00 & 0,00 & 0,5134 & 0,00 & 0,00 & 0,00 & & \\
\hline 18 & 0,00 & 0,00 & 0,00 & 0,4936 & 0,00 & 0,00 & 0,00 & & \\
\hline 19 & 315,20 & 0,00 & $-315,20$ & 0,4746 & 149,59 & 0,00 & $-149,59$ & & \\
\hline 20 & 0,00 & 0,00 & 0,00 & 0,4564 & 0,00 & 0,00 & 0,00 & & \\
\hline 21 & 0,00 & 0,00 & 0,00 & 0,4388 & 0,00 & 0,00 & 0,00 & & \\
\hline 22 & 315,20 & 0,00 & $-315,20$ & 0,422 & 133,01 & 0,00 & $-133,01$ & & \\
\hline 23 & 0,00 & 0,00 & 0,00 & 0,4057 & 0,00 & 0,00 & 0,00 & & \\
\hline 24 & 0,00 & 0,00 & 0,00 & 0,3901 & 0,00 & 0,00 & 0,00 & & \\
\hline \multirow[t]{3}{*}{25} & 315,20 & 0,00 & $-315,20$ & 0,3751 & 118,23 & 0,00 & $-118,23$ & & \\
\hline & & & & & 1783,40 & 743,52 & $-1039,88$ & 0,0640 & $-66,55$ \\
\hline & & & & KNV & & 2,40 & & & \\
\hline
\end{tabular}


Tabelle A-69: GKRB-2; ZR-WW-WG (zwei Verminderungen von BSV)

\begin{tabular}{|c|c|c|c|c|c|c|c|c|c|}
\hline Jahr & $\begin{array}{l}\text { Kosten } \\
\text { (€/ha) }\end{array}$ & $\begin{array}{l}\text { Nutzen } \\
\text { (€/ha) }\end{array}$ & $\begin{array}{l}\text { NK-Diff. } \\
\text { (€/ha) }\end{array}$ & $\begin{array}{c}\text { DF } \\
\text { (Zins } 4 \% \text { ) }\end{array}$ & $\begin{array}{c}\text { disk. Kosten } \\
\text { (€/ha) }\end{array}$ & $\begin{array}{c}\text { disk. Nutzen } \\
\text { (€/ha) }\end{array}$ & $\begin{array}{c}\text { disk. NK-Diff. } \\
(€ / \mathrm{ha})\end{array}$ & $\begin{array}{c}\text { WGF } \\
\text { (Zins } 4 \% \text { ) }\end{array}$ & $\begin{array}{c}\varnothing \text { jährl. Vorteil } \\
(€ / \mathrm{ha} / \mathrm{a})\end{array}$ \\
\hline 0 & 0,00 & 0,00 & 0,00 & 0,00 & 0,00 & 0,00 & 0,00 & & \\
\hline 1 & 315,20 & 384,10 & 68,90 & 0,9615 & 303,06 & 369,31 & 66,25 & & \\
\hline 2 & 0,00 & 91,10 & 91,10 & 0,9246 & 0,00 & 84,23 & 84,23 & & \\
\hline 3 & 0,00 & 73,30 & 73,30 & 0,889 & 0,00 & 65,16 & 65,16 & & \\
\hline 4 & 315,20 & 647,10 & 331,90 & 0,8548 & 269,43 & 553,14 & 283,71 & & \\
\hline 5 & 0,00 & 91,10 & 91,10 & 0,8219 & 0,00 & 74,88 & 74,88 & & \\
\hline 6 & 0,00 & 73,30 & 73,30 & 0,7903 & 0,00 & 57,93 & 57,93 & & \\
\hline 7 & 315,20 & 263,00 & $-52,20$ & 0,7599 & 239,52 & 199,85 & $-39,67$ & & \\
\hline 8 & 0,00 & 0,00 & 0,00 & 0,7307 & 0,00 & 0,00 & 0,00 & & \\
\hline 9 & 0,00 & 0,00 & 0,00 & 0,7026 & 0,00 & 0,00 & 0,00 & & \\
\hline 10 & 315,20 & 0,00 & $-315,20$ & 0,6756 & 212,95 & 0,00 & $-212,95$ & & \\
\hline 11 & 0,00 & 0,00 & 0,00 & 0,6496 & 0,00 & 0,00 & 0,00 & & \\
\hline 12 & 0,00 & 0,00 & 0,00 & 0,6246 & 0,00 & 0,00 & 0,00 & & \\
\hline 13 & 315,20 & 0,00 & $-315,20$ & 0,6006 & 189,31 & 0,00 & $-189,31$ & & \\
\hline 14 & 0,00 & 0,00 & 0,00 & 0,5775 & 0,00 & 0,00 & 0,00 & & \\
\hline 15 & 0,00 & 0,00 & 0,00 & 0,5553 & 0,00 & 0,00 & 0,00 & & \\
\hline 16 & 315,20 & 0,00 & $-315,20$ & 0,5339 & 168,29 & 0,00 & $-168,29$ & & \\
\hline 17 & 0,00 & 0,00 & 0,00 & 0,5134 & 0,00 & 0,00 & 0,00 & & \\
\hline 18 & 0,00 & 0,00 & 0,00 & 0,4936 & 0,00 & 0,00 & 0,00 & & \\
\hline 19 & 315,20 & 0,00 & $-315,20$ & 0,4746 & 149,59 & 0,00 & $-149,59$ & & \\
\hline 20 & 0,00 & 0,00 & 0,00 & 0,4564 & 0,00 & 0,00 & 0,00 & & \\
\hline 21 & 0,00 & 0,00 & 0,00 & 0,4388 & 0,00 & 0,00 & 0,00 & & \\
\hline 22 & 315,20 & 0,00 & $-315,20$ & 0,422 & 133,01 & 0,00 & $-133,01$ & & \\
\hline 23 & 0,00 & 0,00 & 0,00 & 0,4057 & 0,00 & 0,00 & 0,00 & & \\
\hline 24 & 0,00 & 0,00 & 0,00 & 0,3901 & 0,00 & 0,00 & 0,00 & & \\
\hline \multirow[t]{3}{*}{25} & 315,20 & 0,00 & $-315,20$ & 0,3751 & 118,23 & 0,00 & $-118,23$ & & \\
\hline & & & & & 1783,40 & 1404,51 & $-378,90$ & 0,0640 & $-24,25$ \\
\hline & & & & KNV & & 1,27 & & & \\
\hline
\end{tabular}


Tabelle A-70: GKRB-2; ZR-WW-WG (drei Verminderungen von BSV)

\begin{tabular}{|c|c|c|c|c|c|c|c|c|c|}
\hline Jahr & $\begin{array}{c}\text { Kosten } \\
\text { (€/ha) }\end{array}$ & $\begin{array}{c}\text { Nutzen } \\
\text { (€/ha) }\end{array}$ & $\begin{array}{c}\text { NK-Diff. } \\
\text { (€/ha) }\end{array}$ & $\begin{array}{c}\text { DF } \\
\text { (Zins } 4 \%) \\
\end{array}$ & $\begin{array}{c}\text { disk. Kosten } \\
\text { (€/ha) }\end{array}$ & $\begin{array}{c}\text { disk. Nutzen } \\
\text { (€/ha) }\end{array}$ & $\begin{array}{c}\text { disk. NK-Diff. } \\
(€ / h a)\end{array}$ & $\begin{array}{c}\text { WGF } \\
\text { (Zins } 4 \% \text { ) }\end{array}$ & $\begin{array}{c}\varnothing \text { jährl. Vorteil } \\
(€ / h a / a)\end{array}$ \\
\hline 0 & 0,00 & 0,00 & 0,00 & 0,00 & 0,00 & 0,00 & 0,00 & & \\
\hline 1 & 315,20 & 384,10 & 68,90 & 0,9615 & 303,06 & 369,31 & 66,25 & & \\
\hline 2 & 0,00 & 91,10 & 91,10 & 0,9246 & 0,00 & 84,23 & 84,23 & & \\
\hline 3 & 0,00 & 73,30 & 73,30 & 0,889 & 0,00 & 65,16 & 65,16 & & \\
\hline 4 & 315,20 & 647,10 & 331,90 & 0,8548 & 269,43 & 553,14 & 283,71 & & \\
\hline 5 & 0,00 & 91,10 & 91,10 & 0,8219 & 0,00 & 74,88 & 74,88 & & \\
\hline 6 & 0,00 & 73,30 & 73,30 & 0,7903 & 0,00 & 57,93 & 57,93 & & \\
\hline 7 & 315,20 & 647,10 & 331,90 & 0,7599 & 239,52 & 491,73 & 252,21 & & \\
\hline 8 & 0,00 & 91,10 & 91,10 & 0,7307 & 0,00 & 66,57 & 66,57 & & \\
\hline 9 & 0,00 & 73,30 & 73,30 & 0,7026 & 0,00 & 51,50 & 51,50 & & \\
\hline 10 & 315,20 & 263,00 & $-52,20$ & 0,6756 & 212,95 & 177,68 & $-35,27$ & & \\
\hline 11 & 0,00 & 0,00 & 0,00 & 0,6496 & 0,00 & 0,00 & 0,00 & & \\
\hline 12 & 0,00 & 0,00 & 0,00 & 0,6246 & 0,00 & 0,00 & 0,00 & & \\
\hline 13 & 315,20 & 0,00 & $-315,20$ & 0,6006 & 189,31 & 0,00 & $-189,31$ & & \\
\hline 14 & 0,00 & 0,00 & 0,00 & 0,5775 & 0,00 & 0,00 & 0,00 & & \\
\hline 15 & 0,00 & 0,00 & 0,00 & 0,5553 & 0,00 & 0,00 & 0,00 & & \\
\hline 16 & 315,20 & 0,00 & $-315,20$ & 0,5339 & 168,29 & 0,00 & $-168,29$ & & \\
\hline 17 & 0,00 & 0,00 & 0,00 & 0,5134 & 0,00 & 0,00 & 0,00 & & \\
\hline 18 & 0,00 & 0,00 & 0,00 & 0,4936 & 0,00 & 0,00 & 0,00 & & \\
\hline 19 & 315,20 & 0,00 & $-315,20$ & 0,4746 & 149,59 & 0,00 & $-149,59$ & & \\
\hline 20 & 0,00 & 0,00 & 0,00 & 0,4564 & 0,00 & 0,00 & 0,00 & & \\
\hline 21 & 0,00 & 0,00 & 0,00 & 0,4388 & 0,00 & 0,00 & 0,00 & & \\
\hline 22 & 315,20 & 0,00 & $-315,20$ & 0,422 & 133,01 & 0,00 & $-133,01$ & & \\
\hline 23 & 0,00 & 0,00 & 0,00 & 0,4057 & 0,00 & 0,00 & 0,00 & & \\
\hline 24 & 0,00 & 0,00 & 0,00 & 0,3901 & 0,00 & 0,00 & 0,00 & & \\
\hline \multirow[t]{2}{*}{25} & 315,20 & 0,00 & $-315,20$ & 0,3751 & 118,23 & 0,00 & $-118,23$ & & \\
\hline & & & & & 1783,40 & 1992,13 & 208,73 & 0,0640 & 13,36 \\
\hline
\end{tabular}


Tabelle A-71: Reduzierung des PSM-Einsatzes; Pflanzenschutz -35 \% und Ertrag -6 \%; Standort: 50 dt/ha

\begin{tabular}{|c|c|c|c|c|c|c|c|c|c|}
\hline Jahr & $\begin{array}{c}\text { Kosten } \\
\text { (€/ha) }\end{array}$ & $\begin{array}{c}\text { Nutzen } \\
\text { (€/ha) }\end{array}$ & $\begin{array}{c}\text { NK-Diff. } \\
(€ / \text { ha) }\end{array}$ & $\begin{array}{c}\text { DF } \\
\text { (Zins } 4 \% \text { ) }\end{array}$ & $\begin{array}{c}\text { disk. Kosten } \\
(€ / \mathrm{ha})\end{array}$ & $\begin{array}{c}\text { disk. Nutzen } \\
(€ / \mathrm{ha})\end{array}$ & $\begin{array}{c}\text { disk. NK-Diff. } \\
(€ / h a)\end{array}$ & $\begin{array}{c}\text { WGF } \\
\text { (Zins } 4 \% \text { ) }\end{array}$ & $\begin{array}{c}\varnothing \text { jährl. Vorteil } \\
(€ / \text { ha/a) }\end{array}$ \\
\hline 0 & 0 & 0 & 0,00 & 0,00 & 0,00 & 0,00 & 0,00 & & \\
\hline 1 & 32,60 & 58,20 & 25,60 & 0,9615 & 31,34 & 55,96 & 24,61 & & \\
\hline 2 & 32,60 & 58,20 & 25,60 & 0,9246 & 30,14 & 53,81 & 23,67 & & \\
\hline 3 & 32,60 & 58,20 & 25,60 & 0,889 & 28,98 & 51,74 & 22,76 & & \\
\hline 4 & 32,60 & 58,20 & 25,60 & 0,8548 & 27,87 & 49,75 & 21,88 & & \\
\hline 5 & 32,60 & 58,20 & 25,60 & 0,8219 & 26,79 & 47,83 & 21,04 & & \\
\hline 6 & 32,60 & 58,20 & 25,60 & 0,7903 & 25,76 & 46,00 & 20,23 & & \\
\hline 7 & 32,60 & 58,20 & 25,60 & 0,7599 & 24,77 & 44,23 & 19,45 & & \\
\hline 8 & 32,60 & 58,20 & 25,60 & 0,7307 & 23,82 & 42,53 & 18,71 & & \\
\hline \multirow[t]{2}{*}{9} & 32,60 & 58,20 & 25,60 & 0,7026 & 22,90 & 40,89 & 17,99 & & \\
\hline & & & & & 242,39 & 432,73 & 190,34 & 0,1345 & 25,60 \\
\hline
\end{tabular}


Tabelle A-72: Striegeleinsatz bei Weizen; ein Arbeitsgang

\begin{tabular}{|c|c|c|c|c|c|c|c|c|c|}
\hline Jahr & $\begin{array}{l}\text { Kosten } \\
\text { (€/ha) }\end{array}$ & $\begin{array}{r}\text { Nutzen } \\
\text { (€/ha) }\end{array}$ & $\begin{array}{l}\text { NK-Diff. } \\
\text { (€/ha) }\end{array}$ & $\begin{array}{c}\text { DF } \\
\text { (Zins } 4 \% \text { ) }\end{array}$ & $\begin{array}{c}\text { disk. Kosten } \\
\text { (€/ha) }\end{array}$ & $\begin{array}{c}\text { disk. Nutzen } \\
\text { (€/ha) }\end{array}$ & $\begin{array}{c}\text { disk. NK-Diff. } \\
(€ / h a)\end{array}$ & $\begin{array}{c}\text { WGF } \\
\text { (Zins } 4 \% \text { ) }\end{array}$ & $\begin{array}{c}\varnothing \text { jährl. Vor- } \\
\text { teil } \\
(€ / \text { ha/a) }\end{array}$ \\
\hline 0 & 0,00 & 0,00 & 0,00 & 0,00 & 0,00 & 0,00 & 0,00 & & \\
\hline 1 & 31,31 & 218,40 & 187,09 & 0,9615 & 30,10 & 209,99 & 179,89 & & \\
\hline 2 & 31,31 & 218,40 & 187,09 & 0,9246 & 28,95 & 201,93 & 172,98 & & \\
\hline 3 & 31,31 & 218,40 & 187,09 & 0,889 & 27,83 & 194,16 & 166,32 & & \\
\hline 4 & 31,31 & 218,40 & 187,09 & 0,8548 & 26,76 & 186,69 & 159,92 & & \\
\hline 5 & 31,31 & 218,40 & 187,09 & 0,8219 & 25,73 & 179,50 & 153,77 & & \\
\hline 6 & 31,31 & 218,40 & 187,09 & 0,7903 & 24,74 & 172,60 & 147,86 & & \\
\hline 7 & 31,31 & 218,40 & 187,09 & 0,7599 & 23,79 & 165,96 & 142,17 & & \\
\hline 8 & 31,31 & 218,40 & 187,09 & 0,7307 & 22,88 & 159,58 & 136,71 & & \\
\hline 9 & 31,31 & 218,40 & 187,09 & 0,7026 & 22,00 & 153,45 & 131,45 & & \\
\hline 10 & 31,31 & 218,40 & 187,09 & 0,6756 & 21,15 & 147,55 & 126,40 & & \\
\hline 11 & 31,31 & 218,40 & 187,09 & 0,6496 & 20,34 & 141,87 & 121,53 & & \\
\hline \multirow[t]{3}{*}{12} & 31,31 & 218,40 & 187,09 & 0,6246 & 19,56 & 136,41 & 116,86 & & \\
\hline & & & & & 293,85 & 2049,71 & 1755,86 & 0,1066 & 187,17 \\
\hline & & & & KNV & & 0,14 & & & \\
\hline
\end{tabular}


Tabelle A-73: Striegeleinsatz bei Weizen; vier Arbeitsgänge

\begin{tabular}{|c|c|c|c|c|c|c|c|c|c|}
\hline Jahr & $\begin{array}{l}\text { Kosten } \\
\text { (€/ha) }\end{array}$ & $\begin{array}{l}\text { Nutzen } \\
\text { (€/ha) }\end{array}$ & $\begin{array}{l}\text { NK-Diff. } \\
\text { (€/ha) }\end{array}$ & $\begin{array}{c}\text { DF } \\
\text { (Zins } 4 \% \text { ) }\end{array}$ & $\begin{array}{c}\text { disk. Kosten } \\
\text { (€/ha) }\end{array}$ & $\begin{array}{c}\text { disk. Nutzen } \\
\text { (€/ha) }\end{array}$ & $\begin{array}{c}\text { disk. NK-Diff. } \\
\qquad(€ / \text { ha) }\end{array}$ & $\begin{array}{c}\text { WGF } \\
\text { (Zins } 4 \% \text { ) }\end{array}$ & $\begin{array}{c}\varnothing \text { jährl. Vor- } \\
\text { teil } \\
(€ / h / / a)\end{array}$ \\
\hline 0 & 0,00 & 0,00 & 0,00 & 0,00 & 0,00 & 0,00 & 0,00 & & \\
\hline 1 & 74,24 & 218,40 & 144,16 & 0,9615 & 71,38 & 209,99 & 138,61 & & \\
\hline 2 & 74,24 & 218,40 & 144,16 & 0,9246 & 68,64 & 201,93 & 133,29 & & \\
\hline 3 & 74,24 & 218,40 & 144,16 & 0,889 & 66,00 & 194,16 & 128,16 & & \\
\hline 4 & 74,24 & 218,40 & 144,16 & 0,8548 & 63,46 & 186,69 & 123,23 & & \\
\hline 5 & 74,24 & 218,40 & 144,16 & 0,8219 & 61,02 & 179,50 & 118,49 & & \\
\hline 6 & 74,24 & 218,40 & 144,16 & 0,7903 & 58,67 & 172,60 & 113,93 & & \\
\hline 7 & 74,24 & 218,40 & 144,16 & 0,7599 & 56,41 & 165,96 & 109,55 & & \\
\hline 8 & 74,24 & 218,40 & 144,16 & 0,7307 & 54,25 & 159,58 & 105,34 & & \\
\hline 9 & 74,24 & 218,40 & 144,16 & 0,7026 & 52,16 & 153,45 & 101,29 & & \\
\hline 10 & 74,24 & 218,40 & 144,16 & 0,6756 & 50,16 & 147,55 & 97,39 & & \\
\hline 11 & 74,24 & 218,40 & 144,16 & 0,6496 & 48,23 & 141,87 & 93,65 & & \\
\hline \multirow[t]{2}{*}{12} & 74,24 & 218,40 & 144,16 & 0,6246 & 46,37 & 136,41 & 90,04 & & \\
\hline & & & & & 696,75 & 2049,71 & 1352,96 & 0,1066 & 144,23 \\
\hline
\end{tabular}


Tabelle A-74: Striegeleinsatz bei Weizen; sechs Arbeitsgänge

\begin{tabular}{|c|c|c|c|c|c|c|c|c|c|}
\hline Jahr & $\begin{array}{l}\text { Kosten } \\
\text { (€/ha) }\end{array}$ & $\begin{array}{l}\text { Nutzen } \\
\text { (€/ha) }\end{array}$ & $\begin{array}{l}\text { NK-Diff. } \\
\text { (€/ha) }\end{array}$ & $\begin{array}{c}\text { DF } \\
\text { (Zins } 4 \% \text { ) }\end{array}$ & $\begin{array}{c}\text { disk. Kosten } \\
\qquad(€ / \mathrm{ha})\end{array}$ & $\begin{array}{c}\text { disk. Nutzen } \\
\text { (€/ha) }\end{array}$ & $\begin{array}{c}\text { disk. NK-Diff. } \\
\text { (€/ha) }\end{array}$ & $\begin{array}{c}\text { WGF } \\
\text { (Zins } 4 \% \text { ) }\end{array}$ & $\begin{array}{c}\varnothing \text { jährl. Vor- } \\
\text { teil } \\
(€ / \text { ha/a) }\end{array}$ \\
\hline 0 & 0,00 & 0,00 & 0,00 & 0,00 & 0,00 & 0,00 & 0,00 & & \\
\hline 1 & 102,86 & 218,40 & 115,54 & 0,9615 & 98,90 & 209,99 & 111,09 & & \\
\hline 2 & 102,86 & 218,40 & 115,54 & 0,9246 & 95,10 & 201,93 & 106,83 & & \\
\hline 3 & 102,86 & 218,40 & 115,54 & 0,889 & 91,44 & 194,16 & 102,72 & & \\
\hline 4 & 102,86 & 218,40 & 115,54 & 0,8548 & 87,92 & 186,69 & 98,76 & & \\
\hline 5 & 102,86 & 218,40 & 115,54 & 0,8219 & 84,54 & 179,50 & 94,96 & & \\
\hline 6 & 102,86 & 218,40 & 115,54 & 0,7903 & 81,29 & 172,60 & 91,31 & & \\
\hline 7 & 102,86 & 218,40 & 115,54 & 0,7599 & 78,16 & 165,96 & 87,80 & & \\
\hline 8 & 102,86 & 218,40 & 115,54 & 0,7307 & 75,16 & 159,58 & 84,43 & & \\
\hline 9 & 102,86 & 218,40 & 115,54 & 0,7026 & 72,27 & 153,45 & 81,18 & & \\
\hline 10 & 102,86 & 218,40 & 115,54 & 0,6756 & 69,49 & 147,55 & 78,06 & & \\
\hline 11 & 102,86 & 218,40 & 115,54 & 0,6496 & 66,82 & 141,87 & 75,05 & & \\
\hline \multirow[t]{3}{*}{12} & 102,86 & 218,40 & 115,54 & 0,6246 & 64,25 & 136,41 & 72,17 & & \\
\hline & & & & & 965,35 & 2049,71 & 1084,35 & 0,1066 & 115,59 \\
\hline & & & & KNV & & 0,47 & & & \\
\hline
\end{tabular}


Tabelle A-75: Verzicht der Ausbringung von PSM, Weizen, Nutzen niedrig

\begin{tabular}{|c|c|c|c|c|c|c|c|c|c|}
\hline Jahr & $\begin{array}{c}\text { Kosten } \\
\text { (€/ha) }\end{array}$ & $\begin{array}{c}\text { Nutzen } \\
\text { (€/ha) }\end{array}$ & $\begin{array}{c}\text { NK-Diff. } \\
\text { (€/ha) }\end{array}$ & $\begin{array}{c}\text { DF } \\
\text { (Zins } 4 \% \text { ) }\end{array}$ & $\begin{array}{c}\text { disk. Kosten } \\
\text { (€/ha) }\end{array}$ & $\begin{array}{c}\text { disk. Nutzen } \\
(€ / \mathrm{ha})\end{array}$ & $\begin{array}{c}\text { disk. NK-Diff. } \\
(€ / \mathrm{ha})\end{array}$ & $\begin{array}{c}\text { WGF } \\
\text { (Zins } 4 \% \text { ) }\end{array}$ & $\begin{array}{c}\varnothing \text { jährl. Vorteil } \\
(€ / \text { ha/a) }\end{array}$ \\
\hline 0 & 0,00 & 0,00 & 0,00 & 0,00 & 0,00 & 0,00 & 0,00 & & \\
\hline 1 & 252,00 & 166,20 & $-85,80$ & 0,9615 & 242,30 & 159,80 & $-82,50$ & & \\
\hline 2 & 310,75 & 166,20 & $-144,55$ & 0,9246 & 287,32 & 153,67 & $-133,65$ & & \\
\hline 3 & 310,75 & 166,20 & $-144,55$ & 0,889 & 276,26 & 147,75 & $-128,50$ & & \\
\hline 4 & 310,75 & 166,20 & $-144,55$ & 0,8548 & 265,63 & 142,07 & $-123,56$ & & \\
\hline 5 & 310,75 & 166,20 & $-144,55$ & 0,8219 & 255,41 & 136,60 & $-118,81$ & & \\
\hline 6 & 310,75 & 166,20 & $-144,55$ & 0,7903 & 245,59 & 131,35 & $-114,24$ & & \\
\hline 7 & 310,75 & 166,20 & $-144,55$ & 0,7599 & 236,14 & 126,30 & $-109,84$ & & \\
\hline 8 & 310,75 & 166,20 & $-144,55$ & 0,7307 & 227,07 & 121,44 & $-105,62$ & & \\
\hline 9 & 310,75 & 166,20 & $-144,55$ & 0,7026 & 218,33 & 116,77 & $-101,56$ & & \\
\hline 10 & 310,75 & 166,20 & $-144,55$ & 0,6756 & 209,94 & 112,28 & $-97,66$ & & \\
\hline 11 & 310,75 & 166,20 & $-144,55$ & 0,6496 & 201,86 & 107,96 & $-93,90$ & & \\
\hline \multirow[t]{2}{*}{12} & 310,75 & 166,20 & $-144,55$ & 0,6246 & 194,09 & 103,81 & $-90,29$ & & \\
\hline & & & & & 2859,93 & 1559,80 & $-1300,13$ & 0,1066 & $-138,59$ \\
\hline
\end{tabular}


Tabelle A-76:Verzicht der Ausbringung von PSM, Weizen, Nutzen hoch

\begin{tabular}{|c|c|c|c|c|c|c|c|c|c|}
\hline Jahr & $\begin{array}{c}\text { Kosten } \\
\text { (€/ha) }\end{array}$ & $\begin{array}{l}\text { Nutzen } \\
(€ / \text { ha) }\end{array}$ & $\begin{array}{c}\text { NK-Diff. } \\
\text { (€/ha) }\end{array}$ & $\begin{array}{c}\text { DF } \\
\text { (Zins } 4 \%)\end{array}$ & $\begin{array}{c}\text { disk. Kosten } \\
\text { (€/ha) }\end{array}$ & $\begin{array}{c}\text { disk. Nutzen } \\
\text { (€/ha) }\end{array}$ & $\begin{array}{c}\text { disk. NK-Diff. } \\
(€ / h a)\end{array}$ & $\begin{array}{c}\text { WGF } \\
\text { (Zins } 4 \text { \%) }\end{array}$ & $\begin{array}{c}\varnothing \text { jährl. Vorteil } \\
\text { (€/ha/a) }\end{array}$ \\
\hline 0 & 0,00 & 0,00 & 0,00 & 0,00 & 0,00 & 0,00 & 0,00 & & \\
\hline 1 & 252,00 & 270,60 & 18,60 & 0,9615 & 242,30 & 260,18 & 17,88 & & \\
\hline 2 & 310,75 & 270,60 & $-40,15$ & 0,9246 & 287,32 & 250,20 & $-37,12$ & & \\
\hline 3 & 310,75 & 270,60 & $-40,15$ & 0,889 & 276,26 & 240,56 & $-35,69$ & & \\
\hline 4 & 310,75 & 270,60 & $-40,15$ & 0,8548 & 265,63 & 231,31 & $-34,32$ & & \\
\hline 5 & 310,75 & 270,60 & $-40,15$ & 0,8219 & 255,41 & 222,41 & $-33,00$ & & \\
\hline 6 & 310,75 & 270,60 & $-40,15$ & 0,7903 & 245,59 & 213,86 & $-31,73$ & & \\
\hline 7 & 310,75 & 270,60 & $-40,15$ & 0,7599 & 236,14 & 205,63 & $-30,51$ & & \\
\hline 8 & 310,75 & 270,60 & $-40,15$ & 0,7307 & 227,07 & 197,73 & $-29,34$ & & \\
\hline 9 & 310,75 & 270,60 & $-40,15$ & 0,7026 & 218,33 & 190,12 & $-28,21$ & & \\
\hline 10 & 310,75 & 270,60 & $-40,15$ & 0,6756 & 209,94 & 182,82 & $-27,13$ & & \\
\hline 11 & 310,75 & 270,60 & $-40,15$ & 0,6496 & 201,86 & 175,78 & $-26,08$ & & \\
\hline \multirow[t]{2}{*}{12} & 310,75 & 270,60 & $-40,15$ & 0,6246 & 194,09 & 169,02 & $-25,08$ & & \\
\hline & & & & & 2859,93 & 2539,61 & $-320,32$ & 0,1066 & $-34,15$ \\
\hline
\end{tabular}


Tabelle A-77: Kompostausbringung, Variante 1, Standort 4,6 t/ha

\begin{tabular}{|c|c|c|c|c|c|c|c|c|c|}
\hline Jahr & $\begin{array}{c}\text { Kosten } \\
\text { (€/ha) }\end{array}$ & $\begin{array}{l}\text { Nutzen } \\
\text { (€/ha) }\end{array}$ & $\begin{array}{c}\text { NK-Diff. } \\
\text { (€/ha) }\end{array}$ & $\begin{array}{c}\text { DF } \\
\text { (Zins } 4 \% \text { ) }\end{array}$ & $\begin{array}{c}\text { disk. Kosten } \\
(€ / \mathrm{ha})\end{array}$ & $\begin{array}{c}\text { disk. Nutzen } \\
\text { (€/ha) }\end{array}$ & $\begin{array}{c}\text { disk. NK-Diff. } \\
(€ / h a)\end{array}$ & $\begin{array}{c}\text { WGF } \\
\text { (Zins } 4 \% \text { ) }\end{array}$ & $\begin{array}{c}\varnothing \text { jährl. Vorteil } \\
(€ / h / a)\end{array}$ \\
\hline 0 & 0,00 & 0,00 & 0,00 & 0,00 & 0,00 & 0,00 & 0,00 & & \\
\hline 1 & 144,00 & 44,33 & $-99,67$ & 0,9615 & 138,46 & 42,62 & $-95,83$ & & \\
\hline 2 & 0,00 & 5,91 & 5,91 & 0,9246 & 0,00 & 5,46 & 5,46 & & \\
\hline 3 & 0,00 & 5,88 & 5,88 & 0,889 & 0,00 & 5,23 & 5,23 & & \\
\hline 4 & 0,00 & 5,86 & 5,86 & 0,8548 & 0,00 & 5,01 & 5,01 & & \\
\hline 5 & 0,00 & 5,85 & 5,85 & 0,8219 & 0,00 & 4,81 & 4,81 & & \\
\hline 6 & 0,00 & 5,84 & 5,84 & 0,7903 & 0,00 & 4,62 & 4,62 & & \\
\hline 7 & 0,00 & 5,83 & 5,83 & 0,7599 & 0,00 & 4,43 & 4,43 & & \\
\hline 8 & 0,00 & 4,73 & 4,73 & 0,7307 & 0,00 & 3,46 & 3,46 & & \\
\hline 9 & 0,00 & 4,58 & 4,58 & 0,7026 & 0,00 & 3,22 & 3,22 & & \\
\hline 10 & 0,00 & 4,45 & 4,45 & 0,6756 & 0,00 & 3,01 & 3,01 & & \\
\hline 11 & 0,00 & 4,31 & 4,31 & 0,6496 & 0,00 & 2,80 & 2,80 & & \\
\hline 12 & 0,00 & 4,18 & 4,18 & 0,6246 & 0,00 & 2,61 & 2,61 & & \\
\hline 13 & 0,00 & 4,06 & 4,06 & 0,6006 & 0,00 & 2,44 & 2,44 & & \\
\hline 14 & 0,00 & 3,94 & 3,94 & 0,5775 & 0,00 & 2,28 & 2,28 & & \\
\hline 15 & 0,00 & 3,82 & 3,82 & 0,5553 & 0,00 & 2,12 & 2,12 & & \\
\hline 16 & 0,00 & 3,70 & 3,70 & 0,5339 & 0,00 & 1,98 & 1,98 & & \\
\hline 17 & 0,00 & 3,59 & 3,59 & 0,5134 & 0,00 & 1,84 & 1,84 & & \\
\hline 18 & 0,00 & 3,49 & 3,49 & 0,4936 & 0,00 & 1,72 & 1,72 & & \\
\hline 19 & 0,00 & 3,38 & 3,38 & 0,4746 & 0,00 & 1,60 & 1,60 & & \\
\hline 20 & 0,00 & 3,28 & 3,28 & 0,4564 & 0,00 & 1,50 & 1,50 & & \\
\hline 21 & 0,00 & 3,18 & 3,18 & 0,4388 & 0,00 & 1,40 & 1,40 & & \\
\hline 22 & 0,00 & 3,09 & 3,09 & 0,422 & 0,00 & 1,30 & 1,30 & & \\
\hline 23 & 0,00 & 2,99 & 2,99 & 0,4057 & 0,00 & 1,21 & 1,21 & & \\
\hline 24 & 0,00 & 2,90 & 2,90 & 0,3901 & 0,00 & 1,13 & 1,13 & & \\
\hline \multirow[t]{3}{*}{25} & 0,00 & 2,82 & 2,82 & 0,3751 & 0,00 & 1,06 & 1,06 & & \\
\hline & & & & & 138,46 & 108,85 & $-29,61$ & 0,0640 & $-1,89$ \\
\hline & & & & KNV & & 1,27 & & & \\
\hline
\end{tabular}


Tabelle A-78: Kompostausbringung, Variante 2, Standort 4,6 t/ha

\begin{tabular}{|c|c|c|c|c|c|c|c|c|c|}
\hline Jahr & $\begin{array}{c}\text { Kosten } \\
\text { (€/ha) }\end{array}$ & $\begin{array}{c}\text { Nutzen } \\
\text { (€/ha) }\end{array}$ & $\begin{array}{c}\text { NK-Diff. } \\
\text { (€/ha) }\end{array}$ & $\begin{array}{c}\text { DF } \\
\text { (Zins } 4 \%)\end{array}$ & $\begin{array}{c}\text { disk. Kosten } \\
(€ / \mathrm{ha})\end{array}$ & $\begin{array}{c}\text { disk. Nutzen } \\
(€ / h a)\end{array}$ & $\begin{array}{c}\text { disk. NK-Diff. } \\
(€ / h a)\end{array}$ & $\begin{array}{c}\text { WGF } \\
\text { (Zins } 4 \% \text { ) }\end{array}$ & $\begin{array}{c}\varnothing \text { jährl. Vorteil } \\
\text { (€/ha/a) }\end{array}$ \\
\hline 0 & 0,00 & 0,00 & 0,00 & 0,00 & 0,00 & 0,00 & 0,00 & & \\
\hline 1 & 186,00 & 44,33 & $-141,67$ & 0,9615 & 178,84 & 42,62 & $-136,22$ & & \\
\hline 2 & 0,00 & 5,91 & 5,91 & 0,9246 & 0,00 & 5,46 & 5,46 & & \\
\hline 3 & 0,00 & 5,88 & 5,88 & 0,889 & 0,00 & 5,23 & 5,23 & & \\
\hline 4 & 0,00 & 5,86 & 5,86 & 0,8548 & 0,00 & 5,01 & 5,01 & & \\
\hline 5 & 0,00 & 5,85 & 5,85 & 0,8219 & 0,00 & 4,81 & 4,81 & & \\
\hline 6 & 0,00 & 5,84 & 5,84 & 0,7903 & 0,00 & 4,62 & 4,62 & & \\
\hline 7 & 0,00 & 5,83 & 5,83 & 0,7599 & 0,00 & 4,43 & 4,43 & & \\
\hline 8 & 0,00 & 4,73 & 4,73 & 0,7307 & 0,00 & 3,46 & 3,46 & & \\
\hline 9 & 0,00 & 4,58 & 4,58 & 0,7026 & 0,00 & 3,22 & 3,22 & & \\
\hline 10 & 0,00 & 4,45 & 4,45 & 0,6756 & 0,00 & 3,01 & 3,01 & & \\
\hline 11 & 0,00 & 4,31 & 4,31 & 0,6496 & 0,00 & 2,80 & 2,80 & & \\
\hline 12 & 0,00 & 4,18 & 4,18 & 0,6246 & 0,00 & 2,61 & 2,61 & & \\
\hline 13 & 0,00 & 4,06 & 4,06 & 0,6006 & 0,00 & 2,44 & 2,44 & & \\
\hline 14 & 0,00 & 3,94 & 3,94 & 0,5775 & 0,00 & 2,28 & 2,28 & & \\
\hline 15 & 0,00 & 3,82 & 3,82 & 0,5553 & 0,00 & 2,12 & 2,12 & & \\
\hline 16 & 0,00 & 3,70 & 3,70 & 0,5339 & 0,00 & 1,98 & 1,98 & & \\
\hline 17 & 0,00 & 3,59 & 3,59 & 0,5134 & 0,00 & 1,84 & 1,84 & & \\
\hline 18 & 0,00 & 3,49 & 3,49 & 0,4936 & 0,00 & 1,72 & 1,72 & & \\
\hline 19 & 0,00 & 3,38 & 3,38 & 0,4746 & 0,00 & 1,60 & 1,60 & & \\
\hline 20 & 0,00 & 3,28 & 3,28 & 0,4564 & 0,00 & 1,50 & 1,50 & & \\
\hline 21 & 0,00 & 3,18 & 3,18 & 0,4388 & 0,00 & 1,40 & 1,40 & & \\
\hline 22 & 0,00 & 3,09 & 3,09 & 0,422 & 0,00 & 1,30 & 1,30 & & \\
\hline 23 & 0,00 & 2,99 & 2,99 & 0,4057 & 0,00 & 1,21 & 1,21 & & \\
\hline 24 & 0,00 & 2,90 & 2,90 & 0,3901 & 0,00 & 1,13 & 1,13 & & \\
\hline \multirow[t]{3}{*}{25} & 0,00 & 2,82 & 2,82 & 0,3751 & 0,00 & 1,06 & 1,06 & & \\
\hline & & & & & 178,84 & 108,85 & $-69,99$ & 0,0640 & $-4,48$ \\
\hline & & & & KNV & & 1,64 & & & \\
\hline
\end{tabular}


